\title{
THE RELATIONSHIP BETWEEN CASTING DISTORTION, MOLD FILLING AND INTERFACIAL HEAT TRANSFER IN SAND MOLDS
}

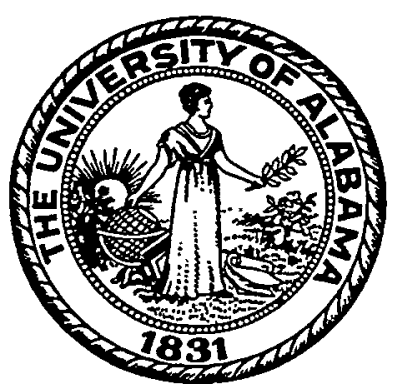

K.A. Woodbury, J.K. Parker and T.S. Piwonka Metal Casting Technology Center

The University of Alabama

Tuscaloosa, Alabama

Y. Owusu

Florida Agricultural and Mechanical University/ Florida State University College of Engineering Tallahassee, Florida

September 30, 1999 


\section{The Relationship between Casting Distortion, Mold Filling and Interfacial Heat Transfer in Sand Molds}

DE-FC07-95ID13363

September 30, 1999

K.A. Woodbury, J.K. Parker and T.S. Piwonka Metal Casting Technology Center The University of Alabama

Tuscaloosa, AL

Y. Owusu

Florida Agricultural and Mechanical University/

Florida State University College of Engineering Tallahassee, FL 


\section{DISCLAIMER}

This report was prepared as an account of work sponsored by an agency of the United States Government. Neither the United States Government nor any agency thereof, nor any of their employees, make any warranty, express or implied, or assumes any legal liability or responsibility for the accuracy, completeness, or usefulness of any information, apparatus, product, or process disclosed, or represents that its use would not infringe privately owned rights. Reference herein to any specific commercial product, process, or service by trade name, trademark, manufacturer, or otherwise does not necessarily constitute or imply its endorsement, recommendation, or favoring by the United States Government or any agency thereof. The views and opinions of authors expressed herein do not necessarily state or reflect those of the United States Government or any agency thereof. 


\section{DISCLAIMER}

Portions of this document may be illegible in electronic image products. Images are produced from the best available original document. 


\section{EXECUTIVE SUMMARY}

\subsection{Introduction}

Dimensional accuracy in metal castings has taken on increased importance in the last decade as methods of controlling cast structure have improved. There is an emphasis on molding accuracy, and, today, on understanding the way alloy solidification affects casting accuracy.

At the same time, the use of solidification simulation programs is expanding rapidly in the metalcasting industry. Well over half of the castings produced in the United States are produced in foundries that routinely use commercial solidification simulation programs. As the use of these programs has proliferated, there has been an increased emphasis in making them as accurate as possible. Ten years ago foundrymen were satisfied to have approximate solutions to casting solidification problems; today accuracy is required. This means using accurate values of thermal parameters involved in metal casting.

Dimensional accuracy and accurate values of thermal parameters are linked in the establishment of interfacial heat transfer coefficients. There is indirect evidence of this ${ }^{1}$ in evidence from actual castings, and foundrymen the world over know that dimensions vary when the casting orientation or gating is changed. The reason that varying the gating or the orientation of the casting in the mold affects dimensions was explained by Campbell ${ }^{2}$, who pointed out that rapid heat transfer between the metal and mold (as would be the case in the first part of the casting to fill) would cause the metal there to solidify sooner than metal that filled later. The metal that solidifies sooner is stronger sooner, and thus will pull on the later-solidifying metal, causing it to distort.

In the same way, parts of the mold that have a higher interfacial heat transfer coefficient than other parts of the mold will cause the metal there to solidify sooner, and will similarly affect the casting dimensions. Because of the growing understanding of the importance of interfacial heat transfer, The University of Alabama, with the help of Florida A\&M University and a team of foundries, investigated the role of interfacial heat transfer in affecting dimensions.

\subsection{Theoretical Underpinnings}

Evidence that interfacial heat transfer plays an important role in casting distortion has been, up to now, indirect. An ambitious program undertaken by the Green Sand Committee of the American Foundrymen's Society in the early 1970's to determine the causes of casting distortion found some sixty-five different factors that could conceivably have some effect." They proposed "that this summary of effects will be analyzed to determine which are controllable and which are not, and which are important, and which are not." Unfortunately, a year later, after they had taken dimensional data on castings from the same pattern poured in fifteen different foundries, they concluded that there was 
no correlation between casting and molding factors and the dimensional accuracy of the casting produced. ${ }^{3}$

The implementation of modern high pressure molding and advanced statistical process control methods has substantially decreased dimensional variation that arises from mold and core making processes. ${ }^{4}$ The application of these production techniques clearly shows that major improvements in dimensional accuracy can be made by adhering to good practice and properly controlling mold making materials and operations. Nevertheless, casting distortion continues to occur. Clearly, factors other than the mold affect casting dimensions. In recent years it has been suggested that gating and pouring practice may have an effect, as the way a mold fills affects the way the casting solidifies: the metal that comes to rest in the mold first begins to solidify first. This means that it is stronger before the rest of the casting, so it shrinks earlier, and therefore pulls the rest of the casting in its direction.

A further implication of this is that areas of more effective heat transfer will solidify before areas where heat transfer is less efficient. Since heat transfer must occur across the interface between the metal and the mold, the interfacial heat transfer coefficient becomes important. With an improved understanding of interfacial heat transfer, it should be possible to design molds, using the appropriate molding materials and gating systems, that minimize casting distortion caused by differential solidification.

A fairly impressive number of studies of interfacial heat transfer have been carried out. In studies using a permanent mold, ${ }^{5}$ a gap was found to form between the metal and the mold during solidification. This occurs when the metal begins to solidify; as it solidifies it contracts. The mold, however, is still hot from the heat transferred from the solidifying casting, and it does not contract. The formation of the gap is accompanied by a ten-fold decrease in the value of the interfacial heat transfer coefficient. The gap is found to form in dry sand as well in aluminum ${ }^{6}$ and in cast iron. ${ }^{7}$ Gravity also has been found to influence the formation of a gap ${ }^{8}$ as the force of gravity is expected to hold the casting down against the mold surface, leading to the preferential formation of a gap on the upper surfaces of the casting.

The formation of a gap has been found to affect casting distortion, especially in continuously cast steel slabs. ${ }^{9,10}$ When the gap forms, cooling of the metal slows appreciably. This means that the resistance to deformation of the metal where the gap forms is also substantially reduced, as the metal in that region remains at a higher temperature (where resistance to deformation is less than at room temperature), and it can be pulled or distorted by the cooler, stronger metal which remains against the mold surface.

This analysis, developed for the simple shapes of continuously cast slabs, also applies to the complex geometries found in commercial shaped castings, such as those poured in green sand, resin-bonded sand, or lost foam. Air gaps are expected to form between the mold and the casting as the casting cools, and shrinks away from the mold. However, because of gravity, there will be places in the casting where the gap does not 
form. The metal in these areas will solidify faster, and, as in the case for slabs, actually deform the hotter, weaker metal adjacent to the air gaps. This has been explained in detail by Campbell. ${ }^{2}$

One implication from this argument is that in unbonded sand molds, gaps should not form. This is because the sand, as it expands, should expand in all directions, including against the metal. Thus castings poured into unbonded molds should solidify more evenly, and should have less distortion. In fact, this has been reported to be the case. $^{11}$

These results provide strong evidence that air gap formation and heat transfer at the mold/metal interface is a significant factor in controlling casting dimensional reproducibility. A number of recent models of casting distortion have neglected gap formation ${ }^{12,13,14}$ because of the difficulty in of including it in the calculations. Indeed, most solidification simulation programs either ignore interfacial heat transfer, or treat it as constant throughout solidification, thus ignoring the possible formation of a gap.

It is clear that more information is needed on the physics of interfacial heat transfer and gap formation. The objective of this program was to determine more accurate values for interfacial heat transfer coefficients, study gap formation in detail, and correlate casting distortion with the physics of interfacial heat transfer.

\subsection{Experimental Program}

The experimental program we carried out consisted of four parts:

1) Determination of interfacial heat transfer coefficients in aluminum alloy/sand mold systems.

2) Measurement of gap formation during solidification of aluminum alloy/resin bonded sand mold, using eddy currents as a non-contact method.

3) Determination of the effect of molding media, gating, casting thickness and casting orientation on the dimensional accuracy of sand castings.

4) Measurement of residual stresses on an un-heat treated casting before and after gate removal.

In the first part of the program, plate castings 12 " 12 " were poured in the Ray. L. Farabee Metalcasting Laboratory at The University of Alabama. These castings varied in thickness, from $1 / 4$ " to 1 ", and they were poured in the horizontal, 30,45 , and $60^{\circ}$ to the horizontal, and the vertical position. Thermal measurements were made and eddy current detectors were used to measure the formation of a gap between the metal and the mold material. These experiments were described fully in the previous annual reports. ${ }^{15}$

The alloy was A356 alloy. It was not degassed prior to pouring, nor were grain refiners or modifiers used. This was deliberate, in that we wanted to study interfacial heat transfer and gap formation without the confusion that shrinkage could cause. 
In the remaining part of the program a casting was designed that had a number of features that would easily demonstrate the effects of varying gating, orientation in the mold, casting thickness, and mold material. These molding methods used were resinbonded sand, and the lost foam process using both silica sand and low-expansion sand at commercial foundries. Each casting was then to be dimensionally inspected using a coordinate measuring machine at another commercial foundry. Unfortunately, no foundry was able to pour green sand castings, and we were forced to abandon this part of the project.

The resin-bonded sand castings were poured by General Motors Powertrain Operations in Saginaw, MI. Low expansion sand lost foam castings were poured by Mercury Marine Corporation in Fond du Lac, WI, and silica sand lost foam castings were poured by Willard Industries in Cincinnati, $\mathrm{OH}$. The castings were then sent to CMI Technical Center in Warren, MI for layout on their Coordinate Measurement Machine. The results from those measurements were sent to Florida A\&M University for analysis.

In the fourth part of the program, we submitted one of the castings to the High Temperature Materials Laboratory at Oak Ridge National Laboratory for Neutron Radiation measurements of residual stresses in the casting before and after the gating was removed.

\subsection{Results and Conclusions}

Details of the experimental program and its findings are presented in four detailed reports that follow this executive summary. These reports describe in detail the experimental effort, the data developed during the investigations, and the reasons for our conclusions from this work. Our results are summarized below:

Part I - Interfacial Heat Transfer

1. Thermocouples implanted in low conductivity media (such as sand) form preferential heat flow paths and can significantly alter the temperature field. Our calculations indicate that errors in readings could be on the order of $50 \mathrm{C}$. The severity of this condition increases as the section size of the casting decreases. Thermocouples should run parallel to the isotherms (e.g, parallel to the mold surface) in mold to minimize this error.

2. An inverse heat conduction methodology is capable of determining the interfacial heat transfer coefficients in resin-bonded sand castings. The method assumes a purely conductive mold medium, which is an approximation. The evolution and migration of gases decomposed from the resin appears to play a significant role in the process.

3. Our results indicate that, for horizontally oriented castings, the heat transfer coefficients during resin-bonded sand casting of aluminum (A356) are on the order of $600 \mathrm{~W} / \mathrm{m}^{2}-\mathrm{K}$. For section thicknesses of $12.7 \mathrm{~mm}$ or greater, the interfacial heat transfer coefficient is approximately constant during the solidification process. For 
thinner sections, the heat transfer coefficient varies through most of the solidification process, and can double in magnitude.

4. For vertically-oriented plate castings, the heat transfer coefficients are highly variable, but appear to be on the same order of magnitude $\left(600 \mathrm{~W} / \mathrm{m}^{2}-\mathrm{K}\right)$. This suggests that simple surface orientation itself does not appear to dramatically affect heat transfer coefficients. For the thickest section, it does appear that there is a trend for increasing heat transfer coefficients during the solidification. Also, differences were found on coefficients on the upper and lower surfaces of horizontal castings, which we attribute to the role of gases from resin decomposition.

5. We have developed an inverse heat conduction algorithm to compute the heat fluxes from castings in green sand. The heat transfer coefficients obtained from the algorithm are not reproducible, and we are continuing work on a heat transfer coefficient-based method.

Part II:

Our results showed that no gap formed during the pouring of most of the castings in this investigation. Neither the gap formation measurements, nor the thermal data were what was expected from the formation of a gap. We looked at the microstructures of the castings poured horizontally, and found extensive columnar grain growing from the cope surface, but only short columnar grains, which quickly changed to equiaxed grains, growing from the drag surface. This indicates very strongly that thermal gradients in the drag were lower than those in the cope. This, in turn, indicates that the heat transfer from the cope surface of the casting was greater than from the drag surface.

We had expected just the opposite phenomenon. We assumed that gravity would force the solidifying metal down onto the drag surface of the mold, and that a clear gap would form on the cope surface. That did not happen. We suspect that by not degassing the metal we counteracted the effect of metal shrinkage, and that no shrinkage took place on the cope surface, as extensive microporosity was found in the castings. As for the lack of effective heat transfer on the drag surface, we attribute this to the collection of gas underneath the solidifying casting. This has been encountered previously. We were also curious about what would happen to the gap on the surfaces of castings poured vertically. We found that gap formation, if any, was not predictable, nor could we find a pattern to gaps, if any, formed on castings poured at a $30^{\circ}, 45^{\circ}$, or $60^{\circ}$ angles.

Our conclusion is that there was no evidence that gaps due to alloy shrinkage actually form in aluminum alloys poured in resin-bonded sand molds. Note that our experimental method deviates substantially from that of past investigators. However, we have been careful to examine as many variables that could affect the accuracy of the instrumentation of our method, and we find it hard to believe that the method is inherently incorrect. Our results call into question the traditional view of gap formation during solidification, and strongly call for further research. 
Part III:

The statistical analysis confirmed that the type of gating systems, casting orientation, molding process used, and casting thickness influence the dimensions of the casting. The factorial analysis of variance determined the effect of each factor on each feature (length, height, thickness, parallelism, casting angle, and flatness). The test of means was an effective method for determining the factor level effects.

For all of the castings, the thickness levels, orientation and gating significantly contributed to the distortion of the features. For all of the resin-bonded castings, $3 / 4$ and $1 / 2$ inch thickness played a significant role in casting distortion. Gating and orientation also played crucial roles in the distortion. Orientation down, $3 / 4$ inch thickness, and top gating affected the lost foam silica sand castings. The thickness level $(3 / 4,1 / 2,1 / 4$ inch) most significantly affected the lost foam low expansion castings compared with the orientation and gating.

Resin bonded sand, orientation down, and top-gating mostly affected all of the $1 / 4$ inch castings. The molding process (mold type) is the most significant factor affecting the casting distortion. The deviation from nominal analysis indicated that the lost foam silica sand process had comparatively less deviation from the designed nominal values, while the lost foam low expansion sand process varied the least from the calculated nominal values. Resin bonded sand castings deviated the most from the designed and calculated nominal values. The most significant contributor to the distortion of all of the castings was resin-bonded sand. Also, the ANOVA analysis indicated that side gating did not significantly contribute to the distortion of the castings.

\section{Part IV:}

1.0 The slightly compressive strains in tangential and axial directions in the gate were released after the runner was removed from the casting. The fact that a compressive strain in the gate closest to the fill of the runner was measured suggests that this gate solidified second. This hypothesis is supported by the increased opening on cutting at the location of the other gates.

2.0 The tangential component of strain in the crown was investigated at three different depths to examine the effect of the opening the gate on removing the runner. In the as-cast specimen the strains went from slightly compressive at the inside to slightly tensile at the outside. The data after the runner was removed showed a different trend, with the more relaxed strains closer to the inside of the casting. This is consistent with the measured opening of the casting when the runner was removed. Thus the strain distribution is modified (it was actually relieved or shifted to more compressive in the direction we examined) by the runner removal, particularly in the crown. 
3.0 The overall low measured value of the strains in the casting are likely a consequence of a strain-relief mechanism at work during solidification. One possibility is that there is a thermal stress relief occurring during the slow cooling in the mold.

\section{References}

${ }^{1}$ G. DiSylvestro, "Dimensional Stability of Castings Made in High Density Green Sand Molding: Phase I - Factors Which Affect Dimensional Stability," AFS Transactions, vol. 84 (1976), p. 745.

2 J. Campbell, Castings, Butterworth-Heinemann Ltd., Oxford, UK, 1992, p. 128.

${ }^{3}$ A.B. Draper, "Meeting the Dimensional Standards of Tomorrow," AFS Transactions, vol. 85 (1977), p. 577.

${ }^{4}$ T.S.V. Rao and H.M. Roshan, "Studies on Dimensional Accuracy and Consistency in High Pressure Molded Castings," AFS Transactions, vol. 96 (1988), p. 37.

${ }^{5}$ T.X. Hou and R.D. Pehlke, "Determination of Mold/Metal Heat Transfer and Simulation of Solidification of an Aluminum-13\% Silicon Casting," AFS Transactions, vol. 96 (1988), p. 129.

${ }^{6}$ Y. Nishida, W. Droste and S. Engler, "The Air Gap Formation Process at the Casting Mold Interface and the Heat Transfer Mechanism Through the Gap," Met. Trans., vol. 17B (1986), p. 833.

${ }^{7}$ X.C. Zeng and R.D. Pehlke, "Analysis of Heat Transfer at Metal-Sand Boundaries and Computer Simulation of a Gray Iron Casting," AFS Transactions, vol. 93 (1985), p. 275. ${ }^{8}$ H. Huang, J.L. Hill and J.T. Berry, "A Free Thermal Contraction Method for Modeling the Heat Transfer Coefficient at the Casting/Mold Interface," International Journal of Cast Metals Research, vol. 5, no. 4 (1993), p. 212.

${ }^{9}$ R.H. Tien and O. Richmond, "Theory of Maximum Tensile Stresses in the solidifying Shell of a Constrained Rectangular Casting," J. Appl. Mechanics, vol. 49 (1982), p. 481. ${ }^{10}$ O. Richmond, L.G. Hector and J.M. Friday, "Growth Instability during Non-Uniform Directional Solidification of Pure Metals," J. Appl, Mechanics, vol. 57 (1990), p. 529.

${ }^{11}$ R.J. Donahue, "Lost Foam Defects in Aluminum-Silicon Alloys: Eliminate Them with Technological Innovations," presented at the Expendable Pattern Casting Technology Conference, September 21-23, 1993, Birmingham, AL.

${ }^{12}$ J. Guan and P.R. Sahm, "Berechnung der Restspannungen Abgekulter Gussstucke mit Hilfe der Finite-Elemente-Methode," Geissereiforchung, vol. 43, no. 1 (1991), p. 10.

${ }^{13} \mathrm{~J}$. Guan and P.R. Sahm, "Numerische Untersuchung der thermishen Spannungen in realen 3D-Gussbauteilen," Giesserei, vol. 79, no. 8 (1992), p. 318.

${ }^{14}$ J. Guan, G.W. Dieckues and P.R. Sahm, "Analysis of residual stresses and cracking of $\gamma$-TiAl castings," Intermetallics, Elsevier Science Limited, 1994, p. 89.

${ }^{15}$ K.A. Woodbury, J.K. Parker, T.S. Piwonka and Y.A. Owusu, "Relationship Between Casting Distortion, Mold Filling and Interfacial Heat Transfer," Report DOE/ID/13363/1, Report DOE/ID/2. 


\title{
The Relationship between Casting Distortion, Mold Filling and Interfacial Heat Transfer in Sand Molds
}

\author{
DE-FC07-95ID13363 \\ FINAL REPORT - PART 1 \\ September 30, 1999 \\ K.A. Woodbury, Q. Ke, Y. Chen, and T.S. Piwonka \\ Metal Casting Technology Center \\ The University of Alabama \\ Tuscaloosa, AL
}

INTERFACIAL HEAT TRANSFER MEASUREMENTS 


\begin{abstract}
Accurate knowledge of the interfacial heat transfer coefficients between a casting and its mold can significantly improve computer simulations for casting process design, and minimize design cycle time. As casting section sizes decrease, the interfacial heat transfer coefficient plays a larger role in determining the overall solidification rate. This report details the results from a three-year investigation to determine the interfacial heat transfer coefficients during sand casting of aluminum (A356). To obtain these results, two series of instrumented plate castings were poured, and temperature data collected from these castings were used to solve the inverse heat conduction problem. Significant findings from this investigation include values for the magnitude and variation of the heat transfer coefficient during solidification for resin-bonded sand molds for three different section sizes. Other notable results from the project include the development of a special algorithm to determine the interfacial heat transfer coefficient in green sand and the identification of significant temperature measurement error due to wire lead heat loss when using thermocouple thermometry.
\end{abstract}

\title{
1.0 INTRODUCTION
}

Increased competitiveness in casting requires reduced scrap and a lower design cycle time. For this reason, many foundries are using computer simulations to prototype new castings before the first metal is poured. This strategy allows exploration of different gating and risering schemes and facilitates optimization of the casting. This computer-based design cycle replaces the old practice of cut-and-try in the foundry, which is expensive in terms of both labor and materials, and is generally more time consuming.

Computer simulations for solidification have attained a high level of maturity, and today can simulate filling time as well as compute the time to solidify throughout the casting. This allows foundrymen to predict the probable final metallurgical structure of the part. The physics of the process of solidification within the casting itself has been studied extensively and is well understood. The physics of solidification have been incorporated accurately into several commercially available computer codes (e.g., ProCast, Magma).

A significant player in the overall simulation is the environment surrounding the cast part, that is, the mold. The sand mold is largely treated as a purely conductive medium, and, provided the thermal properties of the sand are available, the modeling of heat flow in the mold itself is not considered to be difficult.

Of some importance is the interface between the metal and the mold. Figure 1 shows the interface between the metal and the mold, represented as a distinct gap. The physical dimensions of this gap can vary, but even in the case of no visually apparent gap the phenomenon suggested by the temperature distribution in the figure is present. The temperature drop from the surface of the metal to the surface of the mold is produced by an interfacial heat transfer coefficient 
between the metal and the mold. This heat transfer coefficient is determines the heat flow from the casting at any instant in time as

$$
q=h\left(T_{\text {metal }}-T_{\text {mold }}\right)
$$

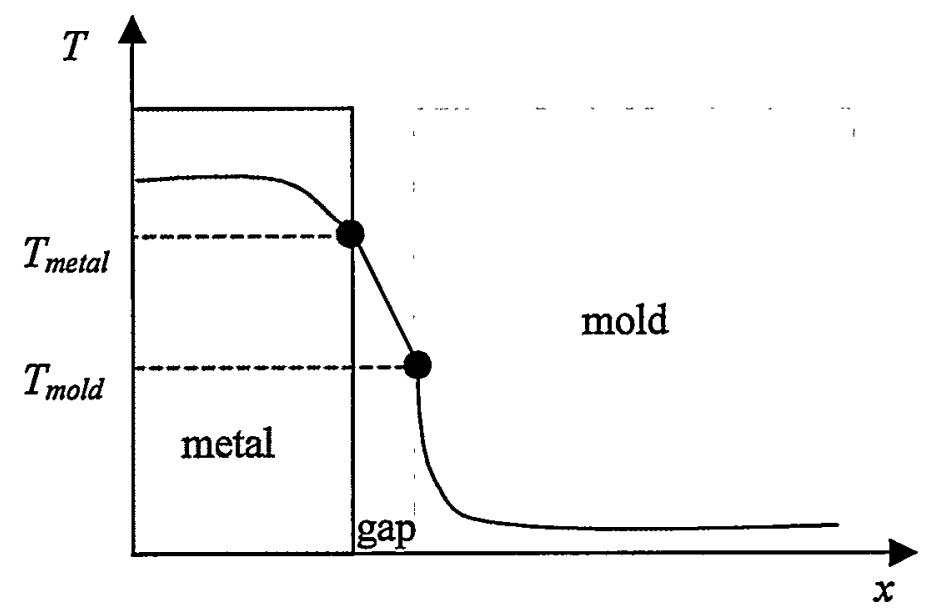

Figure 1. Schematic of interface showing temperature distribution

For thicker castings, the magnitude of the heat transfer coefficient is of lesser importance, as the overall solidification time is long. But, as section size decreases, and solidification times decrease, the resistance to heat flow imposed by this interfacial heat transfer coefficient becomes more significant in the overall process.

This report details a three year investigation to determine the interfacial heat transfer coefficients typical of aluminum castings in sand molds. The general approach will be outlined, including a description of the castings and instrumentation used, along with an explanation of the data analysis method used in the study. The results are presented next, in two parts, reviewing some preliminary results and then results from resin-bonded sand castings. Experiences from green sand work will also be reported. 


\subsection{GENERAL APPROACH}

In order to measure interfacial heat transfer coefficients during casting, several instrumented castings were made. We wanted to obtain results on simple, but industrial sized, castings. Furthermore, we wanted to use the simplest and least expensive instrumentation possible. By using inverse methods (e.g., Beck, 1984) our results could be obtained using simple thermocouple thermometry. Each of these facets of the approach is explained below.

\subsection{Castings}

A simple geometry was selected which would be amenable to analysis using onedimensional parameterization. A broad flat surface was desired, so that heat flow perpendicular to the surface direction would be nearly one-dimensional A $300 \mathrm{~mm}$ (12") square surface was selected, and the required thermal instrumentation was subsequently installed near the center of this face. Three different casting thicknesses were chosen, ranging from rather thick, $25.4 \mathrm{~mm}$ (1") through nominal, $12.7 \mathrm{~mm}$ (1/2"), and a section that was approaching thin, $6.35 \mathrm{~mm}\left(1 / 4^{\prime \prime}\right)$. Figure 2 shows one of the finished plate castings in the intermediate thickness. The "footprint" of the thermal instrument package can be seen in the middle of the plate.

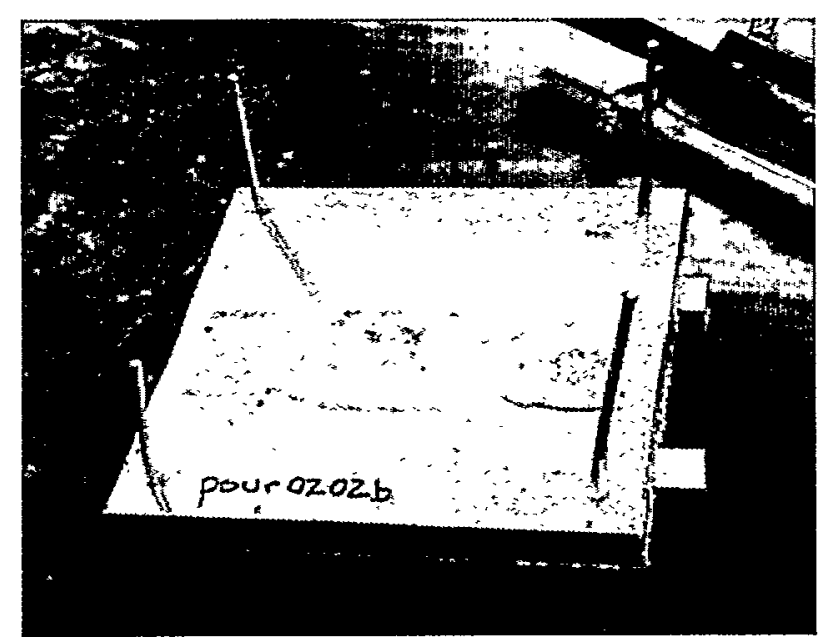

Figure 2. Finished plate casting in the intermediate $(12.7 \mathrm{~mm})$ thickness

\subsection{Instrumentation}

In order to determine the interfacial heat transfer coefficient using inverse methods, we need to measure the temperature of the surface of the solidifying metal and the temperature within the sand mold. We developed a first generation instrument pack that was used in the first sequence of castings. Based on some results and observations from this sequence of tests, a second generation instrument pack was developed.

\subsubsection{First Generation}

The thermocouples were embedded into the sand material by first implanting them in a small instrumentation pack, $75 \mathrm{~mm}$ X $75 \mathrm{~mm}$ X $100 \mathrm{~mm}$ (3" X 3" x 4"). Initially three 
thermocouples were used: one at a nominal $5 \mathrm{~mm}$ depth from the surface and a second at a nominal $15 \mathrm{~mm}$ depth. The third thermocouple was allowed to protrude from the instrument pack surface, so that when the instrument pack is integrated into the mold this third thermocouple extended into the plate cavity. When molten metal was poured into the mold, a simple "twopoint" thermocouple was formed by the electrically conductive molten aluminum and the two thermocouple wires. This measured the solidifying plate surface temperature during casting and solidification. Figure 3 shows the preparation of an early instrument package. Later instrument packs used thermocouples with glass overbraid insulation unlike the rigid refractory sleeves illustrated in the figure.

Note that the thermocouples are installed perpendicular to the surface of the casting. This practice was found to be inferior to a parallel installation, as will be discussed below.
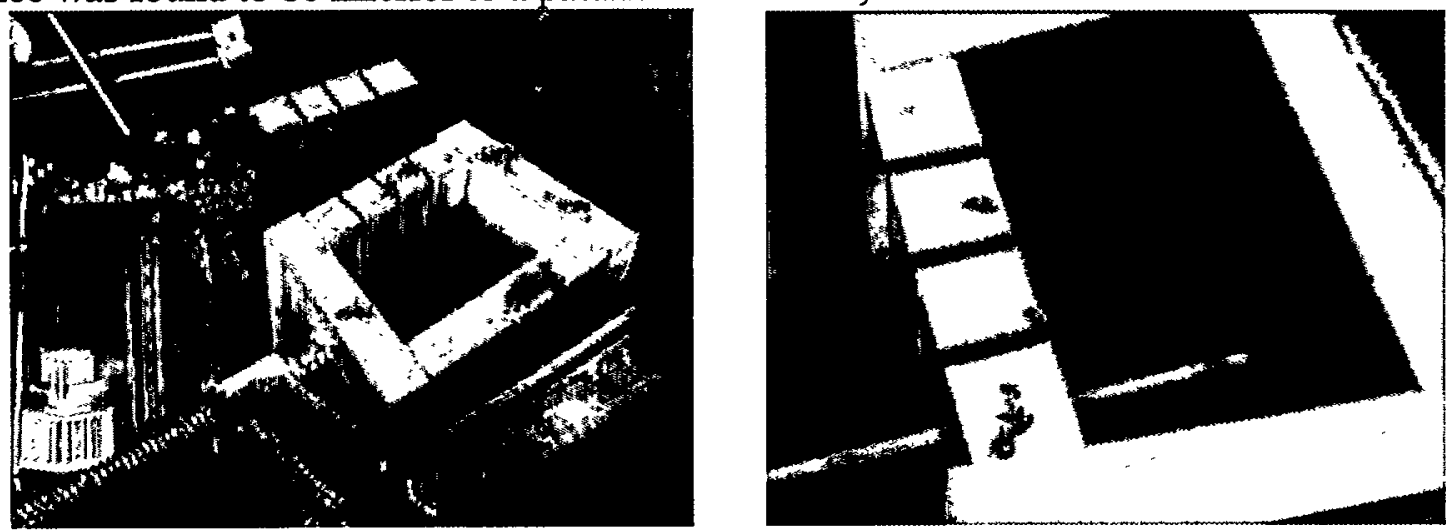

Figure 3. Preparation of a First Generation Thermal Instrument Package

Discussions with Prof. J. V. Beck of Michigan State University led to his suggestion that installation of the thermocouples perpendicular to the isotherms can lead to large errors in the indicated temperature (Beck, 1998).

Figure 4 depicts the two possible types of thermocouple installations: "straight" and "Lshaped". When the conductivity of the wire is much greater than that of the surrounding material (about two orders of magnitude in the present case), then the wire presents a preferentially conductive path for heat flow from the surface (Beck, 1962). This results in a temperature depression in the immediate vicinity of the wire, as depicted in both of the schematics in Figure 4. In the "L-shaped" installation, the tip of the wire is bent so that it is parallel to the local temperature isotherms. In this case, the tip of the thermocouple extends into the region of constant temperature, which is the desired indicated temperature.

This phenomenon was investigated both experimentally and numerically in our program. Figure 5 shows the experimental results from a casting where both types of thermocouples (straight and L-shaped) were installed in a mold. Care was exercised so that the depths of the straight and bent thermocouples were at the same approximate depth from the interface. (The actual depths were determined using a micrometer depth gage after the casting was cooled and removed from the mold). Note that the two " $L$ " thermocouples (at $5.1 \mathrm{~mm}$ and $5.2 \mathrm{~mm}$ ) have virtually an identical response up until elapsed time of 250 seconds, then they begin to differ. The difference at this time is associated with a change in heat transfer at the top surface of the casting, 
which can also be seen the in the other pair of curves. The two "straight" thermocouples also have responses similar to each other, but the one noted at $5.8 \mathrm{~mm}$ depth is always at a lower temperature than the one at $5.3 \mathrm{~mm}$. The important point of all this is the difference between the rate of response of the " 22 " thermocouples, which are at the same nominal depth as the " 11 " thermocouples, and therefore should read the same temperature as the " $\mathrm{x} 1$ " thermocouples. Note that the straight (x1) thermocouples always indicate a lower temperature than the "L" ( $\mathrm{x} 2)$ thermocouples, consistent with the explanation given via Figure 4.

We also performed computer simulations on the "straight" thermocouples to quantify the probable magnitude of the temperature depression at the tip of a wire imbedded in a sand medium. A simple axisymmetric finite element model of a wire surrounded by sand and subjected to heating at the surface was used. The heating imposed at the surface was taken from an experiment we performed. The "error" was defined as the temperature at the same depth from the surface as the wire minus the temperature at the tip of the wire. The resulting error as a function of time is shown in Figure 6 for a $25 \mathrm{~mm}$ thick casting section. Note that the farther the thermocouple is from the surface, the less the deterministic measurement error will be, however, it will never be zero (unless wire with the same properties as sand can be found).
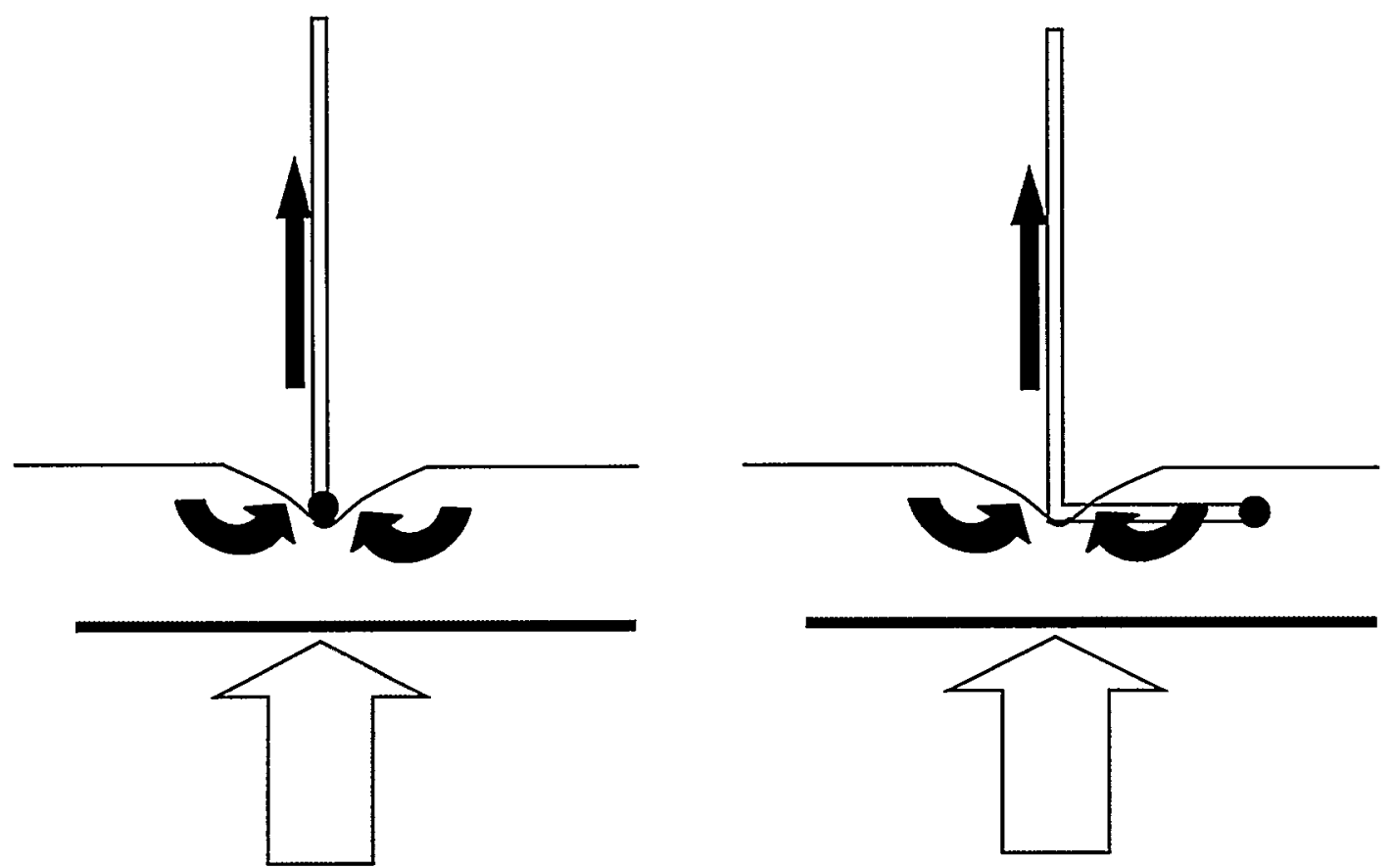

Figure 4. Illustration of junction location relative to distorted temperature field for straight (left) and "L-shaped" (right) thermocouples

\subsubsection{Second Generation}

These observations led to the development of a new instrumentation package, and a second series of castings. The new instrument package used thermocouples with a $50 \mathrm{~mm}$ length parallel to the cast surface, and used two surface thermocouples to measure the temperature of the solidifying metal. Figure 7 shows a picture of a second generation instrument pack. The lead 
wire exiting the side of the instrument pack indicates that the thermocouple junction inside the pack has about a $50 \mathrm{~mm}$ length of wire that was parallel to the casting. Note that there were now two "two-point" thermocouples protruding from the front of the pack (visible at the top and bottom of the surface in Figure 7) which would each provide an independent indication of the solidifying metal temperature.

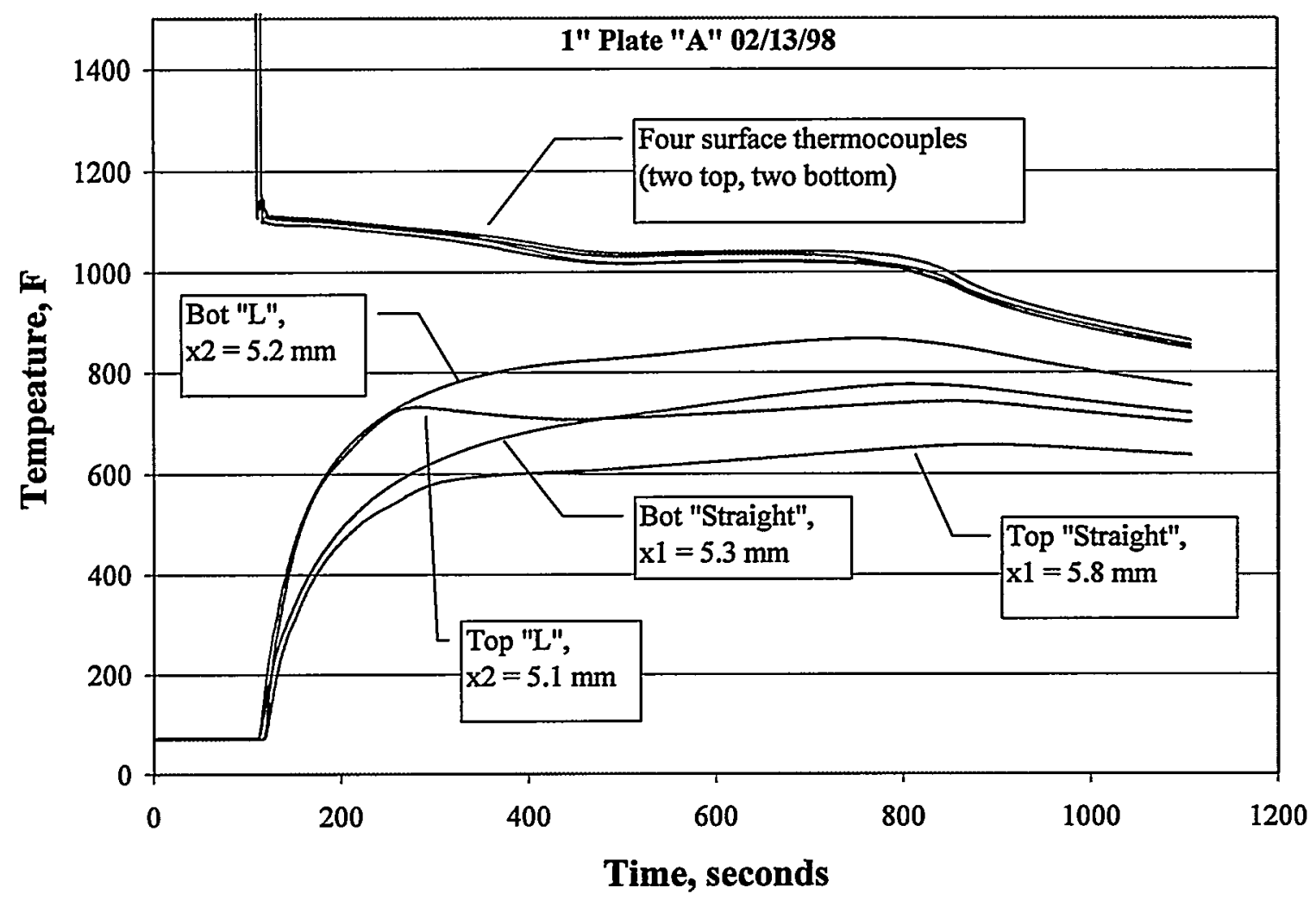

Figure 5. Temperature traces comparing "straight" versus "L-shaped" thermocouples

\subsubsection{Analysis}

The general approach taken to make the heat transfer measurements is based on an inverse heat conduction solution (Beck, et al., 1985) for the surrounding mold medium. Figure 8 depicts a section of a casting, showing the metal (below) the sand (above), and several indicated temperature measurement points. Also suggested by the schematic is the existence of a gap between the metal and the mold, and the heat transfer across that gap. Using temperature measurements in the sand, and assuming that the thermal properties of the sand are completely known, inverse heat conduction methods are used to find the heat transfer $q(t)$ at the interface. If the surface temperature of the solidifying metal is also measured, then the interfacial heat transfer coefficient can be determined. Another approach is to formulate the inverse problem so that the heat transfer coefficient $h(t)$ is determined directly. Both approaches will be explored below, with the latter one ultimately being utilized. 


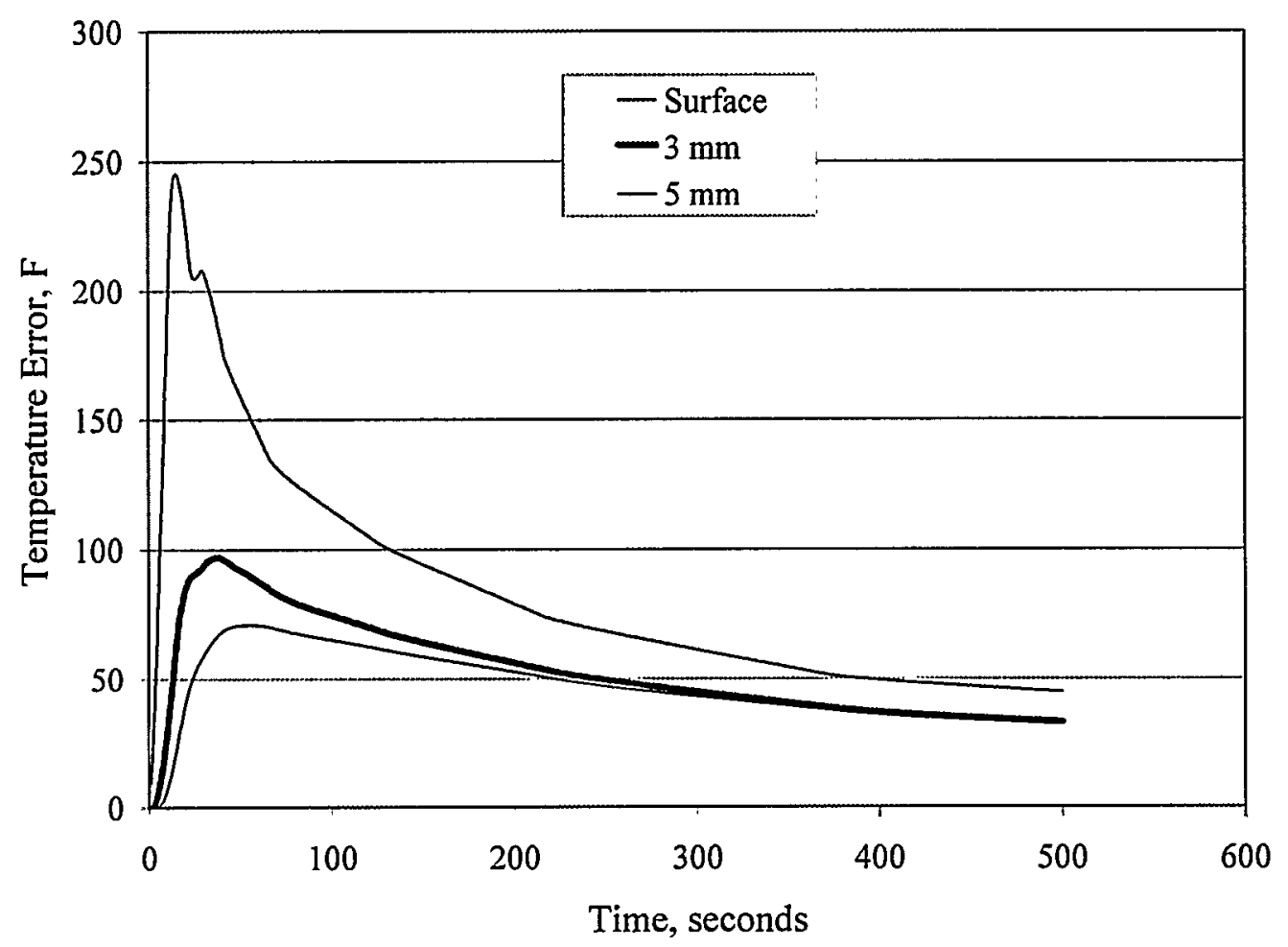

Figure 6. Deterministic thermocouple errors computed from finite element simulation

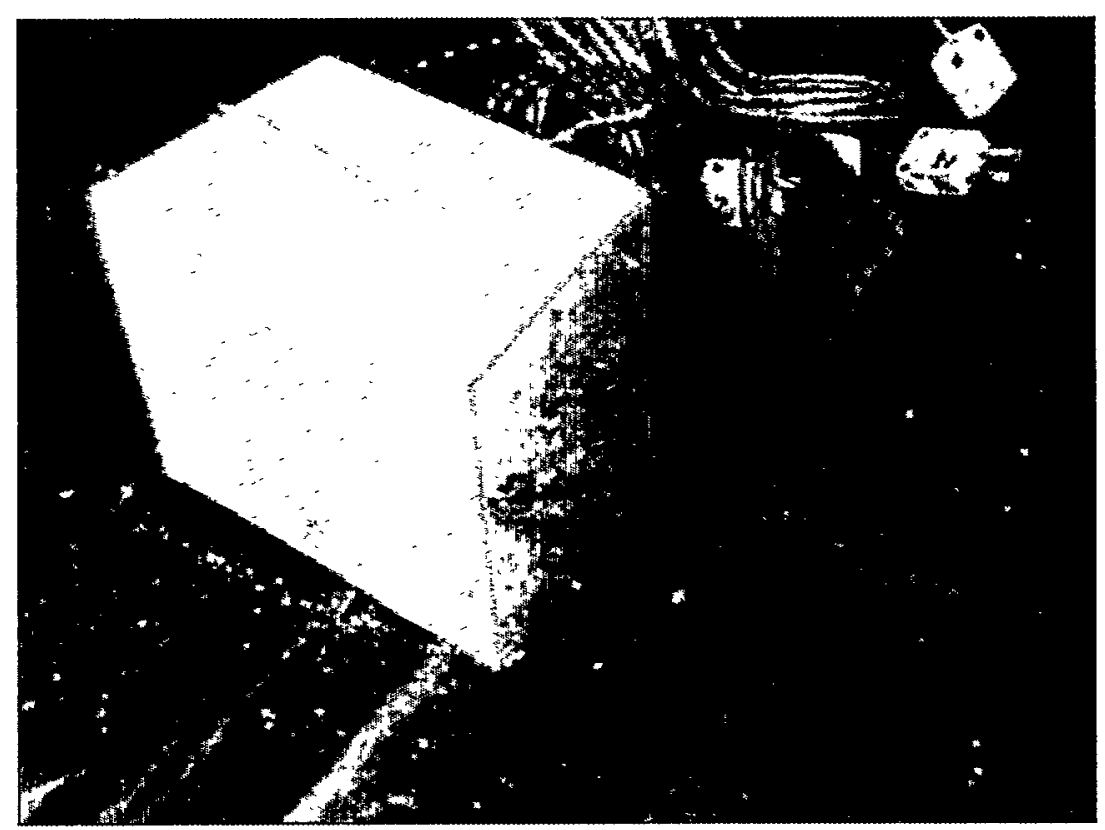

Figure 7. New instrument package. Note thermocouple lead wires exiting from the side of the pack, and two surface thermocouple wires protruding from the front. 


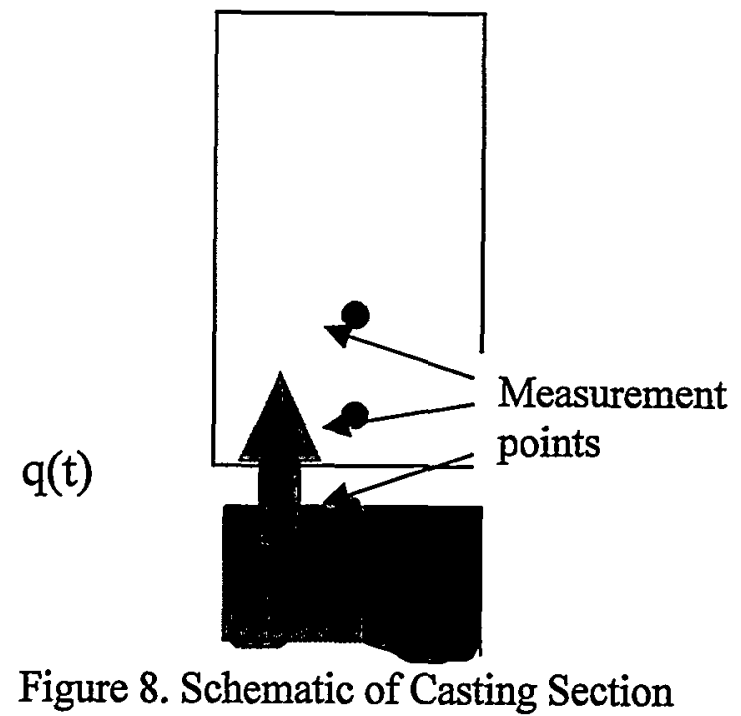




\subsection{Inverse Problems}

The inverse problem of determining the unknown surface heat flow may be understood by comparison with the corresponding forward (or "usual" problem). The "usual" problem of heat conduction may be summarized as follows:

Given: Complete description of system physics

All material properties

Initial system configuration

All external loads on system at all times

Find: System configuration at all subsequent times (the values that you would measure).

The inverse problem may be summarized as follows:

Given: Complete description of physics

All material properties

Initial system configuration

Measurements at known locations at known times

Find: External loads at all times

Thus, the solution of both the forward and the inverse problem require a complete knowledge of the physics, as well as accurate knowledge of the material properties.

The solution to inverse problems is the subject of many articles and books (Beck, Blackwell, and St. Clair (1985), Murio (1993), Hensel (1991)). The mathematical nature of inverse problems is well understood: they are mathematically ill-posed because their solutions do not continuously depend on the data. Specifically, small errors in the input to the inverse problem (the material properties and the measurements) can cause large or violent deviations in the solution of the inverse problem (in this case the unknown heat flux).

The mathematical model is taken as the diffusive heat conduction equation. This is approximate, even for the case of resin-bonded sand, but even more so for the green sand. In resin-bonded sands, the heat from the casting produces evolution of gases from the resin, and these gases are driven away from the hot surface and can carry some energy with them. In the case of green sand, the water binder evaporates and migrates and re-condenses, carrying with it a significant amount of energy. The assumption of a diffusive process is a simplification, but one which greatly simplifies the inverse problem.

In order to apply the inverse method, the thermal properties of the medium must be known at each point in space and time. This variation in space and is handled by allowing the thermal conductivity $k$ and the volumetric heat capacity $C$ to be functions of the local temperature.

Kubo and Pehlke (1985) published a study including thermal properties of silica sand. The properties used in the present effort are derived from Kubo and Pehlke and are summarized in Table 1. The formula for specific heat is that recommended by Kubo and Pehlke (1985) for silica sand, while that for thermal conductivity is the result of a curve-fit to data for dry bentonite bonded silica sand. The temperature for the formulae in Table 1 is in degrees Kelvin. 
Table 1. Thermal Properties of Resin Bonded Sand

\begin{tabular}{|l|l|}
\hline Property (units) & Relation \\
\hline Density $\left(\mathrm{kg} / \mathrm{m}^{3}\right)$ & 1500 \\
\hline Thermal Conductivity $(\mathrm{W} / \mathrm{m}-\mathrm{C})$ & $2.63(\mathrm{~T} / 1000)^{3}+5.59(\mathrm{~T} / 1000)^{2}-4.08(\mathrm{~T} / 1000)+1.9$ \\
\hline Specific Heat $(\mathrm{kJ} / \mathrm{kg}-\mathrm{C})$ & $0.782+0.000571 \mathrm{~T}-18800 / \mathrm{T}^{2}$ \\
\hline
\end{tabular}

\subsection{Heat Flux Approach}

In this approach, which was used in the first sequence of castings, the external heat flux history is considered unknown in the inverse problem. After the heat flux history is determined, the interfacial heat transfer coefficient is found by making use of the metal temperature measured at the casting surface. This method, and a sample of results obtained using this method, will be presented in the following sections.

\subsubsection{Method}

This method was detailed in a paper presented at the $102^{\text {nd }}$ Congress of the American Foundrymen's Society (Woodbury, et al., 1998).

The function estimation problem of finding the unknown surface heat flux may be stated mathematically as follows. For one-dimensional flow of heat in a solid, conservation of energy requires that:

$$
C(T) \frac{\partial T}{\partial t}=\frac{\partial}{\partial x}\left(k(T) \frac{\partial T}{\partial x}\right)
$$

where $C(T)$ is the temperature-dependent volumetric heat capacity (density-specific heat product $C(T)=\rho(T) c_{p}(T)$ ), and $k(T)$ is the temperature dependent thermal conductivity of the material. It is assumed that these thermal properties of the material are completely known.

The initial condition for Equation (2) is

$$
T(x, 0)=g(x)(3)
$$

where the function $g(x)$ is known and typically is a constant value. The boundary conditions for Equation (2) are:

$$
\begin{gathered}
q_{L}=-\left.k \frac{\partial T}{\partial x}\right|_{x=L}=0 \\
q_{0}(t)=-\left.k \frac{\partial T}{\partial x}\right|_{x=0}=f_{1}(t) .
\end{gathered}
$$


In Equation (5), the function $f_{1}(t)$ is NOT known, but is to be determined in the solution of the problem. Supplemental information is provided by measurement of the temperature history at a location $x^{*}$ :

$$
T\left(x^{*}, t\right)=f_{2}(t)
$$

The function $f_{2}(t)$ is not known continuously, but can be found at discrete instants in time using digital data acquisition. These are the temperatures from the thermocouples in the instrumented castings. Note that in this approach, only data from within the mold are used to infer the heat flux into the mold. In the heat transfer coefficient method (to be described later) the temperature of the solidifying metal also enters this computation.

By using an appropriate discretization technique (we use finite elements), the discrete form of the partial differential equation (2) can be written as

$$
[\mathrm{A}]\left\{T^{n+1}\right\}=\left\{R_{1}\left(q_{0}{ }^{n+1}\right)\right\}+\left\{R_{2}\left(q_{0}{ }^{n}\right)\right\}+\left\{R_{3}\left(T^{n}\right)\right\} .
$$

The sequential function specification method for solving inverse function estimation problems consists of (1) assuming a functional form for the unknown function and (2) estimating the components of the unknown function sequentially.

In the present case, the continuous function $q(t)$ is assumed to be represented by a piecewise constant function. An arbitrary time is denoted by $t^{n+1}$, and the corresponding value of heat flux is $q\left(t^{n+1}\right)$. The time corresponding to the occurrence of the heat flux is shifted from the time of the measurement by $\Delta t / 2$.

The sequential estimation procedure requires computation of the "unknown" value of $q\left(t^{n+1}\right)$ in Equation (7) at each step. In its simplest form, the sequential computation allows for a direct computation of each component $q\left(t^{n+1}\right)$ in an "exact matching" fashion. This exact matching (one $q$ value for every measured $T$ value) is the classic formulation of Stoltz (1960) and is extremely sensitive to noise in the input data. This sensitivity to noise is a manifestation of the inherent ill-posedness of the problem, and is made worse by small computational time steps. This ill-posedness can be addressed by regularizing using the now-classic method of Beck. Beck's innovation (Beck, 1968) was to include a few extra measurements from the next $r$ future time steps. In order to add this extra data and not introduce new unknown components of $\{\mathbf{q}\}$ into the problem, Beck suggests letting these components of $\{\mathbf{q}\}$ corresponding to the future times be held temporarily constant at the current time step. That is, let

$$
q^{n+1}=q^{n+2}=\cdots=q^{n+r-1}
$$

This introduction of future times data lends stabilization to the algorithm, but also introduces bias into the computation of $q^{n+1}$. Specifically, the values of $\{\mathbf{q}\}$ during transitions are smoothed; thus sharp transitions can be lost if a value of $r$ which is too large is specified. 
This use of future times data and the assumption embodied in Equation (8) results in an overdetermined problem in the discrete finite element equations (represented by Equation (7)). That is, there are now $r$ equations for the single value of the unknown $q^{n+1}$. This is accommodated by choosing $q^{n+1}$ to minimize the sum of the squared differences between the model-computed values of temperature (given by the solution of Equation (7)) and the experimentally-measured values (represented by $f_{2}(t)$ in Equation (6)). Thus the function to be minimized is

$$
S\left(q^{n+1}\right)=\sum_{i=1}^{i=r}\left(T\left(x^{*}, t^{n+i}, q^{n+1}\right)-f_{2}\left(t^{n+i}\right)\right)^{2}
$$

To handle the non-linear dependence of the solution for $T(x, t)$ on the thermal properties $C(T)$ and $k(T)$, the solution is linearized about a nominal assumed value for $q^{n+1}$ at each time step. Thus, a value for the heat flux, say $q_{k}^{n+1}$, is assumed to be known and a correction $\Delta q_{k}$ is sought so that

$$
q_{k+1}^{n+1}=q_{k}^{n+1}+\Delta q_{k}^{n+1}
$$

Note that these iterations are performed at each time step, so that the time superscript $n+1$ is unchanging. This linearization technique is that which Junkins (1978) refers to as Gaussian Least Squares Differential Correction, and Beck and Arnold (1977) refer to as Gauss Method of Minimization. The resulting update equation for the heat flux component $\Delta q_{k}$ is

$$
\Delta q_{k}=\left[\mathbf{X}^{\mathrm{T}} \mathbf{X}\right]^{-1} \mathbf{X}^{T}\left\{T\left(x^{*}, q_{k}^{n+1}\right)-f_{2}\right\} .
$$

In Equation (11), the length of the vector $\left\{T\left(x^{*}, q_{k}^{n+1}\right)-f_{2}\right.$ is $r$, the number of future times used, and the notation $T\left(x^{*}, q_{k}^{n+1}\right)$ only emphasizes that the currently assumed value of $q_{k}^{n+1}$ is used to generate the solution. In Equation (11), the $\mathbf{X}$ matrix is the sensitivity matrix which relates the measured response $T\left(x^{*}, t\right)$ to the unknown heat flux component $q_{k}{ }^{n+1}$. Mathematically, the components of the $\mathbf{X}$ matrix are the partial derivatives of the solution $T\left(x^{*}, t\right)$ to the unknown heat flux component $q_{k}^{n+1}$. The components of the $\mathbf{X}$ matrix will vary with time, only because of the non-linearity presented by the properties $C(T)$ and $k(T)$. In the present formulation, the $\mathbf{X}$ matrix is recomputed at each time step by a direct sensitivity coefficient method (Tseng, et al, 1995, Woodbury and Premanand, 1996).

Once all the values of $q(t)$ have been computed sequentially, the temperature field at all points in space and time can be computed from solution of the direct heat conduction problem represented by Equations (2)-(5). This is important, as the temperature at the surface of the mold will be necessary to compute the heat transfer coefficient. Specifically, the heat transfer coefficient will be computed from the following relation: 


$$
h=\frac{q}{T_{\text {metal }}-T_{\text {mold }}}
$$

where $T_{\text {metal }}$ is the surface temperature of the solidifying casting, and $T_{\text {mold }}$ is the surface temperature of the mold.

\subsubsection{Typical Results}

Heat fluxes and heat transfer coefficients were obtained using the method outlined above. A set of results for heat fluxes for the $25 \mathrm{~mm}$ plates are seen in Figure 9 (bottom surfaces) and Figure 10 (top surfaces). The results in both cases are extremely reproducible; the results shown are from four different plates cast on two different days. The results for the bottom surface were reproduced to a high degree, but the top surface results were a bit more variable.

Figures 11 and 12 show the corresponding heat flux computed from Equation (12) after the heat flux history is found. Note the that coefficients from the bottom surface (Figure 11) are very well reproduced, but those from the top (Figure 12) vary wildly from one case to another.

\subsubsection{Problems}

The lack of reproducibility in the heat transfer coefficient results in Figure 12 is obviously a concern. The main reason cited for this behavior is related to NOT using the measured solidifying plate temperature in the calculation of $q(t)$. The heat flux estimate is made without regard to the "source" temperature, $T_{\text {metal }}(t)$. This can lead to a calculation of $T_{\text {mold }}(t)$ which is inconsistent with the measured source temperature. The data for $T_{\text {metal }}(t)$ are available and can be used to get a better estimate of $h(t)$. 
1.0" Horizontal Plates (Bots) 08/13/98 + 08/25/98

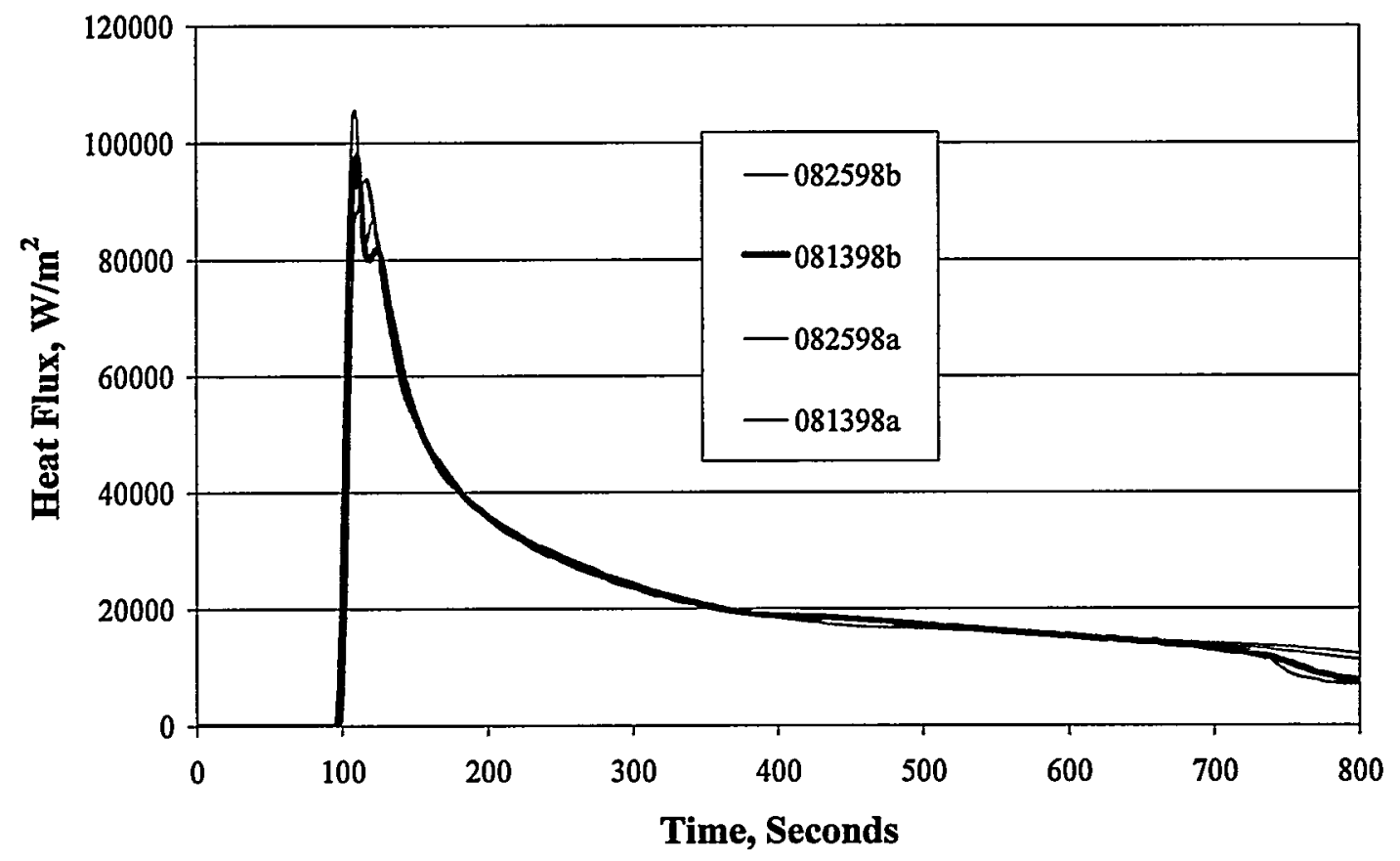

Figure 9. Heat fluxes obtained from bottom surfaces of $25 \mathrm{~mm}$ horizontal plates using heat flux formulation

1.0" Horizontal Plates (Tops) 08/13/98 + 08/25/98

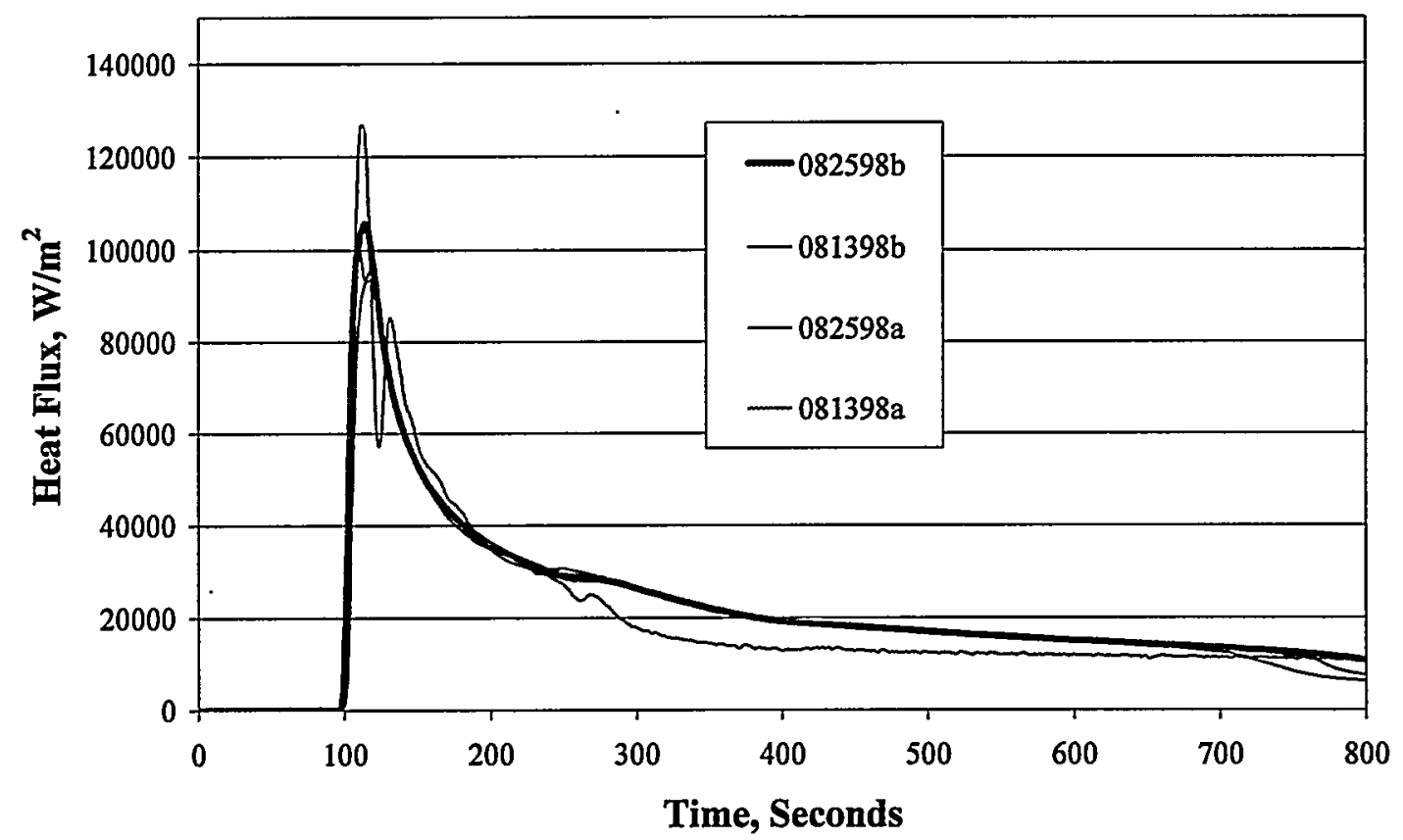

Figure 10. Heat Fluxes from top surfaces of $25 \mathrm{~mm}$ horizontal plates using heat flux formulation 


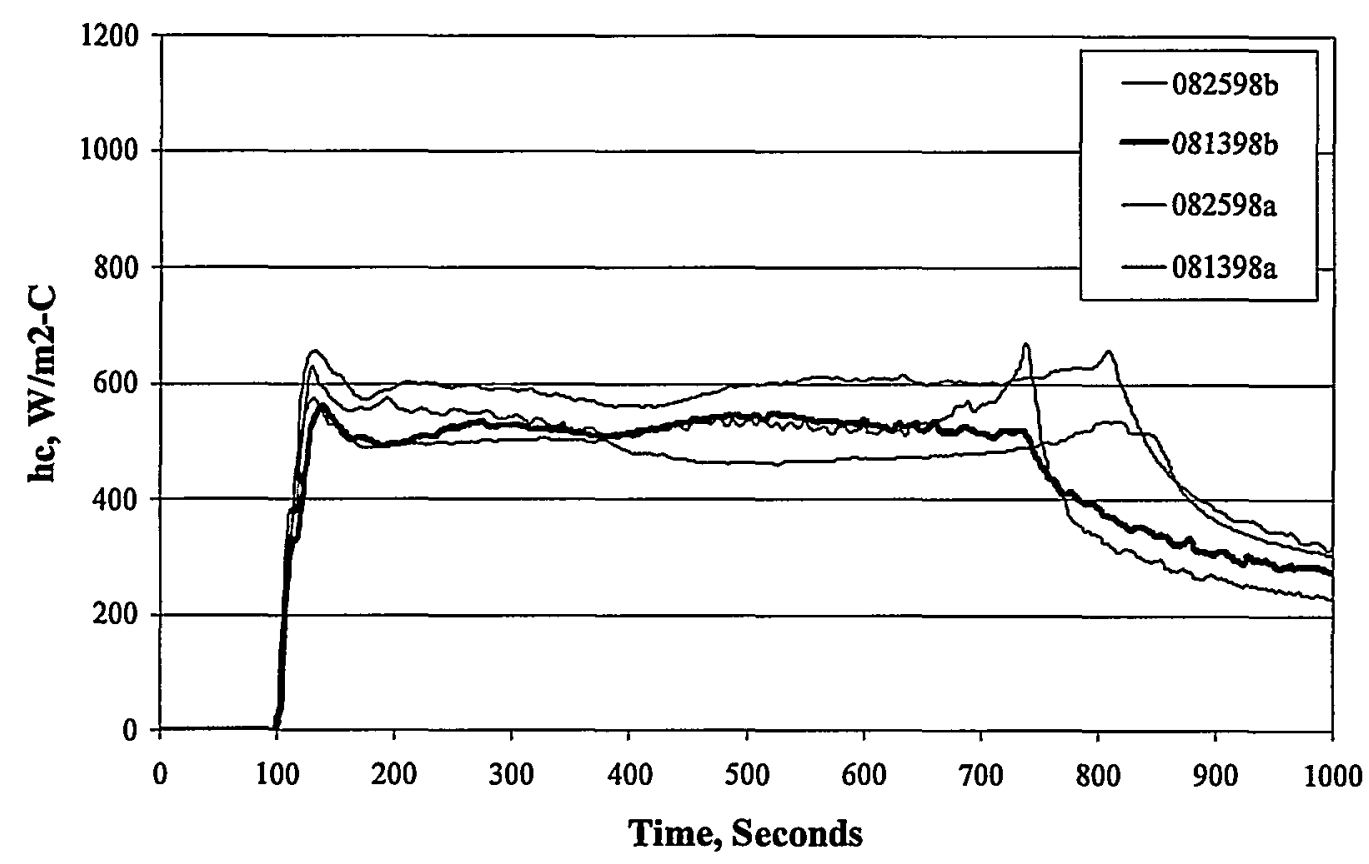

Figure 11. Heat Transfer Coefficients from bottom surface of $25 \mathrm{~mm}$ plates using heat flux formulation

1.0" Horizontal Plates (Tops) 08/13/98 + 08/25/98

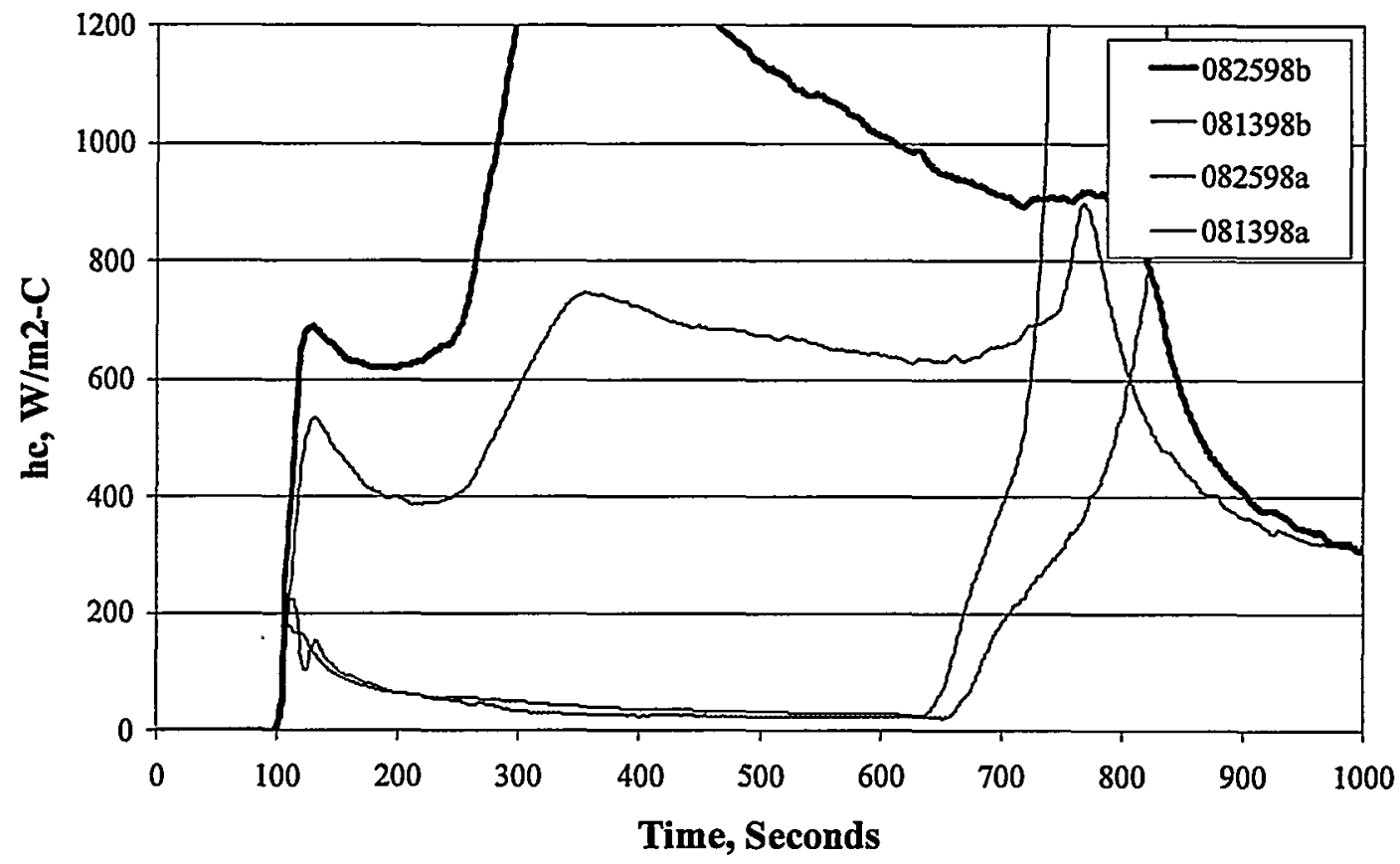

Figure 12. Heat Transfer Coefficients from top surfaces of $25 \mathrm{~mm}$ plates using heat flux formulation 


\subsection{Direct Coefficient Approach}

To alleviate the variability in the results such as those seen in Figure 12, a modified inverse heat conduction analysis was adopted. The basic idea in this approach is to make use of as much experimental evidence as possible for each casting. A side benefit is that the desired interfacial heat transfer coefficient is the direct outcome of the analysis.

\subsubsection{Method}

The conduction of heat in solids is governed by Equation (2). For a body heated by a heat transfer coefficient at the left end $(x=0)$ and insulated at the right end $(x=L)$, the appropriate boundary conditions can be expressed as:

$$
\begin{gathered}
-\left.k \frac{\partial T}{\partial x}\right|_{x=0}=h(t)\left(T_{\infty}(t)-T(0, t)\right) \\
-\left.k \frac{\partial T}{\partial x}\right|_{x=L}=0
\end{gathered}
$$

A suitable initial condition for this problem is:

$$
\left.T(x, 0)\right|_{t=0}=T_{0}
$$

The problem defined by Equation (2) and Equations (13)-(15) is the forward or direct problem. Given the appropriate values of $h(t), T_{\infty}(t)$, and the properties $k$ and $C$, the temperature in the domain for all non-zero values of time can be found at all $x$ locations.

In order to conduct the estimation for the heat transfer coefficient, the sensitivity of the measurement to the unknown parameter must be found. The sensitivity coefficient is the derivative of the observed quantity (in this case, the temperature) with respect to the unknown quantity (in this case the heat transfer coefficient):

$$
X_{h}=\frac{\partial T}{\partial h} .
$$

A mathematical problem to compute these sensitivity coefficients can be found by taking partial derivatives of the problem defined by Equation (2) and Equations (13)-(15) with respect to the heat transfer coefficient. In so doing, we make the assumption that, at each time step, the heat transfer coefficient is constant for the next several time steps. That is, $h(t)=h_{c}$ :

$$
C(T) \frac{\partial X_{h}}{\partial t}=\frac{\partial}{\partial x}\left(k(T) \frac{\partial X_{h}}{\partial x}\right)
$$




$$
\begin{aligned}
-\left.k \frac{\partial X_{h}}{\partial x}\right|_{x=0}= & \left(T_{\infty}(t)-T(0, t)\right)+h_{c} X_{h}(0, t) \\
& -\left.k \frac{\partial X_{h}}{\partial x}\right|_{x=L}=0
\end{aligned}
$$

To find $h(t)$ in the inverse problem, we use the data from both of the surface thermocouples on each surface of the plate to determine $T_{\infty}(t)$. Specifically, $T_{\infty}(t)$ is computed as the average of the two thermocouples imbedded in the surface of the plate. At each time step, the sensitivity problem (Equations (17)-(19)) is solved, and the resulting sensitivity matrix $\mathbf{X}$ is used in a modification of Equation (11) to compute the heat transfer coefficient at each time step. We use all the available experimental data, which includes not only the two plate surface temperature measurements, but also, in general, two subsurface (mold) thermocouple readings.

\subsubsection{Typical Results}

The results obtained using the heat transfer coefficient formulation for the same cases presented earlier are seen in Figures 13 and 14. The results in Figure 13 are very similar to those in Figure 11, but those in Figure 14 are more consistent (to each other) than those in Figure 12. There is, however, a notable variability in the results for heat transfer coefficient for the upper surfaces of the horizontal plate (Figure 14). Also shown in Figures 13 and 14 are the average values of the four cases presented in each figure. The average is shown as the heavy black line. These average values are the ones we report for each case.

1.0" Horizontal Plates (Bots) 08/13/98 + 08/25/98

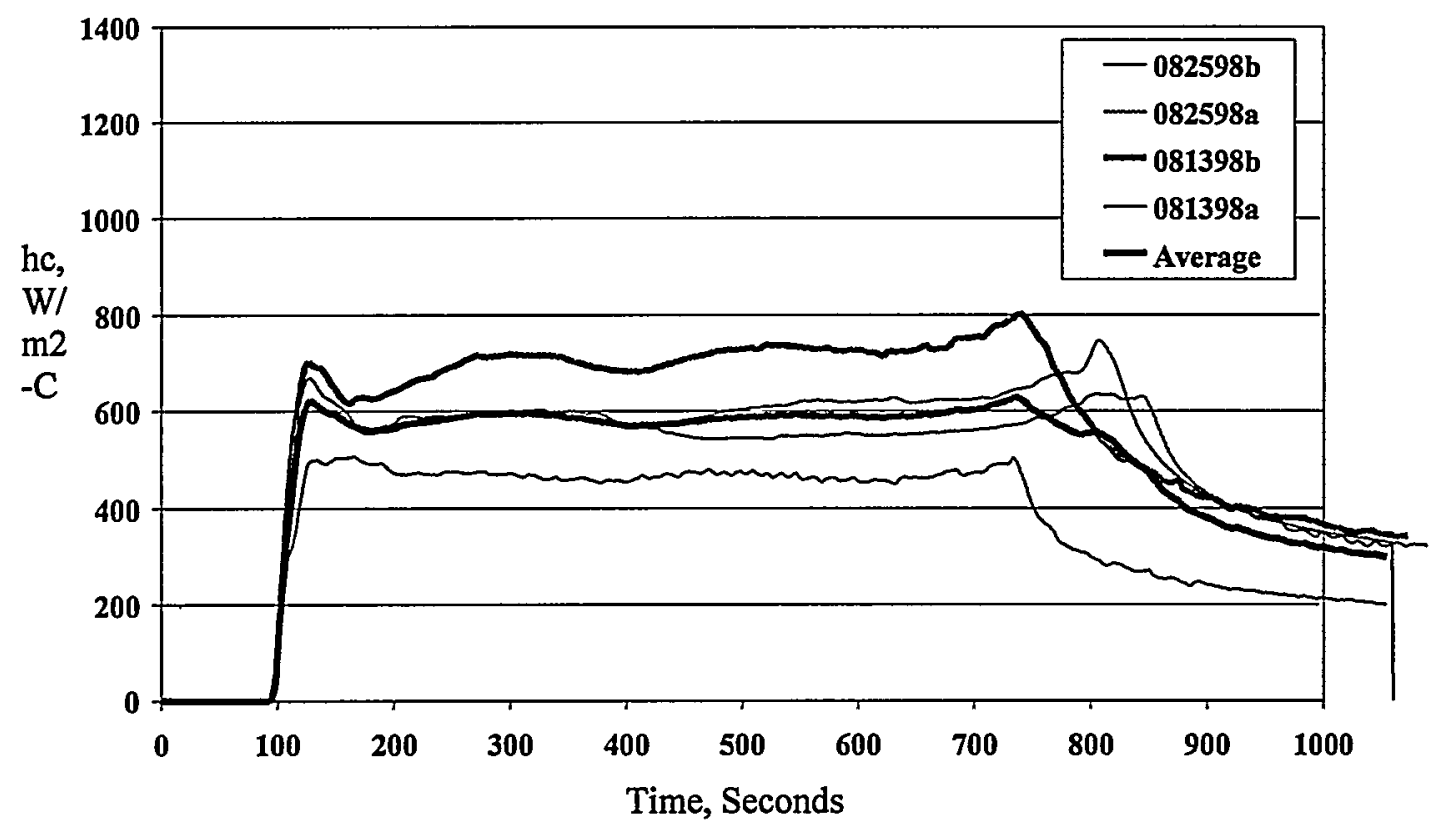

Figure 13. Heat Transfer Coefficients from bottom surface of $25 \mathrm{~mm}$ plates using heat transfer coefficient formulation 


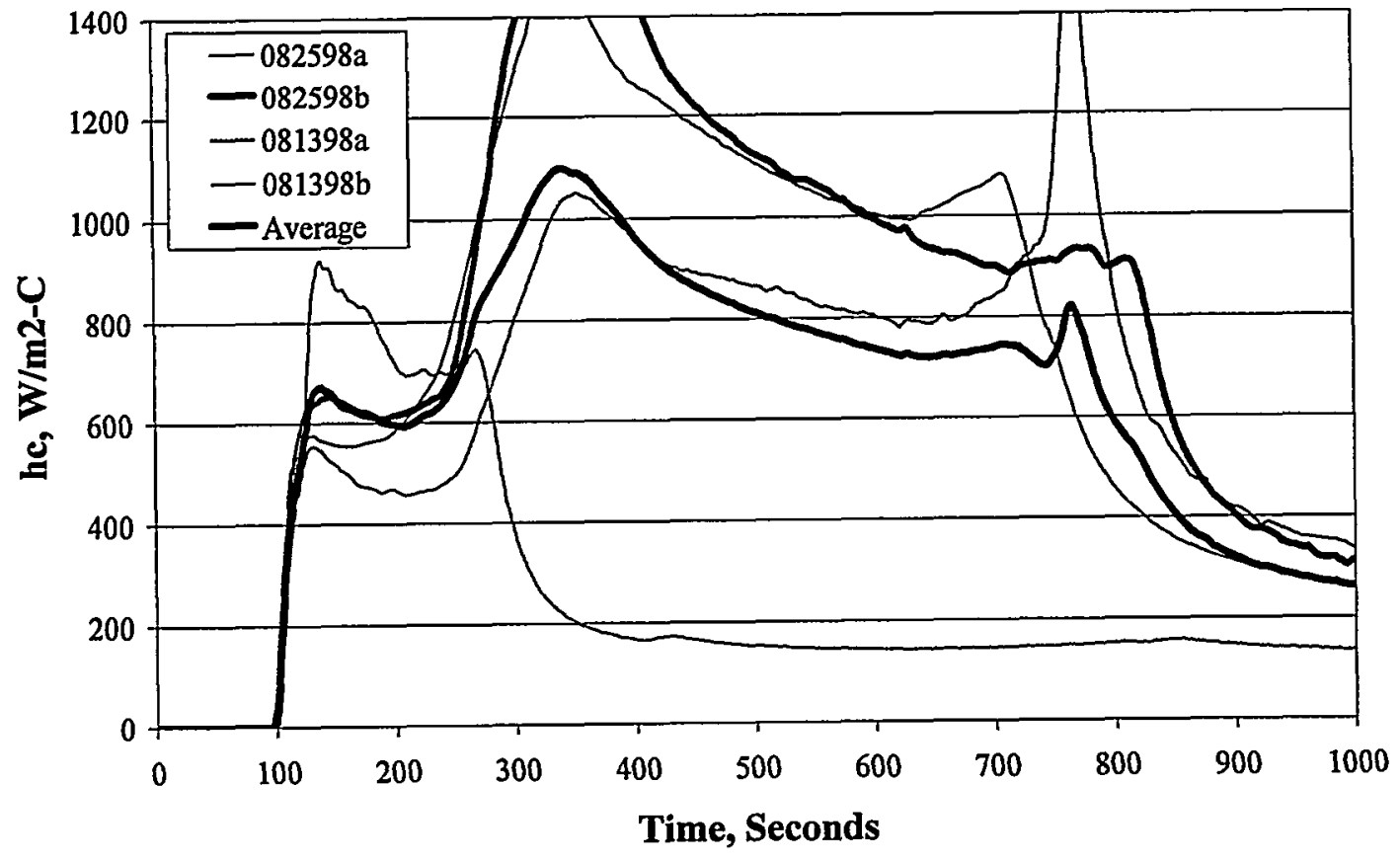

Figure 14. Heat Transfer Coefficients from top surface of $25 \mathrm{~mm}$ plates using heat transfer coefficient formulation 


\subsection{Preliminary Castings}

The information in this section is included for largely for purposes of documenting the history of the project. An ambitious matrix of castings was outlined and these castings were begun in the spring of 1997. During the production of the castings, several problems were identified and subsequently corrected. In the end, a second, smaller, matrix of castings was devised and the second series of castings was poured in 1998.

The original casting matrix required four replicates of castings in two thicknesses $(6.35 \mathrm{~mm}$ and $25.4 \mathrm{~mm}$ ) in four orientations (horizontal, 30 degrees, 60 degrees, and vertical). Thus a total of $4 \times 2 \times 4=32$ castings were in the matrix.

\subsection{Typical Results}

Results typical of those obtained from the preliminary series are seen in Figures 15 and 16 for the $25 \mathrm{~mm}$ casting. Figure 15 shows the heat flux results (obtained using the heat fluxbased inverse problem solution) from a horizontal casting. The heat fluxes from the top and bottom are similar to each other, with that from the top being a bit lower than that from the bottom over most of the solidification.

Figure 16 shows the corresponding heat transfer coefficient for this case. Note there is a wider discrepancy between the top and bottom surfaces.

\subsection{Problems}

We began to look for problems in the procedure and analysis primarily because the heat transfer coefficients were not repeatable from one pour to the next. Some problems that were found have been mentioned previously in this report. The problems found were:

1. Thermocouples installed perpendicular to isotherms, leading to artificially low readings. This was addressed by the second generation instrument package.

2. Poor digital resolution (12-bit) of the temperature data which leads to "noisy" heat flux and heat transfer coefficient values. This was addressed by using a higher resolution (16bit) data acquisition system.

3. Use of heat flux-based inverse algorithm which failed to take advantage of all the experimental evidence from the casting

4. Use of a single surface temperature reading. Adding a redundant reading allowed us to use an average value, or provides the critical data if one of the sensors fails. 


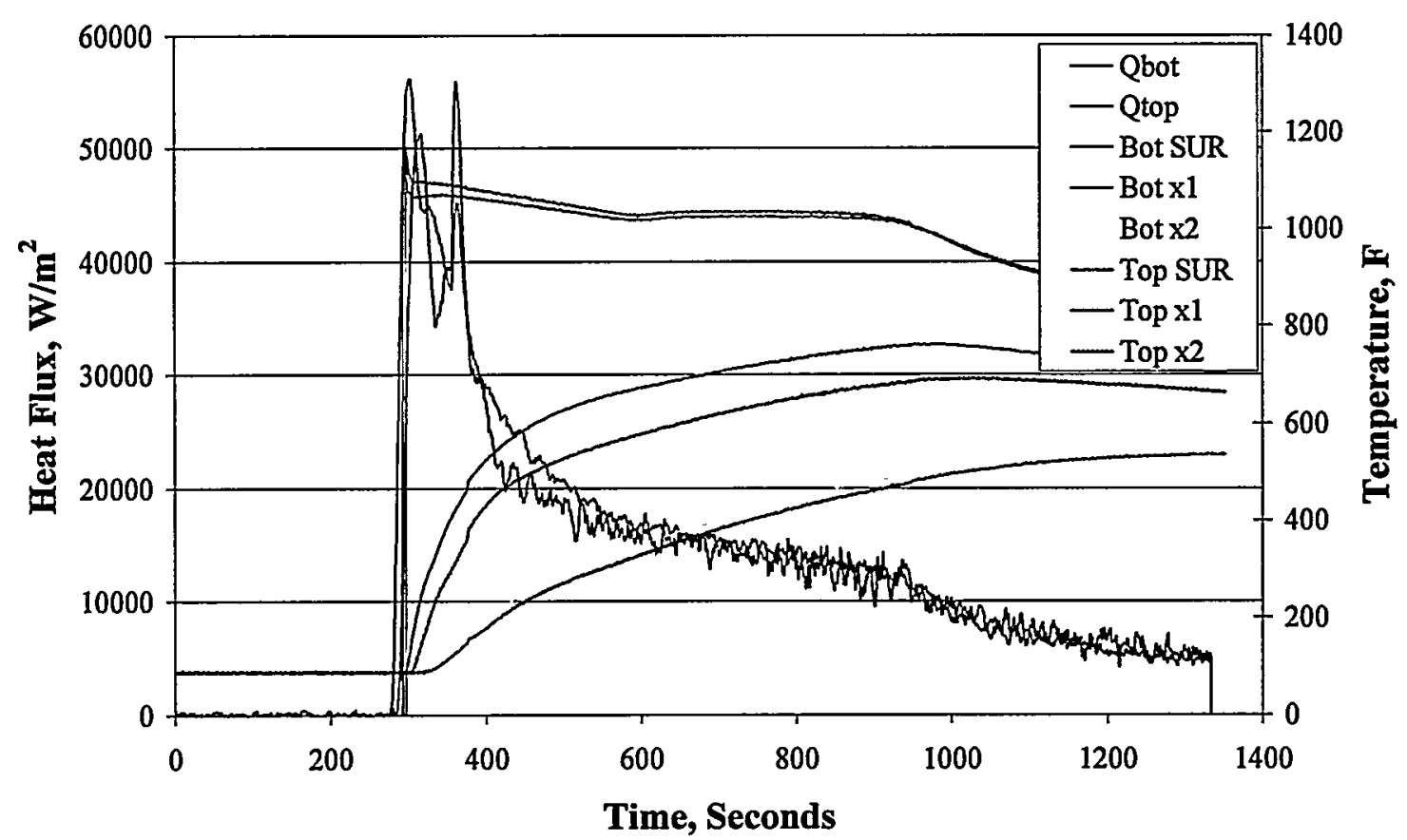

Figure 15. Typical heat flux results for horizontal plate from first sequence of pours

1.0" Plate 07/15/97

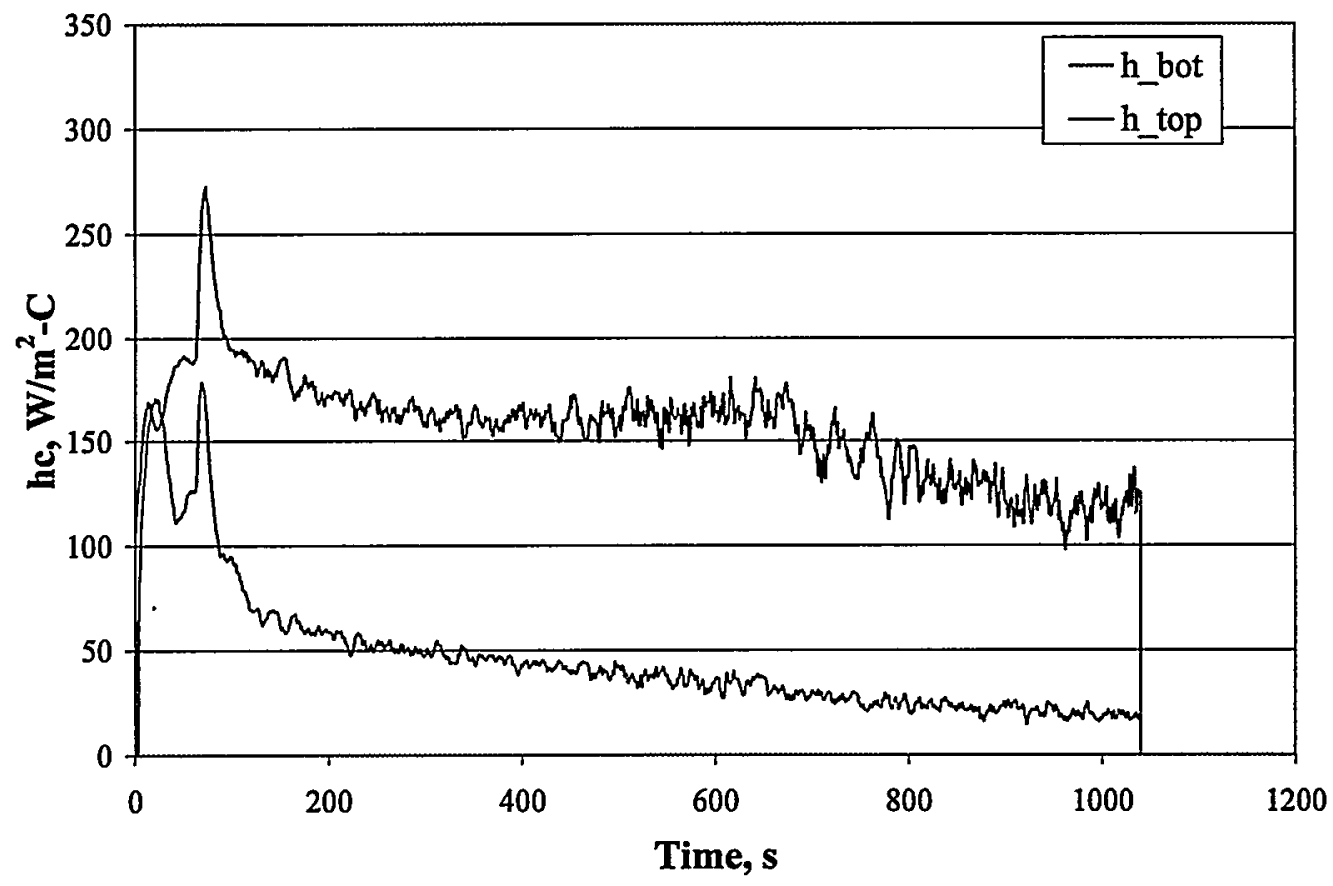

Figure 16. Typical heat transfer coefficient from first series of pours 


\subsection{Resin-Bonded Sand}

The resolution of the issues listed above in the midst of the initial matrix of pours led to a need for a second series of castings. Our experience with the first set of pours indicated that the thickness of the section was more important than the surface orientation, so we decided to add another plate of intermediate thickness to the test matrix.

\subsection{Pouring Matrix}

We decided to pour four replicas of each of three thicknesses, $6.35 \mathrm{~mm}(1 / 4$ "), $12.7 \mathrm{~mm}$ $\left(1 / 2^{\prime \prime}\right)$, and $25.4 \mathrm{~mm}\left(1^{\prime \prime}\right)$ in three different orientations (horizontal, 45 degrees, and vertical). This requires $4 \times 3 \times 3=36$ castings total.

Table 2 shows the pouring matrix, and the "Case Name", which includes the date of the pour as part of the name. Additionally, the plates are poured two at a time (in quick succession) and thus an "a" or " $b$ " is appended to the case name to differentiate the plates. Those marked with a dark gray box had fatal errors that precluded the use of the data from that case. The alloy was not de-gassed prior to pouring, nor were grain refiners or modifiers used in making the castings. All castings were analyzed for chemistries, and all were within the commonly accepted alloy specification. Our purpose was to study the thermal properties of the interface, not to produce castings having high mechanical properties.

Table 2. Pouring Matrix

\begin{tabular}{llll} 
Notes & Case Name & Configuration & $\begin{array}{l}\text { data } \\
\text { interval }\end{array}$ \\
\hline DAQ problem & pour061798a & $0.25^{\prime \prime} 45$ degrees & 1.0 \\
DAQ problem & pour061798b & $0.50^{\prime \prime} 45$ degree & 1.0 \\
Bleed out & pour061898a & $0.25^{\prime \prime} 45$ degree & 1.0 \\
& pour061898b & $0.50^{\prime \prime} 45$ degree & 1.0 \\
& pour062498a & $0.25^{\prime \prime} 45$ degree & 0.2 \\
DAQ Stopped & pour062498b & $0.50^{\prime \prime} 45$ degree & 0.2 \\
pour062598a & $0.25^{\prime \prime} 45$ degree & 0.2 \\
& pour062598a & $0.50^{\prime \prime} 45$ degree & 0.2 \\
pour070198a & $1.0^{\prime \prime} 45$ degree & 1.0 \\
pour070198b & $1.0^{\prime \prime} 45$ degree & 1.0 \\
pour070298a & $1.0^{\prime \prime} 45$ degree & 1.0 \\
pour070298b & $1.0^{\prime \prime} 45$ degree & 1.0 \\
pour071598a & $0.25^{\prime \prime}$ vertical & 0.2 \\
pour071598b & $0.25^{\prime \prime}$ vertical & 0.2 \\
pour071598c & $0.50^{\prime \prime}$ vertical & 0.2 \\
pour071598d & $0.50^{\prime \prime}$ vertical & 0.2 \\
pour071798a & $0.25^{\prime \prime}$ vertical & 0.2 \\
pour071798b & $0.50^{\prime \prime}$ vertical & 0.2 \\
pour071798c & $0.25^{\prime \prime}$ vertical & 0.2
\end{tabular}




\begin{tabular}{llll} 
Notes & Case Name & Configuration & $\begin{array}{l}\text { data } \\
\text { interval }\end{array}$ \\
\hline & pour071798d & $0.50^{\prime \prime}$ vertical & 0.2 \\
pour072398a & $1.0^{\prime \prime}$ vertical & 1.0 \\
pour072398b & $1.0^{\prime \prime}$ vertical & 1.0 \\
& pour072498a & $1.0^{\prime \prime}$ vertical & 1.0 \\
pour072498b & $1.0^{\prime \prime}$ vertical & 1.0 \\
pour072998a & $0.25^{\prime \prime}$ Horizontal & 0.2 \\
pour072998b & $0.5^{\prime \prime}$ Horizontal & 0.2 \\
pour073098a & $0.25^{\prime \prime}$ Horizontal & 0.2 \\
pour073098b & $0.5^{\prime \prime}$ Horizontal & 0.2 \\
pour081298a & $0.25^{\prime \prime}$ Horizontal & 0.2 \\
popped & pour081298b & $0.5^{\prime \prime}$ Horizontal & 0.2 \\
pour081398a & $1.0^{\prime \prime}$ Horizontal & 1.0 \\
pour081398b & $1.0^{\prime \prime}$ Horizontal & 1.0 \\
pour082498a & $0.25^{\prime \prime}$ Horizontal & 0.2 \\
pour082498b & $0.5^{\prime \prime}$ Horizontal & 0.2 \\
pour082598a & $1.0^{\prime \prime}$ Horizontal & 1.0 \\
pour082598b & $1.0^{\prime \prime}$ Horizontal & 1.0
\end{tabular}

\subsection{Results}

Results were obtained for most of the orientations and thicknesses. However, due to the problems with many of the $6.35 \mathrm{~mm}$ and $12.7 \mathrm{~mm}$ plates in the 45 degree inclination, results are not reported for these configurations.

\subsubsection{Heat Transfer Coefficients Versus Time for Each Orientation}

Figures 17 through 30 show the heat transfer coefficients obtained using the direct heat transfer coefficient approach in the inverse problem for many of the cases listed in Table 2. In each figure, the four lines in color are the results from individual tests, and the solid black line is the average of the other curves.

In general, the heat transfer coefficients in Figures 17 through 30 are on the order of $600 \mathrm{~W} / \mathrm{m}^{2}-\mathrm{K}$. These compare favorably with the only work from the literature we found dealing with aluminum castings in resin-bonded sand molds (Hwang, et al., 1994). Hwang, et al made a flat casting ( $150 \mathrm{~mm}$ by $150 \mathrm{~mm}$ by $30 \mathrm{~mm}$ ) of A356 aluminum. Measurements were obtained both in the solidifying metal and in the sand mold at nominal distances of $3 \mathrm{~mm}, 7 \mathrm{~mm}$, and $13 \mathrm{~mm}$ from the casting. Using an inverse procedure, they computed the interfacial heat transfer coefficient during the process. The values they report range from a maximum of $712 \mathrm{~W} / \mathrm{m}^{2}-\mathrm{C}$ to $586 \mathrm{~W} / \mathrm{m}^{2}-\mathrm{C}$.

In general, the results for horizontal castings (Figs.25 through 30) are much more repeatable than those from the vertical configuration (Figs. 19 through 24). In the vertical 


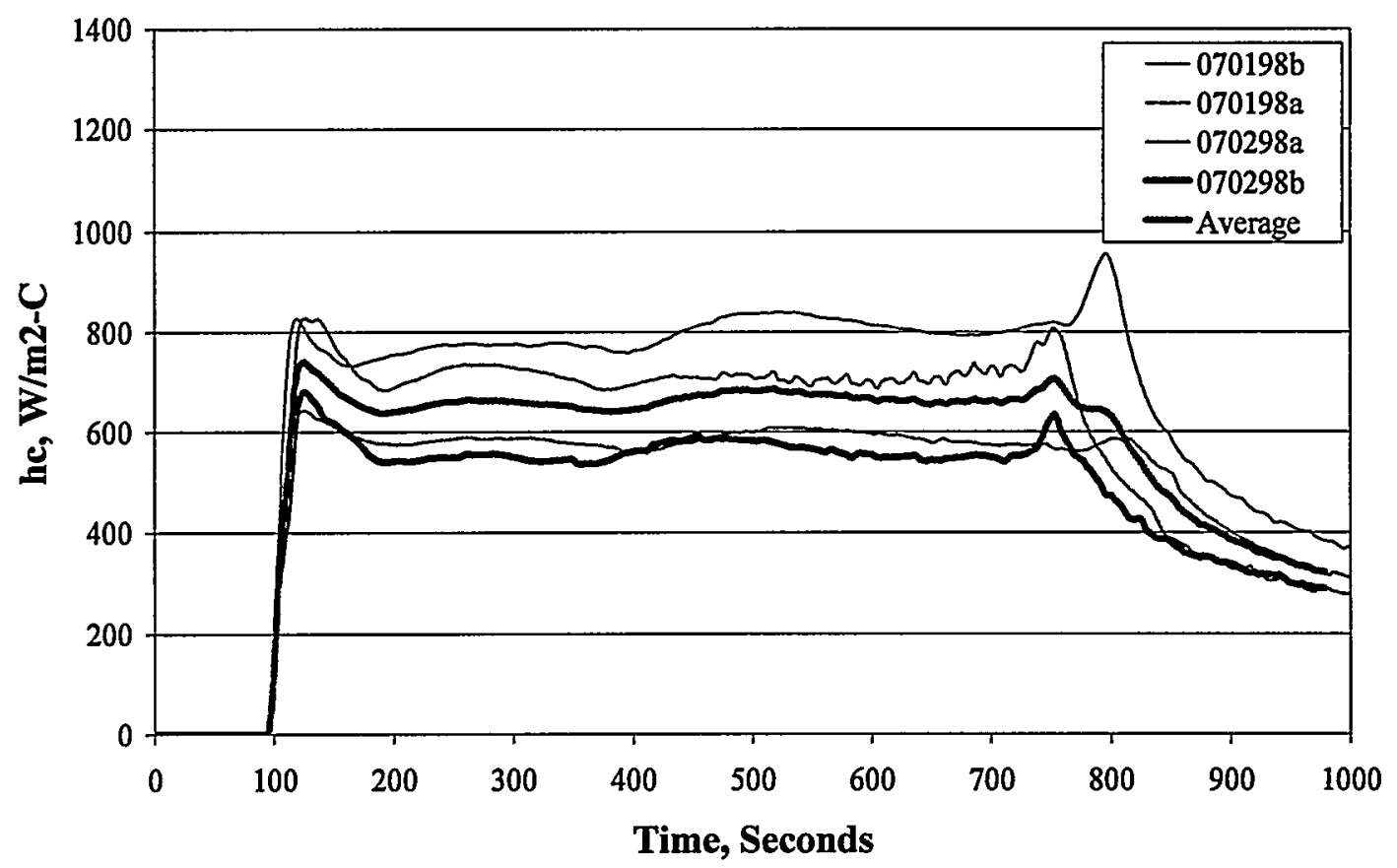

Figure 17. Heat Transfer Coefficients from Bottom Surface of $25.4 \mathrm{~mm}$ plates (45 degree inclination)

1.0" Plates Poured at 45 Degrees (Tops) 07/01/98 + 07/02/98

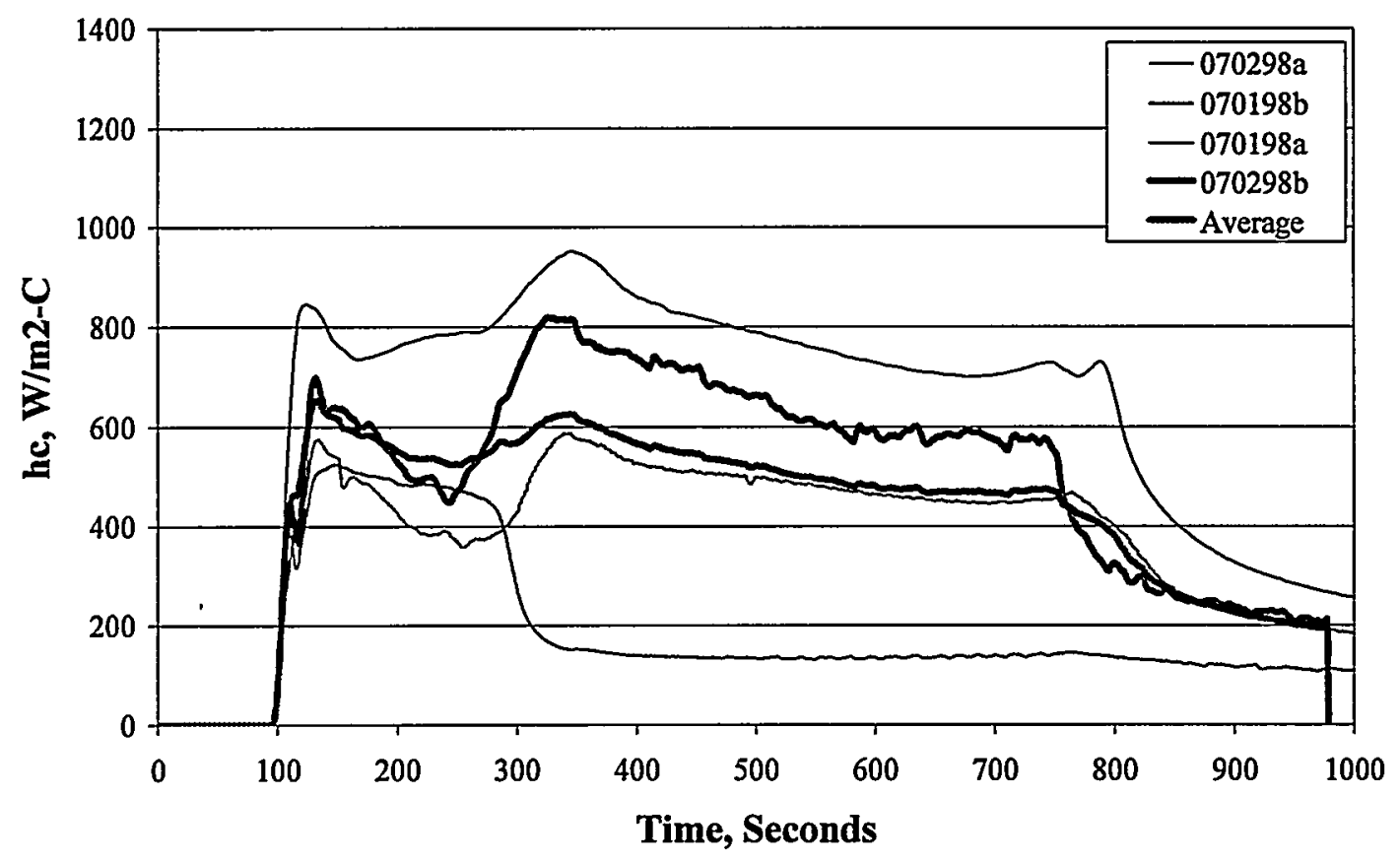

Figure 18. Heat Transfer Coefficients from Top Surface of $25.4 \mathrm{~mm}$ plates (45 degree inclination) 


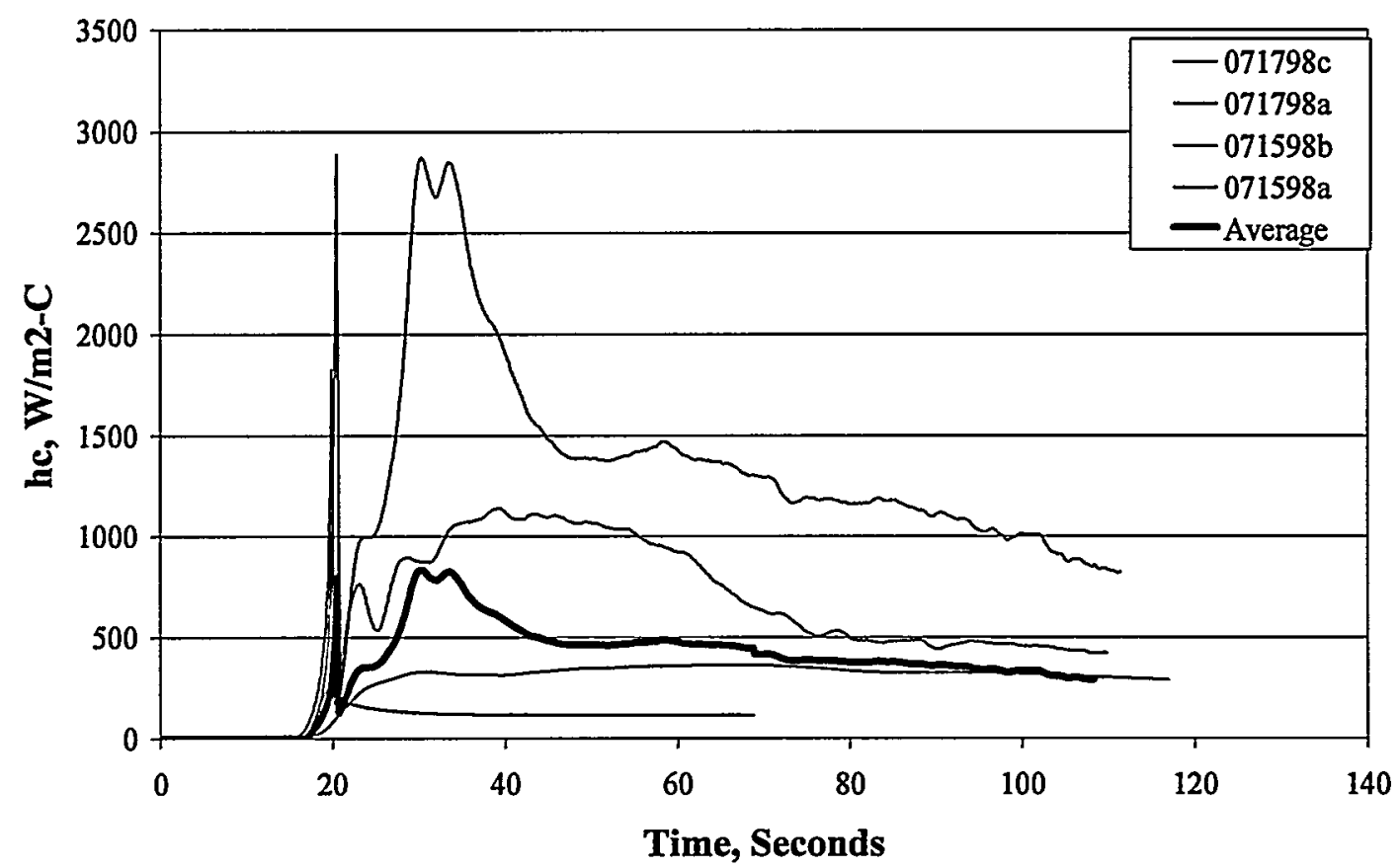

Figure 19. Heat Transfer Coefficients from "Bottom" Surface of $6.35 \mathrm{~mm}$ plates (vertical orientation)

0.25" Vertical Plates (Tops) 07/15/98 + 07/17/98

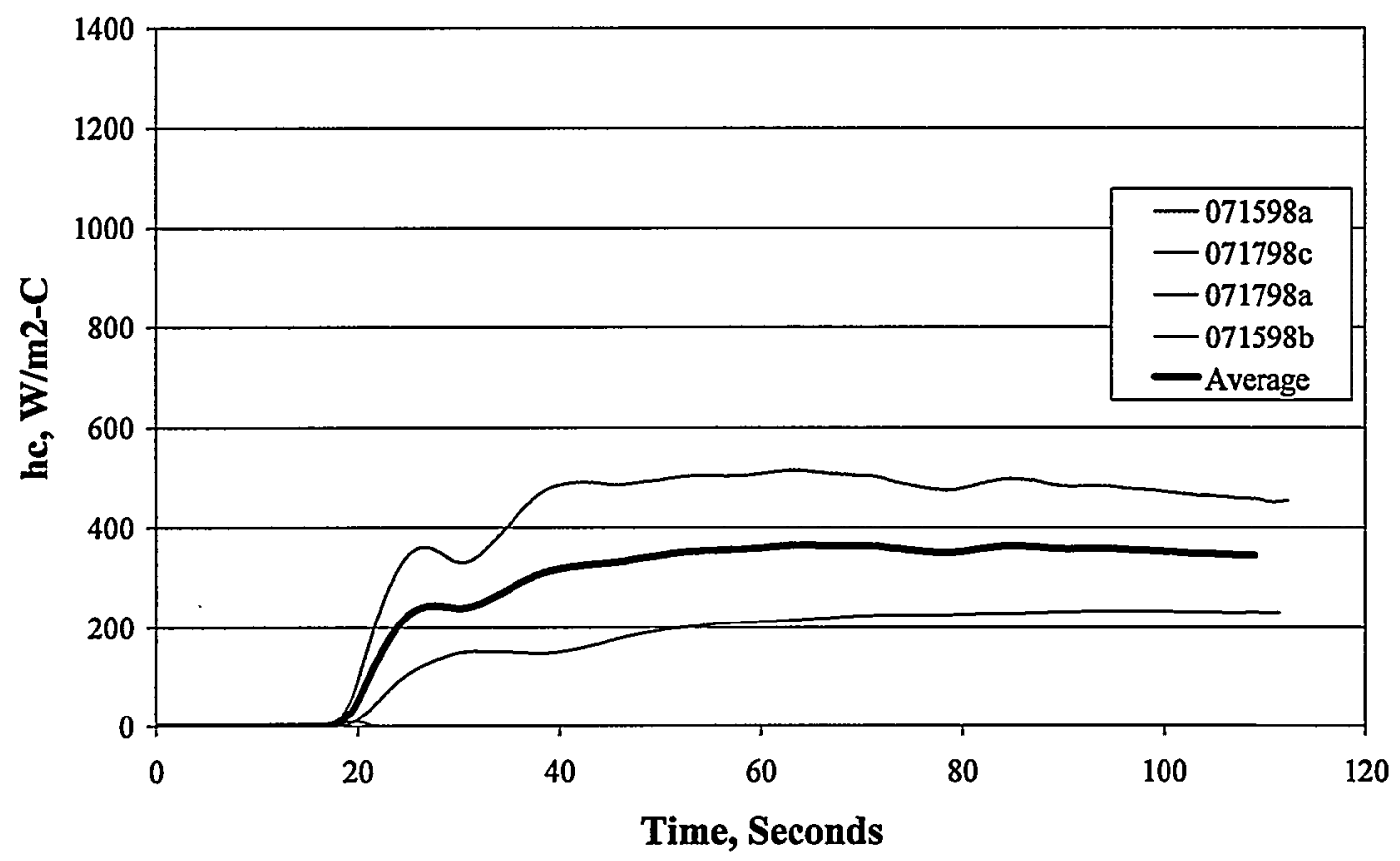

Figure 20. Heat Transfer Coefficients from "Top" Surface of $6.35 \mathrm{~mm}$ plates (vertical orientation) 


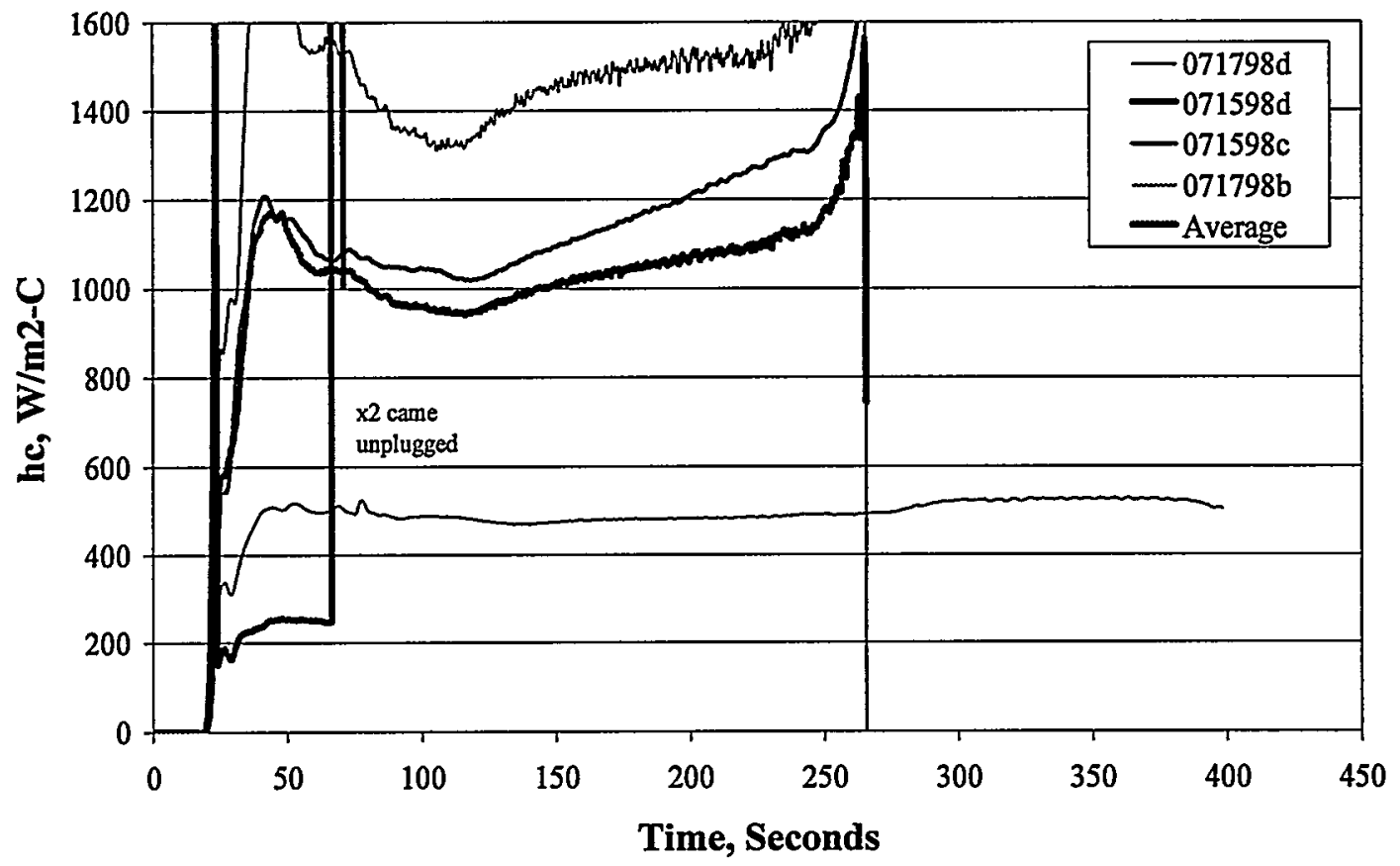

Figure 21. Heat Transfer Coefficients from "Bottom" Surface of $12.7 \mathrm{~mm}$ plates (vertical orientation)

0.5" Vertical Plates (Tops) 07/15/98 + 07/17/98

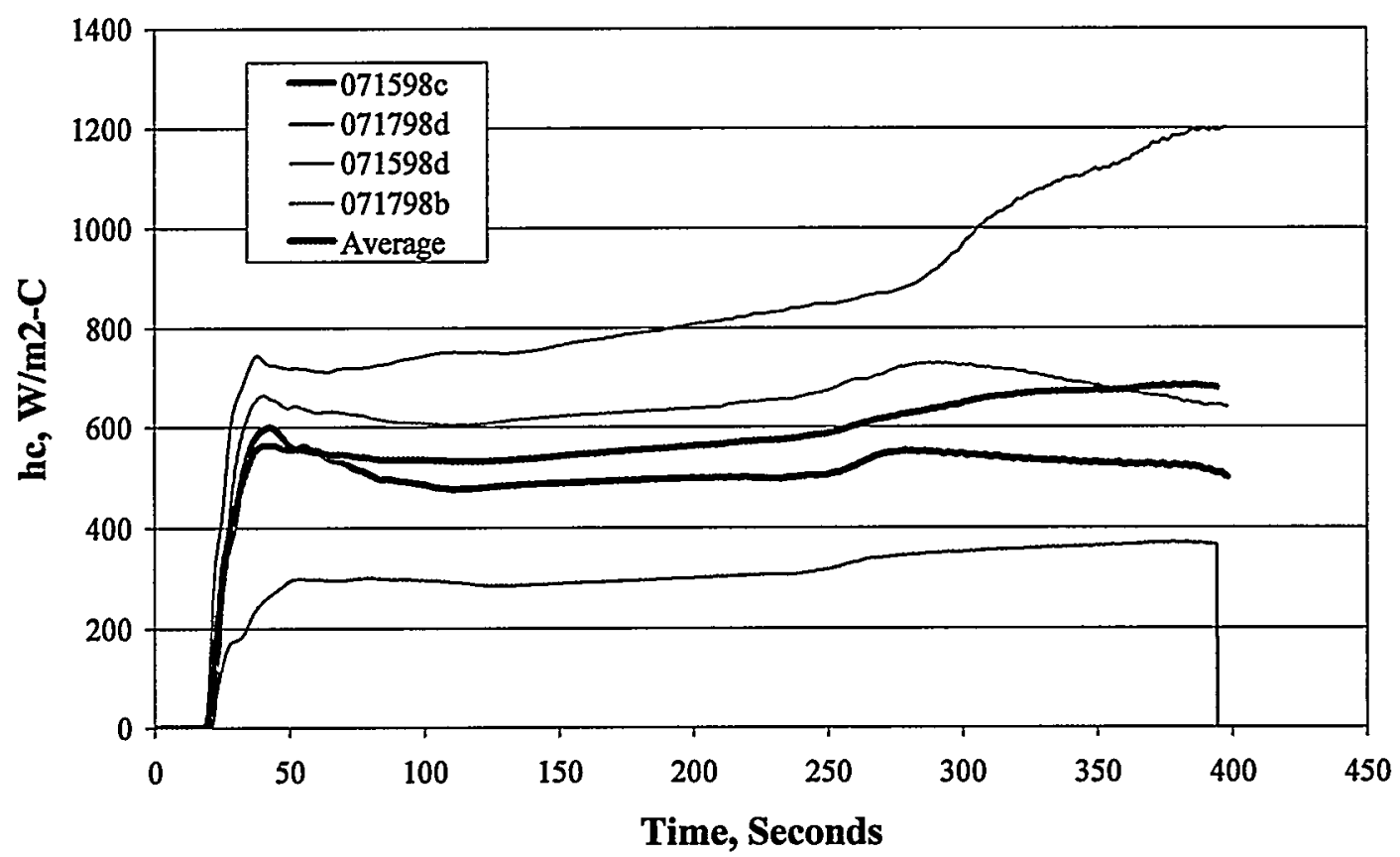

Figure 22. Heat Transfer Coefficients from "Top" Surface of $12.7 \mathrm{~mm}$ plates (vertical orientation) 


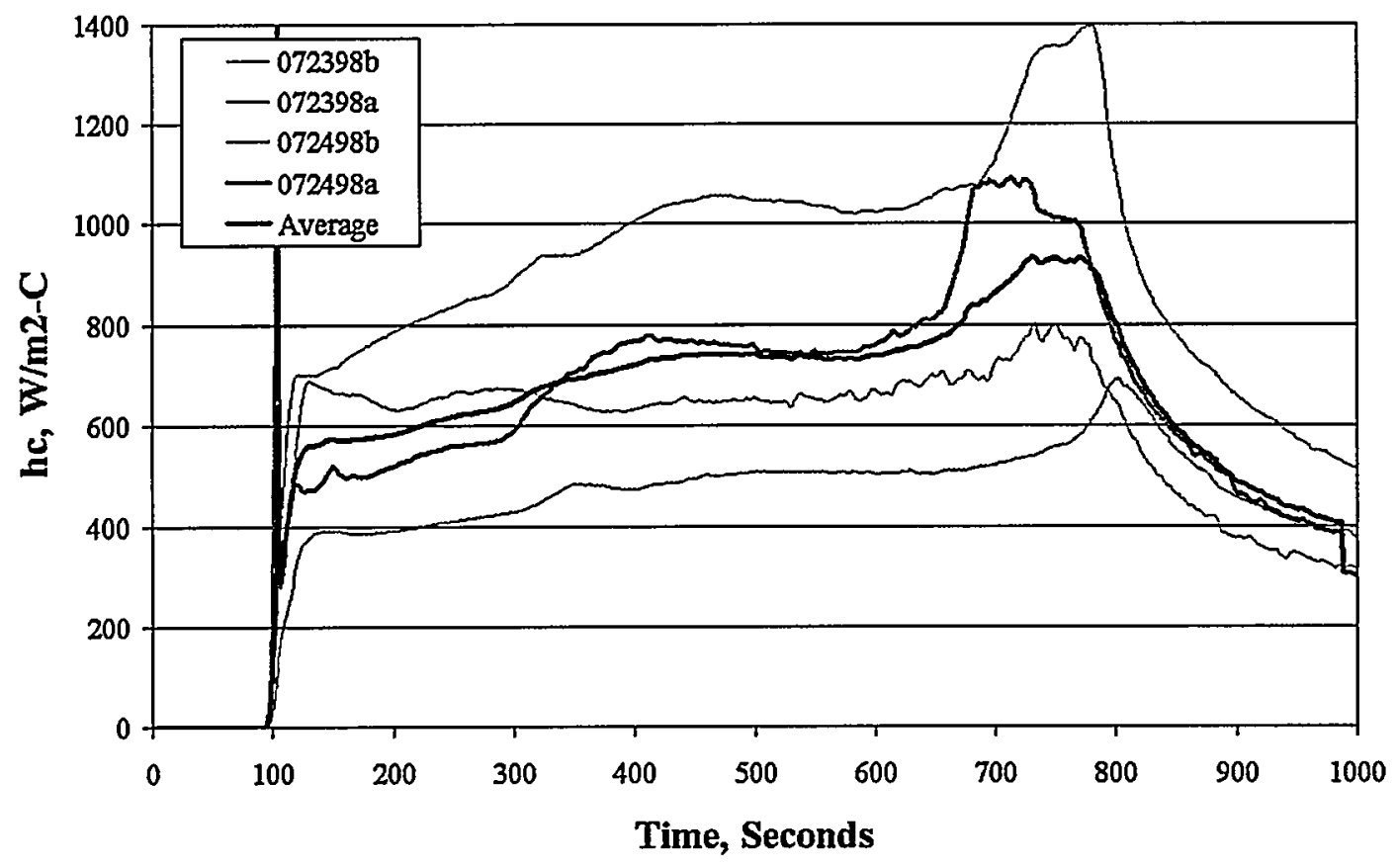

Figure 23. Heat Transfer Coefficients from "Bottom" Surface of $25.4 \mathrm{~mm}$ vertical plates 1.0" Vertical Plates (Tops) 07/23/98 + 07/24/98

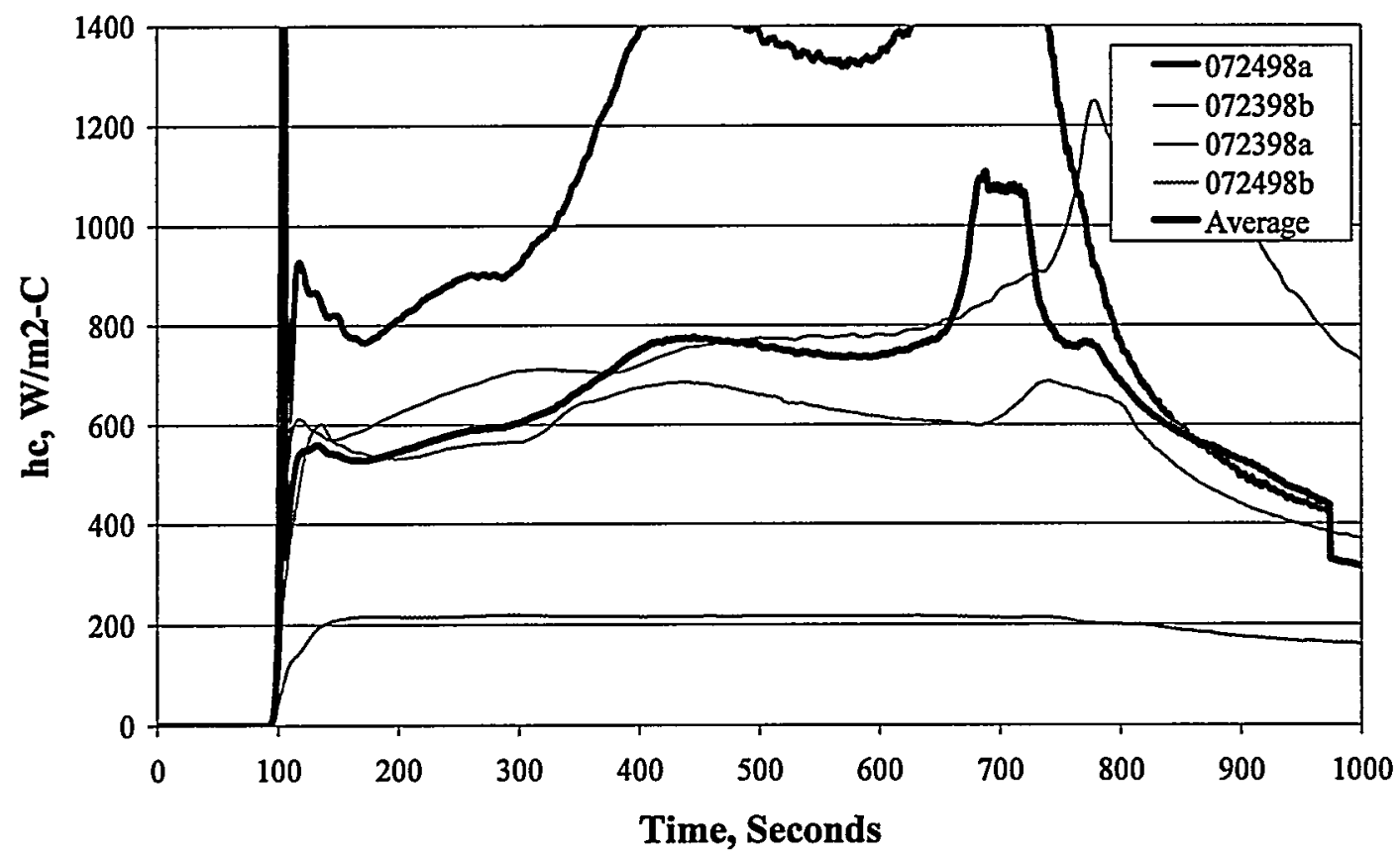

Figure 24. Heat Transfer Coefficients from "Top" Surface of $25.4 \mathrm{~mm}$ vertical plates 
0.25" Horizontal Plates (Bots) 07/29/98 + 07/30/98 + 08/12/98 +08/24/98

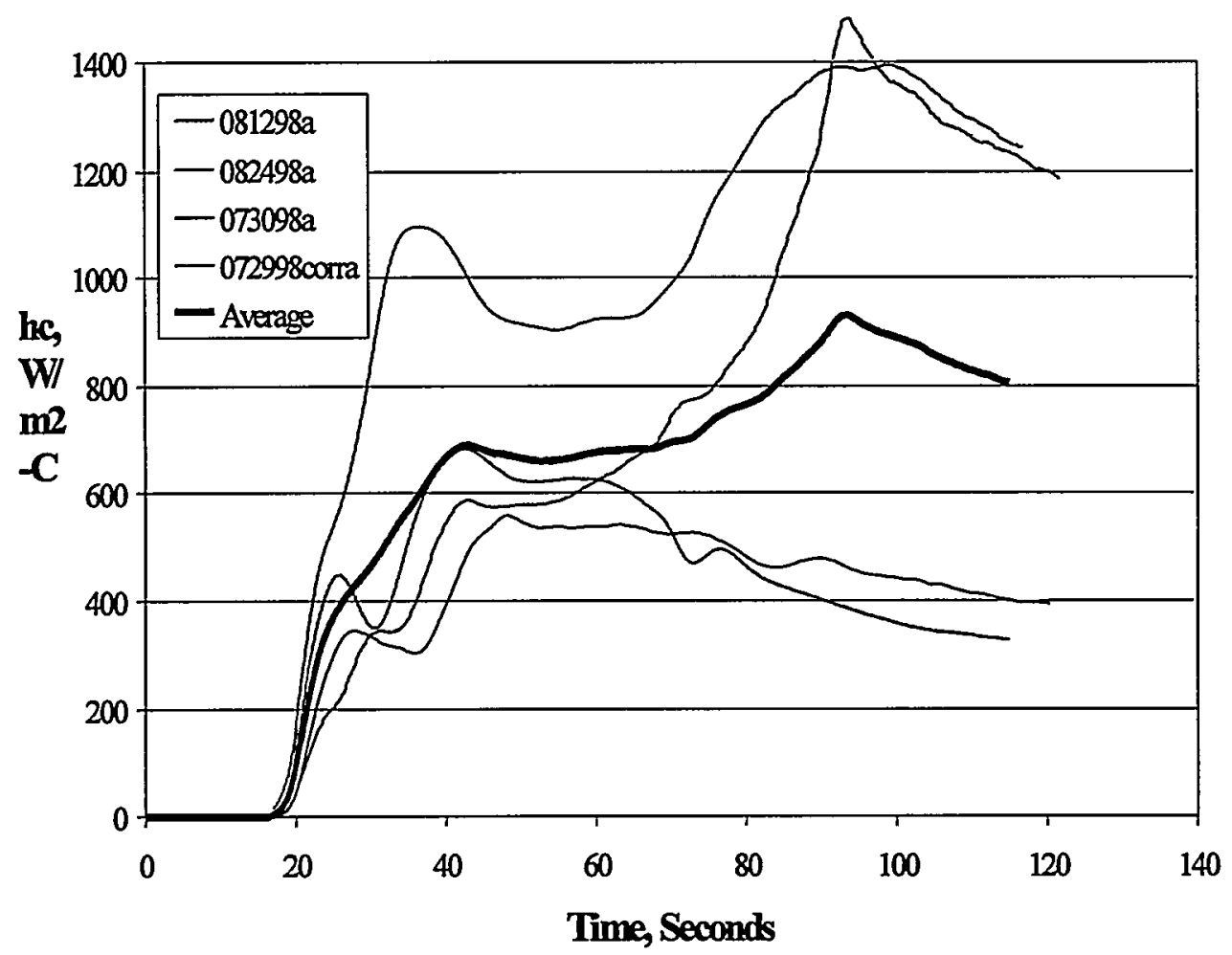

Figure 25. Heat Transfer Coefficients from Bottom Surface of $6.35 \mathrm{~mm}$ horizontal plates

0.25" Horizontal Plates (Tops) 07/29/98 + 07/30/98 + 08/12/98 + 08/24/98

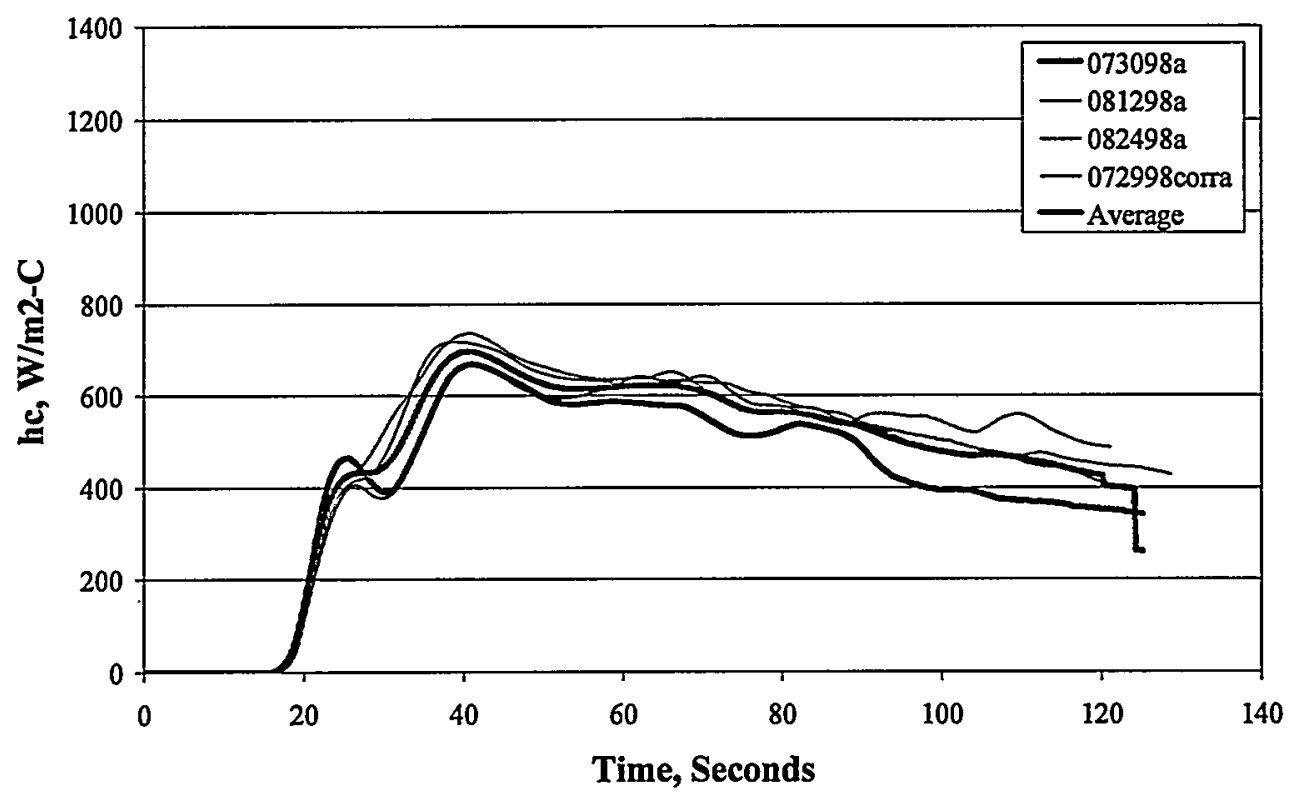

Figure 26. Heat Transfer Coefficients from Top Surface of $6.35 \mathrm{~mm}$ horizontal plates 
0.50" Horizontal Plates (Bots) 07/29/98 + 07/30/98 + 08/12/98 + 08/24/98

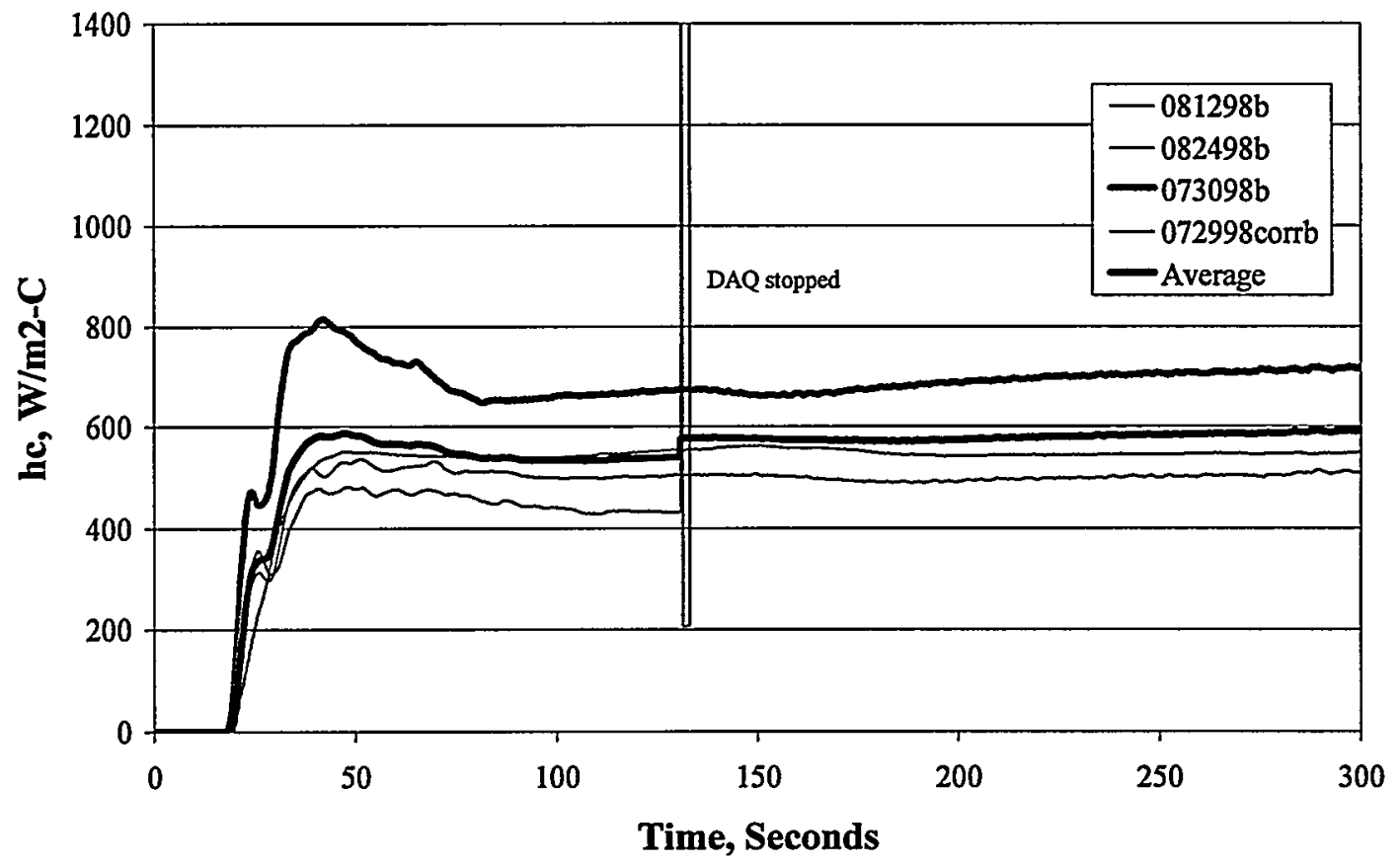

Figure 27. Heat Transfer Coefficients from Bottom Surface of $12.7 \mathrm{~mm}$ horizontal plates

0.50" Horizontal Plates (Tops) 07/29/98 + 07/30/98 + 08/12/98 + 08/24/98

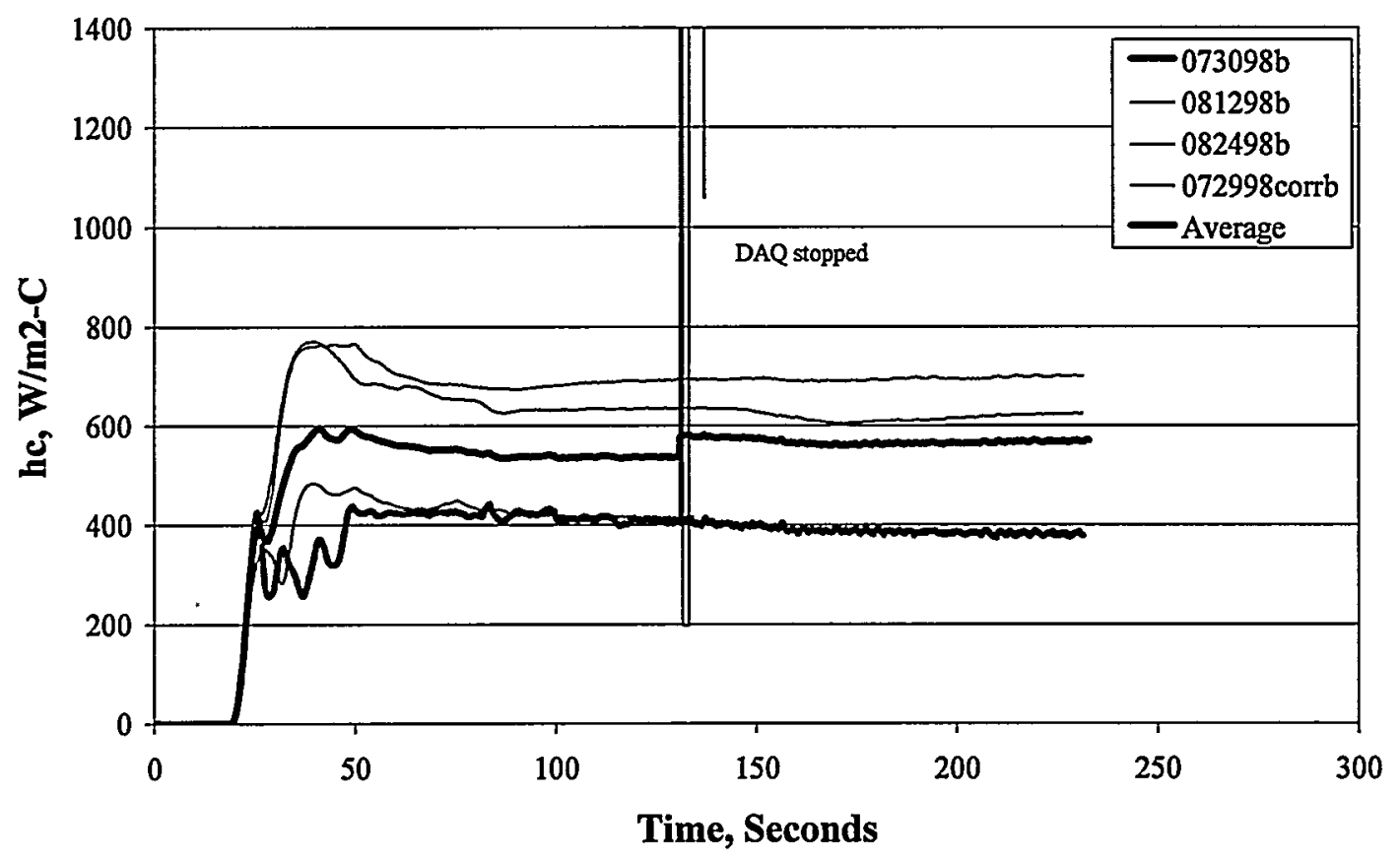

Figure 28. Heat Transfer Coefficients from Top Surface of $12.7 \mathrm{~mm}$ horizontal plates 


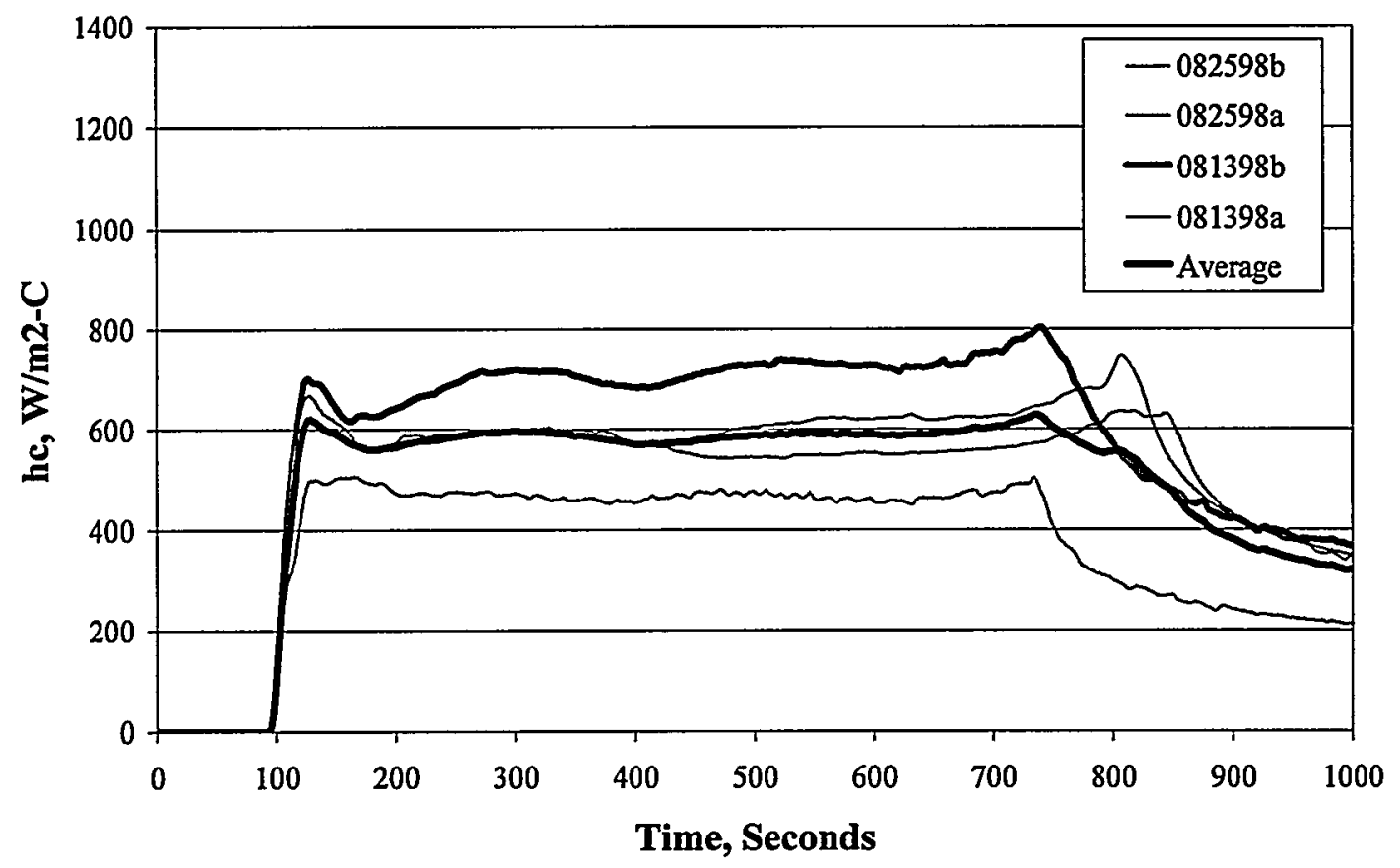

Figure 29. Heat Transfer Coefficients from Bottom Surface of $25.4 \mathrm{~mm}$ plates (horizontal orientation)

1.0" Horizontal Plates (Tops) 08/13/98 + 08/25/98

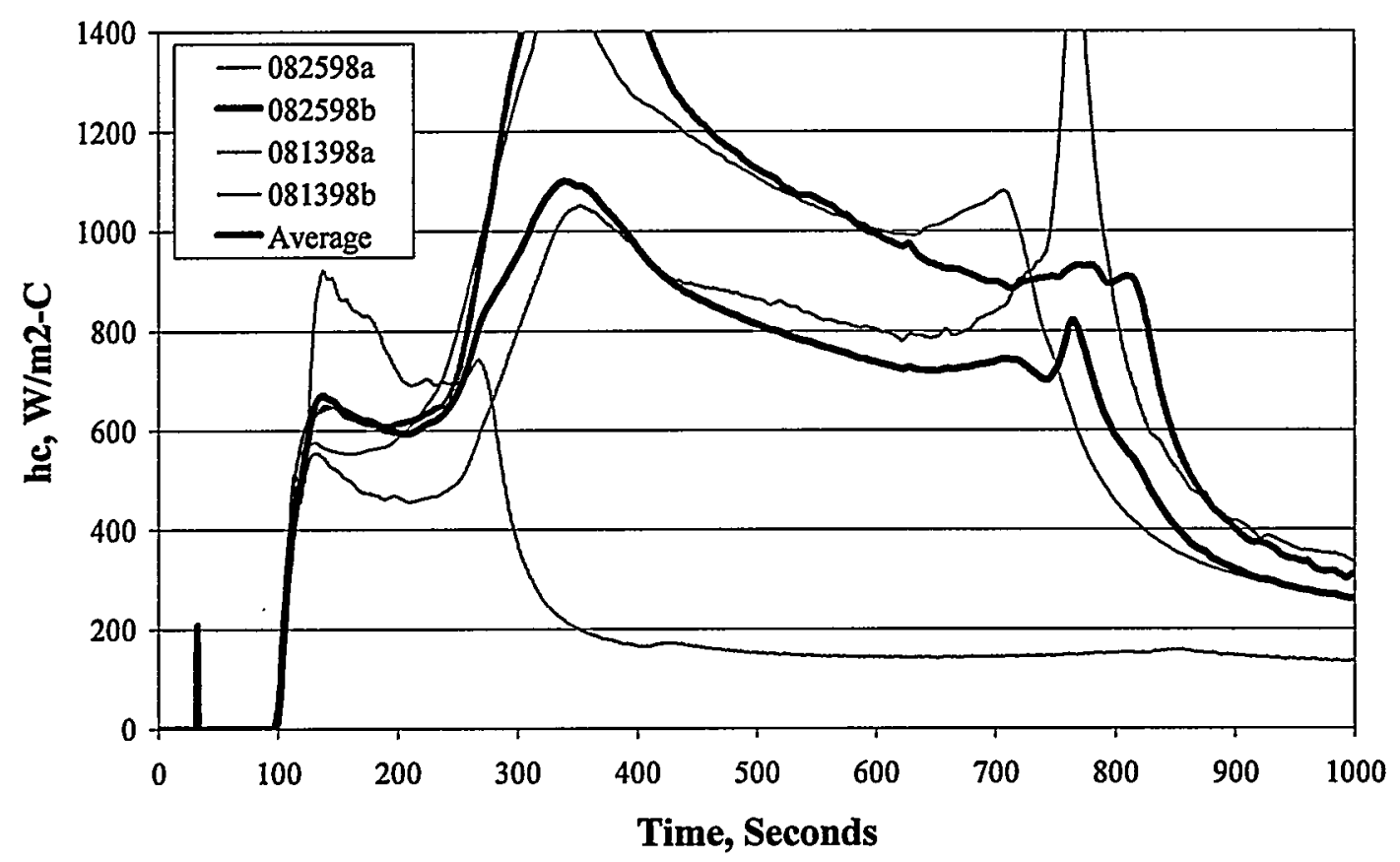

Figure 30. Heat Transfer Coefficients from Top Surface of $25.4 \mathrm{~mm}$ plates (horizontal orientation) 
configuration, the designation of "Top" and "Bottom" are not totally arbitrary, and refer to which half of the mold was built first (the "Bottom" was built first). The molds for these vertical castings were different from those for the horizontal and angled castings, and were gated from the top. The heat transfer coefficients for the vertical castings have a highly random character, as the filling of the mold can be different, depending on the angle of entry of the pouring stream into the mold, and the exact attitude of the mold (none were exactly 90 degrees to the gravity field), etc. On average, the heat transfer coefficients for the thickest sections tended to increase during solidification in vertically oriented plates, from $600 \mathrm{~W} / \mathrm{m}^{2}-\mathrm{K}$ to $700 \mathrm{~W} / \mathrm{m}^{2}-\mathrm{K}$ (from 100 to 700 seconds in Fig. 23 and 24). For the thinnest section, the heat transfer coefficient appears to be nearly constant around $500 \mathrm{~W} / \mathrm{m}^{2}-\mathrm{K}$ (Fig. 19) or $400 \mathrm{~W} / \mathrm{m}^{2}-\mathrm{K}$ (Fig. 20). For the intermediate thickness, the heat transfer coefficient in Fig. 22 suggests the value is nearly constant during solidification at about $550 \mathrm{~W} / \mathrm{m}^{2}-\mathrm{K}$.

A similarity can be noted between the interfacial heat transfer coefficients on the top surface of the $25.4 \mathrm{~mm}$ castings in the 45 degree (Fig. 18) and the horizontal (Fig. 30) configurations. In each case, on three out of four pours, the coefficient increased initially, then generally increased further after about 150 seconds. However, on one of the four pours, the heat transfer coefficient increased initially, then decreased after about 150 to 200 seconds. This decrease in heat transfer coefficient, which occurs on the upper surface of heavy sectioned castings about $25 \%$ of the time, may be associated with the formation of a gap (see Part II of this report).

\subsubsection{Heat Transfer Coefficients Versus Solidifying Metal Temperature}

The results depicted in Figures 17 through 30 are useful to gage the repeatability of the castings, but are not particularly useful for any computational purpose. In order to facilitate implementation into a computer program, the average values of interfacial heat transfer coefficient were plotted against the averaged value of the solidifying metal surface temperature. (This will be the average value of the four times two surface thermocouples on each side of the plate).

The results for the horizontal configuration can be seen in Figures 31, 32, and 33 for the thinnest, intermediate, and thickest sections, respectively. An interesting trend can be noted from these figures. For the thinnest section, the coefficients are the same until about the middle of the temperature range $(560 \mathrm{C}$ or so). Then the bottom coefficient remains high while the top decreases. For the intermediate thickness, the coefficients are about the same throughout the range for the top and bottom surfaces. But for the thicker section, the coefficients at the higher and lower temperatures are similar, but in the center the heat transfer coefficient on the top is much larger than on the bottom. The reason for this increased coefficient in the middle of the range for the thicker section is believed to be the evolution of significant amounts of gases from the resin in the thicker section. These warm gases are free to rise through the porous media above the plate, but form a stable layer below the plate. Thus, there is enhanced heat transfer from the upper surface due to the gas motion, and this results in a higher apparent heat transfer coefficient to account for the extra energy. This phenomenon does not occur at sections thinner than 
$12.7 \mathrm{~mm}$, as the total energy of the molten metal is significantly less.

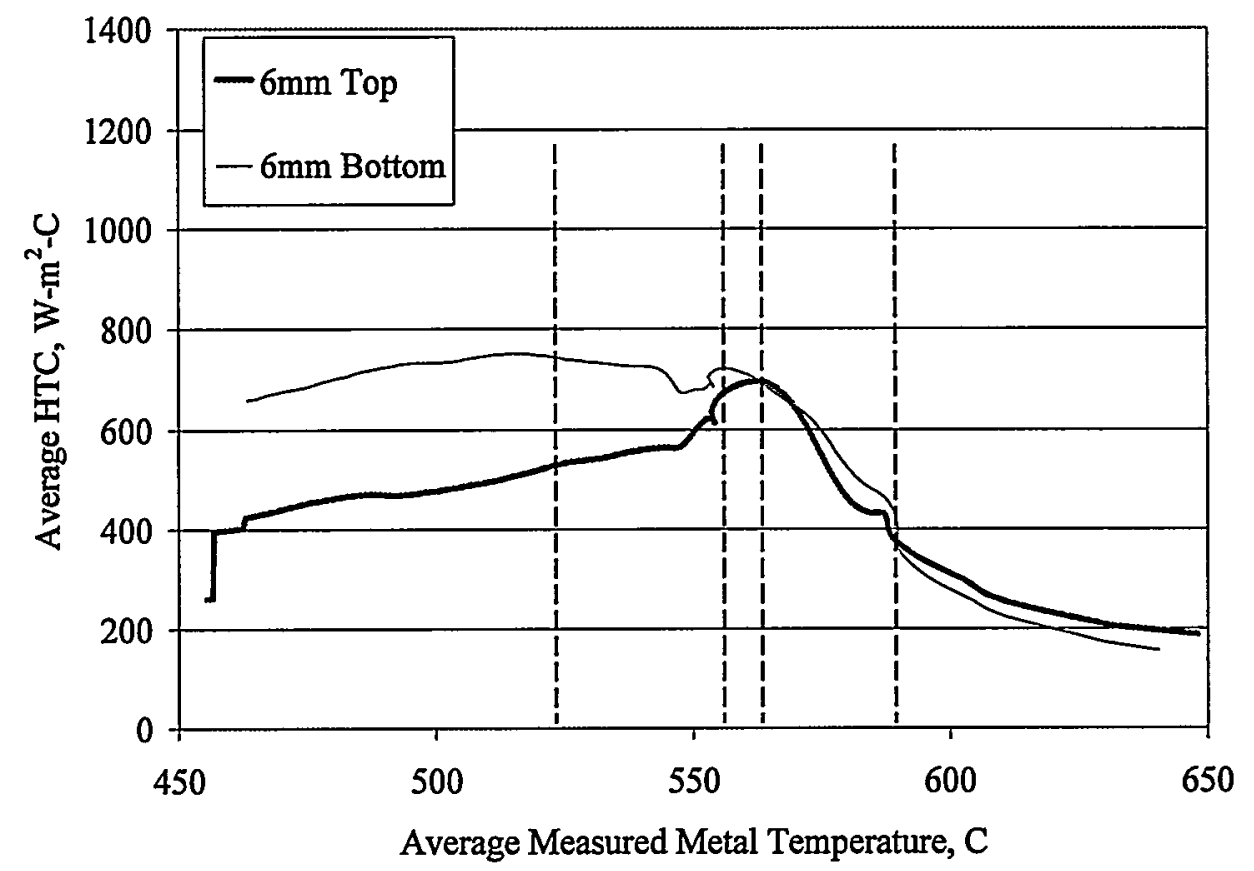

Figure 31. Average heat transfer coefficients for both surfaces of $6.35 \mathrm{~mm}$ casting (horizontal configuration)

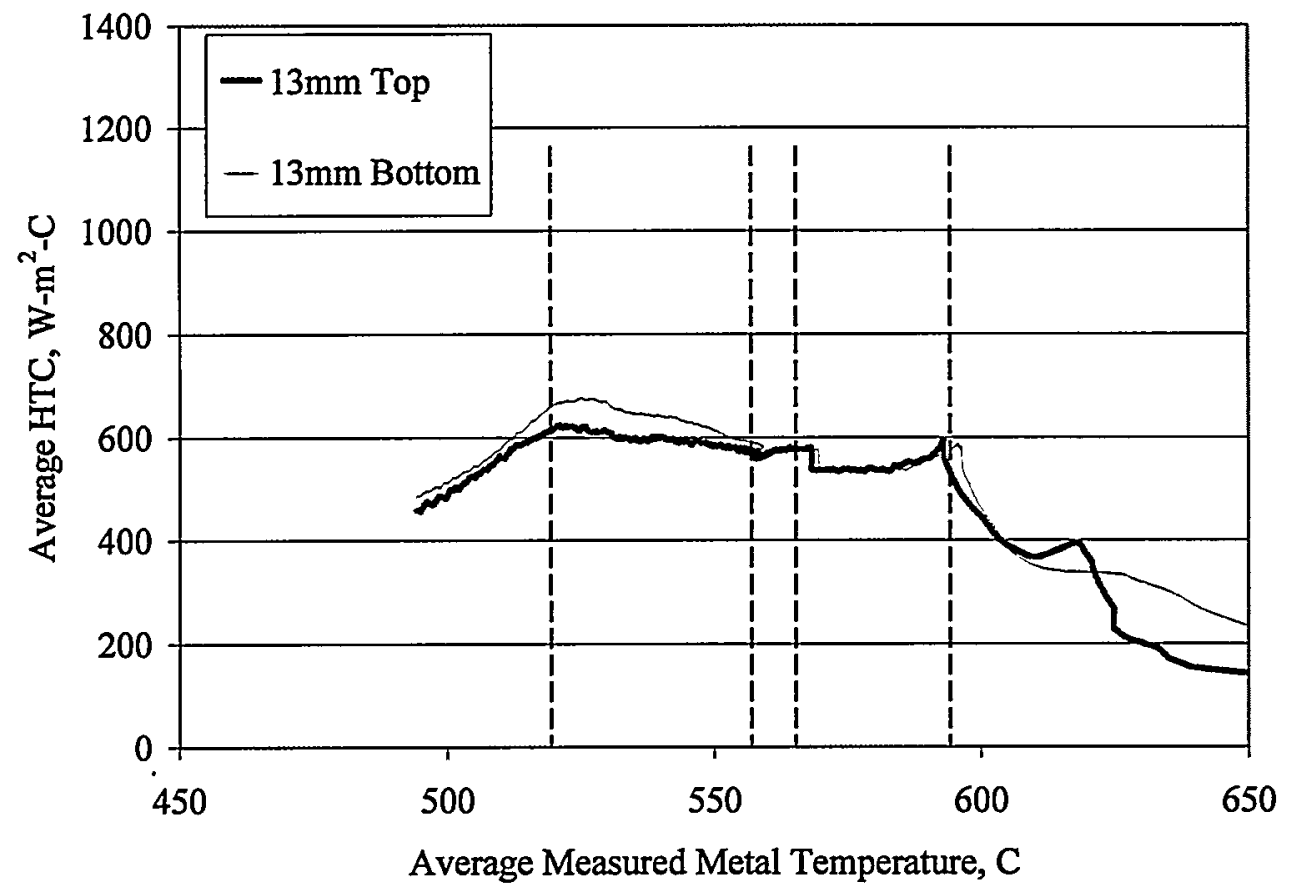

Figure 32. Average heat transfer coefficients for both surfaces of $12.7 \mathrm{~mm}$ casting (horizontal configuration) 


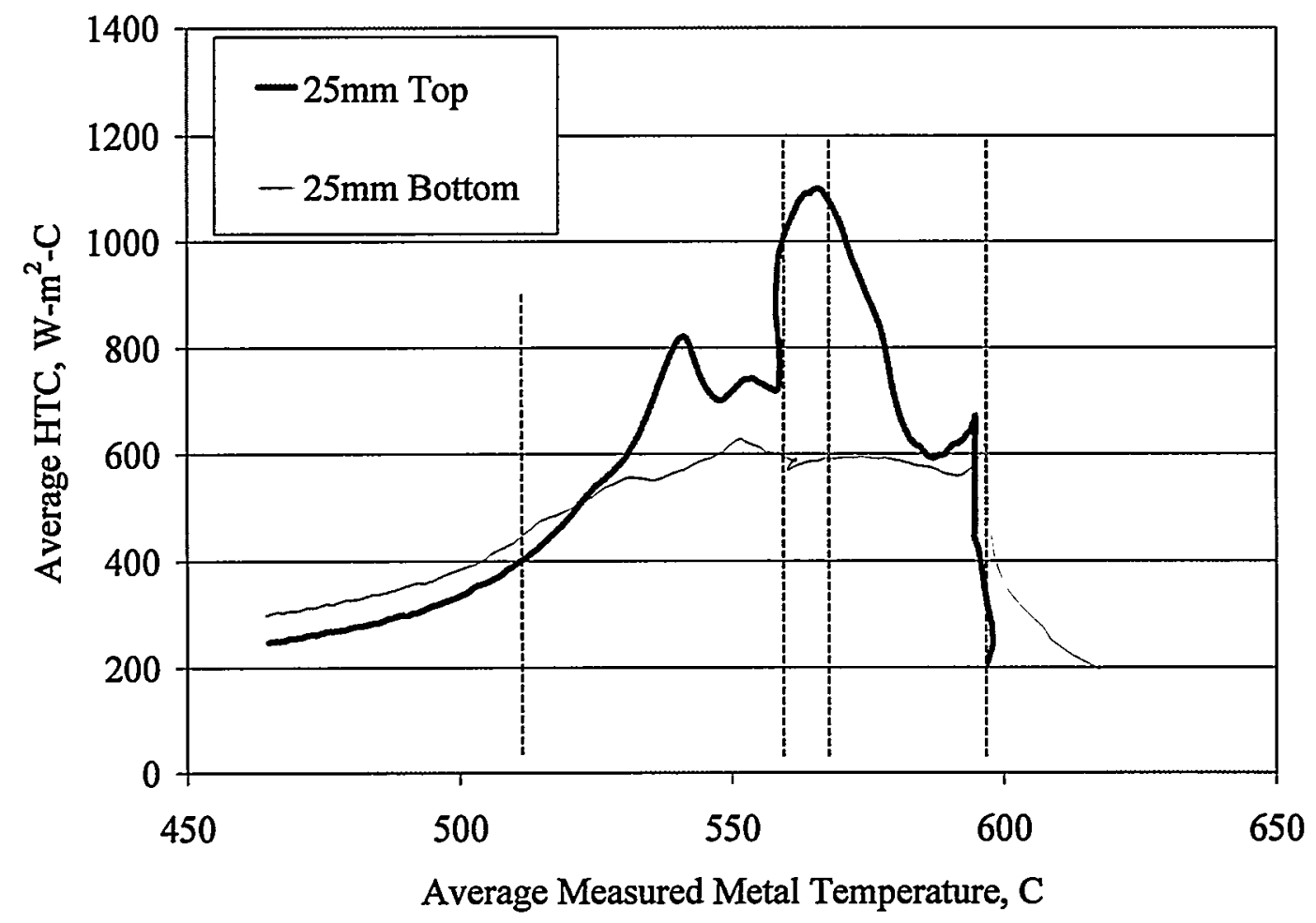

Figure 33. Average heat transfer coefficients for both surfaces of $25.4 \mathrm{~mm}$ casting (horizontal configuration)

\subsubsection{Solidification Process}

Some insight into the variation of the heat transfer coefficient during solidification can be gained by constructing the first derivative of the cooling curve. These are shown in Figures 33 through 35 for the three thicknesses in the horizontal configuration. On the right scale in each of these figures and on the upper curves the temperature histories of the solidifying plates are shown. These cooling curves are the average of all the thermocouples from the four plates. Note that the top and bottom surfaces are nearly duplicated for each case. The first derivative of these cooling curves was taken numerically using a simple finite difference technique, and these derivatives are shown as the lower curves in the figures (the left scale provides the magnitude).

In this cooling curve, there is some initial violent transient, but soon after the pouring begins the curve begins to follow a characteristic shape (see, for example, Backerud, et al, 1990, pages 3-6). The key points of reference are the beginning of solidification (nucleation of aluminum grains) which is marked by the first maximum (at about 23 seconds in Figure 33), the end of the dendritic growth phase at the first minimum (near $t=40$ seconds in Figure 33), and the completion of solidification marked by the last minimum (at about $t=90$ seconds in Figure 33). Also of note is the increase in the cooling rate caused by the nucleation of eutectic silicon crystals, which occurs at about $t=48$ seconds in Figure 33 . 


\subsection{5 inch Horizontal Plate}

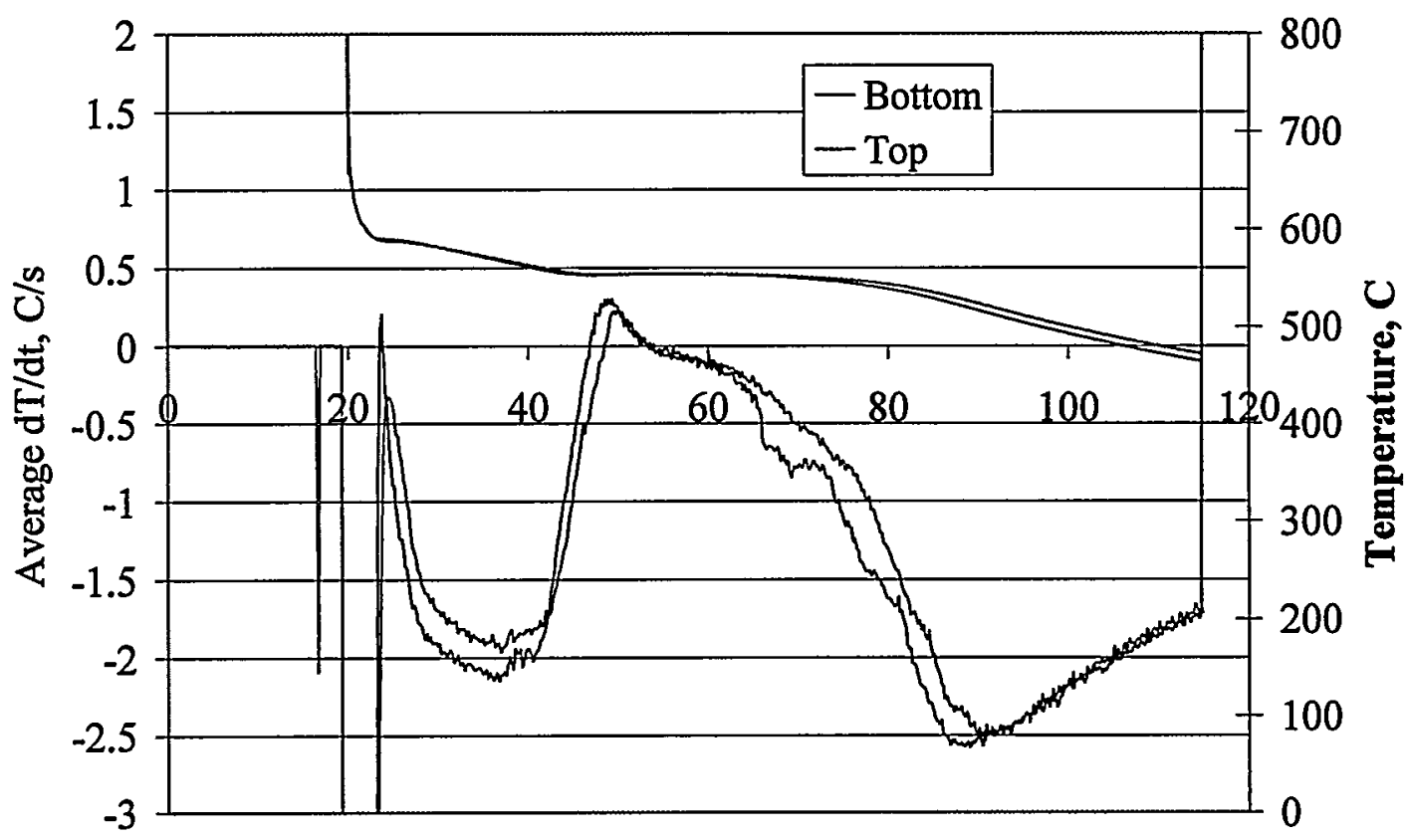

Time, $s$

Figure 34. Average cooling curves (upper lines, right scale) and their first derivatives (left scale) for $6.35 \mathrm{~mm}$ plates

\subsection{0 inch Horizontal Plate}

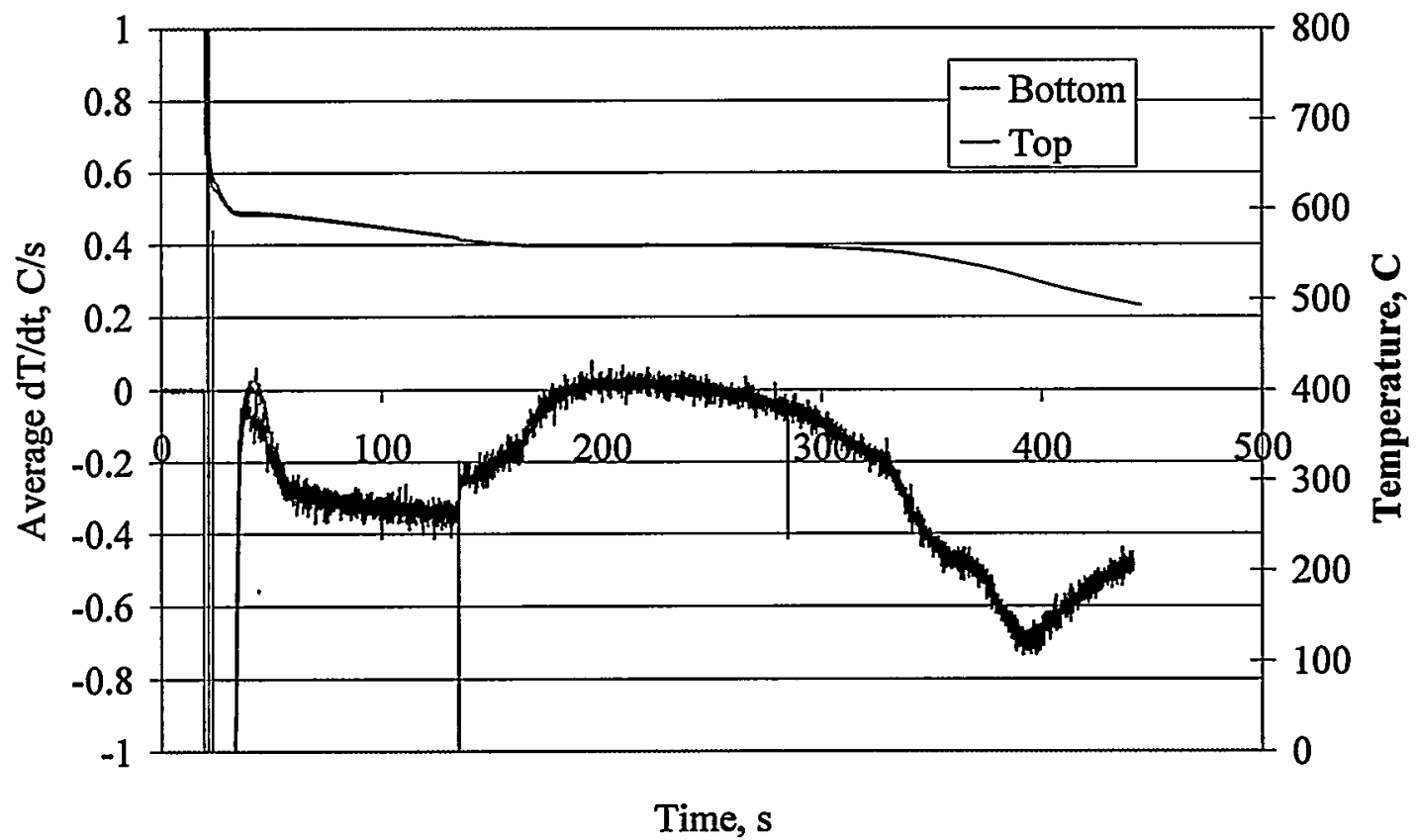

Figure 35. Average cooling curves (upper lines, right scale) and their first derivatives (left scale) for $12.7 \mathrm{~mm}$ plates 


\section{0 inch Horizontal Plate}

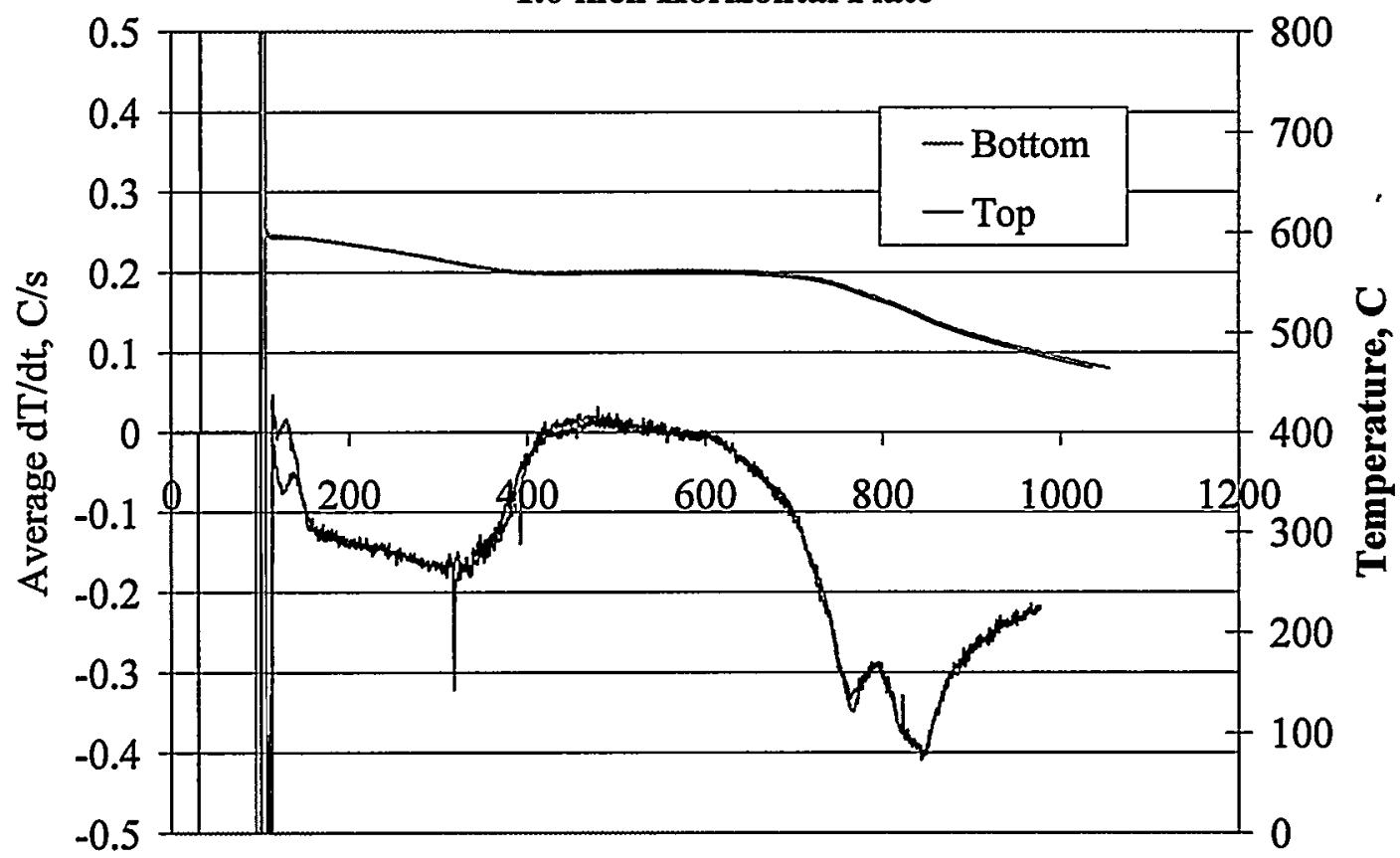

Time, $\mathbf{s}$

Figure 36. Average cooling curves (upper lines, right scale) and their first derivatives (left scale) for $25.4 \mathrm{~mm}$ plates

Table 3. Times and temperatures of significant points during solidification for each plate thickness (based on horizontal plates)

\begin{tabular}{|l|l|l|l|}
\hline & $6.35 \mathrm{~mm}$ & $12.7 \mathrm{~mm}$ & $25.4 \mathrm{~mm}$ \\
\hline $\begin{array}{l}\text { Beginning of } \\
\text { Solidification }\end{array}$ & $3 \mathrm{secs} / 588 \mathrm{C}$ & $1.5 \mathrm{secs} / 592 \mathrm{C}$ & $4 \mathrm{secs} / 595 \mathrm{C}$ \\
\hline $\begin{array}{l}\text { End of Dendritic } \\
\text { Growth }\end{array}$ & $20.3 \mathrm{secs} / 561 \mathrm{C}$ & $112 \mathrm{secs} / 563 \mathrm{C}$ & $228 \mathrm{secs} / 566 \mathrm{C}$ \\
\hline $\begin{array}{l}\text { End of Nucleation } \\
\text { of Silicon Crystals }\end{array}$ & $29.4 \mathrm{secs} / 554 \mathrm{C}$ & $172 \mathrm{secs}$ / 558 C & $337 \mathrm{secs} / 558 \mathrm{C}$ \\
\hline $\begin{array}{l}\text { End of } \\
\text { Solidification }\end{array}$ & $69 \mathrm{secs} / 525 \mathrm{C}$ & $377 \mathrm{secs} / 517 \mathrm{C}$ & $740 \mathrm{secs} / 510 \mathrm{C}$ \\
\hline
\end{tabular}

Table 3 shows the location of the key features of the solidification process for the three thicknessess abstracted from Figures 34 through 36 . Both the time since pouring began and the temperature at which the event occurs are noted in the table. The temperatures at which these events occur are marked with a vertical dashed line in Figures 31 through 33.

It is interesting to note that the maximums in the heat transfer coefficients for both the thickest and thinnest sections correspond to the release of energy associated with the appearance of silicon in the alloy. The reason for the lack of appearance of a peak in the intermediate thickness is not obvious. 
Note that for both surfaces of the $12.7 \mathrm{~mm}$ plate and the lower surface of the $25.4 \mathrm{~mm}$ plate that the heat transfer coefficient is approximately constant during solidification. However, for the $6.35 \mathrm{~mm}$ plate, results from both surfaces indicate that the heat transfer coefficient changes during most of the solidification process. 


\subsection{Values of Heat Transfer Coefficient During Solidification}

We propose the following ad hoc models for the interfacial heat transfer coefficient during solidification. These models use the temperature of the solidifying surface as the correlating parameter, and are therefore well-suited for implementation into computer programs or simulations.

The models we propose are in the form of piecewise linear functions. These functions are specified as tabular data (temperature, heat transfer coefficient pairs) and are modelled after the horizontal configurations depicted in Figures 31 through 33. Table 4 gives the defining pairs for each of the thicknesses considered in this study.

Table 4. Heat Transfer Coefficient Models for three section thicknesses. Temperature is in degrees Celsius and heat transfer coefficient is in W/m $\mathrm{m}^{2}-\mathrm{C}$

\begin{tabular}{|c|c|c|c|c|c|c|c|}
\hline \multicolumn{4}{|c|}{$25 \mathrm{~mm}$ thickness } & \multicolumn{4}{|c|}{$13 \mathrm{~mm}$ thickness } \\
\hline \multicolumn{2}{|c|}{ Bottom } & \multicolumn{2}{|l|}{ Top } & \multicolumn{2}{|c|}{ Bottom } & \multicolumn{2}{|l|}{ Top } \\
\hline$T$ & $h$ & $T$ & $H$ & $T$ & $h$ & $T$ & $H$ \\
\hline 595 & 600 & 595 & 600 & 592 & 560 & 592 & 560 \\
\hline 566 & 600 & 585 & 600 & 580 & 535 & 580 & 535 \\
\hline 558 & 600 & 566 & 1090 & 563 & 580 & 563 & 580 \\
\hline 510 & 435 & 558 & 730 & 558 & 580 & 558 & 580 \\
\hline \multirow[t]{3}{*}{465} & 300 & 535 & 730 & 517 & 670 & 517 & 600 \\
\hline & & 510 & 395 & 495 & 490 & 495 & 460 \\
\hline & & 465 & 250 & & & & \\
\hline \multicolumn{4}{|c|}{$6 \mathrm{~mm}$ thickness } & & & & \\
\hline \multicolumn{2}{|c|}{ Bottom } & \multicolumn{2}{|l|}{ Top } & & & & \\
\hline$T$ & $h$ & $T$ & $h$ & & & & \\
\hline 588 & 420 & 588 & 420 & & & & \\
\hline 561 & 705 & 561 & 705 & & & & \\
\hline 554 & 725 & 554 & 660 & & & & \\
\hline 525 & 750 & 545 & 565 & & & & \\
\hline \multirow[t]{2}{*}{465} & 665 & 525 & 530 & & & & \\
\hline & & 465 & 430 & & & & \\
\hline
\end{tabular}

The highlighted cells in Table 4 correspond to the points of the solidification process identified in Table 3 and noted in Figures 31 through 33. In some cases, additional points needed to be added to define the heat transfer coefficient model. These are the unshaded boxes in Table 4. 


\subsection{Green Sand}

Castings in green sand are important in the foundry industry. Part of our research effort was directed toward this important process. The computation of heat transfer coefficients in green sand is complicated significantly by the evaporation, movement, and condensation of water in the sand matrix.

\subsection{Analysis}

We developed a simple inverse heat conduction method for use in green sand based on the zonal model of Tsai (1986). In this approach, we analyzed the dry sand zone only, but considered the effect of the moving moisture wall on the overall process. The procedure is detailed a conference publication (Woodbury and Ke, 1999).

\subsubsection{Zonal Model}

The zonal model is stated in detail by Tsai, et al. (1986). Figure 37 shows the different regions in green sand. The analysis for each of these zones is described below.

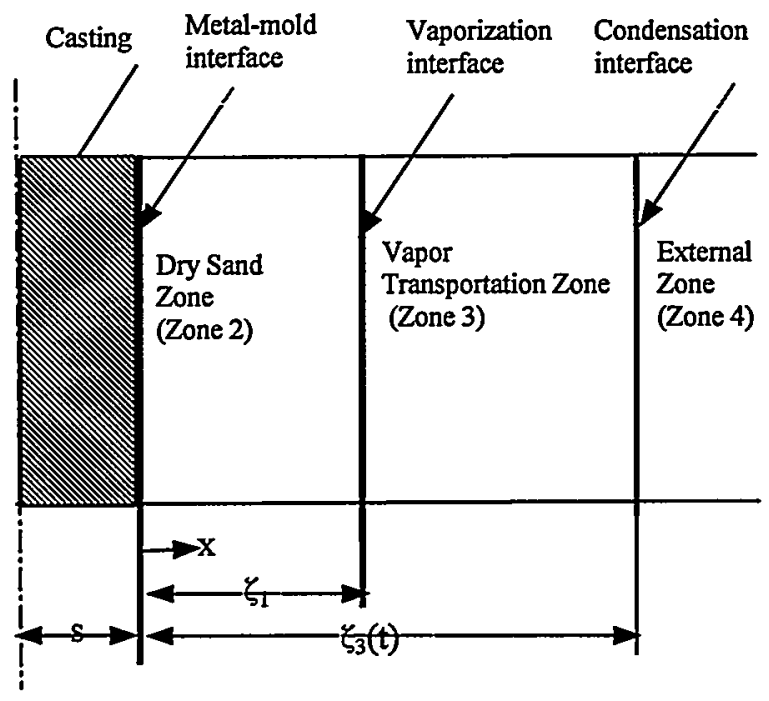

Figure 37. Schematic representation of different zones in green sand

In zone 1 , the molten metal solidifies and releases heat. This heat release is not important in the inverse algorithm, so its description is omitted here. Further details are available in Tsai, et al. (1986).

In zone 2 , the dry sand zone, simple one dimensional transient heat conduction problem must be solved, but the location of the right boundary location changes with time. The mathematical description for this zone is:

$$
C_{2}(T) \frac{\partial T_{2}}{\partial t}=k_{2}(T) \frac{\partial^{2} T_{2}}{\partial x^{2}} \quad \text { in } 0 \square x \square \square_{1}(t)
$$




$$
\begin{array}{lr}
-k_{2} \frac{\partial T_{2}}{\partial x}=q(t) & \text { on } x=0 \\
T_{2}=T_{c} & \text { on } x=\square_{1}(t) \\
-k_{2} \frac{\partial T_{2}}{\partial x}=L \rho_{2} W \frac{\partial \zeta_{1}}{\partial t} & \text { on } x=\square_{1}(t)
\end{array}
$$

In zone 3, the vapor transportation zone, the temperature remains constant and the governing equation in this zone is simple,

$$
T_{3}=T_{c},
$$

where $T_{c}$ is vaporization temperature of water.

The water content in the vapor transportation zone is assumed to be:

$$
W=W_{0}+\left\{W_{0} C_{W}+\left(100-W_{0}\right) C_{d}\right\}\left(T_{c}-T_{2 i}\right) / L .
$$

from

Using an energy balance equation, the vapor transportation interface can be calculated

$$
\zeta_{3}(t)=\frac{W}{W-W_{0}} \zeta_{1}(t) \cdot(23)
$$

The external zone can be treated as a semi-infinite region. However, it is not important in the inverse algorithm as the sensor cannot be located in this zone.

The inverse problem is solved similar to that for the purely diffusive case. That is, the sensitivity of the measurement to the surface heat flux is computed mathematically by differentiating Eqs. (20) and solving the associated sensitivity problem. These sensitivity coefficients are used in a modified form of Eq. (11) to compute the surface heat flux. Details are provided in Woodbury and $\mathrm{Ke}(1999)$.

\subsection{Effect of Moisture Movement}

A very important phenomena inherent to moisture-bearing porous media such as green sand molds is that the sensitivity coefficient will be zero for some time for sensor locations away from the heated surface. It makes the inverse algorithm very challenging. The reason for this is the presence of the vaporization region that masks the effect of the surface heat flux. As heat is added continuously to green sand, the vaporization interface and the condensation interface will move with time.

The reason for the initial zero value of $\mathbf{X}$ for subsurface sensors can be shown as follows. At first, the sensor will be in the external zone. In this zone, the mathematical descriptions are:

$$
\begin{array}{lr}
\frac{\partial T_{4}}{\partial t}=a_{4} \frac{\partial^{2} T_{4}}{\partial x^{2}} & \text { in } \square_{2}(t) \square x \square \square \\
T_{4}=T_{c} & \text { on } x=\square_{2}(t) \\
T_{4}=T_{i} \text { on } x=\square &
\end{array}
$$


It contains no information about the left boundary heat flux. The derivative of the above equations with respect to $q_{0}$ is zero, this means the temperature is independent of the heat flux, so $X_{q_{0}}$ is zero.

As the condensation interface moves and reaches the sensor location, the sensor falls into the vapor transport zone. In this zone, no matter how large the heat flux is, the temperature still remains constant. Its value is equal to the vaporization temperature of water, so that the temperature is also independent of the heat flux added at the left boundary and the sensitivity remains zero. Only after the vaporization interface passes over the sensor will the sensor be located in the dry sand zone and from then on, the sensitivity will be non-zero and it will increase with time.

In summary, the best place for the sensor to be located is on the active surface. If the sensor is located below the surface, there will be a period of time (proportional to the depth of the sensor) when no information about heat flux is available. There will be loss of information of the heat flux until the sensor falls into the dry sand zone.

Woodbury and Ke (1999) showed that a heat flux based inverse solver can give good results for the heat flux at the surface if the temperature sensor is located near the active surface. We are in the process of developing a heat transfer coefficient-based inverse heat conduction algorithm to compute the heat fluxes directly.

\subsection{Results}

We have obtained some results from experimental data for castings in green sand. Figure 38 shows one of these cases. Note that the heat flux is visually smooth, and that the small oscillations in the heat flux at around 200 seconds translate into noticeable sawtooths in the heat transfer coefficient. Also, we see variability in the results for repeated cases not unlike that seen in resin-bonded sand. Therefore, we are in the process of perfecting a heat transfer coefficientbased inverse algorithm to compute these results. These results will be the subject of a research report to the American Foundrymen's Society in 2000. 


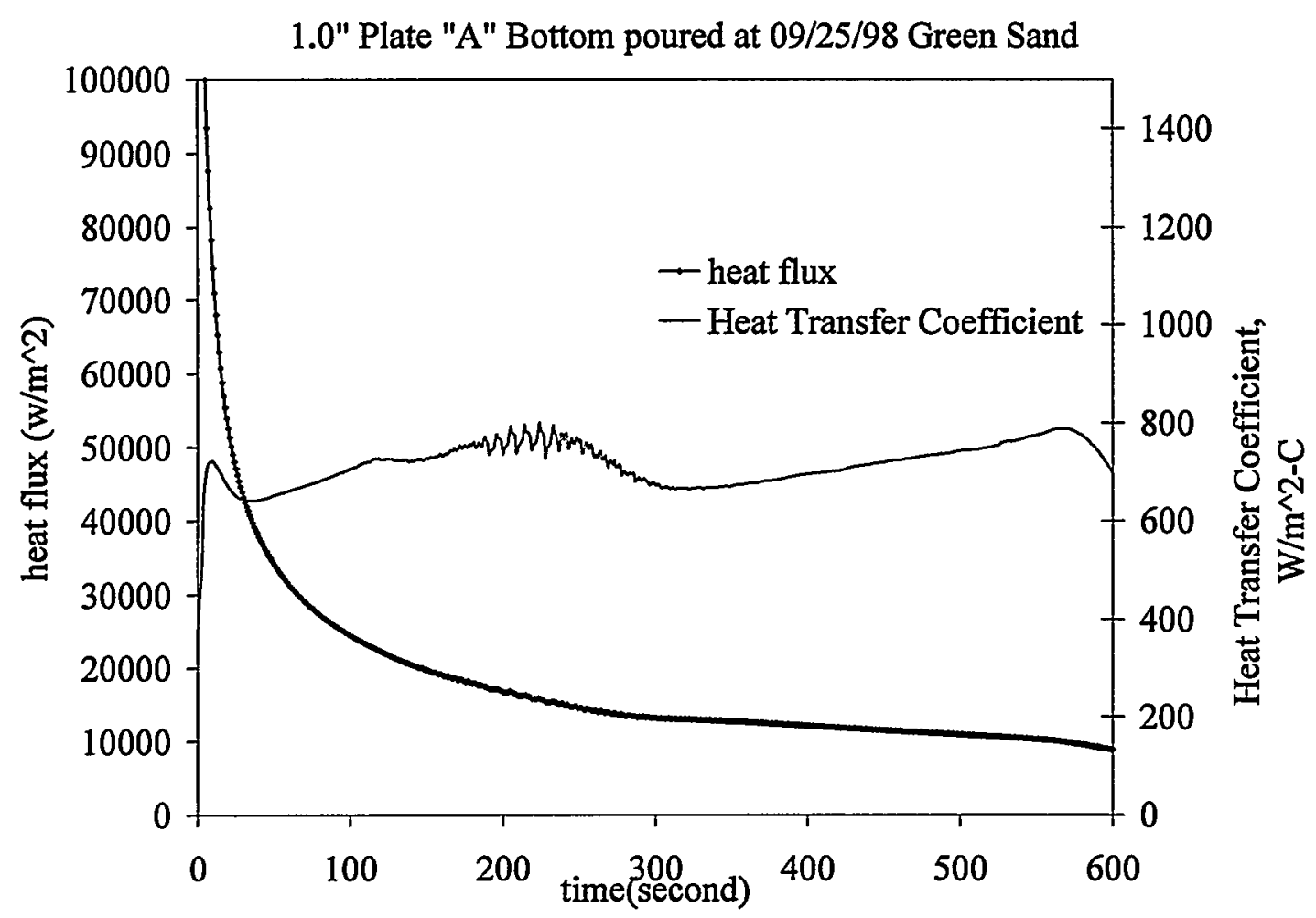

Figure 38. Results from Heat Flux Based Analysis for green sand casting 


\subsection{Conclusions}

Thermocouples implanted in low conductivity media (such as sand) form preferential heat flow paths and can significantly alter the temperature field. Our calculations indicate that errors in readings could be on the order of $50 \mathrm{C}$. Thermocouples should run parallel to the isotherms in mold to minimize this error.

We have developed an inverse heat conduction methodology to determine the interfacial heat transfer coefficients in resin-bonded sand castings. The method assumes a purely conductive mold medium, which is an approximation. The evolution and migration of gases decomposed from the resin appears to play a significant role in the process.

Our results indicate that for horizontally oriented plates, the heat transfer coefficients during resin-bonded sand casting of aluminum (A356) are on the order of $600 \mathrm{~W} / \mathrm{m}^{2}-\mathrm{K}$. For section thicknesses of $12.7 \mathrm{~mm}$ or greater, the interfacial heat transfer coefficient is approximately constant during the solidification process. For thinner sections, the heat transfer coefficient varies through most of the solidification process, and it can nearly double in magnitude.

For vertically-oriented plate castings, the heat transfer coefficients are highly variable, but appear to be on the same order of magnitude $\left(600 \mathrm{~W} / \mathrm{m}^{2}-\mathrm{K}\right)$. This suggests that simple surface orientation itself does not appear to dramatically affect heat transfer coefficients. For the thickest section, it does appear that there is a trend for increasing heat transfer coefficients during solidification. Also, differences were found on coefficients on the upper and lower surfaces of horizontal castings, which we attribute to the role of gases from resin decomposition.

We have developed an inverse heat conduction algorithm to compute the heat fluxes from castings in green sand. The heat transfer coefficients obtained from the algorithm are not reproducible, and a heat transfer coefficient-based method is being perfected. 


\subsection{References}

Bäckerud, L., Chai, G., and Tamminen, J., 1990, Solidification Characteristics of Aluminum Alloys - Volume 2, Foundry Alloys, AFS/Skanaluminum.

Beck, James V., "Thermocouple Temperature Disturbances in Low Conductivity Materials," Transactions of the ASME: Journal of Heat Transfer, May, 1962, pp. 124-132.

Beck, J. V., 1968, "Surface Heat Flux Determination using an Integral Method," Nuclear Engineering and Design, Vol. 7, pp. 170-178.

Beck, J. V., and Arnold, K. J., 1977, Parameter Estimation in Engineering and Science, John Wiley, New York.

Beck, J. V., Blackwell, B., and St. Clair, C., 1987, Inverse Heat Conduction: Ill-posed Problems, Wiley.

Beck, J. V., 1998, private communication.

Hensel, E., 1991, Inverse Theory and Applications for Engineers, Prentice-Hall.

Hwang, J.-C., Chuang, H.-T., Jong, S.-H., and Hwang, W.-S., "Measurement of Heat Transfer Coefficient at Metal/Mold Interface During Casting," AFS Transactions, Vol. 102, 94-16, 1994, pp. 877-883.

Junkins, J. L., 1978, An Introduction to Optimal Estimation of Dynamical Systems, Sijthoff \& Noordhoff, The Netherlands.

Kubo, K. and Pelkhe, R. D., 1985, "Thermal Properties of Molding Sands," AFS Transactions, 85-66, pp. 44.

Stoltz, G., 1960, "Numerical Solution to an Inverse Problem of Heat Conduction for Simple Shapes," ASME Journal of Heat Transfer, Vol. 82, pp. 20-26.

Tsai, H. L., Chiang, K. C., Chen, T. S.; 1986, "Movement of Moisture Front and Alloy Solidification in Green Sand Casting," AFS Transactions, pp. 191-196.

Tseng, A. A., Chen, T. C., and Zhao, F. Z., 1995, "Direct Sensitivity Coefficient Method for Solving Two-Dimensional Inverse Heat Conduction Problems by Finite-Element Scheme," Numerical Heat Transfer, Part B, Vol. 27, pp. 291-307

Murio, D. A., 1993, The Mollification Method and the Numerical Solution of Ill-Posed Problems, Wiley-Interscience.

Woodbury, K. A., and Premanand B., 1996, "Finite Element Solution of the 2D-IHCP: Application to Steady-State Problems," Second International Conference on Inverse Problems in Engineering, Engineering Foundation, June 9-14, Le Croisic, France.

Woodbury, K. A., Chen, Y., Parker, J. K., and Piwonka, T. S., 1998, "Measurement of Interfacial Heat Transfer Coefficients between Aluminum Castings and Resin-bonded Sand Molds," AFS Transactions, 98-116, pp 705-711. 
Woodbury, K. A., and Ke, Q., "A boundary Inverse Heat Conduction Problem with phase change for moisture-bearing porous medium," presented at the Third International Conference on Inverse Problems in Engineering, Port Ludlow, WA, June 13-18, 1999. 
DOE/ID/13363-F

Part II

\title{
The Relationship between Casting Distortion, Mold Filling and Interfacial Heat Transfer in Sand Molds
}

\author{
DE-FC07-95ID13363 \\ FINAL REPORT - PART II \\ GAP FORMATION MEASUREMENTS
}

J.K. Parker and T.S. Piwonka

Metal Casting Technology Center

The University of Alabama

Tuscaloosa, AL

September 30, 1999 


\begin{abstract}
The development of an inductive, eddy-current proximity sensor for noncontact measurement of the gap formation has been the focus of this portion of the project. The high temperature $\left(\sim 1000^{\circ} \mathrm{F}\right)$ encountered during the initial stages of the foundry pours has a significant detrimental effect on the output of the proximity sensor. A lengthy development effort significantly reduced this temperature sensitivity. The results of the experiments were such that we were unable to determine the formation of a gap between the metal and the mold as solidification progressed. These results are consistent with the results from the interfacial heat transfer measurement work (see Part I of this report).
\end{abstract}

\title{
1.0 INTRODUCTION
}

\subsection{Objective}

Traditional wisdom has long maintained that an air gap is often formed at the moldcasting interface during solidification of metals. The presence of this air gap would greatly reduce the heat transfer between the metal and mold materials. An air gap is considered to be formed chiefly by relative movements between the casting and the mold caused by the thermal contraction and expansion during solidification of the metal. The interfacial heat transfer coefficients during the solidification of castings would be affected by the formation of a gap between the mold and casting. The objective of this research was to determine the thickness of the air gap formation as a function of time. High precision measuring devices with $\pm 0.0025 \mathrm{~mm}$ resolution over an approximately $0.25 \mathrm{~mm}$ total gap width $( \pm 0.0001$-inch resolution over 0.010 inch total gap) were desired for the measurement of the gap formation. The high temperature environment due to the presence of the molten metal further greatly complicates the measurement of the gap formation.

\subsection{Constraints on the Measurement System}

The gap formation measurement can be achieved either by contact methods or by noncontact methods. The contact method of measuring the gap formation is done by using two quartz rods, one fused to the metal specimen and other to the mold. The quartz rods project through the exterior of the casting where relative displacement measurements are made. These external position measurements are typically made with either dial indicators [7] or with linear variable differential transformers (LVDTs) [8]. The main disadvantage of this method is that they are not suitable for on-line measurements in a production facility as they need special preparation for the casting mold (drilling hole, mounting rods, attaching LVDTs or dial gages, etc.). This method may not be accurate, because the presence of both friction and clearance between the quartz rod and the hole in the mold could have localized effects on the formation of the air gap and its measurement, and also because the quartz rod chills metal prematurely.

In the non-contact methods of the gap formation measurement, the above problem can be eliminated. Typical non-contact methods of displacement measurement employ capacitance gages or eddy current sensors. Of all the non-contact displacement measurement methods, eddy current techniques are relatively inexpensive compared to other techniques [1], are suitable for 
accurate measurements, and have the capability of operating under extreme conditions. Because of the variation in the inductance of the coil, eddy current methods are used to determine material homogeneity and other material characteristics like surface flaws as a non-destructive testing technique. The flow of eddy current is impeded by the material's electric resistance, which reflects characteristics such as hardness and chemical composition. Therefore eddy current measurements reveal various properties of materials [12]. They can also be used as proximity or displacement measuring sensors. The simple construction of the sensor makes it easy to manufacture. The eddy current method of gap formation measurement involves the induction of eddy currents in a conductive test object by a suitable distribution of impressed currents and the detection of the resultant field [2].

The points discussed above motivated the selection of the eddy current technique for the determination of the gap formation as a function of time.

\subsection{Literature Review}

A brief review of literature available for both eddy current proximity sensors and air gap formation in castings is given below.

Eddy current techniques are among the oldest methods of checking surface flaws and other material properties like hardness and chemical composition [12]. Theoretical analysis of eddy current impedance in semi-infinite medium was done by Cheng [3]; the main interest of this study was directed towards the reflected impedance of the coil due to the presence of a semiinfinite medium. Dodd and Deeds [4] developed an analytical closed form solution to the eddy current probe-coil problems. This was a theoretical approach towards the eddy current techniques. Their theoretical solution was experimentally verified by constructing coils, which were fabricated by winding the wire on an annular section with a rectangular cross-section. Thickness and conductivity of metallic layers from eddy current measurements are presented by Moulder, Uzal, and Rose [1]. The eddy current probe developed by them is an air-core circular coil with rectangular cross-section. The reason for not employing a coil of such shape in the present research is that the winding of the magnet wire over such coil is a difficult task to achieve. Experimental measurements of the eddy current signal in a conducting half space in the presence of flaws were done by Long, Toomsawasdi, and Zaman [2]. The experiment aims at investigation of change in impedance of the eddy current coil near a conducting half space as a function of lift-off distance.

Bissonnette and Cloutier [5] presented a method of air gap measuring system, in which the main point of interest was to monitor the air gap in hydroelectric generators using the capacitance techniques.

The selection of a suitable switching circuit has been to determine the resistance and frequency simultaneously in the study of the effects of the temperature on the eddy current proximity sensor. Masters [6] developed a circuit for temperature compensation of eddy current probes to determine the gap in bearings of cryogenic machines.

The contact method of determination of gap formation was demonstrated by Shahverdi, Farhadi, Karimitaheri, Davami, and Asgari [7]. This method of gap formation determination is 
done by using two quartz rods; however, the results obtained may not be accurate because of the presence of friction or clearance between the mold and the quartz rods. Nishida, Droste, and Engler [8] developed an apparatus for measurement of casting and mold displacement. This method also employs quartz rods in the experiments. This method was tested on both cylindrical and flat castings. The gap formation in aluminum- $13 \%$ silicon cylindrical castings was evaluated by Hou and Pehlke [9]. They present the gap width at various times in a 3.6 inch diameter cylindrical casting. A dried silica sand mold was used.

\subsection{Air Gap Formation Measurement Methods}

Contact and non-contact methods are discussed below, with emphasis on eddy current technique of gap formation measurement.

\subsubsection{Contact Methods}

The traditional method of measuring the gap formation with the contact method approach is done by using two quartz rods, one fused to the metal specimen and other to the mold. These quartz rods project through the exterior of the casting, where the relative displacement measurements are made typically with either dial indicators or linear variable differential transformers (LVDTs). The experimental procedure, using the contact method, employed to measure the displacement of the metal and the mold during solidification is discussed in this section.

The layout of the experimental setup, used to measure the gap formation using the contact method, is shown in Figure 1.1.
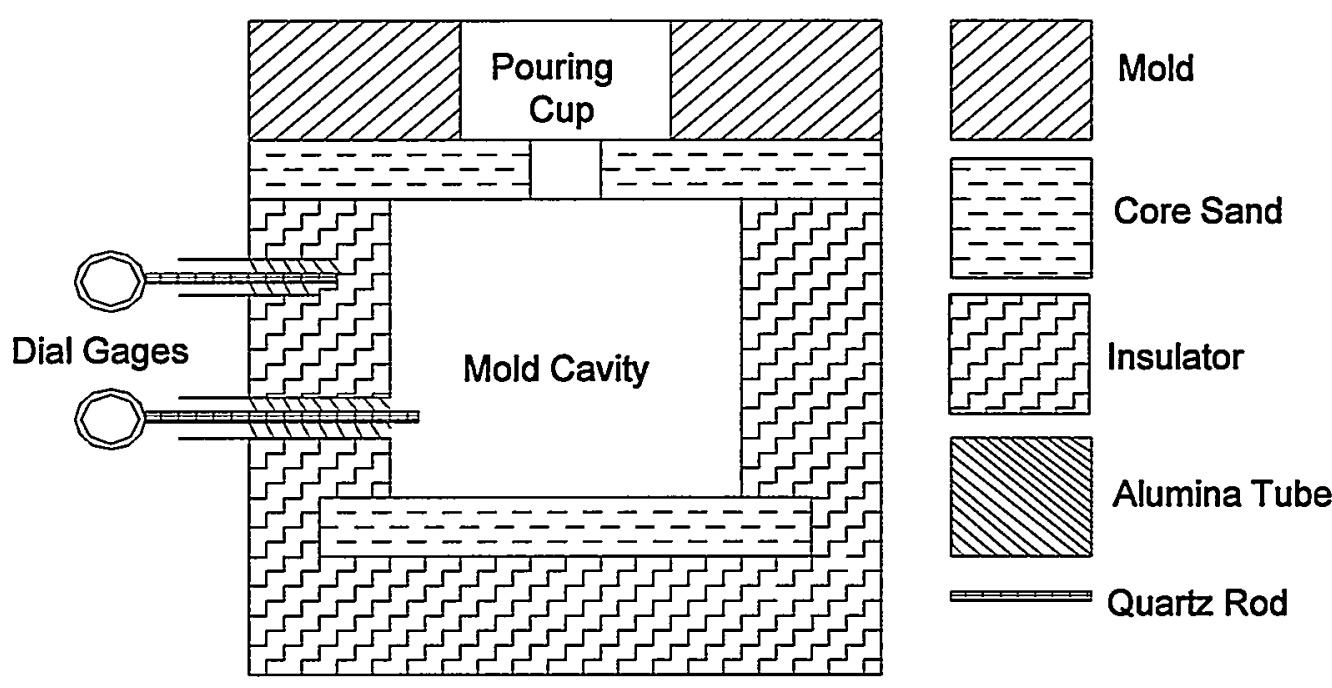

Figure 1.1. Gap measuring technique using contact methods

The experimental setup incorporates two quartz probes that are inserted into the mold and casting. Each of the quartz rods is connected to a sensitive dial gage. The interfacial gap is derived from the expansion of the mold and the contraction of the solidified metal. The width of the gap is measured as the difference between the location of the casting and the inner surfaces of the mold, which varies with time [7]. 
As mentioned earlier, this method of measuring the width of gap formation is not suitable for on-line measurements in a production facility, as it needs special preparation of the mold. This method may not be accurate due to the presence of clearance or friction between the quartz probes and the hole in the mold and also due to the chilling effect of the sensor.

With these potential problems in mind, it appears that the ideal way to perform air gap measurement is to use a non-contact sensor that can measure distances in extreme conditions. A very promising technology, which uses the eddy current effects on an inductive coil probe, was selected for the measurement of gap formation.

\subsubsection{Eddy Current Methods}

The layout of the experimental setup, used to measure the gap formation using the eddy current technique, is shown in the Figure 1.2. The eddy current sensor is buried in the sand mold and the molten alloy is poured into the mold. The sensor initially registers the presence of the metal and gives an output of a particular frequency as a function of distance $\mathrm{D}_{1}$. As the metal cools, the gap is formed between the metal and the mold. The metal moves away from the sensor (denoted by $\mathrm{D}_{2}$ in the Figure) and the output of the sensor changes accordingly. The output of the sensor frequency and the time are recorded continuously. These data are correlated to the lab calibration data, and the formation of the air gap as a function of time is calculated. The difference between $D_{1}$ and $D_{2}$ will be the gap formed. Note that this assumes the only movement between the sensor and the casting is due to gap formation.

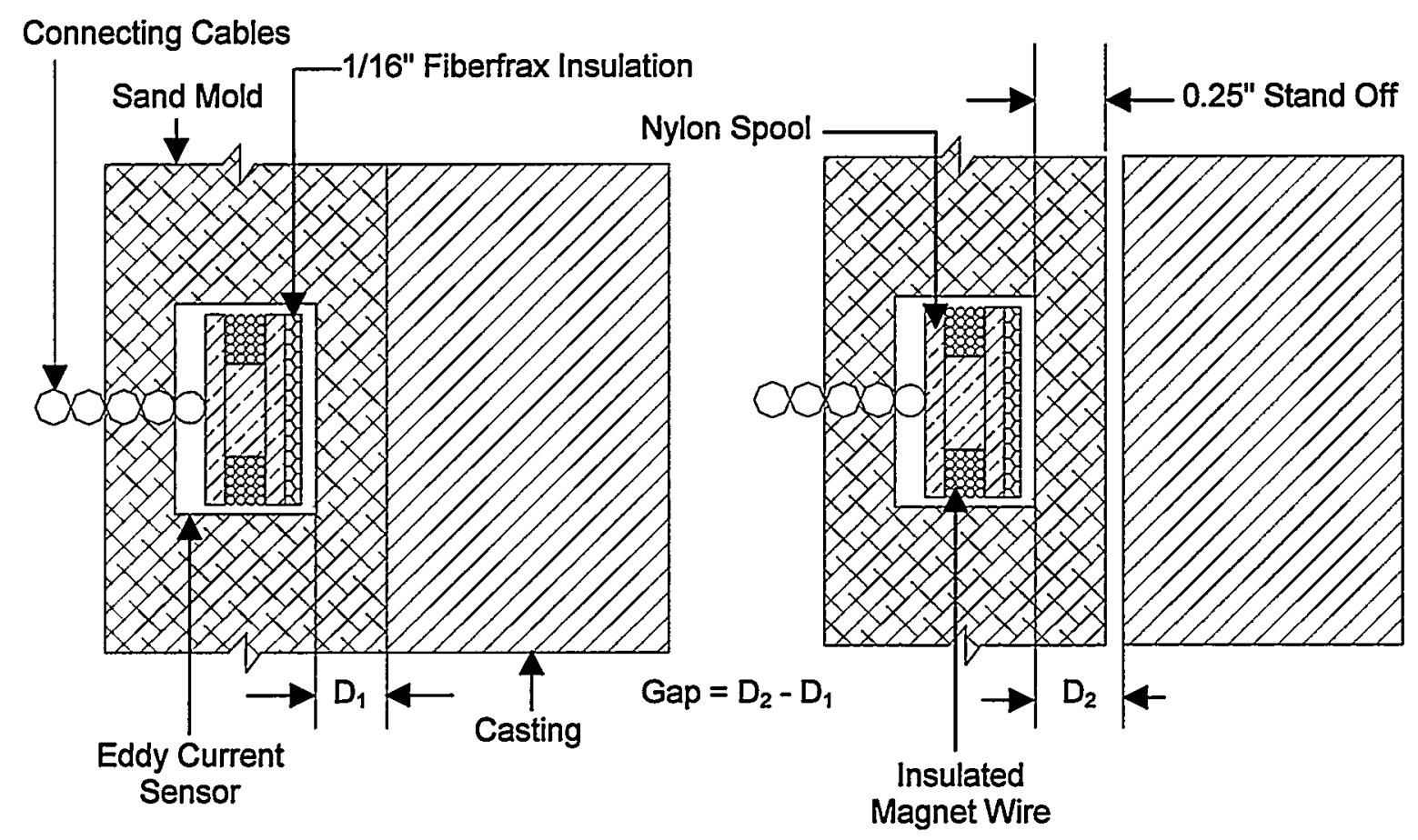

Figure 1.2. Eddy current sensor technique of gap formation measurement

The simplest eddy current sensor is simply a coil of fine gage wire. Eddy current sensors are radio frequency $(\mathrm{RF})$ inductive devices. A tuned RLC oscillator circuit is formed in part by 
the inductance of the sensor. Some authors [3] use the term "impedance," which takes into account the resistance, capacitance, and inductance of the coil. When the coil of wire carrying a time-varying current is placed in the proximity of metals, an eddy current is induced in the material. Then there will be a pronounced change in the inductance of the coil, which in turn changes the frequency of oscillation of current. The magnitude of change of inductance depends on the factors like shape and size of the coil, number of turns of the wire wound, and the gage of the wire used. The depth of penetration of eddy current on a conductive target is explained by the skin-effect principle, which states that an $\mathrm{AC}$ magnetic field penetrates a conductor approximately one skin depth, $\delta=1 / \sqrt{\pi \mu \sigma f}$, where $\mu$ is magnetic permeability of the conductor, $\sigma$ is volume electrical conductivity of the conductor, and $f$ is frequency. For aluminum, the skin depth is evaluated as shown below [13]:

$$
\begin{aligned}
& \mu_{0}=\text { permeability of free space; } \mu_{0}=4 \pi \times 10^{-7} \mathrm{H} / \mathrm{m}, \\
& \mu_{\mathrm{r}}=\text { relative permeability of aluminum; } \mu_{\mathrm{r}}=1, \\
& \mu=\mu_{0} \times \mu_{\mathrm{r}}, \\
& \sigma=34.4 \times 10^{6} \mathrm{~S} / \mathrm{m}, \\
& f=48 \times 10^{3} \mathrm{~Hz},
\end{aligned}
$$

Substituting these values in the above equation, the skin depth, $\delta$, for aluminum is $0.391 \mathrm{~mm}$, which is nearly equal to 0.015 inch.

When a time-varying current is applied to coiled wire (sensor), a magnetic field is generated. When this sensor is brought near the conductive target, the magnetic field is disrupted. This generates "eddy" currents in the conductive target. These eddy currents generate their own magnetic field, which interacts with the original, modifying the inductance of the originating coil. The oscillation frequency of the RLC is therefore a function of the inductance, which is in turn a function of the distance from the conductive surface. The oscillation frequency of the RLC circuit is measured with a precision $10 \mathrm{MHz}$ counter, and the resulting measurement is correlated to distance from the conductive surface by an extensive calibration process.

\subsection{Outline of Report}

The details of design and fabrication of the eddy current proximity sensor are discussed in Chapter 2. A brief overview of the electrical and electronic circuitry employed in the operation of the sensor and the experiments that were conducted to arrive at the operating frequency of the sensor are presented in Chapter 3. The calibration process for relating sensor output to position is detailed in Chapter 4. The experiments conducted to determine sensor repeatability are also presented in this chapter. The installation of the sensors in the "sensor packs" and in the casting molds is covered in Chapter 5. Results from typical foundry pours are shown in Chapter 6, along with a discussion of several possible sources of error. Thickness measurements and green sand results are also presented in Chapter 6. The Appendix contains experimental results for twenty test cases conducted during the summer of 1998. 


\subsection{DEVELOPMENT OF THE SENSOR AND THE CIRCUITRY}

This chapter presents the design and the development of the eddy current proximity sensor for the measurement of the gap formation in aluminum castings.

\subsection{Coil Fabrication}

Proximity sensor coils are inductors, which are nothing but multiple coils of wire wound around a spool. When a time-varying voltage is applied to this coiled wire, a magnetic field is induced. When the sensor coil is brought in the vicinity of a metallic object, there will be pronounced change in the magnetic field and the inductance of the coil.

\subsubsection{Coil Winder}

The proximity sensor coils are made using a coil winder, which wraps the wire around a spool in a controlled, uniform, and preset fashion. The coil winding process is shown in the following setup in Figure 2.1. The working of the components of the coil winding setup is explained in the following paragraphs.

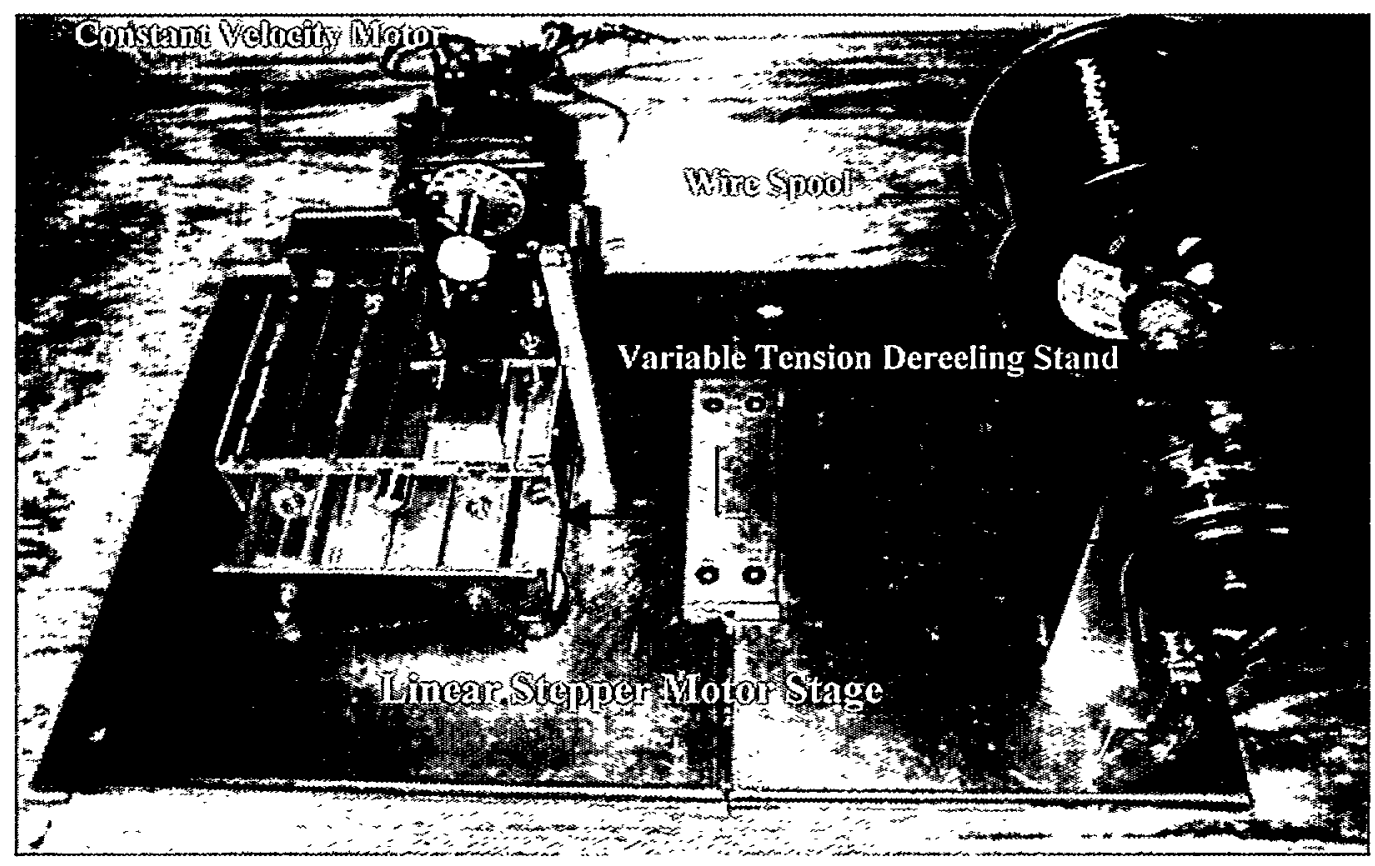

Figure 2.1. Coil winding setup

The coil winder shown in the Figure 2.1 is a small scale device that winds the wire around the spool, based on user-defined parameters like the number of turns to be wound, gage of the wire, and the width of the spool. The coil winding apparatus was designed and constructed by Cooper and Johns [11].

The coil winder consists of a variable tensioning dereeling stand, which can accept multiple sizes of source spools and can accommodate many different sizes of wire gages. 
Tension on the wire can be adjusted to prevent backlash and wire breakage using the variable tensioning mechanism. The variable tensioning dereeling stand is coupled with a constant velocity motor, which is in turn mounted on a linear stepper motor stage. The target spool is mounted along the shaft, which is fitted into the coupler of the constant velocity motor. Wire is pulled off the source spool by the constant velocity motor and is guided on to the target spool by the moving stepper motor stage back and forth to wind the wire over the coil evenly. Wrapping begins when the motor is turned on. A saw cut in the spool allows the wire to be inserted from the top and to lie flat along the winding surface. The notched circumference allows the shaft to be easily cut off after the winding process is done [11].

\subsubsection{Spool}

Nylon was selected as the material for the spool since it can withstand elevated temperatures and also can be machined easily. Figure 2.2 shows the design of the spool.

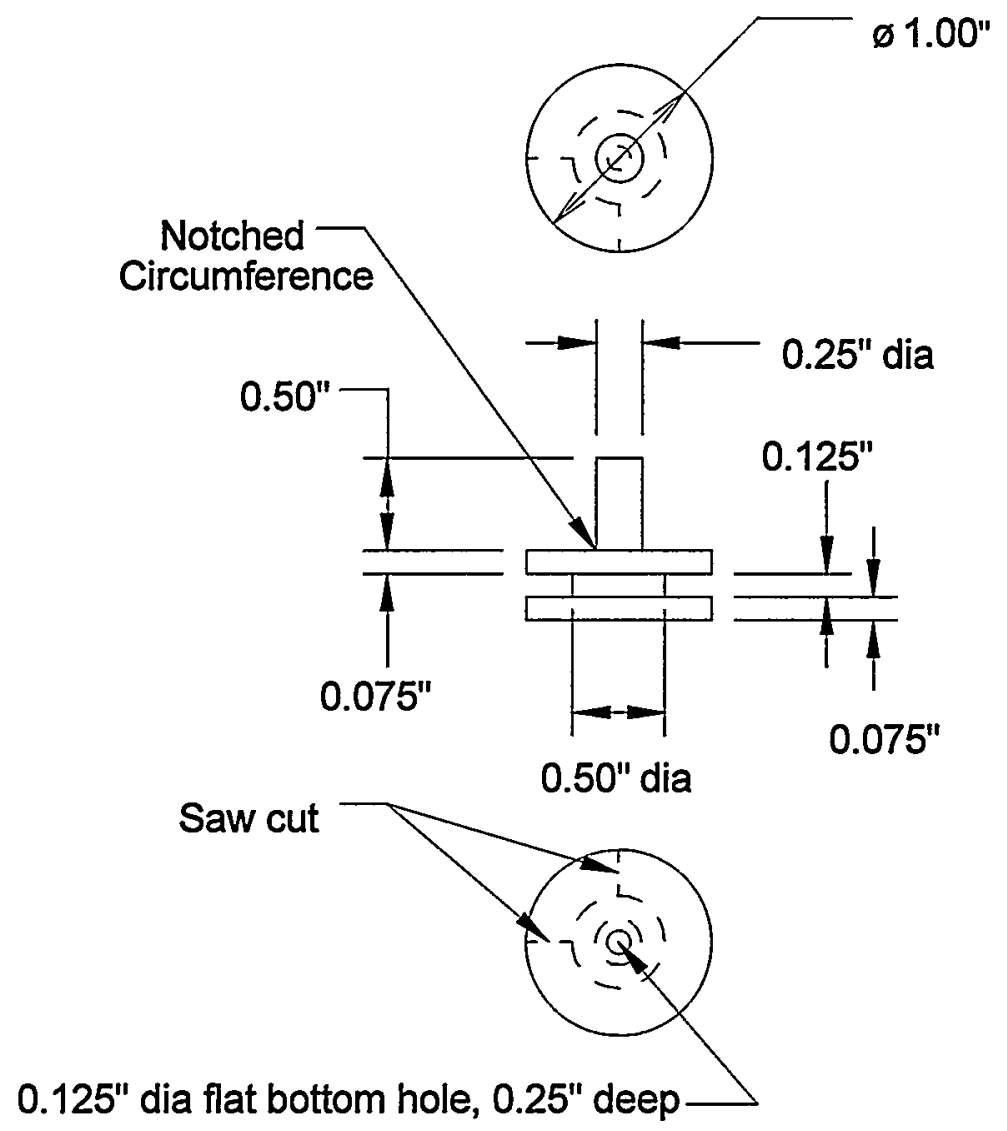

Figure 2.2. Nylon spool for proximity sensor

\subsubsection{Constant Velocity Motor}

The coupler on which the spool is fitted is bonded to a perforated disc that, along with an optical sensor, serves as a counter for the number of turns of the motor. As the disc rotates through the optical sensor, the sensor reads the changes between open and closed beam detection. The disc is fitted on to the shaft of the constant velocity motor. The assembly of the 
spool, coupler, disc, optical sensor, and the constant velocity motor mounts to the stepper motor, as shown in Figure 2.3.

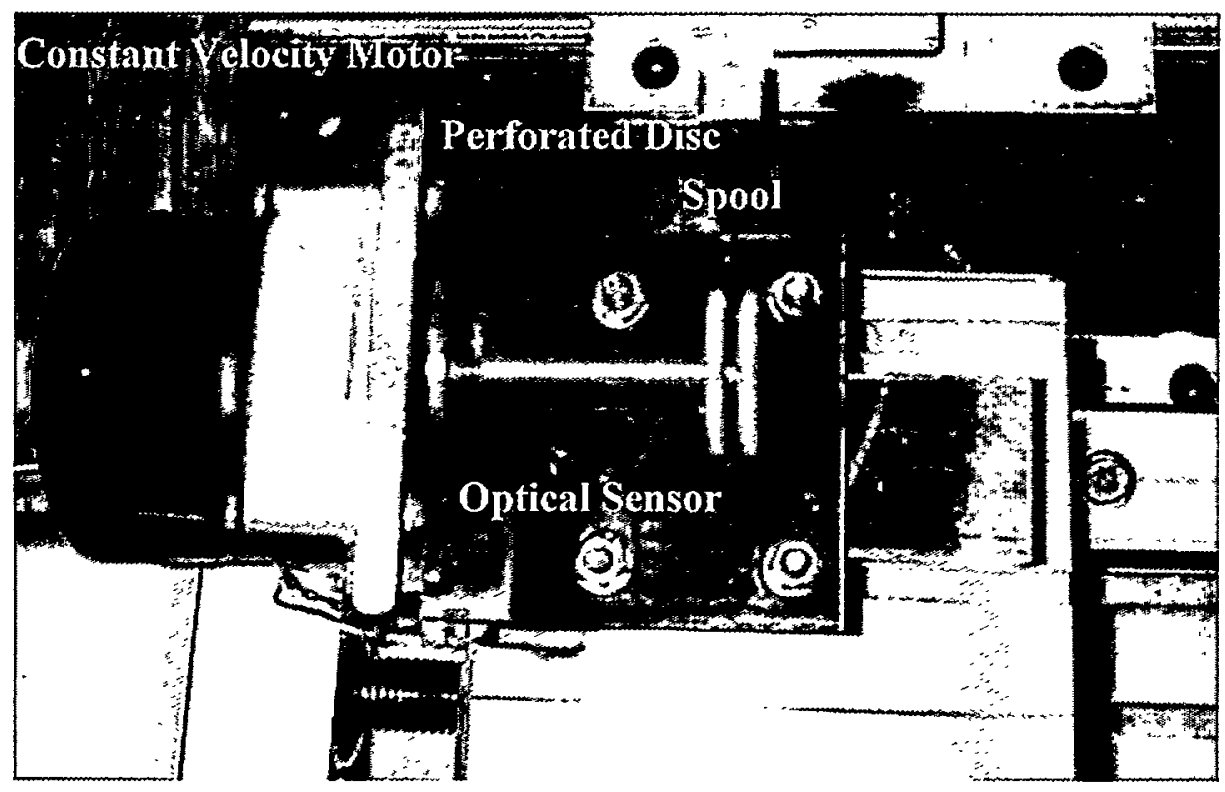

Figure 2.3. Spool winding assembly

The stepper motor moves back and forth according to the user-defined parameters at a slow, controlled and continuous rate, which is critical for the delicate winding of the coils. When the winding process begins, the constant velocity motor turns and the stepper motor moves the stage back and forth to wrap the wire on the spool smoothly and evenly. The winding process is computer controlled using a code written in Microsoft Quick Basic. The user inputs the size of the spool, gage of wire, and the number of turns desired. Then the program uses subroutines to calculate the speed and the distance traveled by the stepper motor stage as well as when to stop the stepper motor, and the constant velocity motor upon completion of the winding based on the user inputs.

\subsection{Sensor Fabrication}

The coil of magnet wire produced by the process described above forms the core of the proximity sensor. From the earliest days of this research, it was recognized that the coil must be protected from the foundry environment. The nylon spool/magnet wire coil combination was encased in a high-temperature ceramic material. The development process followed to generate the final sensor design is detailed below.

\subsubsection{Sensor Development}

The observed results from several foundry pours clearly indicated a significant temperature effect on the proximity sensor output. This observation has lead to the development of five new sensor designs with lowered temperature sensitivity. The first modification incorporated an insulating air gap between the sensor coil and the ceramic housing, shown in Figure 2.4. Results from one of the calibration tests (over a 0.050 inch range) are shown in Figure 2.5. The hot plate was turned on at about the 150 sample point, and its temperature was increased to $900^{\circ} \mathrm{F}$. The proximity sensor remained relatively insensitive to temperature changes 
up to an internal coil temperature of about $300^{\circ} \mathrm{F}$. However, there was no drastic change in the sensor output at the $350^{\circ} \mathrm{F}$ point, as had been seen in the early foundry results. The air gap appeared to be somewhat effective in extending the time frame over which the proximity sensor would work in the foundry pours. Ultimately this approach was abandoned due to the difficulty in fabricating the air gap sensor.

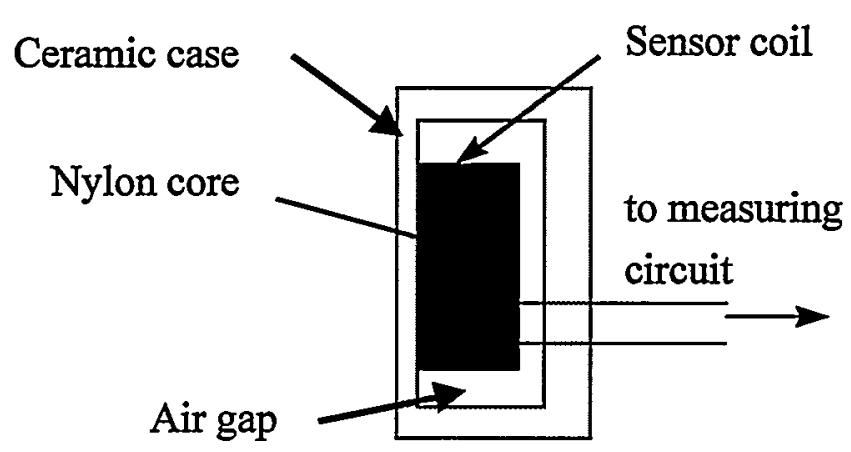

Figure 2.4. Air gap sensor

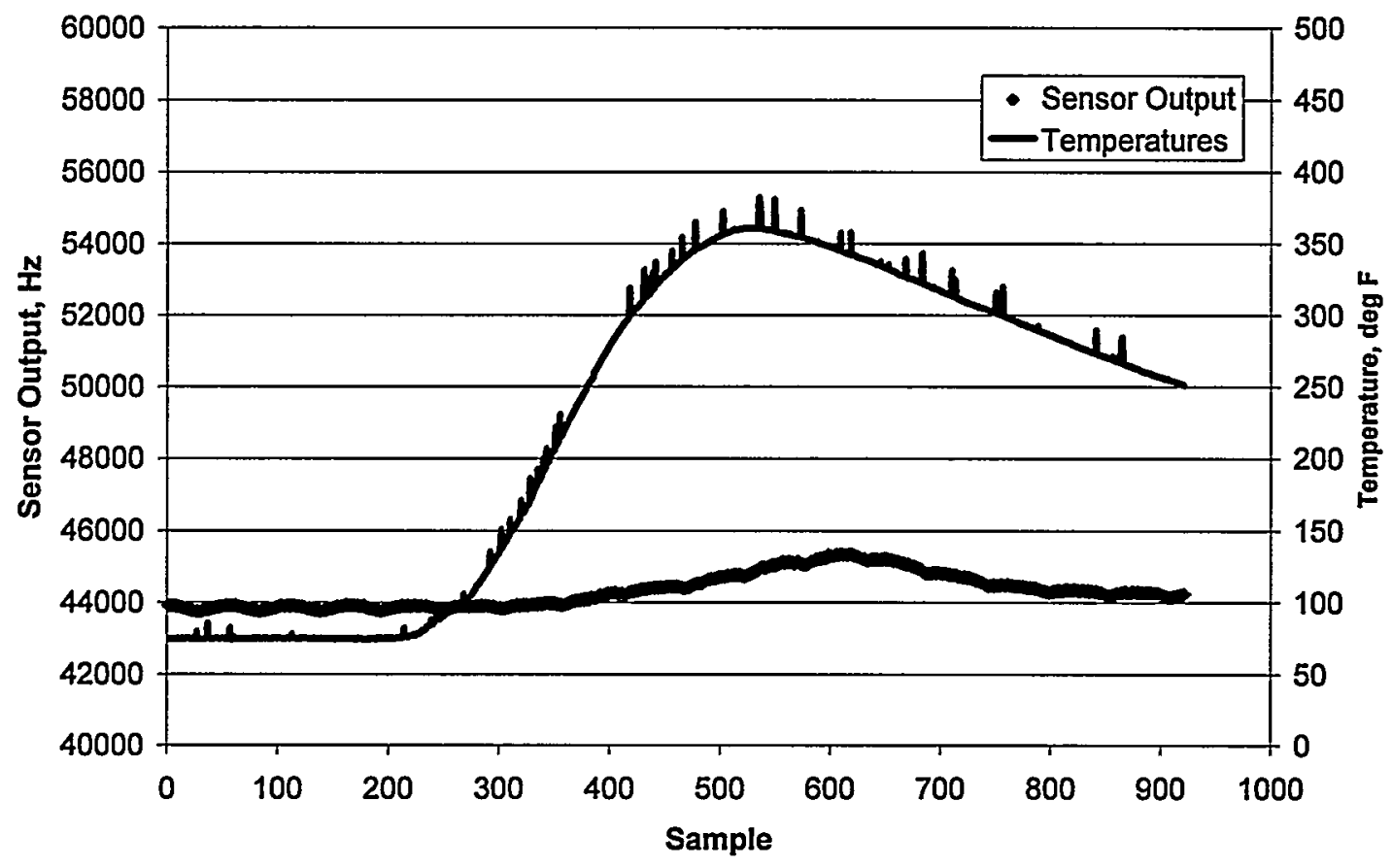

Figure 2.5. Hot plate test - air gap sensor

A second modified proximity sensor design used 200 turns of 36 gauge wire with a thicker Teflon $\circledast$ insulation rated to $200{ }^{\circ} \mathrm{C}$ (the normal sensor uses 500 turns of 34 gauge wire with an insulation rated to $180{ }^{\circ} \mathrm{C}$ ). The smaller number of turns was due to the increased thickness of the Teflon $\circledast$ insulation. Results from a hot plate calibration test are shown in Figure 2.6. Note the higher operating frequency of the sensor - this resulted from lower coil inductance created by having only 200 turns of wire on the coil. The calibration test was conducted in a similar fashion to the one discussed above. Note that similar results were obtained in that no large temperature effect on the sensor was observed. This may have resulted from the fact that 
the sensor did not reach the critical $350^{\circ} \mathrm{C}$ temperature because it had thicker wire insulation. A small temperature effect on the sensor output can be observed in Figure 2.6. For this sensor, the 0.050 inch calibration "stroke" gives a sensor output change on the order of $400 \mathrm{~Hz}$. The $175^{\circ} \mathrm{F}$ internal coil temperature increase gave a sensor output change on the order of $2600 \mathrm{~Hz}$ - about 6-7 times that of the calibration stroke. This sensor design was also ultimately abandoned because of difficulty in fabricating and handling it and the extremely fragile 36 gauge wire.

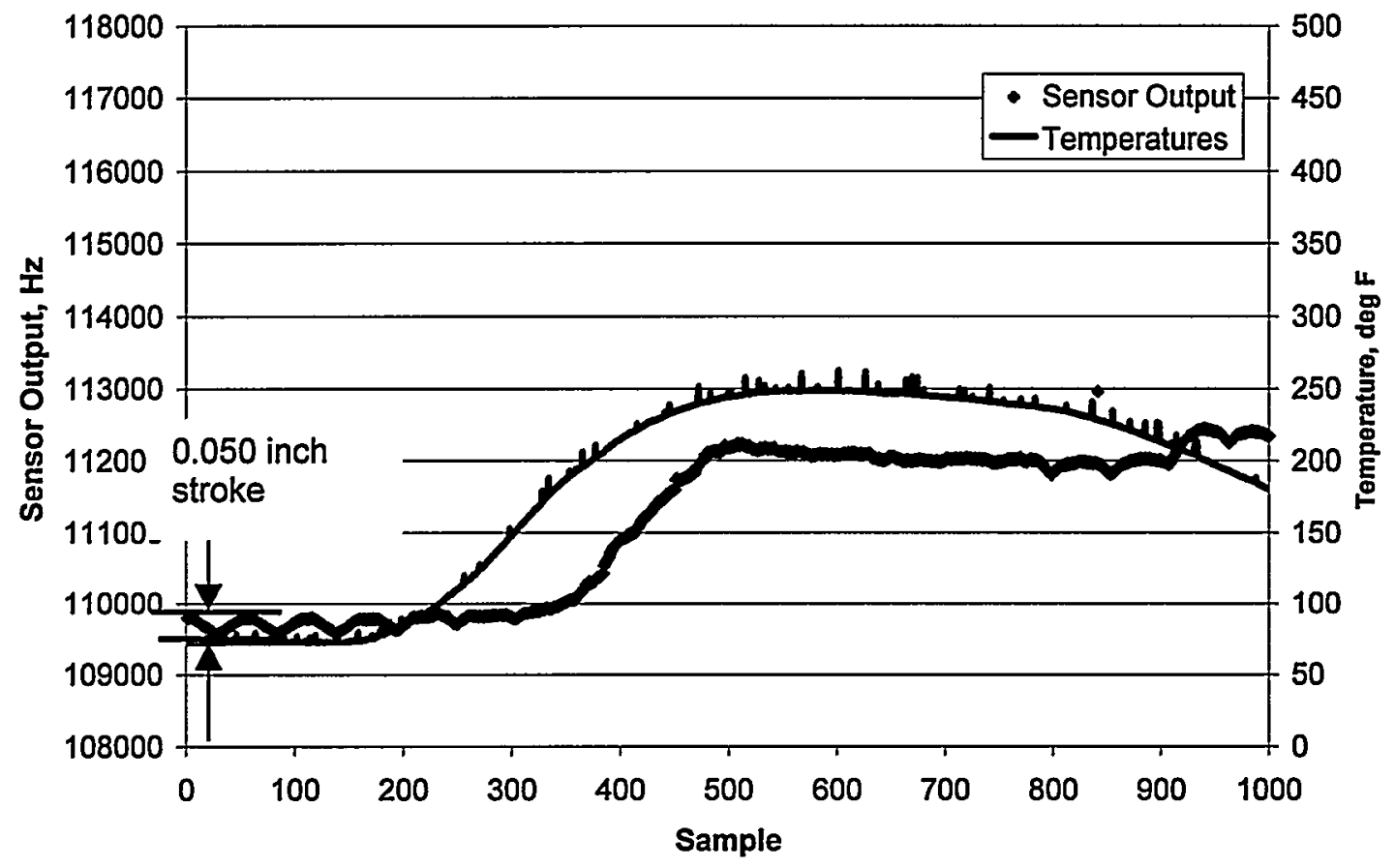

Figure 2.6. Hot plate test -36 gage wire sensor

The results from the second sensor design with higher temperature rated insulation looked promising. Three additional types of magnet wire with higher rated insulation were purchased,

- \#34 AWG HAPT Class 200C magnet wire (MWS Wire) - rated to $200^{\circ} \mathrm{C}$

- \#34 AWG HML Class 220C magnet wire (MWS Wire) - rated to $220^{\circ} \mathrm{C}$

- \#33 AWG Heavy Allex magnet wire (Essex Express) - rated to $220^{\circ} \mathrm{C}$.

Proximity sensors were wound and tested from the first two types of magnet wire listed. The third type of high temperature wire (\#33 AWG Heavy Allex) was not tested extensively. The heavier gauge wire allowed only 275 turns on the standard spool, which greatly reduced the sensor's sensitivity.

Static hot-plate (laboratory) test results for the other two types of wire are shown below in Figures 2.7 and 2.8. In this test the hot plate was maintained at $900^{\circ} \mathrm{F}$. A proximity sensor mounted in the sand pack was then placed on the hot plate. The sensor was then heated by the direct contact between the sand pack and the hot plate. As shown in the figures below, both types of wire have greatly extended temperature ranges over the standard $180^{\circ} \mathrm{C}$ wire that was previously used. The $200^{\circ} \mathrm{C}$ rated wire allowed more turns (500 vs. 450$)$ than the $220^{\circ} \mathrm{C}$ rated 
wire due to the slightly thinner insulation. This larger number of turns gave a better position (gap) sensitivity.

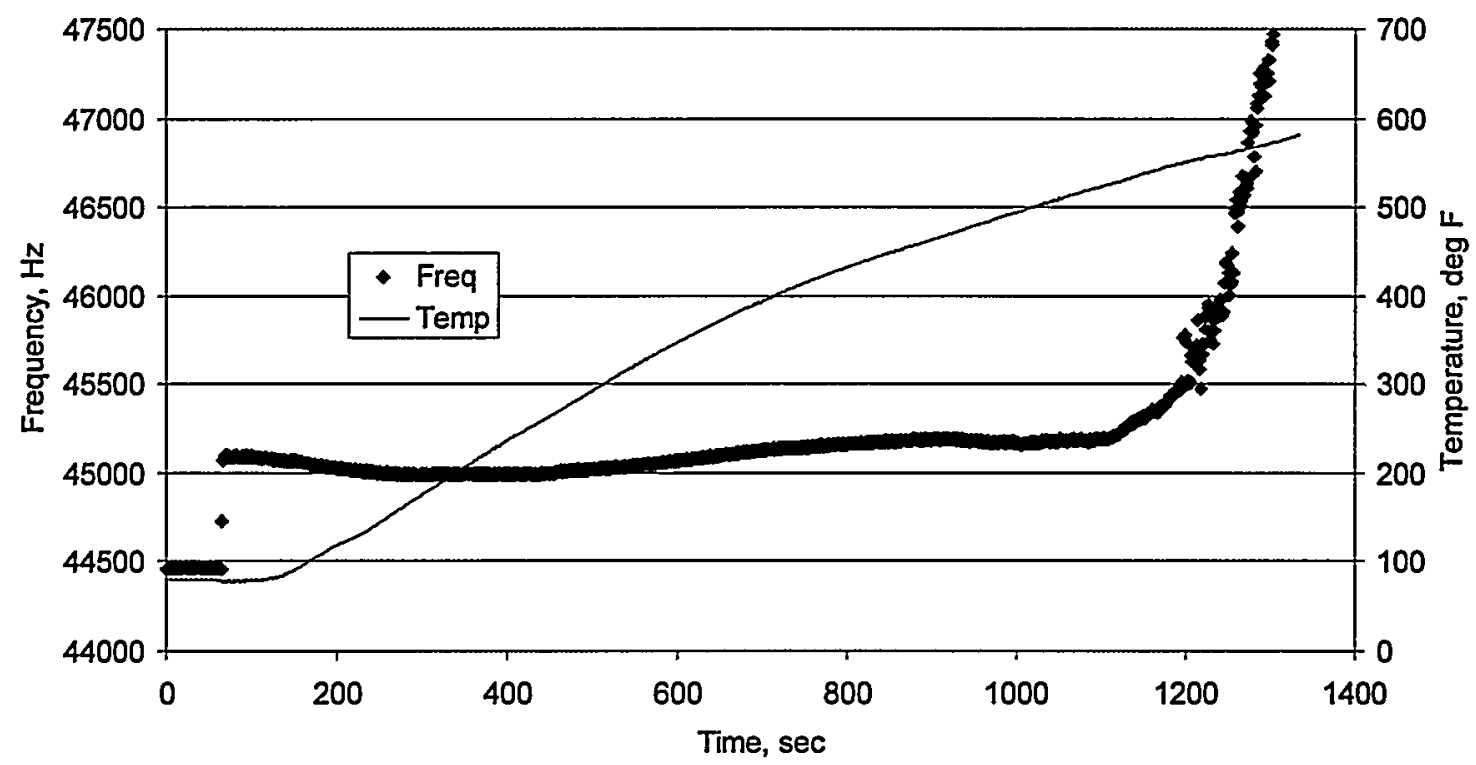

Figure 2.7. Hot plate test results ( 34 gage wire, $200^{\circ} \mathrm{C}$ rating)

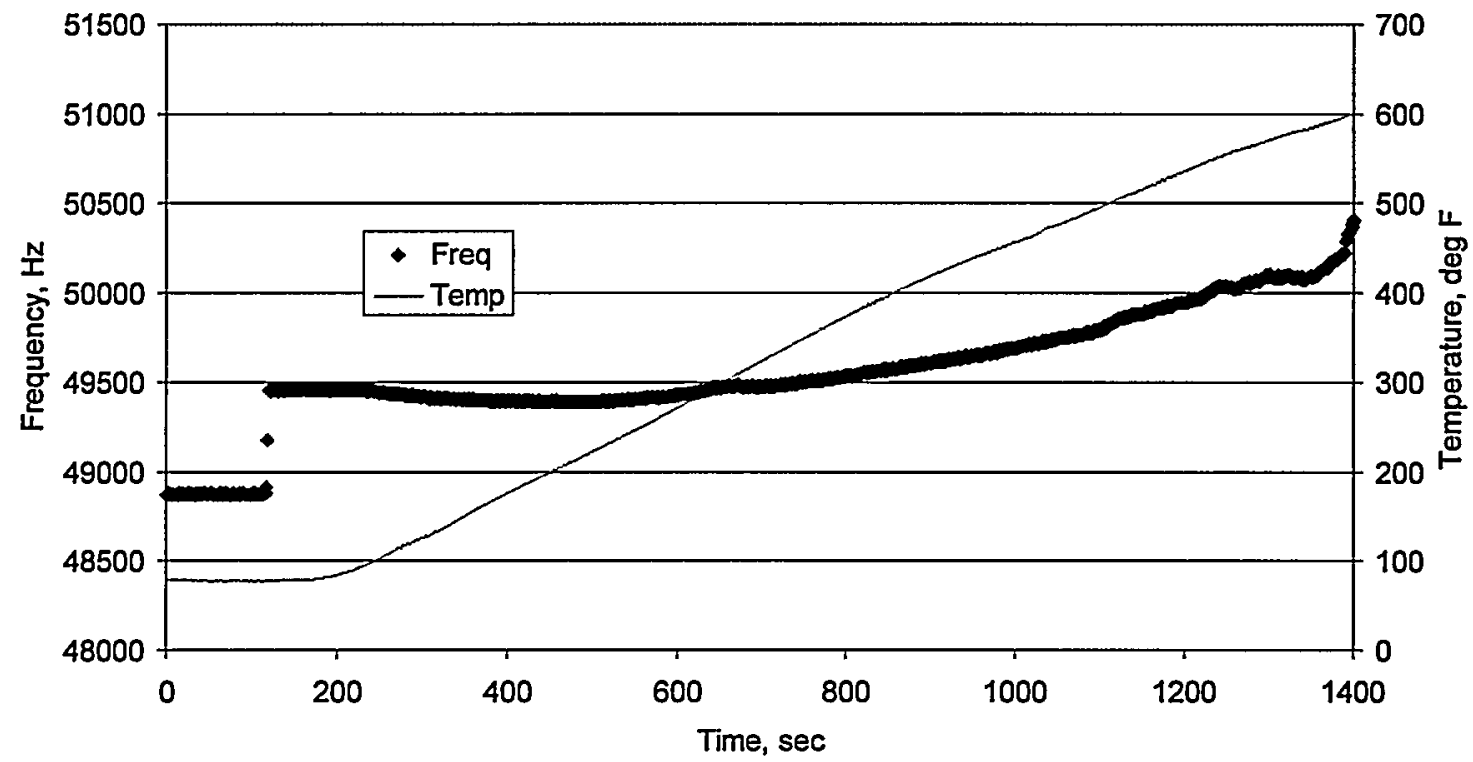

Figure 2.8. Hot plate test results ( 34 ga wire, $220^{\circ} \mathrm{C}$ rating)

The fifth (and final) experimental proximity sensor design used a 1/16 inch $(1.6 \mathrm{~mm})$ thick sheet of Cotronics \#300-40 ceramic paper inserted between the nylon sensor spool and the face of the ceramic casting of the proximity sensor, shown in Figure 12. This ceramic paper has a rated thermal conductivity of approximately $0.38-0.60 \mathrm{Btu} / \mathrm{hr}-\mathrm{ft}^{2}-{ }^{\circ} \mathrm{F} / \mathrm{in}$, which is $10 \%$ of the Cotronics Rescor 750 ceramic. The ceramic paper insulation was used instead of the air gap insulation, which proved to be too difficult to manufacture repeatably. Figure 2.9 shows a hot 
plate test for a proximity sensor made in this configuration. The slope of the temperature line is less than that of Figure 2.8, which indicates that the ceramic paper is somewhat effective in reducing the temperature rise of the proximity sensor. This is the final proximity sensor design.

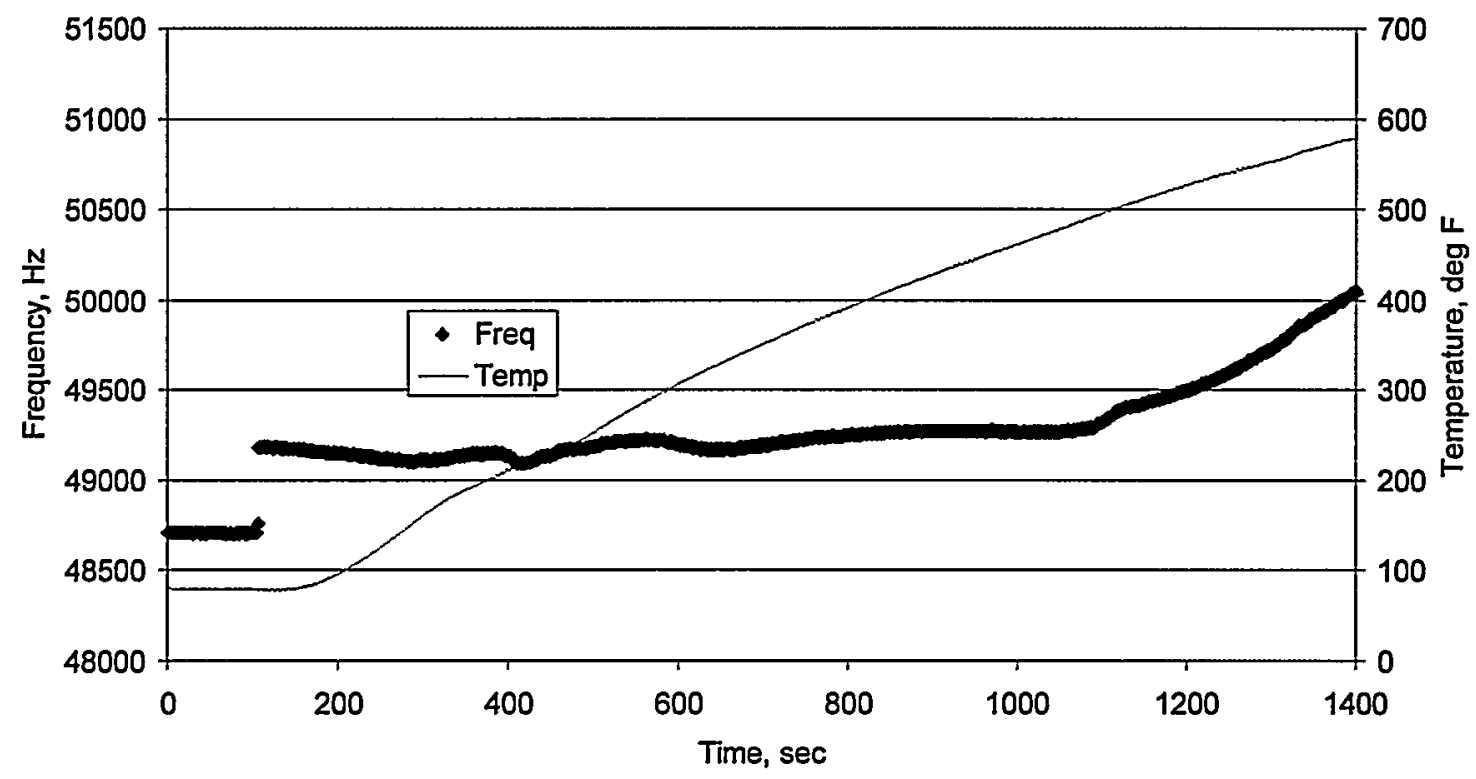

Figure 2.9. Hot plate test results ( 34 ga wire, $220^{\circ} \mathrm{C}$ Rating, ceramic paper insulation)

\subsubsection{Final Sensor Design}

The eddy current sensor was fabricated by winding 450 turns of the 34-gage CLASS HML $220^{\circ} \mathrm{C}$ magnet wire on the nylon spool. Once the coil winding process was complete, the spool was removed and the shaft is cut off. The two leads coming out of the spool were soldered to the connecting cable and the connector socket soldered to the other end of the cable. A 1/16inch thickness of Fiberfrax paper was cut into a 1-inch disc and glued in front of the spool to provide thermal insulation for the sensor. The spool was then placed in the mold, as shown in Figure 2.10, and the mold was then filled with Cotronics Rescor ${ }^{\circledR} 750$ high temperature resistant ceramic material. After the spool was covered with the ceramic material, it was placed on the vibrating table to allow the air bubbles to escape and eliminate porosity. Figure 2.11 shows a completed sensor as well as two intermediate stages.

The ceramic was allowed to dry for about 24 hours in the mold. The sensor was then cured by placing it in a preheated oven at $200^{\circ} \mathrm{F}$ for about 5 hours to allow the moisture to escape. Once the sensor was ready, it was calibrated in the laboratory by continuously recording the sensor output while varying the distance of the sensor from zero inches to a distance of one inch, in steps of 0.004 inch, using an aluminum plate as the target. This calibration provided the reference data to be used later in casting experiments. 

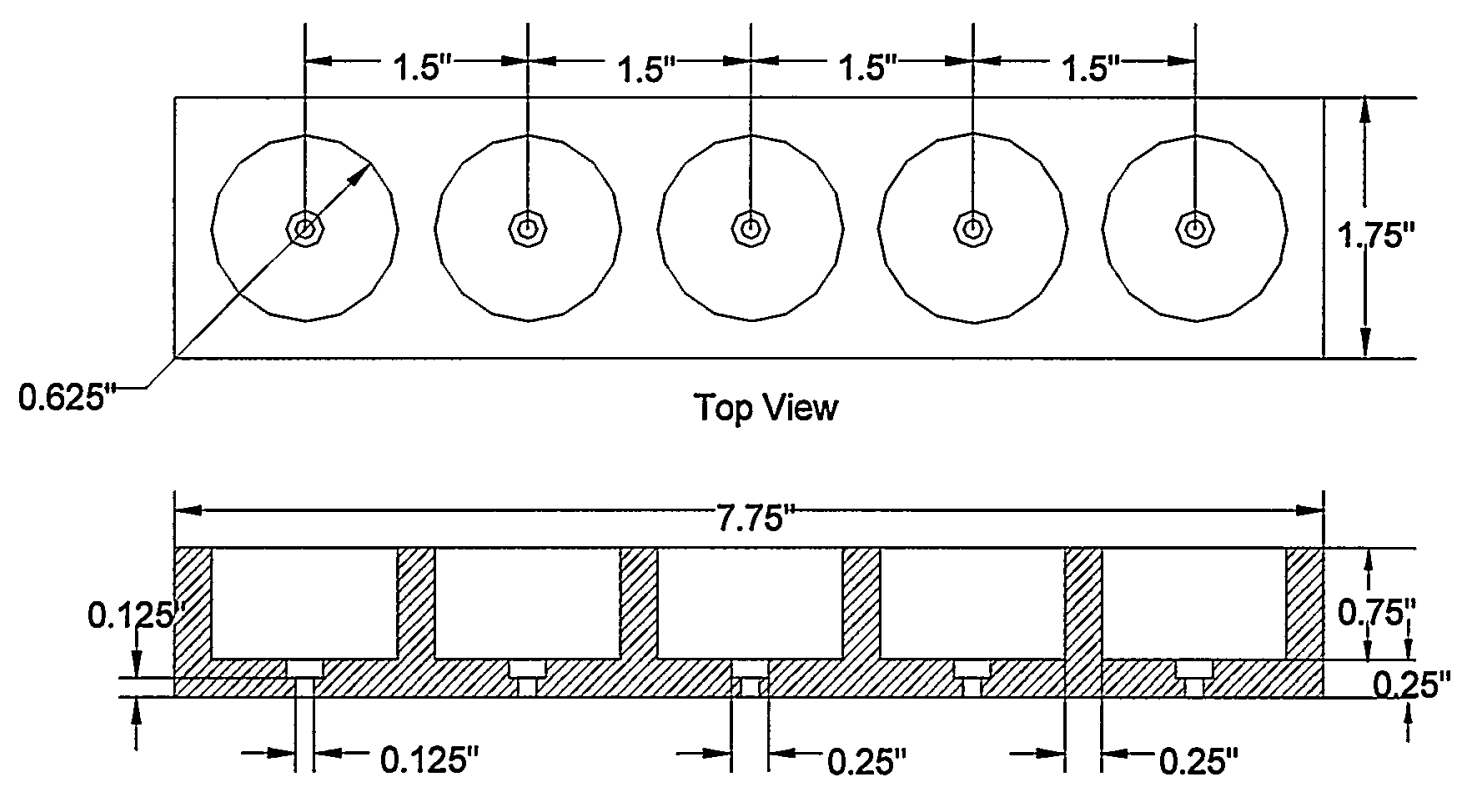

Half Sectional Front View

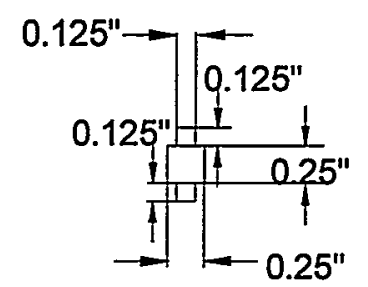

Pin

Figure 2.10. Sensor ceramic mold

Sensor coil (450 turns of $34 \mathrm{ga}, 220^{\circ} \mathrm{C}$ insulation)

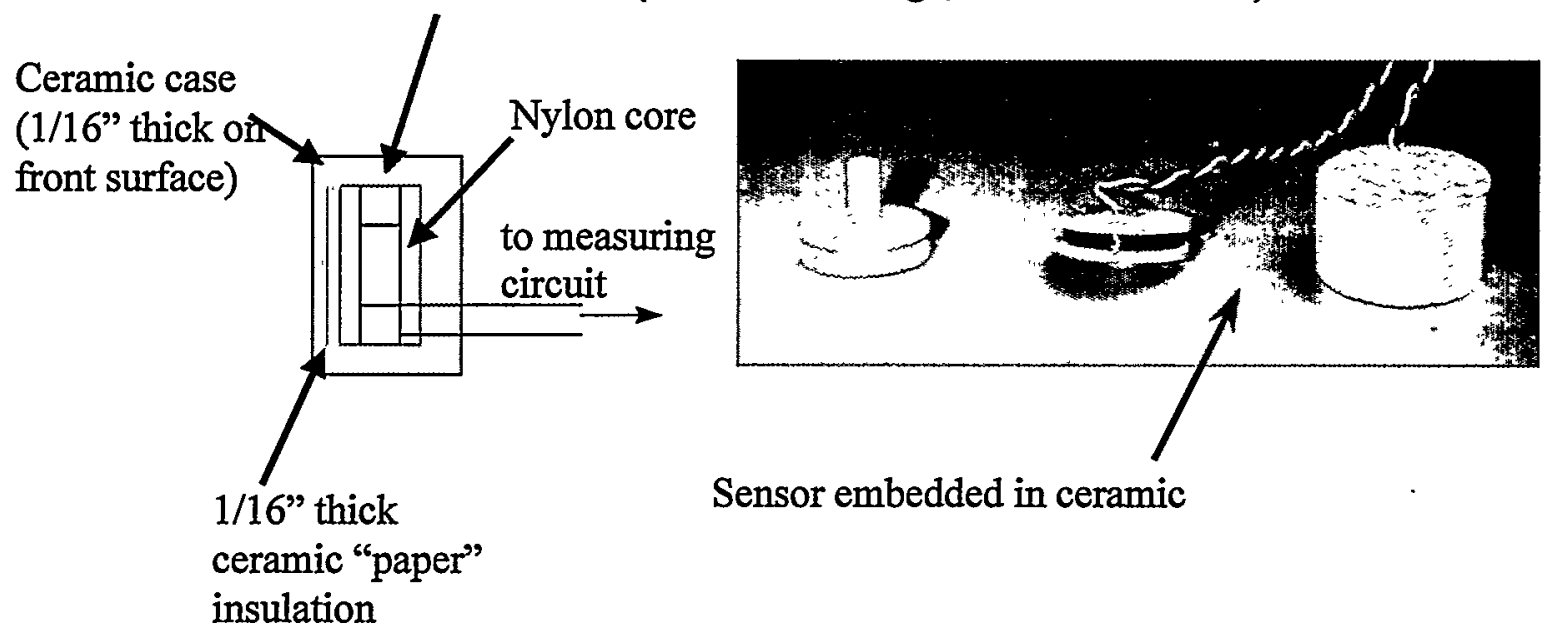

Figure 2.11. Final proximity sensor design 


\subsection{Effect of Moisture on the Sensor Output}

An early series of laboratory hot plate calibration tests uncovered a phenomenon that had occasionally corrupted our proximity sensor results. A proximity sensor mounted in a sand pack was placed on the hot plate. The sensor was then heated by direct contact between the sand pack and the hot plate. As shown in Figure 2.12 below, on the $1^{\text {st }}$ test a rapid increase in the sensor output occured at a sensor temperature of about $200-220^{\circ} \mathrm{F}$. The same sensor pack was tested on the following day $\left(2^{\text {nd }} T\right.$ Test), and the sensor output "spike" at these temperatures was not present. We reasoned that there could sometimes be residual moisture in the ceramic material surrounding the proximity sensor coil to cause this output spike. For this reason, we instituted a curing cycle to thoroughly dry the sensors before using them in the lab or the foundry. A representative foundry test ( $1 / 2$ inch plate thickness, 45 degree angle) is shown in Figure 2.13. Close observation of the temperature profile indicates that there is a small change in slope just below the $200-220^{\circ} \mathrm{F}$ point, which indicates that the sensors were still not entirely dry even after the curing process.

Sensor "curing" tests developed during early June 1998 were conducted with a vent hole present in the sensor. This vent hole allowed moisture from the curing ceramic to escape from the sensor. Thermo-gravimetric testing results for the curing process are shown below in Figure 2.14. The "unplugged" sensors have the vent hole and are clearly more thoroughly dried than the "plugged" sensors without the vent.

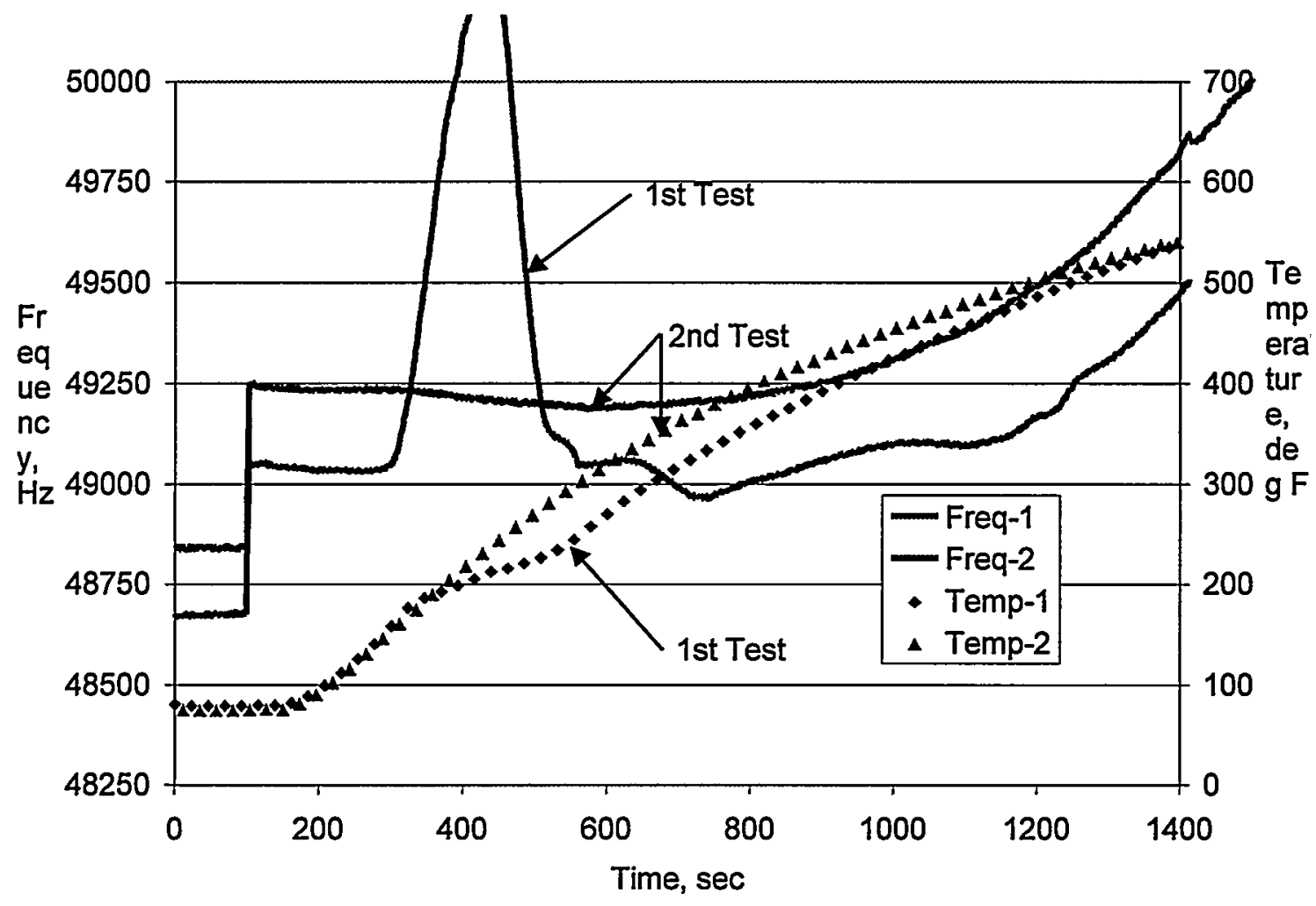

Figure 2.12. Hot plate test results, sensor \#527-1 


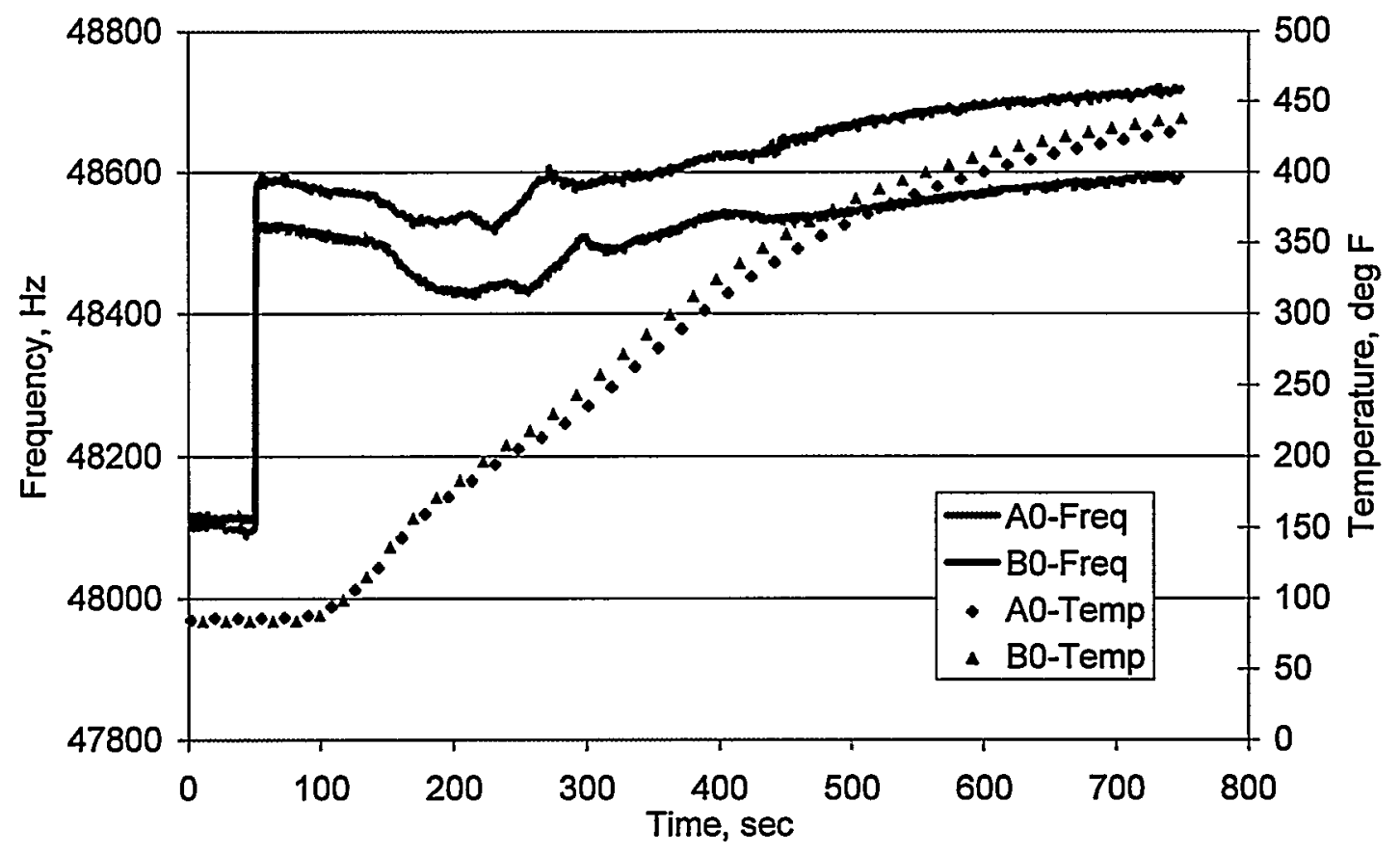

Figure 2.13. Foundry test, $6 / 24 / 98$ (1/2 inch plate, 45 degree angle) 


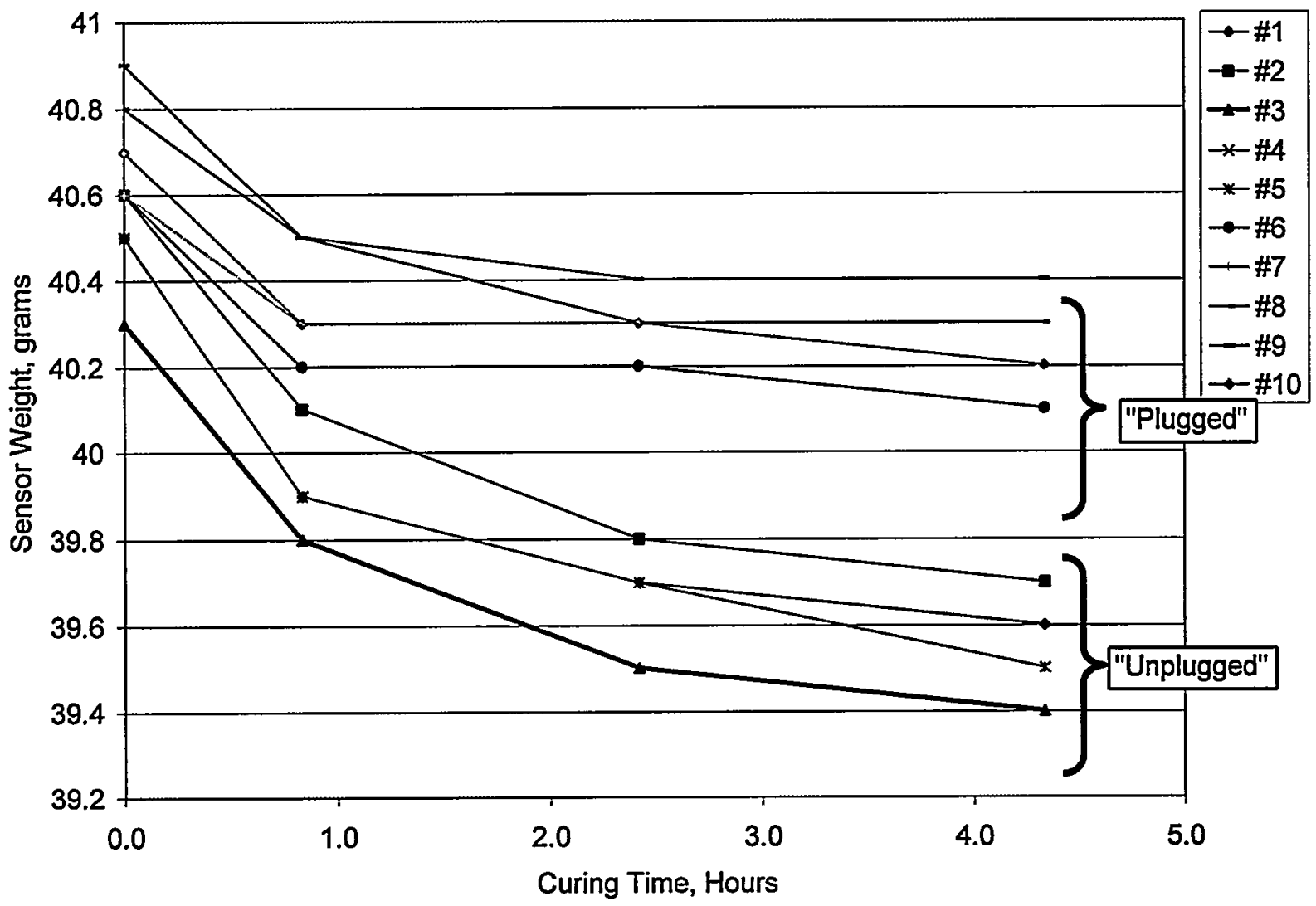

Figure 2.14. Thermo-gravimetric testing results 


\subsection{PROXIMITY SENSOR ELECTRONICS}

\subsection{Electrical Circuitry}

The electrical circuit employed for operation of the sensor is discussed in this section. The circuit is shown in Figure 3.1.

The sensor coil acts as an inductor $(\mathrm{L})$ in the circuit. Two capacitors $(\mathrm{C} 1$ and $\mathrm{C} 2)$ combine with the inductor to form an LC oscillator. The transistor switches from ON to OFF (and vice versa) based on the amplitude of the LC circuit waveform. The circuit shown in Figure 2.15 thus forms essentially a square wave generator, with an oscillation frequency strongly dependent on the inductance of the sensor coil. Circuit frequency is accurately measured using a Guide Technology GT-100 universal counter board installed in a $166 \mathrm{MHz}$ Pentium computer.

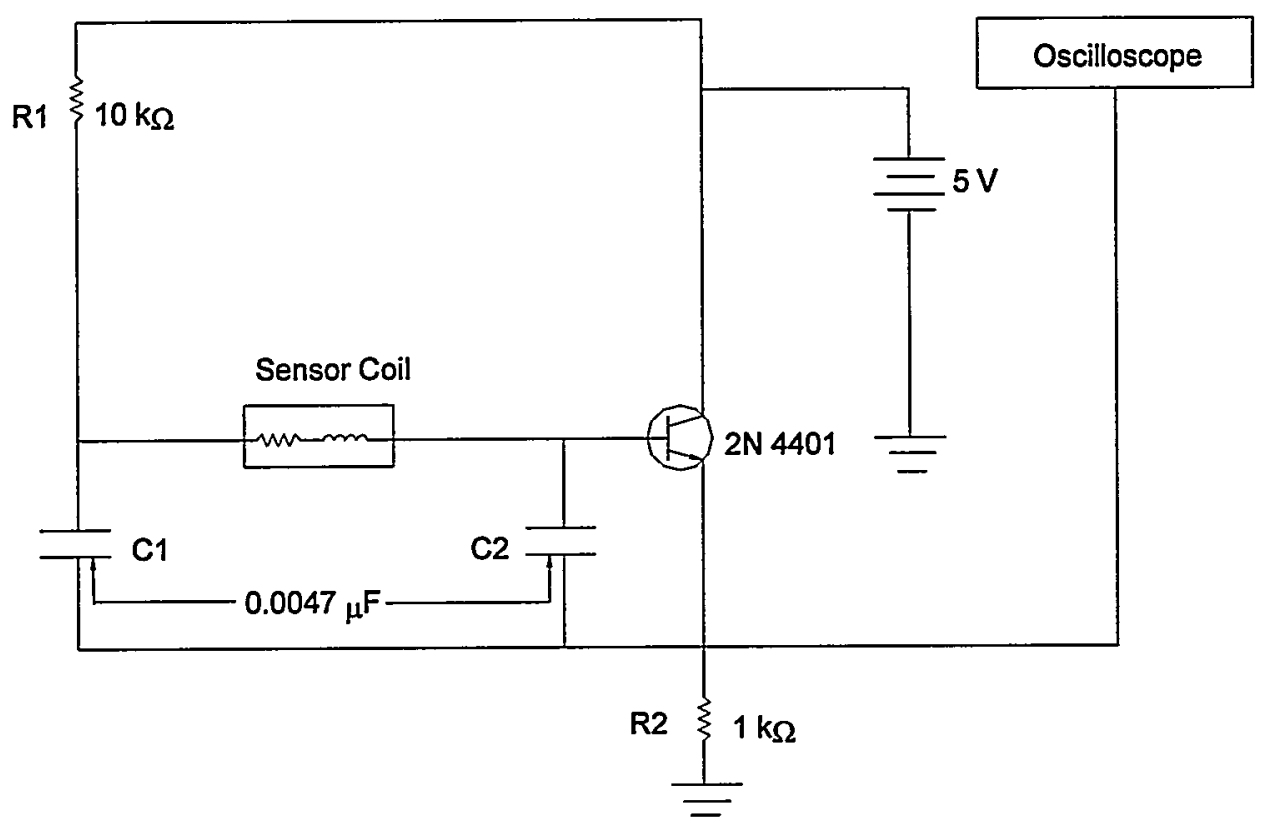

Figure 3.1. Proximity sensor electrical circuit

\subsection{Operating Frequency}

The output frequency of the sensor depends on the values of capacitance and resistance of the capacitor and the resistor shown in the circuit diagram in Figure 3.1. A series of tests was conducted varying the values for the capacitance and resistance in the circuit to arrive at an optimum value for resistance and capacitance. However the value of $\mathrm{R} 2$ in the circuit was maintained throughout as $1 \mathrm{k} \Omega$. The value of $\mathrm{C} 1$ and $\mathrm{C} 2$ in the circuit was kept the same for each set of experiments.

The circuit was assembled on a breadboard and the sensor was connected as shown in the circuit diagram. The sensor was initially placed on the aluminum plate such that there was no gap between the sensor and the aluminum plate. A set of 100 samples of frequency was taken. This procedure was repeated by moving the sensor to a distance of 0.1 inch and 0.2 inch. Finally, the target plate was removed and again 100 samples of frequency were taken. 
The values of capacitance $\mathrm{C} 1$ and $\mathrm{C} 2$ were kept constant at $0.0022 \mu \mathrm{F}$, and $\mathrm{R} 2$ was maintained as $1 \mathrm{k} \Omega$. The value for $\mathrm{R} 1$ was changed for each set of readings; data are shown in Figure 2.16. It can be observed from the plot that as the resistance is increased from $8.2 \mathrm{k} \Omega$ to 30 $\mathrm{k} \Omega$, the sensor output decreases slightly by approximately $1000 \mathrm{~Hz}$. However the trend of frequency change is almost same for different values of the resistance. It is also observed that the sensitivity of the sensor (the difference between the sensor output at the initial and final distances) is essentially equal for different resistors.

The results of the tests are summarized and shown in Table 3.1.

\section{Table 3.1. Summary of sensor output for different values of C1, C2, and R1}

\begin{tabular}{|c|c:c|c|}
\hline $\mathrm{C} 1 . \mathrm{C} 2$ & $\mathrm{R} 1$ & Nominal Frequency at O" & Change in Frequenc| \\
\hline 0.0022 & 8.2 & 66277.5 & 3432.1 \\
\hline 0.0022 & 10 & 65845.5 & 3406.3 \\
\hline 0.0022 & 20 & 65260.2 & 3442.7 \\
\hline 0.0022 & 30 & 65186.3 & 3437.6 \\
\hline 0.0044 & 8.2 & 47775.3 & 2508.9 \\
\hline 0.0044 & 10 & 47639.6 & 2530.3 \\
\hline 0.0044 & 20 & 47355.4 & 2477.5 \\
\hline 0.0044 & 30 & 47361.2 & 2469.0 \\
\hline 0.0047 & 8.2 & 46004.3 & 2415.6 \\
\hline 0.0047 & 10 & 45865.5 & 2419.8 \\
\hline 0.0047 & 20 & 45627.8 & 2395.0 \\
\hline 0.0047 & 30 & 45640.1 & 2380.2 \\
\hline 0.0094 & 8.2 & 33123.7 & 1729.1 \\
\hline 0.0094 & 10 & 33022.0 & 1693.9 \\
\hline 0.0094 & 20 & 32983.5 & 1721.5 \\
\hline 0.0094 & 30 & 33070.6 & 1727.9 \\
\hline
\end{tabular}

The third column in the table shows the sensor output when the target material is in contact with the sensor. The last column in the table is the difference in the sensor output with the metal surface in contact with the sensor and the sensor output when the metal is absent in front of the sensor. It is seen that the sensitivity of the sensor (the last column in the above table) is almost the same for each set of $\mathrm{C} 1$ and $\mathrm{C} 2$ values. Hence any nominal value for R1 can be selected.

The standard deviation of the sensor output was high for the higher values of resistance. Hence, a nominal value for the resistor R1 was selected as $10 \mathrm{k} \Omega$. It was found that seen that the standard deviation for the values of $0.0047 \mu \mathrm{F}$ for $\mathrm{C} 1$ and $\mathrm{C} 2$ was low; hence this value was adopted for $\mathrm{C} 1$ and $\mathrm{C} 2$ for the working of the sensor. 


\subsection{Switching and Sampling Procedure}

Early foundry tests indicated that there was an increase in the sensor output as the sensor was heated in the mold. The sensor output when the sensor was heated was beyond the normal range of operation. Our concept for the proximity sensor system is to measure both inductance (which is a function of the gap and the coil temperature) and the coil's resistance (which is a function of the coil temperature alone). This requires a "switching" circuit that alternately connects the proximity sensor coil to the inductance and resistance measuring circuits. A new set of sensor electronics was designed, fabricated, and tested in both the lab and the foundry. The new electronics use much smaller electromechanical relays mounted near the sensors. This arrangement greatly reduced the crosstalk between the two sensors mounted in the same casting. It also greatly reduced the noise induced in the temperature readings used for heat flux calculations. A final benefit is that a total of four sensors can be measured simultaneously, which improved our productivity in the foundry pours.

Static hot plate tests were conducted in the lab to observe the effect of temperature on the sensor output. The sensor was packed in sand; this is referred to as a "sensor pack," which is explained in detail in Chapter 4. The stand-off distance of 0.25 inch was maintained in front of sensor. The sensor was then placed on a static hot plate and insulated on all sides. The hot plate was electrically heated to $900^{\circ} \mathrm{F}$, and the frequency recorded continuously. The behavior of sensor when it was heated is shown in Figure 3.2.

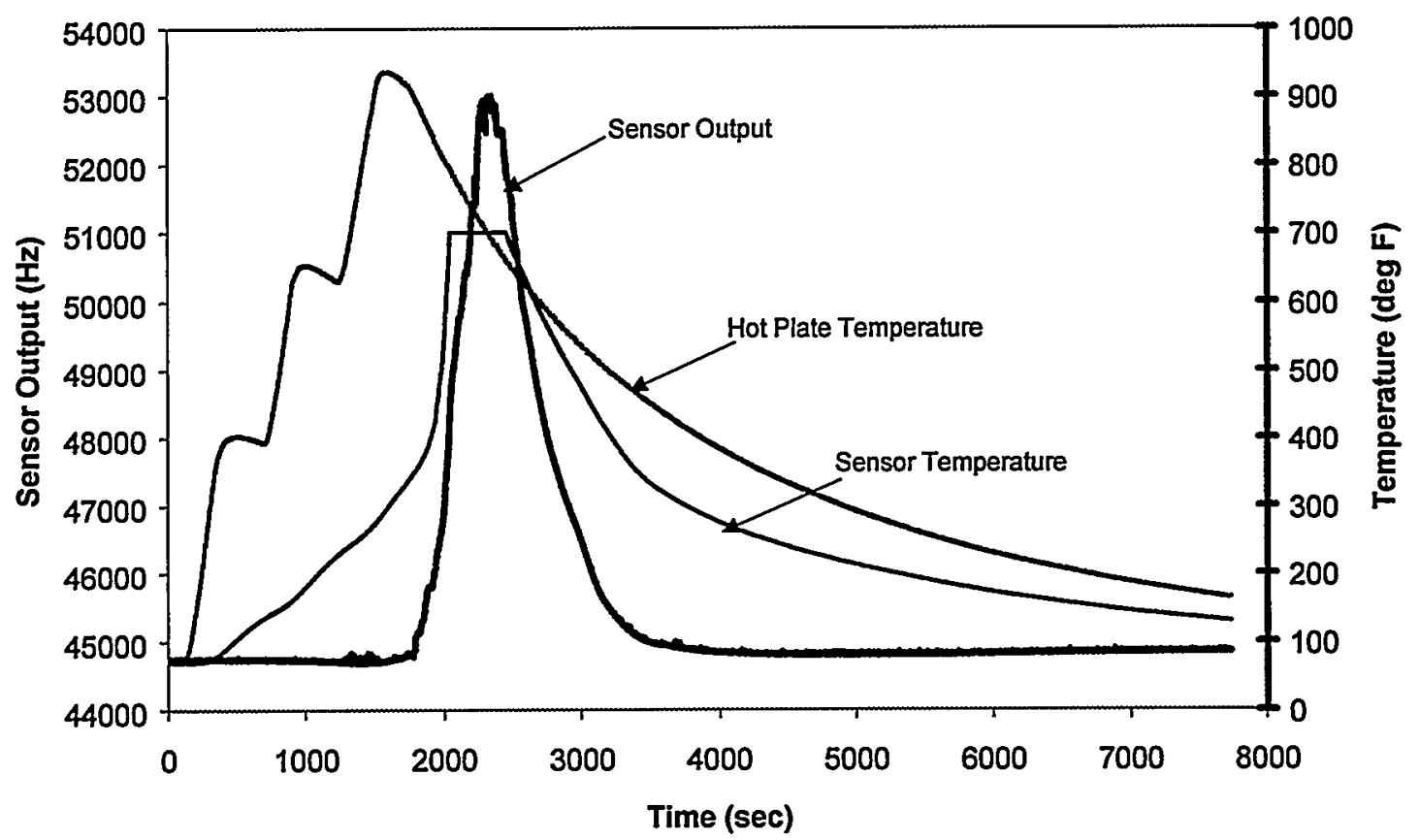

Figure 3.2. Temperature effect on proximity sensor - hot plate test

The data for the plot shown in the Figure 3.2 are based on an early sensor design with 500 turns of standard 34-gage magnet wire without the Fiberfrax insulation in front of the spool. The hot plate was heated from room temperature to $900^{\circ} \mathrm{F}$. The temperature of $900{ }^{\circ} \mathrm{F}$ in three stages was maintained for about 300 seconds and the hot plate was turned off to allow the sensor 
pack to cool to room temperature. It can be observed that at about the 1,700-second mark there was a rapid increase in the sensor output from about $44,700 \mathrm{~Hz}$ to $52,800 \mathrm{~Hz}$. It can also be seen that there was a time lag of about 600 seconds for the sensor to be heated to maximum after the hot plate reached its maximum temperature of $900^{\circ} \mathrm{F}$. When the sensor was allowed to cool, the sensor output decreased to about $44,700 \mathrm{~Hz}$ after approximately 5,000 seconds. This clearly demonstrated that there was an effect of temperature on the sensor. Since the peak in sensor output occurred after the hot plate temperature peaked, the internal sensor temperature was determined to be the primary reason for the increase in the sensor output. Note that the flat area in the peak of the sensor temperature was due to saturation of the op-amp circuit.

The eddy current proximity sensor was placed in the mold at about 0.25 inch distance from the surface of the mold cavity. This distance was sufficient for the sensor to be heated when the molten metal was poured into the mold cavity. On heating, the resistance of the wire coil changes. Since there was a change in resistance, there was a need to keep track of the resistance of wire along with the output frequency of the sensor, in order to know the temperature of the sensor. The sensor (in series with a $1-\mathrm{k} \Omega$ resistance) forms one leg in a Wheatstone Bridge circuit; the output voltage of the Wheatstone circuit changes according to the change in the resistance of the coil. The voltage is calibrated to calculate the temperature of the sensor. The temperature calibration is explained in detail in a later chapter.

To record the voltage and the sensor output simultaneously, a switching and sampling procedure was adopted. The switching circuit is shown in Figure 3.3.

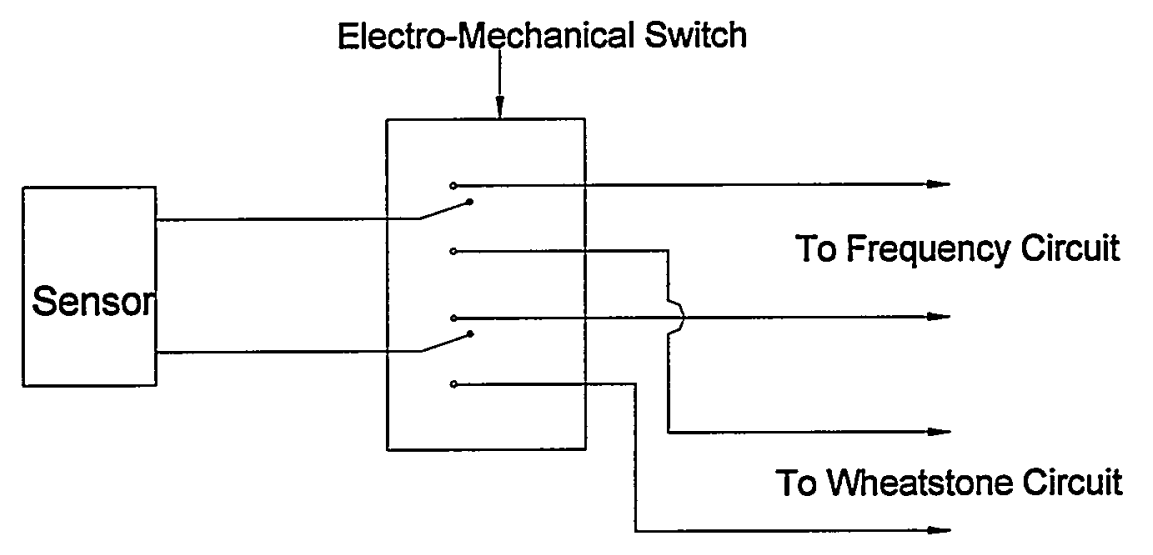

Figure 3.3. Switching circuit for temperature / frequency measurement

The terminals of the sensor coil are connected to the Wheatstone bridge, which is the resistance measuring circuit, and also to the frequency measuring circuit through an electromechanical switch. The electro-mechanical switch consists of a relay, which switches between the frequency measuring circuit and the Wheatstone bridge. In this way, both the frequency and the resistance of the coil can be simultaneously measured. 


\subsection{Wheatstone Bridge}

The Wheatstone bridge employed to measure the change in the resistance of the sensor coil is shown in Figure 3.4. The Wheatstone bridge is a circuit that is commonly used to make sensitive measurements of changes in resistance. The bridge is "balanced" when the voltage is zero across the bridge at room temperature. Typically a Wheatstone bridge consists of four resistors arranged in a diamond orientation. Resistors of $1.022 \mathrm{k} \Omega$ constitute the three arms of the bridge and a $1-\mathrm{k} \Omega$ resistor connected in series with the proximity sensor coil forms the fourth $\mathrm{arm}$. The resistance of the sensor coil is around $22 \Omega$, hence the bridge is nearly balanced at room temperature. An input $\mathrm{DC}$ voltage of 9 volts is applied between the top and bottom of the diamond, and the output voltage is measured across the middle. The Wheatstone bridge output (a DC voltage) is read by a Computer Boards CIO-DAS800 12 bit data acquisition board.

When molten aluminum is poured in the mold cavity, transfer of heat from the metal to the sensor heats the sensor. This increases the sensor's resistance, which creates a large imbalance in the bridge, causing a change in the output voltage of the previously balanced bridge. This unbalance causes a different voltage to appear across the middle of the bridge. The change in the output voltage is measured and converted to obtain the temperature of the sensor.

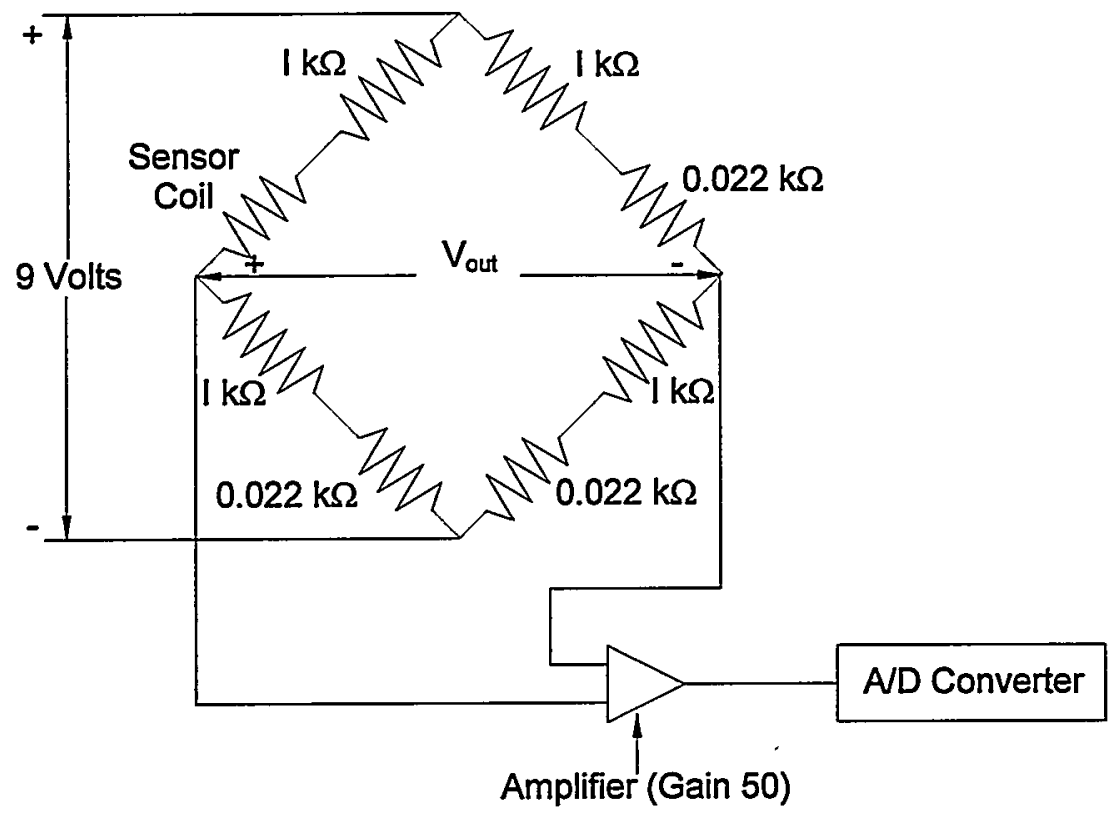

Figure 3.4. Wheatstone bridge for temperature measurement

Thus, the sensor output frequency and the temperature of the sensor can be alternately measured using the above-described circuits. Figure 3.5 shows a schematic of the complete frequency and resistance measurement system. 


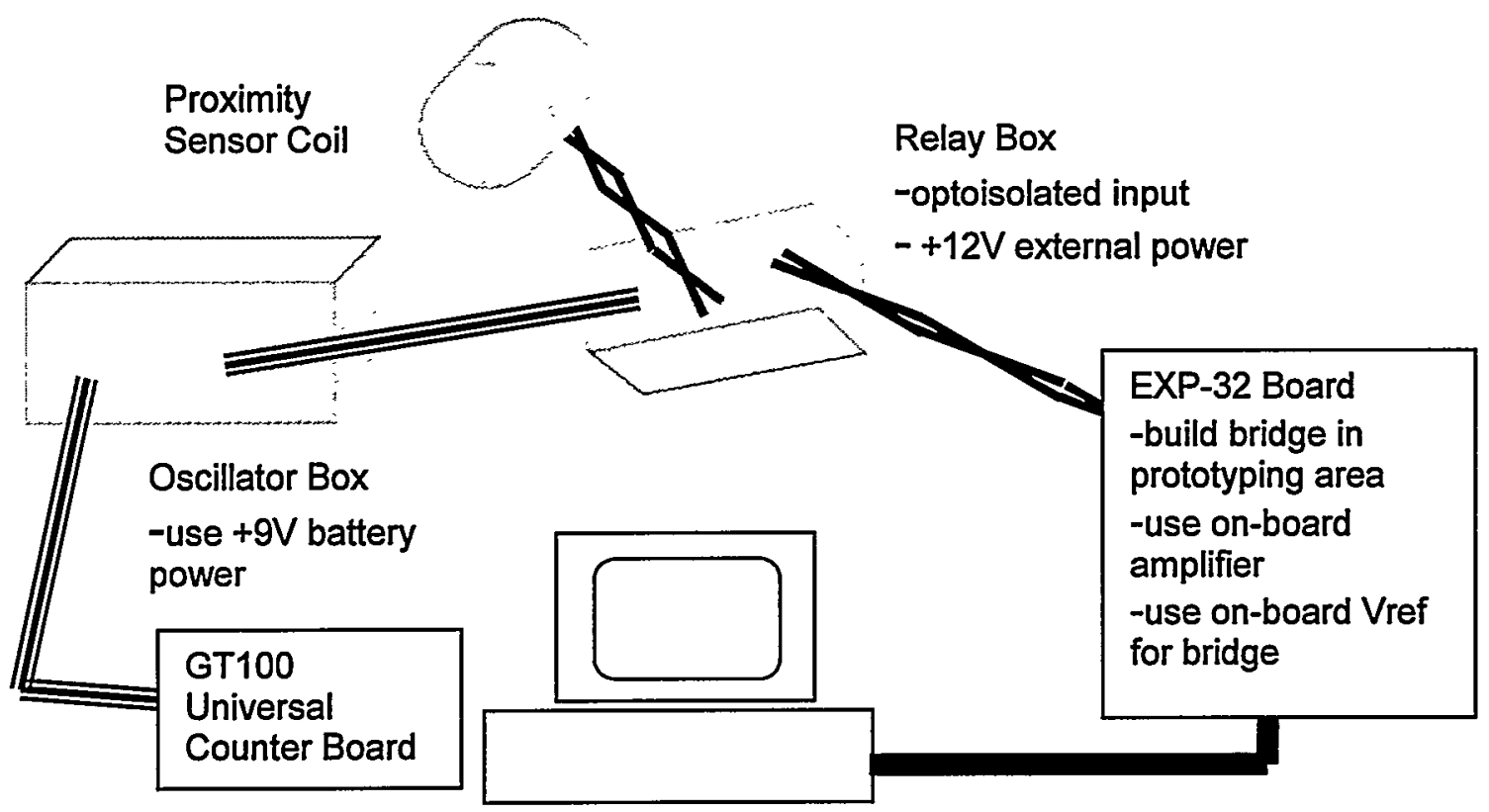

Figure 3.5. Proximity sensor frequency and resistance measurement system 


\subsection{SENSOR CALIBRATION}

The sensor fabrication process was discussed in the previous chapter. Once the fabrication process was complete, the sensor was calibrated in order to know its output frequency when it was placed at different distances from the aluminum target. Since the output frequency of each sensor was slightly different, all the sensors had to be calibrated before they could be used to measure the gap formation in castings. This chapter deals with the sensor calibration procedure and the determination of the 'calibration equation' of the sensor. Repeatability of the proximity sensors is also discussed in this chapter.

\subsection{Calibration Procedure}

The setup for frequency calibration of the sensor is shown in Figure 4.1.

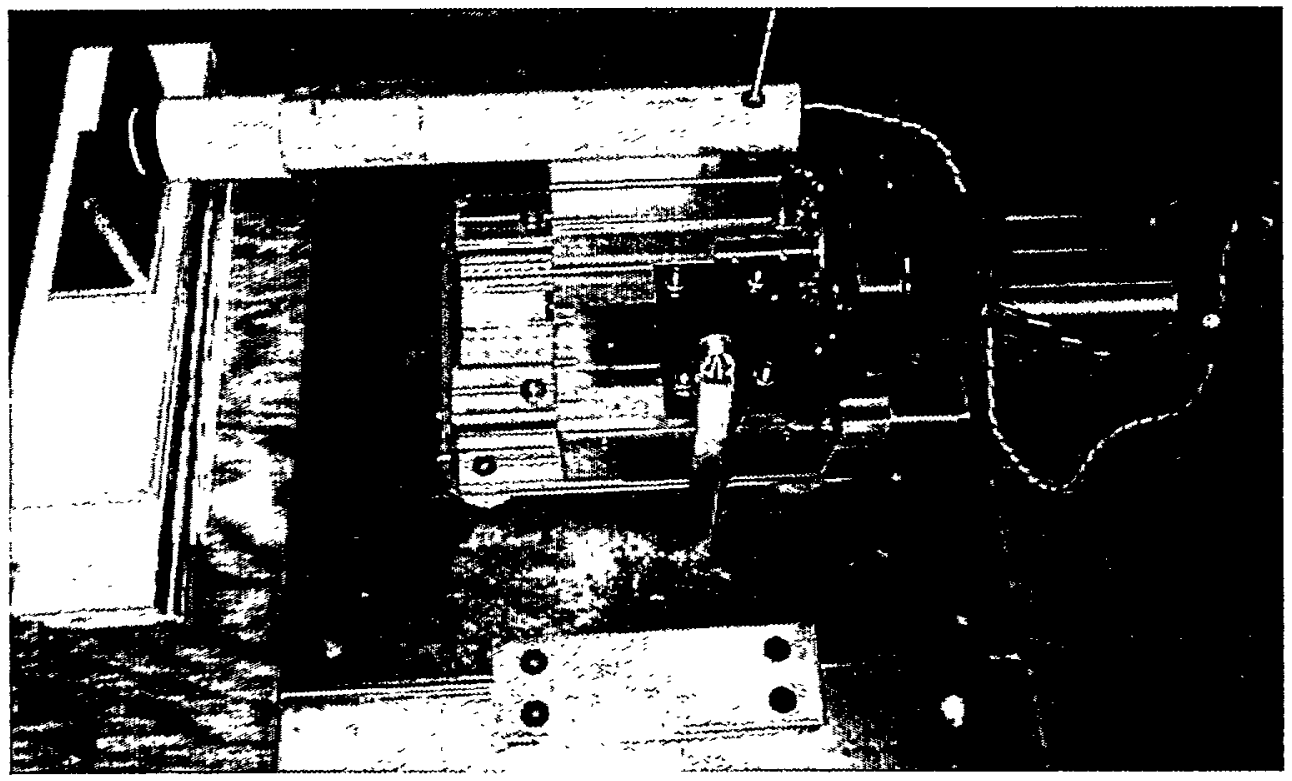

Figure 4.1. Sensor calibration setup

The sensor was mounted on to one end of a rigid plastic tube. The other end of the tube was fixed to the moving stage of the stepper motor. The sensor was then connected to the frequency measuring circuit. An aluminum plate was positioned in front of the sensor to serve as the target for the sensor. The calibration test procedure is

1. zero the sensor coil by making contact with an aluminum target,

2. move the sensor coil away from the target by 5 to 20 steps (each step is about $0.0048 \mathrm{~mm}$ or 0.00019 inch),

3. wait approximately 1 second,

4. take 5 to 10 readings ( $\sim 50 \mathrm{msec}$ per measurement) from the frequency measuring circuit and record results in a data file,

5. record the actual position of the sensor with a digital dial indicator and record the position results in a data file,

6. repeat steps $2-5$ until the sensor has traveled the length of the test range, then

7. repeat steps $2-6$ as the sensor is moved back towards the target. 
Typical sensor behavior as it was moved away from the target is shown in the Figure 4.2.

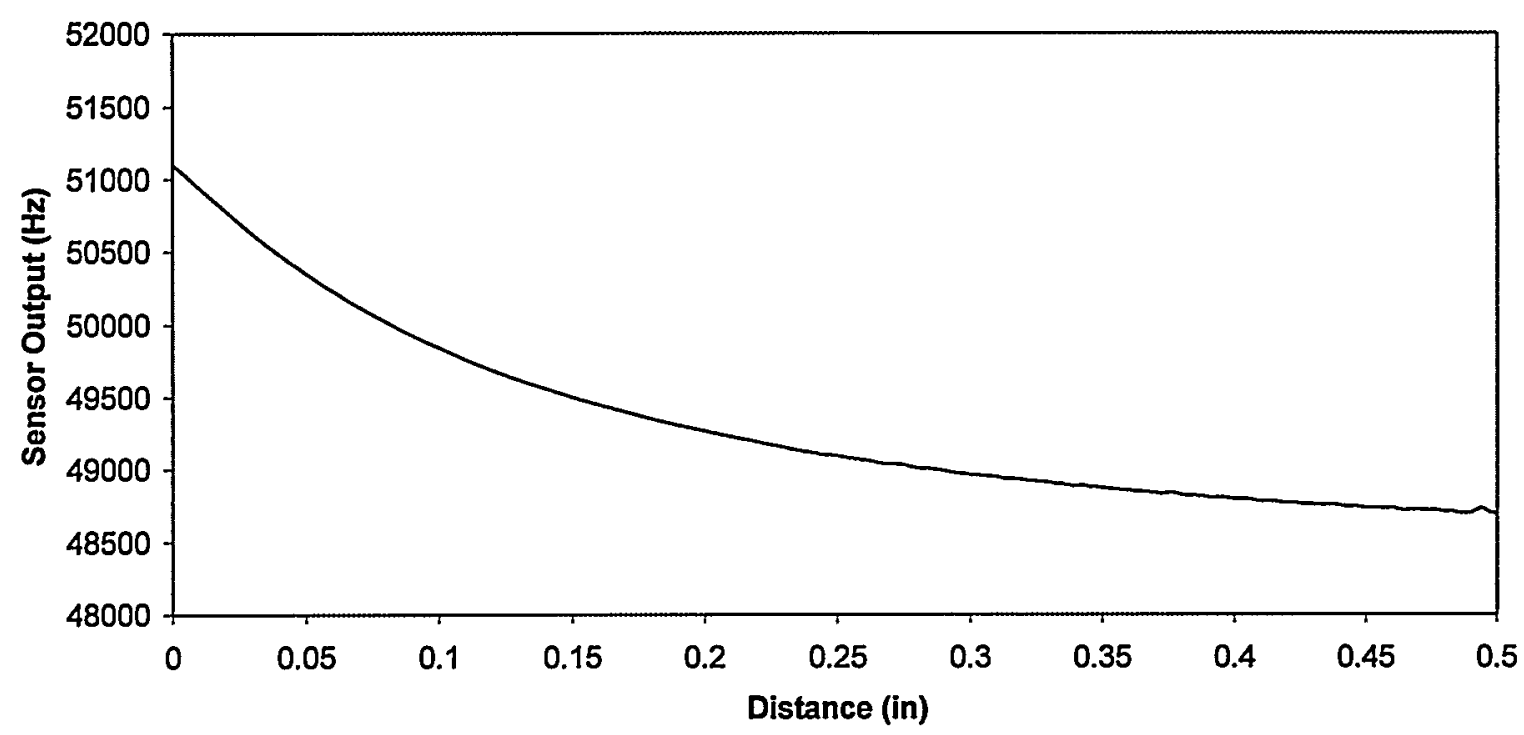

Figure 4.2. Typical behavior of the sensor as moved away from the target

It can be seen from the above plot that, as the sensor was moved away from the target to a distance of 0.5 inch, the output frequency of the sensor decreased from about $51,000 \mathrm{~Hz}$ to about $48,700 \mathrm{~Hz}$. When the sensor was near the aluminum target, it had large impedance. As the distance between the sensor and the target was increased, the impedance decreased [2] and the output frequency also decreased accordingly.

The calibration data were then exported to Microsoft Excel. The sensor was initially at a distance of 0.25 inch from the metal surface in the sand mold. The gap formation was very small, hence the calibration data of interest were around 0.2 inch to 0.35 inch. Therefore, the distance from 0.22 inch to 0.35 inch and the corresponding sensor output were plotted with the distance on $\mathrm{x}$-axis and sensor output on $\mathrm{y}$-axis. A best-fit curve of second order, to fit the data was then plotted. The equation of the best-fit curve was the calibration equation of the sensor. The calibration equation described the output of the sensor as a function of its distance from aluminum target. The determination of the calibration equation of the sensor is shown in Figure 4.3.

From foundry data the output of the sensor was recorded as a function of time. From the lab calibrations, the calibration equation was determined. The coefficients of the terms in the calibration equation were among the parameters used in analysis of the data to obtain the gap formation as a function of time. 


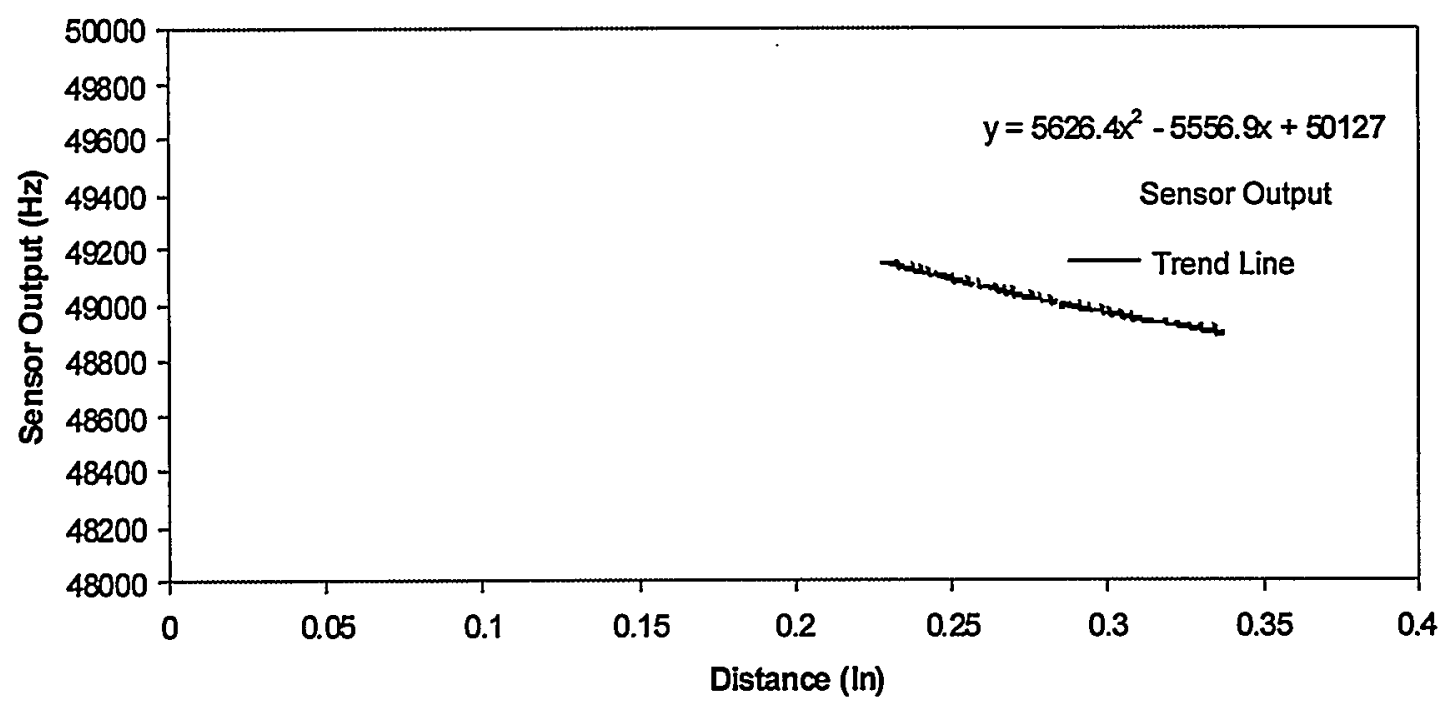

Figure 4.3. Determination of the "Calibration equation" of the sensor

\subsection{Measured Position from Sensor Frequency}

The determination of the distance between the sensor and the mold-metal interface is discussed in this section. In the foundry application, the time and the sensor output (frequency) are recorded continuously. As described earlier, the equation of the sensor output frequency is fitted to a second order curve of the form:

$$
f=f_{0}+p_{1} D+p_{2} D^{2}
$$

where $D$ is the distance between the sensor and the metal calibration surface, $f$ is the measured sensor frequency, and $f_{0}, p_{1}$, and $p_{2}$ are the curve fit parameters uniquely determined for each sensor via calibration. The equation can be rewritten as

$$
p_{2} D^{2}+p_{1} D+\left(f_{0}-f-\Delta f\right)=0
$$

There is some "drift" in the sensor output frequency between the lab calibration and the use of the sensor in the foundry. The $\Delta f$ in the equation above is applied to ensure that the initial computed position of the sensor is at the known stand-off distance (usually 0.25 inch). The equation derived above is a quadratic equation in $D$. Given a measured value of $f$, the equation can be solved to obtain the distance, $D$, of the metal from the surface of the sensor.

\subsection{Sensor Repeatability}

Repeatability is the ability of the sensor to give the same output, over and over again, for the same input. It is very important that the sensor is highly repeatable. When the sensor is calibrated again and again, it should have the same output frequency for the same position. If the sensor is not repeatable, then the correlation of lab data and foundry data are incorrect. To show the repeatability of the sensors, test calibrations were carried out. A sensor was mounted on the stepper motor and it was calibrated several times using the above-described calibration 
procedure. The results are plotted as shown in Figure 4.4. It can be seen from the plot in Figure 4.4 that the trend of change of sensor output with the increase in the distance is almost the same. The plot for each of the iterations is essentially parallel.

Since the sensor is at a distance of 0.25 inch in the mold, and the width of gap formation is considered to be narrow, the data of interest is in the range of 0.22 inch to $0.35 \mathrm{inch}$. The data for the above plot in this region has been plotted as shown in Figure 4.5.

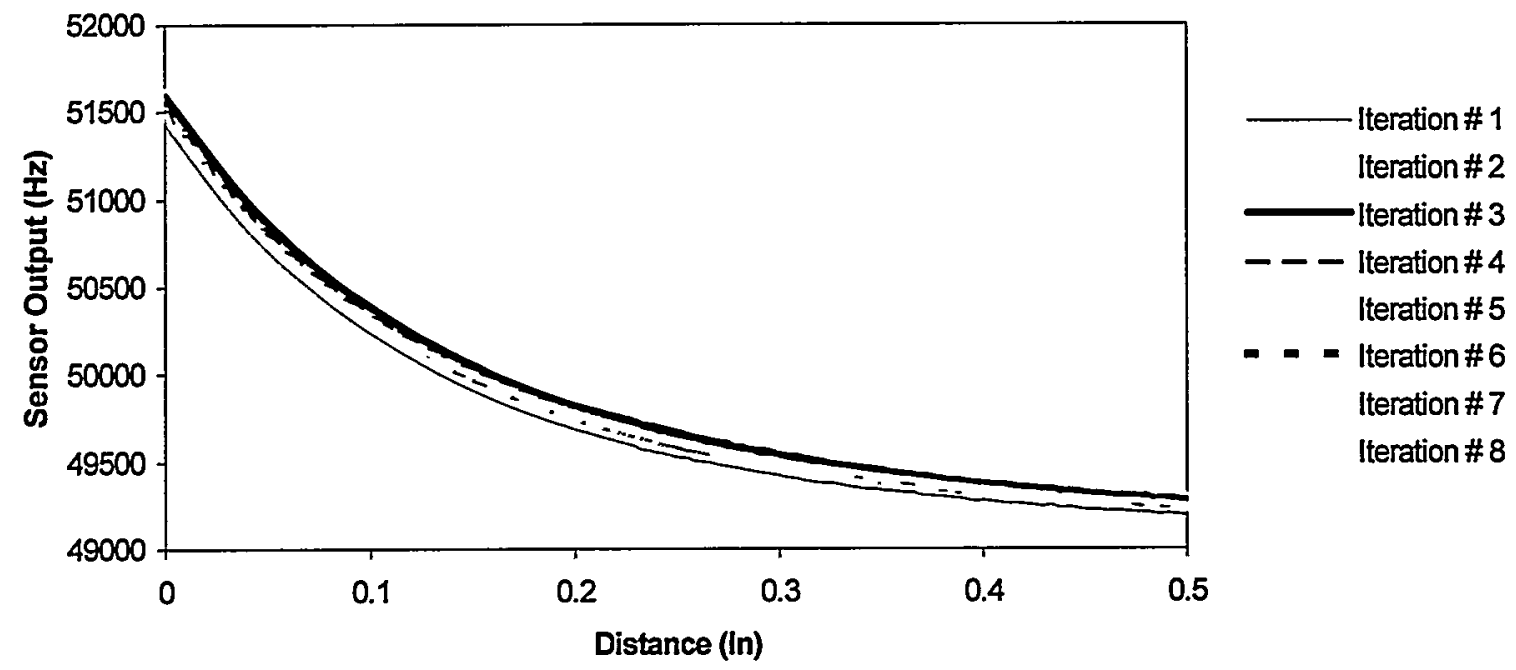

Figure 4.4. Plot of Sensor Output for a series of iterations

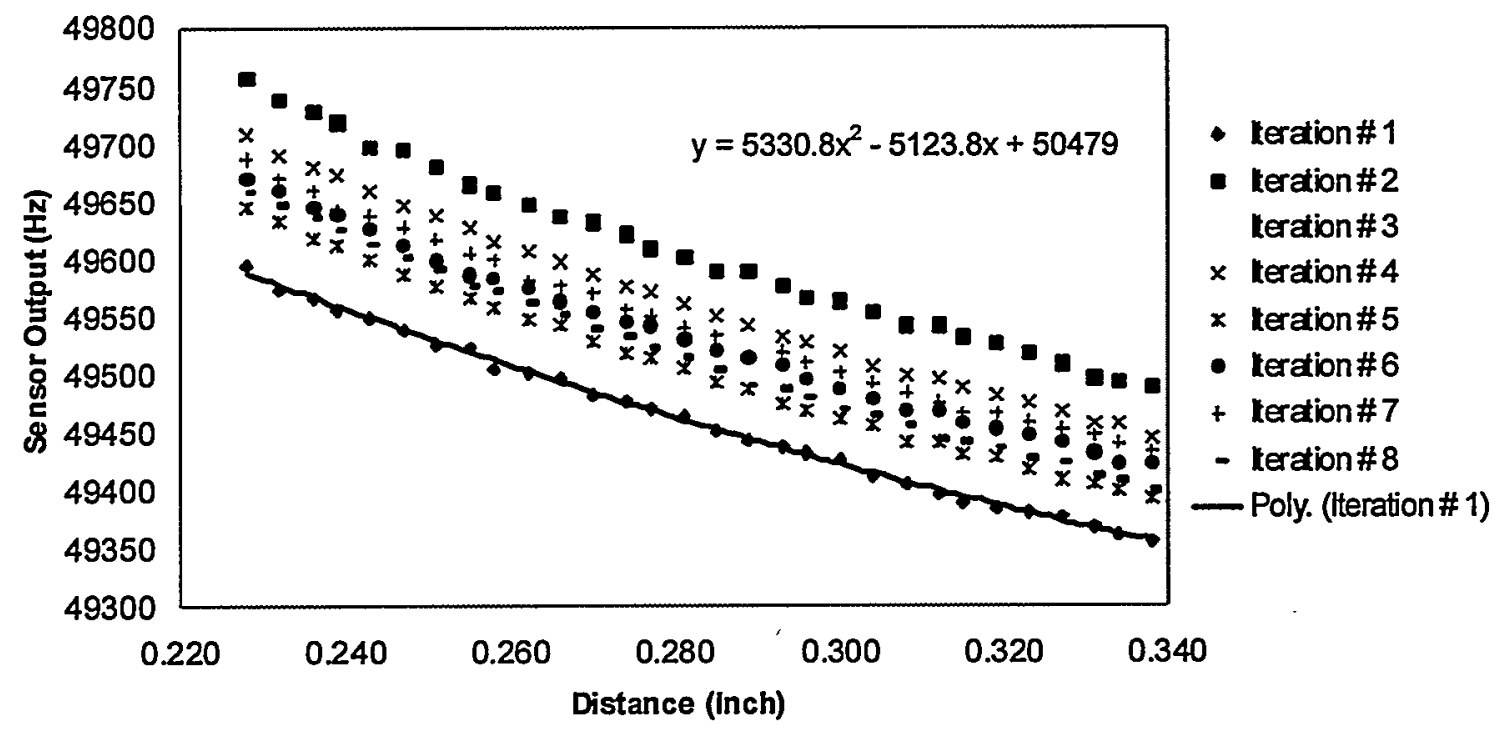

Figure 4.5. Repeatability test results

A trend line of second order has been fitted to the data of the first iteration, which is the worst case. The standard deviation at each of the positions has been calculated, and the average 
of these standard deviations was $46 \mathrm{~Hz}$. The following calculations have been made to determine the range of error in absolute position at 0.25 inch:

Slope of curve for first iteration at 0.250 inch $=-2458 \mathrm{~Hz} /$ inch

Sensitivity $=1 /$ Slope $=-0.0004068 \mathrm{inch} / \mathrm{Hz}$

Standard deviation $\sigma=46 \mathrm{~Hz}$

$\Rightarrow( \pm 2 \sigma) *(1 /$ Slope $)= \pm 0.037$ inch

There is a $95 \%$ confidence that any future absolute distance calculated from the calibration curve will be within \pm 0.037 inch of the true value. This is a relatively large error and indicates that the current design of the proximity sensor is not very accurate for absolute position measurements.

The distance corresponding to each of the frequency measurements for iterations \#2 through \#8 was determined using the trend line equation of the first iteration. This data represents the estimated distances, $D_{\text {EST. }}$. The difference of values of $D_{\text {EST }}$ and the $D_{\text {EST }}$ at a true 0.25 inch have been calculated and represent an estimated delta position, $\Delta_{\text {EST. }}$ The true delta position is indicated by $\Delta_{\mathrm{TRUE}}$, i.e.,

$$
\Delta_{\text {TRUE }}=\text { True position }-0.250 \text { inch. }
$$

The error is the difference between $\Delta_{\mathrm{EST}}$ and $\Delta_{\mathrm{TRUE}}$, which is plotted in Figure 4.6 versus true distance.

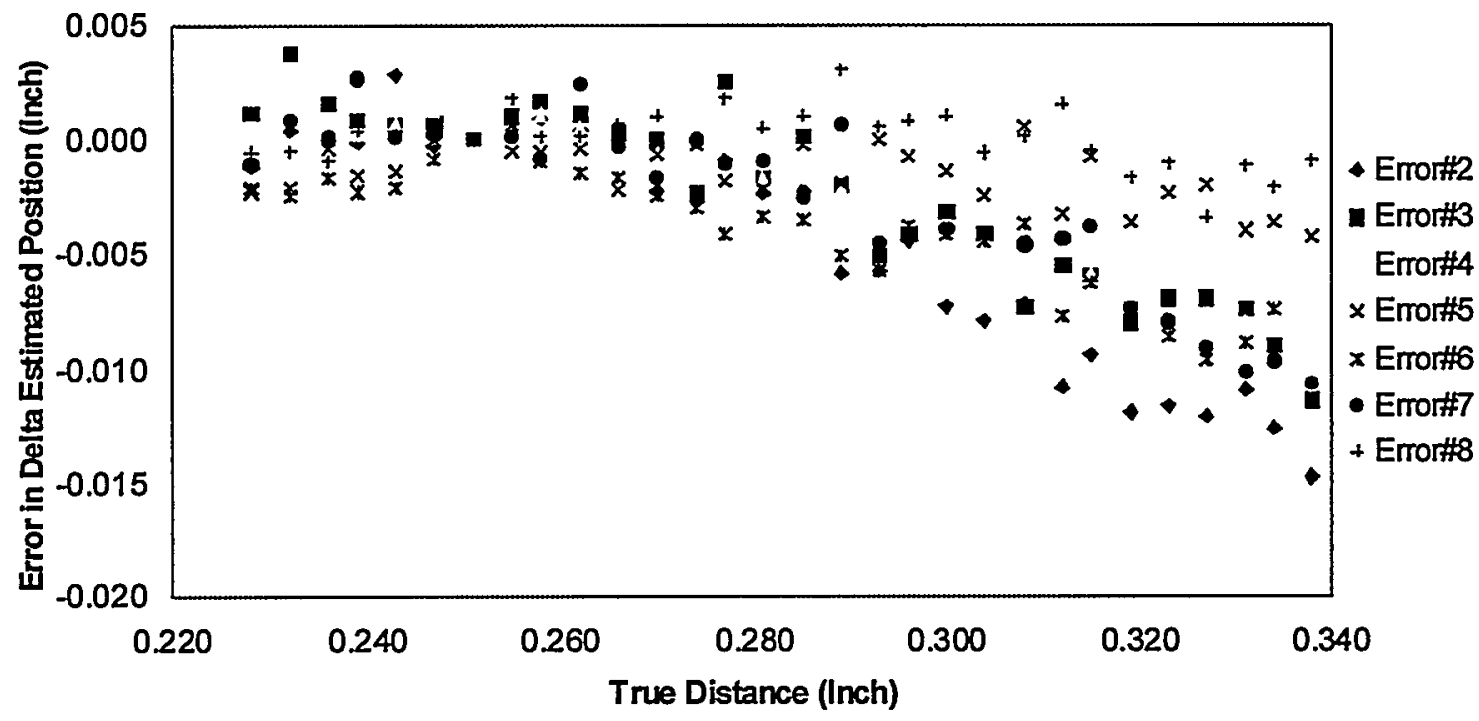

Figure 4.6. Error in delta position

The average standard deviation of the data shown in the above plot over the range of true distance from 0.220 to 0.280 was calculated to be 0.00115 inch:

Standard deviation $\sigma=0.00115$ inch

$$
\Rightarrow( \pm 2 \sigma)= \pm 0.0023 \text { inch }
$$

This value of \pm 0.0023 inch indicates that the current design of the proximity sensor is reasonably accurate for small relative position measurements. 


\subsection{Temperature Calibration of the Sensor}

Significant temperature effects on the output of the proximity sensor were observed in both the foundry and the lab tests. In both environments the effect was relatively minor at first, but caused a rapid and large increase in the sensor output after prolonged exposure. It was important to know the temperature of the sensor at which this rapid increase of the output was encountered. A temperature equation that uses output voltage of the Wheatstone bridge to calculate the temperature of sensor, is derived below.

When the sensor in the mold is heated, the resistance of the sensor wire coil changes. Since the sensor in series with 1-k $\Omega$ resistance forms one of the arms in the Wheatstone circuit, the output voltage of the Wheatstone circuit changes according to the change in the resistance of the coil. This output voltage is used to calculate the temperature of the sensor.

The sensor is fabricated by winding 450 turns of the 34-gage CLASS HML $220^{\circ} \mathrm{C}$ magnet wire on a nylon spool. This is typically about 85 feet of wire. The resistance of the 34gage wire is $266 \Omega$ per 1,000 feet at $77^{\circ} \mathrm{F}$ and $307 \Omega$ per 1,000 feet at $149{ }^{\circ} \mathrm{F}$ [14]. The plot of the resistance is shown in Figure 4.7.

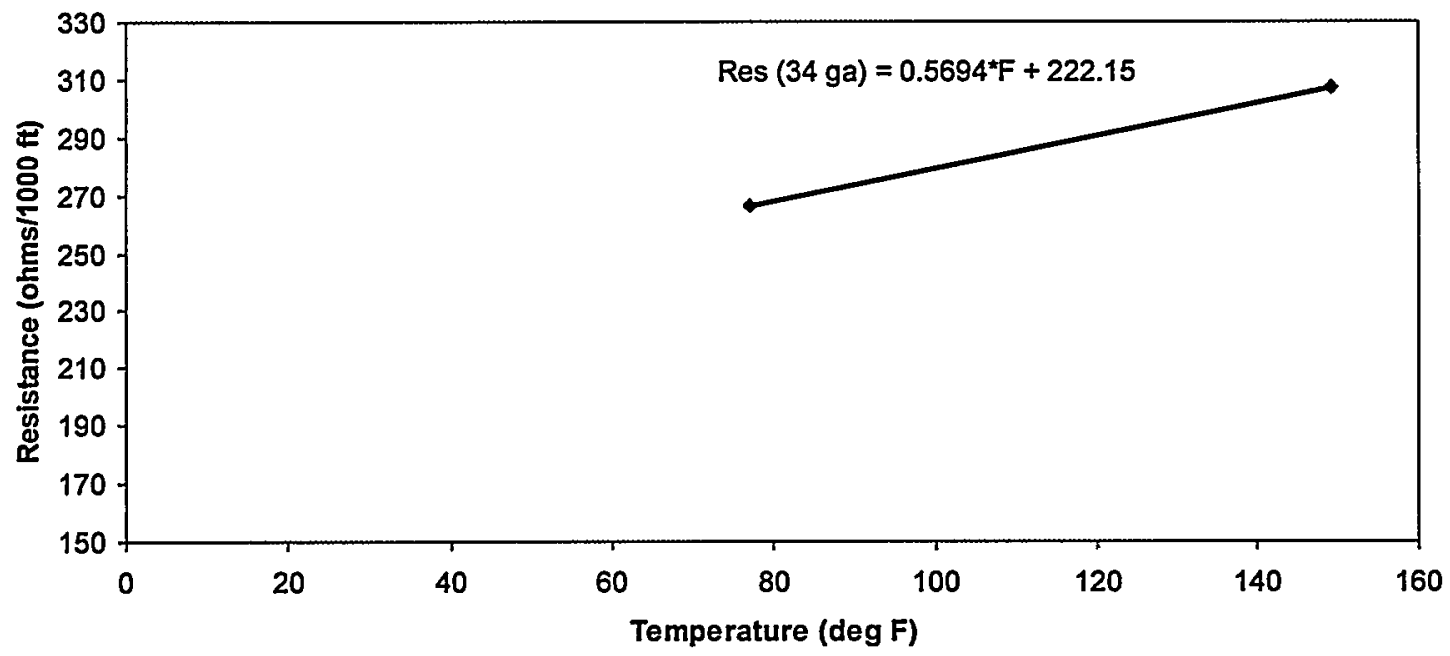

Figure 4.7. Plot of resistance per 1,000 feet of wire vs temperature

A trend line has been plotted between these two points. The equation that describes the relation between resistance per 1,000 feet of wire and temperature is

$$
\text { Resistance per } 1,000 \text { feet }=0.5694 * \mathrm{~F}+222.15
$$

The resistance of the wire per 1,000 feet derived from this equation is $240.4 \Omega$ per 1,000 feet at $32^{\circ} \mathrm{F}$ and $342.9 \Omega$ per 1,000 feet at $212^{\circ} \mathrm{F}$. As mentioned earlier, the length of wire coil is about 85 feet; therefore, the resistance of the coil at $32^{\circ} \mathrm{F}\left(\mathrm{R}_{32}\right)$ is $20.4 \Omega$, and the resistance of the coil at $212^{\circ} \mathrm{F}\left(R_{212}\right)$ is $29.1 \Omega$. So, the change in resistance $\Delta R=R_{212}-R_{32}=8.7 \Omega$. The ratio of change in voltage $\Delta \mathrm{V}$ to the bridge voltage $\mathrm{V}$, is given by:

$$
\Delta \mathrm{V} / \mathrm{V}=(1 / 4) *(\Delta \mathrm{R} / \mathrm{R}) * \mathrm{G}
$$


where, $G$ is the gain of the amplifier $=50$. The $1 / 4$ term is used for a quarter bridge configuration, where only one of the four legs of the Wheatstone bridge is active.

The corresponding change in voltage $\Delta \mathrm{V}$ is given by the equation:

$$
\begin{aligned}
\Delta \mathrm{V} & =(1 / 4) *(\Delta \mathrm{R} / \mathrm{R}) * \mathrm{~V} * \mathrm{G} \\
\Rightarrow \Delta \mathrm{V} & =(1 / 4) *(8.7 / 1025) * 9 * 50
\end{aligned}
$$

Therefore, $\Delta \mathrm{V}=0.95625$ Volts. The change in temperature $\Delta \mathrm{T}=212^{\circ} \mathrm{F}-32^{\circ} \mathrm{F}=180^{\circ} \mathrm{F}$. Hence, the ratio of change in temperature to the change in voltage is $\Delta \mathrm{T} / \Delta \mathrm{V}=188^{\circ} \mathrm{F}$ per Volt:

$$
\begin{gathered}
\Rightarrow 188 *\left(\mathrm{~V}_{0}-\mathrm{V}_{1}\right)=\left(\mathrm{T}_{0}-\mathrm{T}_{1}\right) \\
\Rightarrow \mathrm{T}_{1}=\left(188 * \mathrm{~V}_{1}\right)+\left(\mathrm{T}_{0}-188 * \mathrm{~V}_{0}\right)
\end{gathered}
$$

where, $T_{1}$ is the present temperature of the sensor

$T_{0}$ is the initial temperature of the sensor

$V_{1}$ is the present output voltage of the Wheatstone bridge

$\mathrm{V}_{0}$ is the initial output voltage of the Wheatstone bridge.

The factor $\left(\mathrm{T}_{0}-188 * \mathrm{~V}_{0}\right)$ is a constant quantity and was adjusted by trimming the potentiometer that is connected between the top and the bottom of the Wheatstone bridge (Figure 2.19) at the beginning of the experiment. Therefore,

$$
\mathrm{T}_{1}=\left(188 * \mathrm{~V}_{1}\right)+\text { constant }
$$

Using the above relationship, the temperature of the sensor could be calculated provided the output voltage of the Wheatstone bridge was known.

\subsection{Resistance-Temperature Validation}

Static hot plate tests were conducted in the laboratory to verify the equation derived in the above section. Four thermocouples were glued to the front and the back surface of the nylon spool before encasing it in the ceramic material. The design of the sensor with thermocouples is shown in Figure 4.8. The sensor was then covered with ceramic material.

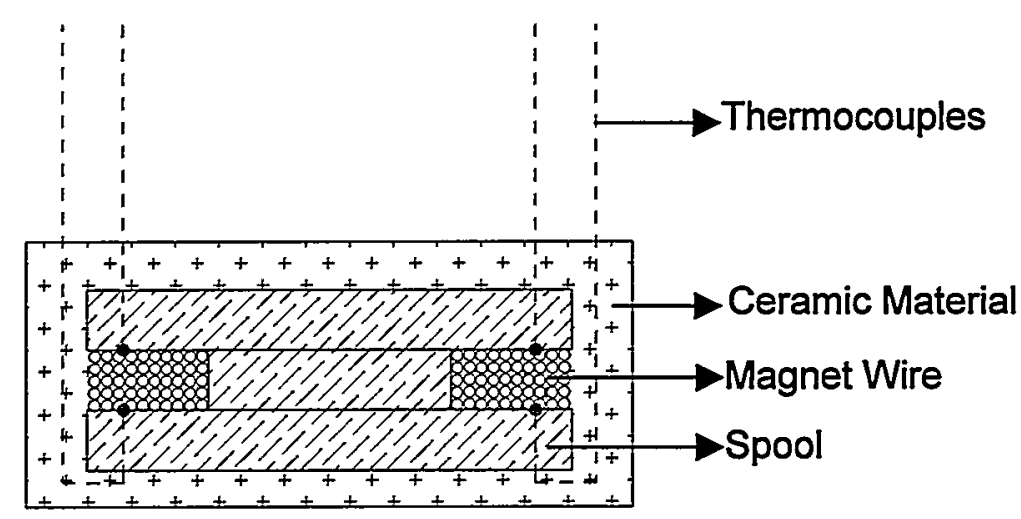

Figure 4.8. Schematic of the sensor with thermocouples 
A typical sensor pack was made with a stand-off distance of 0.25 inch using this sensor. A static hot plate test was conducted with this sensor pack, as follows:

The heater plate was turned on and allowed to heat to a temperature of $900^{\circ} \mathrm{F}$. The output voltage from the Wheatstone bridge and the readings on the four thermocouples were recorded continuously. Then after about 150 seconds, the sensor pack was placed on the heater plate. The temperature recorded by the four thermocouples and the calculated temperature of the sensor were plotted, as shown in Figure 4.9.

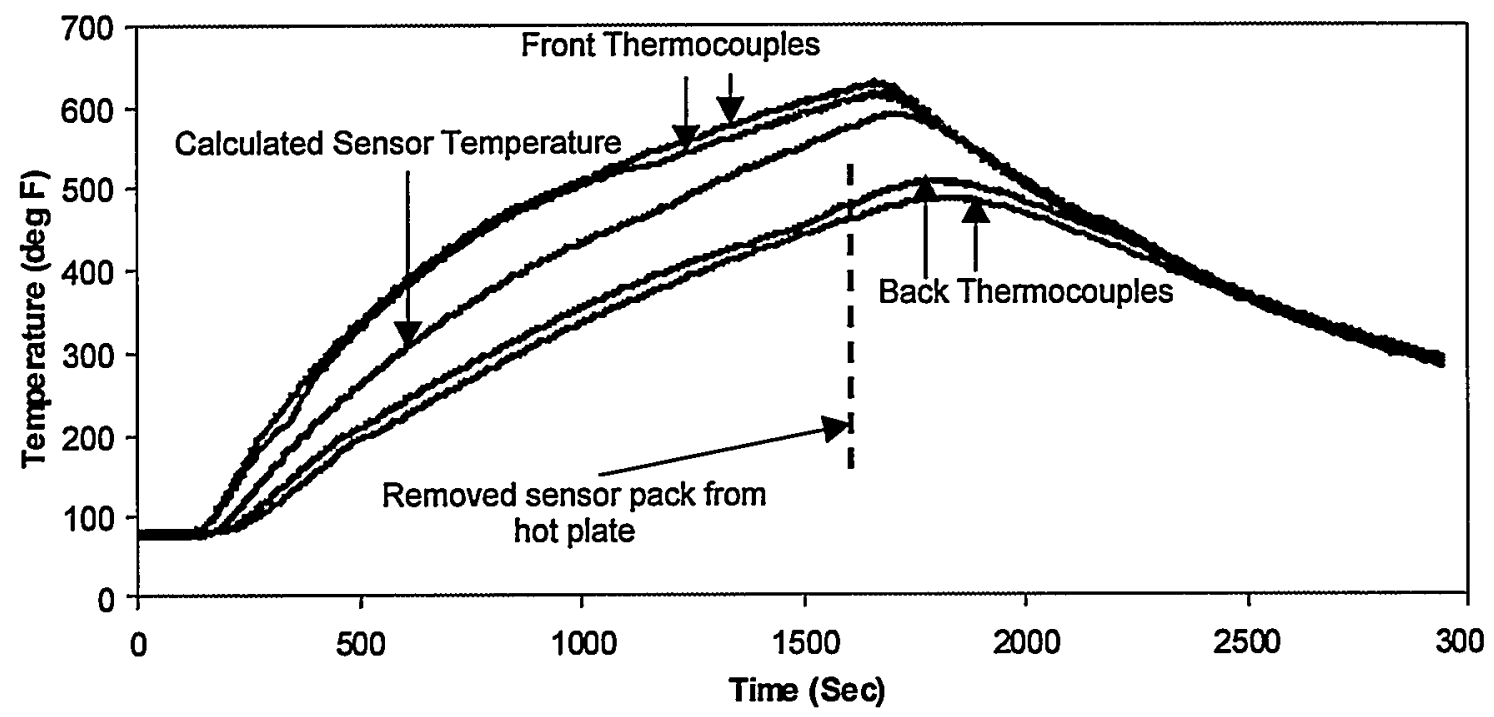

Figure 4.9. Sensor-thermocouple calibration

It can be seen from the above plot that the temperature recorded by the thermocouples that were located nearer to the surface of the heater plate, referred to as front thermocouples, increased from about $80^{\circ} \mathrm{F}$ to $615^{\circ} \mathrm{F}$ at the end of 1670 seconds after the start of the test. The temperature recorded by the thermocouples that were located away from the surface of the heater plate, referred to as back thermocouples, increased from about $80^{\circ} \mathrm{F}$ to $500^{\circ} \mathrm{F}$ at the end of 1800 seconds after the start of the test. The sensor temperature was also calculated using the output voltage from the Wheatstone bridge as detailed in section 4.4. The calculated sensor temperature is also plotted in Figure 4.10. It can be seen that the calculated sensor temperature is approximately equal to the average of the temperatures recorded by the four thermocouples. The average of the readings on four thermocouples has been calculated. This value and the value of calculated sensor temperature has been plotted against time. The plot is shown in Figure 4.10.

It can be seen from the above plot that, in the useful range of sensor operation (when the temperature of the sensor is below $450^{\circ} \mathrm{F}$ ), the average of the four thermocouple readings and the calculated sensor temperature are approximately equal. Hence the calculated temperature of the sensor can be taken as bulk temperature of the sensor. Therefore, the formula derived in section 4.3 can be used to determine the bulk temperature of the sensor, provided the output voltage from the Wheatstone bridge is given. 


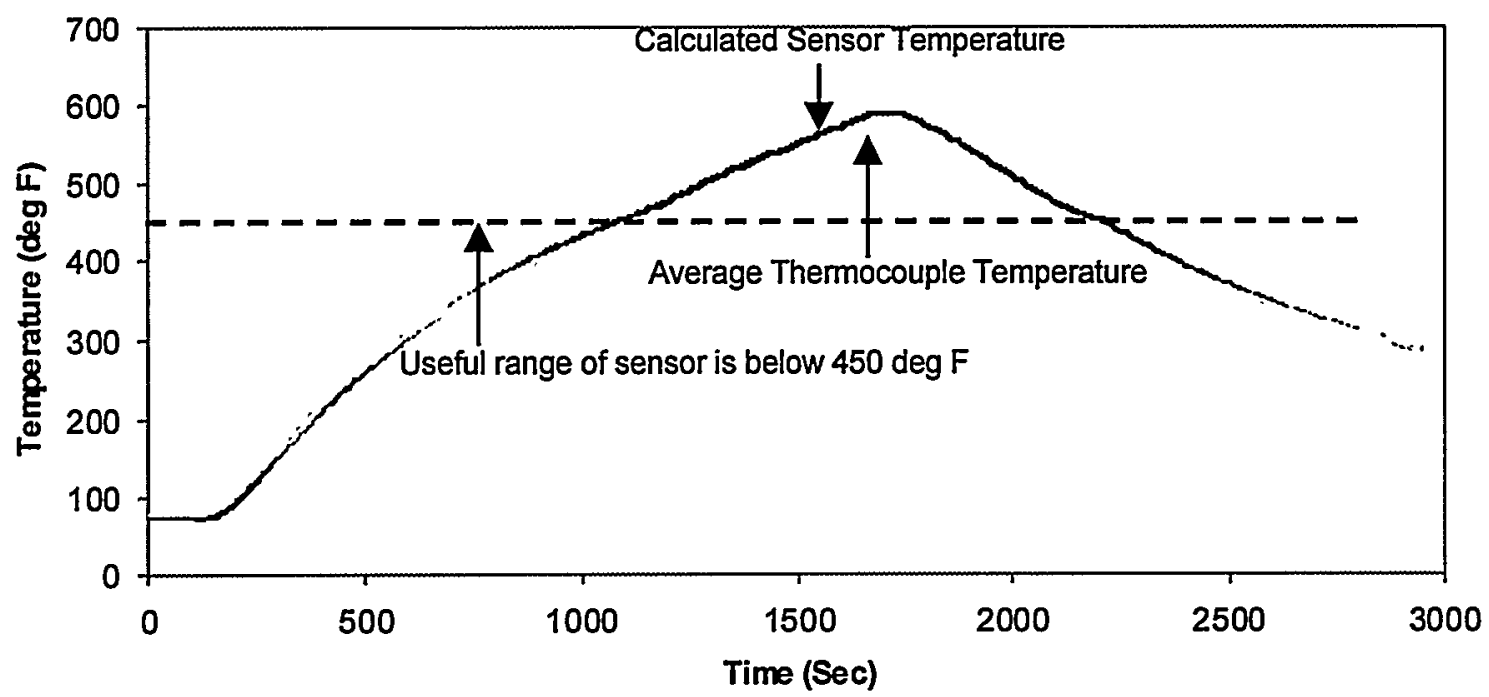

Figure 4.10. Average thermocouple temperature and calculated sensor temperature

\section{6 "Hot Plate" Calibration}

The sensor calibration discussed in section 4.1 was conducted with a constant, room temperature target. "Hot plate" calibrations were also conducted where the output of the sensor and the output voltage from the Wheatstone bridge were recorded continuously. The readings of the thermocouples located on the hot plate were also recorded. The behavior of the sensor in the hot plate calibration is presented in this section.

A typical sensor pack has been made with the sensor. A stand-off distance of 0.25 inch of resin-bonded sand was maintained in front of the sensor. The sensor was fastened on the stepper motor base and then aligned against the hot plate such that the surface of the sensor pack was in contact with the hot plate, as shown in Figure 4.11. The hot plate was turned on, and the calibration procedure was performed by moving the sensor to a distance of 0.08 inch from the hot plate, and then retracting the sensor pack towards the hot plate. Initially the temperature of the hot plate was maintained at $80^{\circ} \mathrm{F}$. The sensor calibration procedure was iterated three times and then the temperature on the hot plate was increased to $300^{\circ} \mathrm{F}$ and 6 iterations were done. This procedure was repeated by increasing the temperature of the hot plate to $600^{\circ} \mathrm{F}$ and $900^{\circ} \mathrm{F}$. The output of the sensor and the output voltage from the Wheatstone bridge were recorded continuously. The data are plotted in Figure 4.12.

Figure 4.12 shows that there was a drop in the sensor output as it was moved away from the hot plate. The peak of the sensor output curve is the output of the sensor when the sensor pack is in contact with the hot plate. The valley of the curve is the sensor output when the sensor pack is at a distance of 0.08 inch from the hot plate. The temperature of the sensor is calculated from the output voltage of the Wheatstone bridge. The first three iterations were made while maintaining the temperature of about $80^{\circ} \mathrm{F}$ on the hot plate. The sensor output at the peak is about $49,560 \mathrm{~Hz}$, and it is about $49,400 \mathrm{~Hz}$ when it is at a distance of 0.08 inch from the hot plate. The next six iterations were with a temperature of $300^{\circ} \mathrm{F}$ on the hot plate. 


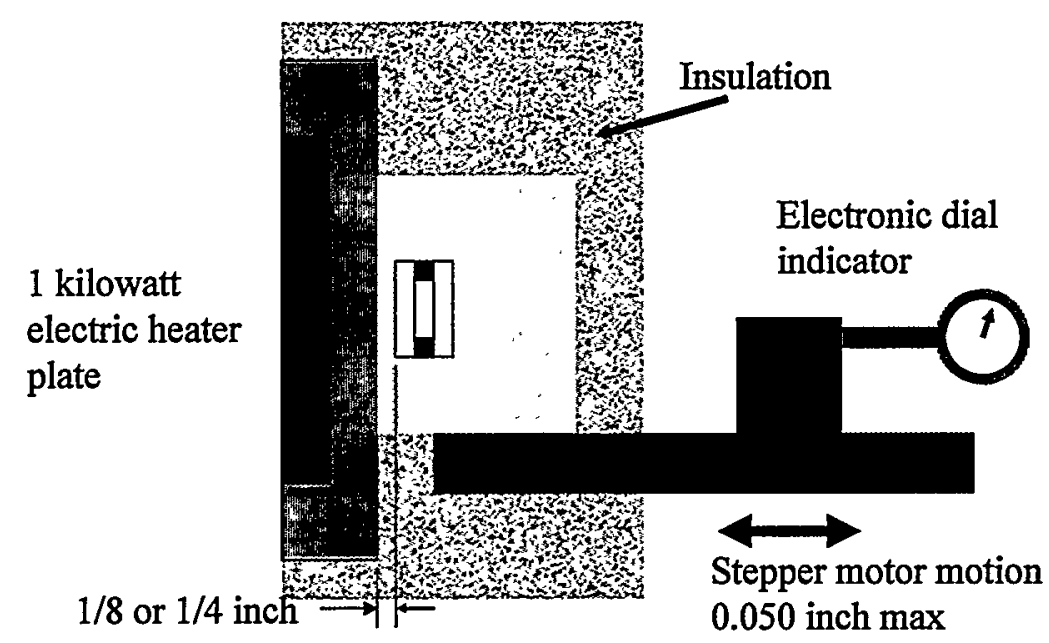

Figure 4.11. Hot-plate calibration test setup

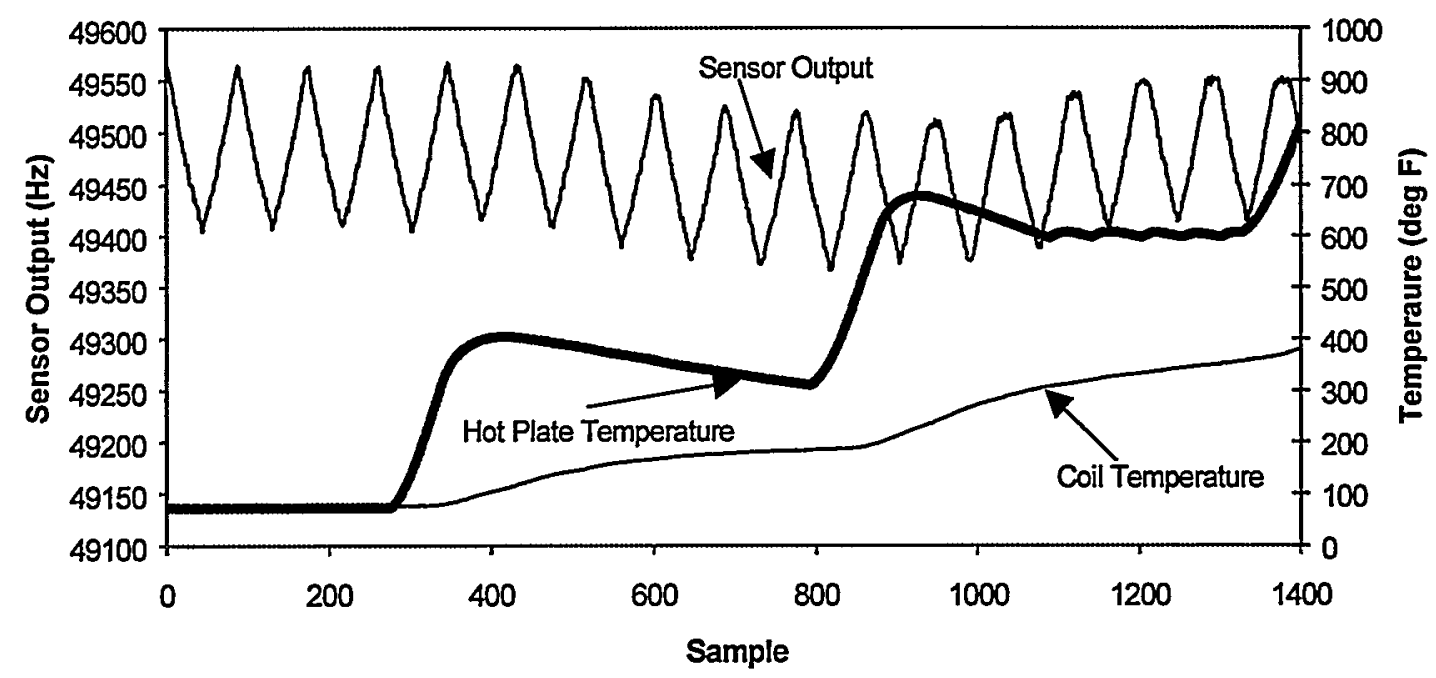

Figure 4.12. Position-temperature calibration of the sensor

It can be seen that the temperature of the sensor increased from $80^{\circ} \mathrm{F}$ to about $185^{\circ} \mathrm{F}$ at the end of these six iterations. During this period the whole calibration curve shifted down by about $50 \mathrm{~Hz}$. This downward shift in sensor output frequency will be discussed in the next section. The temperature of the hot plate was then increased to $600{ }^{\circ} \mathrm{F}$ and six more iterations were carried out. The temperature of the sensor increased to about $350^{\circ} \mathrm{F}$. At this point the sensor output curve decreased until the temperature of the sensor was about $200^{\circ} \mathrm{F}$. After the temperature of the sensor increased beyond about $200^{\circ} \mathrm{F}$ there was a shift of the calibration curve upwards. Finally, the hot plate temperature was increased to $900^{\circ} \mathrm{F}$ and six iterations were performed. The temperature of the sensor increased to about $390^{\circ} \mathrm{F}$. The calibration curve also shifted upwards. When the temperature of the sensor crossed about $400^{\circ} \mathrm{F}$, the sensor output increased beyond the normal range of operation, where the test was terminated. 
From the test described in the above paragraph, it is clear that the sensor output initially decreases continuously till the temperature of the sensor reaches about $280^{\circ} \mathrm{F}$. If the temperature of the sensor is increased to about $380^{\circ} \mathrm{F}$, the sensor output increases constantly. When the temperature of the sensor increases above about $380^{\circ} \mathrm{F}$, the sensor output increases beyond the normal range of operation. The effective data that can be considered for analysis from the proximity sensor is the data where the temperature of the sensor is less than $400^{\circ} \mathrm{F}$.

Several attempts were made to develop a temperature correction factor to adjust the proximity sensor frequency data temperature-dependant behavior described above. These efforts met with limited success, and were eventually abandoned based on the analysis of several foundry test pours. One part of the temperature calibration which may explain the initial drop in sensor output during the hot plate test is described in the next section.

\subsection{Sand Pack Expansion}

Figure 4.13 shows the results of several "static" hot-plate tests. In each of these tests a sensor sand pack was placed on the hot plate surface, which was pre-heated to $900^{\circ} \mathrm{F}$.

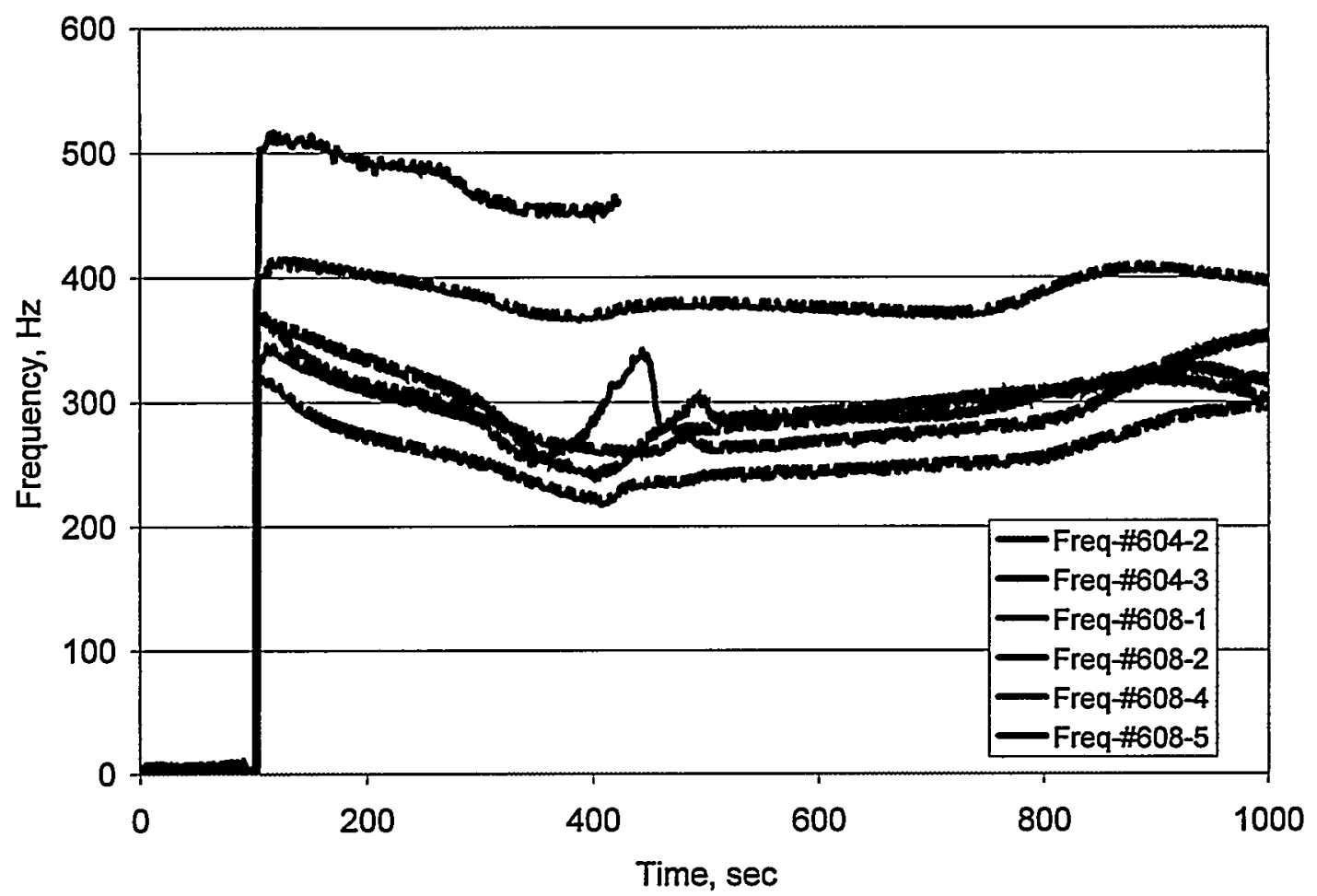

Figure 4.13 Results of static hot-plate tests 
Frequency is displayed in the "delta" mode, where the initial "no target" output of the sensor is subtracted from each subsequent reading. Note that all six sensor outputs have roughly the same drop in frequency over the first 350-400 seconds of the test. This drop in sensor output would correspond to the sensor moving away from the surface of the hot plate. Since the sensor pack rested on the hot plate surface, movement experienced by the sensor pack would be due to thermal expansion of the 0.25 inch layer of resin-bonded sand between the sensor and the hotplate surface.

Another series of tests was conducted to further document the initial sand pack expansion. The test apparatus is shown in Figure 4.14. In these tests one electronic dial indicator was used to correlate the movement of the sensor pack (due to thermal expansion) and the frequency measured by the sensor. The second dial indicator was used to subtract the expansion of the "hot plate" from the overall movement of the sensor pack. Thermocouples were mounted to the rod connecting the second dial indicator and the aluminum disk. The temperatures measured from these thermocouples were used to determine the thermal expansion of the rod during the test. Figure 4.15 defines the lengths $L$ used in the data reduction for these tests.

From the geometry defined by Figure 4.15,

$$
L=L_{1}+L_{\text {rod }}+L_{\text {alum }}=L_{2}+L_{\text {pack }}+L_{\text {alum }}
$$

Each of the lengths (except for $L$ ) consists of both an initial length and a change in length, so

$$
\left(L_{1}+\Delta L_{1}\right)+\left(L_{\text {rod }}+\Delta L_{\text {rod }}\right)+\left(L_{\text {alum }}+\Delta L_{\text {alum }}\right)=\left(L_{2}+\Delta L\right)_{2}+\left(L_{\text {pack }}+\Delta L_{\text {pack }}\right)+\left(L_{\text {alum }}+\Delta L_{\text {alum }}\right)
$$

Subtracting the initial lengths from both sides of the equation and rearranging gives, where

$$
\Delta L_{\text {pack }}=\Delta L_{1}-\Delta L_{2}+\Delta L_{\text {rod }}
$$

$\Delta L_{\text {pack }}$ is the desired "true" expansion of the sensor pack,

$\Delta L_{\mathrm{I}} \quad$ is the change in length of the sensor pack measured by the $1^{\text {st }}$ dial indicator,

$\Delta L_{2}$ is the change in length of the rod measured by the $2^{\text {nd }}$ dial indication, and

$\Delta L_{\text {rod }}$ is the change in length of the rod estimated from the thermocouple measurements.

Figure 4.16 shows typical results from one of these tests. Power was applied to the hot plate heater at the 100 second point. Due to an unknown reason, the sensor pack very quickly "shrinks" before beginning a relatively constant growth due to thermal expansion. The sensor coil frequency tracks both the initial "shrinkage" of the pack and the subsequent growth up to the 700 second point. The sensor pack continues growing until it levels off at approximately 900 seconds. Note that the sensor frequency begins to increase at the 700 second point (which indicates that the sensor is moving closes to the hot plate), while the sensor pack appears to continue "growing" for another 200 seconds. Similar results were obtained in other tests. 
The results of the temperature calibration efforts for the proximity sensors were at best inconclusive. Figure 4.13, which shows static results, demonstrates just how much variability was experienced between sensors - even under the tightly controlled lab tests. Foundry test results displayed even more variability, which prevented accurate temperature calibration of the proximity sensors used to measure the air gap formation.

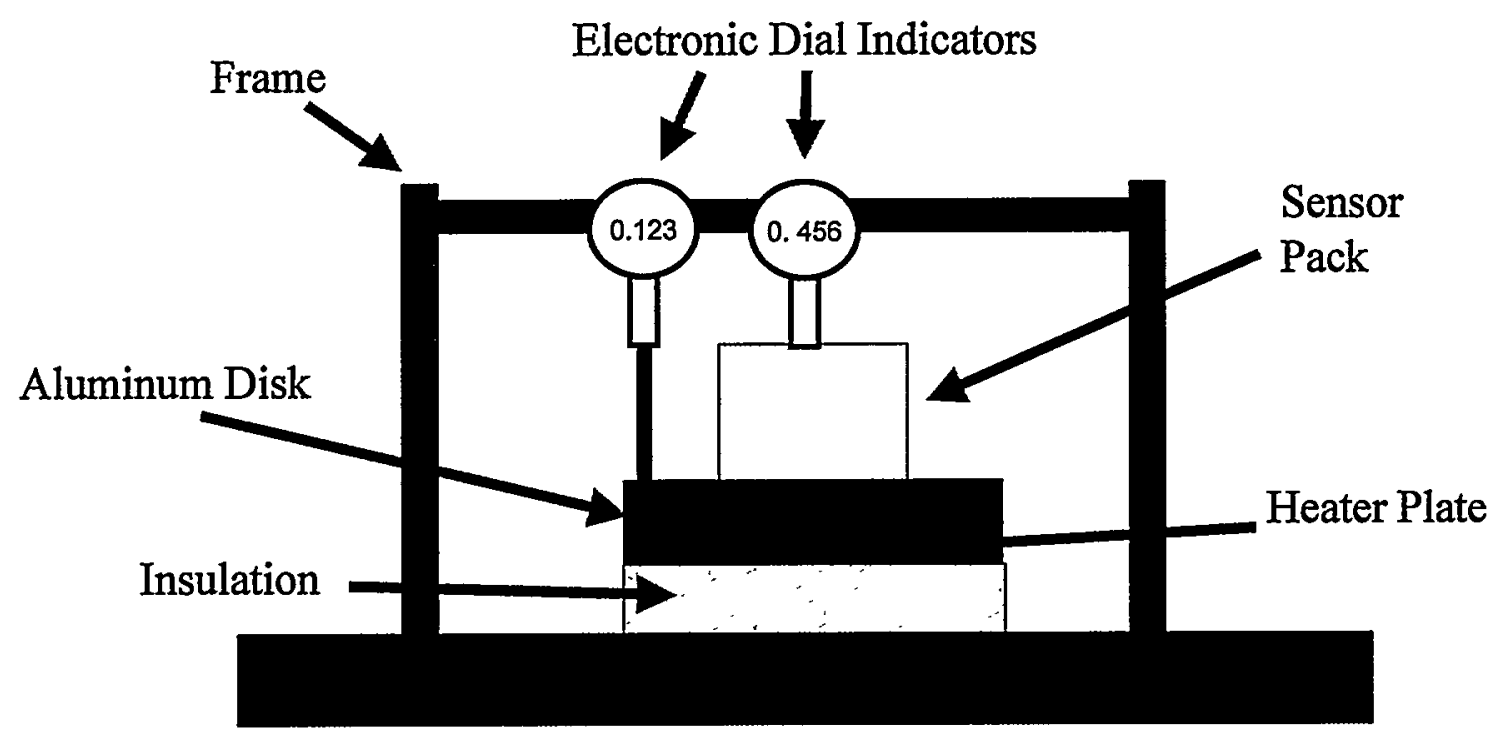

Figure 4.14. Sensor pack expansion test

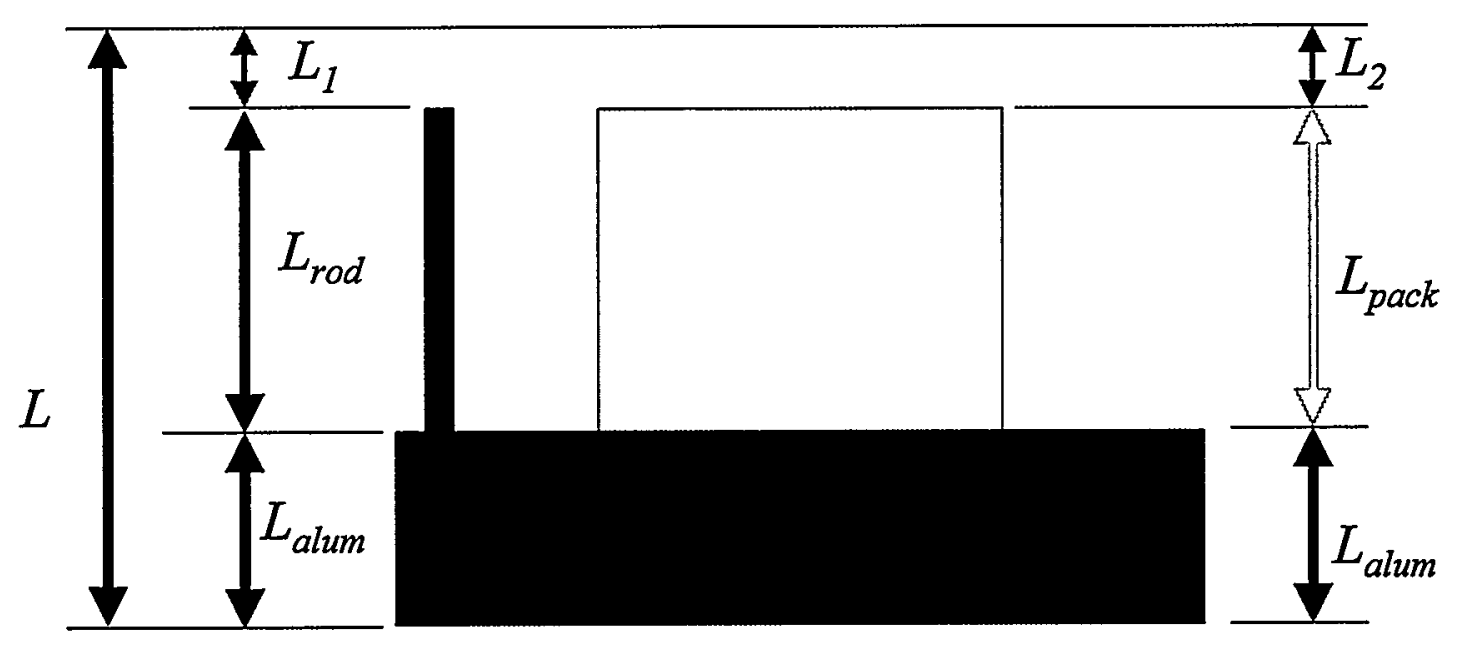

Figure 4.15. Static hot-plate test definitions 


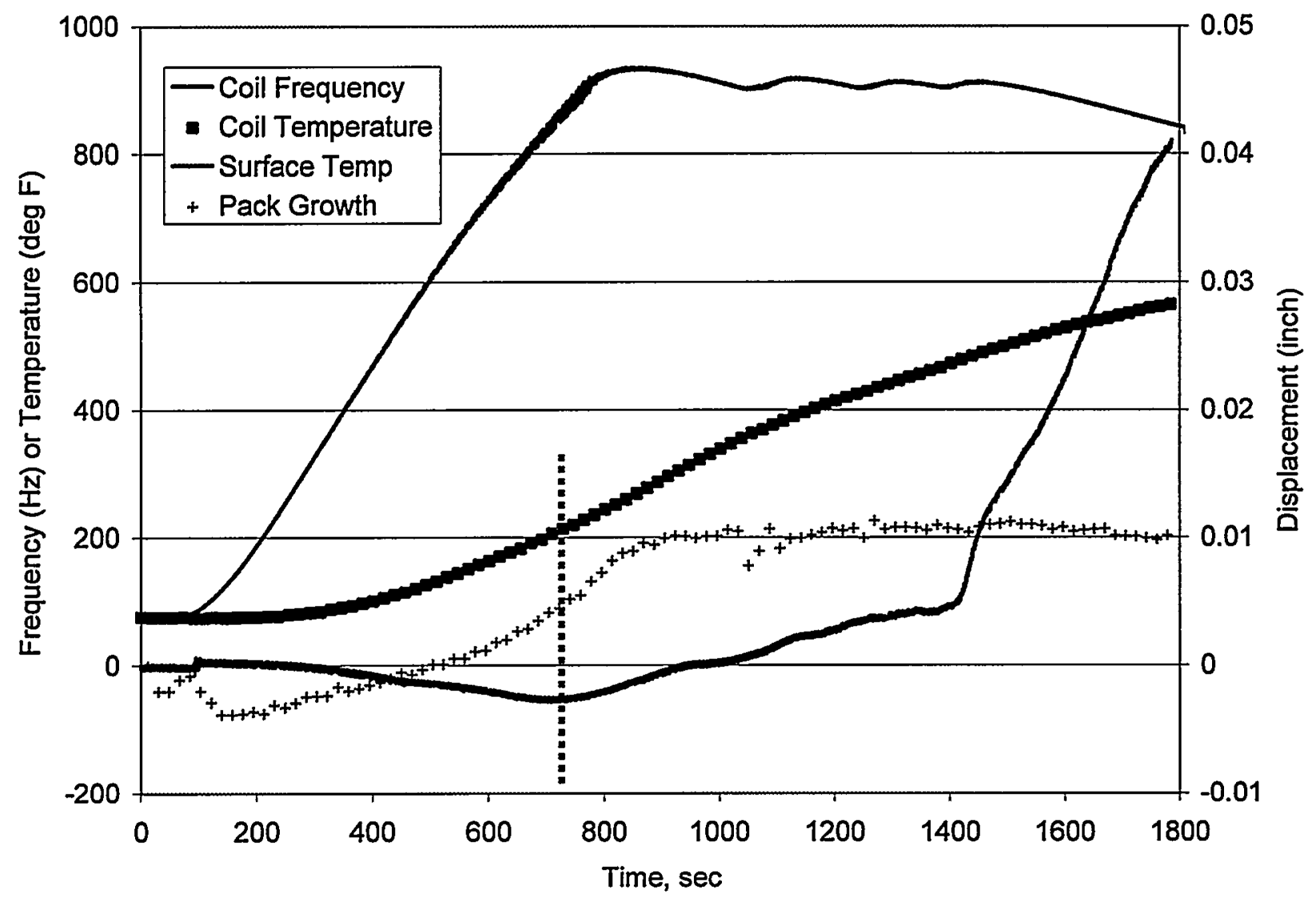

Figure 4.16. Sensor pack expansion test results 


\subsection{FOUNDRY TEST SETUP}

This chapter describes the positioning of the sensor in the sand mold and process of acquiring the data using the sensor for the gap formation measurement in the foundry. Typical foundry pour data are also presented in this chapter.

\subsection{Sensor Packs}

When the sensor is placed in the mold cavity, it is desirable to have the surface of the sensor parallel to the mold cavity surface. The sensor should be placed as close as possible to the metal, in order to obtain good data. If the sensor is too close to the surface of the mold cavity, the sensor is heated up quickly that the output frequency of the sensor is beyond its normal range of operation. Hence a nominal stand-off distance of 0.25 inch was selected. To control the standoff distance, "sensor packs" were made and placed in the mold cavity. The sensor pack was a 3-inch cube made out of sand with the sensor embedded in it. They were made using a split wooden box with a bottom plate. The bottom plate was designed with a step of about 0.25 inch that fit into the split box. The wooden box could be split in the middle to facilitate easy removal of the sensor pack from it after the sand sets down. The sensor was placed in the split box as shown in Figure 5.1.

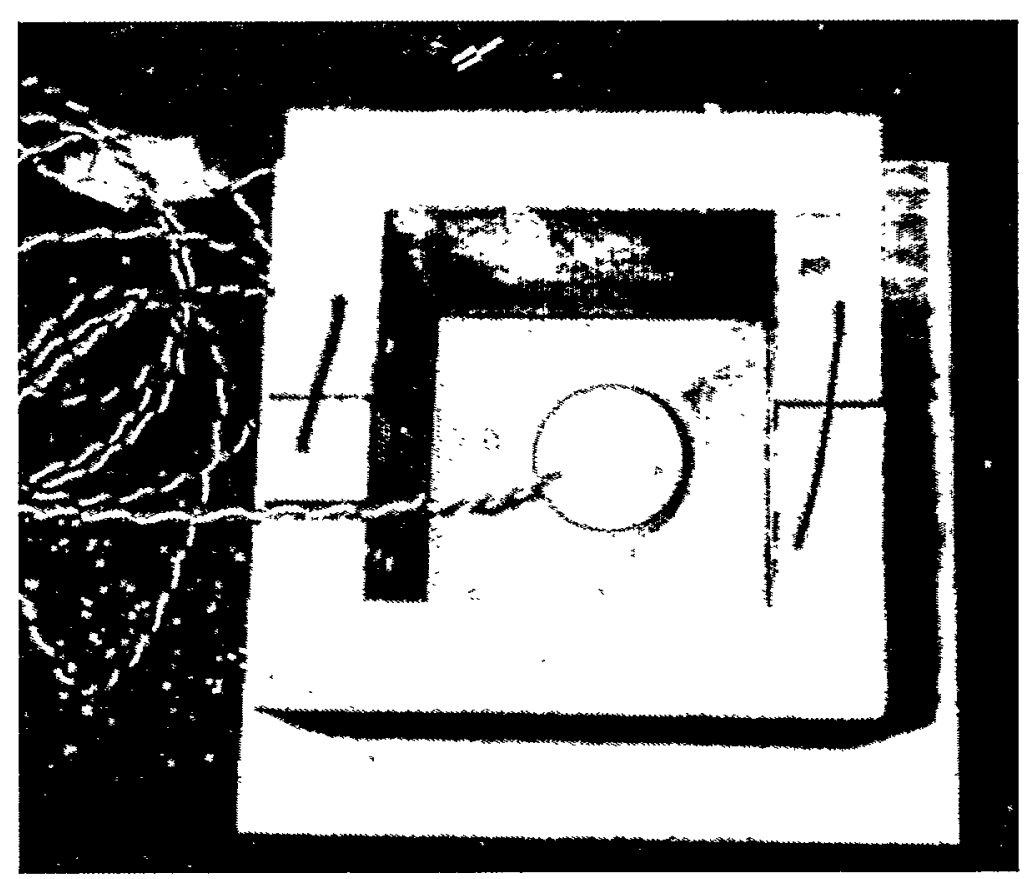

Figure 5.1. Positioning of the sensor in the split box

The sand was then put into the box and thoroughly packed. The box was then turned over and the bottom plate taken off. As shown in Figure 5.2 the sensor is positioned at a stand-off distance and is aligned parallel to the surface. 


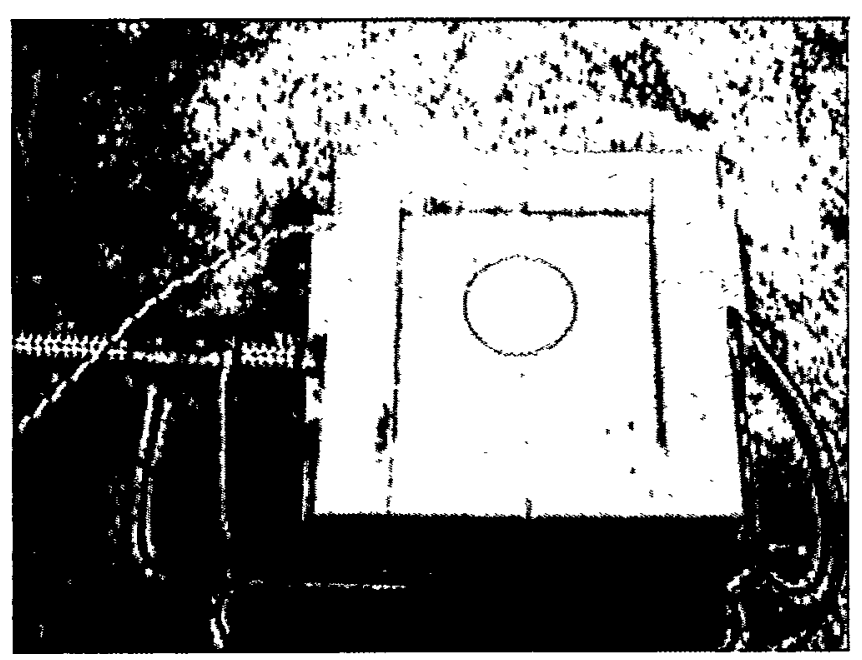

Figure 5.2. Sensor at standoff distance

The sensor was then packed with sand from this side and sand allowed to set for about 10 minutes. The sensor pack was then taken off from the split box and placed in the mold cavity. The finished sensor pack is shown in Figure 5.3. A faint parting line can be seen on the left side of the finished sensor pack. This line shows the thickness of the resin-bonded sand in front of the proximity sensor.

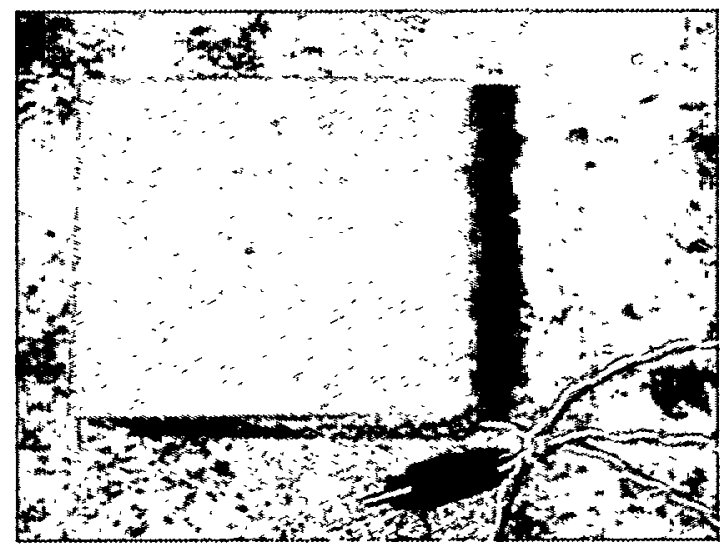

Figure 5.3. Finished sensor pack

\section{2 Positioning of the Sensor in the Mold}

The sensor pack was then placed beside the thermocouple packs in the mold and the mold filled with the sand. The positions of the sensor packs in the mold is shown in Figure 5.4. The mold surface from inside with two sensor packs besides a thermocouple pack is shown in Figure 5.5. 


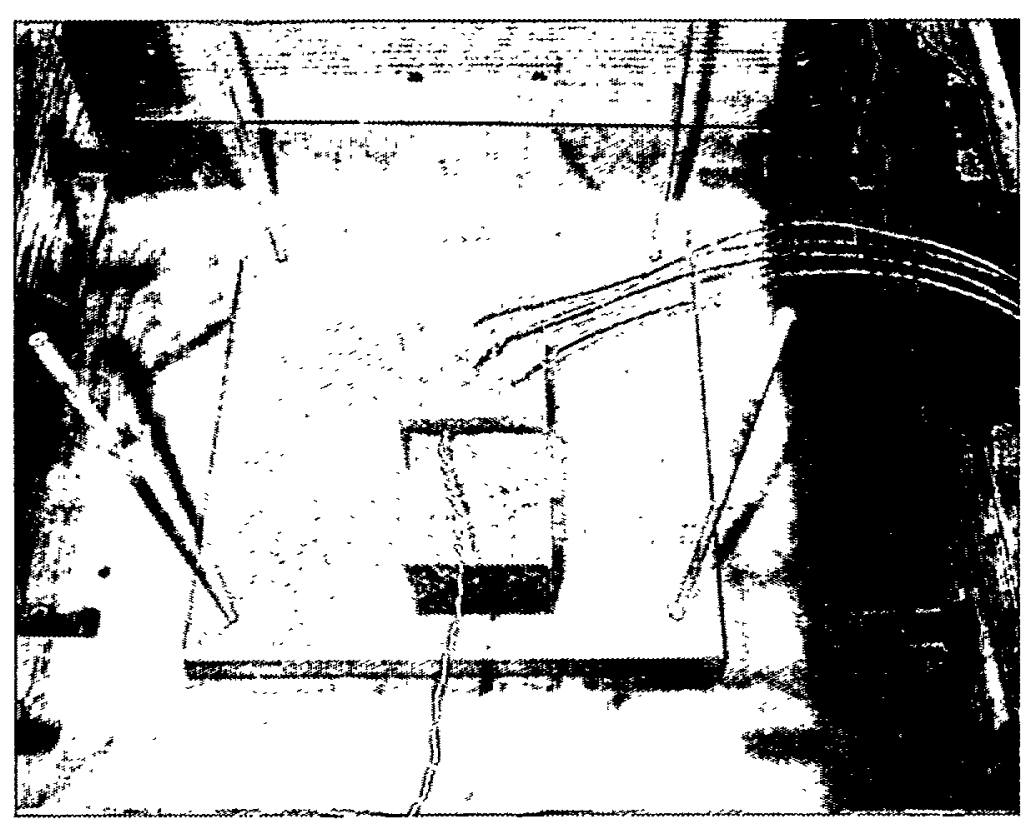

Figure 5.4. Position of the sensor pack in the mold

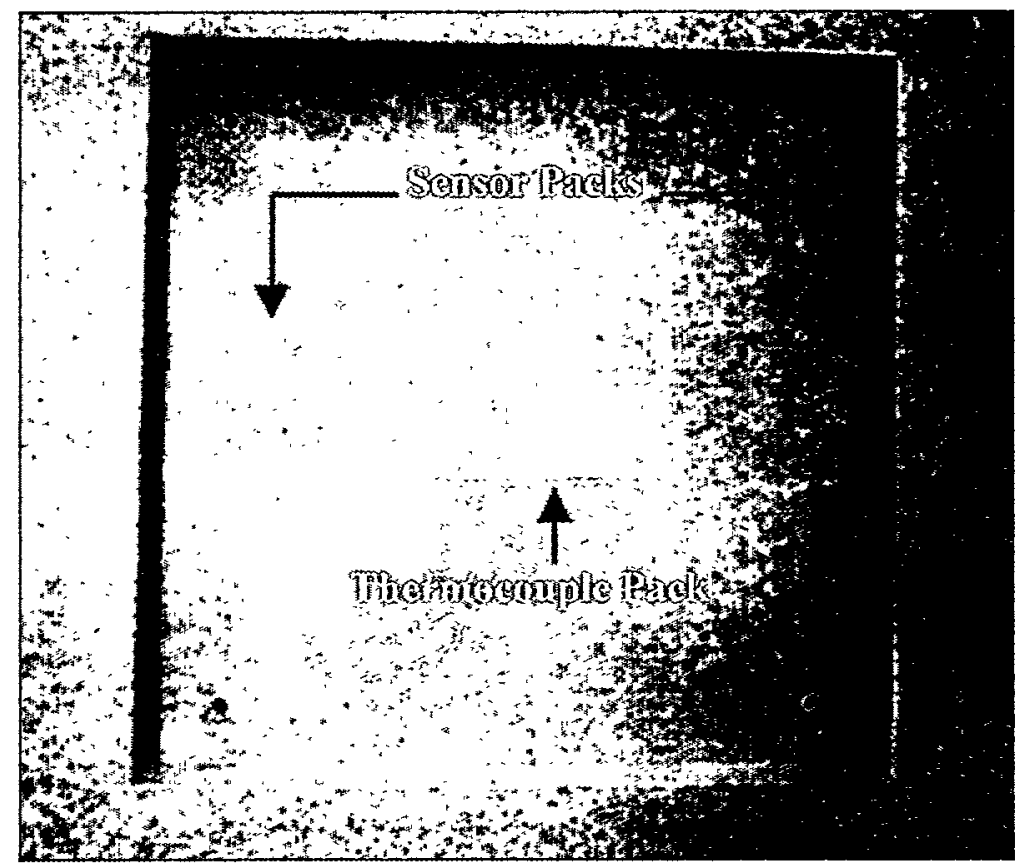

Figure 5.5. Mold cavity as seen from inside

Once the mold making was completed, the sensor was connected to the measuring circuit, which in turn was connected to the computer. The measurement of output frequency of the sensor was computer controlled by a program written in Microsoft Quick Basic.

Before the mold is filled with the molten metal, the sensor does not detect the presence of any metal and the output frequency is low. Once the mold was filled with the molten metal in the region of the sensor, the sensor registered an initial distance of the metal, and there was a sudden 
increase in the output frequency. Measurements were made for 500 to 1000 seconds depending on the thickness of the casting. The resistance of the sensor was measured simultaneously to calculate the temperature of the sensor. 


\subsection{RESULTS - FOUNDRY TEST DATA}

\subsection{Typical Foundry Results}

Typical foundry pour data for a 1-inch-thick aluminum plate are shown in Figure 6.1. The molten aluminum was poured into the mold cavity at about 780 seconds after the start of collection of data. The proximity sensor output frequency (both the bottom and the top sensors) increased suddenly by about $400 \mathrm{~Hz}$. Note that the bottom sensor frequency increased before the top sensor, which would be expected from the arrangement of the mold. The output from each sensor then began a slow decline over the next 150 seconds to about the 900-950 second mark. This drop in the sensor output occurred because thermal expansion of the sand in front of the sensor increased the distance between the sensor and the metal surface. At this point in the test, the output frequency increased due to thermal effects on the sensor. Figures 6.2 and 6.3 show additional measured and computed data for the same test pour shown in Figure 6.1. Proximity sensor temperature and frequency results for twenty test cases from the summer of 1998 are given in the Appendix.

As described earlier, we were never able to correlate the observed temperature effects on the sensor with repeatable laboratory results. The curves labeled "Bottom Gap" and "Top Gap" were computed using the room temperature calibration curves, thus are not true indications of any measured air gap. However, some useful trends and anomalous behaviors were determined by the proximity sensors.

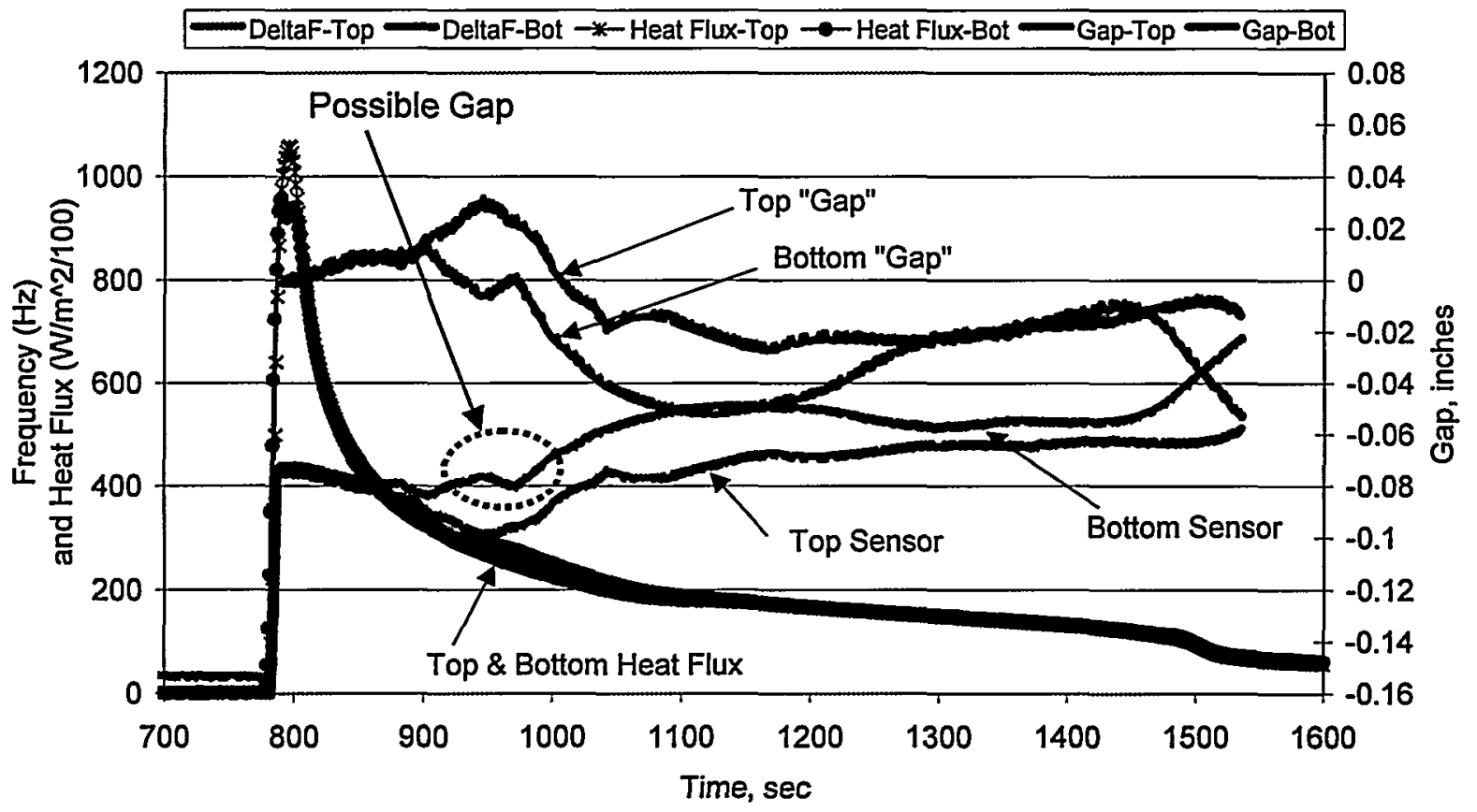

Figure 6.1. Results for pour $8 / 25 / 98 \mathrm{~b}$ 


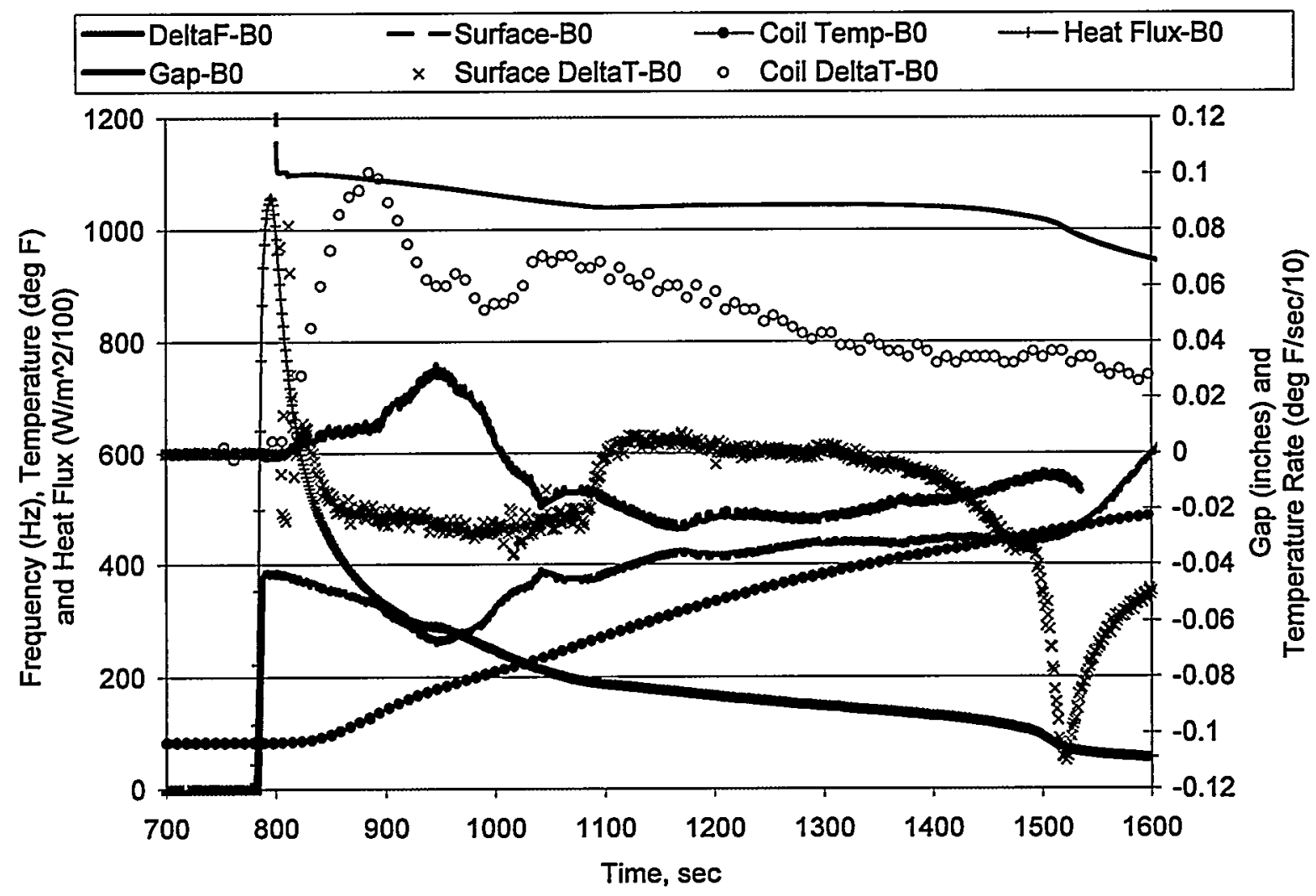

Figure 6.2. Additional data for top sensor, pour $8 / 25 / 98 \mathrm{~b}$

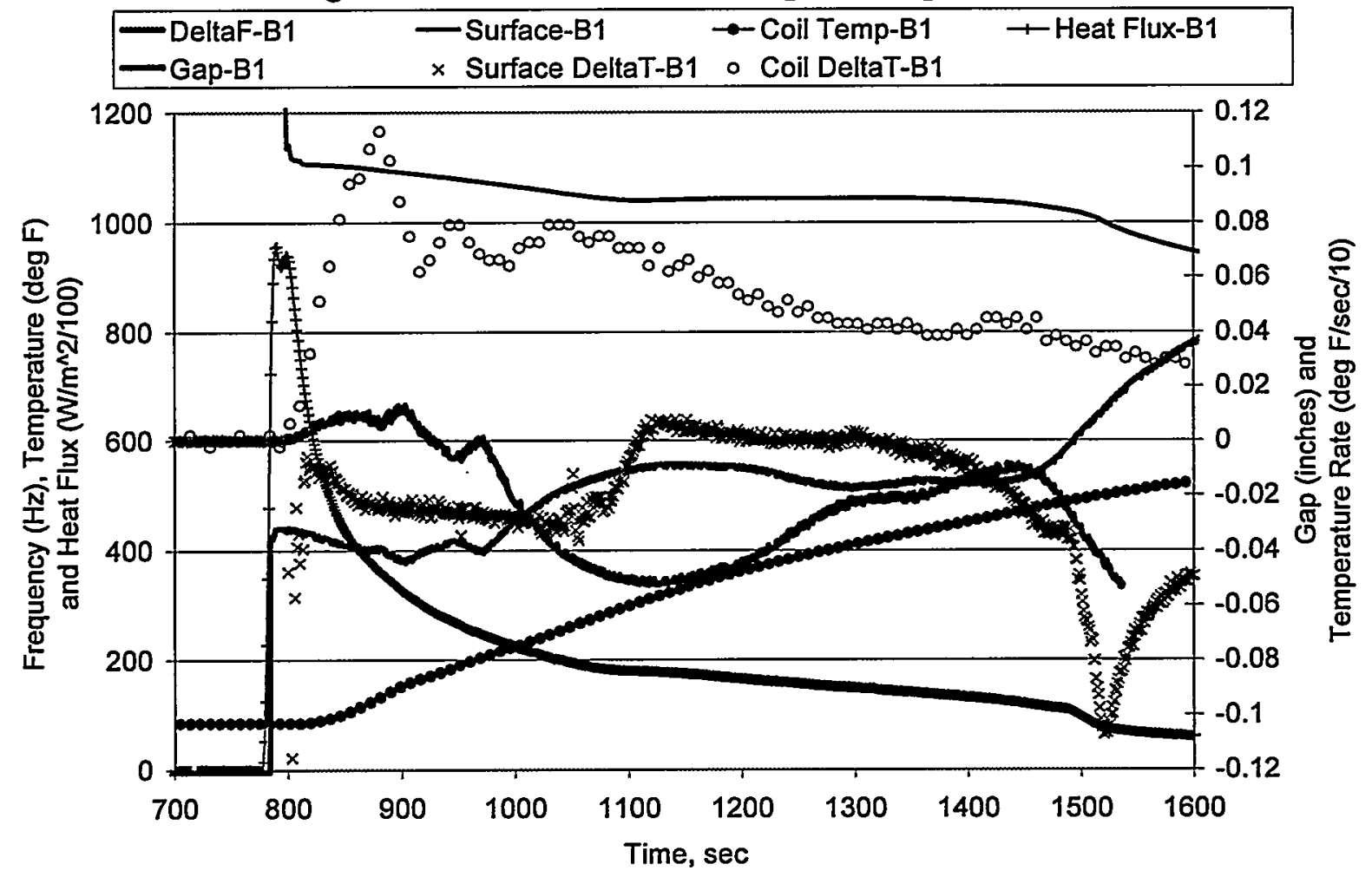

Figure 6.3. Additional data for bottom sensor, pour $8 / 25 / 98 \mathrm{~b}$ 


\subsection{Observations of Initial Slope}

Another set of experimental results for 1 inch, resin-bonded castings is shown in Figure 6.4. The item of interest is the initial slope of the proximity sensor output. The initial slopes of the proximity sensor output in foundry tests fall into one of two patterns:

- both "down" (believed to be due to sand expansion immediately after the pouring of the hot metal, seen in Figure 6.1), and

- one sensor slope "down," but the other sensor slope is "up" (seen in Figure 6.4).

This phenomenon was observed several times in the foundry pours (see the Appendix). It does not appear to be caused by temperature effects on the sensor, since the sensor had not had time to heat significantly. There are also no observed correlations found between sensor values, the slopes of the sensor output, or changes in the sensor slope with heat fluxes or heat transfer coefficients. Lab calibrations of the sensor packs always show a "down" slope, due to expansion of the sand in front of the sensor. The most likely reason for the occasional "up" slope is that the sensor output is valid, i.e., the sensor is moving (slightly) closer to the metal surface. This is most likely due to a breakdown in the sand surface in front of the sensor. This phenomenon violates one of the key assumptions (Section 1.4.2) for the use of the proximity sensor technique in measuring gap formation in sand castings.

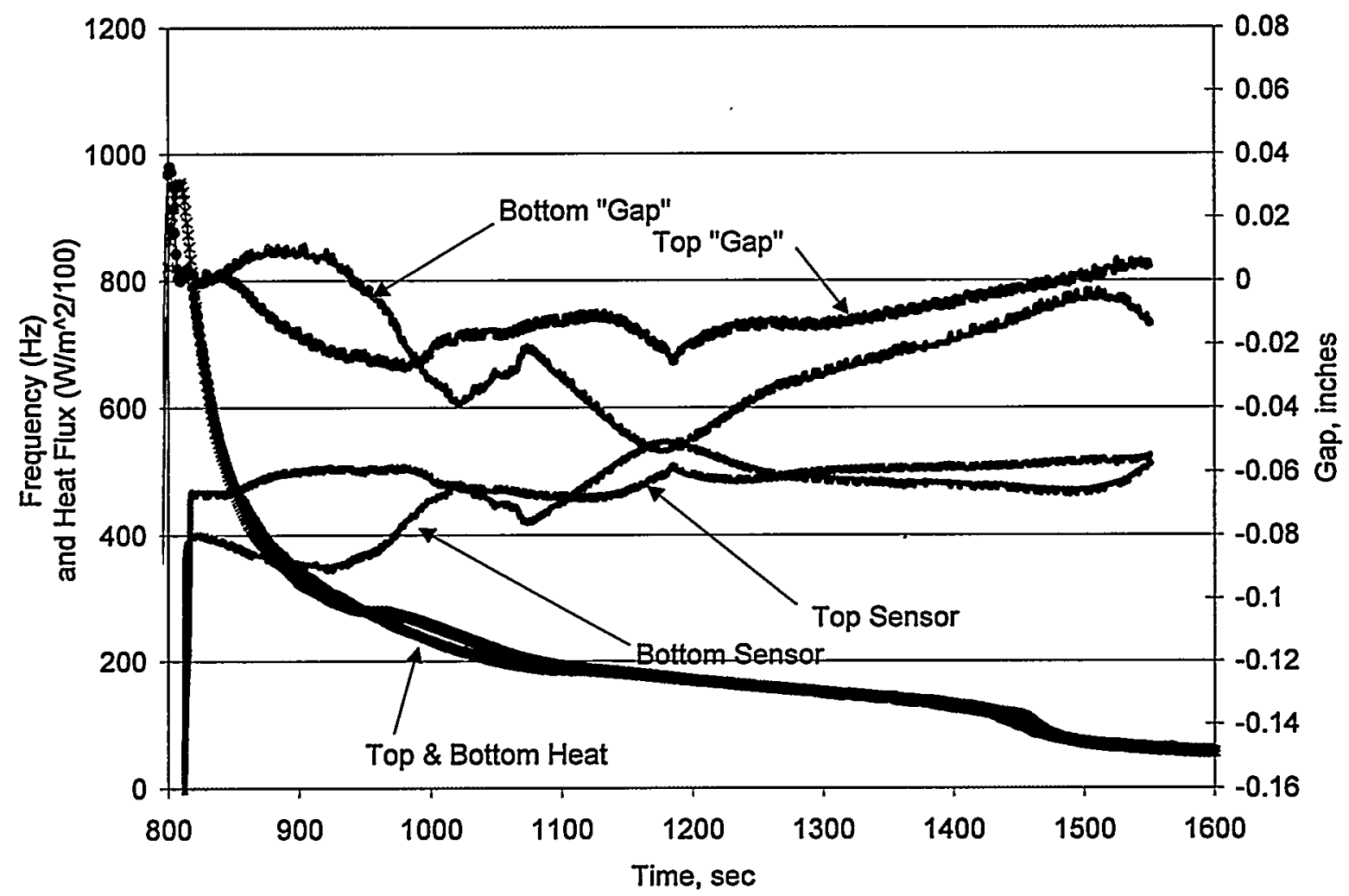

Figure 6.4 Results from pour 8/13/98b. 


\subsection{Possible Sources of Error for Proximity Sensor}

The observed variations in the proximity sensor output in the foundry pours from what was anticipated from the lab calibration tests could be due to any one (or combinations) of several factors discussed below.

The depth of penetration of magnetic field in a target depends on the magnetic permeability of medium between the sensor and the target. Due to the high temperature environment around the sensor in the foundry, the permeability of the medium may vary constantly, hence the sensor output also changes accordingly.

A closely related phenomenon is the "skin depth" effect. It is possible that the skin depth is not sufficient during the early parts of the pour, since the proximity sensor does not "see" the metal more than about 0.015 inch $(0.4 \mathrm{~mm})$ past the surface. If the metal surface under the sensor were subject to "freezing" and re-melting which changed this skin depth, then the sensor output would change without dimensional (i.e., a "gap") changes.

In the lab calibrations, the sensor is calibrated against an aluminum plate at room temperature. When the sensor initially senses the metal when placed in the mold, the aluminum is at a temperature of about $1,000^{\circ} \mathrm{F}$. Hence there is no exact correlation between the lab calibration data and the data obtained form the foundry test pours.

The conductivity of aluminum also changes as it cools from liquid to solid. Therefore there might be variation of sensor output due to the change in conductivity of aluminum. If this parameter varies greatly from $900^{\circ} \mathrm{F}$ (the temperature of the lab benchtop tests) to $1000+^{\circ} \mathrm{F}$ (temperatures in the foundry pours), then the lab calibrations would not capture the true behavior of the sensor in the foundry environment.

Localized irregularities in the casting surface (our sand pack surface is mildly rougher than other surfaces on the casting) create a variable "average" distance for the sensor.

In the foundry application, the front surface of the sensor is at a high temperature and the back surface of the sensor is at a relatively lower temperature. Therefore, there is a thermal gradient across the front and back surfaces of the sensor. In the data analysis, the sensor temperature is an average or "bulk" temperature of the sensor determined from the entire coil of magnet wire. This might also cause anomalies in the sensor output, since this heating effect might not be repeatable in the foundry tests.

\subsection{Casting Thickness}

The thicknesses of 36 resin-bonded castings made during the summer have been measured. Figure 6.5 shows the grid spacing and labels for the 25 locations where the thickness was measured.

Figure 6.6 shows the results for the minimum, average, and maximum thickness' for 12 nominally $1 / 4$ inch thick castings. The difference between the minimum and maximum thickness varies from 0.040 to $0.089 \mathrm{inch}$. The same pattern was used for all 12 castings. There was no consistent pattern to the location of the maximum thickness, although it was never in the row closest to the gating. 


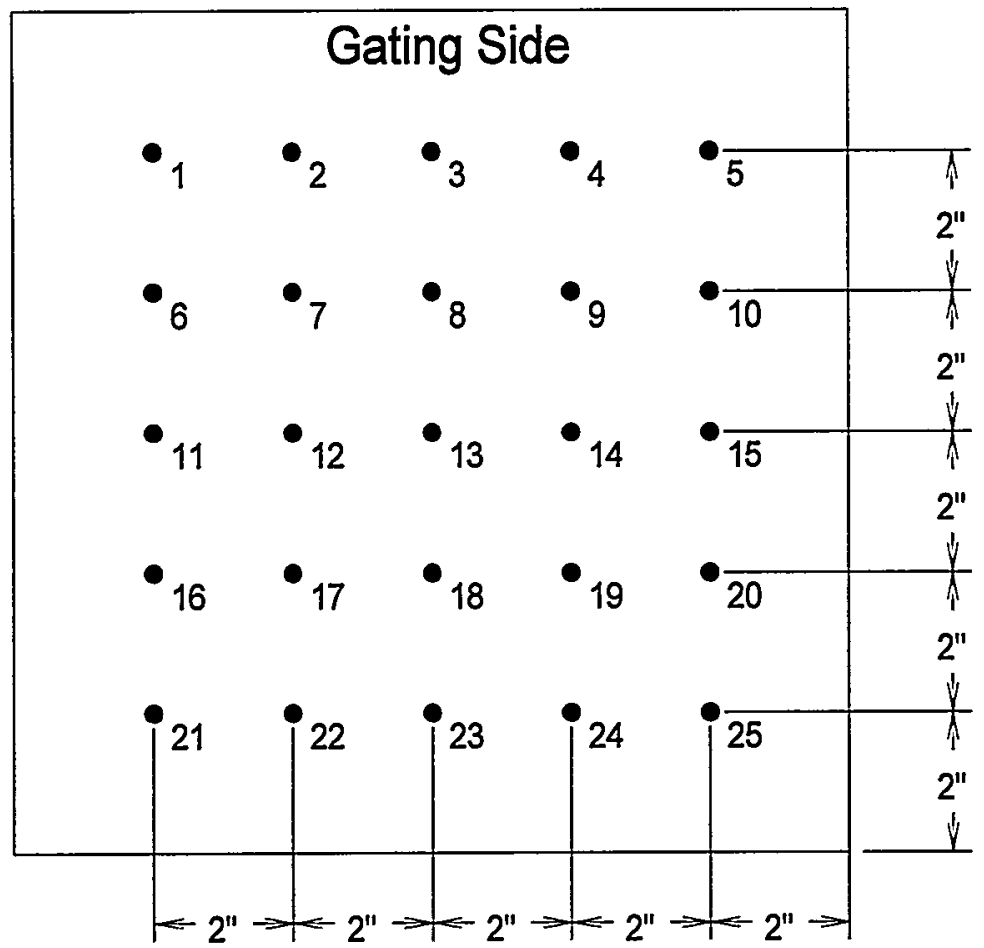

Figure 6.5. Grid spacing and labels for thickness measurement

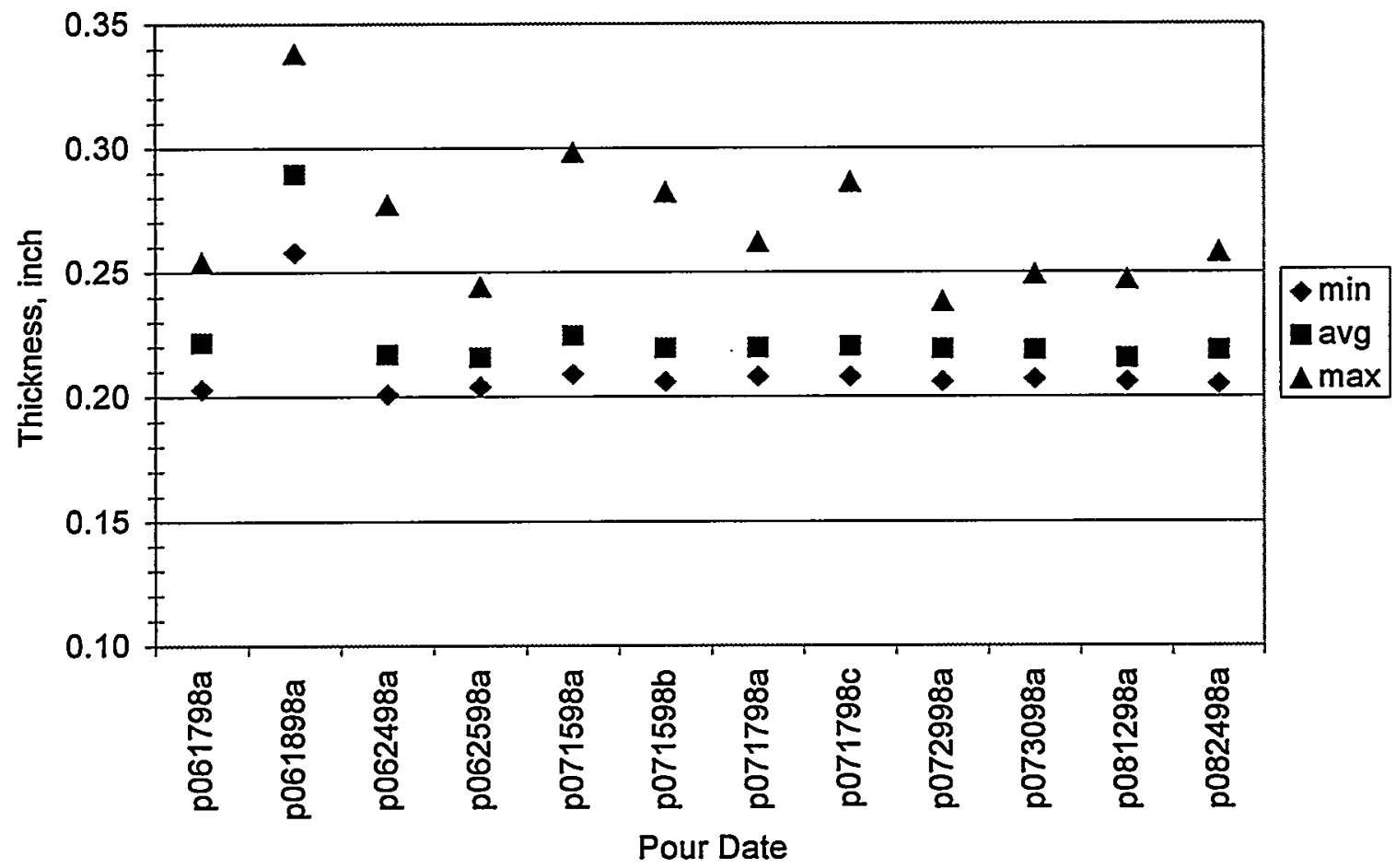

Figure 6.6. Measured plate thickness, nominal 0.25 inch 
Figure 6.7 shows the results for the minimum, average, and maximum thickness for 12 nominally $1 / 2$ inch thick castings. The difference between the minimum and maximum thickness varies from 0.044 to $0.080 \mathrm{inch}$. The results from the 4 pours in the middle (p071598a,b and p071798a,b) were for the vertical orientation. The same pattern was used for all 12 castings.

Figure 6.8 shows the results for the minimum, average, and maximum thickness for 12 nominally 1 inch thick castings. The difference between the minimum and maximum thickness varies from 0.050 to $0.089 \mathrm{inch}$. Two different patterns were used on each date. Note that one of the castings is consistently 0.060 to 0.080 inch thicker than the other casting. The maximum thickness for the 1 inch castings typically occurred at either location \#23 (7 times) or location $\# 15$ (3 times).

Because the sensors did not reveal the formation of a gap, we expended a great deal of effort to calibrate them and compensate for any possible errors. Even so, we did not see the results we expected. We are forced to consider the possibility that the sensors may indeed have worked properly, and gap formation is neither as predictable nor as repeatable as has been assumed. The alloy was not degassed before pouring, and was filled with microporosity (the lack of degassing was intentional, so that we were not confused with shrinkage changes in volume instead of gap formation). In some cases the microstructure showed that the bottom of the casting cooled more slowly than the top, a counter-intuitive result, as we assumed that gravity would force the metal against the lower surface of the mold. However, if gas released from the resin in the drag on pouring collected on the bottom surface of the casting, it could have served as an insulator, and forced the gap to occur where it was not expected. Indeed, the microstructure of the castings strongly suggests that no large gap formed reproducibly.

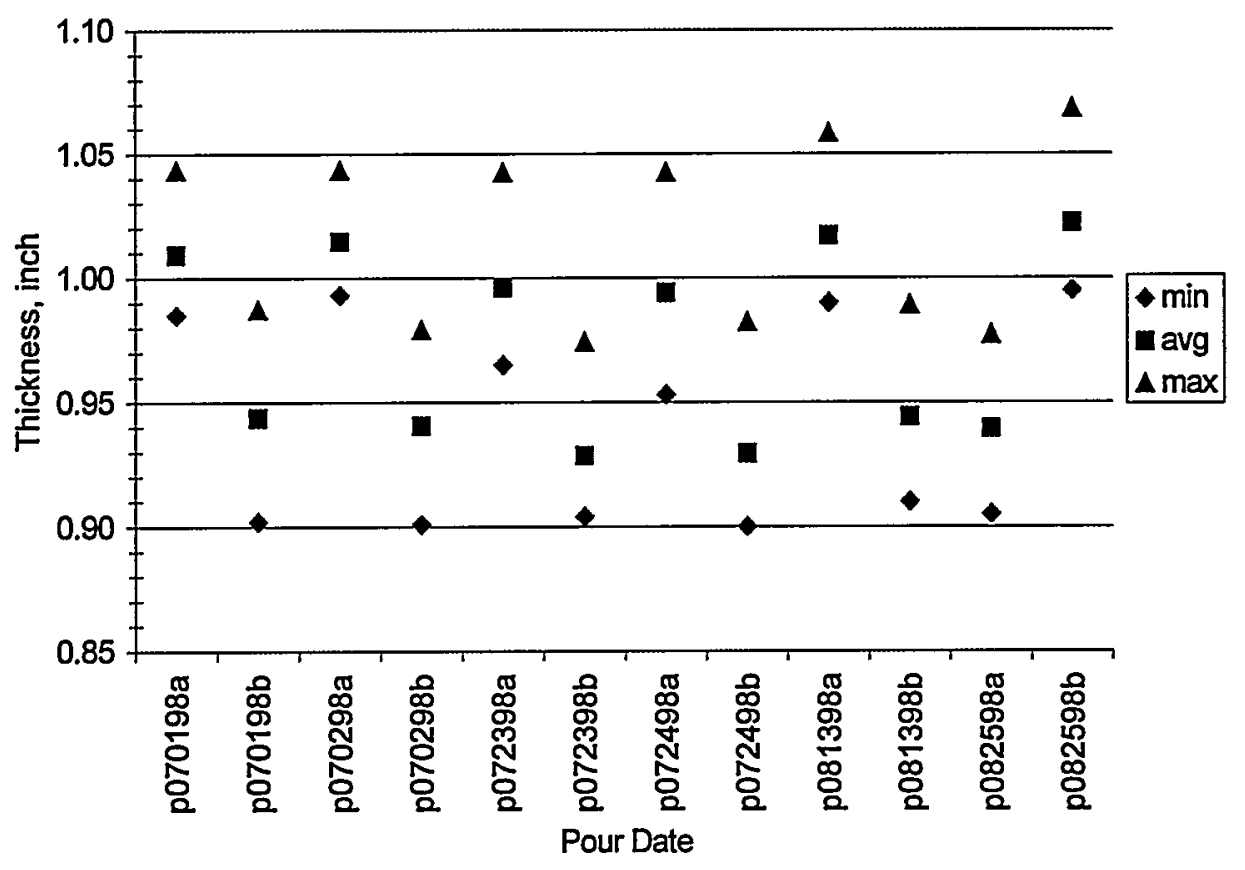

Figure 6.7. Measured plate thickness, nominal 0.50 inch 


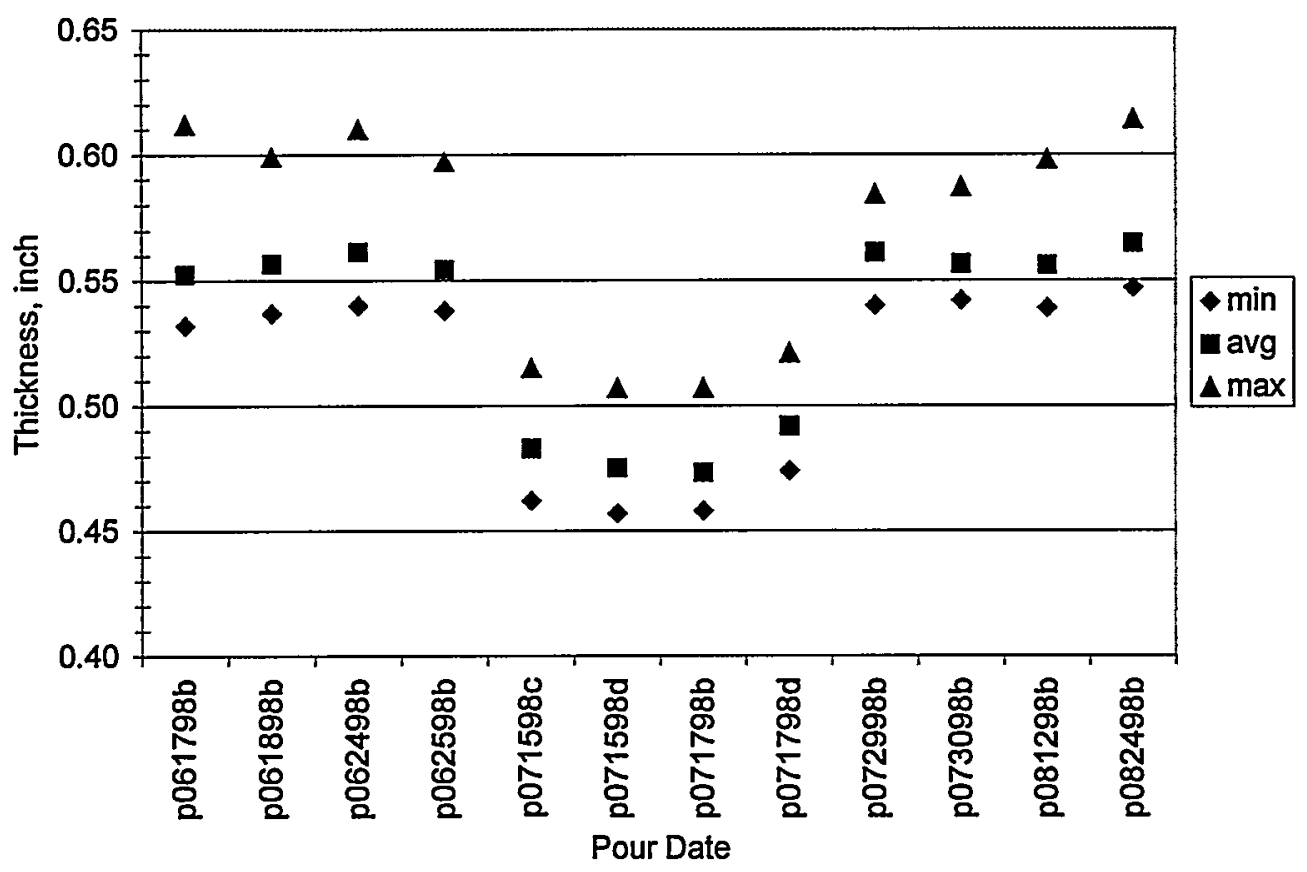

Figure 6.8. Measured plate thickness, nominal 1.00 inch

If gap formation is erratic (and we cannot determine this definitely from the results of this investigation, although our results with the eddy-current method and the microstructure strongly suggest it), then it will be necessary to re-consider the mechanism of gap formation. Castings poured on slants or vertically did not indicate that gaps were being formed reproducibly either. It appears that the most that can be said for the question of gap formation is that our results call into question the current theories.

\subsection{Does A Gap Form?}

Our data strongly suggests that no gap formed during the pouring of most of the castings in this experiment. Neither the gap formation measurements, nor the thermal data were what was expected from the formation of a gap. We looked at the microstructures of the castings poured horizontally, and found extensive columnar grain growing from the cope surface, but only short columnar grains, which quickly changed to equiaxed grains, growing from the drag surface. This indicates very strongly that thermal gradients in the drag were lower than those in the cope. This, in turn, indicates that the heat transfer from the cope surface of the casting was greater than from the drag surface.

We had expected to see just the opposite phenomenon. We assumed that gravity would force the solidifying metal down onto the drag surface of the mold, and that a clear gap would form on the cope surface. That did not happen. We suspect that by not degassing the metal we counteracted the effect of metal shrinkage, and that no shrinkage took place on the cope surface. As for the lack of effective heat transfer on the drag surface, we attribute this to the collection of 
gas underneath the solidifying casting. This has been encountered previously [15]. We were also curious about what would happen to the gap on the surfaces of castings poured vertically. We found that gap formation, if any, was not predictable, nor could we find a pattern to gaps, if any, formed on castings poured at $30^{\circ}, 45^{\circ}$ or $60^{\circ}$ angles.

Our conclusion is that there is no evidence that gaps due to alloy shrinkage actually form in aluminum alloys poured in resin-bonded sand molds. Note that our experimental method deviates substantially from that of past investigators, and the fact that we did not degas the metal may have affected our results, as the castings contained considerable amounts of microporosity. However, we have been careful to examine as many variables that could affect the accuracy of the instrumentation of our method as we could identify, and we find it hard to believe that the method we used for gap measurement is inherently incorrect. Our results call into question the traditional view of gap formation during solidification, and strongly call for further research into the question.

\subsection{Green Sand Results}

The vast majority of the proximity sensor work conducted as part of this research was done with resin-bonded castings. Figure 6.9 shows results from the eddy-current proximity sensor on a foundry pour with green sand. The moisture present in the green sand appears to greatly affect the sensor output. This is consistent with our earlier observations of the need to thoroughly dry or cure the sensors before use in the foundry. For this reason, we have concluded that reliable results cannot obtained from the green sand casting pours using the eddy current technique as we practiced it. 


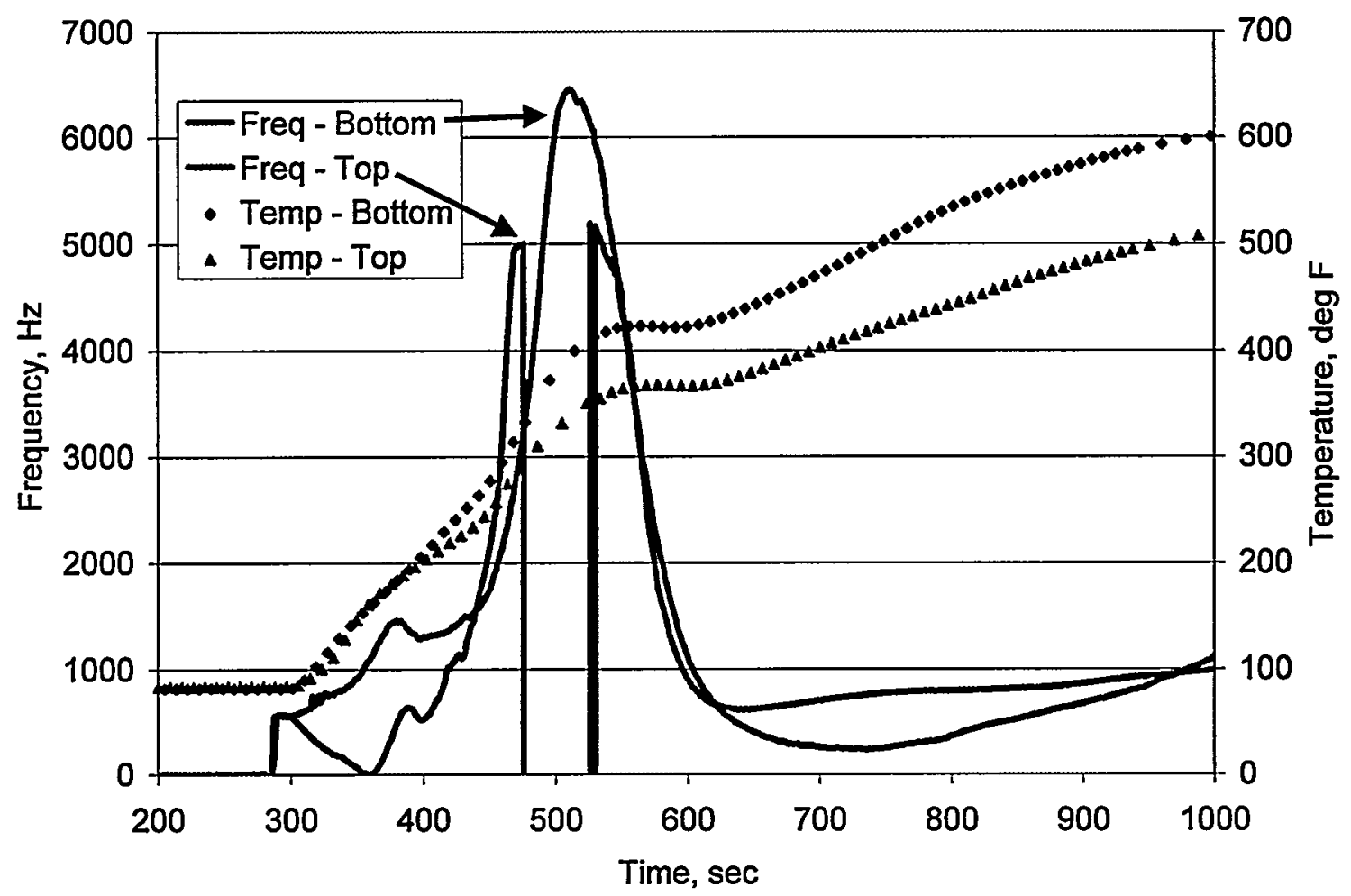

Figure 6.9. Foundry test, 9/25/98 (1 inch, green sand)

\subsection{REFERENCES}

1. John C. Moulder, Erol Uzal and James H. Rose, "Thickness and conductivity of metallic layers from eddy current measurements," Review of Scientific Instruments, Vol. 63, No. 6, 1992, p. 3455.

2. Stuart A. Long, Sompongse Toomsawasdi and Afroz J. M. Zaman, "Experimental measurements of the eddy current signal due to a flawed, conducting half space," Review of Progress in Quantitative Nondestructive Evaluation, Donald O. Thompson and Dale E. Chimenti, Vol. 3A, Plenum Press, New York, 1982, p. 3455.

3. David H. S. Cheng, "The reflected impedance of a circular coil in the proximity of a semiinfinite medium," IEEE Transactions on Instrumentation and Measurement, Vol. IM-14, No. 3, 1965, p. 107.

4. Dodd C. V. and Deeds W. E., "Analytical solutions to eddy-current probe-coil problems," Journal of Applied Physics, Vol. 39, No. 6, 1968, p. 2829.

5. Marc Bissonnette P. Eng and Marius Cloutier, "Air gap measuring system," Proceedings of the First International Machinery Monitoring and Diagnostics Conference, Sep 11-14 1989, Las Vegas, NV, 1989, p. 261. 
6. Robert M. Masters, "Technique for temperature compensation of eddy-current proximity probes," NASA Technical Paper 2880, 1989.

7. Shahverdi H. R., Farhadi F., Karimitaheri A., Davami P. and Asgari K., "A mathematical model for air-gap formation at the metal-mould interface in the computer simulation of Al12\%Si in a sand mould," Cast Metals, Vol. 6, No. 4, 1994, p. 231.

8. Nishida Y., Droste W. and Engler S., "The air-gap formation process at the casting-mold interface and the heat transfer mechanism through the gap," Metallurgical Transactions, Vol.17B, 1986, p. 833.

9. Hou T. X. and Pehlke R. D., "Determination of mold-metal interfacial heat transfer and simulation of solidification of an Aluminum-13\% Silicon casting," AFS Transactions, Vol.96, 1988, p. 129.

10. Das S. and Paul A. J., "Determination of metal-mold interfacial heat transfer coefficients for castings using a solution technique for inverse problems based on the boundary element method," Modeling of Casting, Welding and Advanced Solidification Processes VI, Piwonka T. S., Voller V. and Katgerman L., The Minerals, Metals \& Materials Society, Warrendale, PA, 1993, p. 647.

11. Andy Cooper and Michael Johns, "Manufacture of proximity sensor coils," Report of ME490 design team, Department of Mechanical Engineering, The University of Alabama, 1997.

12. McGraw-Hill Encyclopedia of Science \& Technology, $6^{\text {th }}$ Ed., Vol. 5, 1987, p. 589.

13. Alexander D. Khazan, Transducers and Their Elements: Design and Application, PTR Prentice Hall, Englewood Cliffs, NJ, 1994.

14. Theodore Baumeister, Eugene A. Avallone and Theodore Baumeister III, Marks' Standard Handbook for Mechanical Engineers, $8^{\text {th }}$ Ed., McGraw-Hill book company, p.15-7.

15. J.T. Berry, Mississippi State University, private communication, August, 1999. 
APPENDIX 


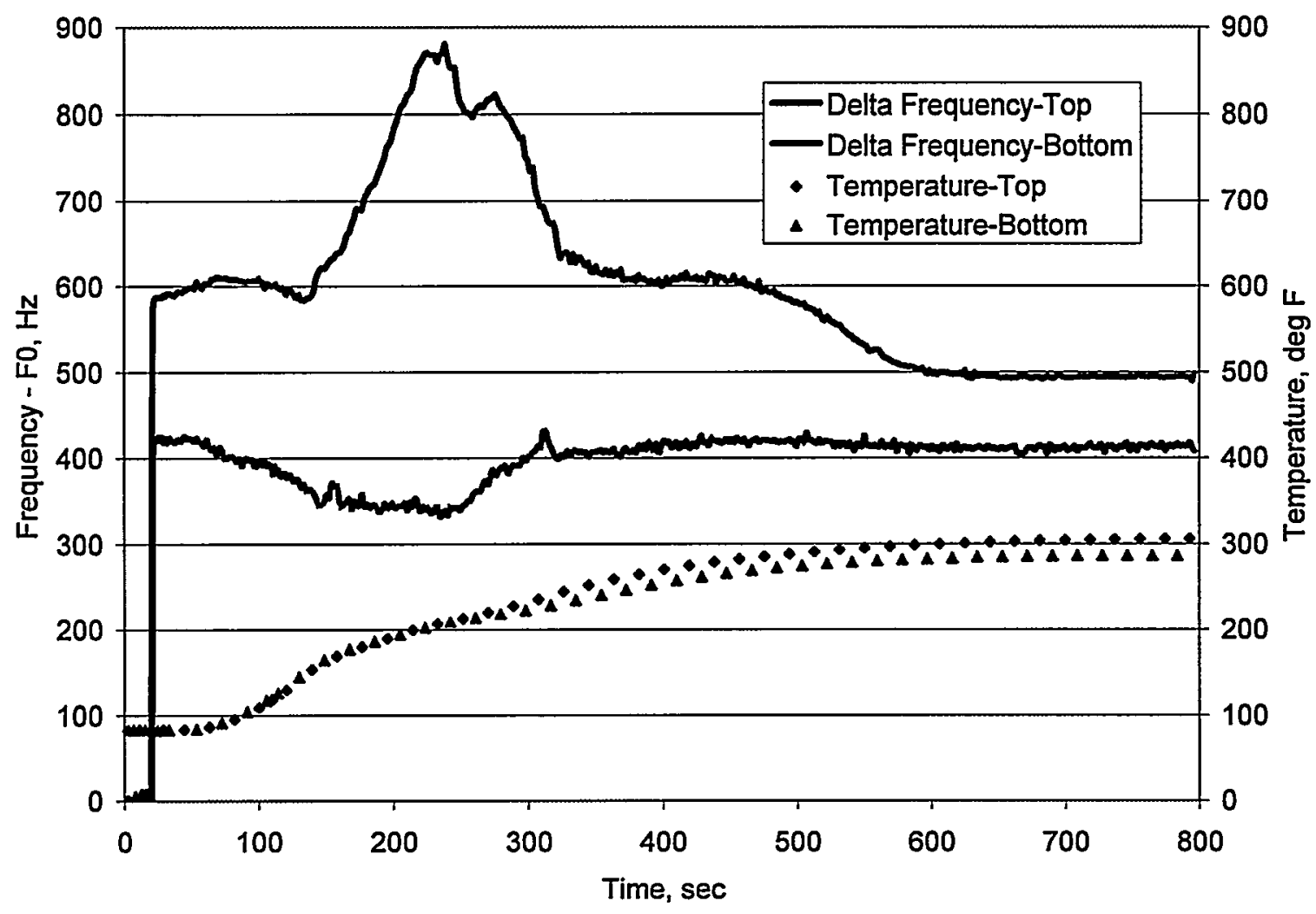

Figure A-1. Pour 061798a, 0.25 inch, horizontal 


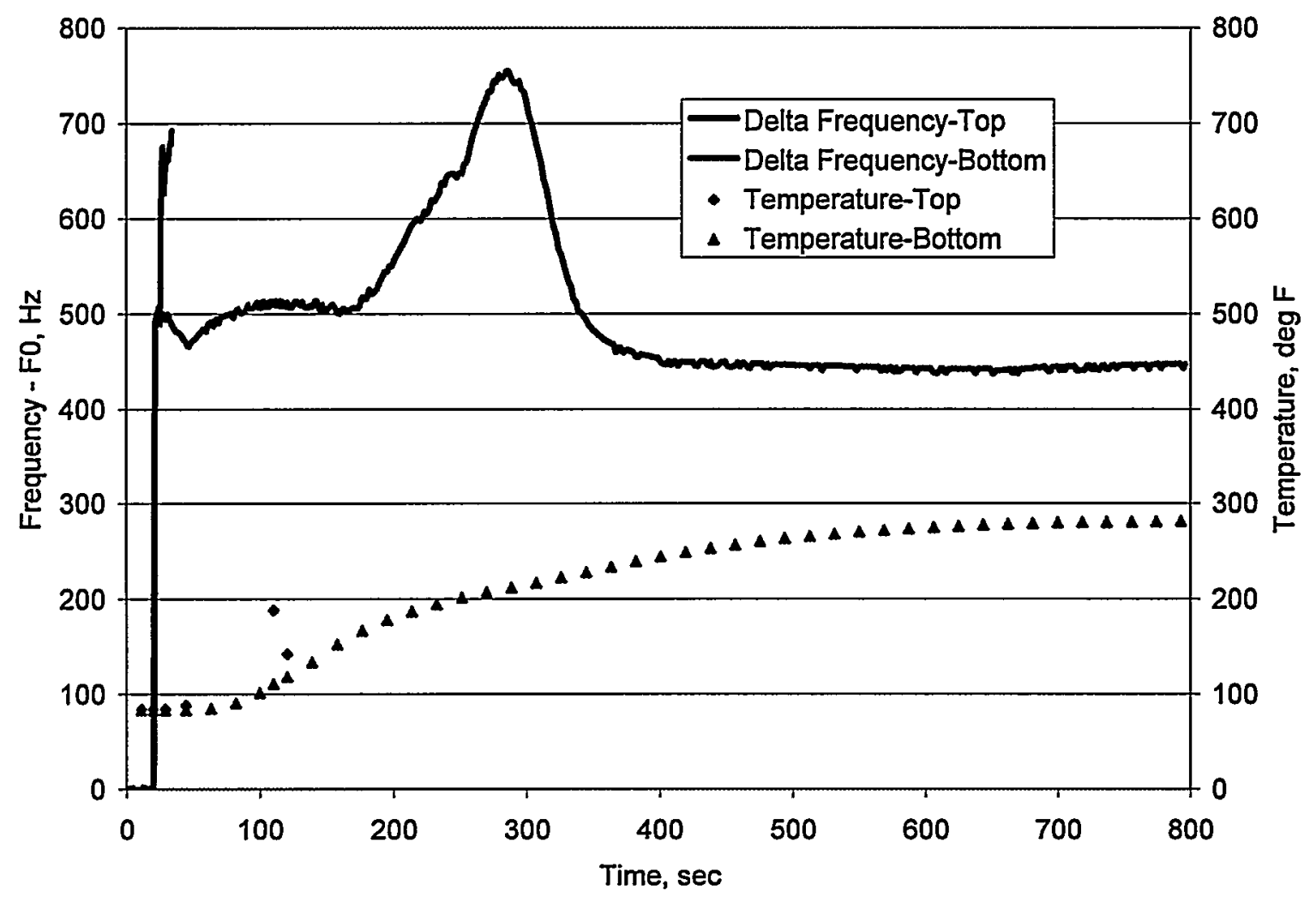

Figure A-2. Pour 061798a, 0.25 inch, horizontal 


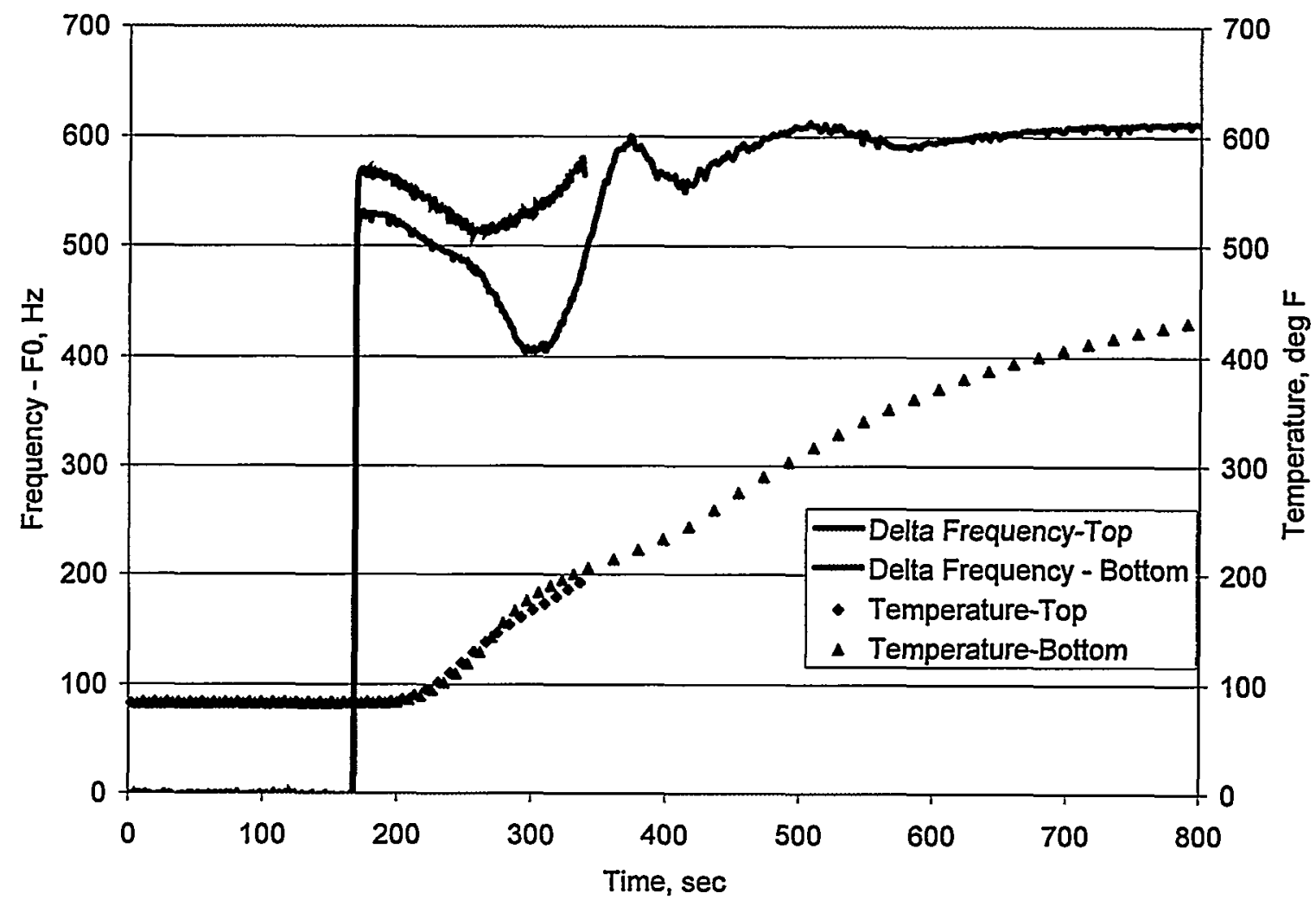

Figure A-3. Pour 061798b, 0.5 inch, horizontal

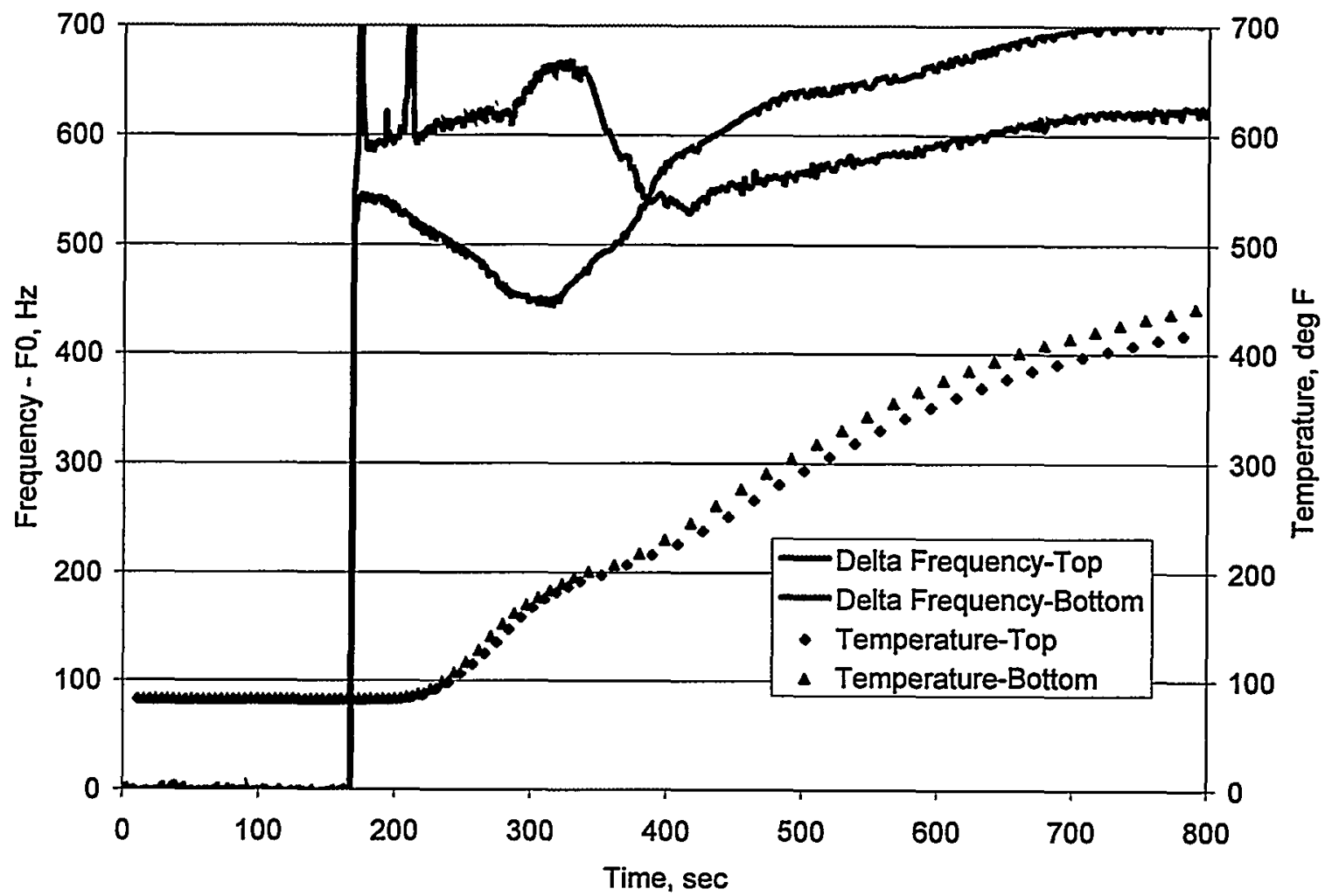

Figure A-4. Pour 061798b, 0.5 inch, horizontal 


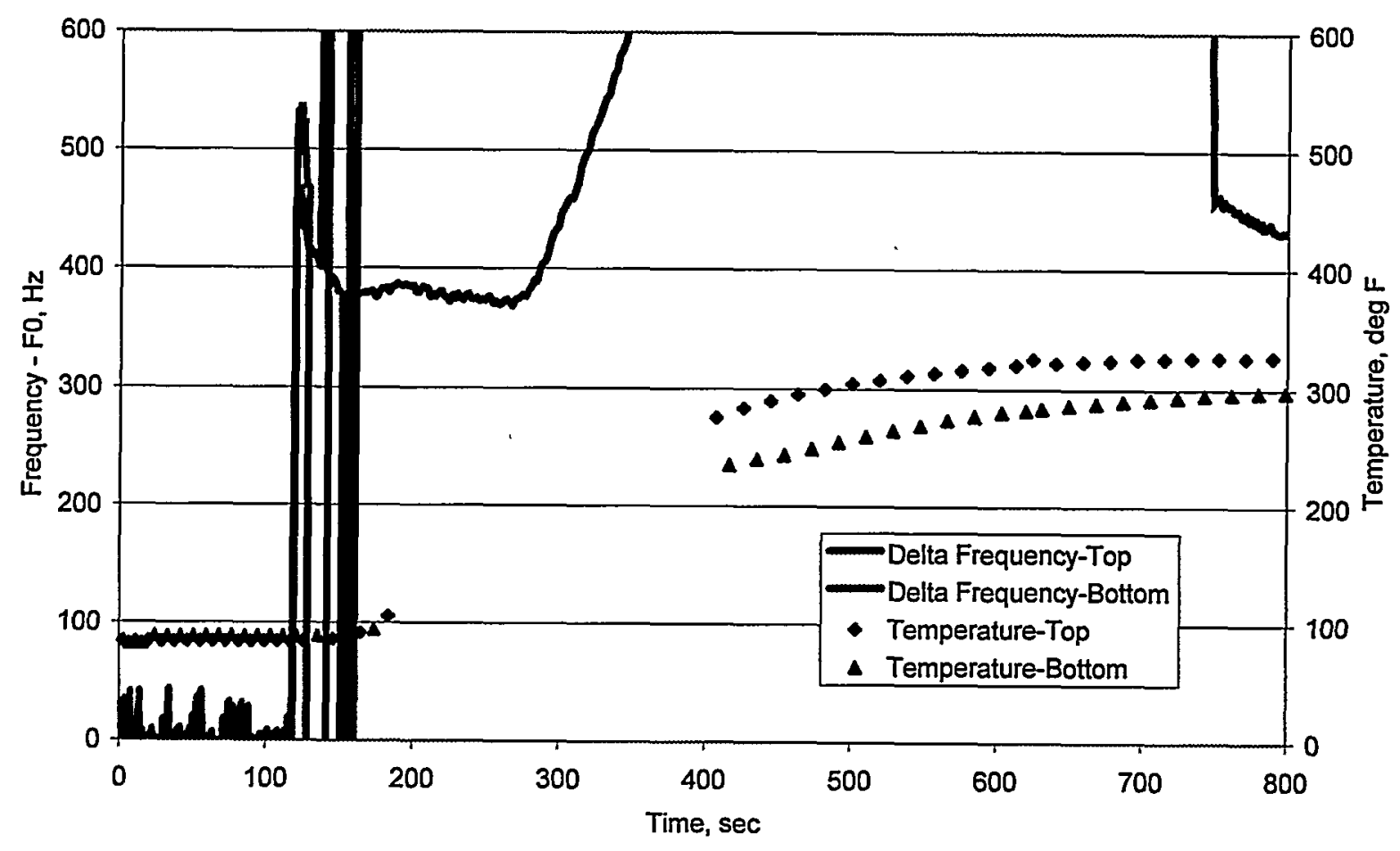

Figure A-5. Pour 061898a, 0.25 inch, horizontal

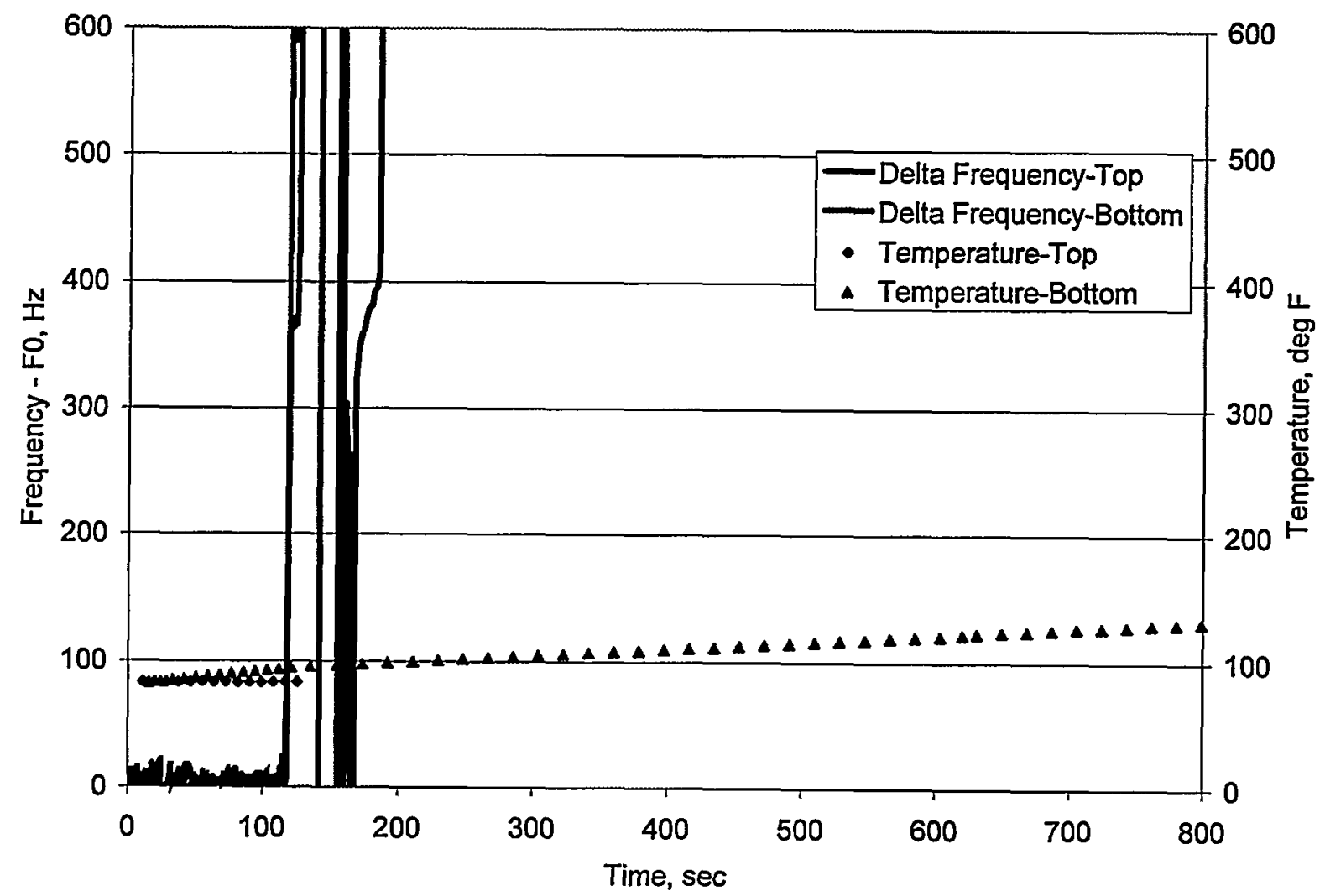

Figure A-6. Pour 061898b, 0.25 inch, horizontal 


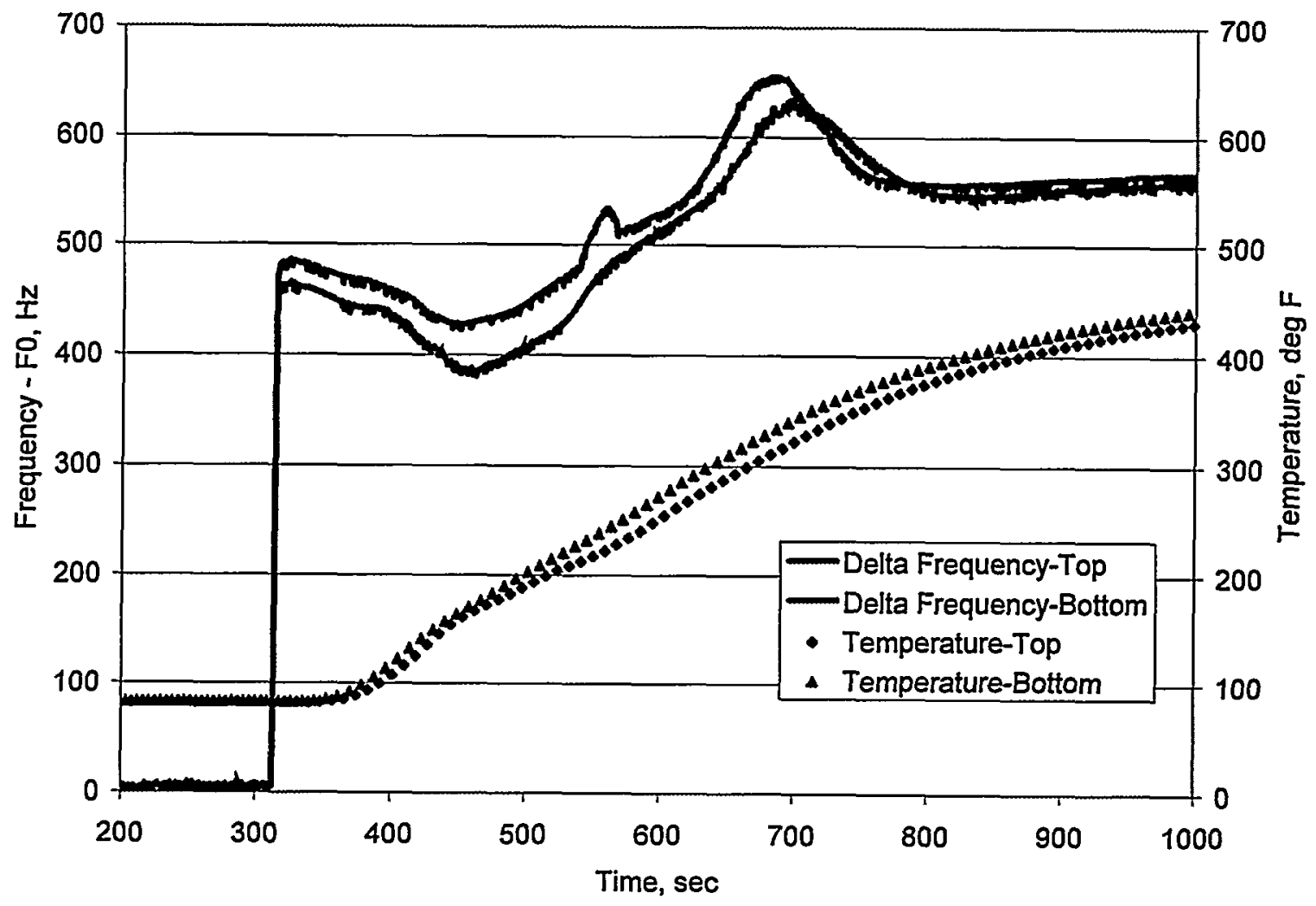

Figure A-7. Pour 061898b, 0.5 inch, horizontal

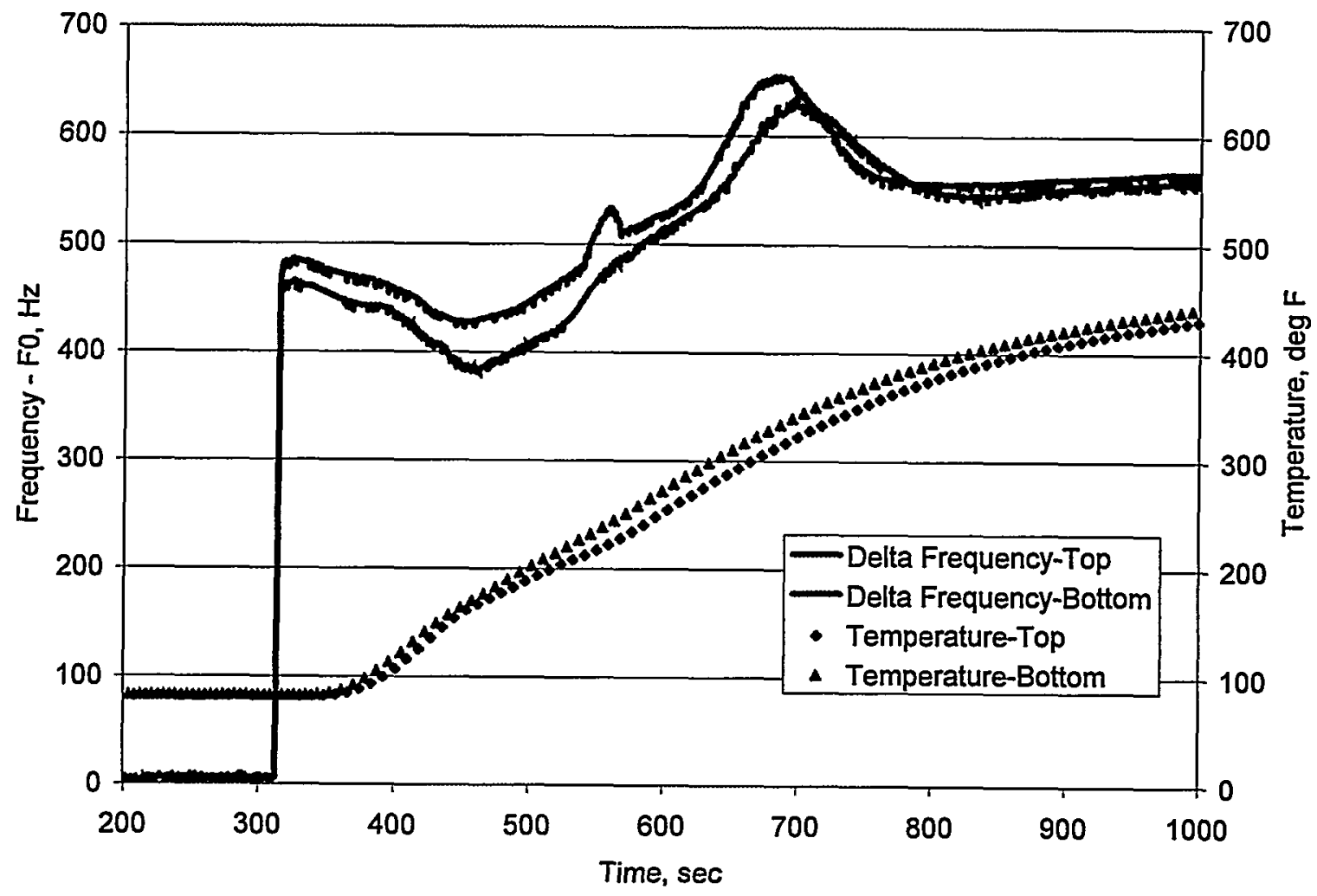

Figure A-8. Pour 061898b, 0.5 inch, horizontal 


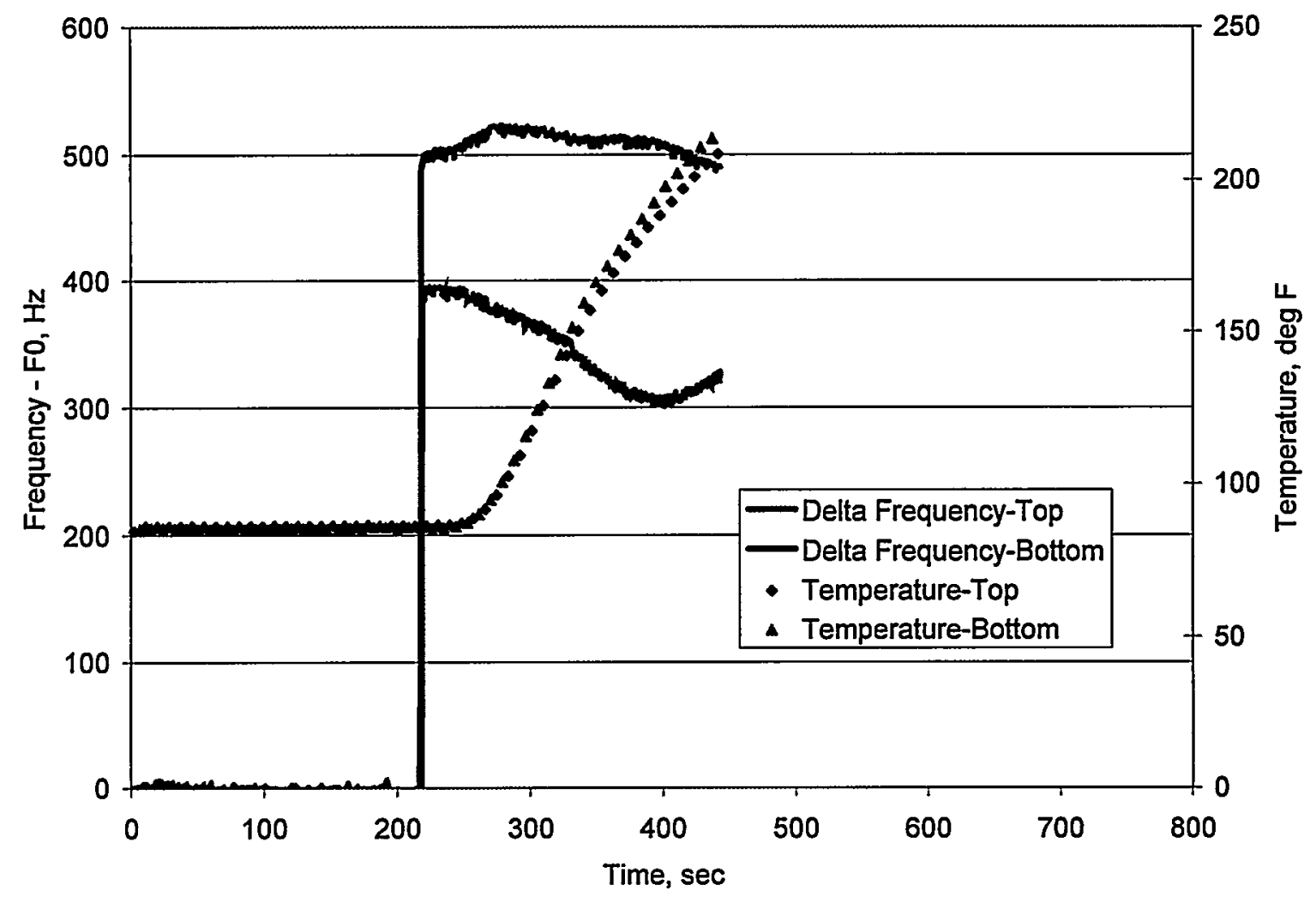

Figure A-9. Pour 062498a, 0.25 inch, horizontal

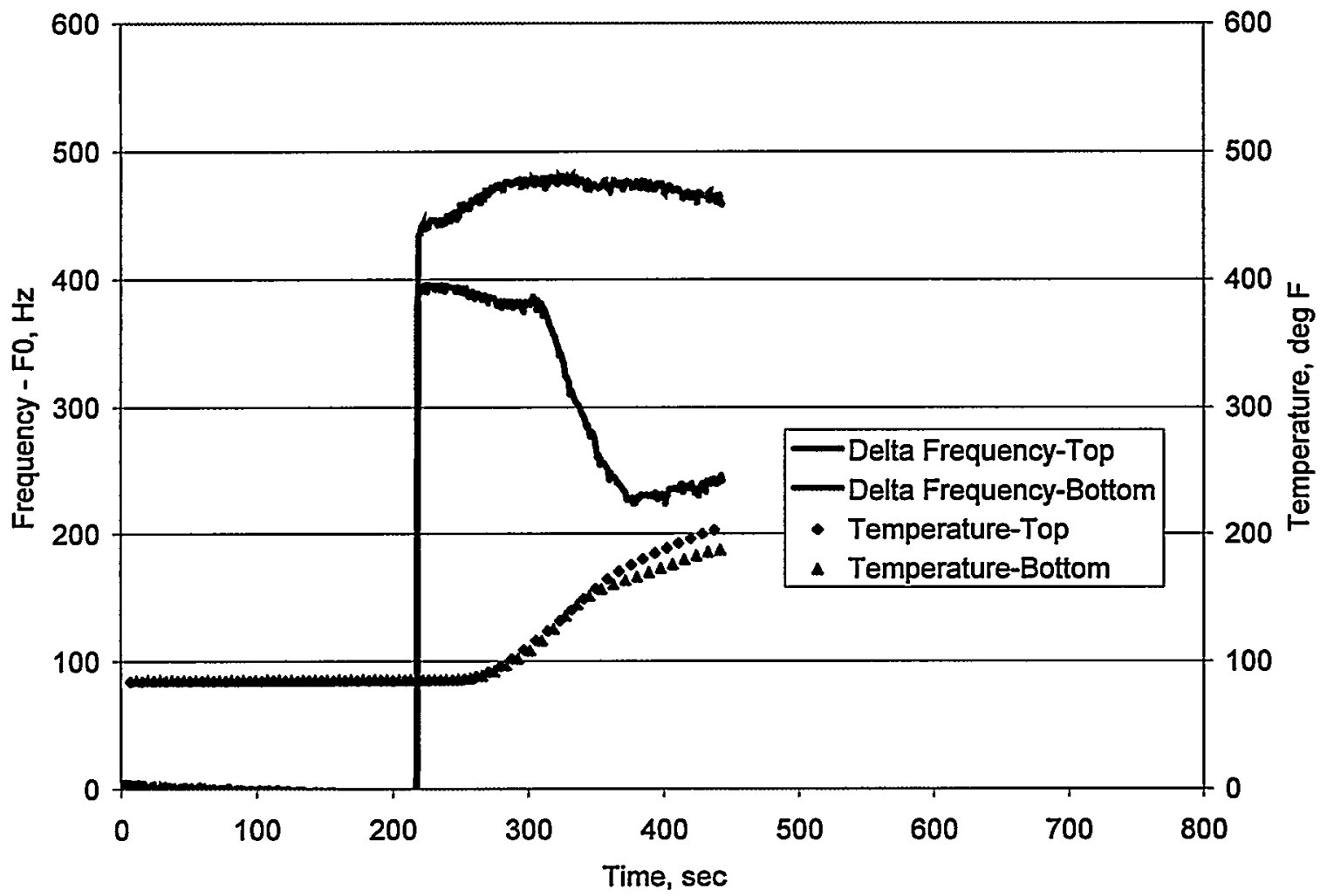

Figure A-10. Pour 062498a, 0.25 inch, horizontal 


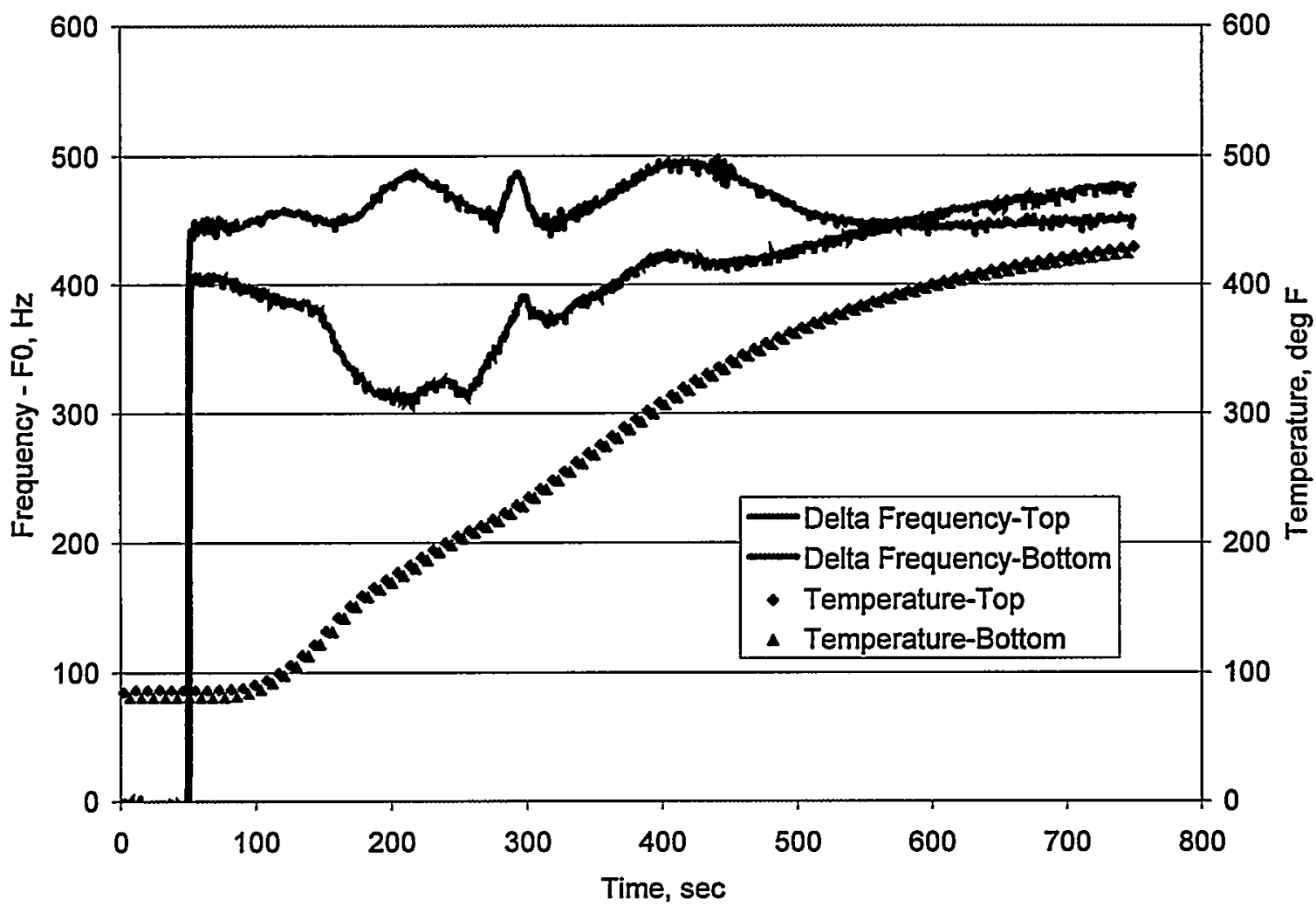

Figure A-11. Pour 062498b, 0.5 inch, horizontal

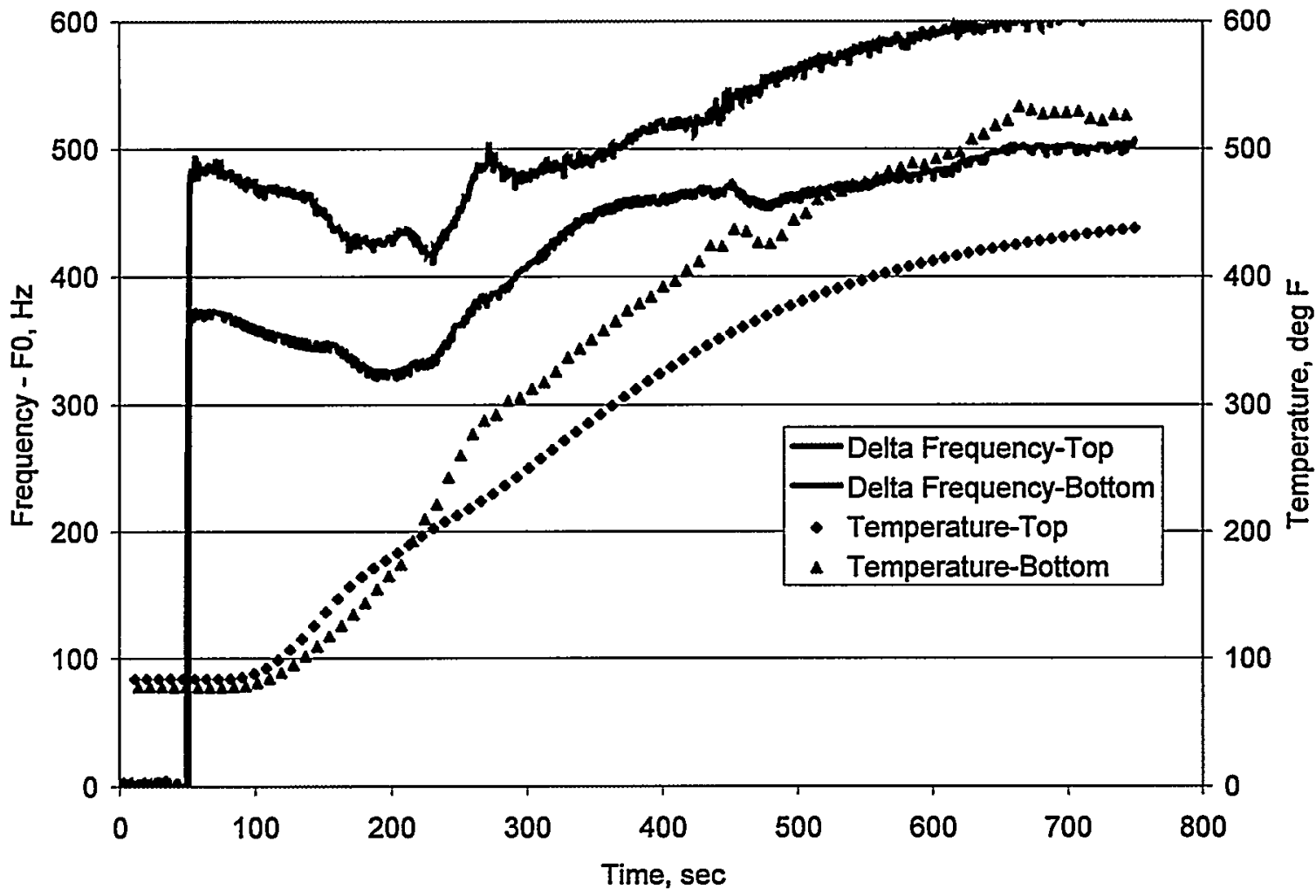

Figure A-12. Pour 062498b, 0.5 inch, horizontal 


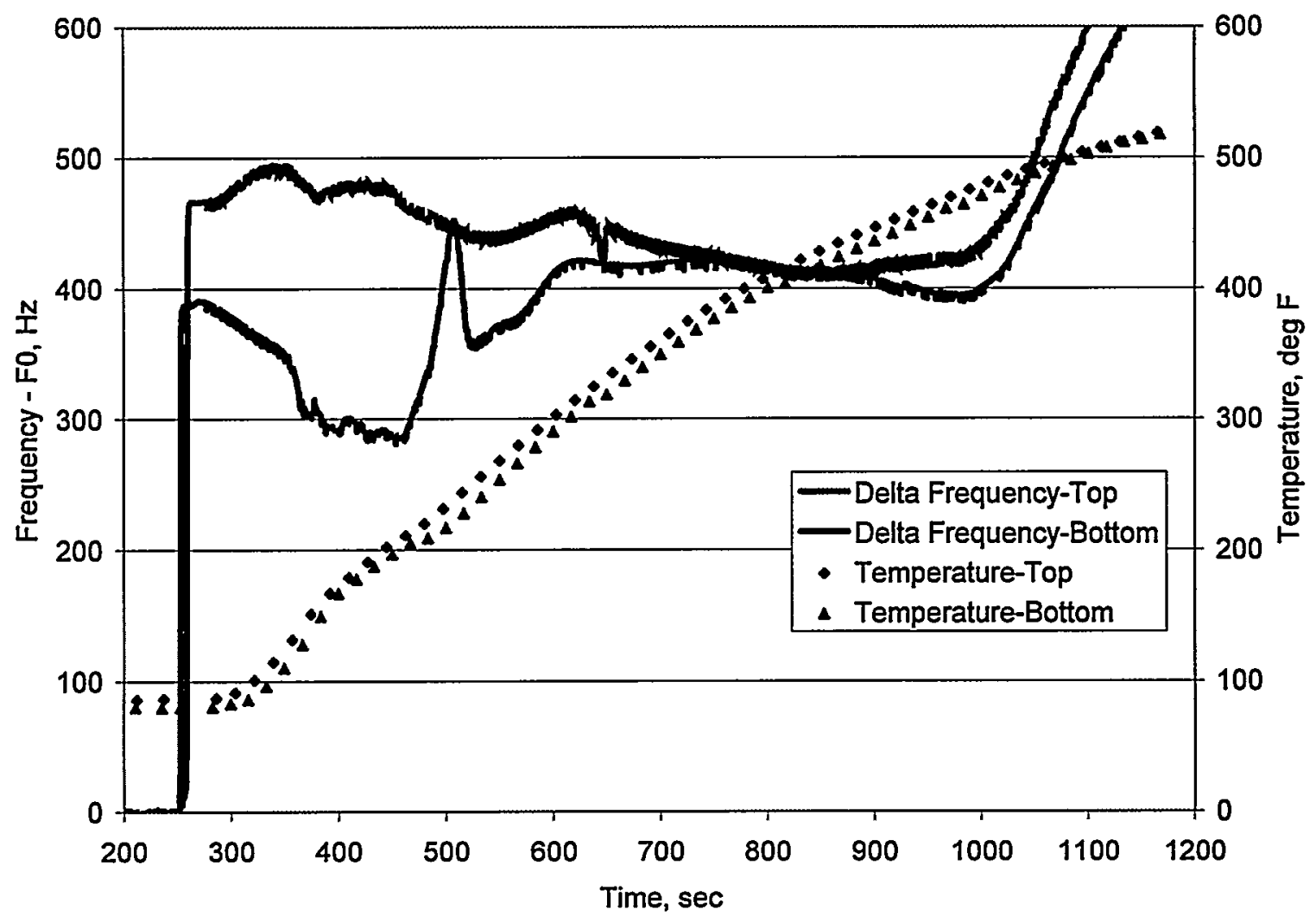

Figure A-13. Pour 070198, 1.0 inch, horizontal

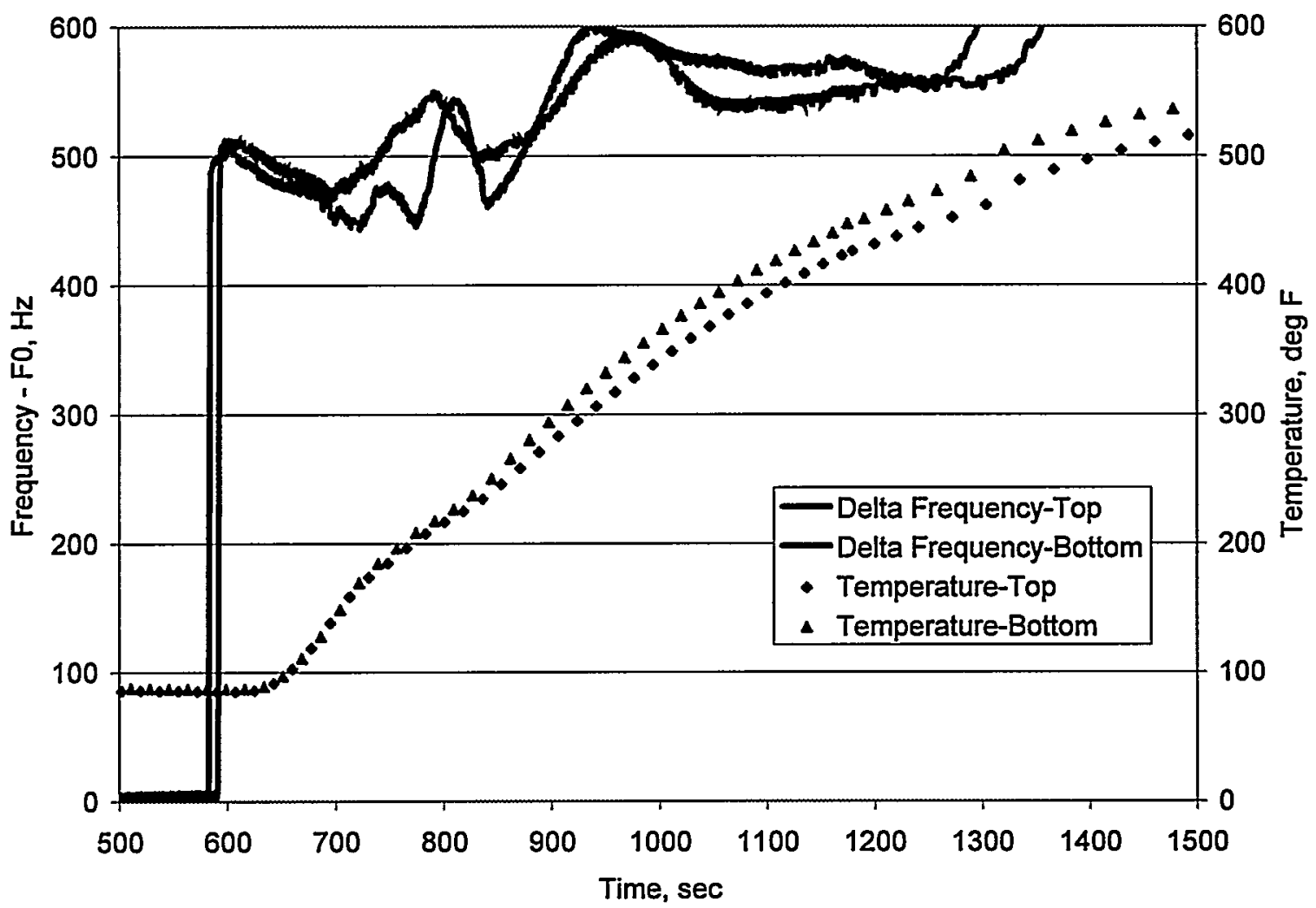

Figure A-14. Pour 070198, 1.0 inch, horizontal 


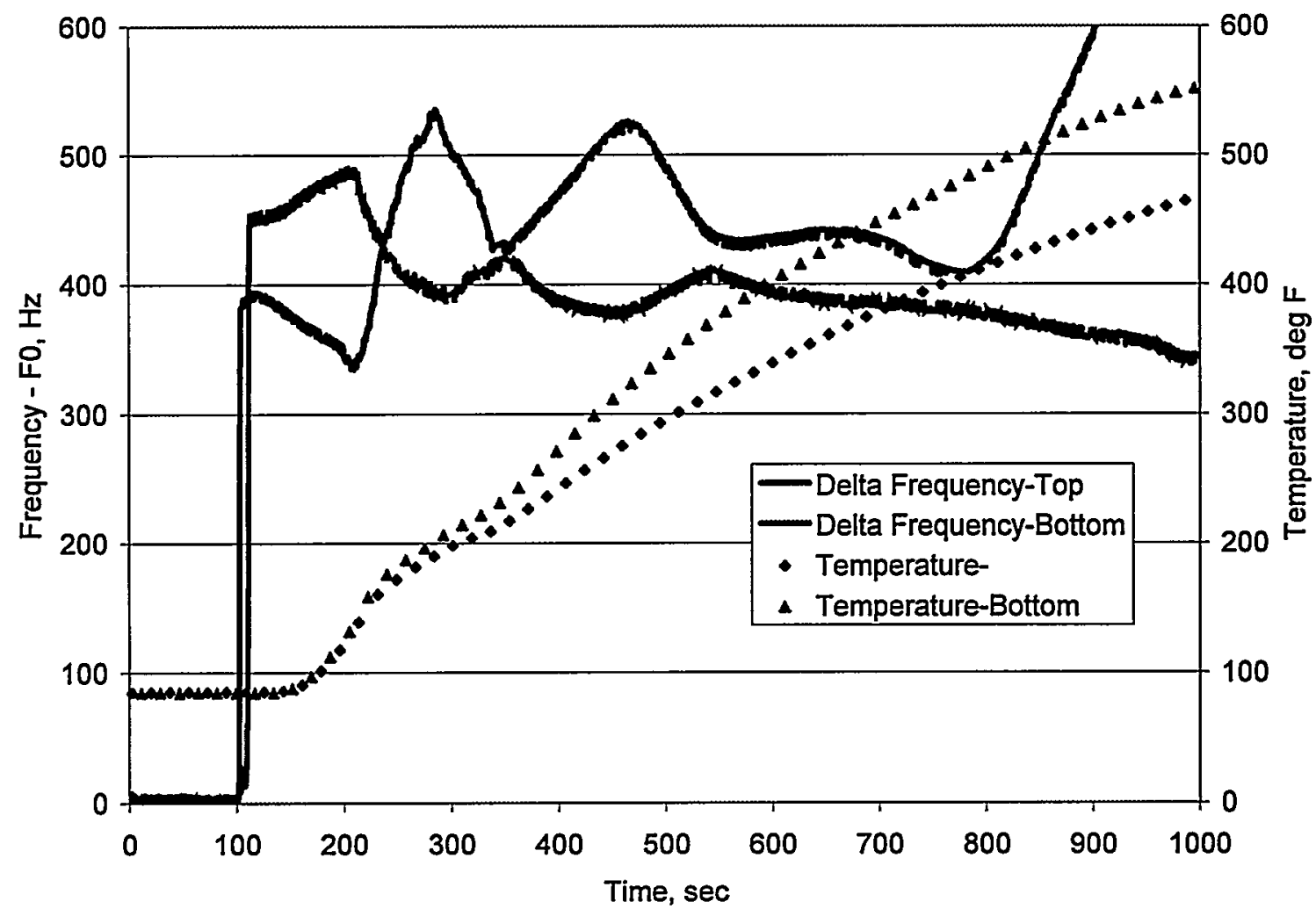

Figure A-15. Pour 070298, 1.0 inch, horizontal

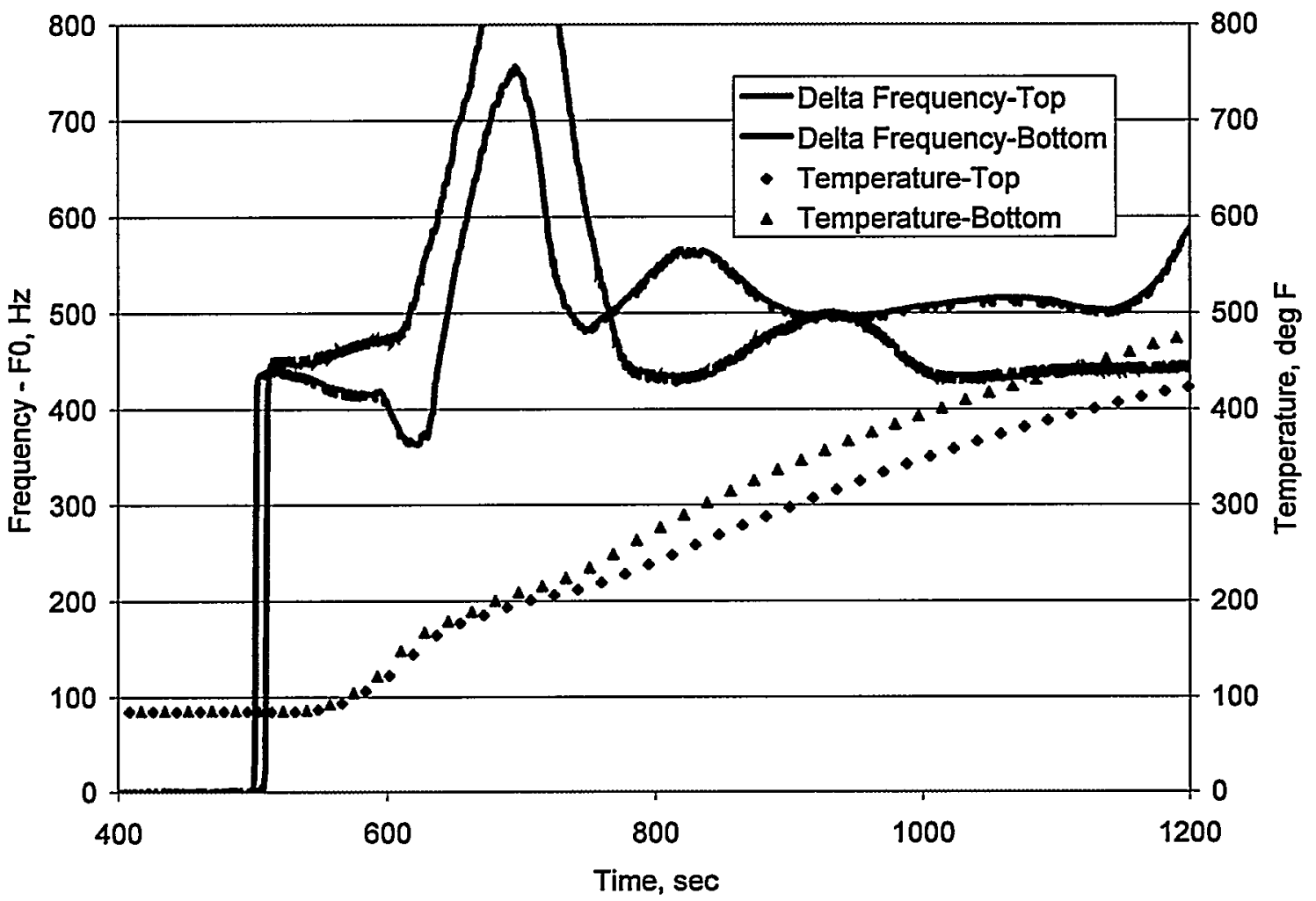

Figure A-16. Pour 070298, 1.0 inch, horizontal 


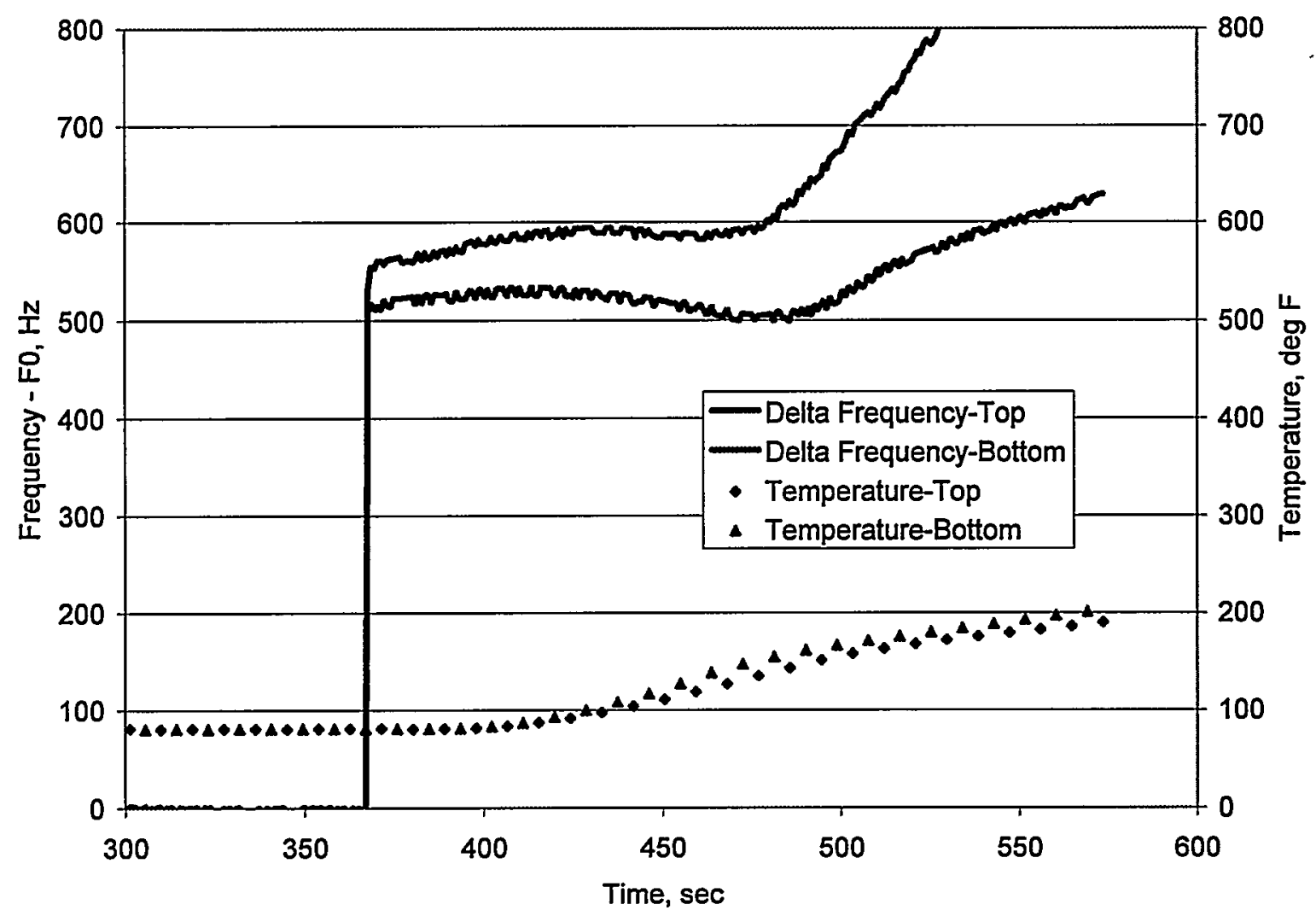

Figure A-17. Pour 071598a, 0.25 inch, horizontal

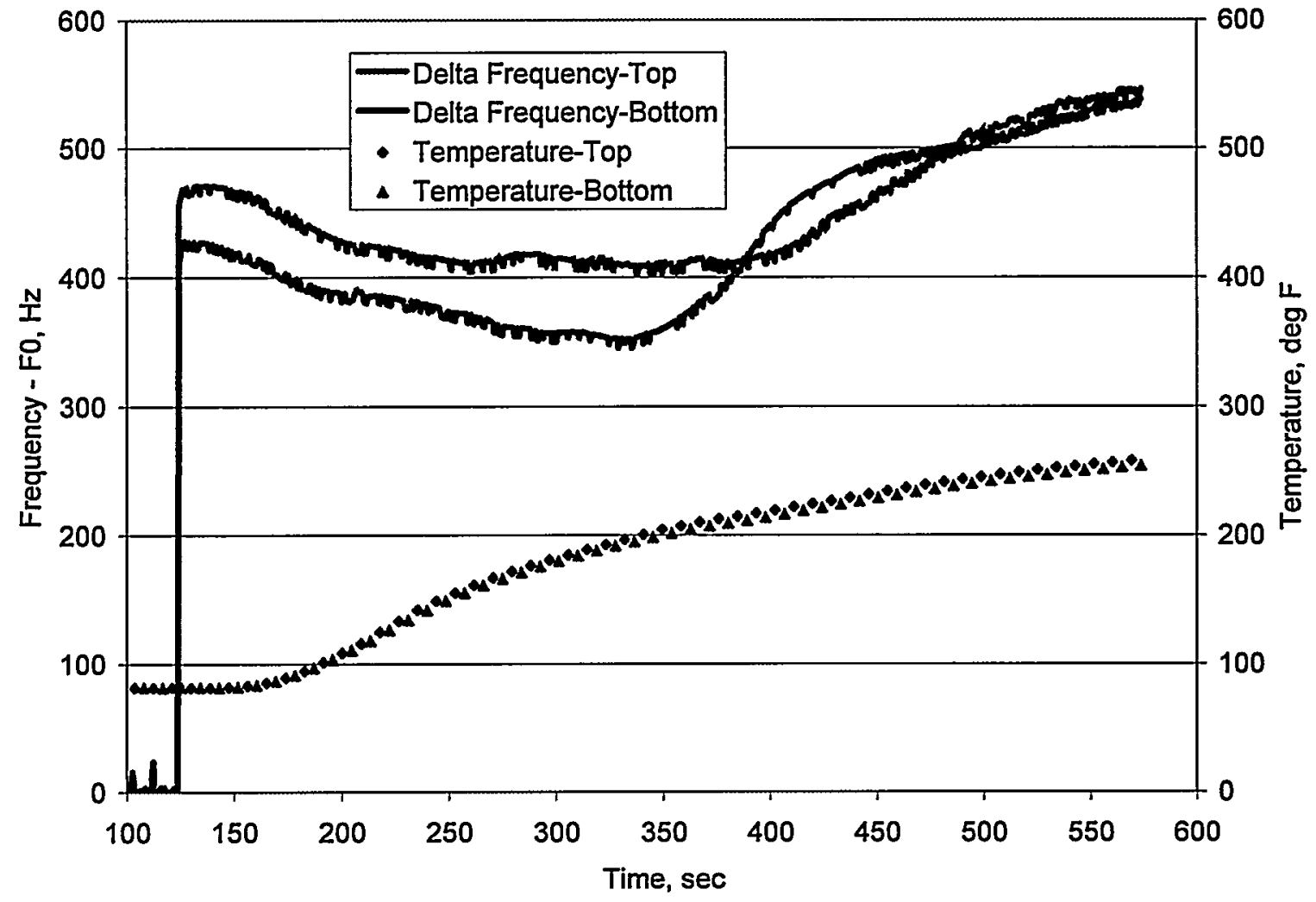

Figure A-18. Pour 071598a, 0.25 inch, horizontal 


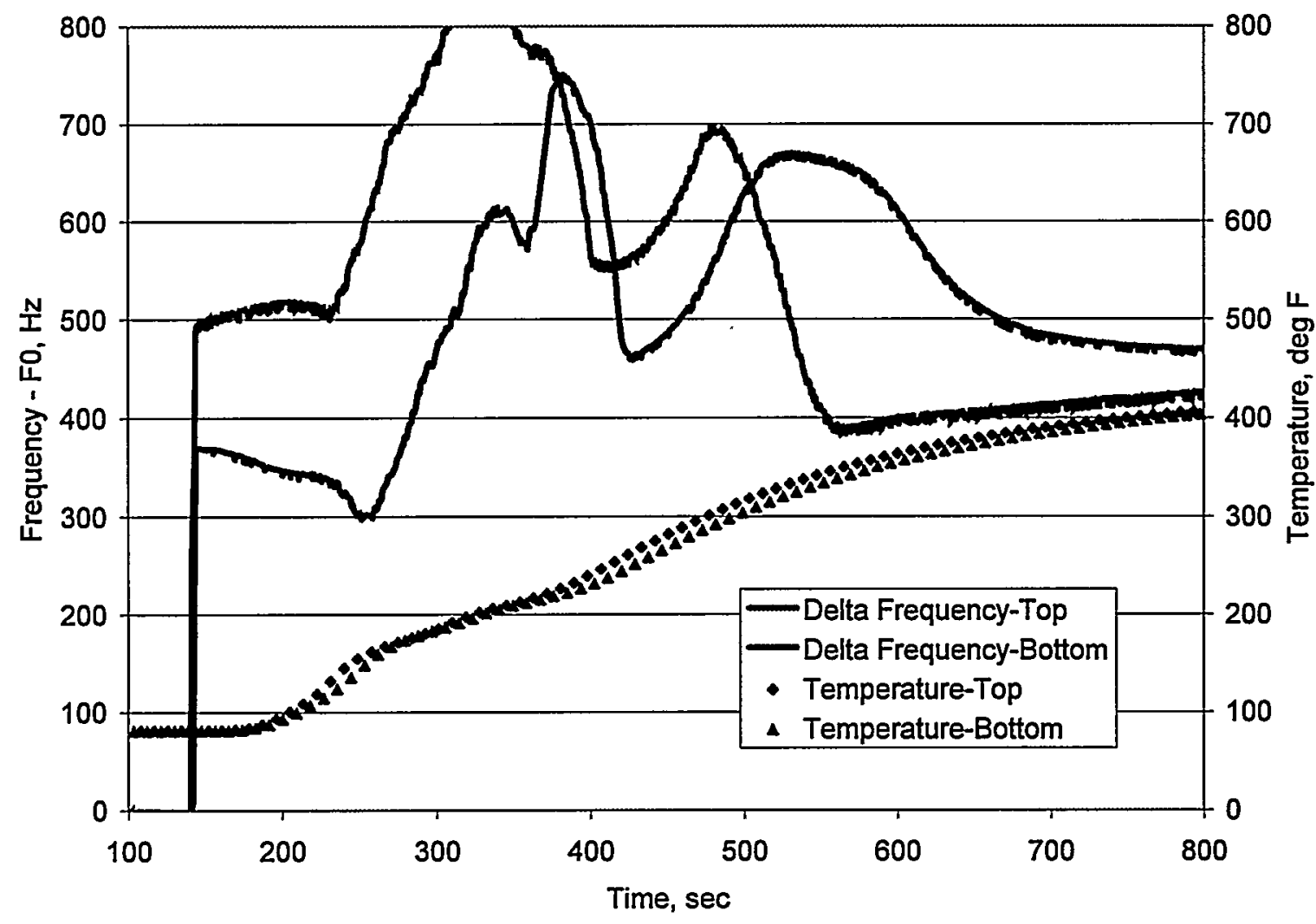

Figure A-19. Pour 071598b, 0.5 inch, horizontal

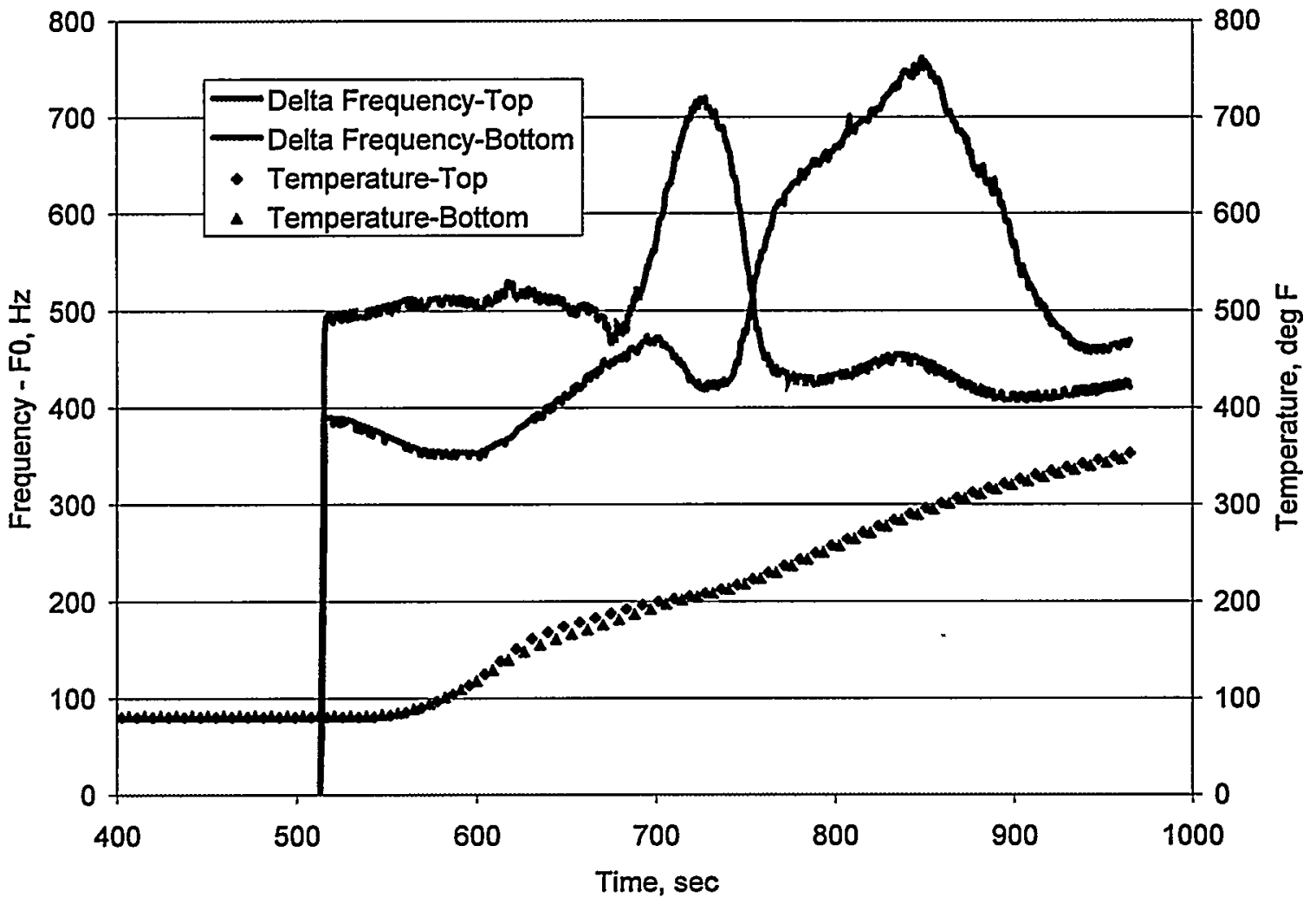

Figure A-20. Pour 071598b, 0.5 inch, horizontal 


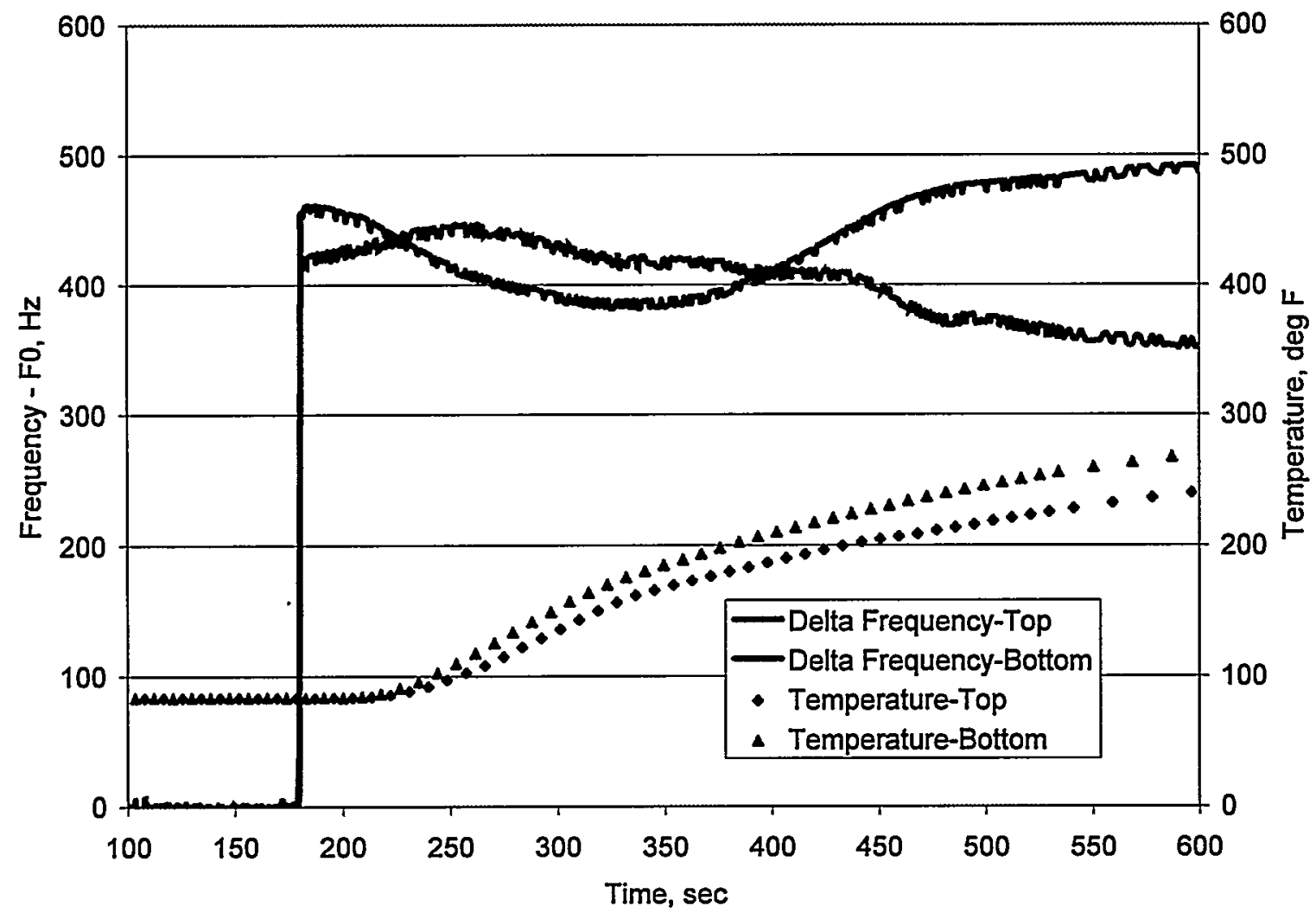

Figure A-21. Pour 071798a, 0.25 inch, horizontal

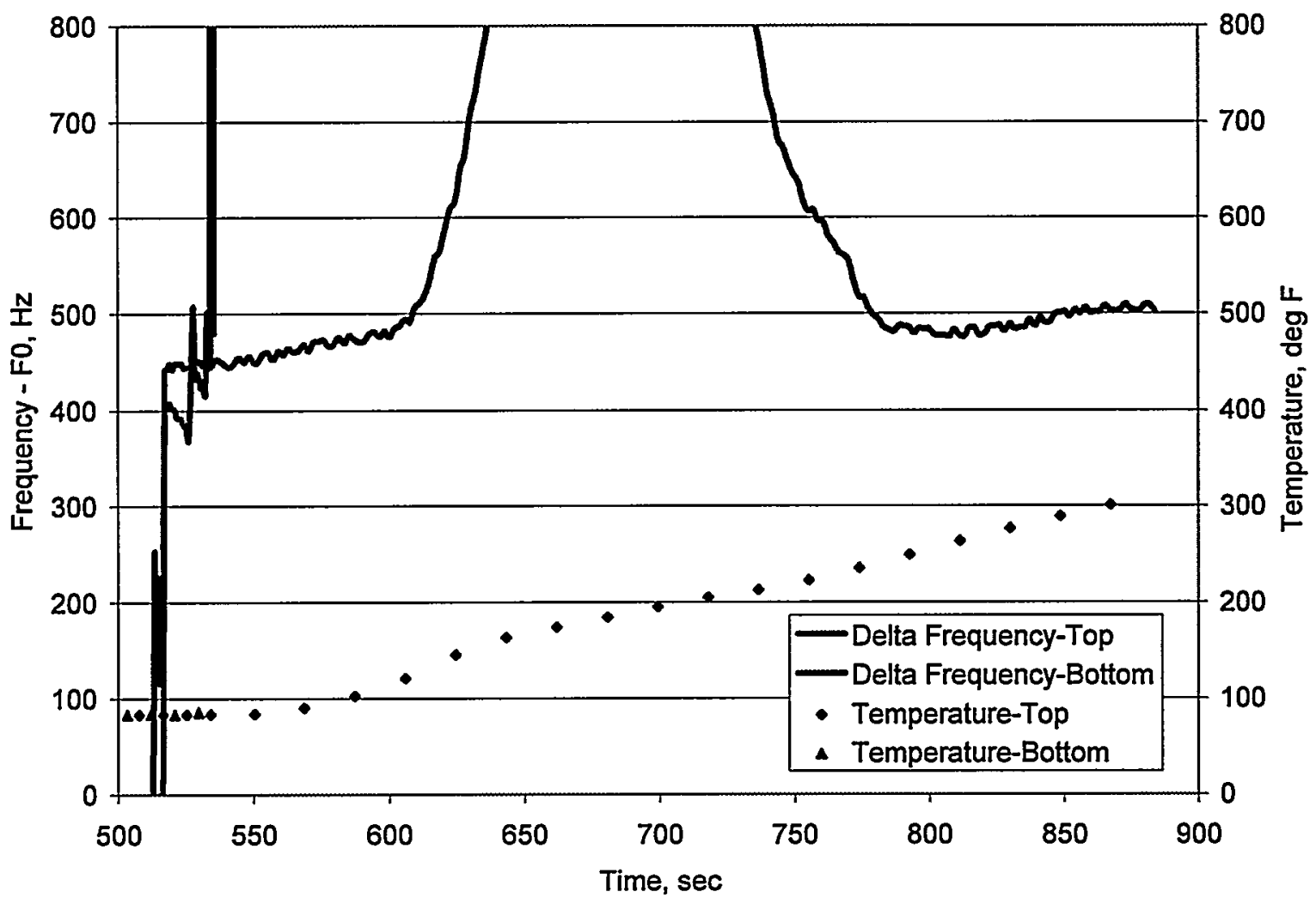

Figure A-22. Pour 071798a, 0.5 inch, horizontal 


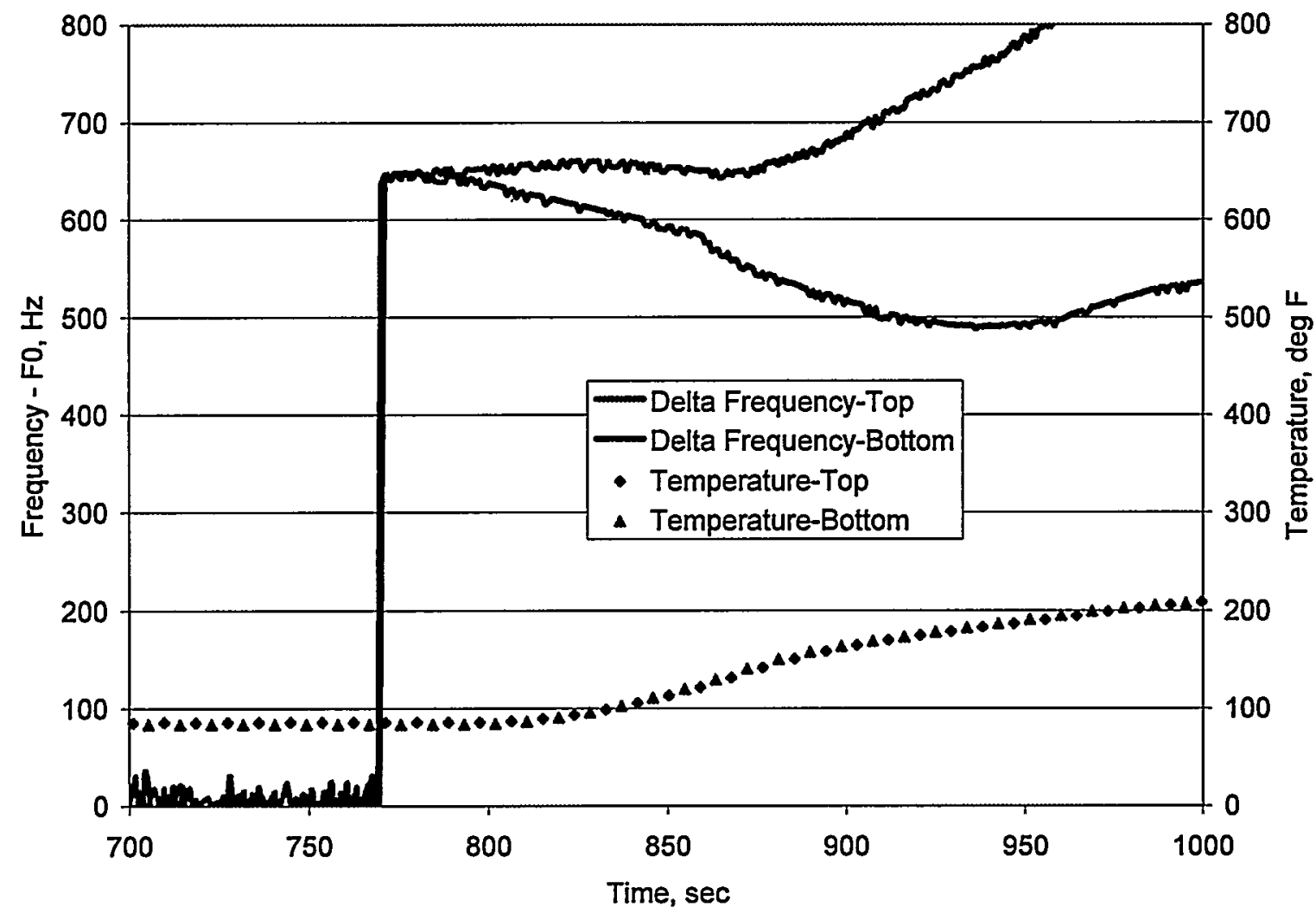

Figure A-23. Pour 071798b, 0.25 inch, horizontal

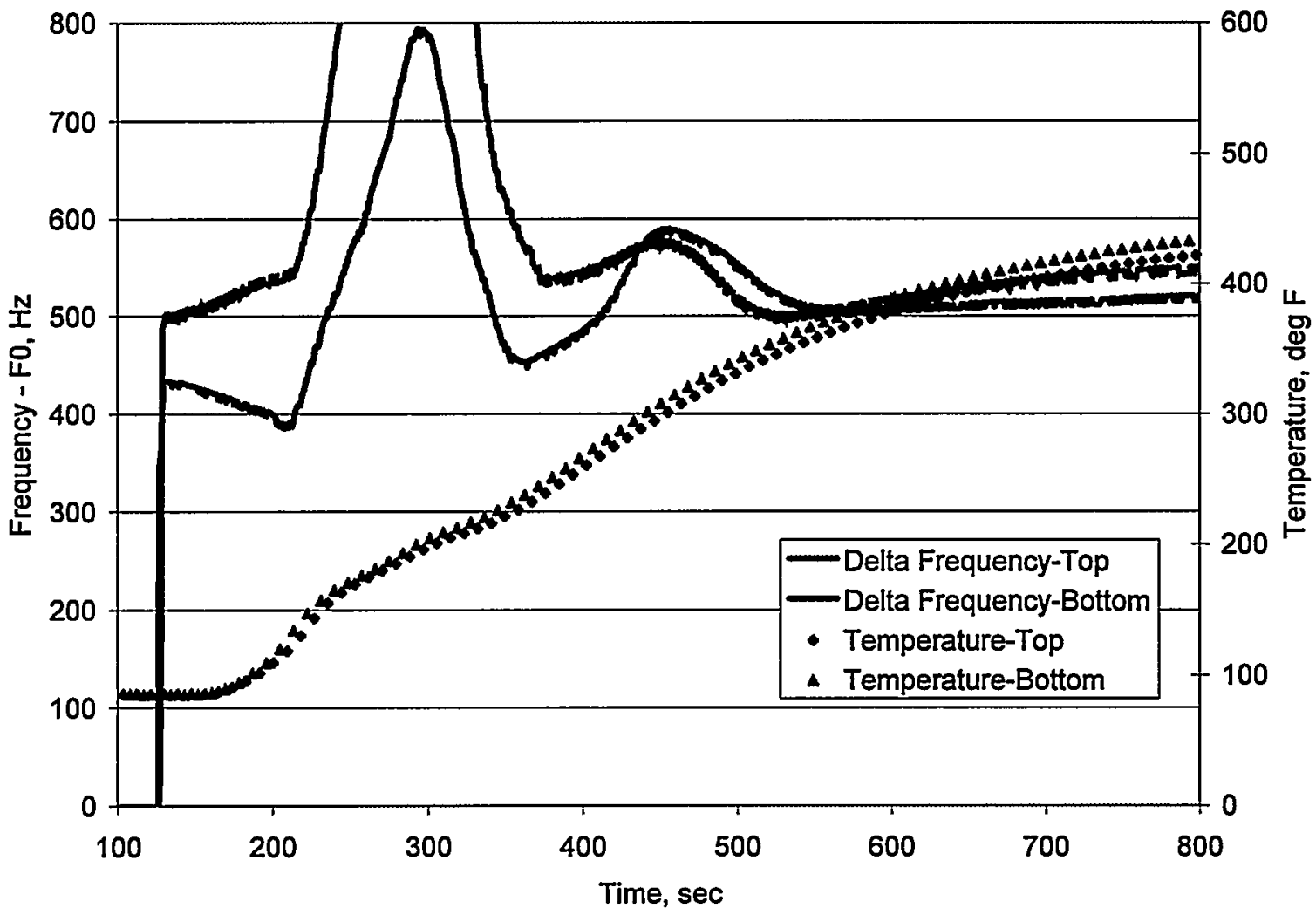

Figure A-24. Pour 071798b, 0.5 inch, horizontal 


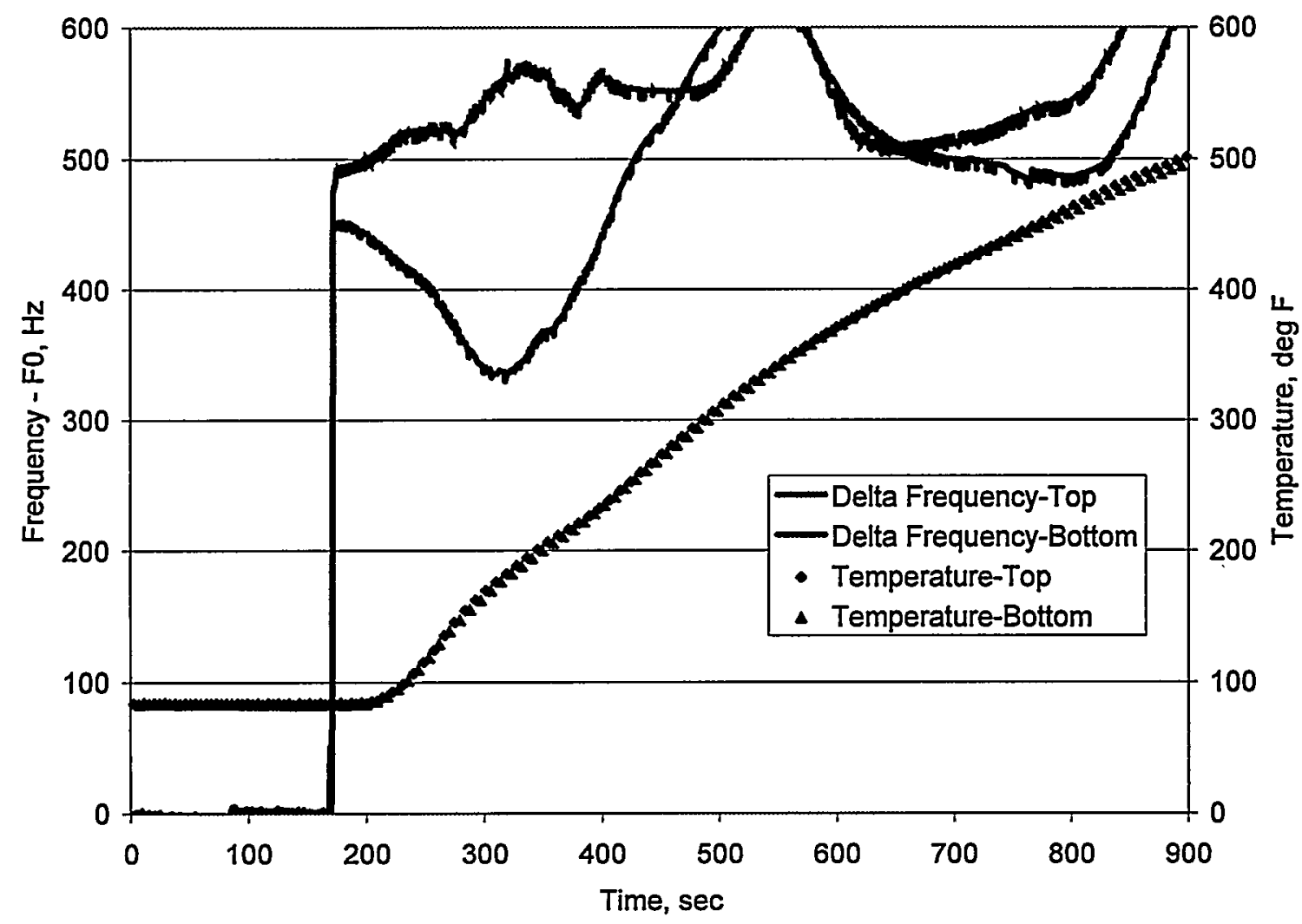

Figure A-25. Pour 072398, 1.00 inch, horizontal

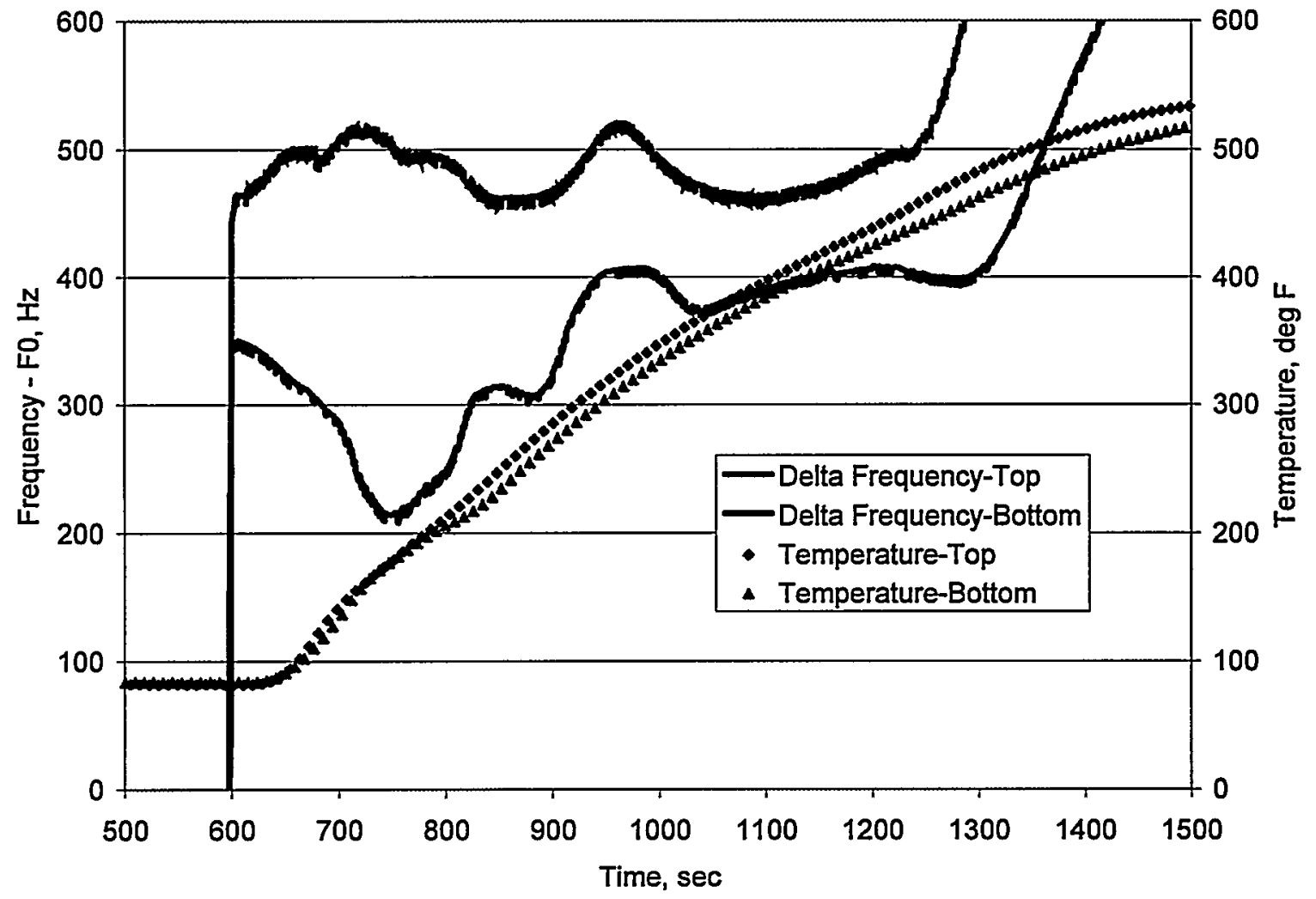

Figure A-26. Pour 072398, 1.00 inch, horizontal 


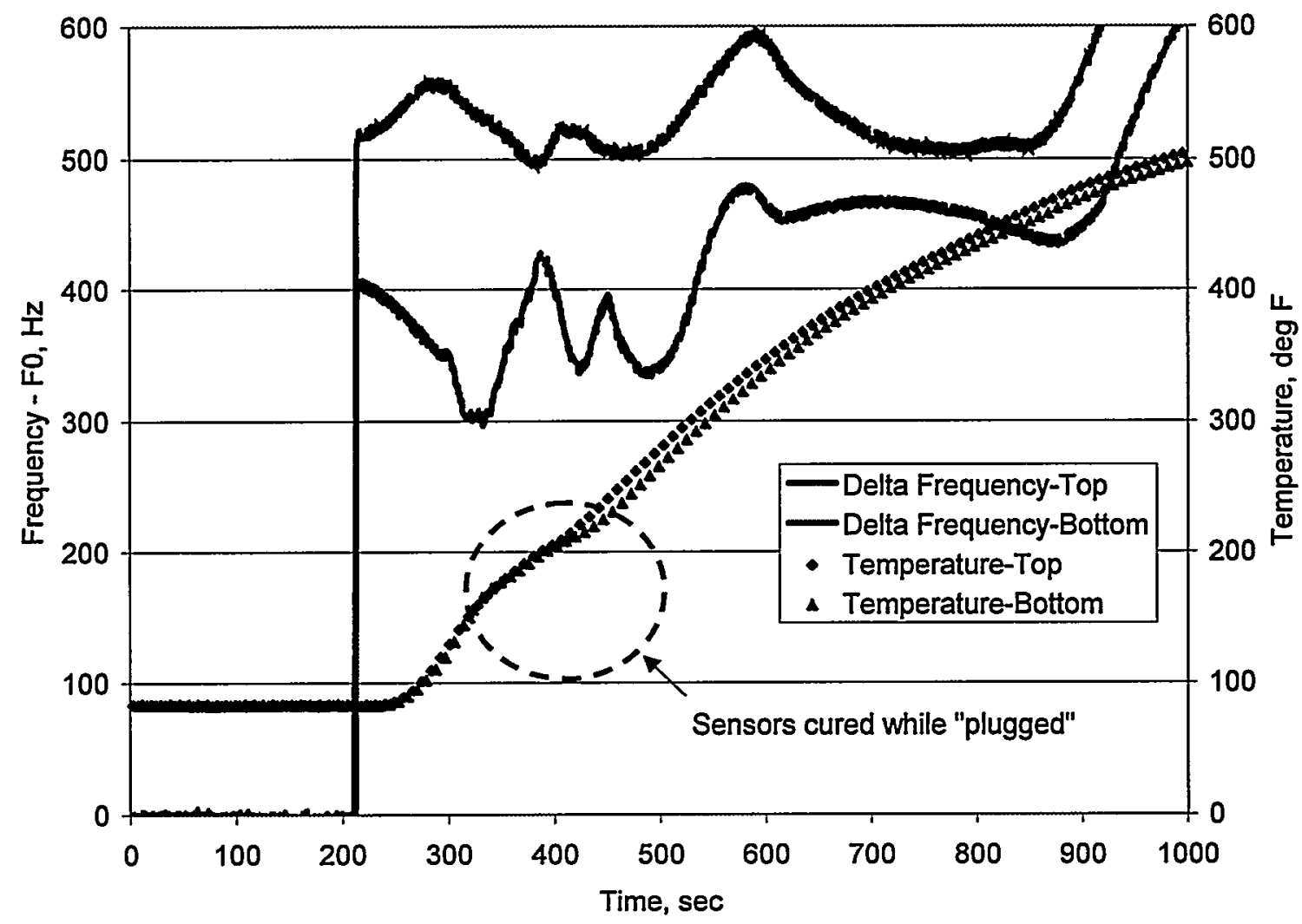

Figure A-27. Pour 072498, 1.00 inch, horizontal

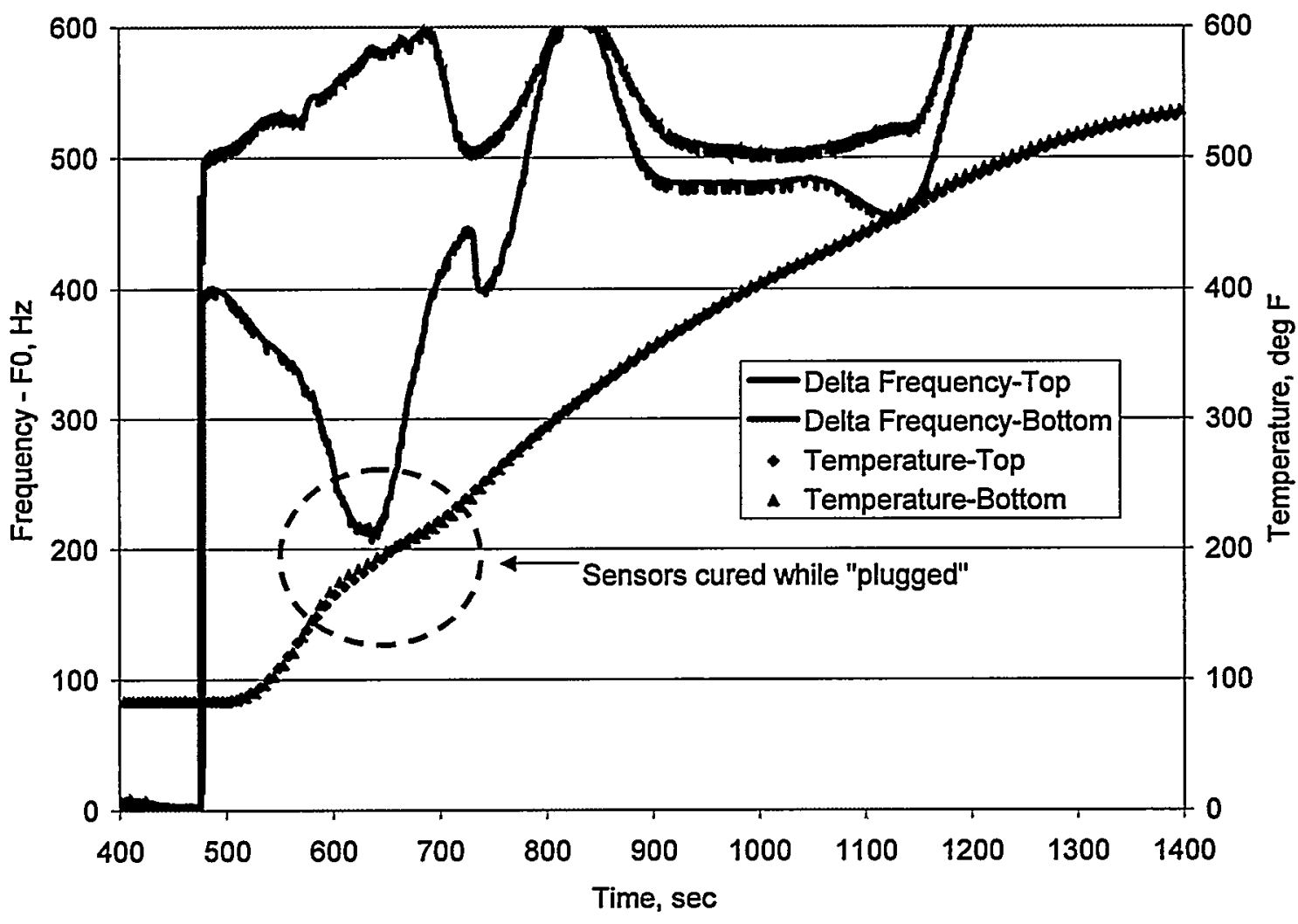

Figure A-28. Pour 072498, 1.00 inch, horizontal 


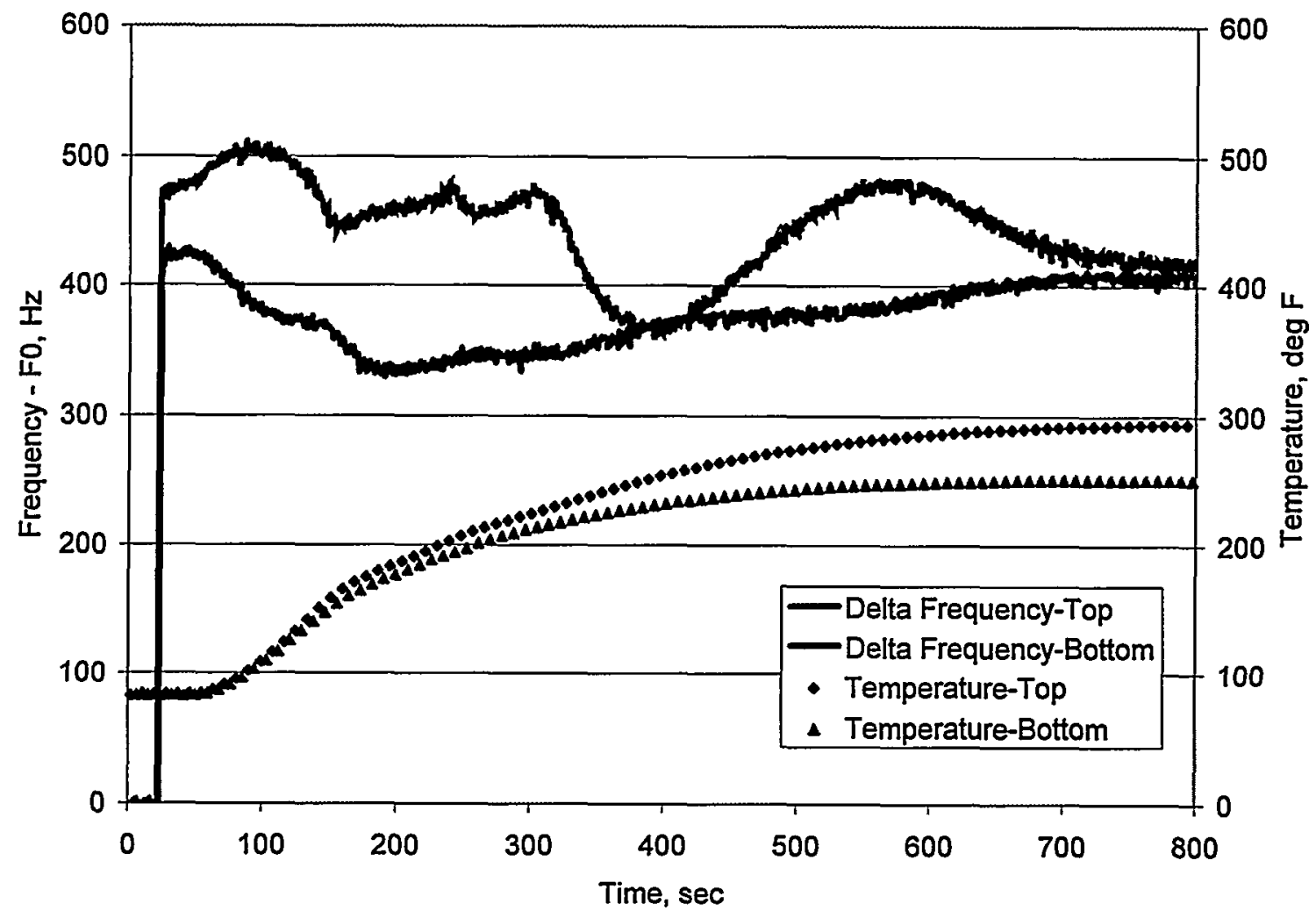

Figure A-29. Pour 072998, 0.25 inch, horizontal

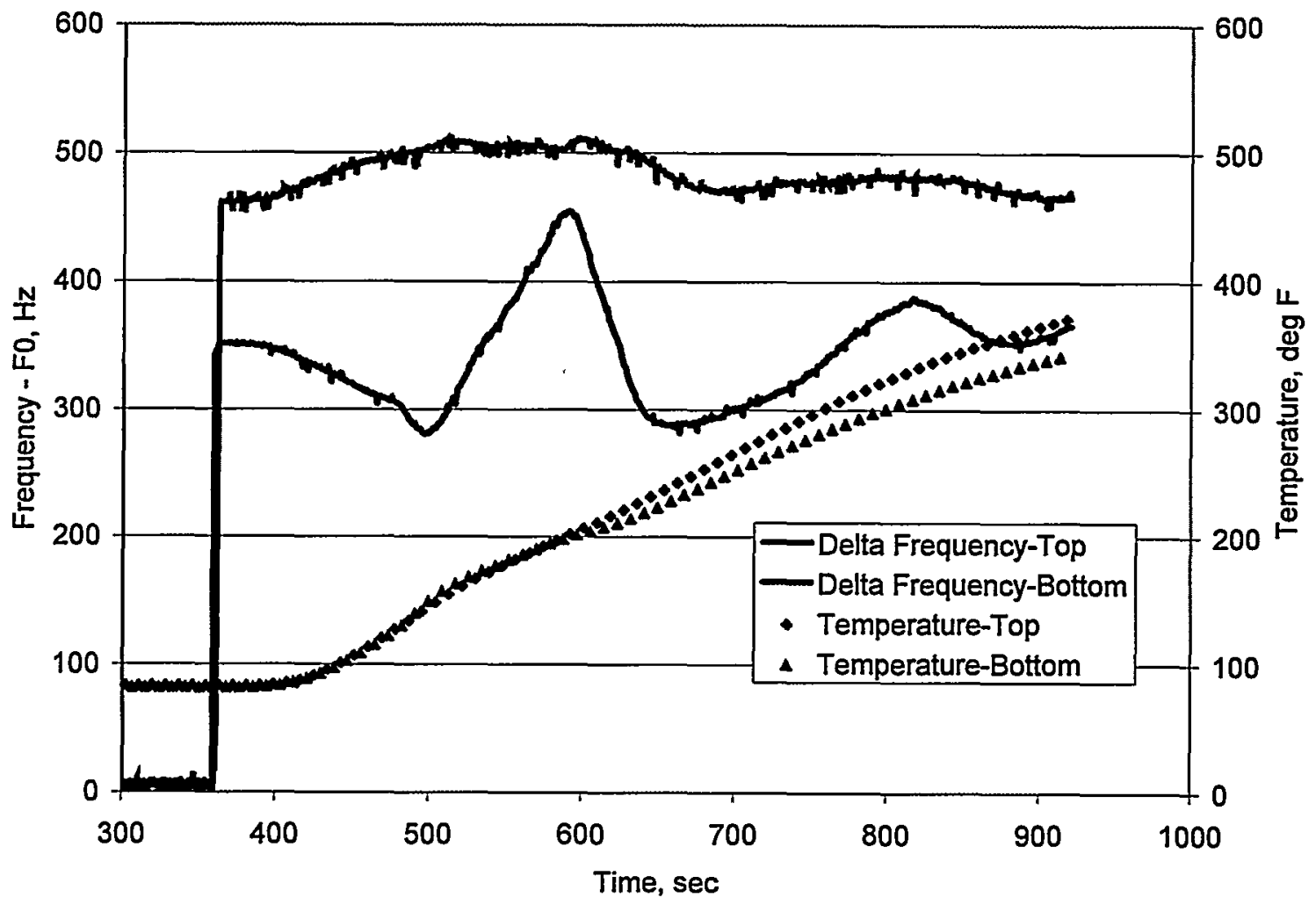

Figure A-30. Pour 072998, 0.50 inch, horizontal 


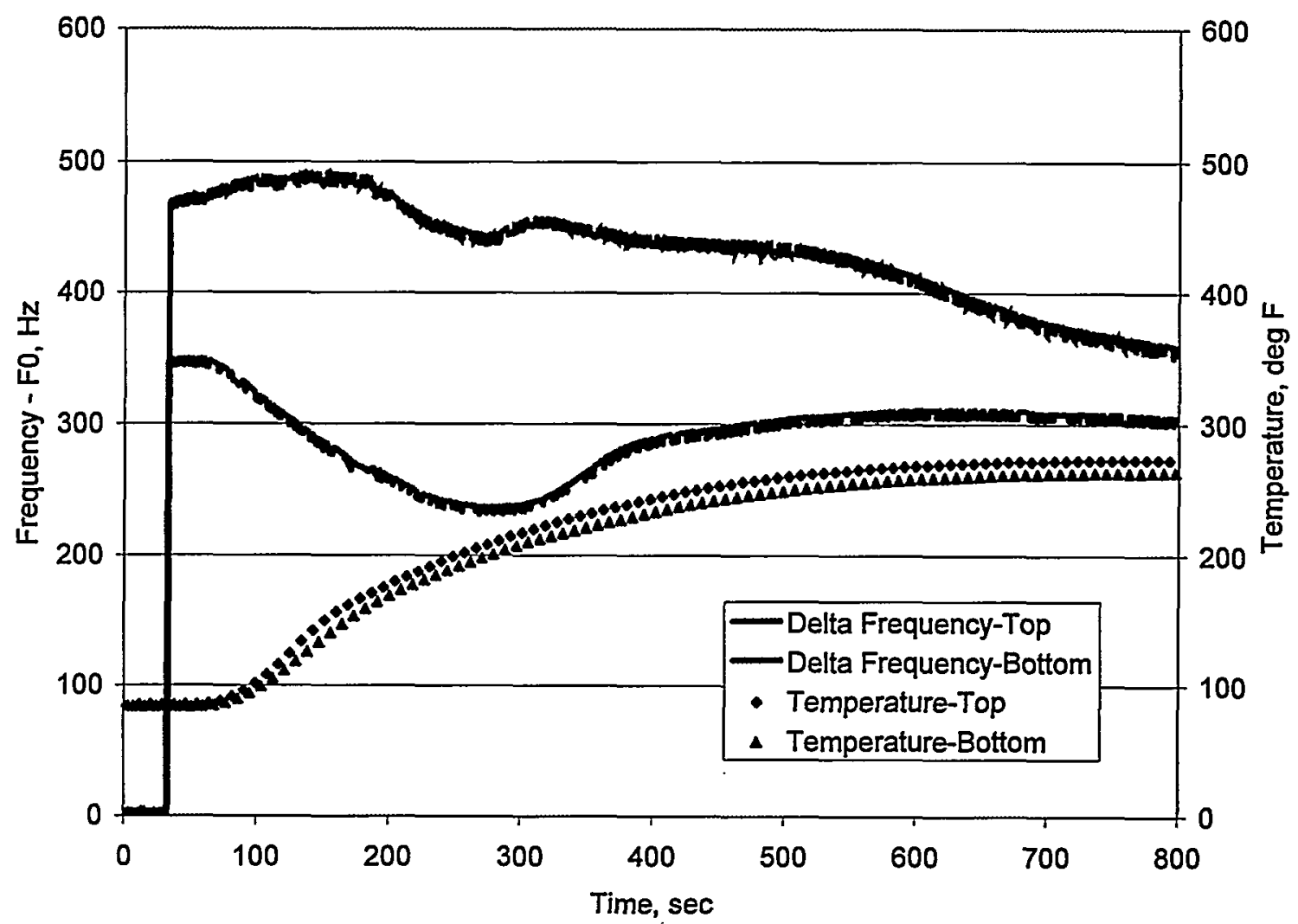

Figure A-31. Pour 073098, 0.25 inch, horizontal

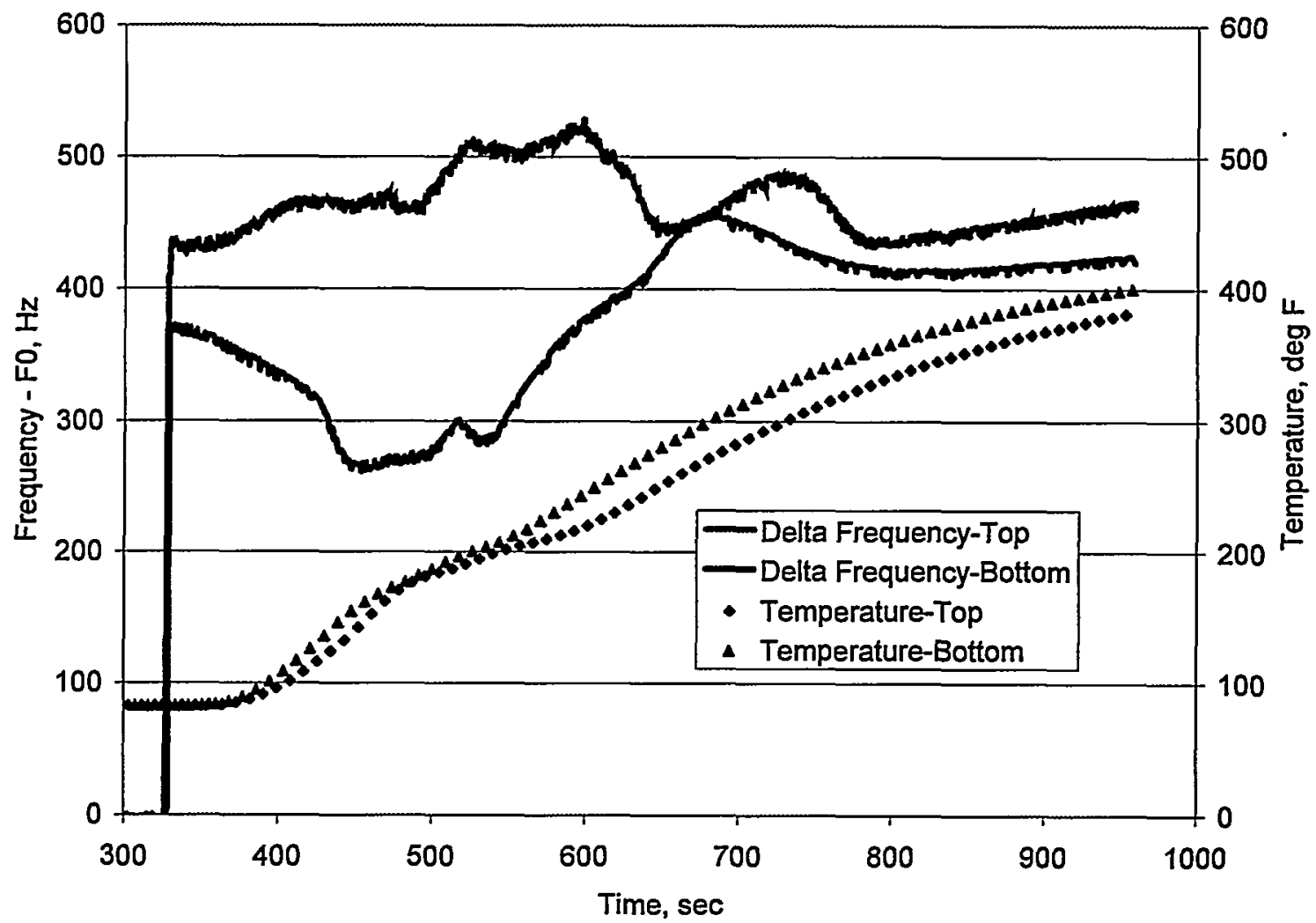

Figure A-32. Pour 073098, 0.50 inch, horizontal 


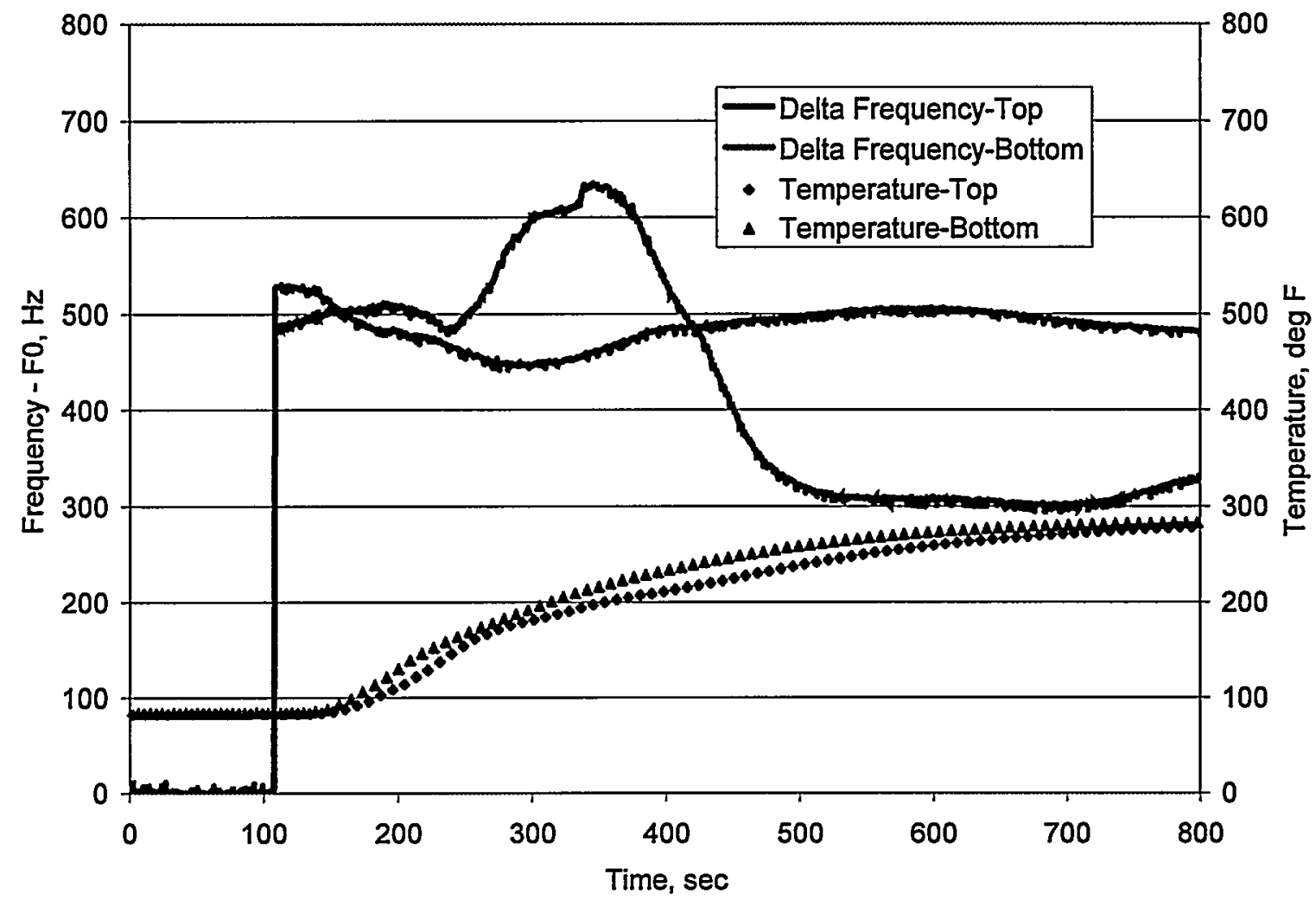

Figure A-33. Pour 081298, 0.25 inch, horizontal

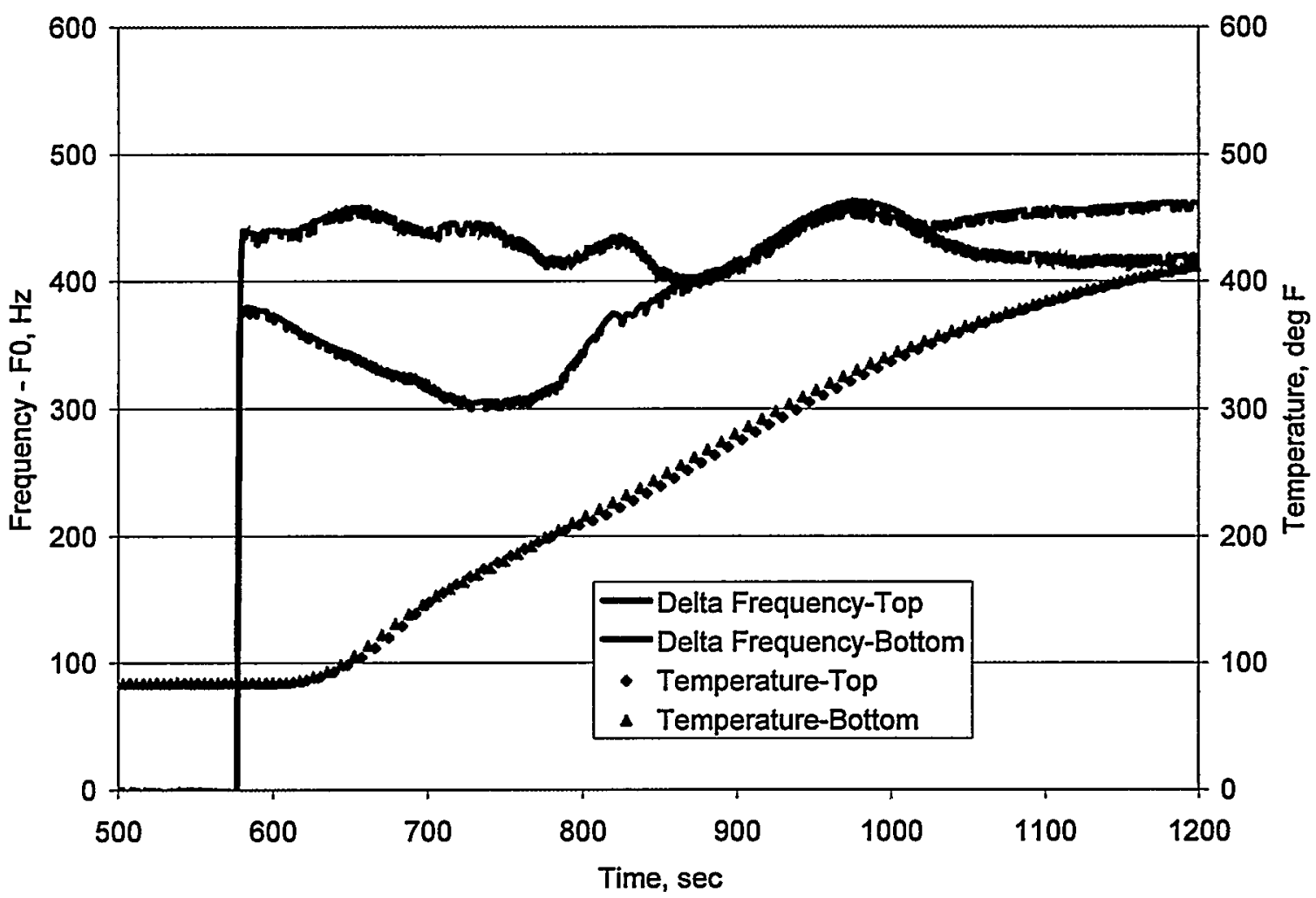

Figure A-34. Pour 081298, 0.50 inch, horizontal 


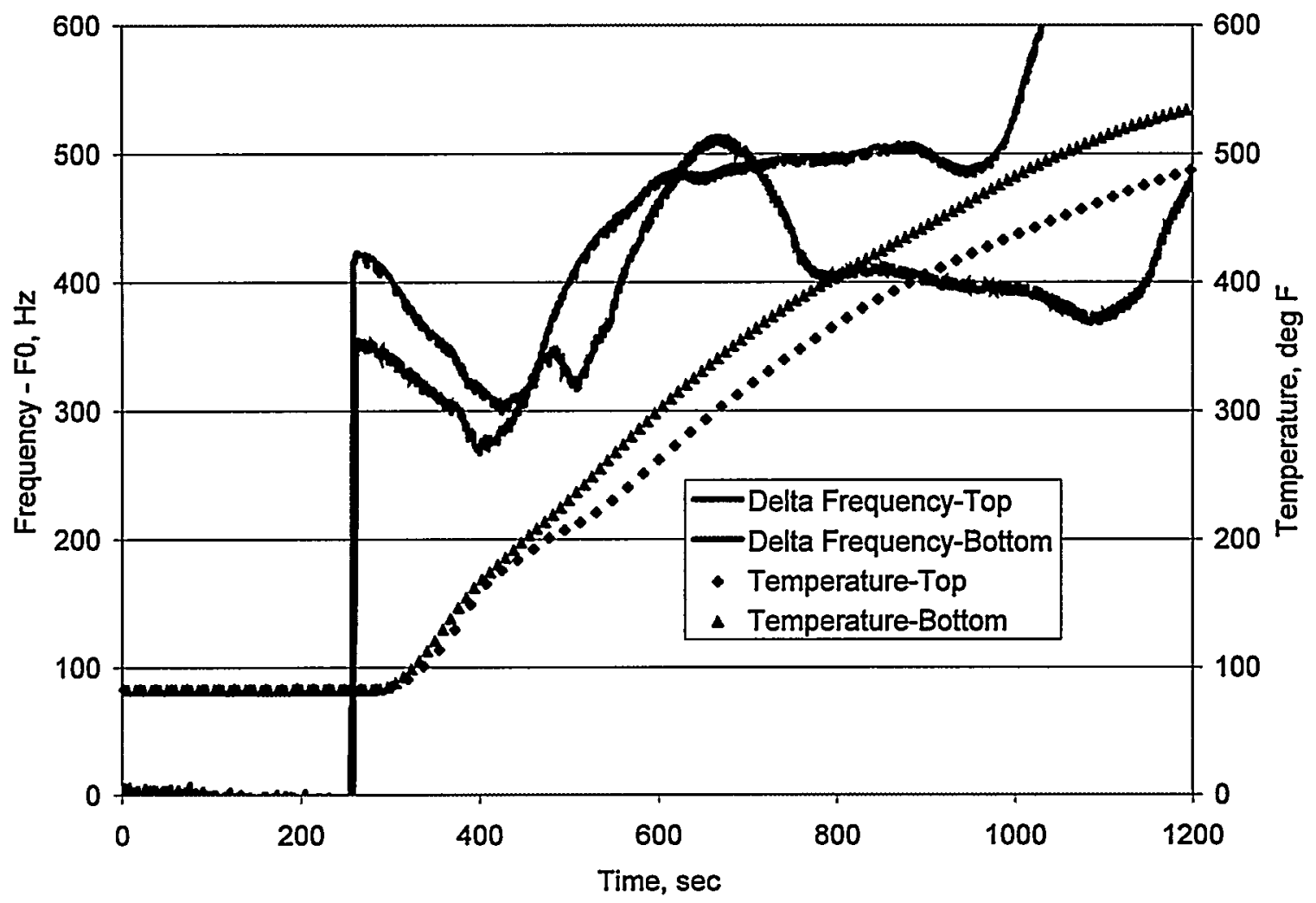

Figure A-35. Pour 081398, 1.00 inch, horizontal

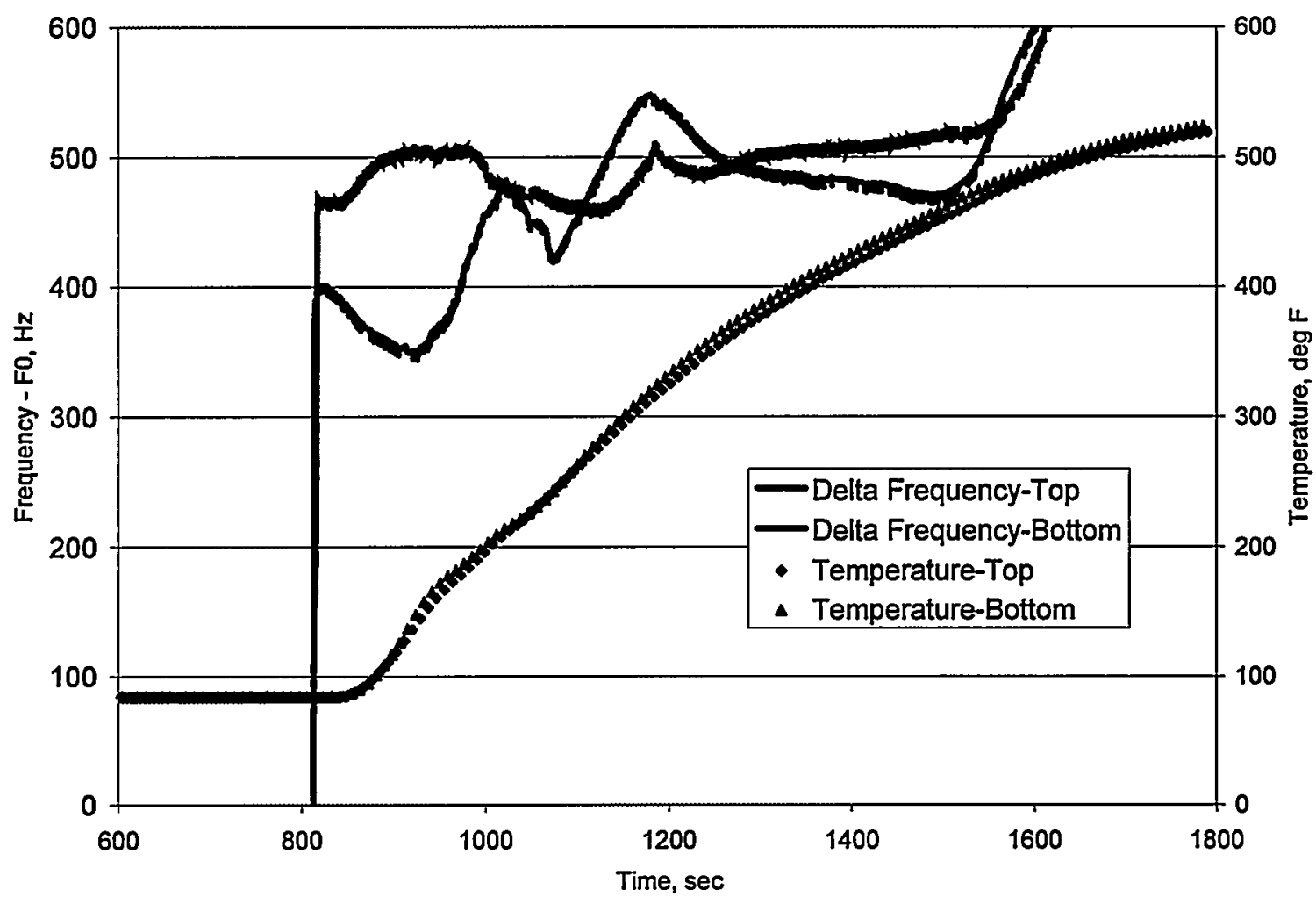

Figure A-36. Pour 081398, 1.00 inch, horizontal 


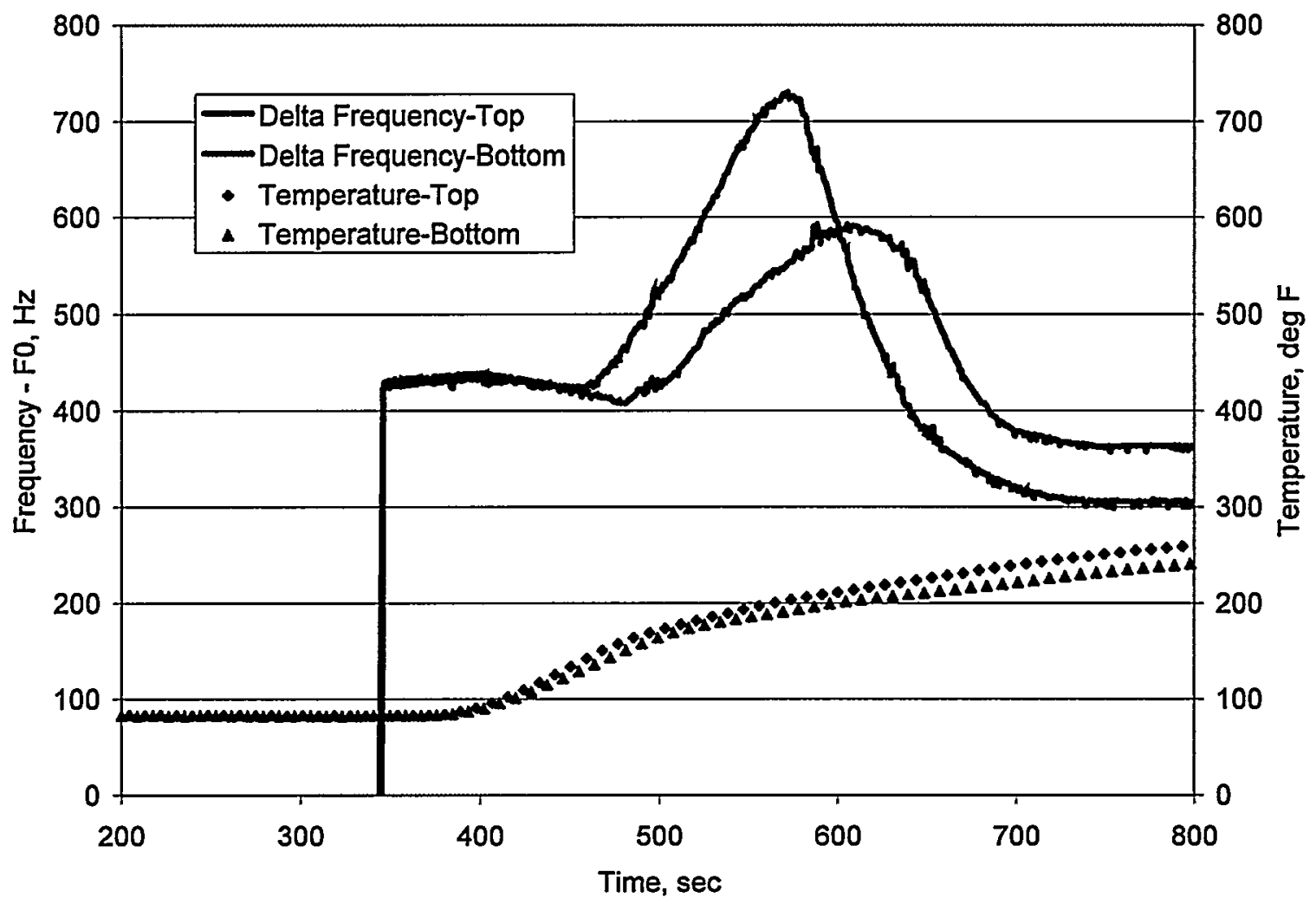

Figure A-37. Pour 082498, 0.25 inch, horizontal

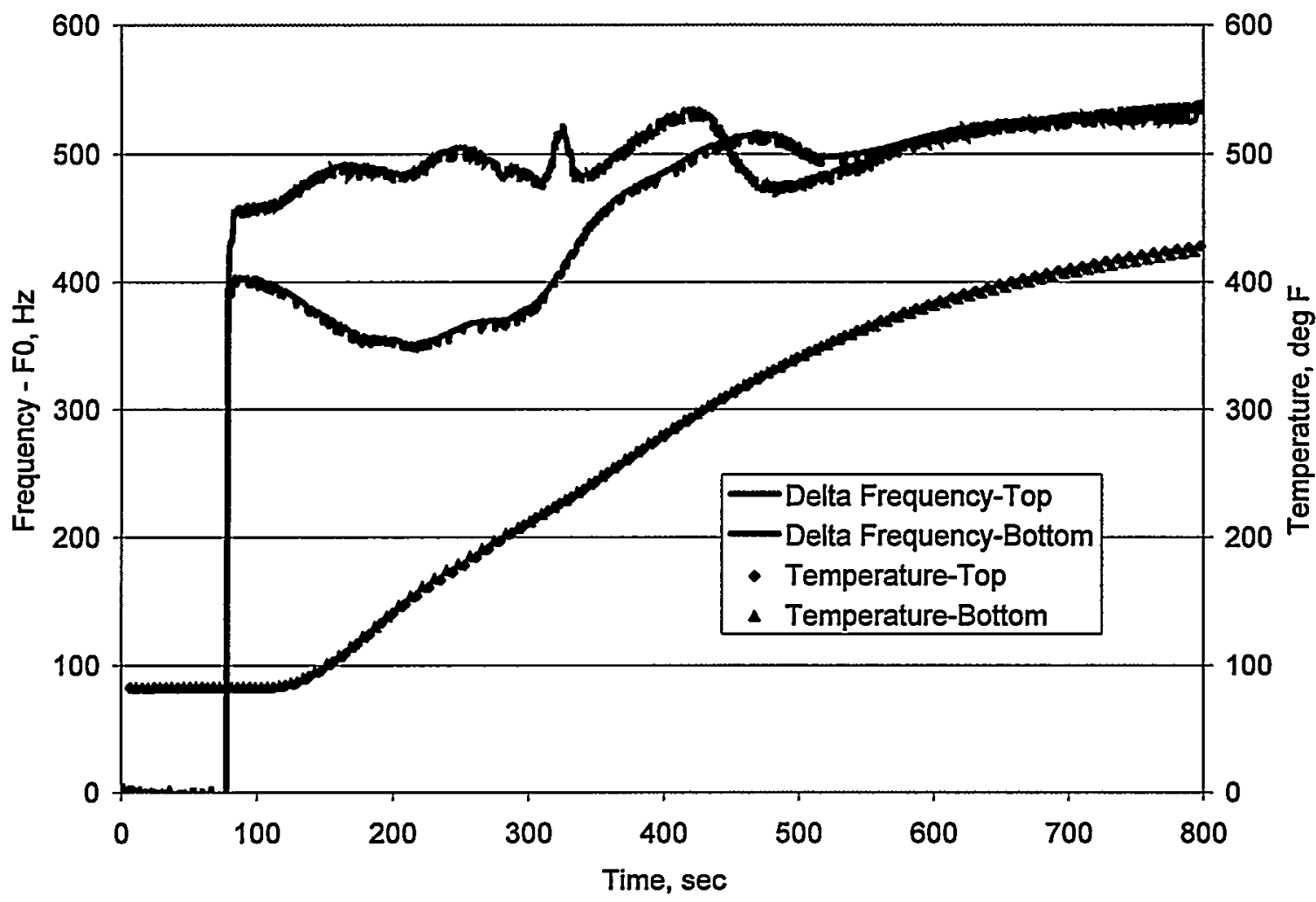

Figure A-38. Pour 082498, 0.50 inch, horizontal 


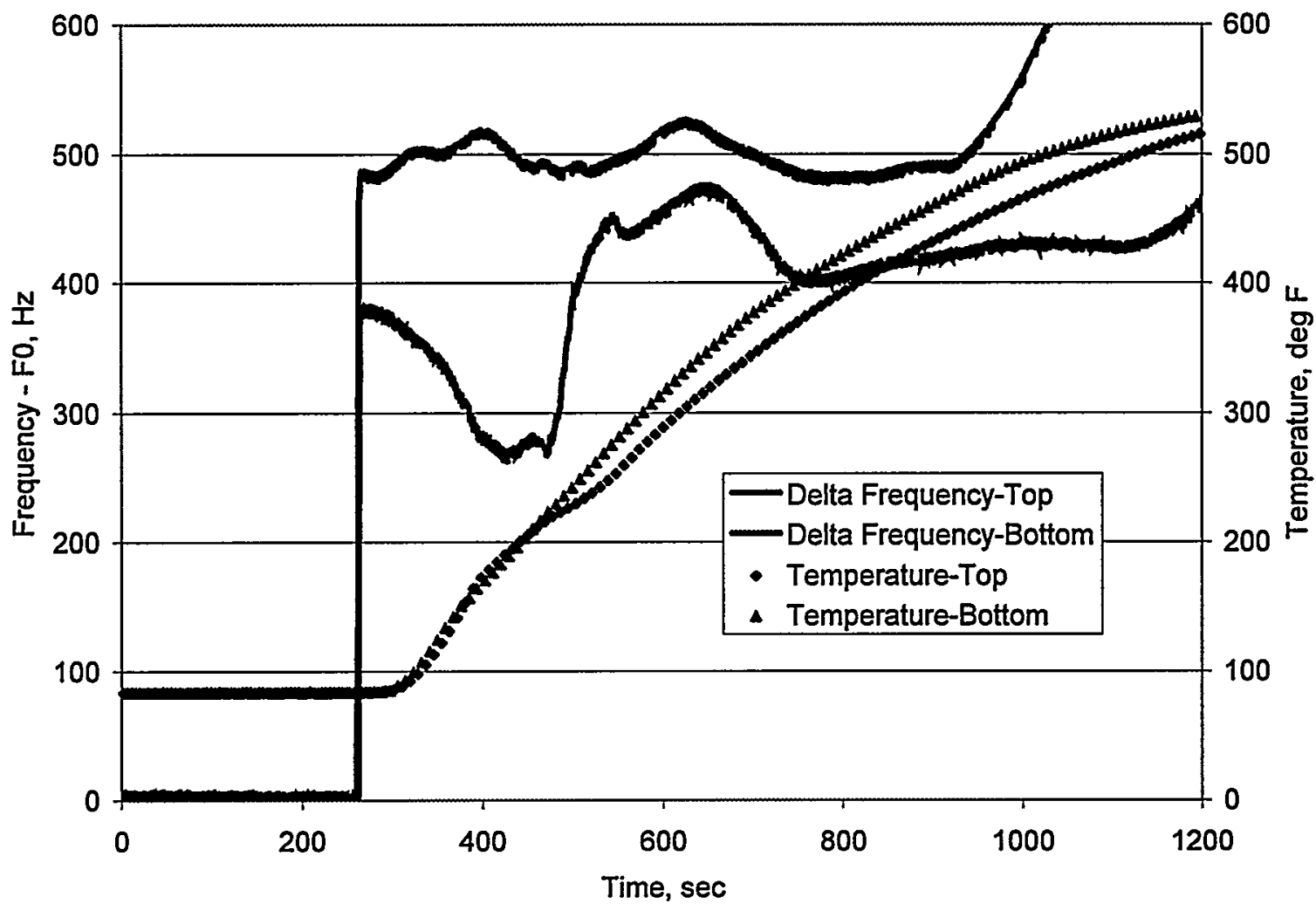

Figure A-39. Pour 082598, 1.00 inch, horizontal

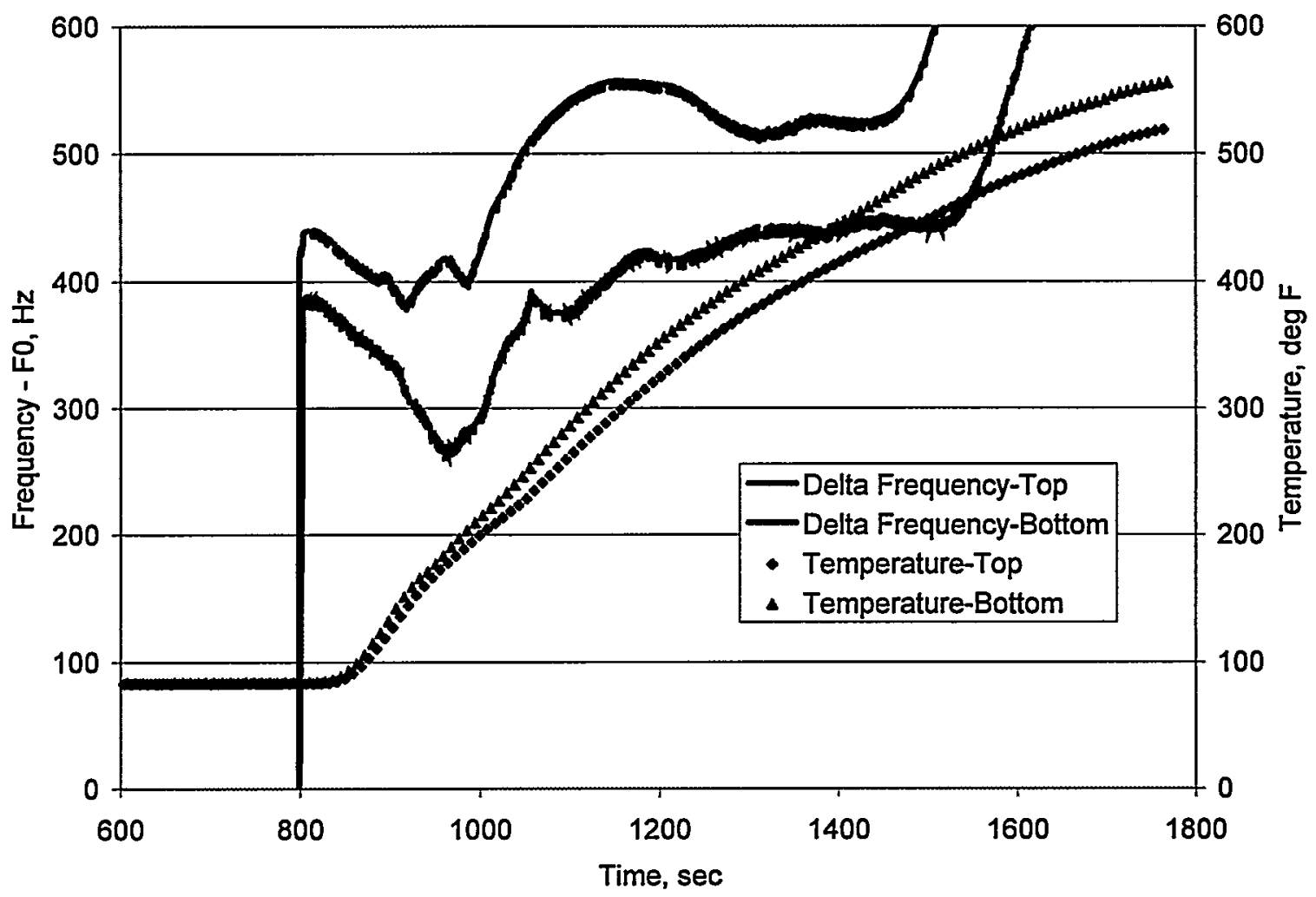

Figure A-40. Pour 082598, 1.00 inch, horizontal 
DOE/ID/13363-F

Part III

\title{
The Relationship between Casting Distortion, Mold Filling and Interfacial Heat Transfer in Sand Molds
}

DE-FC07-95ID13363

FINAL REPORT - PART III

\begin{abstract}
APPLICATION OF STATISTICAL METHODS FOR ANALYZING THE RELATIONSHIP BETWEEN CASTING DISTORTION, MOLD FILLING, AND INTERFACIAL HEAT TRANSFER IN SAND MOLDS
\end{abstract}

September 30, 1999

\author{
YAW A. OWUSU, $\mathrm{PhD}$ \\ Associate Professor \& Fulbright Fellow \\ FAMU-FSU College of Engineering \\ Florida A \& M University \\ Tallahassee, Florida
}

Contributing Graduate Students:

Tarsha Dargan

Casimiro Batista

Kevin Hayes 


\section{TABLE OF CONTENTS}

TABLE OF CONTENTS

LIST OF FIGURES

Page

LIST OF TABLES

LIST OF ABBREVIATIONS AND PARAMETER DEFINITIONS

EXECUTIVE SUMMARY

CHAPTER 1. INTRODUCTION

1.1 Introduction of Statistical Analysis of Point Cloud Data

from Coordinate Measuring Machine

1.1.1 Description of Problem

1.1.2 Benefits of Research

1.1.3 Project Description

1.1.4 Objective

ii

iv

$\mathrm{v}$

vi

vii

1

$$
1
$$

CHAPTER 2. LITERATURE SURVEY 7

2.1 Sand Molding Processes 7

2.1.1 Resin Bonded Sand Molding Process

2.1.2 Lost Foam Casting Process 9

2.2 Statistical Methods for Evaluation of Geometric and Dimensional Variations

2.2.1 Linear Dimensions 11

2.2.2 Surface Flatness Evaluation 11

2.2.3 Flatness Error using Non-linear Optimization technique 12

2.2.4 Evaluation of Parallel Surfaces 13

2.2.5 Calculation of Tapered Angle 13

2.3 Statistical Analysis of Dimensional Data 14

2.3.1 Analysis of Variance $\quad 15$

2.3.2 The F Test 15

2.3.3 Test on Means 17

2.3.3.1 Duncan's Multiple Range Test 17

2.3.3.2 Tukey's Least Significance Difference(TSD) 19

2.3.3.3 Newman Keul's Test 19

2.3.3.4 Scheffe's Test 20 
CHAPTER 3. DATA COLLECTION, EVALUATION AND ANALYSIS 22

3.1 Data Collection $\quad 26$

3.2 Data Analysis 26

3.2.1 Flatness Evaluation 29 .

3.2.2 Evaluation of Parallelism 29

3.2.3 Calculation of Tapered Angle 31

3.2.4 Calculation of Casting Dimensions 33

CHAPTER 4. METHOD IMPLEMENTATION AND ANALYSIS 36

4.1 Statistical Analysis $\quad 36$

CHAPTER 5. RESULTS 41

5.1 Data Analysis 41

5.2 Statistical Analysis Results $\quad 41$

5.2.1 Ranking of Significant Factors $\quad 50$

5.2.2 Analysis of Variation from Nominal 52

CHAPTER 6. CONCLUSIONS

6.1 Future Work 56

REFERENCES

APPENDIX A: Experimental Design of the Project 60 APPENDIX B : ANOVA and Newman Keuls Tables 71

APPENDIX C: Bullseye Plots $\quad 176$ 


\section{LIST OF FIGURES}

Figure

Page

Schematic of Orientation and Gating Systems $\quad 6$

Sand Mold and its Main Features $\quad \mathbf{8}$

Flatness Error Evaluation using Nonlinear Optimization Techniques 112

Parallelism Error between Two Planes 14

Parameters to Compute Tapered Angle $\quad 14$

Down Orientation-Gating Bottom 24

Orientation Side-Side Gating 25

Up Orientation-Top Gating 25

Four View Drawing of the Casting (Mailbox) 27

Mailbox Casting: Linear Distances and Point Cloud from CMM 28

Surface Plot of Flatness $\quad 42$

Box Plot of Left Flange Flatness of all 0.25 inch castings 43

Histogram of Left Casting Angle of Resin Bonded Castings 44

Scatter Plot of Parallelism of all 0.25 inch castings 45

"Bullseye" Plot 53 


\section{LIST OF TABLES}

Factors and Levels Analyzed for Effect on Parameter Variations 4 Symbolic Analysis of Variance Table $\quad 16$

Experimental Design of Project 23

Sample CMM Point Cloud Data from CMI 27

Design Nominal Values $\quad 28$

Flatness Worksheet $\quad 30$

Parallelism Calculation Worksheet $\quad 32$

Parallelism Index $\quad 33$

Casting Angle Calculation Worksheet 34

Height, Length, and Thickness Calculation Worksheet 35

Factorial Analysis of Variance of Left Flange Flatness of all 0.25 inch Castings $\quad 38$

Factorial Analysis of Variance of Height of Lost Foam Low

Expansion castings ( $0.25 \mathrm{inch}) \quad 39$

Factorial Analysis of Variance: Left Wall Flatness Resin Bonded

Castings ( 0.25 inch)

Factorial Analysis of Variance: Parallelism of Lost Foam

Silica Sand castings ( 0.25 inch)

Newman-Keuls Test of Means on Mold Type of the Left Flange

Flatness Data (all 0.25 inch)

Newman-Keuls Test of Means on Gating System of the Lost Foam

Low Expansion Parallelism Data (all 0.25 inch)

Significant Contributors to Casting Distortion (0.25 inch) 47

Significant Contributors to Casting Distortion $(0.50 \mathrm{inch}) \quad 48$

Significant Contributors to Casting Distortion (0.75 inch) 49

Significant Contributors to Casting Distortion (all) 51

Deviation from Design Nominal Values $\quad 54$

Deviation from Calculated Nominal Values $\quad 54$ 


\section{LIST OF NOMENCLATURE AND PARAMETER DEFINTIONS}

\section{List of Nomenclature}

CMM: $\quad$ Coordinate Measuring Machine

RBS:

LFLE:

LFSS:

ANOVA:

Nominal:
Resin Bonded Sand

Lost Foam Low Expansion Sand

Lost Foam Silica Sand

Analysis of Variance

Calculated Nominal Values obtained from CMM data.

Bullseye: Bullseye charts provide pictorial descriptions of the parameter variations from the calculated nominal values

G: $\quad$ Gating

$\mathrm{O}: \quad$ Orientation

T: Thickness

M: $\quad$ Mold Type/Process

\section{Parameter Definitions}

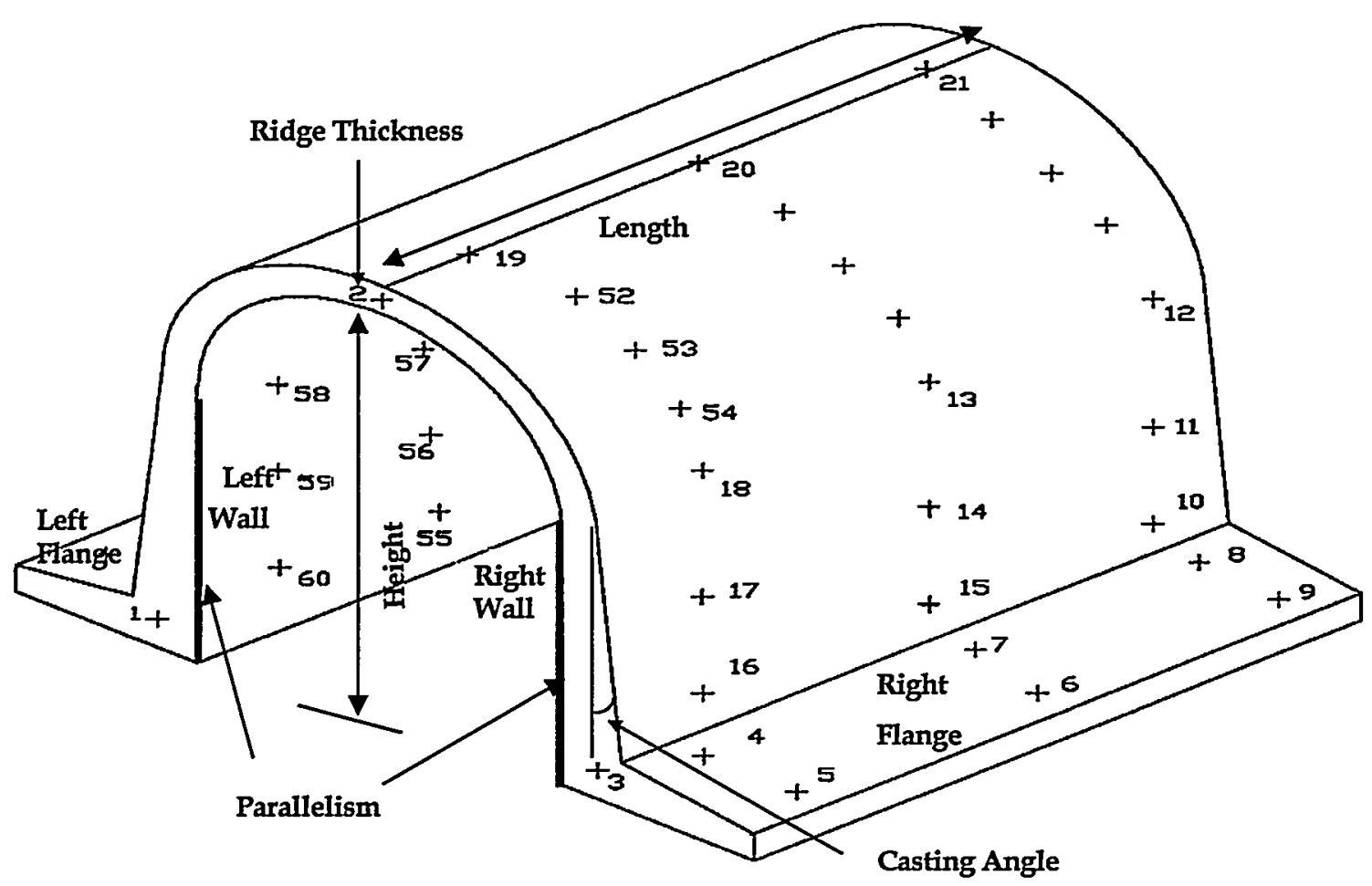




\section{EXECUTIVE SUMMARY}

This report presents a statistical method of evaluating geometric variations of casting products using point cloud data measured on a coordinate measuring machine (CMM to evaluate differences in dimensional and accuracy of test castings as affected by gating system, molding process, section thickness, and casting orientation. Feature parameters of flatness, parallelism, tapered angle, overall length, and height of test casting were obtained from CMM point cloud data and these were utilized to identify the relative importance of the factors that contribute to the dimensional inaccuracies. The scope of this study included three casting thicknesses: $1 / 4$ in., $1 / 2$ in., and $3 / 4$ in. Three sand molding processes for the research included: resin-bonded sand, lost foam silica sand, and lost foam low expansion sand.

US Department of Energy (DOE) and through the Cast Metals Coalition, the American Foundrymen's Society supported this research work. The research was carried out jointly by The University of Alabama, Florida A \& M University in Tallahassee, General Motors Company, Mercury Marine Company, Willard Industries, and CMI Technical Center.

The summary of the research results is as follows:

1. CMM point cloud data have been statistically analyzed successfully to evaluate casting distortion.

2. This research has established that casting distortion is influenced by the following parameters: casting thickness, orientation in the mold, gating system, and type of molding process.

3. From the analyses, it was found that resin bonded sand process, top gating, and orientation down contributed most to the casting distortion. 


\subsection{INTRODUCTION}

The focus of this research task was to evaluate the dimensional variations that exist as gating system, molding process, casting thickness, and orientation of the casting are varied.

The objective of a manufacturing system is to produce products of specific shapes, sizes, finishes, and complexity. The quality of the manufactured part is the measure of the extent to which a part conforms to the needs or desires of the customer or user. Quality assurance is the mechanism that is responsible for maintaining consistent product quality. Under the umbrella of quality assurance is quality control. Statistical quality control techniques have allowed castings to greatly improve over the past twenty years, yet controlling casting dimensions continues to be a challenge.

\subsection{Description of Problem}

Casting distortion is thought to be caused by non-uniform cooling in various sections of complex geometric surfaces. This phenomenon has been successfully modeled; however, role of non-uniform heat transfer on casting distortion has not been successfully modeled.

It is assumed that the parts of the casting that solidify first will become stronger than those that solidify later. The parts of the casting that solidify first will be the ones with the coldest metal. They may also be those sections of the casting that come to rest first in the mold cavity. All of these factors are governed by the way the casting fills. That, in turn, is governed by the gating system and the casting orientation. Casting thickness may also affect it. The objectives of this work were:

1) To present a statistical approach for evaluating the effect of specific factors (gating system, molding process, casting thickness, and orientation within the mold) on casting inaccuracy.

2) To possibly relate these factors to the heat transfer coefficient at the mold-metal interface.

\subsection{Project Description}

Florida A \& M University applied statistical methods to analyze the relationship between casting distortion, mold filling, and interfacial heat transfer in sand molds. This analysis involved preprocessing the point cloud data, calculating the dimensional and form parameters such as: length, thickness, height, flatness, tapered angle, and parallelism. Statistical analysis was performed on these parameters to determine the effect of such factors as gating $(\mathrm{G})$, orientation $(0)$, thickness $(T)$, and molding process (type) $(M)$ on the parameter variations. There were three levels for each factor (see Table 1.1). They included: Gating: top, bottom, and side; Orientation: up, down, and side (as shown in Figure 1.2); Thickness: $1 / 4$ in., $1 / 2$ in., $3 / 4$ in.; Molding process: resin bonded sand, lost foam silica sand, and lost foam low expansion sand. The pictorial presentations of the gating and orientation systems are shown in Figure 1.2. 
Table 1.1 Factors and Levels Analyzed for Effect on Parameter Variations

\begin{tabular}{|c|c|c|}
\hline & Variable & Level of \\
\hline \multirow[t]{3}{*}{1} & Rốtding Method (M): & $\begin{array}{l}\text { Racticos } \\
\text { Resin bonded sand molding }\end{array}$ \\
\hline & & mestholam molding with silica bonded sand \\
\hline & & mith low expansion (carbon)sand molding \\
\hline \multirow[t]{3}{*}{2} & Gating System & motphgded system \\
\hline & $(G):$ & Bottom-gated \\
\hline & & sratemated system \\
\hline 3 & Casting Orientation $(\mathrm{O})$ : & Up, Down, \\
\hline 4 & Casting Thickness (T): & S4, dezway 3/4 inch. \\
\hline
\end{tabular}

\subsection{Objective}

1. To use point cloud data generated from a Coordinate Measuring Machine (CMM) to calculate dimensional and form parameters.

2. To use statistical methods to evaluate the effect of gating system, molding process, casting thickness, and orientation on casting distortion. 

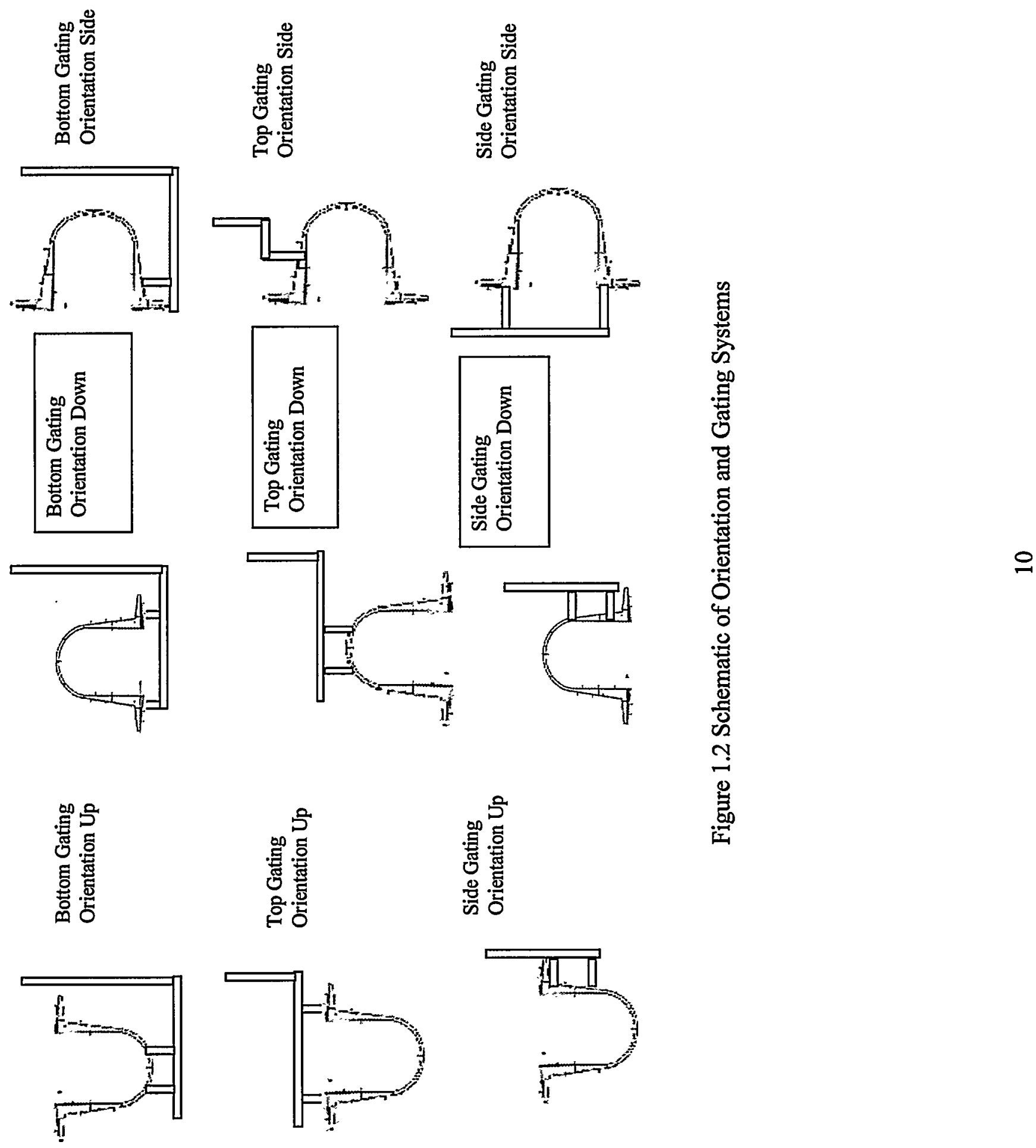


\subsection{TECHNICAL BACKGROUND}

\subsection{Statistical Methods for Evaluation of Geometric and Dimensional Variations}

There is a great deal of research in the area of tolerance evaluation or metrology of manufactured parts. Minimum zone is a popular method of determining tolerance deviations. The methodology of minimum zone is to calculate the maximum dimensional error by locating the minimum deviation among all the sampled coordinates using various optimization schemes. This method requires that the entire deviation space be examined. However, the CMM assigns point clouds to a sample of the data. Therefore, errors often occur. For example, all sampled deviations may be found to be within tolerance, but since all the data have not been examined, some non-sampled deviations may in fact be out of bounds. Therefore, minimum zone is not an effective method for tolerance evaluation of CMM sampled data [Menq et. al, 1990].

Sobh and his colleagues attempted a probabilistic approach [Sobh et al, 1994]. In their research they constructed geometric entities using uncertainty models for the objects that contained noisy measurements and proceeded to do reasoning on the uncertain geometries. Menq and his research team's method assumed normally distributed points in which the sampling space is related to the process capability [Menq et al., 1990]. This method proves that the sampling intensity (number of sampled points) does not depend on the tolerance or manufacturing alone, but depends on a combination of both factors. Thus, the number of sampled points is reduced as the process capability increases.

Kendall and Moran studied the theories of stochastic geometry [Kendall and Moran 1963]. They examined a method of choosing distributions on geometric elements that provide a consistent interpretation of physical geometric elements.

\subsubsection{Linear Dimensions}

The Euclidean distance is the linear distance between two coordinate points $\left(\mathrm{x}_{1}, \mathrm{y}_{1}, \mathrm{z}_{1}\right)$ and

$\left(\mathrm{x}_{2}, \mathrm{y}_{2}, \mathrm{z}_{2}\right)$. It is calculated using equation 2.1.

$$
\operatorname{DIST}=\operatorname{SQRT}\left[\left(\mathrm{x}_{2}-\mathrm{x}_{1}\right)^{2}+\left(\mathrm{y}_{2}-\mathrm{y}_{1}\right)^{2}+\left(\mathrm{z}_{2}-\mathrm{z}_{1}\right)^{2}\right]
$$

In this expression, DIST is the desired linear distance, SQRT is the square root, and $\mathrm{x}, \mathrm{y}$, $\mathrm{z}$ are the coordinates for points 1 and 2 .

\subsubsection{Surface Flatness Evaluation}

Since sampled inspection does not measure all data points, it is possible to infer that a surface is flat when it actually is not. A result of all the measured sampled points being within the tolerance limit that does not necessarily indicate a flat surface. If any point is outside the tolerance, the surface is not considered flat since it is assumed that the CMM machine has negligible measurement error. Therefore it is necessary to calculate the variance of the sample points to see if the variation of the surface is acceptable. 


\subsubsection{Flatness Error Evaluation using Non-linear Optimization Technique}

Flatness is defined as "a condition of a surface having all elements in one plane" according to ANSI Y14.5M standards. This method of calculating flatness error of the casting is similar to a mathematical model developed by Cheraghi et al [Cheraghi et al,1996]. This method uses a non-linear optimization approach to calculate the exact values of flatness errors as defined by the ANSI Y14.5M standards on geometric dimensioning and tolerance. The measurements are taken in $x, y, z$ coordinates on the walls' surface with the $z$-axis representing the direction in which the flatness will be measured. The $z$ coordinates measure the variation on the surface of the casting as shown in Figure 2.1.

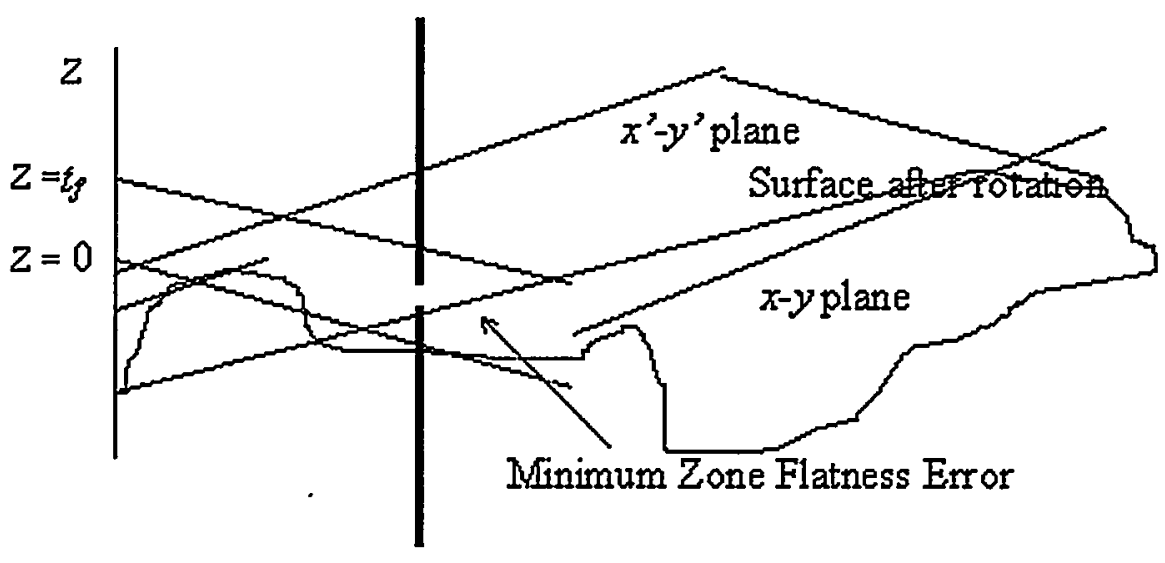

Figure 2.1 Flatness Error Evaluation using Non-linear Optimization Technique

To obtain the exact value of the surface flatness, two planes parallel to the $x-y$ plane (representing the ideal surface of the walls) a distance of $t_{f}$ apart must be found to define the flatness tolerance zone.

The nonlinear optimization problem is expressed as:

$$
\begin{aligned}
& \text { Minimize } t_{f}=-J_{\min }+J_{\max } \\
& \text { Subject to: } \\
& J_{i=t_{f}} \\
& J_{i}=0 \quad i=1,2, \ldots, n
\end{aligned}
$$

where, $J_{i}=-x_{i} \cos \theta \sin \gamma+y_{i} \sin \theta+z_{i} \cos \theta \cos \gamma+z$ and $t_{f}$ is the distance representing the flatness error.

This method provides exact flatness error values and has a short computational time.

\subsubsection{Evaluation of Parallel Surfaces}


To evaluate the parallelism error of surfaces, it is necessary to find the distance between the points on opposite locations of the planes that are being evaluated. From these distances, the difference between the shortest distance and the longest distance gives the parallelism error. Mathematically, it is expressed as

$$
\varepsilon_{\text {parallelism }}=\max \left(\text { dist }_{A B}\right)-\min \left(\text { dist }_{A B}\right)
$$

where, $\varepsilon_{\text {parallelism }}$ is the difference between the maximum distance between planes $A$ and $B$. The distance is computed using equation 2.1. Figure 2.2 illustrates the parallelism error between two planes.

\subsubsection{Calculation of the Tapered Angle}

$$
\text { The Euclidean distance, equation 2.1, and } \tan \theta=\frac{\text { opposite side }}{\text { adjacent side }}
$$

was used to calculate the tapered angle; yielding

$$
\cos \theta=\frac{\sqrt{\left(x_{1}-x_{0}\right)^{2}+\left(y_{1}-y_{0}\right)^{2}+\left(z_{1}-z_{0}\right)^{2}}}{\sqrt{\left(x_{3}-x_{2}\right)^{2}+\left(y_{3}-y_{2}\right)^{2}+\left(z_{3}-z_{2}\right)^{2}}}
$$

Figure 2.3 shows the parameters used in this equation. These angles are based on the assumption that the walls of the casting are fairly straight.

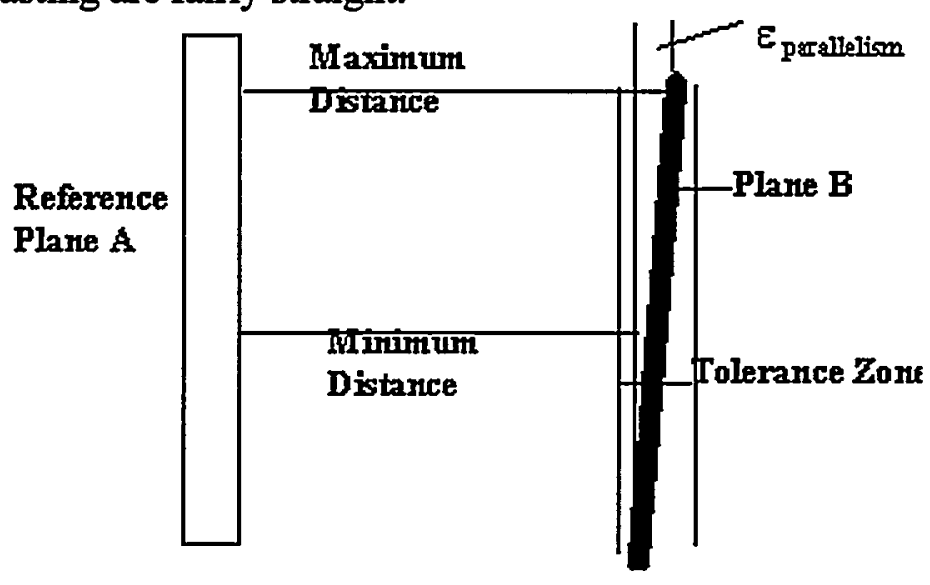

Figure 2.2 Parallelism Error between Two Planes 


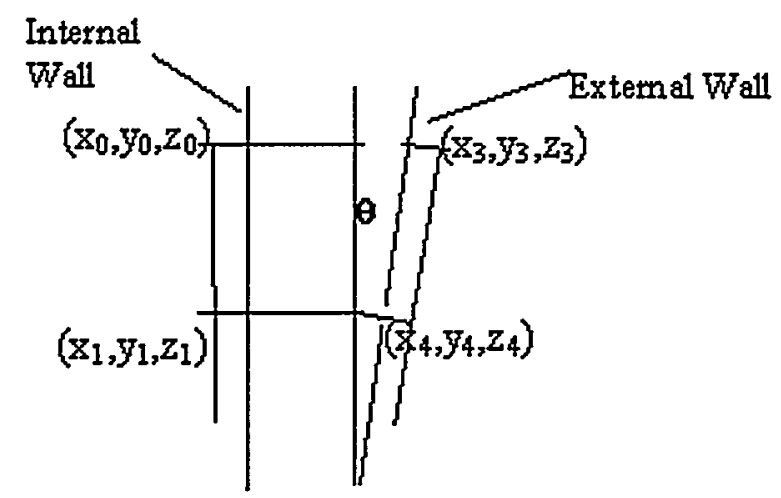

Figure 2.3 Parameters to Compute Tapered Angle

\subsection{Statistical Analysis of Dimensional Data}

The statistical analysis of the dimensional data includes the factorial analysis of variance and the tests on means.

\subsubsection{Analysis of Variance}

Analysis of variance (ANOVA) procedures separate the variation observable on a response into two basic components: variation due to assignable causes and random or chance variation. Assignable causes refer to known or suspected sources of variation that could be corrected during the conduct of the experiment. Random or chance variation includes the effects of all other sources that could not be controlled or measured during the experiment except by statistical modeling. The generic statistical model for the sand casting dimensional data is

$$
y_{i j k l r}=\mu_{i j k l}+e_{i j k l .}
$$

In this equation, $y_{i j k l r}$ is the measured dimensions of the sand casting due to the different factor level combinations. The number of repeat tests are represented by $r=1,2,3$. The levels for the molding method are represented by $i=1,2,3$. Similarly, $j=1,2,3 ; k=1,2,3 ; l=1,2,3$, represent the levels for the gating system, the casting orientation and thickness, respectively. In this model $\mu_{i j k l}$ is the effect of the assignable causes and $e_{i j k l}$ is the random error of the experiment. The symbolic analysis of variance for the four factors is the shown in Table 2.1.

\subsubsection{The F -Test}

In order to find out if the differences on casting dimensions are due to assignable causes or random error, the F-test was implemented. The F-test distribution function tests the hypothesis that a particular main effect or interaction is zero. The corresponding F-ratio should be around one, since both the numerator and the denominator of the F-statistic are estimating the same quantity, which is the error variance. If the null hypothesis is false, the numerator mean square will tend to be larger that the error mean square. Thus, large F-ratios lead to rejection of the hypothesis of no factor effects. The F-test applies under the assumptions that both sampled populations are normally distributed, and the samples are random and independent. 
Table 2.1. Symbolic Analysis of Variance Table

\begin{tabular}{|c|c|c|c|c|}
\hline $\begin{array}{c}\text { Source of } \\
\text { Variation }\end{array}$ & $\begin{array}{c}\text { Sum of } \\
\text { Squares }\end{array}$ & $\begin{array}{c}\text { Degrees of } \\
\text { Freedom }\end{array}$ & $\begin{array}{c}\text { Mean } \\
\text { Square }\end{array}$ & F-Value \\
\hline $\mathrm{A}$ & $\mathrm{SS}_{\mathrm{A}}$ & $\mathrm{a}-1$ & $\mathrm{SS}_{\mathrm{A}} / \mathrm{df}(\mathrm{A})$ & $\mathrm{MS}_{\mathrm{A}} / \mathrm{MS}_{\mathrm{E}}$ \\
\hline $\mathrm{B}$ & $\mathrm{SS}_{\mathrm{B}}$ & $\mathrm{b}-1$ & $\mathrm{SS}_{\mathrm{B}} / \mathrm{df}(\mathrm{B})$ & $\mathrm{MS}_{\mathrm{B}} / \mathrm{MSE}$ \\
\hline $\mathrm{C}$ & $\mathrm{SS}_{\mathrm{C}}$ & $\mathrm{c}-1$ & $\mathrm{SS}_{\mathrm{C}} / \mathrm{df}(\mathrm{C})$ & $\mathrm{MS}_{\mathrm{C}} / \mathrm{MS}_{\mathrm{E}}$ \\
\hline $\mathrm{D}$ & $\mathrm{SS}_{\mathrm{D}}$ & $\mathrm{d}-1$ & $\mathrm{SS}_{\mathrm{D}} / \mathrm{df}(\mathrm{D})$ & $\mathrm{MS}_{\mathrm{D}} / \mathrm{MS}_{\mathrm{E}}$ \\
\hline $\mathrm{AB}$ & $\mathrm{SS}_{\mathrm{AB}}$ & $(\mathrm{a}-1)(\mathrm{b}-1)$ & $\mathrm{SS}_{\mathrm{AB}} / \mathrm{df}(\mathrm{AB})$ & $\mathrm{MS}_{\mathrm{AB}} / \mathrm{MS}_{\mathrm{E}}$ \\
\hline $\mathrm{AC}$ & $\mathrm{SS}_{\mathrm{AC}}$ & $(\mathrm{a}-1)(\mathrm{c}-1)$ & $\mathrm{SS}_{\mathrm{Ad}} / \mathrm{df}(\mathrm{AC})$ & $\mathrm{MS}_{\mathrm{AC}} / \mathrm{MS}_{\mathrm{E}}$ \\
\hline $\mathrm{AD}$ & $\mathrm{SS}_{\mathrm{AD}}$ & $(\mathrm{a}-1)(\mathrm{d}-1)$ & $\mathrm{SS}_{\mathrm{AD}} / \mathrm{df}(\mathrm{AD})$ & $\mathrm{MS}_{\mathrm{AD}} / \mathrm{MS}_{\mathrm{E}}$ \\
\hline $\mathrm{BC}$ & $\mathrm{SS}_{\mathrm{BC}}$ & $(\mathrm{b}-1)(\mathrm{c}-1)$ & $\mathrm{SS}_{\mathrm{Bd}} / \mathrm{df}(\mathrm{BC})$ & $\mathrm{MS}_{\mathrm{BC}} / \mathrm{MS}_{\mathrm{E}}$ \\
\hline $\mathrm{BD}$ & $\mathrm{SS}_{\mathrm{BD}}$ & $(\mathrm{b}-1)(\mathrm{d}-1)$ & $\mathrm{SS}_{\mathrm{BD}} / \mathrm{df}(\mathrm{BD})$ & $\mathrm{MS}_{\mathrm{BD}} / \mathrm{MS}_{\mathrm{E}}$ \\
\hline $\mathrm{CD}$ & $\mathrm{SS}_{\mathrm{CD}}$ & $(\mathrm{c}-1)(\mathrm{d}-1)$ & $\mathrm{SS}_{\mathrm{CD}} / \mathrm{df}(\mathrm{CD})$ & $\mathrm{MS}_{\mathrm{CD}} / \mathrm{MS}_{\mathrm{E}}$ \\
\hline $\mathrm{ABC}$ & $\mathrm{SS}_{\mathrm{ABC}}$ & $(\mathrm{a}-1)(\mathrm{b}-1)(\mathrm{c}-1)$ & $\mathrm{SS}_{\mathrm{ABd}} / \mathrm{df}(\mathrm{ABC})$ & $\mathrm{MS}_{\mathrm{ABC} / \mathrm{MS}}$ \\
\hline $\mathrm{ABD}$ & $\mathrm{SS}_{\mathrm{ABD}}$ & $(\mathrm{a}-1)(\mathrm{b}-1)(\mathrm{d}-1)$ & $\mathrm{SS}_{\mathrm{ABD}} / \mathrm{df}(\mathrm{ABD})$ & $\mathrm{MS}_{\mathrm{ABD}} / \mathrm{MS}_{\mathrm{E}}$ \\
\hline $\mathrm{ACD}$ & $\mathrm{SS}_{\mathrm{ACD}}$ & $(\mathrm{a}-1)(\mathrm{c}-1)(\mathrm{d}-1)$ & $\mathrm{SS}_{\mathrm{ACD}} / \mathrm{df}(\mathrm{ACD})$ & $\mathrm{MS}_{\mathrm{ACD}} / \mathrm{MS}_{\mathrm{E}}$ \\
\hline $\mathrm{BCD}$ & $\mathrm{SS}_{\mathrm{BCD}}$ & $(\mathrm{b}-1)(\mathrm{c}-1)(\mathrm{d}-1)$ & $\mathrm{SS}_{\mathrm{BD}} / \mathrm{df}(\mathrm{BCD})$ & $\mathrm{MS}_{\mathrm{BDC}} / \mathrm{MS}_{\mathrm{E}}$ \\
\hline $\mathrm{ABCD}$ & $\mathrm{SS}_{\mathrm{ABCD}}$ & $(\mathrm{a}-1)(\mathrm{b}-1)(\mathrm{c}-1)$ & $\mathrm{SS}_{\mathrm{ABCD}} / \mathrm{df}(\mathrm{ABC})$ & $\mathrm{MS}_{\mathrm{ABCD}} / \mathrm{MS}_{\mathrm{E}}$ \\
\hline Error & $\mathrm{abcd}(\mathrm{m}-1)$ & $(\mathrm{d}-1)$ & & \\
\hline Total & $\mathrm{abcdm}-1$ & $\mathrm{SS} \mathrm{E}$ & $\mathrm{SS}_{\mathrm{E}} / \mathrm{df}(\mathrm{Error})$ & \\
\hline
\end{tabular}

Mathematically the F-ratio is expressed

$$
F=\left(s_{1}^{2} / \sigma_{2}^{2}\right) /\left(s_{2}{ }^{2} / \sigma_{1}^{2}\right) .
$$

In this equation, $s^{2}$ is the sample variance and $\sigma^{2}$ is the population variance. This equation is reduced to the ratio of the sample variances because it is assumed that the population variances are equal. Therefore, their ratio is unity.

The hypothesis tests procedure as follows:

One-tailed test

$$
\begin{aligned}
& H_{o}: \sigma_{2}^{2}=\sigma_{1}^{2} \\
& H_{a}: \sigma_{1}^{2}<\sigma_{2}^{2}
\end{aligned}
$$

Test statistic:

$F=s_{1}^{2} / s_{2}^{2}$

Rejection region:

$$
\mathrm{F}>\mathrm{F}_{\alpha}
$$

Two-tailed test

$$
\begin{aligned}
& H_{o}: \sigma_{2}^{2}=\sigma_{1}^{2} \\
& H_{a}: \sigma_{1}^{2} \neq \sigma_{2}^{2}
\end{aligned}
$$

Test statistic:

$$
F=s_{1}{ }^{2} / s_{2}{ }^{2} \text { when } s_{1}{ }^{2}>s_{2}{ }^{2}
$$$$
F=s_{2}^{2} / s_{1}^{2} \text { when } s_{2}^{2}>s_{1}^{2}
$$

Rejection region:

$$
\begin{array}{ll}
F>F_{\alpha / 2} & \text { when } s_{1}{ }^{2}>s_{2} \\
\text { or } F>F_{\alpha / 2} & \text { when } s_{1}{ }^{2}>s_{2}
\end{array}
$$

where $F_{\alpha}$ and $F_{\alpha / 2}$ are based on $v_{1}=$ numerator degrees of freedom and $v_{2}=$ denominator degrees of freedom; $v_{1}$ and $v_{2}$ are the degrees of freedom for the numerator and denominator sample variances, respectively.

\subsubsection{Tests on Means}


If the F-test results in the rejection of the null hypothesis, the treatment means are equal. Yet, it is required to know which of the means differ and by how much. Confidence intervals can be placed on the difference between the various pairs of treatment means in an experiment.

For $\mathrm{p}$ treatment means, then

$$
c=p(p-1) / 2 .
$$

where $c$ pairs of means that can be compared. If it is necessary to have $100(1-\alpha)$ percent confidence that each of the $c$ confidence intervals contains the true difference, multiple intervals will have to be formed using a smaller value of $\alpha$ than would a single interval. There are a number of procedures available for making multiple comparisons. Among the procedures are Duncan's multiple range test, Tukey's procedure, Newman-Keuls test and Scheffe's test. All of these test of means yield similar results. Yet, due to preference, the Newman-Keul Range Test of Means was used in this research for the analysis.

\subsubsection{Newman-Keuls Range Test}

Application of the Newman-Keuls range test requires that the averages be ordered from smallest to largest. Each based on sample size $\mathrm{n}$. They are considered significantly different if

where

$$
\left|\overline{y_{i}}-\bar{y}_{j}\right|>N_{p} \text {, }
$$

$$
\mathrm{N}_{\mathrm{p}}=\mathrm{q}\left(\alpha_{\mathrm{p}} ; \mathrm{p}, v\right)\left(\mathrm{MS}_{\mathrm{E}} / \mathrm{n}\right)^{1 / 2} \text {, }
$$

and $q\left(\alpha_{p} ; p, v\right)$ is the studentized-range critical point based on comparing the largest and the smallest of $\mathrm{p}$ averages, $\mathrm{MS}_{\mathrm{E}}$ is the mean squared error based on $v$ degrees of freedom, and the experimentwise significance level is $\alpha_{p}$ and $n$ is the number of observations in the treatment being compared. The experimentwise significance level is related to a comparisonwise level $\alpha$ through equation 2.23

$$
\alpha_{p}=1-(1-\alpha)^{p-1} .
$$

In this procedure, the two most extreme averages are compared first. The difference between the largest and the smallest of $\mathrm{p}=\mathrm{k}$ factor-level or interaction averages is compared using $\mathrm{N}_{\mathrm{k}}$ in equation 2.22 with the experimentwise significance level of $\alpha_{\mathrm{k}}$. If these averages are not found significantly different with $\mathrm{k}=\mathrm{p}$, testing stops and all the averages are declared not significantly different at the $100 \alpha_{\mathrm{k}}$ percent significance level. This is equivalent to non-rejection of $\mathrm{H}_{0}: \mu_{1}=\mu_{2}=\ldots=\mu_{\mathrm{k}}$. If the two extreme averages are significantly different, testing continues.

The next step is to compare the largest average with the second smallest and the smallest average with the second largest, each test using equations 2.22 and 2.23 with $\mathrm{p}=\mathrm{k}-1$. If neither of these tests are statistically significant, testing ceases and only the two extreme averages are judged significantly different. If one or both of the tests are statistically significant, testing continues with the group(s) of averages for which the two extremes have been declared significantly different. Testing continues in this fashion until no further significant differences are obtained. 


\subsection{DATA COLLECTION, EVALUATION AND ANALYSIS}

In this project, the effect of gating, thickness, orientation and mold process on form and dimensional errors is analyzed. The four variable factors involved in the investigation are shown in Table 3.1. The four variable factors have three levels each and each level has three replications. The letters $\mathrm{M}, \mathrm{G}, \mathrm{O}$, and $\mathrm{T}$ as defined above represent the variable factors. The subscripts 1,2 , and 3 represent levels of the factors and the X's represent three replications at each level. The design of experiment tables for the calculated parameters are located in Appendix A.

The statistical analysis used in this investigation includes Analysis of Variance (ANOVA) for full factorial randomized experiment. Randomized complete block design technique was used for controlling experimental variability due to parallelism. Newman-Keuls Test of Means was performed to obtain the effect of differences of means for the sample data.

The data received for the resin-bonded castings included three orientations: opening up, opening down and opening laterally. The opening up and opening down castings had two gating systems, top and bottom. The opening laterally castings used four gating systems: top, bottom, and two side gating systems, one filling through the flanges and the other side gating filling through the top ridge.

All the orientation and gating combinations were cast at three different thicknesses. There were three replicates made of each casting combination, but not all proved viable. There were no side-gated data received from the resin-bonded sand castings (rbs). The data received for the lost foam with low expansion sand molding method and lost foam with silica sand molding (lfle and lfss) included all three orientations, gating systems (top, bottom, and side), and three levels of thickness $(1 / 4,1 / 2,3 / 4$ inch). There were three replicates made of each combination.

Figure 3.1 a-f shows pictures of the orientation and gating systems examined in this research work. The ANOVA was performed on the three data sets. In addition to the analysis of variance, Newman-Keuls statistical tests of means were performed where statistical significance was observed. Finally, a group of charts including scatter plots, histograms, normal probability diagrams and box plots were used graphically to confirm the analytical results from ANOVA and Test of Means.

Scatter plots made it possible to visualize the trend of the data. Similarly, the histograms revealed the frequency distribution of the data. 


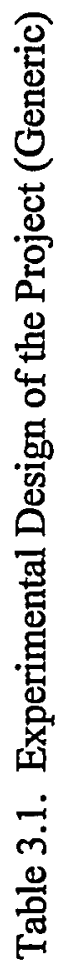

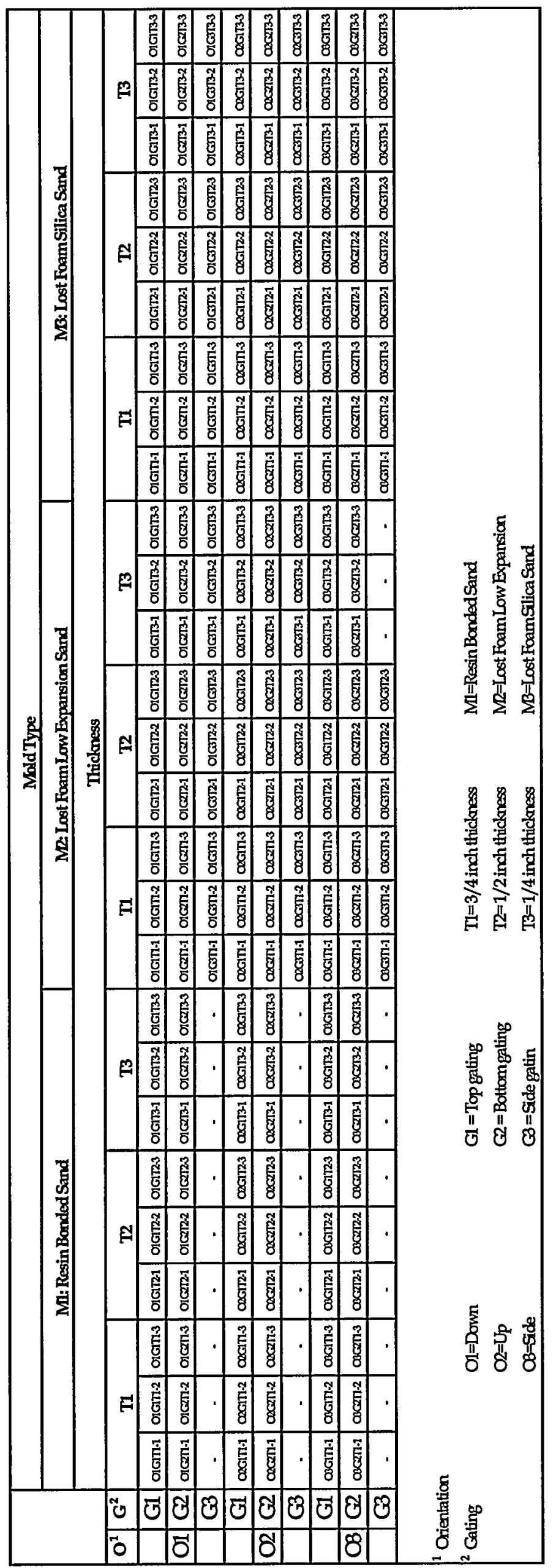

a 

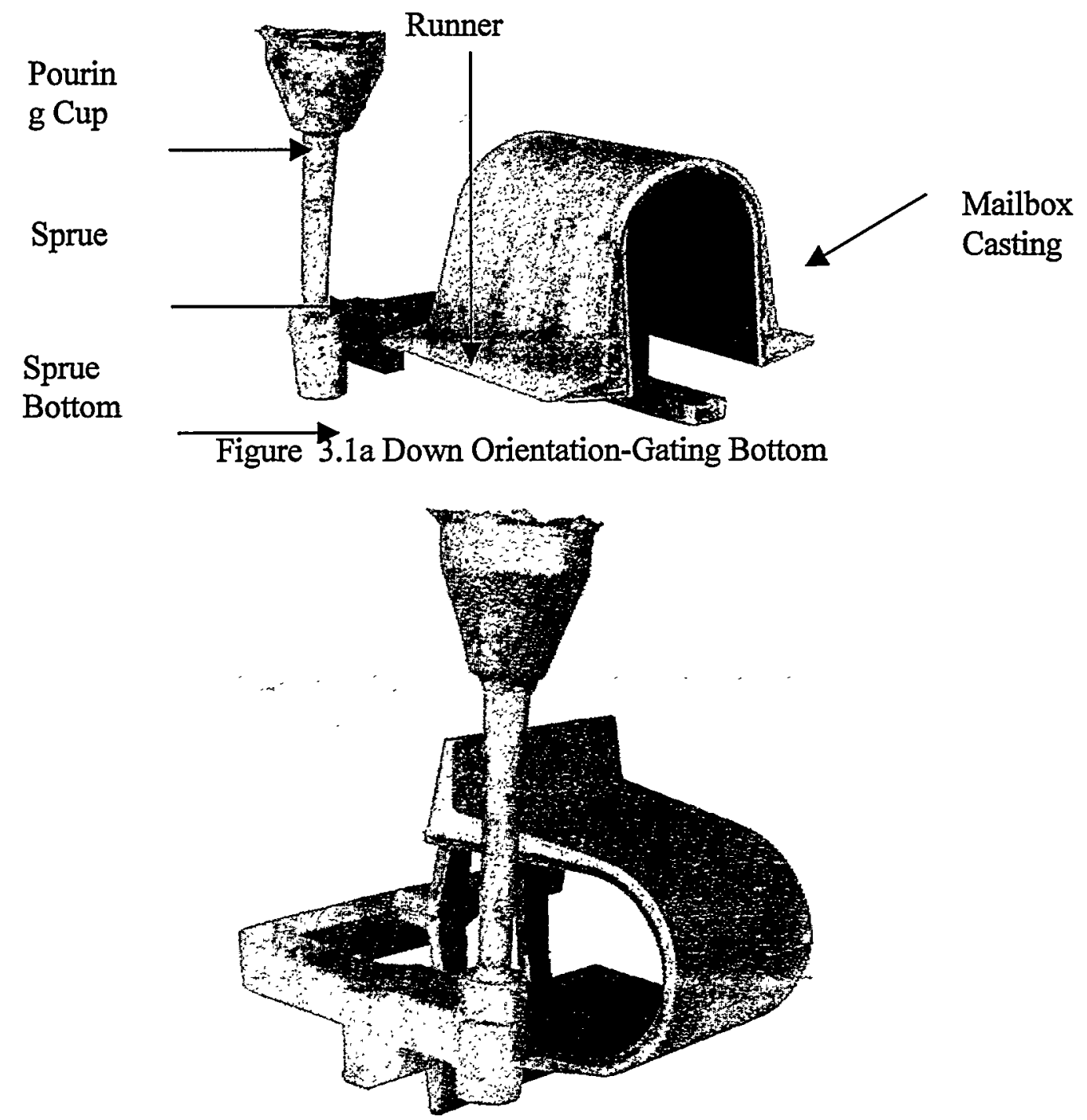

Figure 3.1b Orientation Side-Side Gating 


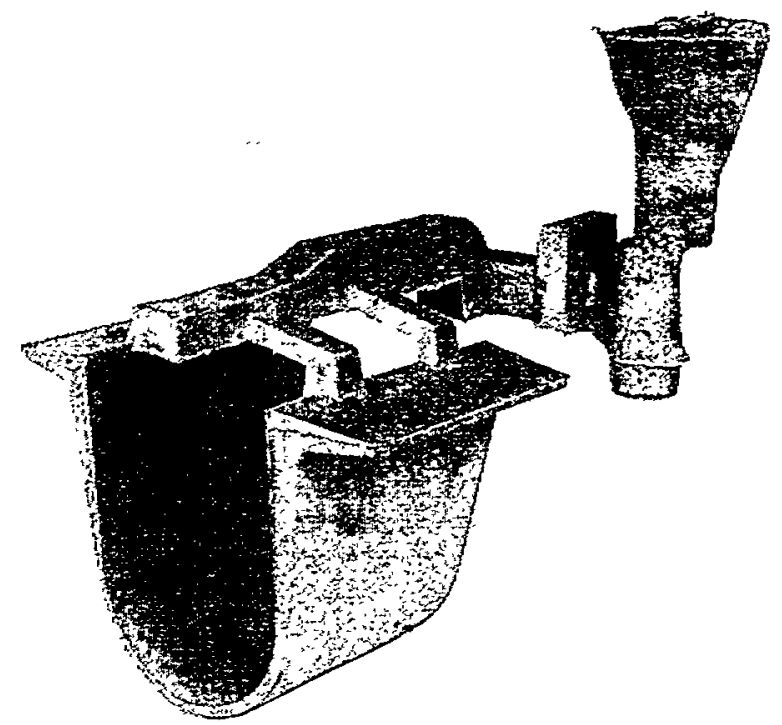

Figure 3.1c Up Orientation-Top Gating

The normal probability diagrams showed whether the data normally distributed. The box plot was used to view the range of the data points and its distribution around the mean. In order to study the variation of the distortion on sand castings, casting quality characteristics were analyzed. These characteristics include flatness, thickness, parallelism, casting angle, length, and height. Figure 3.2 is a four-view drawing of the casting.

\subsection{Data Collection: Preprocessing of Data for Statistical Analysis}

CMI Technical Center (CMI) used a Coordinate Measuring Machine to measure the point cloud data. Table 3.2 shows a sample of the raw point cloud data sent from CMI. In order to get the data into a functional format it was necessary to preprocess the data. below:

The steps used in preprocessing the data before conducting statistical analysis are as listed

1. The point cloud data were received in comma delimited form in Microsoft Excel files.

2. The files were edited so that only the point cloud coordinates were in the files. These coordinates were arranged in a long row with each set of $x, y, z$ coordinates after the other $\left(\mathrm{x}_{1}, \mathrm{y}_{1}, \mathrm{z}_{1}, \mathrm{x}_{2}, \mathrm{y}_{2}, \mathrm{z}_{2, \ldots}, \ldots\right.$.

3. The files were opened in a text editor and separated into the ordered triple sets so that when viewed in Excel, there were three columns of data corresponding to the coordinate $\operatorname{axes}(x, y, z)$. There were 180 rows and 3 columns.

4. When opened in Excel, the data were separated into 3 sections with 60 points in each. Each section contained the points for each replicate $(1,2,3)$.

5. The data were broken down into the individual replicates on separate worksheets within each workbook.

6. Each set of point cloud data for the replicates were divided up into the respective geometrical entity represented (e.g. edge points, right flange, inner left wall, etc.) 
7. Each file (workbook) is representative of one casting orientation, gating, thickness, and sand type.

\subsection{Data Analysis}

Data Analysis is the evaluation of the data variations, associated with the calculated parameters, such as: flatness, casting tapered angle, parallelism, and linear distances (length, thickness, and height) of the castings. Figure 3.3 indicates pictorially the linear distances that were calculated for the project. The hash marks indicate where the Coordinate Measuring Machine (CMM) measured surface coordinates for the point cloud data.

\section{Table 3.2 Sample CMM Point Cloud Data from CMI}

\begin{tabular}{|c|c|c|c|c|c|c|c|c|}
\hline $\begin{array}{l}\text { Part Program } \\
\text { Inspector } \\
\text { AFSWS } \\
\text { MLBX }\end{array}$ & $\begin{array}{l}\text { mlbx75 } \\
\text { JS }\end{array}$ & & & & & & & \\
\hline Nominal & -0.46267 & 2.46322 & -0.35968 & 0 & 2.15707 & 8.41401 & -0.76627 & 0 \\
\hline \#NAME? & 0 & 0 & 0 & 0.5 & 0 & 0 & 0 & 0.5 \\
\hline \#NAME? & 0 & 0 & 0 & 0.5 & 0 & 0 & 0 & 0.5 \\
\hline Seq\# Datatype & IX & IY & $1 \mathrm{Z}$ & 1DS & $2 X$ & $2 Y$ & $2 Z$ & 2DS \\
\hline $6 / 10 / 9720: 08$ & -0.46508 & 2.465562 & -0.34047 & 0.019518 & 2.15495 & 8.416134 & -0.74892 & 0.017639 \\
\hline $6 / 10 / 9720: 35$ & -0.46586 & 2.46634 & -0.33414 & 0.025951 & 2.147165 & 8.423862 & -0.686 & 0.081507 \\
\hline $6 / 10 / 9720: 54$ & -0.46648 & 2.466955 & -0.32912 & 0.031037 & 2.151773 & 8.419288 & -0.72324 & 0.043705 \\
\hline
\end{tabular}

Table 3.3 shows the design nominal values as provided in conjunction with the project.

Table 3.3 Design Nominal Values

\begin{tabular}{|l|c|}
\hline Parameters & Design Nominal Values \\
\hline Length(inch) & $6^{+1}$ \\
\hline Height (inch) & $8^{+1}$ \\
\hline Parallelism (inch) & 0 \\
\hline Ridge Thickness (inch) & $1 / 4,1 / 2,3 / 4$ \\
\hline Flatness (inch) & 0 \\
\hline Casting Angle (degrees) & $8^{+2}$ \\
\hline
\end{tabular}




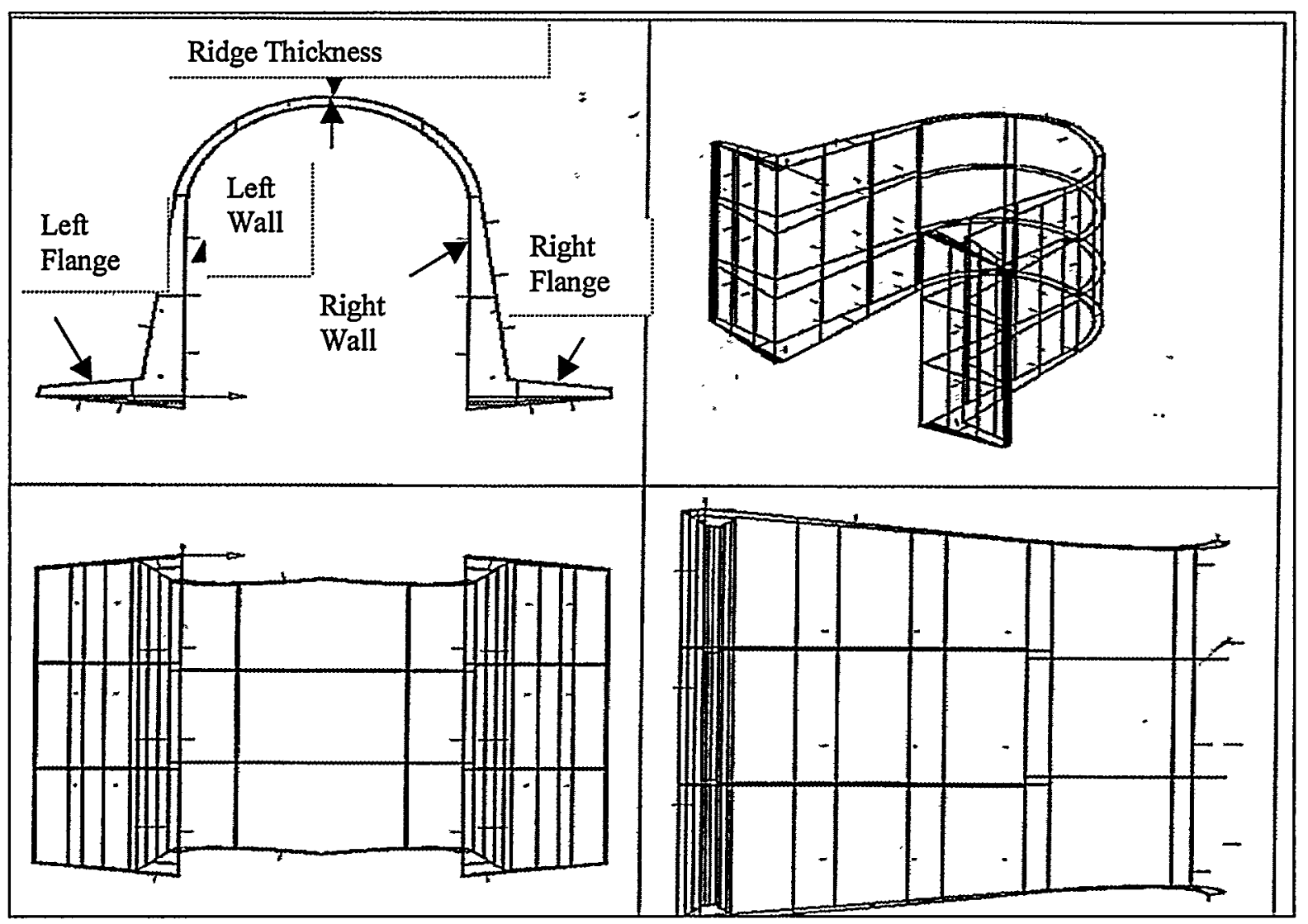

Figure 3.2 Four View Drawing of the Casting (Mailbox) 


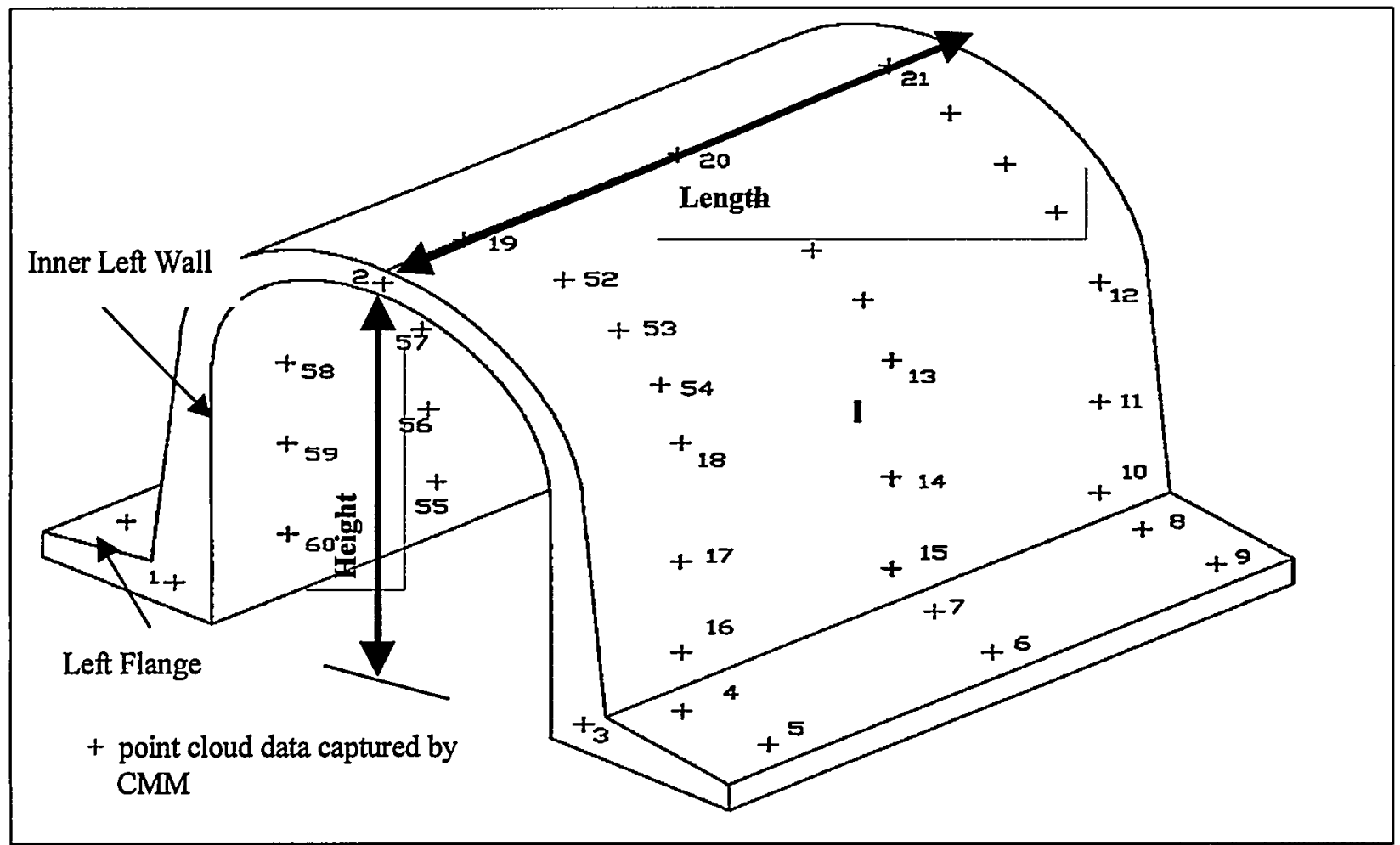

Figure 3.3 Mailbox Casting: Linear Distances and Point Cloud from CMM

\subsubsection{Flatness Evaluation}

The flatness of the plane surfaces was calculated using the nonlinear optimization method as detailed in the literature survey section. The nonlinear program was coded in $\mathrm{C}$ programming language. This method requires that the evaluation plane be relatively parallel with the $\mathrm{xz}$ plane, meaning that the flatness deviation(error) is measured in the y direction. Therefore, some of the data needed to be rearranged. The arrangement of data for the flanges was fine, but the data for the inner walls of the casting were in the yz plane and needed to be manipulated. This was remedied simply by switching the $\mathrm{x}$ and $\mathrm{y}$ columns (basically a rotation of axes) in the data files. In order for the program to read the data, the data must be in a text file, so the raw data for the flanges and walls were copied from Excel and placed into a text file so that the program could read the data. The flatness indices generated by the program were stored in text files and copied back into Excel.

Table 3.4 shows a sample flatness worksheet for the 0.25 inch casting after preprocessing and flatness calculation. The Table provides the left and right flatness indices for the casting flanges and inner walls. See Figures 3.2 and 3.3 for the location of the parameters (ridge thickness, flanges, walls, height, and length).

\subsubsection{Evaluation of Parallelism}


The first step in calculating the parallelism was to place the point cloud data representing the inner walls into the Parallelism worksheet within each workbook. Then the data points were sorted for each replicate so that the points from the right inner wall lined up next to the points on the left inner wall (mirror points). Then the distance between these mirror points was calculated using the Euclidean distance formula as given in equation 2.1.

The average of the difference between the maximum and minimum distances of each replicate is the parallelism index. The index was calculated automatically in Excel by writing a simple cell formula. Table 3.5 is a Parallelism Calculation Worksheet and Table 3.6 shows the calculated indices.

\subsubsection{Calculation of Tapered Angle}

Equation 2.6 requires that the distance in the $y$-axis be relatively the same on the inner and outer walls in order to maintain the trigonometric relationship. This can be seen in Figure 2.4. Further, if the vertical distance is represented by the $y$-axis, it can be seen that $\left(y_{1}-y_{0}\right)$ must equal $\left(y_{3}-y_{2}\right)$ for the cosine relationship to hold. On viewing the data (inner and outer wall data pasted into the Casting Angle worksheet), the y-coordinates of the inner wall were found to be a greater distance apart in the $y$ direction than the y-coordinates on the outer wall. Consequently, if the points were input directly into equation 2.6 , there would be a mathematical error since the inverse cosine of a number greater than one does not exist. Therefore, an additional point on the inner wall was determined using linear interpolation. This would give an inner wall $y$ displacement similar to the y displacement of the outer wall making equation 2.6 valid. The linear interpolation equation requires that two points be known and that one of the coordinates for the desired point be known.

The $y$ coordinate was the desired coordinate, therefore the $\mathrm{x}$ coordinate was the point to be interpolated. Given this, the linear interpolation equation is shown in equation 3.1:

$$
x_{m}=\frac{\left(x_{u}-x_{l}\right)\left(y_{m}-y_{l}\right)}{\left(y_{u}-y_{l}\right)}+x_{l}
$$

where $u, l$, and $m$ represent the upper, lower and middle points, respectively. This formula was entered into the Casting Angle worksheet to determine the normalized point that would be input into equation 2.6. Equation 2.6 was entered into the casting angle worksheet and the cell formula interpolated the point and determined the casting angle index. The angle was converted to degrees from radians my multiplying the angle by $180 / \pi$. Table 3.7 shows the Casting Angle Calculation worksheet. 
Table 3.4 Flatness Worksheet ( 0.25 inch)

\begin{tabular}{|l|c|c|c|}
\hline \multicolumn{4}{|c}{ Left Flange Flatness } \\
OG & \multicolumn{1}{c}{ Rep 1 } & \multicolumn{1}{c}{ Rep2 } & \multicolumn{1}{c|}{ Rep3 } \\
\cline { 2 - 4 } DT & 0.0129354 & 0.0136618 & 0.0146739 \\
DB & 0.0324497 & 0.0217598 & 0.0142373 \\
\cline { 2 - 4 } LT & 0.0087811 & 0.0093092 & 0.0245206 \\
LB & 0.0072153 & 0.0277153 & 0.0169051 \\
\cline { 2 - 4 } UT & 0.011571 & 0.0063659 & 0.0125229 \\
UB & 0.0095053 & 0.0360345 & 0.0109011 \\
DT & 0.0089608 & 0.005837 & 0.0073989 \\
DB & 0.0067354 & 0.005149 & 0.0081379 \\
LT & 0.0072122 & 0.0046309 & 0.0107616 \\
LB & 0.0115883 & 0.0095767 & 0.0069717 \\
UT & 0.0135152 & 0.0101367 & 0.0080434 \\
UB & 0.0057487 & 0.0087922 & 0.0031266 \\
DT & 0.0050286 & 0.0072522 & 0.0092289 \\
DB & 0.0058943 & 0.0218564 & 0.0048638 \\
\cline { 2 - 4 } LT & 0.0087732 & 0.0084919 & 0.004029 \\
LB & 0.0014278 & 0.0035927 & 0.0099568 \\
\cline { 2 - 4 } UT & 0.0060703 & 0.0029883 & 0.0052404 \\
\cline { 2 - 4 } UB & 0.0033837 & 0.0108975 & 0.0104584 \\
\hline & & &
\end{tabular}

\begin{tabular}{|c|c|c|c|}
\hline \multicolumn{4}{|c|}{ Right Flange Flatness } \\
\hline OG & $\operatorname{Rep} 1$ & $\operatorname{Rep2}$ & Rep3 \\
\hline DT & 0.0366162 & 0.0744574 & 0.0362902 \\
\hline DB & 0.181809 & 0.122948 & 0.0581749 \\
\hline $\mathbf{L T}$ & 0.0074081 & 0.0403775 & 0.0530523 \\
\hline LB & 0.0187794 & 0.0421317 & 0.0316486 \\
\hline UT & 0.134544 & 0.0736808 & 0.044145 \\
\hline UB & 0.013127 & 0.0510237 & 0.0300487 \\
\hline DT & 0.619931 & 0.0392332 & 0.3295821 \\
\hline DB & 0.0196367 & 0.0237013 & 0.030751 \\
\hline $\mathbf{L T}$ & 0.0800546 & 0.0778565 & 0.12408 \\
\hline LB & 0.0195263 & 0.0390204 & 0.0630544 \\
\hline UT & 0.0081539 & 0.0104232 & 0.0196142 \\
\hline $\mathbf{U B}$ & 0.0901868 & 0.0302396 & 0.029533 \\
\hline DT & 0.0322928 & 0.0419266 & 0.0396022 \\
\hline DB & 0.0304407 & 0.0243834 & 0.0185112 \\
\hline LT & 0.0897477 & 0.0236101 & 0.0687879 \\
\hline LB & 0.0041387 & 0.0509327 & 0.0217184 \\
\hline UT & 0.0210578 & 0.0236701 & 0.0094043 \\
\hline UB & 0.0529513 & 0.06347 & 0.0760583 \\
\hline
\end{tabular}

\section{Left Wall Flatmess}

\begin{tabular}{|c|c|c|c|c|c|c|c|}
\hline OG & Rep1 & $\operatorname{Rep2}$ & $\operatorname{Rep} 3$ & OG & Rep1 & $\operatorname{Rep2}$ & Rep3 \\
\hline DT & 0.010207 & 0.0078542 & 0.0136745 & DT & 0.025197 & 0.0132632 & 0.093224 \\
\hline DB & 0.0182163 & 0.0116467 & 0.0071281 & DB & 0.0320878 & 0.0400243 & 0.177846 \\
\hline LT & 0.0105275 & 0.0179948 & 0.0183624 & $\mathbf{L T}$ & 0.0226669 & 0.0414157 & 0.0503888 \\
\hline LB & 0.0094711 & 0.0188516 & 0.0291162 & $\mathbf{L B}$ & 0.0427694 & 0.0601225 & 0.118547 \\
\hline UT & 0.0142193 & 0.0260697 & 0.0157151 & UT & 0.0119405 & 0.0046077 & 0.106884 \\
\hline UB & 0.007994 & 0.0110085 & 0.0081764 & UB & 0.0571237 & 0.0484715 & 0.0245318 \\
\hline DT & 0.0166189 & 0.0167874 & 0.0167032 & $\mathbf{D T}$ & 0.0542135 & 0.027195 & 0.0407043 \\
\hline DB & 0.0068196 & 0.00742 & 0.0051805 & DB & 0.0166039 & 0.0239692 & 0.0061564 \\
\hline LT & 0.0167121 & 0.0058158 & 0.0097162 & LT & 0.018055 & 0.0122786 & 0.0289178 \\
\hline $\mathbf{B}$ & 0.0177619 & 0.0048409 & 0.0232826 & $\mathbf{L B}$ & 0.0310197 & 0.0198727 & 0.0147619 \\
\hline UT & 0.01681 & 0.0132178 & 0.0200619 & $\mathbf{U T}$ & 0.0324574 & 0.0311875 & 0.0146966 \\
\hline UB & 0.0257171 & 0.014637 & 0.0246025 & UB & 0.0506253 & 0.110769 & 0.065259 \\
\hline DT & 0.0073194 & 0.0099229 & 0.0078465 & DT & 0.0102034 & 0.0107045 & 0.0156121 \\
\hline DB & 0.0133748 & 0.0053432 & 0.0132569 & DB & 0.0303278 & 0.0450339 & 0.0387015 \\
\hline LT & 0.0065861 & 0.0092626 & 0.0183574 & $\mathbf{L T}$ & 0.0845289 & 0.088799 & 0.114245 \\
\hline LB & 0.0085532 & 0.0075857 & 0.0137874 & LB & 0.0180655 & 0.0432391 & 0.0280375 \\
\hline UT & 0.0079739 & 0.0116383 & 0.0063942 & UT & 0.0356369 & 0.0134392 & 0.0129704 \\
\hline UB & 0.0089708 & 0.0144176 & 0.0134642 & UB & 0.100449 & 0.0697465 & 0.0300765 \\
\hline
\end{tabular}

Definitions: O: Orientation

D: Orientation Down

U: Orientation Up

G: Gating

T: Top

B: Bottom

L: Orientation Lateral(Side) 


\subsubsection{Calculations of Casting Dimensions}

The equations for calculating the linear distances (i.e. the thickness of the castings at the top ridge, height, and the length of the castings) were entered into the CMM point cloud data worksheet. The worksheet contained the data for all three replicates. The distances were calculated using the linear distance formula $\left(\mathrm{x}_{2}-\mathrm{x}_{1}\right)$. The length was calculated using the $\mathrm{z}$ distance between the center edge point (EP) and back edge point (BEP) for each replicate. The formula is $\left(\mathrm{EP}_{\mathrm{z}}-\mathrm{BEP}_{\mathrm{z}}\right)$. The height is calculated by using the following formula:

$$
\text { Average }\left(R p_{y} \text {-Average }\left(\min \left(F p_{y}\right)\right)\right. \text {. }
$$

where $\mathrm{RP}_{\mathrm{y}}$ denotes the ridge points in the $\mathrm{y}$-direction and $\min \mathrm{FP}_{\mathrm{y}}$ denotes the lowest flange points in the y-direction. The thickness was calculated by averaging the y-distance of the ridge points and inner walls and taking the difference. Table 3.8 is a Height, Length, and Thickness calculation worksheet. 


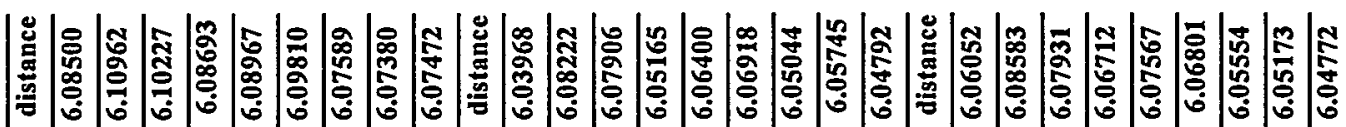

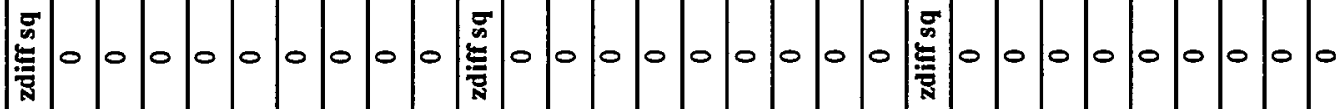

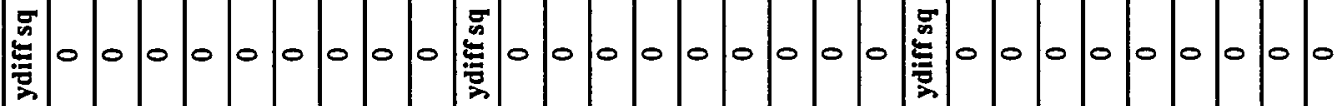

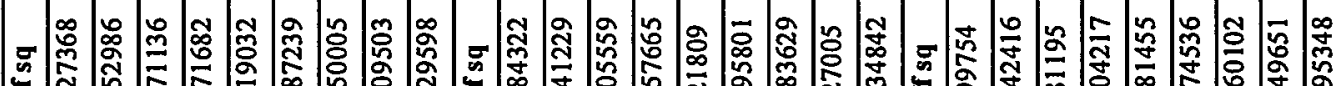

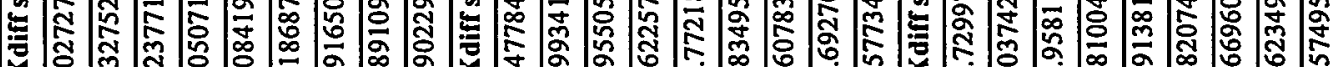
蛋

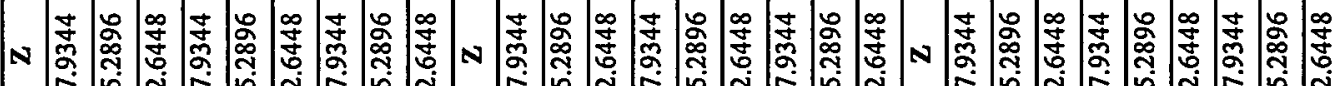

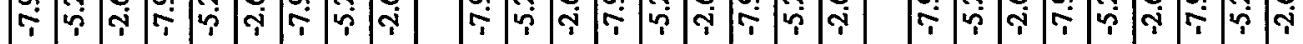

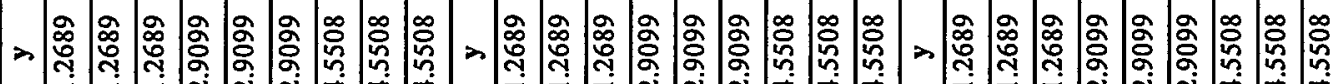

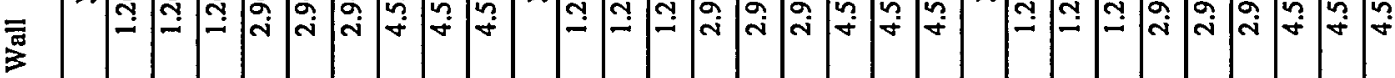

范

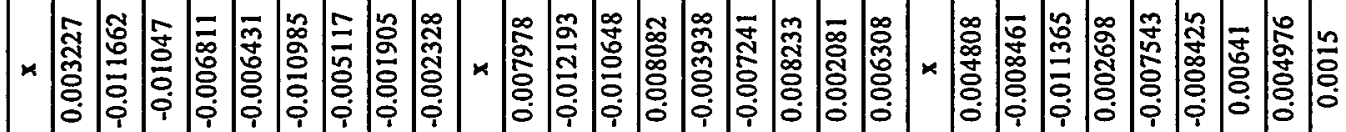

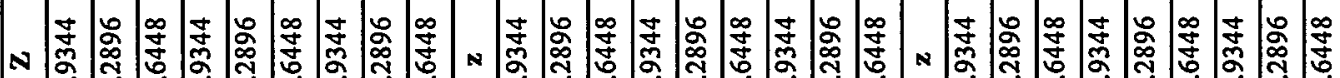

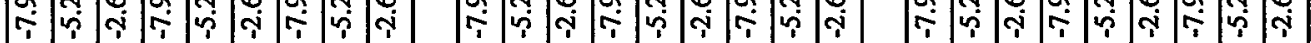

高

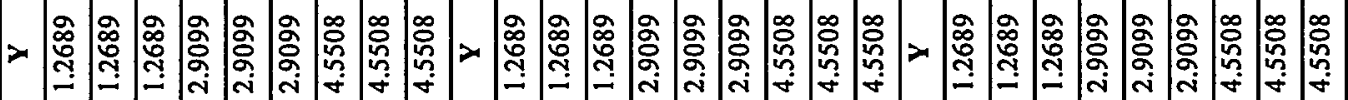

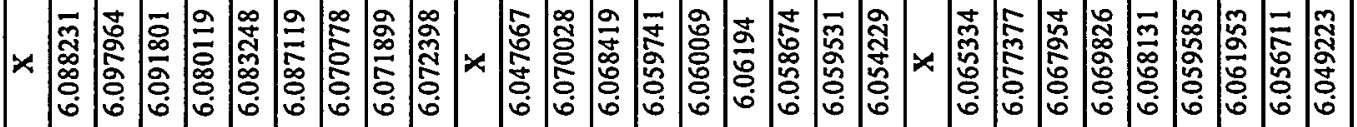


Table 3.6 Parallelism Index

\begin{tabular}{|c|c|c|c|}
\hline Replicate & Max dist & min dist & parallelim \\
\hline 1 & 6.109626 & 6.073804 & $\mathbf{0 . 0 3 5 8 2 2}$ \\
\hline 2 & 6.082221 & 6.039689 & $\mathbf{0 . 0 4 2 5 3 2}$ \\
\hline 3 & 6.085838 & 6.047723 & $\mathbf{0 . 0 3 8 1 1 5}$ \\
\hline
\end{tabular}

Table 3.7 Casting Angle Calculation Worksheet

\begin{tabular}{|c|c|c|c|c|c|c|c|c|c|}
\hline \multicolumn{9}{|c|}{$\begin{array}{l}\text { Right Casting Angle Calculation(Replicate 1) } \\
\text { Replicate } 1\end{array}$} & $\begin{array}{c}\text { Casting } \\
\text { Angle }\end{array}$ \\
\hline 6.820275 & 2.026755 & -7.8129 & 6.088231 & 1.2689 & -7.9344 & 6.088231 & 1.2689 & -7.9344 & 10.12 \\
\hline 6.627267 & 3.52851 & -7.8129 & 6.080119 & 2.9099 & -7.9344 & 6.0723776 & 4.25 & -7.9344 & \\
\hline 6.42457 & 5.029088 & -7.8129 & 6.070778 & 4.5508 & -7.9344 & & & & \\
\hline 6.422561 & 5.028841 & -5.2653 & 6.071899 & 4.5508 & -5.2896 & 6.097964 & 1.2689 & -5.2896 & 10.12 \\
\hline 6.621888 & 3.52785 & -5.2653 & 6.083248 & 2.9099 & -5.2896 & 6.074288 & 4.25 & -5.2896 & \\
\hline 6.815204 & 2.026132 & -5.2653 & 6.097964 & 1.2689 & -5.2896 & & & & \\
\hline 6.814022 & 2.025987 & -2.7177 & 6.091801 & 1.2689 & -2.6448 & 6.091801 & 1.2689 & -2.6448 & 10.12 \\
\hline 6.623176 & 3.528008 & -2.7177 & 6.087119 & 2.9099 & -2.6448 & 6.0741764 & 4.25 & -2.6448 & \\
\hline 6.416798 & 5.028133 & -2.7177 & 6.072398 & 4.5508 & -2.6448 & \multicolumn{3}{|c|}{ Right Casting Angle(Rep1) } & \\
\hline $\begin{array}{l}\text { Right Cast } \\
\text { Replicate } 1\end{array}$ & \multicolumn{9}{|c|}{$\begin{array}{l}\text { Right Casting Angle Calculation(Replicate 1) } \\
\text { Replicate } 1\end{array}$} \\
\hline-0.351678 & 5.020136 & -7.8129 & -0.005117 & 4.5508 & -7.9344 & 0.003227 & 1.2689 & -7.9344 & 10.15 \\
\hline-0.519308 & 3.515252 & -7.8129 & -0.006811 & 2.9099 & -7.9344 & -0.0043522 & 4.25 & -7.9344 & \\
\hline-0.674892 & 2.008901 & -7.8129 & 0.003227 & 1.2689 & -7.9344 & & & & \\
\hline-0.681185 & 2.009674 & -5.2653 & -0.011662 & 1.2689 & -5.2896 & -0.011662 & 1.2689 & -5.2896 & 10.14 \\
\hline-0.519442 & 3.515268 & -5.2653 & -0.006431 & 2.9099 & -5.2896 & -0.0027993 & 4.25 & -5.2896 & \\
\hline-0.351543 & 5.020119 & -5.2653 & -0.001905 & 4.5508 & -5.2896 & & & & \\
\hline-0.357016 & 5.020791 & -2.7177 & -0.002328 & 4.5508 & -2.6448 & -0.01047 & 1.2689 & -2.6448 & 10.14 \\
\hline-0.524397 & 3.515877 & -2.7177 & -0.010985 & 2.9099 & -2.6448 & -0.0030742 & 4.25 & -2.6448 & \\
\hline-0.687111 & 2.010401 & -2.7177 & -0.01047 & 1.2689 & -2.6448 & \multirow{2}{*}{\multicolumn{3}{|c|}{ Left Casting Angle(Rep1) }} & \\
\hline & & & & & & & & & 10.15 \\
\hline
\end{tabular}


Table 3.8 Height, Length, and Thickness Calculation Worksheet

\begin{tabular}{|c|c|c|c|c|c|c|c|c|c|c|}
\hline & \multicolumn{10}{|c|}{ Length } \\
\hline & & \multicolumn{2}{|c|}{ Edge points(z-values) } & & \multicolumn{3}{|c|}{ back edge points(z-values) } & & & \\
\hline & Replicate 1 & Replicate 2 & Replicate 3 & Replicate 1 & Replicate 2 & Replicate 3 & & & & \\
\hline & -0.447461 & -0.373175 & -0.376152 & -9.258259 & -9.1825 & -9.194202 & Length 1 & Length 2 & Length 3 & \\
\hline \multirow[t]{9}{*}{ middle } & -0.774418 & -0.779279 & -0.763303 & -8.566546 & -8.52613 & -8.520512 & 7.79 & 7.75 & 7.76 & \\
\hline & -0.414694 & -0.342567 & -0.356066 & -9.2738 & -9.185958 & -9.183904 & & & & \\
\hline & & & & & & & & & & \\
\hline & \multicolumn{10}{|c|}{ Height } \\
\hline & \multicolumn{3}{|c|}{ ridge points(y-values) } & \multicolumn{3}{|c|}{ right flange(y-values) } & & \multicolumn{2}{|c|}{ left flange(y-values) } & \\
\hline & Replicate 1 & Replicate 2 & Replicate 3 & Replicate 1 & Replicate 2 & Replicate 3 & Replicate 1 & Replicate 2 & Replicate 3 & \\
\hline & 8.818637 & 8.776808 & 8.805258 & -0.038625 & -0.119717 & -0.085829 & -0.125554 & -0.131836 & -0.118149 & \\
\hline & 8.831355 & 8.783336 & 8.812826 & -0.037679 & -0.119969 & -0.079898 & -0.122384 & -0.124316 & -0.116732 & \\
\hline & 8.843286 & 8.789744 & 8.820552 & -0.021643 & -0.147657 & -0.079773 & -0.114303 & -0.127921 & -0.114424 & \\
\hline \multirow[t]{12}{*}{ average } & 8.83109267 & 8.783296 & 8.8128787 & & & & & & & \\
\hline & & & & & & & & & & \\
\hline & \multicolumn{3}{|c|}{ Ridge points(y-values),averages } & \multicolumn{3}{|c|}{ Flange average } & Height1 & Height2 & Height3 & \\
\hline & 8.83718933 & \begin{tabular}{|l|}
8.78652 \\
\end{tabular} & 8.8167153 & -0.076698 & -0.128569333 & -0.099134167 & 8.91 & 8.92 & 8.92 & \\
\hline & & & & & & & & & & \\
\hline & & & & & Thickness & & & & & \\
\hline & & $\begin{array}{c}\text { ridge points (y- } \\
\text { values) }\end{array}$ & & & \multicolumn{2}{|c|}{ Inner wall ridge(y-values) } & & & & \\
\hline & Replicate 1 & \begin{tabular}{|l|} 
Replicate 2 \\
\end{tabular} & \begin{tabular}{|l|} 
Replicate 3 \\
\end{tabular} & Replicate 1 & Replicate 2 & Replicate 3 & Thickness 1 & Thickness 2 & Thickness 3 & \\
\hline & 8.818637 & 8.776808 & 8.805258 & 8.520976 & 8.464262 & 8.50427 & 0.297661 & 0.312546 & 0.300988 & \\
\hline & 8.831355 & 8.783336 & 8.812826 & 8.548627 & 8.477321 & 8.517008 & 0.282728 & 0.306015 & 0.295818 & \\
\hline & 8.843286 & 8.789744 & 8.820552 & 8.548036 & 8.47589 & 8.51755 & 0.29525 & 0.313854 & 0.303002 & \\
\hline & & & & & & Thickness & 0.29 & 0.31 & 0.30 & \\
\hline
\end{tabular}




\subsection{METHOD OF IMPLEMENTATION AND STATISTICAL ANALYSIS}

The implementation of the methodology involved a series of steps. Initially, the data was preprocessed. Then, the form parameters were calculated from the point cloud data collected from the castings. The analysis of variance (ANOVA) statistical analysis was then performed. Finally, the Newman-Keuls Test of Means was performed. The characteristic parameters of the castings that were analyzed in this research are summarized in the following statements.

1. Flatness of the flanges and inner walls of the casting

2. Parallelism of the inside walls of the casting

3. The tapered angle of the outside walls of the casting

4. Casting height

5. Ridge thickness

6. Length of the top ridge of the casting

\subsection{Statistical Analysis}

The statistical analysis was performed in order to determine the effect of the factors (gating, orientation, thickness, and sand molding process) on the form parameters. Tables $4.1-$ 4.4 are samples of the factorial analysis of variance (ANOVA) performed on the data. Samples of the remaining Tables are located in Appendix C. The ANOVA results were analyzed for significant contributors at the 95 percent confidence level.

The index analyzed in Table 4.1 is the left flange flatness of the entire set of $1 / 4$ inch casting data. According to the analysis of variance (ANOVA) of this index, the mold type (M) factor and orientation-mold type (OM) factor interaction are affecting the left flange flatness at the 95 percent confidence level. Newman-Keuls is performed on the mold type (M) due to the significance indicated at the mold factor; thus, mold type $(M)$ has a more significant contribution to the distortion than orientation (O). Table 4.2 is the ANOVA for the height of the $1 / 4$ inch lost foam low expansion castings. According to the table, neither gating $(G)$ or orientation $(O)$ significantly affects the height of the lost foam low expansion castings; thus, no test of means was performed. Table 4.3 is the ANOVA for the left wall flatness of the resin-bonded castings. The factor interaction of gating-orientation (GO) is significantly affecting the left wall flatness. Since there was no significance indicated for the single factors ( $G$ and $O)$, both gating $(G)$ and orientation $(\mathrm{O})$ were affecting left wall flatness equally; therefore, the Newman-Keuls test was unnecessary. Table 4.4 is the ANOVA for the parallelism of the lost foam silica sand castings. The factor interaction of gating and orientation (GO) and gating (G) are significantly affecting the parallelism of the lost foam silica sand castings. Since there was significance for the single factor gating, gating has a greater effect on the parallelism error. Newman-Keuls must be performed in this case.

The Newman-Keuls test of mean was performed on the significant factors found in the analysis of the ANOVA tables at the 95 percent confidence level. The purpose of the test of means is to determine which factor levels have the greatest effect on the distortion. Tables 4.5 and 4.6 are the Newman-Keuls test of means ( 5 percent significance level) performed based on 
the analysis of ANOVA Tables 4.1-4.4. Table 4.5 is the Newman-Keuls on the mold type of all of the $1 / 4$ " left flange flatness data. The Newman-Keuls test of means indicates that M1 is the mold factor level that is contributing to the left flange flatness error of all of the $1 / 4$ inch casting data; therefore, resin-bonded sand is the mold type contributing to the left flange flatness error for this data set. Basically the way the table is interpreted is M1 is more significant than M2, M1 is more significant than $M 3$, and M2 is more significant than M3; therefore, M1 is most significant. Table 4.6 is the Newman-Keuls on the gating of the $1 / 4$ inch lost foam silica sand parallelism data. The test of means indicates that top gating (G1) is the gating type contributing most to the $1 / 4$ inch lost foam silica sand parallelism error. The analysis performed on the $1 / 2$ " and $3 / 4$ " data is similar. The full results of the ANOVA and Newman-Keuls test of means will be discussed in section 5.0 (below).

Table 4.1 Factorial Analysis of Variance: Left Flange Flatness of all 1/4 inch castings

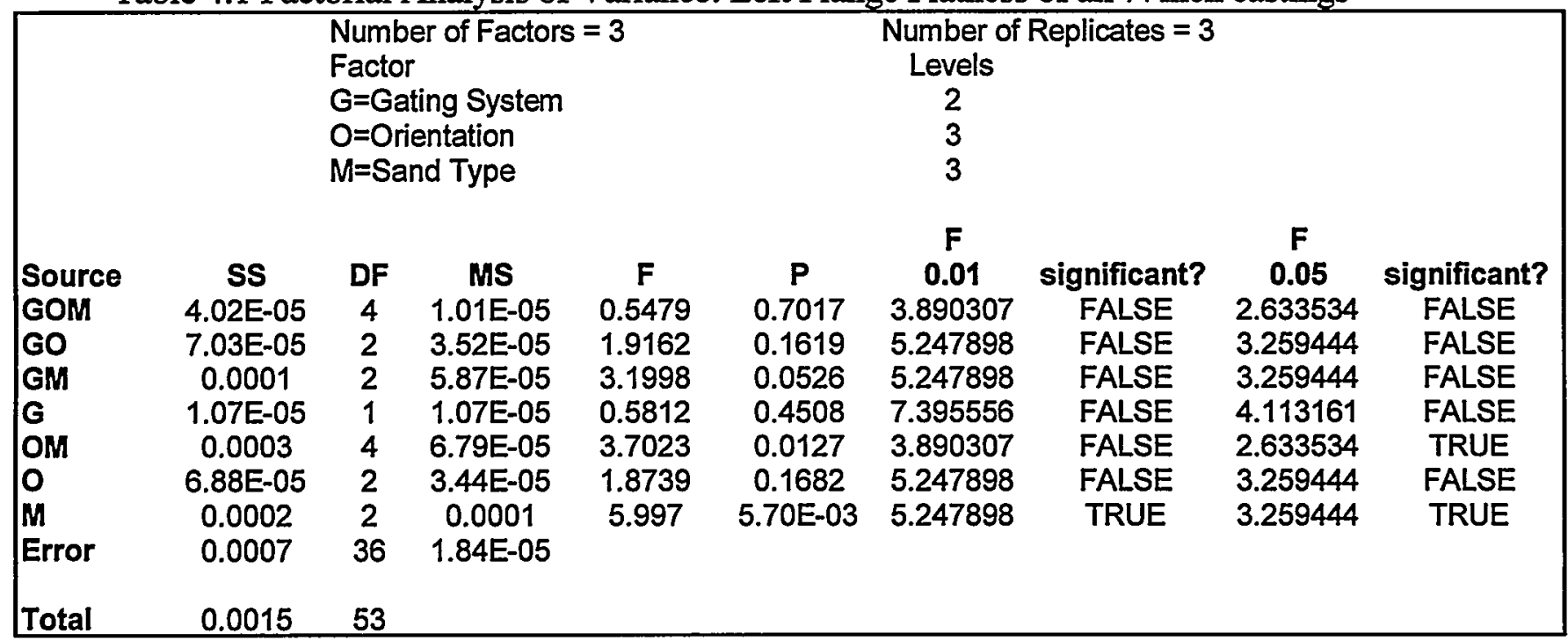

Table 4.2 Factorial Analysis of Variance of Height of Lost Foam Low Expansion Castings ( $1 / 4$ in.)

\begin{tabular}{|c|c|c|c|c|c|c|c|c|c|}
\hline \multirow[b]{2}{*}{$\begin{array}{l}\text { Source } \\
G 0 \\
G \\
G \\
0 \\
\text { Error }\end{array}$} & \multirow[b]{2}{*}{$\begin{array}{c}\text { SS } \\
0.0096 \\
0.0083 \\
0.0088 \\
0.026\end{array}$} & \multicolumn{3}{|c|}{$\begin{array}{l}\text { Number of Factors }=2 \\
\text { Factor } \\
G=\text { Gating } \\
O=\text { Orientation }\end{array}$} & \multicolumn{5}{|c|}{$\begin{array}{l}\text { Number of Replicates }=3 \\
\text { Levels } \\
2 \\
3\end{array}$} \\
\hline & & $\begin{array}{c}\text { DF } \\
2 \\
1 \\
2 \\
12\end{array}$ & $\begin{array}{c}\text { MS } \\
0.0048 \\
0.0083 \\
0.0044 \\
0.0022\end{array}$ & $\begin{array}{c}F \\
2.2083 \\
3.809 \\
2.0252\end{array}$ & $\begin{array}{c}P \\
0.1525 \\
0.0747 \\
0.1747\end{array}$ & $\begin{array}{c}\mathbf{F} \\
\mathbf{0 . 0 1} \\
6.926598 \\
9.330279 \\
6.926598\end{array}$ & $\begin{array}{l}\text { significant? } \\
\text { FALSE } \\
\text { FALSE } \\
\text { FALSE }\end{array}$ & $\begin{array}{c}F \\
0.05 \\
3.88529 \\
4.747221 \\
3.88529\end{array}$ & $\begin{array}{c}\text { significant? } \\
\text { FALSE } \\
\text { FALSE } \\
\text { FALSE }\end{array}$ \\
\hline Total & 0.0526 & 17 & & & & & & & \\
\hline
\end{tabular}

Table 4.3 Factorial Analysis of Variance: Left Wall Flatness of Resin Bonded Castings( $1 / 4$ in.) 


\begin{tabular}{|c|c|c|c|c|c|c|c|c|c|}
\hline \multirow[b]{2}{*}{$\begin{array}{l}\text { Source } \\
\text { GO } \\
\text { G } \\
\text { O } \\
\text { Error }\end{array}$} & \multirow[b]{2}{*}{$\begin{array}{c}\text { SS } \\
0.0002 \\
4.67 \mathrm{E}-05 \\
2.33 \mathrm{E}-06 \\
0.0002\end{array}$} & \multicolumn{3}{|c|}{$\begin{array}{l}\text { Number of Factors }=2 \\
\text { Factor } \\
\text { G=Gating } \\
\text { O=Orientation }\end{array}$} & \multicolumn{3}{|c|}{$\begin{array}{l}\text { Number of Replicates }=3 \\
\text { Levels } \\
2 \\
3\end{array}$} & \multirow[b]{2}{*}{$\begin{array}{c}\mathbf{F} \\
\mathbf{0 . 0 5} \\
3.88529 \\
4.747221 \\
3.88529\end{array}$} & \multirow[b]{2}{*}{$\begin{array}{c}\text { significant? } \\
\text { TRUE } \\
\text { FALSE } \\
\text { FALSE }\end{array}$} \\
\hline & & $\begin{array}{c}\text { DF } \\
2 \\
1 \\
2 \\
12\end{array}$ & $\begin{array}{c}\text { MS } \\
0.0001 \\
4.67 E-05 \\
1.17 E-06 \\
2.06 E-05\end{array}$ & $\begin{array}{c}\mathbf{F} \\
5.4367 \\
2.2668 \\
0.0566\end{array}$ & $\begin{array}{c}P \\
P \\
0.0209 \\
0.158 \\
0.9452\end{array}$ & $\begin{array}{c}\mathbf{F} \\
\mathbf{0 . 0 1} \\
6.926598 \\
9.330279 \\
6.926598\end{array}$ & $\begin{array}{l}\text { significant? } \\
\text { FALSE } \\
\text { FALSE } \\
\text { FALSE }\end{array}$ & & \\
\hline Total & 0.0005 & 17 & & & & & & & \\
\hline
\end{tabular}

Table 4.4 Factorial Analysis of Variance: Parallelism of Lost Foam Silica Sand Castings( $(1 / 4 \mathrm{in}$.)

\begin{tabular}{|c|c|c|c|c|c|c|c|c|c|}
\hline \multirow[b]{2}{*}{$\begin{array}{l}\text { Source } \\
\text { GO } \\
\text { G } \\
\text { O } \\
\text { Error }\end{array}$} & \multirow[b]{2}{*}{$\begin{array}{l}\text { SS } \\
0.0208 \\
0.0227 \\
0.0042 \\
0.0259\end{array}$} & \multicolumn{3}{|c|}{$\begin{array}{l}\text { Number of Factors } \\
\text { Factor } \\
G=\text { Gating } \\
O=\text { Orientation }\end{array}$} & \multicolumn{5}{|c|}{$\begin{array}{l}\text { Number of Replicates } \\
\text { Levels } \\
2 \\
3\end{array}$} \\
\hline & & $\begin{array}{c}\text { DF } \\
4 \\
2 \\
2 \\
18\end{array}$ & $\begin{array}{c}\text { MS } \\
0.0052 \\
0.0114 \\
0.0021 \\
0.0014\end{array}$ & $\begin{array}{c}\mathbf{F} \\
3.6063 \\
7.8834 \\
1.4484\end{array}$ & $\begin{array}{c}\mathbf{P} \\
0.0251 \\
0.0035 \\
0.2611\end{array}$ & $\begin{array}{c}0.01 \\
4.57902 \\
6.0129 \\
6.0129\end{array}$ & $\begin{array}{l}\text { significant? } \\
\text { FALSE } \\
\text { TRUE } \\
\text { FALSE }\end{array}$ & $\begin{array}{c}0.05 \\
2.92775 \\
3.55456 \\
3.55456\end{array}$ & $\begin{array}{c}\text { significant? } \\
\text { TRUE } \\
\text { TRUE } \\
\text { FALSE }\end{array}$ \\
\hline Total & 0.0736 & 26 & & & & & & & \\
\hline
\end{tabular}

Table 4.5 Newman-Keuls Test of Means on Mold Type of the Left Flange Flatness Data(1/4 in.)

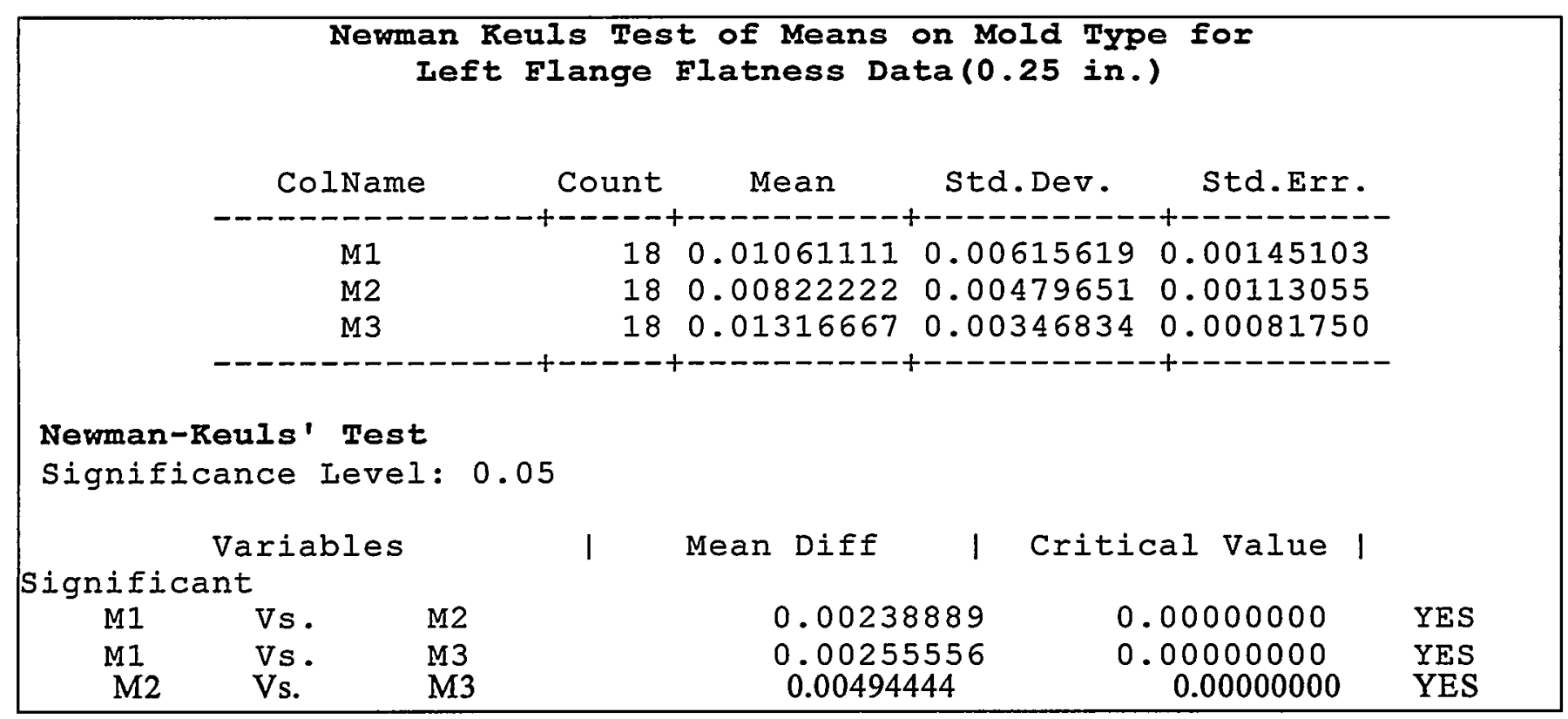


Table 4.6 Newman-Keuls Test of Means on Gating System of Lost Foam Low Expansion Parallelism Data( $1 / 4$ in.)

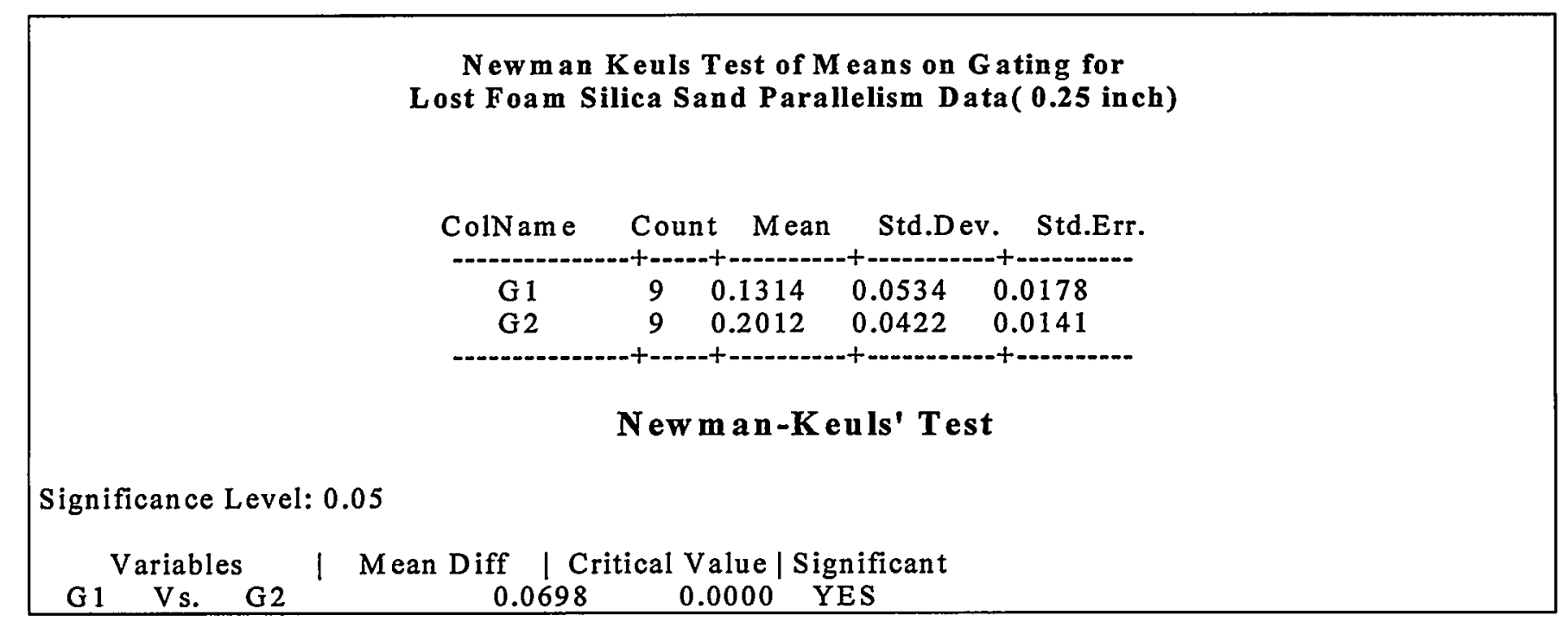




\subsection{RESULTS}

\subsection{Data Analysis}

From the charts and statistical analysis, there were differences noted between the nominal and calculated values. Overall, the nominal values of the resin bonded casting parameters varied more than the lost foam processes parameters. The lost foam silica sand casting parameters varied the least. Samples of the figures used in these analyses included surface plots of flatness (Figure 5.1), box plots (Figure 5.2), histograms (Figure 5.3), scatter plots (Figure 5.4), and bullseye plots (Figure 5.5). The surface plot of flatness shows the three-dimensional variations in flatness. Box plots show the spread of the data around the mean. Histograms show the normal distribution features of the data. Scatter Plots show the two-dimensional variation in the data. Bullseye Plots were used to display how well the process "hit" the target. It is a visual representation of the variation of the calculated value from nominal value.

\subsection{Statistical Analysis Results}

The statistical analysis demonstrates that the factors investigated (gating, orientation, sand mold type, and thickness) definitely affect casting distortion. Factorial analysis of variance was performed on all of the data for each molding processes separately and collectively. The analysis was performed on three orientation levels (down, up, and left), three thickness levels $(3 / 4$, $1 / 2$, and $1 / 4$ inch) and three molding process levels (resin bonded sand, lost foam low expansion, and lost foam silica sand). Two gating systems (top and bottom) were analyzed for all three-sand types and analysis for variations due to side gating was performed solely on the lost foam processes (since this was the only side gating data available). Newman-Keuls test of means was performed on the significant factors as determined by the ANOVA. The results of the analyses are summarized in Tables 5.1-5.4. The tables outline the significant contributors to the distortion on the parameters (left wall flatness, height, etc.) at $\alpha=5$ percent level). In the tables, ALL indicates that "all" factor levels contributed equally to the casting distortion. The dash "-_" means that from the ANOVA analysis, the effect of the factor was not significant. The complete set of factorial analysis of variance (ANOVA) and Newman-Keuls test of means is located in Appendix B.

Table 5.1 shows the results of the analysis performed on all of the $1 / 4$ inch castings. According to the analysis, the significant contributors to the distortion are the resin-bonded sand, orientation down and top gating (See Table 5.1 a). From Table $5.1 \mathrm{~b}$ and d, it can be seen that the $1 / 4$ inch lost foam silica sand and resin bonded sand castings are distorted by orientation down and top gating, while the distortion of the low expansion castings are primarily due to orientation down (Table $5.1 \mathrm{c}$ ). Table 5.2a-d shows the analysis performed on all of the $1 / 2$ inch castings. The analysis indicated that all gating levels equally affected the height and right flange flatness of the 
lost foam silica sand castings, left flange flatness and left wall flatness of the resin bonded castings and the right flange flatness of the of the lost foam low expansion castings. All orientation levels equally affected the right flange flatness of the lost foam silica sand castings, height of the resin bonded castings, and length, parallelism, left wall flatness, and right wall flatness and right flange flatness of the lost foam low expansion castings. Top gating affected the right wall flatness of the resin-bonded castings and orientation up affected the length of the lost foam low expansion castings. The analysis performed on the $3 / 4$ inch castings indicated that orientation down and top gating characterized the distortion of the lost foam silica sand castings (See Table 5.3). The low

expansion and resin bonded castings are equally affected by all orientation and gating levels. The low expansion castings are mostly affected by the orientation.

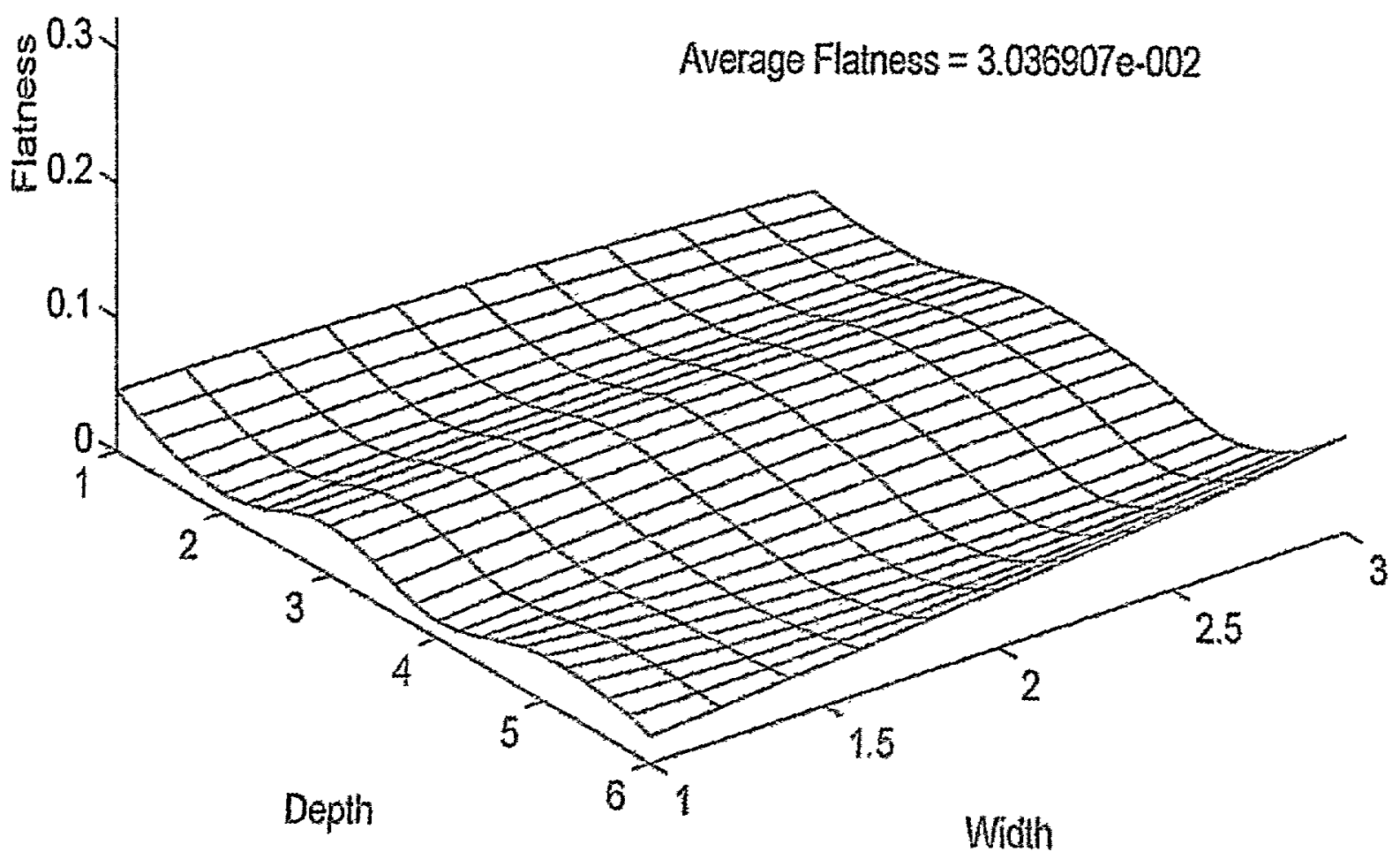

Figure 5.1. Surface Plot of Flatness 


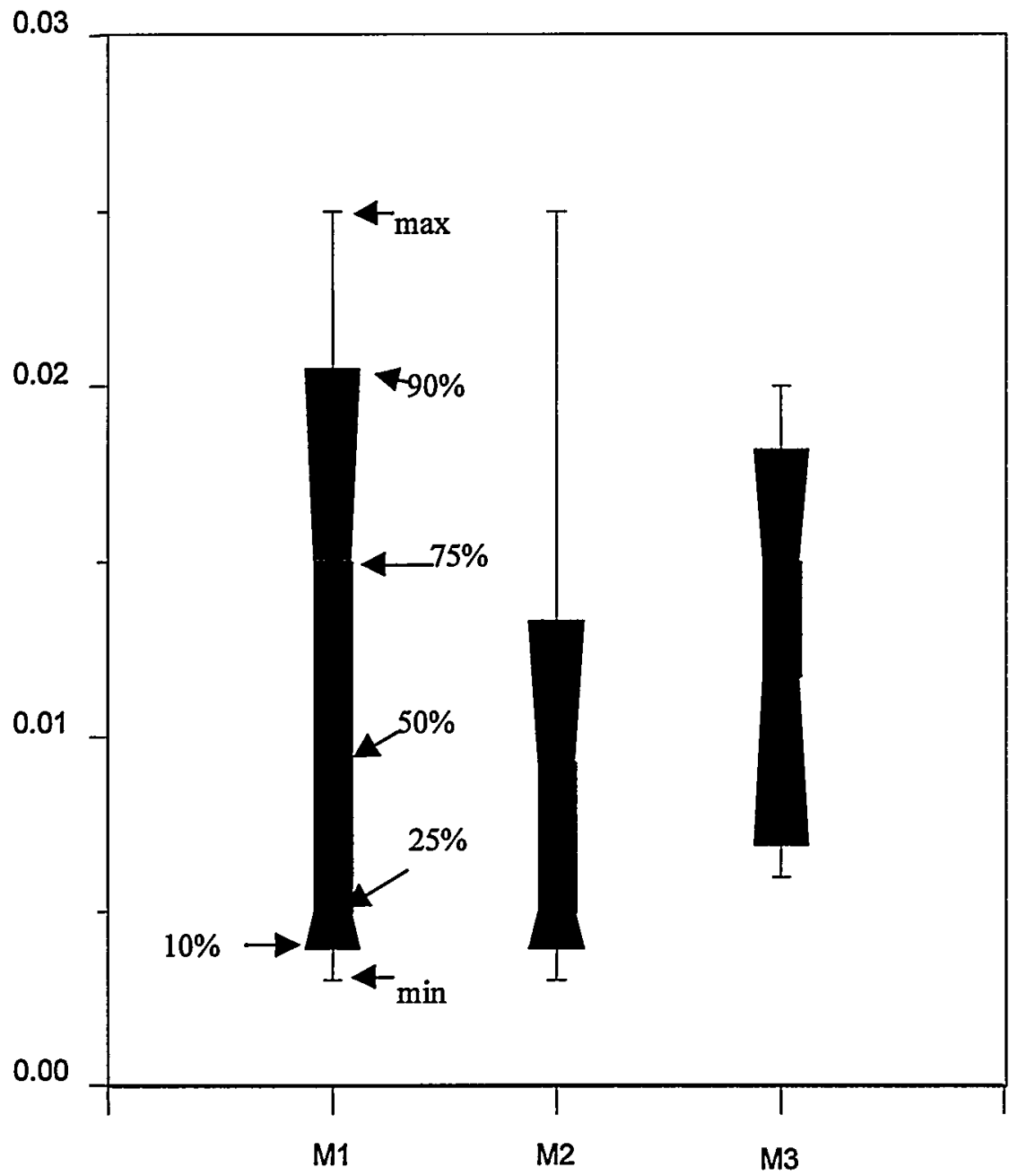

Figure 5.2 Box Plot of Left Flange Flatness of all 0.25 inch castings

M1: Resin Bonded Sand

M2: Lost Foam Low Expansion

M3: Lost Foam Low Expansion

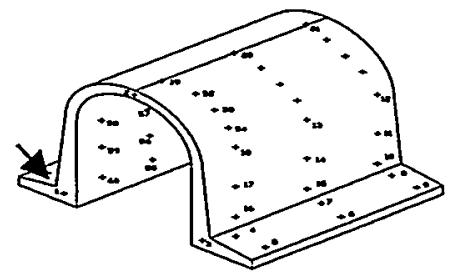


Histogram of Left Casting Angle of RBS 0.25 inch Castings

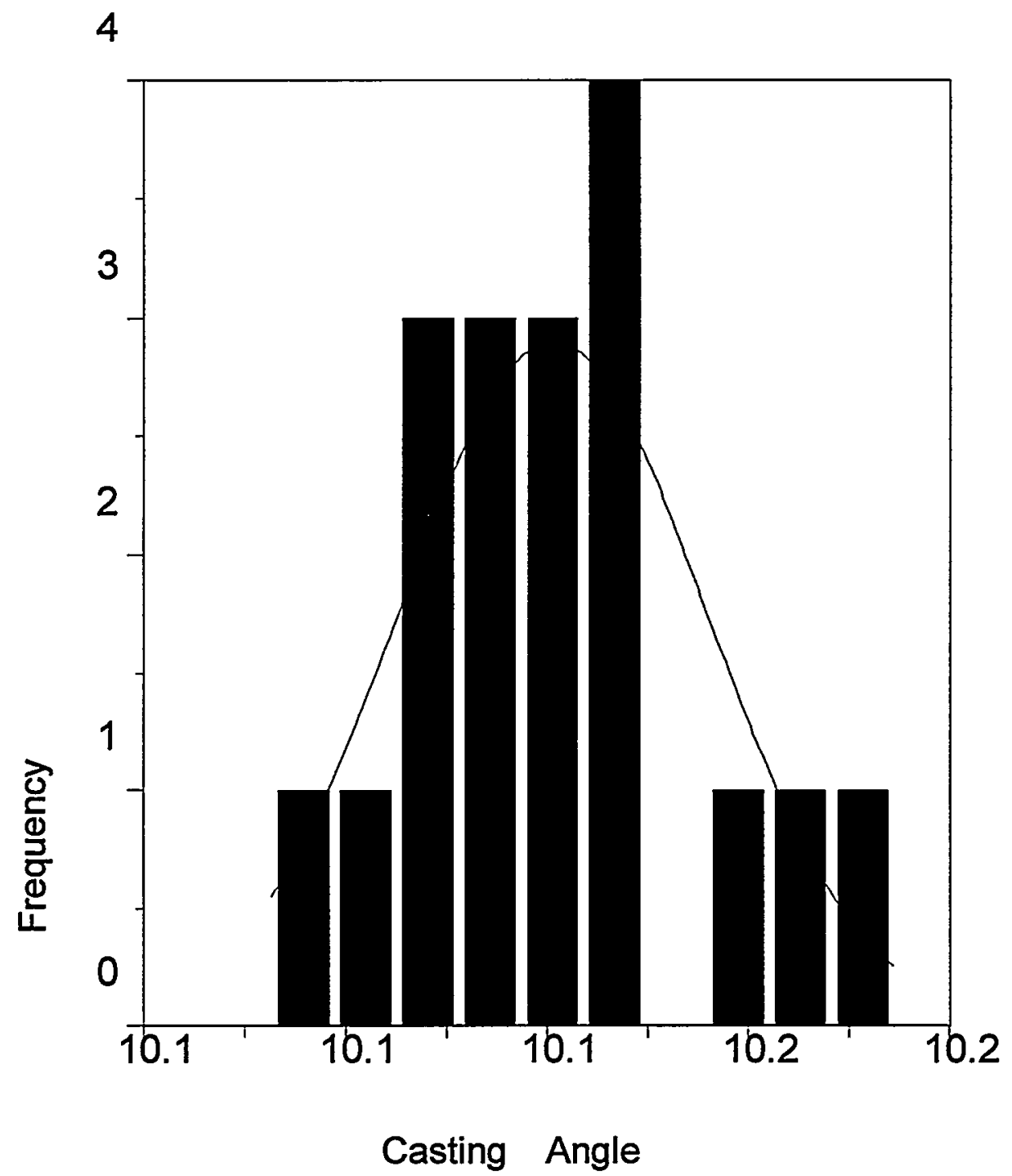

Figure 5.3 Histogram of Left Casting Angle Resin Bonded Sand ( 0.25 inch)

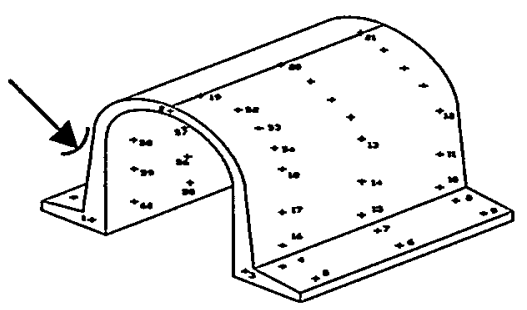




\section{Scatter Plot of Parallelism of all 0.25 inch Castings}

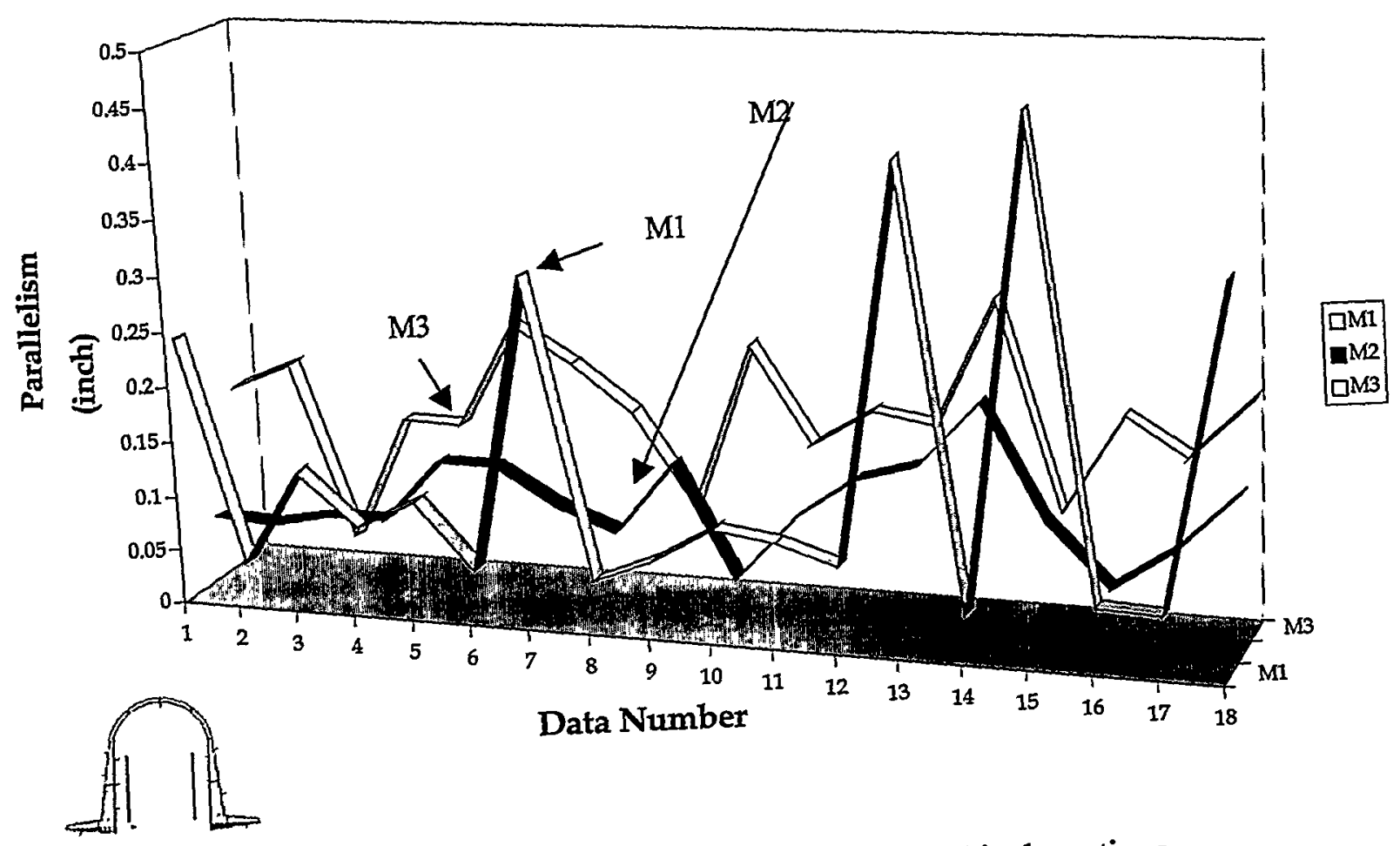

Figure 5.4 Scatter Plot of Parallelism of all 0.25 inch castings

(M1: Resin Bonded Sand; M2: Lost Foam Low Expansion; M3: Lost Foam Silica Sand) 
Table 5.1 Significant Contributors to Distortion of $1 / 4$ inch Casting $(\alpha=0.05)$

(Results of Statistical Analysis)

\begin{tabular}{|l|c|c|c|}
\hline \multicolumn{4}{|c|}{ a: All 0.25 inch castings } \\
\hline Characteristic & Gating & Orientation & Mold Type \\
\hline Thickness & - & - & - \\
\hline Height & - & - & Resin Bond \\
\hline Length & - & Down & Resin Bond \\
\hline Parallelism & - & - & LF Silica \\
\hline Left Casting Angle & - & - & - \\
\hline Right Casting Angle & - & - & Resin Bond \\
\hline Left Flange Flatness & - & - & Resin Bond \\
\hline Right Flange Flatness & - & - & Resin Bond \\
\hline Left Wall Flatness & Top ${ }^{2}$ & Down & Resin Bond \\
\hline Right W all Flatness & Top & Down & - \\
\hline
\end{tabular}

\begin{tabular}{|l|c|c|}
\hline \multicolumn{3}{|c|}{ c: Lost Foam Low Expansion } \\
\hline Characteristic & Gating & Orientation \\
\hline Thickness & - & - \\
\hline Height & - & - \\
\hline Length & - & Down \\
\hline Parallelism & - & - \\
\hline Left Casting Angle & - & - \\
\hline Right Casting Angle & - & - \\
\hline Left Flange Flatness & - & - \\
\hline Right Flange Flatness & - & Down \\
\hline Left W all Flatness & - & - \\
\hline Right Wall Flatness & - & - \\
\hline
\end{tabular}

\begin{tabular}{|c|c|c|c|c|c|}
\hline \multicolumn{3}{|c|}{\begin{tabular}{|c|} 
b: Lost Foam Silica Sand \\
\end{tabular}} & \multicolumn{3}{|c|}{ d: Resin Bonded Sand } \\
\hline Characteristic & Gating & Orientation & \begin{tabular}{|l|} 
Characteristic \\
\end{tabular} & Gating & Orientation \\
\hline Thickness & Top & Down & \begin{tabular}{|l} 
Thickness \\
\end{tabular} & - & Down \\
\hline Height & - & Down & Height & Top & - \\
\hline Length & - & Down & Length & - & - \\
\hline Parallelism & Top & - & Parallelism & - & - \\
\hline Left Casting Angle & - & - & Left Casting Angle & - & - \\
\hline Right Flange Flatness & - & - & Right Flange Flatness & - & - \\
\hline Left Wall Flatness & - & - & Left Wall Flatness & Top & Down \\
\hline \multirow[t]{2}{*}{ Right Wall Flatness } & - & - & Right Wall Flatness & Top & - \\
\hline & \multicolumn{3}{|c|}{$\begin{array}{l}\text { Notes } \\
\text { Bolded Item indicates the "Most Significant" Factor } \\
\text { Superscript indicates importance rating (rank) } \\
\text { ALL indicates that all of the factor levels affect the parameter equally } \\
\text { Dash (-) indicates that the factor had no significant contribution }\end{array}$} & & \\
\hline
\end{tabular}


Table 5.2 Significant Contributors to Distortion on 0.50 inch Castings $(\alpha=0.05$ ) (Results of Statistical Analysis)

\begin{tabular}{|l|c|c|c|}
\hline \multicolumn{4}{|c|}{ a: $\mathbf{l l}$ 0.50 inch Castings } \\
\hline Characteristic & Gating & Orientation & Mold Type \\
\hline Thickness & - & - & ALL \\
\hline Height & & & Resin Bonded \\
\hline Length & - & ALL & Resin Bonded \\
\hline Parallelism & - & - & Low-Expan. \\
\hline Left Casting Angle & - & - & Resin Bonded \\
\hline Right Casting Angle & - & - & - \\
\hline Left Flange Flatness & - & - & - \\
\hline Right Flange Flatness & ALL & - & LF Silica \\
\hline Left Wall Flatness & ALL & ALL & ALL \\
\hline Right Wall Flatness & - & - & Low-Expan. \\
\hline
\end{tabular}

\begin{tabular}{|l|c|c|}
\hline \multicolumn{3}{|c|}{ c: Resin Bonded Sand } \\
\hline Characteristic & Gating & Orientation \\
\hline Thickness & - & - \\
\hline Height & - & ALL \\
\hline Length & - & ALL \\
\hline Parallelism & - & ALL \\
\hline Left Casting Angle & - & - \\
\hline Right Casting Angle & - & - \\
\hline Left Flange Flatness & ALL & - \\
\hline Right Flange Flatness & - & - \\
\hline Left Wall Flatness & ALL & ALL \\
\hline Right Wall Flatness & Top & ALL \\
\hline
\end{tabular}

\section{b: Lost Foam Silica Sand}

\begin{tabular}{|l|c|c|}
\hline \multicolumn{3}{|c|}{ b: Lost Foam Silica Sand } \\
\hline Characteristic & Gating & Orien tation \\
\hline Thickness & - & - \\
\hline Height & ALL & - \\
\hline Length & - & - \\
\hline Parallelism & - & - \\
\hline Left Casting Angle & - & - \\
\hline Right Casting Angle & - & - \\
\hline Left Flange Flatness & - & - \\
\hline Right Flange Flatness & ALL & ALL \\
\hline Left Wall Flatness & - & - \\
\hline Right Wall Flatness & - & - \\
\hline
\end{tabular}

d: Lost Foam Low Expansion

\begin{tabular}{|l|c|c|}
\hline Characteristic & Gating & Orientation \\
\hline Thickness & - & - \\
\hline Height & - & - \\
\hline Length & - & Up \\
\hline Parallelism & - & - \\
\hline Left Casting Angle & - & - \\
\hline Right Casting Angle & - & - \\
\hline Left Flange Flatness & - & - \\
\hline Right Flange Flatness & ALL & ALL \\
\hline Left Wall Flatness & - & - \\
\hline Right Wall Flatness & - & - \\
\hline
\end{tabular}

Notes

Bolded Item indicates the "Most Significant" Factor

Superscript indicates importance rating (rank)

ALL indicates that all of the factor levels affect the parameter equally

Dash (-) indicates that the factor had no significant contribution

Table 5.3 Significant Contributors to Distortion on 0.75 inch castings $(\alpha=0.05)$

(Results of Statistical Analysis 


\begin{tabular}{|l|c|c|c|}
\hline \multicolumn{4}{|c|}{ a: A ll 0.75 inch castings } \\
\hline Characteristic & Gating & Orientation & Mold Type \\
\hline Thickness & - & - & Resin-Bond \\
\hline Height & - & ALL & Resin-Bond \\
\hline Length & - & - & Resin-Bond \\
\hline Parallelism & - & - & Resin-Bond \\
\hline Left Casting Angle & ALL & & ALL \\
\hline Right Casting Angle & - & ALL & Resin-Bond \\
\hline Left Flange Flatness & - & - & - \\
\hline Right Flange Flatness & - & ALL & ALL \\
\hline Left W all Flatness & - & - & Resin-Bond \\
\hline Right W all Flatness & - & - & Resin-Bond \\
\hline
\end{tabular}

\begin{tabular}{|l|c|c|}
\hline \multicolumn{3}{|c|}{ c: Lost Foam Low Expansion } \\
\hline Characteristic & Gating & Orientation \\
\hline Thickness & A LL & A LL \\
\hline Height & A LL & A LL \\
\hline Length & - & - \\
\hline Parallelism & - & - \\
\hline Left Casting Angle & - & - \\
\hline Right Casting Angle & - & - \\
\hline Left Flange Flatness & - & ALL \\
\hline Right Flange Flatness & - & - \\
\hline Left Wall Flatness & - & ALL \\
\hline Right W all Flatness & - & - \\
\hline
\end{tabular}

\begin{tabular}{|l|c|c|}
\hline \multicolumn{3}{|c|}{ b: Lost Foam Silica Sand } \\
\hline Characteristic & Gating & Orientation \\
\hline Thickness & Top & - \\
\hline Height & - & Down \\
\hline Length & - & Dow n \\
\hline Parallelism & - & Dow n \\
\hline Left Casting Angle & - & - \\
\hline Right Casting Angle & Top & - \\
\hline Left Flange Flatness & - & A LL \\
\hline Right Flange Flatness & - & - \\
\hline Left Wall Flatness & - & ALL \\
\hline Right W all Flatness & - & - \\
\hline
\end{tabular}

\begin{tabular}{|l|c|c|}
\hline \multicolumn{3}{|c|}{ d: Resin Bonded Sand } \\
\hline Characteristic & Gating & Orientation \\
\hline Thickness & - & - \\
\hline Height & ALL & ALL \\
\hline Length & - & - \\
\hline Parallelism & ALL & ALL \\
\hline Left Casting Angle & ALL & ALL \\
\hline Right Casting Angle & - & ALL \\
\hline Left Flange Flatness & ALL & ALL \\
\hline Right Flange Flatness & - & - \\
\hline Left W all Flatness & - & - \\
\hline Right W all Flatness & ALL & - \\
\hline
\end{tabular}

Notes

Bolded Item indicates the "Most Significant" Factor

Superscript indicates importance rating (rank)

ALL indicates that all of the factor levels affect the parameter equally

Dash $(-)$ indicates that the factor had no significant contribution 
Table 5.4 shows the results of the all of the castings. The results for all of the resinbonded sand castings are shown in Table 5.4a. According to the analysis, all orientation configurations equally affected the thickness, height, length, left casting angle, and left flange flatness. The thickness, length, and parallelism was affected by the $3 / 4$ inch thickness, while the $1 / 2$ inch thickness significantly affected the left and right casting angle, and right flange flatness. Top gating contributed significantly to the height distortion. All gating configurations equally affected the left casting angle and left flange flatness.

Table 5.4b shows the results for all of the lost foam low expansion castings. The parallelism and left casting angle were affected by $1 / 2$ inch thickness, the right flange flatness and right wall flatness were affected by the $1 / 4$ inch thickness and the thickness was affected by $3 / 4$ inch thickness. All orientation configuration configurations equally affected the height, length, and right flange flatness. Down orientation significantly contributed to the distortion of the left flange flatness. All gating configurations affected the right flange flatness equally.

Table 5.4c shows the results for all of the lost foam silica sand castings. The thickness, height, parallelism, left casting angle, right casting angle were affected by the $3 / 4$ inch thickness. All thickness levels contributed equally to the right flange flatness, left wall flatness, and right wall flatness. Orientation down affected the thickness, height, length, and right casting angle. Side orientation affected the left flange flatness. Top gating affected the thickness and parallelism. All gating, orientation, and thickness levels affected the right flange flatness.

\subsubsection{Ranking of Significant Factors}

Ranking is a method of indicating the relative importance. Ranking of significant factors indicates the relative importance of a factor. A two step method was utilized for determining the relative importance of the factors.

- Step 1: Finding the difference between F-value and the Fcritical value for the factors (from the ANOVA table) that significantly contributed to the error of a parameter

- Step 2: Determining the relative significance by comparing the differences of each factor affecting a parameter

In Tables 5.1-5.4, bolding and superscripting indicate the rank of the factor as determined by these two steps. For example, upon viewing the significant factors affecting the length of all of the 0.25-inch castings (Table 5.1a), there are two significant factors, orientation and mold type. The bolding of resin-bonded indicates that the mold type factor was most important (from ANOVA) and resin-bonded sand is the factor level with the most contribution to the distortion of the length parameter of all 0.25 -inch castings. In this case only two factors were significant, thus only bolding was used to show the most significant factor and factor level (factor: mold type, level: resin bonded). The left wall flatness error of all of the 0.25-inch castings (Table 5.1a) is due to the gating, orientation, and mold-type. The rank is indicated by superscripts. The order of factor significance, ranked from highest to lowest is sand type, gating, and orientation. From the bolding and superscripting, it is easily shown that mold type contributed the most to the error and 
Table 5.4 Significant Contributors to Distortion on all castings $(\alpha=0.05)$

\begin{tabular}{|l|c|c|c|}
\hline \multicolumn{4}{|c|}{ a: Resin Bonded Sand Castings } \\
\hline Characteristic & Gating & Orientation & Thickness \\
\hline Thickness & - & ALL $^{2}$ & $\mathbf{0 . 7 5}^{1}$ \\
\hline Height & Top $^{1}$ & ALL $^{3}$ & ALL $^{2}$ \\
\hline Length & - & ALL $^{2}$ & $0.75^{1}$ \\
\hline Parallelism & - & - & 0.75 \\
\hline Left Casting Angle & ALL $^{3}$ & ALL $^{2}$ & $\mathbf{0 . 5}$ \\
\hline Right Casting Angle & - & - & 0.5 \\
\hline Left Flange Flatness & ALL $^{1}$ & ALL $^{2}$ & - \\
\hline Right Flange Flatness & - & - & 0.5 \\
\hline Left Wall Flatness & - & - & - \\
\hline Right Wall Flatness & - & - & - \\
\hline
\end{tabular}

\begin{tabular}{|l|c|c|c|}
\hline \multicolumn{4}{|c|}{ c: Lost Foam Low Expansion } \\
\hline Characteristic & Gating & Orientation & hicknes \\
\hline Thickness & - & ALL $^{2}$ & $\mathbf{0 . 7 5}^{1}$ \\
\hline Height & - & & ALL \\
\hline Length & - & ALL $^{2}$ & ALL $^{1}$ \\
\hline Parallelism & - & - & $\mathbf{0 . 5}$ \\
\hline Left Casting Angle & - & - & $\mathbf{0 . 5}$ \\
\hline Right Casting Angle & - & - & - \\
\hline Left Flange Flatness & - & Down $^{2}$ & - \\
\hline Right Flange Flatness & ALL $^{2}$ & ALL $^{3}$ & $\mathbf{0 . 2 5}$ \\
\hline Left Wall Flatness & - & - & - \\
\hline Right Wall Flatness & - & - & 0.25 \\
\hline
\end{tabular}

\begin{tabular}{|c|c|c|c|c|}
\hline \multicolumn{4}{|c|}{$\begin{array}{l}\text { b: Lost Foam Silica Sand } \\
\end{array}$} & \\
\hline Characteristic & Gating & Orientation & Thickness & \\
\hline Thickness & Top $^{2}$ & Down $^{3}$ & $0.75^{1}$ & \\
\hline Height & - & Down $^{2}$ & $0.75^{1}$ & \\
\hline Length & - & Down & - & \\
\hline Parallelism & Top & - & 0.75 & \\
\hline Left Casting Angle & - & - & 0.75 & \\
\hline Right Casting Angle & - & Down $^{2}$ & $0.75^{1}$ & \\
\hline Left Flange Flatness & - & Side & - & \\
\hline Right Flange Flatness & $\mathrm{ALL}^{2}$ & $\mathrm{ALL}^{3}$ & $\mathrm{ALL}^{\prime}$ & \\
\hline Left Wall Flatness & - & - & ALL & \\
\hline \multirow[t]{2}{*}{ Right Wall Flatness } & - & - & ALL & \\
\hline & \multicolumn{4}{|c|}{$\begin{array}{l}\text { Notes } \\
\text { Bolded Item indicates the "Most Significant" Factor } \\
\text { Superscript indicates importance rating (rank) } \\
\text { ALL indicates that all of the factor levels affect the parameter equally } \\
\text { Dash (-) indicates that the factor had no significant contribution }\end{array}$} \\
\hline
\end{tabular}


resin bonded sand is the level or type of mold that contributed the most to the error of all of the 0.25 inch castings.

\subsubsection{Analysis of Variation from Nominal}

The nominal values are listed in Table 5.5. The analysis of variation from nominal indicates how well the process hit the target for the parameters (length, height, etc.). It shows the deviation of the calculated values of the parameters from the targeted values. "Bullseye" plots were used to display the results. The "Bullseye" is the visual representation of the actual values for each characteristic parameter in reference to the nominal values. The average deviation for each parameter of each molding process (mold type) is located on the "Bullseye" chart. Appendix C contains the full set of "Bullseye" plots. A sample is in Figure 5.5. Table 5.6 summarizes the molding process parameter deviations from the actual design nominal values. The design nominal values were those given during product design. Table 5.7 shows the deviation from the calculated nominal values. These calculated nominal values are simply the average calculated values of each parameter from the CMM point cloud data. These were the target values used for the "Bulleyes".

Table 5.5 Nominal Values (Design and Calculated)

\begin{tabular}{|c|c|c|c|c|c|c|c|}
\hline Parameters & Unit & \multicolumn{3}{|c|}{ Design Nominal Values } & \multicolumn{3}{|c|}{ Calculated Nominal Values(CMM) } \\
\hline Thickness & inch & 0.25 & 0.50 & 0.75 & 0.26 & 0.52 & 0.77 \\
\hline Height & inch & & 8 & & & 8.70 & \\
\hline Length & inch & & 6 & & & 6.78 & \\
\hline Parallelism & inch & & 0 & & & 0.10 & \\
\hline Left Casting Angle & degrees & & 8 & & & 10.00 & \\
\hline Right Casting Angle & degrees & & 8 & & & 10.00 & \\
\hline Left Flange Flatness & inch & & 0 & & & 0.01 & \\
\hline Right Flange Flatness & inch & & 0 & & & 0.05 & \\
\hline Left Wall Flatness & inch & & 0 & & & 0.02 & \\
\hline Right Wall Flatness & inch & & 0 & & & 0.03 & \\
\hline
\end{tabular}

Bullseye of Length error of 0.25 inch castings

(Nominal Value: 6 in.)

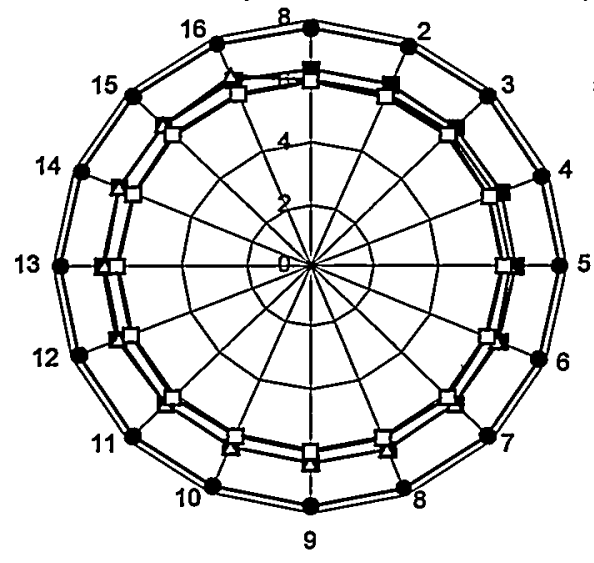

Average Difference RBS $=1.768$ LFLE $=0.4268$

LFSS $=.3523$

Figure 5.5 Bullseye Plot of Length of 0.25 inch castings ( ${ }^{\star}$ RBS: Resin Bonded Sand, LFSS: Lost Foam Silica Sand, LFLE: Lost Foam Low Expansion) 
Table 5.6 Deviation from Design Nominal

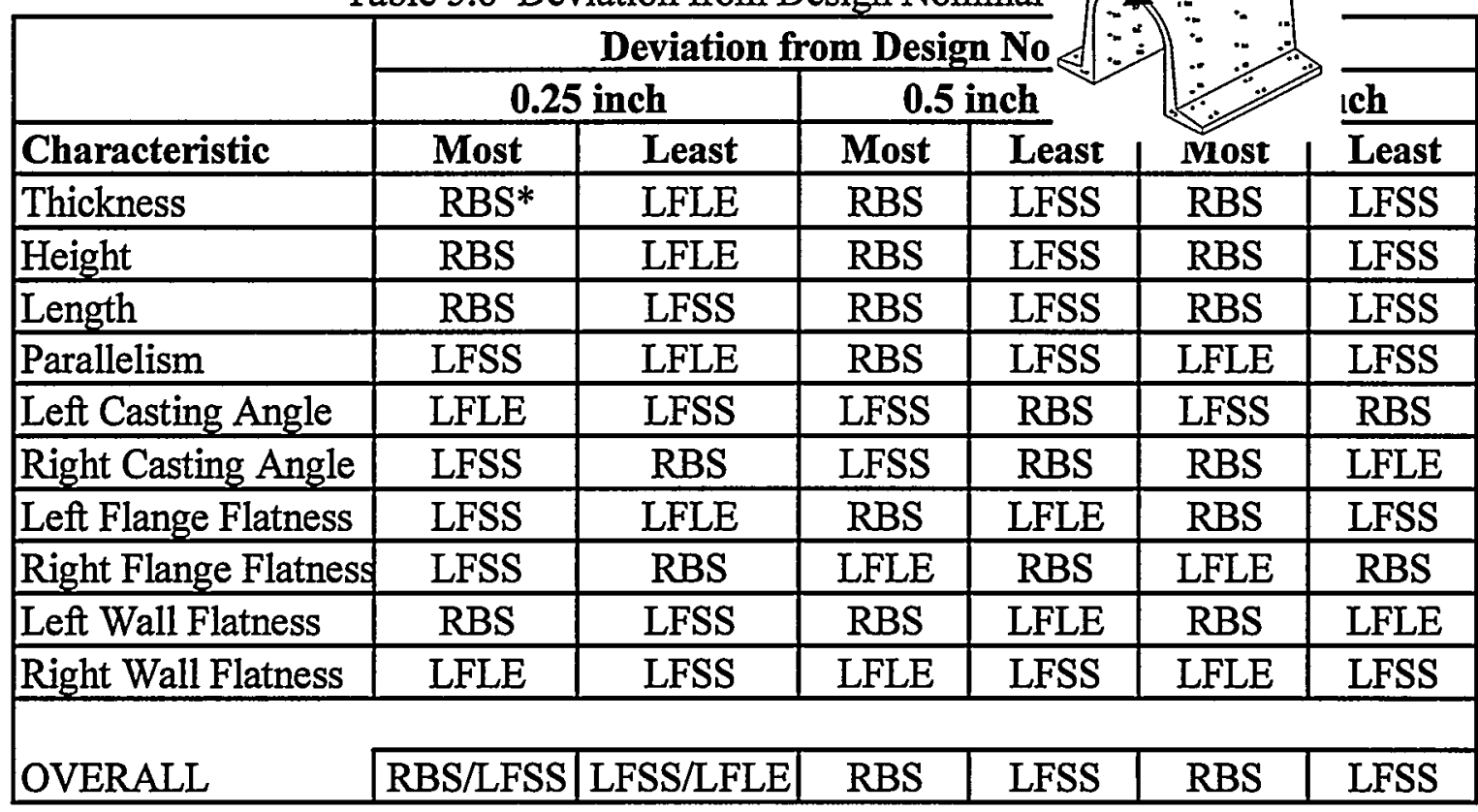

Table 5.7 Deviation from Calculated Nominal Value

\begin{tabular}{|c|c|c|c|c|c|c|}
\hline \multirow{3}{*}{\begin{tabular}{|l} 
\\
Characteristic \\
\end{tabular}} & \multicolumn{6}{|c|}{ Deviation from Calculated Nominal Value } \\
\hline & \multicolumn{2}{|c|}{0.25 inch } & \multicolumn{2}{|c|}{0.5 inch } & \multicolumn{2}{|c|}{0.75 inch } \\
\hline & Most & Least & Most & Least & Most & Least \\
\hline Thickness & RBS & LFSS & RBS & LFLE & RBS & LFLE \\
\hline Height & RBS & LFLE & RBS & LFLE & RBS & LFLE \\
\hline Length & RBS & LFSS & RBS & LFLE & RBS & LFLE \\
\hline Parallelism & $\mathrm{RBS}$ & LFLE & LFSS & RBS & LFSS & LFLE \\
\hline Left Casting Angle & LFLE & LFSS & LFSS & RBS & LFSS & RBS \\
\hline Right Casting Angle & RBS & RBS & LFSS & RBS & $\mathrm{RBS}$ & LFLE \\
\hline Left Flange Flatness & LFLE & LFSS & LFLE & RBS & LFSS & RBS \\
\hline Right Flange Flatness & RBS & LFSS & RBS & LFLE & LFLE & LFSS \\
\hline Left Wall Flatness & $\overline{\mathrm{RBS}}$ & $\overline{\text { LFLE }}$ & $\overline{\mathrm{RBS}}$ & LFSS & RBS & LFSS \\
\hline Right Wall Flatness & RBS & LFLE & LFSS & LFLE & LFLE & RBS \\
\hline Overall & RBS & LFSS & RBS & LFLE & RBS & LFLE \\
\hline
\end{tabular}

\section{*RBS: Resin Bonded Sand, LFSS: Lost Foam Silica Sand, LFLE: Lost Foam Low Expansio}

\subsection{CONCLUSIONS}

The purpose of this work was to use statistical analysis as a viable tool in the foundry industry. The primary objective was to determine the effect of gating, orientation, molding 
process, and thickness on casting distortion. The use of the analysis methodologies in this work has proven that statistical analysis may be used with confidence in the foundry based on the data collected from the coordinate measuring machine (CMM).

The statistical analysis confirmed that the type of gating systems, orientation, molding process, and casting thickness indeed influence the dimensions of the casting. The factorial analysis of variance determined the effect of each factor on each feature (length, height, thickness, parallelism, casting angle, and flatness). The test of means was an effective method for determining the factor level effects. It is noted that there may have been errors introduced to the castings in association with process error, human error, batch error, multiple mold error, etc. Yet, for this analysis, it was assumed that all of the parameter variations found were solely due to the gating, orientation, thickness, and mold type.

For all of the castings, the thickness levels, orientation and gating significantly contributed to the distortion of the features. For all of the resin-bonded castings, $3 / 4$ and $1 / 2$ inch thickness played a significant role in casting distortion. Gating and orientation also played crucial roles in the distortion. Orientation down, $3 / 4$ inch thickness, and top gating affected the lost foam silica sand castings. The thickness level $(3 / 4,1 / 2,1 / 4$ inch) most significantly affected the lost foam low expansion castings compared with the orientation and gating.

Resin bonded sand, orientation down, and top-gating mostly affected all of the $1 / 4$ inch castings. The molding process (mold type) is the most significant factor affecting the casting distortion. The deviation from nominal analysis indicated that the lost foam silica sand method had comparatively less deviation from the designed nominal values, while the lost foam low expansion method varied the least from the calculated nominal values. Resin bonded sand castings deviated the most from the designed and calculated nominal values. The most significant contributor to the distortion of all of the castings was resin-bonded sand. Also, the ANOVA analysis indicated that side gating did not significantly contribute to the distortion of the castings.

\subsection{Future Work}

There are many viable areas of research that may come out of this study. Some suggestions are listed below.

1) This same study may be performed on different ferrous metals such as cast iron and steel.

2) The algorithms used to calculate the dimensional variations may be coded into the coordinate- measuring machine.

3) The understanding of point cloud data combined with neural networks may be used in a foundry setting to determine, classify, alleviate, and remedy dimensional variation.

4) Control and measure errors associated with the each process, batches, etc., so that it is possible to more accurately determine the effect of gating, orientation, thickness, and mold type on the parameter distortion. 


\subsection{REFERENCES}

Batista, Casimiro, "Application of Statistical Methods for Improvement of Metal Casting Dimensional Accuracy in Sand Molds," Masters Thesis, The Florida State University, 1997

Cattanach, Robert., etal, The Handbook for Environmentally Conscious Manufacturing: From Design and Production to Labeling and Recycling, Chicago: 1996.

Cheraghi, S. Hossein, Straightness and flatness tolerance evaluation: and optimization approach, Precision Engineering, New York: Elsevier Science, Inc., 1996.

Dargan, Tarsha, "Statistical Analysis of Point Cloud Data from a Coordinate Measuring Machine and the Application of Green Quality Function Deployment II on Sand Casting Processes," Masters Thesis, Florida Agricultural and Mechanical University,1998.

Dionne, Louis, "Lost Foam Technique for Making Fiberglass Parts", http:// www.ezone.com, October 1998

DiSylvestro, G., "Dimensional Stability of Castings Made in High Density Green Sand Molding: Phase I - Factors Which Affect Dimensional Stability", AFS Transactions, Vol. 84, pp. $745,1976$.

Doyle, Lawrence E, et al., Manufacturing Processes and Materials for Engineers, Prentice-Hall 1985, page 120-173.

Ehrenfield, John, "Moving Toward Loop-Closing Structures: Systems Issues in Achieving Environmentally Conscious Manufacturing", Center for Technology, Policy \& Industrial Development Massachusetts Institute of Technology.

Elmography, W. H., Wu, H. A., Elmography, H. A., "Evaluation of Actual Geometric Tolerance Using Coordinate Measuring Data", Advances in Design and Automation.

EPA, "Environmental Accounting Case Studies: Green Accounting at AT\&T," EPA 742- R-95003

Gess, David, Life Cycle Cost Management, Perspectives, http: www.dfi.com/lccm.html: Autumn 1994

Govers, C.P.M., "What and how about quality function deployment(QFD)," International Journal of Production Economics, Netherlands: 1996

Heine, R. W., Loper Jr., C. R., and Rosenthal, P. C., Principles of Metal Casting, Second Edition, McGraw-Hill Book Company, 1967. 
Henry, J. Glynn, et al., Environmental Science and Engineering, New Jersey: Prentice-Hall, Inc., 1996, pg. 693

Ho K., Pehlke R. D., "Mechanisms of Heat Transfer at a Metal-Mold Interface", AFS Transactions, Vol. 61, pp. 587 - 598, 1984.

Kalpakjian, S., Manufacturing Engineering and Technology, Third Edition, AdisonWesley Publishing Company, New York, 1995.

Masor, R.L., Gunst, R.F., Statistical Design and Analysis of Experiments: with Applications to Engineering and Science, John Wiley \& Sons, New York, 1989.

Menq, C. H., Yau, H. T., Lai, G. Y., Miller, R. A. "Statistical Evaluation of Form Tolerances Using Discrete Measurement Data", Advances in Integrated Product Design and Manufacturing, American Society of Mechanical Engineers, (Production Engineering Division (Publication), PED, Vol. 47, 1990, p. 135-149.

Murphy, T. S. R., and Abdin, S. Z., 1980, "Minimum Zone Evaluation of Surfaces", International Journal of Machine Tool Design and Research, Vol. 20, No. 2, 1989.

Nishida, Y., Journal of Japan Institute of Metals, Vol. 34, pp. 1135 - 1139, 1970.

Owusu, Yaw, "Short Course on Metal Casting Technology , Argonne National Laboratory, Chicago, August 13, 1996.

Owusu, Yaw A., Sodium Silicate Bonding in Foundry Sands, PhD Dissertation, The Pennsylvania State University, 1980.

Owusu, Yaw, "Systems Approach to Concurrent Engineering: Design, Manufacturing, and Product Support," January 1998.

Owusu, Yaw A., and Batista, Casimiro, Statistical Analysis of Casting Distortion using Geometrical and Dimensional Tolerance Evaluation Technique, 1997.

Piwonka, T. S., "Improved Dimensional Accuracy-Is It Worth Anything to The Casting Buyer?” The Crucible, Non-Ferrous Founders Society, pp. 10, March/April 1990.

Piwonka, T. S., "Heat Transfer at the Mold-Metal Interface in Investment Castings", Proceedings of the $41^{\text {st }}$ Annual Technical Meeting, Investment Casting Institute, paper \#15, Chicago, IL, October 1993.

Rosenau, Milton D and Moran, John J, Managing the Development of New Products, New York: Van Nostrand Reinhold, 1993. 
Sobh, T. M., Zhu, X., Brudelin, B., "Analysis of Tolerance for Manufacturing Geometric Objects from Sensed Data", http://www.cs.utah.edu/ sobh/jmachine/paper.html, 1994.

Tien, R. H., and Richmond, O., "Theory of Maximum Tensile Stresses in the Solidifying Shell of a Constrained Rectangular Casting", Journal of Applied Mechanics, Vol. 49, pp. 481, 1982.

Turner, etal, Introduction to Industrial System Engineering, Prentice-Hall, New Jersey: 1978, pp. $73-76$

http://www.tcbi.com/archiesr/secondary/

http://inaba.nrim.go.jp:8080/movie/CAST/cast.html, Metal casting by the sand mold 
APPENDIX A

(Experimental Design of the Project) 


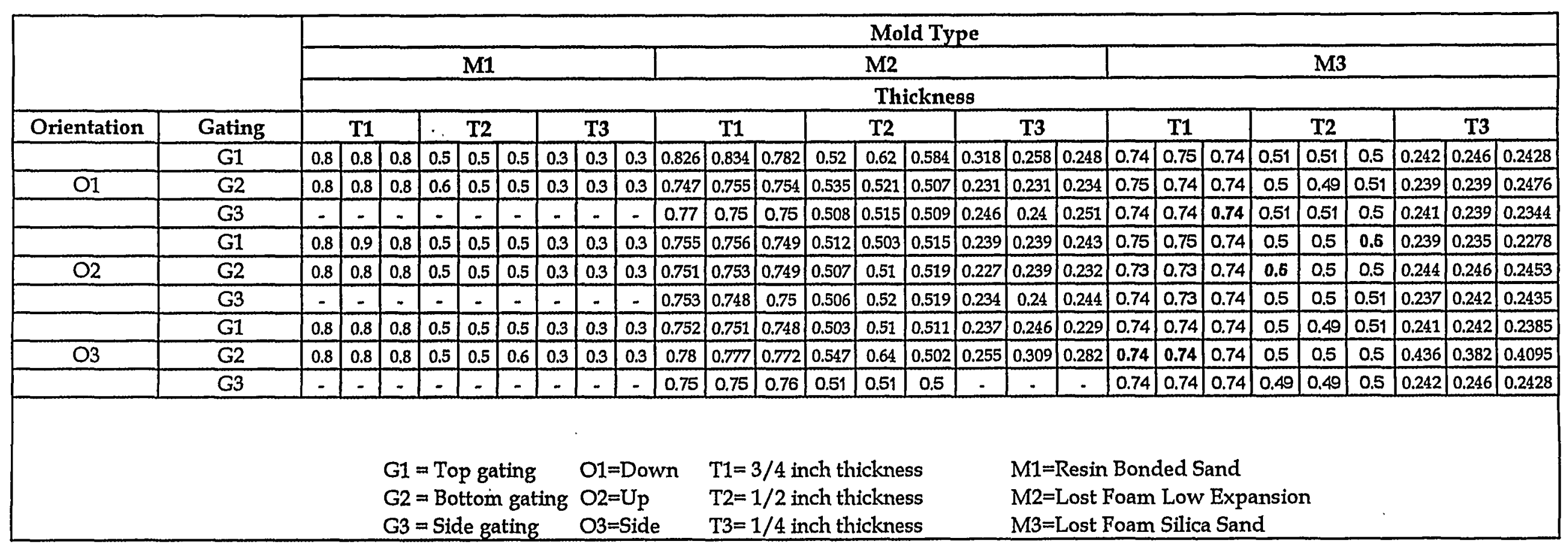

Bold indicates average was calculated to replace missing data

- indicates a full set of missing data 
Table A. 2 Experimental Design of the Project

(Height)

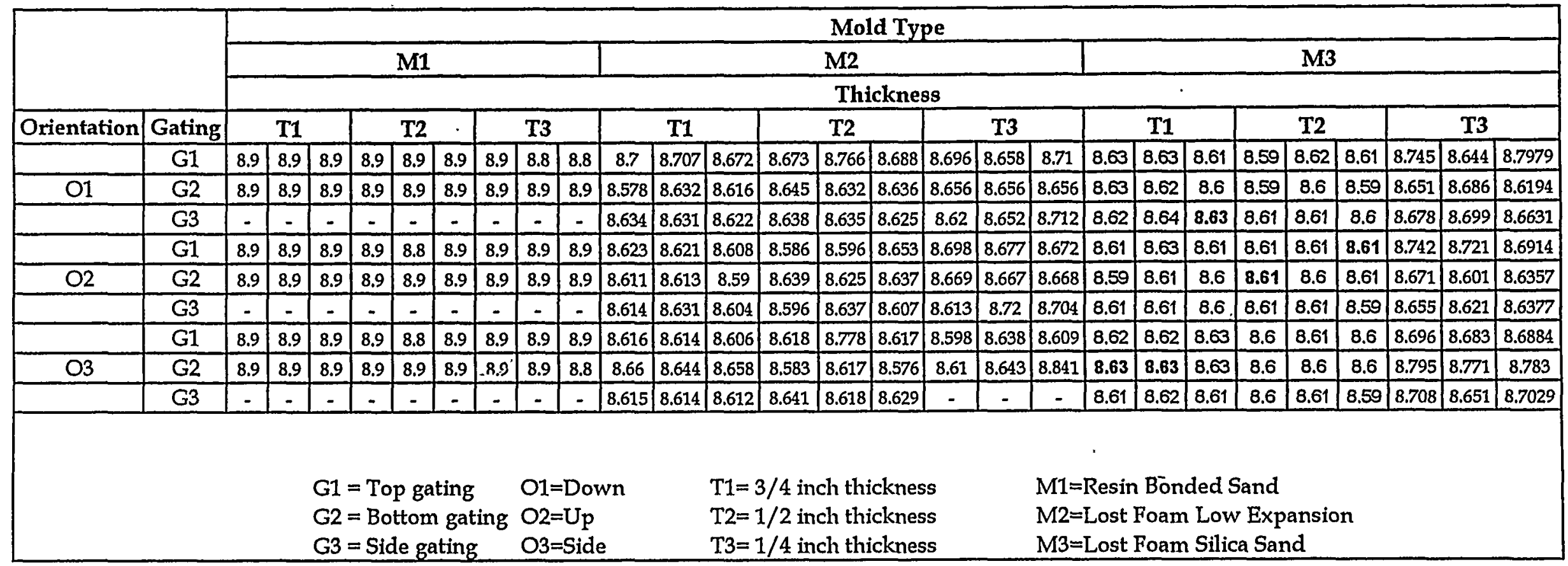

Bold indicates average was calculated to replace missing data

- indicates a full set of missing data 
Table A. 3 Experimental Design of the Project

(Length)

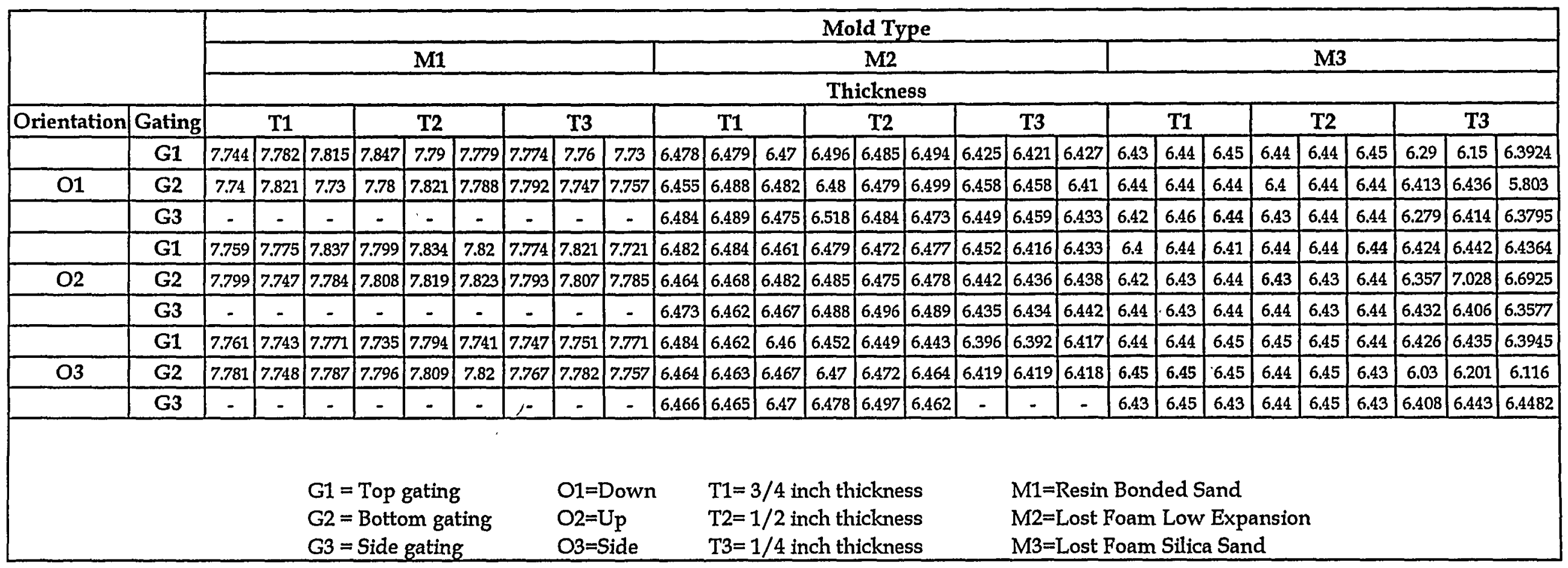

Bold indicates average was calculated to replace missing data

- indicates a full set of missing data 
Table A. 4 Experimental Design of the Project

(Parallelism)

\begin{tabular}{|c|c|c|c|c|c|c|c|c|c|c|c|c|c|c|c|c|c|c|c|c|c|c|c|c|c|c|c|c|}
\hline & & \multicolumn{27}{|c|}{ Mold Type } \\
\hline & & \multicolumn{9}{|c|}{ M1 } & \multicolumn{9}{|c|}{ M2 } & \multicolumn{9}{|c|}{ M3 } \\
\hline & & \multicolumn{27}{|c|}{ Thickness } \\
\hline Orientation & Gating & \multicolumn{3}{|c|}{$\mathrm{T} 1$} & \multicolumn{3}{|c|}{$\mathrm{T} 2$} & \multicolumn{3}{|c|}{ T3 } & \multicolumn{3}{|c|}{$\mathrm{T} 1$} & \multicolumn{3}{|c|}{$\mathrm{T} 2$} & \multicolumn{3}{|c|}{ T3 } & \multicolumn{3}{|c|}{$\mathrm{T} 1$} & \multicolumn{3}{|c|}{ T2 } & \multicolumn{3}{|c|}{ T3 } \\
\hline & G1 & 0.103 & 0.089 & 0.087 & 0.062 & 0.114 & 0.105 & 0.24 & 0.311 & 0.422 & 0.059 & 0.092 & 0.148 & 0.059 & 0.05 & 0.141 & 0.058 & 0.077 & 0.052 & 0.03 & 0.03 & 0.04 & 0.06 & 0.04 & 0.04 & 0.166 & 0.204 & 0.1682 \\
\hline \multirow[t]{3}{*}{$\mathrm{O} 1$} & $\mathrm{G} 2$ & 0.088 & 0.071 & 0.065 & 0.099 & 0.116 & 0.076 & 0.036 & 0.043 & 0.038 & 0.056 & 0.069 & 0.207 & 0.027 & 0.022 & 0.096 & 0.065 & 0.044 & 0.09 & 0.04 & 0.04 & \begin{tabular}{|l|}
0.05 \\
\end{tabular} & 0.02 & 0.03 & 0.03 & 0.189 & 0.163 & 0.2816 \\
\hline & G3 & \begin{tabular}{|l|}
- \\
\end{tabular} & - & - & - & - & - & - & - & - & 0.052 & 0.068 & 0.088 & 0.184 & 0.067 & 0.029 & 0.062 & 0.08 & 0.204 & 0.04 & 0.03 & 0.03 & 0.02 & 0.03 & 0.03 & 0.111 & 0.12 & 0.196 \\
\hline & G1 & 0.056 & 0.067 & 0.058 & 0.07 & 0.122 & 0.067 & 0.124 & 0.065 & 0.466 & 0.066 & 0.136 & 0.105 & 0.141 & 0.072 & 0.061 & 0.047 & 0.064 & 0.03 & 0.05 & 0.05 & 0.06 & 0.03 & 0.03 & 0.03 & 0.029 & 0.082 & 0.0958 \\
\hline \multirow[t]{3}{*}{$\mathrm{O} 2$} & G2 & 0.058 & 0.065 & 0.09 & 0.126 & \begin{tabular}{|l|}
0.11 \\
\end{tabular} & 0.121 & 0.077 & 0.097 & 0.053 & 0.067 & 0.5 & 0.034 & 0.019 & 0.025 & 0.033 & 0.055 & 0.039 & 0.091 & 0.06 & 0.05 & 0.07 & 0.04 & 0.03 & 0.05 & 0.931 & 0.952 & 0.9418 \\
\hline & G3 & -1 & - & - & - & \begin{tabular}{|l|}
- \\
\end{tabular} & - & - & - & - & 0.056 & 0.046 & 0.036 & 0.111 & 0.073 & 0.062 & \begin{tabular}{|l|}
0.04 \\
\end{tabular} & 0.086 & 0.049 & 0.05 & 0.05 & 0.07 & 0.05 & 0.04 & 0.04 & 0.173 & 0.206 & 0.1808 \\
\hline & G1 & 0.048 & 0.039 & 0.034 & 0.054 & 0.071 & 0.059 & 0.105 & 0.089 & 0.05 & 0.123 & 0.093 & 0.087 & 0.077 & 0.054 & 0.07 & 0.036 & 0.054 & 0.068 & 0.02 & 0.04 & 0.02 & 0.07 & 0.05 & 0.03 & 0.142 & 0.143 & 0.1531 \\
\hline \multirow[t]{2}{*}{$\mathrm{O} 3$} & G2 & \begin{tabular}{|l|}
0.088 \\
\end{tabular} & 0.073 & 0.095 & 0.042 & 0.062 & 0.098 & 0.044 & 0.072 & 0.336 & 0.123 & 0.132 & 0.141 & 0.118 & 0.064 & 0.063 & 0.038 & 0.073 & 0.016 & 0.03 & \begin{tabular}{|l|} 
\\
\end{tabular} & \begin{tabular}{|l|}
0.03 \\
\end{tabular} & \begin{tabular}{|l|}
0.03 \\
\end{tabular} & $\begin{array}{ll}0.05 \\
\end{array}$ & 0.03 & 0.237 & 0.176 & 0.206 \\
\hline & G3 & -1 & - & - & - & 4 & 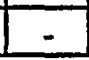 & $1-$ & - & - & 0.075 & 0.025 & 0.06 & \begin{tabular}{|l|}
0.045 \\
\end{tabular} & 0.107 & 0.042 & -1 & $1-1$ & - & 0.05 & 0.04 & 0.05 & 0.04 & 0.05 & 0.06 & 0.095 & 0.186 & 0.1252 \\
\hline & & & & \multicolumn{4}{|c|}{$\begin{array}{l}\text { G1 }=\text { Top gating } \\
\text { G2 = Bottom gating } \\
\text { G3 = Side gating }\end{array}$} & $\begin{array}{l}\mathrm{O} 1=\mathrm{I} \\
\mathrm{O} 2=\mathrm{L} \\
\mathrm{O} 3=\mathrm{S}\end{array}$ & $\begin{array}{l}\text { Down } \\
\text { Jp } \\
\text { Side }\end{array}$ & & \multicolumn{6}{|c|}{$\begin{array}{l}\mathrm{T} 1=3 / 4 \text { inch thickness } \\
\mathrm{T} 2=1 / 2 \text { inch thickness } \\
\mathrm{T} 3=1 / 4 \text { inch thickness }\end{array}$} & & \multicolumn{8}{|c|}{$\begin{array}{l}\text { M1=Resin Bonded Sand } \\
\text { M2=Lost Foam Low Expansion } \\
\text { M3 }=\text { Lost Foam Silica Sand }\end{array}$} & & & \\
\hline
\end{tabular}

Bold indicates average was calculated to replace missing data

- indicates a full set of missing data 
Table A. 5 Experimental Design of the Project

(Left Casting Angle)

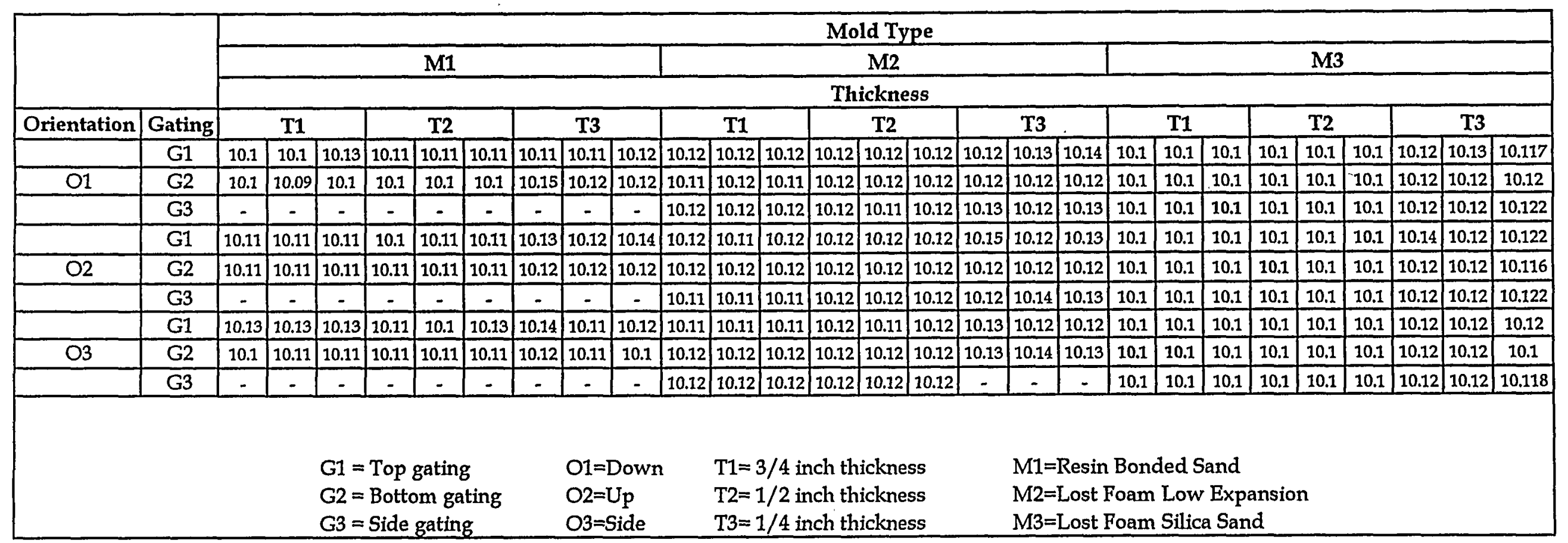

Bold indicates average was calculated to replace missing data

- indicates a full set of missing data 
Table A. 6 Experimental Design of the Project

(Right Casting Angle)

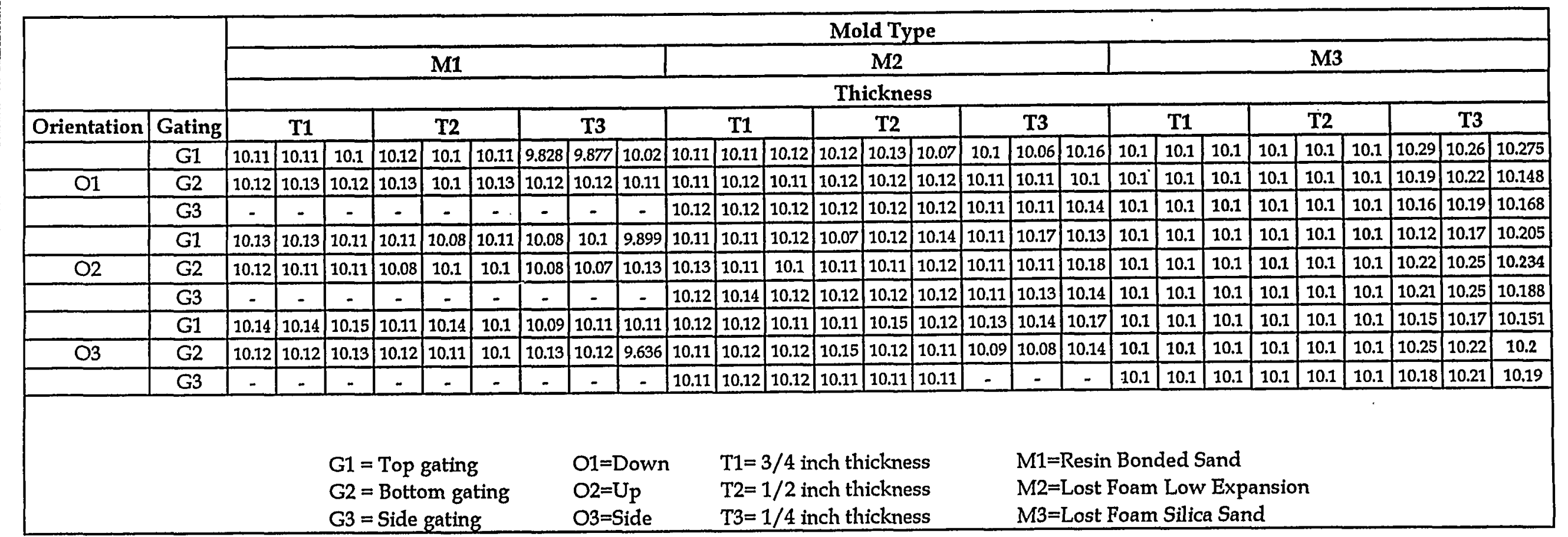

Bold indicates average was calculated to replace missing data

- indicates a full set of missing data 
Table A. 7 Experimental Design of the Project

(Left Flange Flatness)

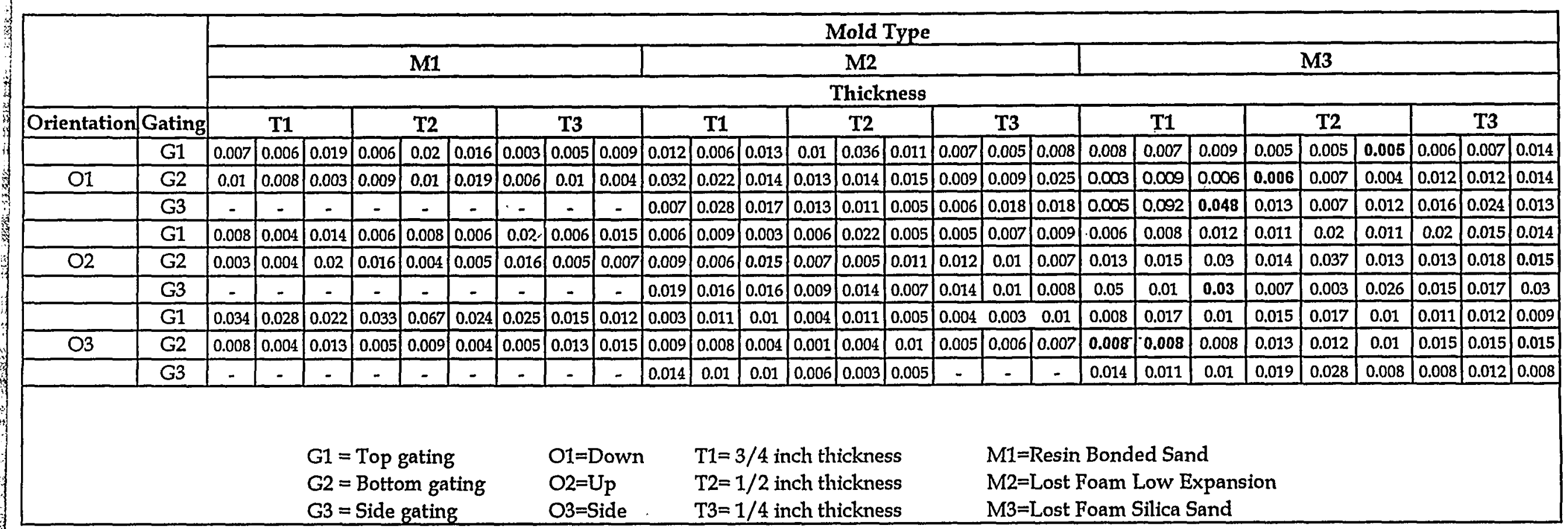

Bold indicates average was calculated to replace missing data

- indicates a full set of missing data 
Table A. 9 Experimental Design of the Project

(Left Wall Flatness)

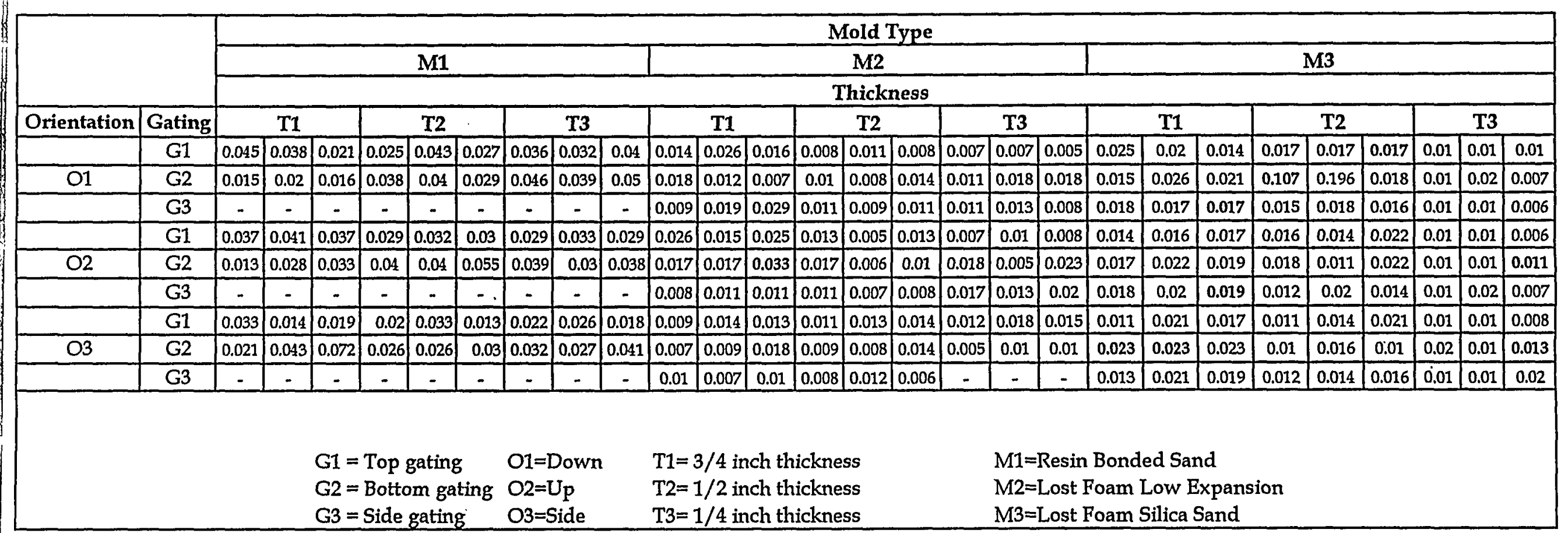

Bold indicates average was calculated to replace missing data

- indicates a full set of missing data 
Table A. 10 Experimental Design of the Project (Right Wall Flatness)

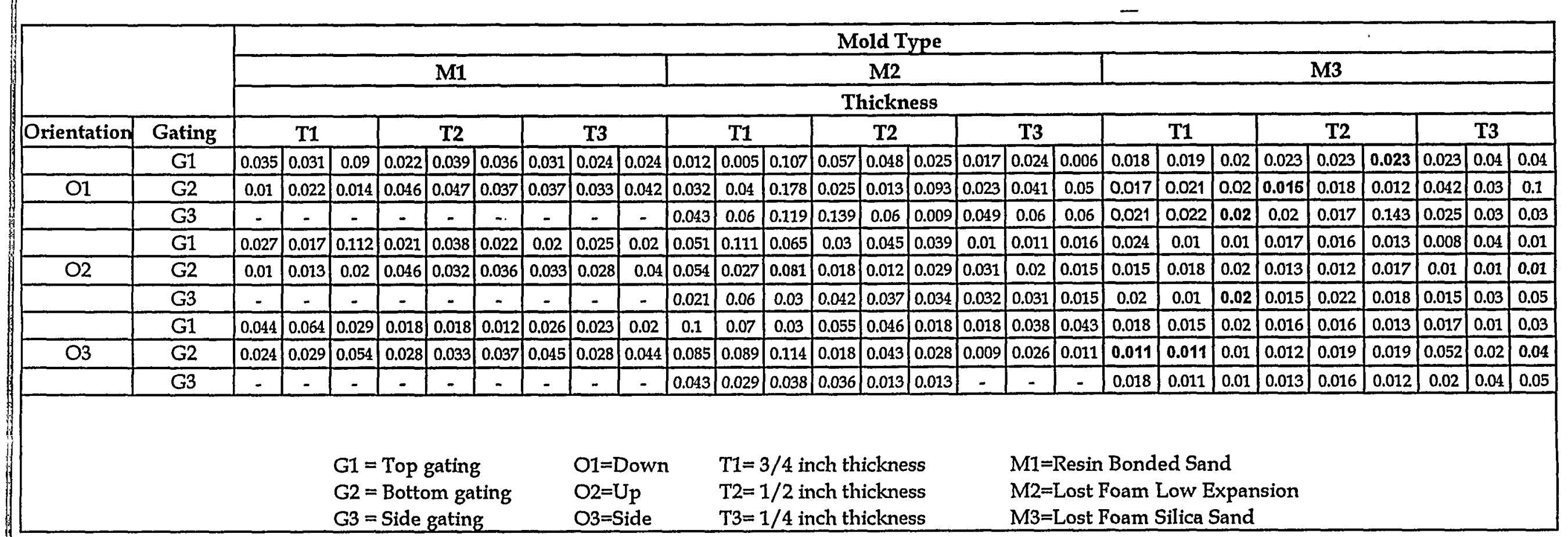

Bold indicates average was calculated to replace missing data - indicates a full set of missing data 
APPENDIX B

(ANOVA and Newman Keuls) 
Table B.1 Factorial Analysis of Variance: Height (all 0.25 inch castings)

\author{
Number of Factors $=3$ \\ Factor \\ $\mathrm{G}=$ Gating System \\ $\mathrm{O}=$ Orientation \\ $\mathrm{M}=$ Sand Type
}

$\begin{array}{lcc}\text { Source } & \text { SS } & \text { DF } \\ \text { GOM } & 0.0384 & 4 \\ \text { GO } & 0.0045 & 2 \\ \text { GM } & 0.0123 & 2 \\ \text { G } & 0.0019 & 1 \\ \text { OM } & 0.022 & 4 \\ \text { O } & 0.0018 & 2 \\ \text { M } & 0.5826 & 2 \\ \text { Error } & 0.0509 & 36 \\ & & \\ \text { Total } & 0.7144 & 53\end{array}$

$0.7144-53$
Number of Replicates $=3$ Levels

2

3

3

\begin{tabular}{|c|c|c|c|c|c|c|}
\hline MS & $\mathbf{F}$ & $\mathbf{P}$ & $\begin{array}{c}F \\
0.01\end{array}$ & significant? & $\begin{array}{c}F \\
0.05\end{array}$ & significant? \\
\hline 0.0096 & 6.7949 & 0.0004 & 3.8903067 & TRUE & 2.6335343 & TRUE \\
\hline 0.0023 & 1.5929 & 0.2173 & 5.2478981 & FALSE & 3.2594443 & FALSE \\
\hline 0.0061 & 4.3312 & 0.0206 & 5.2478981 & FALSE & 3.2594443 & TRUE \\
\hline 0.0019 & 1.3547 & 0.2521 & 7.3955562 & FALSE & 4.1131614 & FALSE \\
\hline 0.0055 & 3.8869 & 0.01 & 3.8903067 & FALSE & 2.6335343 & TRUE \\
\hline 0.0009 & 0.6284 & 0.5392 & 5.2478981 & FALSE & 3.2594443 & FALSE \\
\hline 0.2913 & 205.9536 & $1.96 \mathrm{E}-20$ & 5.2478981 & TRUE & 3.2594443 & TRUE \\
\hline
\end{tabular}


Table B.2 Factorial Analysis of Variance: Length (all 0.25 inch castings)

\author{
Number of Factors $=3$ \\ Factor \\ $\mathrm{G}=$ Gating System \\ $\mathrm{O}=$ Orientation \\ $\mathrm{M}=$ Sand Type
}

Number of Replicates $=3$

Levels

2

3

3

\begin{tabular}{|c|c|c|c|c|c|c|c|c|c|}
\hline & & & & & & $\mathbf{F}$ & & $\mathbf{F}$ & \\
\hline Source & SS & DF & MS & $\mathbf{F}$ & $\mathbf{P}$ & 0.01 & significant? & 0.05 & significant? \\
\hline GOM & 0.1593 & 4 & 0.0398 & 2.6608 & 0.0482 & 3.8903067 & FALSE & 2.6335343 & TRUE \\
\hline GO & 0.0788 & 2 & 0.0394 & 2.6331 & 0.0857 & 5.2478981 & FALSE & 3.2594443 & FALSE \\
\hline $\mathbf{G M}$ & 0.0072 & 2 & 0.0036 & 0.242 & 0.7863 & 5.2478981 & FALSE & 3.2594443 & FALSE \\
\hline $\mathbf{G}$ & $6.02 \mathrm{E}-05$ & 1 & $6.02 E-05$ & 0.004 & 0.9498 & 7.3955562 & FALSE & 4.1131614 & FALSE \\
\hline $\mathbf{O M}$ & .0 .2237 & 4 & 0.0559 & 3.736 & 0.0121 & 3.8903067 & FALSE & 2.6335343 & TRUE \\
\hline $\mathbf{0}$ & $0.1564^{\circ}$ & 2 & 0.0782 & 5.2235 & 0.0102 & 5.2478981 & FALSE & 3.2594443 & TRUE \\
\hline $\mathbf{M}$ & 22.7517 & 2 & 11.3758 & 760.1056 & $3.60 \mathrm{E}-30$ & 5.2478981 & TRUE & 3.2594443 & TRUE \\
\hline Error & 0.5388 & 36 & 0.015 & & & & & & \\
\hline Total & 23.9159 & 53 & & & & & & & \\
\hline
\end{tabular}


Table B.3 Factorial Analysis of Variance: Parallelism (all 0.25 inch castings)

Number of Factors $=3$

Factor

$\mathrm{G}=$ Gating System

$\mathrm{O}=$ Orientation

Number of Replicates $=3$

$M=$ Sand Type

Levels

2

3

$\begin{array}{lccccccccc}\text { Source } & \text { SS } & \text { DF } & \text { MS } & \mathbf{F} & \mathbf{P} & \mathbf{0 . 0 1} & \text { significant? } & \mathbf{0 . 0 5} & \text { significant? } \\ \text { GOM } & 0.066 & 4 & 0.0165 & 2.9176 & 0.0345 & 3.8903067 & \text { FALSE } & 2.6335343 & \text { TRUE } \\ \text { GO } & 0.041 & 2 & 0.0205 & 3.6258 & 0.0368 & 5.2478981 & \text { FALSE } & 3.2594443 & \text { TRUE } \\ \text { GM } & 0.0823 & 2 & 0.0411 & 7.2791 & 0.0022 & 5.2478981 & \text { TRUE } & 3.2594443 & \text { TRUE } \\ \text { G } & 0.0043 & 1 & 0.0043 & 0.7561 & 0.3903 & 7.3955562 & \text { FALSE } & 4.1131614 & \text { FALSE } \\ \text { OM } & 0.0155 & 4 & 0.0039 & 0.6873 & 0.6055 & 3.8903067 & \text { FALSE } & 2.6335343 & \text { FALSE } \\ \text { O } & 0.0173 & 2 & 0.0086 & 1.5261 & 0.2311 & 5.2478981 & \text { FALSE } & 3.2594443 & \text { FALSE } \\ \text { M } & 0.0432 & 2 & 0.0216 & 3.8172 & 0.0314 & 5.2478981 & \text { FALSE } & 3.2594443 & \text { TRUE } \\ \text { Error } & 0.2035 & 36 & 0.0057 & & & & & \end{array}$

Total $\quad 0.4729 \quad 53$ 
Table B.4 Factorial Analysis of Variance: Left Casting Angle (all 0.25 inch castings)

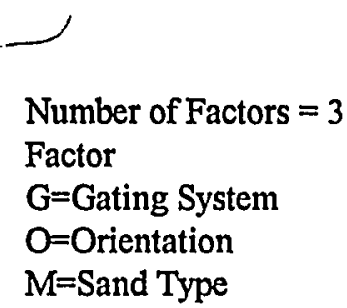

\begin{tabular}{|c|c|c|c|c|c|c|c|c|c|}
\hline & & & & & & $\mathbf{F}$ & & $\mathbf{F}$ & \\
\hline Source & SS & DF & MS & $\mathbf{F}$ & $\mathbf{P}$ & 0.01 & significant? & 0.05 & significant? \\
\hline GOM & 0.0008 & 4 & 0.0002 & 3.3044 & 0.021 & 3.8903067 & FALSE & 2.6335343 & TRUE \\
\hline GO & 0.0004 & 2 & 0.0002 & 3.0927 & 0.0576 & 5.2478981 & FALSE & 3.2594443 & FALSE \\
\hline GM & $4.42 E-06$ & 2 & $2.21 \mathrm{E}-06$ & 0.0349 & 0.9657 & 5.2478981 & FALSE & 3.2594443 & FALSE \\
\hline $\mathbf{G}$ & 0.0001 & 1 & 0.0001 & 1.6661 & 0.205 & 7.3955562 & FALSE & 4.1131614 & FALSE \\
\hline OM & 0.0002 & 4 & $5.77 \mathrm{E}-05$ & 0.9133 & 0.4667 & 3.8903067 & FALSE & 2.6335343 & FALSE \\
\hline $\mathbf{O}$ & $8.16 \mathrm{E}-05$ & 2 & $4.08 \mathrm{E}-05$ & 0.645 & 0.5306 & 5.2478981 & FALSE & 3.2594443 & FALSE \\
\hline M & 0.0004 & 2 & 0.0002 & 3.2351 & 0.051 & 5.2478981 & FALSE & 3.2594443 & FALSE \\
\hline Error & 0.0023 & 36 & $6.32 \mathrm{E}-05$ & & & & & & \\
\hline
\end{tabular}

Number of Replicates $=3$

Levels

2

3

3

Total $\quad 0.0043 \quad 53$ 
Table B.5 Factorial Analysis of Variance: Right Casting Angle (all 0.25 inch castings)

Number of Factors $=3$

Factor

$\mathrm{G}=$ Gating System

$\mathrm{O}=$ Orientation

$\mathrm{M}=$ Sand Type

\begin{tabular}{|c|c|c|c|c|c|c|c|c|c|}
\hline & & & & & & $\mathbf{F}$ & & $\mathbf{F}$ & \\
\hline Source & SS & DF & MS & $\mathbf{F}$ & $\mathbf{P}$ & 0.01 & significant? & 0.05 & significant? \\
\hline GOM & 0.1037 & 4 & 0.0259 & 4.1105 & 0.0076 & 3.8903067 & TRUE & 2.6335343 & TRUE \\
\hline GO & 0.0175 & 2 & 0.0088 & 1.3902 & 0.2621 & 5.2478981 & FALSE & 3.2594443 & FALSE \\
\hline GM & 0.0085 & 2 & 0.0043 & 0.6767 & 0.5146 & 5.2478981 & FALSE & 3.2594443 & FALSE \\
\hline G & 0.0034 & 1 & 0.0034 & 0.5468 & 0.4644 & 7.3955562 & FALSE & 4.1131614 & FALSE \\
\hline $\mathbf{O M}$ & 0.0111 & 4 & 0.0028 & 0.4381 & 0.7802 & 3.8903067 & FALSE & 2.6335343 & FALSE \\
\hline $\mathbf{0}$ & 0.0026 & 2 & 0.0013 & 0.2029 & 0.8173 & 5.2478981 & FALSE & 3.2594443 & FALSE \\
\hline M & 0.2693 & 2 & 0.1346 & 21.3397 & $7.73 \mathrm{E}-07$ & 5.2478981 & TRUE & 3.2594443 & TRUE \\
\hline Error & 0.2271 & 36 & 0.0063 & & & & & & \\
\hline Total & 0.6433 & 53 & & & & & & & \\
\hline
\end{tabular}

Number of Replicates $=3$

Levels

2

3

3 
Table B.6 Factorial Analysis of Variance: Left Flange Flatness (all 0.25 inch castings)

Number of Factors $=3$
Factor
G=Gating System
$O=$ Orientation
$M=$ Sand Type

\begin{tabular}{|c|c|c|c|c|c|c|c|c|c|}
\hline & & & & & & $\mathbf{F}$ & & $\mathbf{F}$ & \\
\hline Source & SS & DF & MS & $\mathbf{F}$ & $\mathbf{P}$ & 0.01 & significant? & 0.05 & significant? \\
\hline GOM & $4.02 \mathrm{E}-05$ & 4 & 1.01E-05 & 0.5479 & 0.7017 & 3.8903067 & FALSE & 2.6335343 & FALSE \\
\hline GO & $7.03 \mathrm{E}-05$ & 2 & $3.52 \mathrm{E}-05$ & 1.9162 & 0.1619 & 5.2478981 & FALSE & 3.2594443 & FALSE \\
\hline GM & 0.0001 & 2 & $5.87 \mathrm{E}-05$ & 3.1998 & 0.0526 & 5.2478981 & FALSE & 3.2594443 & FALSE \\
\hline $\mathbf{G}$ & $1.07 \mathrm{E}-05$ & 1 & $1.07 \mathrm{E}-05$ & 0.5812 & 0.4508 & 7.3955562 & FALSE & 4.1131614 & FALSE \\
\hline OM & 0.0003 & 4 & $6.79 \mathrm{E}-05$ & 3.7023 & 0.0127 & 3.8903067 & FALSE & 2.6335343 & TRUE \\
\hline $\mathbf{0}$ & $6.88 \mathrm{E}-05$ & 2 & $3.44 \mathrm{E}-05$ & 1.8739 & 0.1682 & 5.2478981 & FALSE & 3.2594443 & FALSE \\
\hline $\mathbf{M}$ & 0.0002 & 2 & 0.0001 & 5.997 & $5.70 \mathrm{E}-03$ & 5.2478981 & TRUE & 3.2594443 & TRUE \\
\hline Error & 0.0007 & 36 & $1.84 \mathrm{E}-05$ & & & & & & \\
\hline
\end{tabular}

Number of Replicates $=3$

Levels

2

3

\section{$\mathrm{O}=$ Orientation}

$$
\text { (3) }
$$

.

3

Total $\quad 0.0015 \quad 53$ 
Table B.7 Factorial Analysis of Variance: Right Flange Flatness (all 0.25 inch castings)

\section{Number of Factors $=3$ \\ Factor \\ $\mathrm{G}=$ Gating System \\ $\mathrm{O}=$ Orientation \\ $\mathrm{M}=$ Sand Type}

$\begin{array}{lcccc}\text { Source } & \text { SS } & \text { DF } & \text { MS } & \text { F } \\ \text { GOM } & 0.0009 & 4 & 0.0002 & 0.1602 \\ \text { GO } & 0.0003 & 2 & 0.0002 & 0.1218 \\ \text { GM } & 0.0004 & 2 & 0.0002 & 0.1668 \\ \text { G } & 3.57 E-06 & 1 & 3.57 \mathrm{E}-06 & 0.0027 \\ \text { OM } & 0.0021 & 4 & 0.0005 & 0.3898 \\ \text { O } & 0.0053 & 2 & 0.0026 & 1.9584 \\ \text { M } & 0.0224 & 2 & 0.0112 & 8.297 \\ \text { Error } & 0.0485 & 36 & 0.0013 & \\ & & & & \end{array}$

Number of Replicates $=3$ Levels

2

3

3

$\begin{array}{ccccc} & \mathbf{F} & & \mathbf{F} & \\ \mathbf{P} & \mathbf{0 . 0 1} & \text { significant? } & \mathbf{0 . 0 5} & \text { significant? } \\ 0.9571 & 3.8903067 & \text { FALSE } & 2.6335343 & \text { FALSE } \\ 0.8857 & 5.2478981 & \text { FALSE } & 3.2594443 & \text { FALSE } \\ 0.847 & 5.2478981 & \text { FALSE } & 3.2594443 & \text { FALSE } \\ 0.9592 & 7.3955562 & \text { FALSE } & 4.1131614 & \text { FALSE } \\ 0.8145 & 3.8903067 & \text { FALSE } & 2.6335343 & \text { FALSE } \\ 0.1558 & \mathbf{5 . 2 4 7 8 9 8 1} & \text { FALSE } & 3.2594443 & \text { FALSE } \\ 0.0011 & 5.2478981 & \text { TRUE } & 3.2594443 & \text { TRUE }\end{array}$


Table B.8 Factorial Analysis of Variance: Left Wall Flatness (all 0.25 inch castings)

Number of Factors $=3$
Factor
G=Gating System
O=Orientation
M=Sand Type

Number of Replicates $=3$ Levels

2

3

3

\begin{tabular}{|c|c|c|c|c|c|c|c|c|c|}
\hline & & & & & & $\mathbf{F}$ & & $\mathbf{F}$ & \\
\hline Source & SS & DF & MS & $\mathbf{F}$ & $\mathbf{P}$ & 0.01 & significant? & 0.05 & significant? \\
\hline GOM & 0.0002 & 4 & $5.66 \mathrm{E}-05$ & 2.8754 & 0.0365 & 3.8903067 & FALSE & 2.6335343 & TRUE \\
\hline GO & $3.05 \mathrm{E}-05$ & 2 & $1.52 \mathrm{E}-05$ & 0.7742 & 0.4686 & 5.2478981 & FALSE & 3.2594443 & FALSE \\
\hline GM & $1.00 \mathrm{E}-04$ & 2 & $6.31 \mathrm{E}-05$ & 3.207 & 0.0523 & 5.2478981 & FALSE & 3.2594443 & FALSE \\
\hline G & 0.0003 & 1 & 0.0003 & 13.0988 & 0.0009 & 7.3955562 & TRUE & 4.1131614 & TRUE \\
\hline OM & 0.0004 & 4 & $9.31 \mathrm{E}-05$ & 4.7295 & 0.0036 & 3.8903067 & TRUE & 2.6335343 & TRUE \\
\hline 0 & $1.00 \mathrm{E}-04$ & 2 & $6.86 \mathrm{E}-05$ & 3.4835 & 0.0414 & 5.2478981 & FALSE & 3.2594443 & TRUE \\
\hline M & 0.006 & 2 & 0.003 & 152.4224 & $2.68 \mathrm{E}-18$ & 5.2478981 & TRUE & 3.2594443 & TRUE \\
\hline Error & 0.0007 & 36 & $1.97 \mathrm{E}-05$ & & & & & & \\
\hline
\end{tabular}

$\begin{array}{lll}\text { Total } & 0.0079 & 53\end{array}$ 


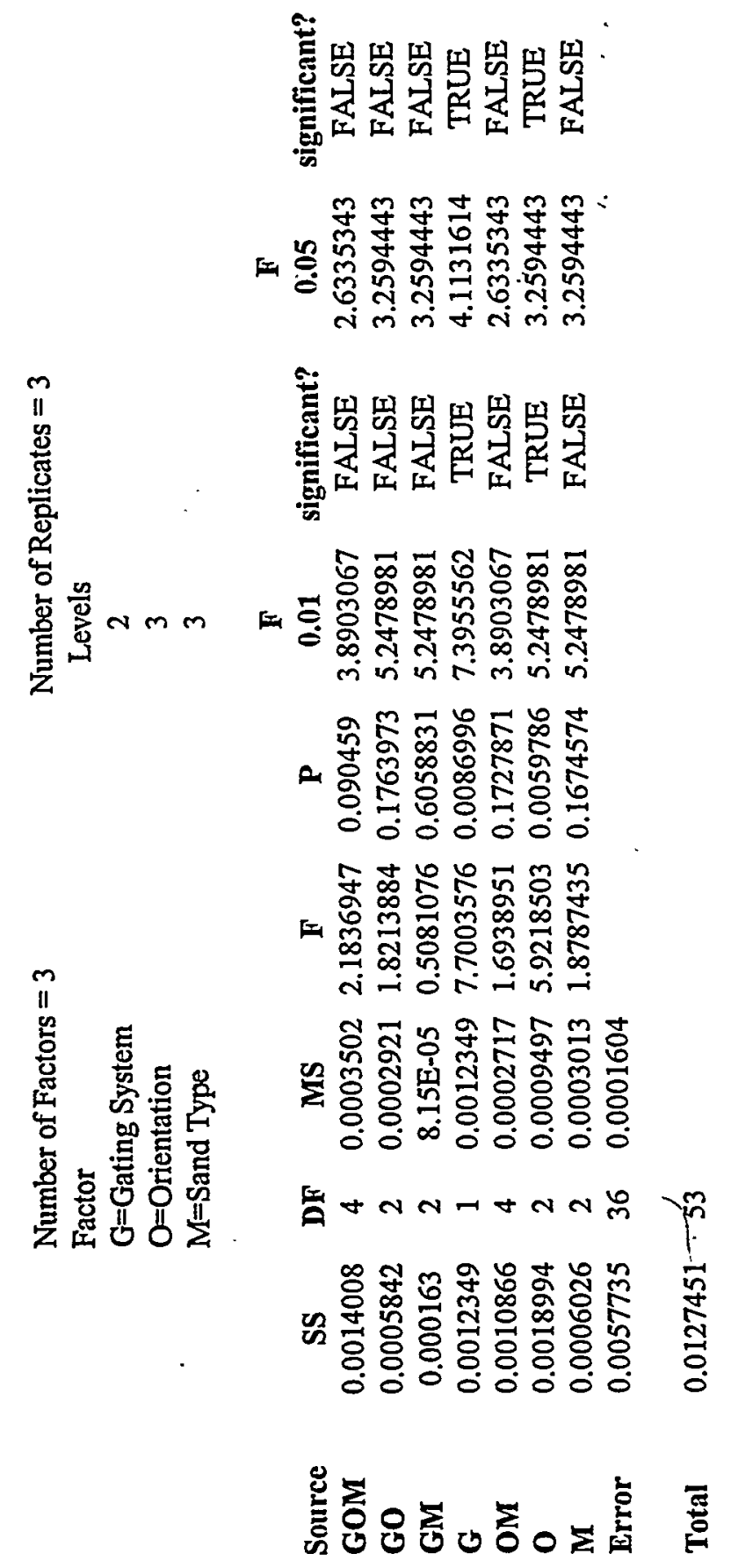


Table B.10 Factorial Analysis of Variance: Thickness

(all 0.50 inch castings)

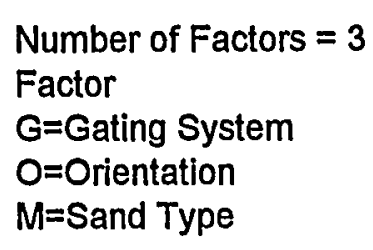

Number of Replicates $=3$

Levels

$$
\begin{aligned}
& 2 \\
& 3 \\
& 3
\end{aligned}
$$

\begin{tabular}{|c|c|c|c|c|c|c|c|c|c|}
\hline & & & & & & $\mathbf{F}$ & & $\mathbf{F}$ & \\
\hline Source & SS & DF & MS & $\mathbf{F}$ & $\mathbf{P}$ & 0.01 & significant? & 0.05 & significant? \\
\hline GOM & 0.004549 & 4 & 0.001137 & 2.272828 & 0.080404 & 3.890307 & FALSE & 2.633534 & FALSE \\
\hline GO & 0.004826 & 2 & 0.002413 & 4.823236 & 0.013935 & 5.247898 & FALSE & 3.259444 & TRUE \\
\hline GM & 0.000316 & 2 & 0.000158 & 0.315865 & 0.731158 & 5.247898 & FALSE & 3.259444 & FALSE \\
\hline $\mathbf{G}$ & 9.86E-05 & 1 & $9.86 \mathrm{E}-05$ & 0.196993 & 0.659816 & 7.395556 & FALSE & 4.113161 & FALSE \\
\hline OM & 0.002707 & 4 & 0.000677 & 1.352614 & 0.269613 & 3.890307 & FALSE & 2.633534 & FALSE \\
\hline 0 & 0.002308 & 2 & 0.001154 & 2.306967 & 0.114102 & 5.247898 & FALSE & 3.259444 & FALSE \\
\hline$M$ & 0.011789 & 2 & 0.005894 & 11.78093 & 1.16E-04 & 5.247898 & TRUE & 3.259444 & TRUE \\
\hline Error & 0.018012 & 36 & 0.0005 & & & & & & \\
\hline Total & 0.044605 & 5 & & & & & & & \\
\hline
\end{tabular}


Table B.11 Factorial Analysis of Variance: Length

(all 0.50 inch castings)

Number of Factors $=3$

Factor

$G=$ Gating System

$\mathrm{O}=$ Orientation

$M=$ Sand Type
Number of Replicates $=3$

Levels

2
3
3

$F$

0.01 significant? 0.05 significant?

3.890307 FALSE 2.633534 FALSE

5.247898 FALSE 3.259444 TRUE

5.247898 FALSE 3.259444 FALSE

7.395556 FALSE 4.113161 FALSE

3.890307 TRUE 2.633534 TRUE

$\begin{array}{llllllllll}0 & 0.002756 & 2 & 0.001378 & 5.695969 & 0.007092 & 5.247898 & \text { TRUE } & 3.259444 & \text { TRUE } \\ \text { M } & 21.65944 & 2 & 10.82972 & 4.48 E+04 & 0.00 E+00 & 5.247898 & \text { TRUE } & 3.259444 & \text { TRUE }\end{array}$

$\begin{array}{llllllllll}0 & 0.002756 & 2 & 0.001378 & 5.695969 & 0.007092 & 5.247898 & \text { TRUE } & 3.259444 & \text { TRUE } \\ \text { M } & 21.65944 & 2 & 10.82972 & 4.48 E+04 & 0.00 E+00 & 5.247898 & \text { TRUE } & 3.259444 & \text { TRUE }\end{array}$

$\begin{array}{llll}\text { Error } & 0.008708 & 36 & 0.000242\end{array}$

Total $\quad 21.68023 \quad 53$ 
Table B.12 Factorial Analysis of Variance: Height

(all 0.50 inch castings)

\begin{tabular}{|c|c|c|c|c|c|c|c|c|c|}
\hline & & $\begin{array}{l}\text { Num } \\
\text { Factc } \\
G=G \\
O=O \\
M=S\end{array}$ & $\begin{array}{l}\text { er of Factor } \\
\text { ting System } \\
\text { entation } \\
\text { nd Type }\end{array}$ & $s=3$ & & $\begin{array}{c}\text { Number of } \\
\text { Levels } \\
2 \\
3 \\
3\end{array}$ & Replicates $=3$ & - & \\
\hline Source & SS & DF & MS & F & $\mathbf{P}$ & 0.01 & significant? & 0.05 & significant? \\
\hline GOM & 0.00848 & 4 & 0.00212 & 2.741141 & 0.043429 & 3.890307 & FALSE & 2.633534 & TRUE \\
\hline GO & 0.002165 & 2 & 0.001083 & 1.399913 & 0.259722 & 5.247898 & FALSE & 3.259444 & FALSE \\
\hline GM & 0.006887 & 2 & 0.003443 & 4.45229 & 0.018716 & 5.247898 & FALSE & 3.259444 & TRUE \\
\hline $\mathbf{G}$ & 0.002061 & 1 & 0.002061 & 2.664954 & 0.111299 & 7.395556 & FALSE & 4.113161 & FALSE \\
\hline OM & 0.005714 & 4 & 0.001429 & 1.847046 & 0.141197 & 3.890307 & FALSE & 2.633534 & FALSE \\
\hline 0 & 0.004513 & 2 & 0.002257 & 2.917886 & 0.066924 & 5.247898 & FALSE & 3.259444 & FALSE \\
\hline & 0.757342 & 2 & 0.378671 & 489.6193 & $7.86 \mathrm{E}-27$ & 5.247898 & TRUE & 3.259444 & TRUE \\
\hline Error & 0.027842 & 36 & 0.000773 & & & & & & \\
\hline Total & 0.815005 & 53 & & & & & & & \\
\hline
\end{tabular}


Table B.13 Factorial Analysis of Variance: Parallelism

(all 0.50 inch castings)

\author{
Number of Factors $=3$ \\ Factor \\ $G=G$ ating System \\ $O=$ Orientation \\ $M=$ Sand Type
}

$\begin{array}{lccccccc}\text { Source } & \text { SS } & \text { DF } & \text { MS } & \text { F } & \text { P } & \mathbf{F} & \\ \text { GOM } & 0.005459 & 4 & 0.001365 & 2.250072 & 0.082858 & 3.890307 & \text { FALSE } \\ \text { GO } & 0.000893 & 2 & 0.000446 & 0.735792 & 0.486192 & 5.247898 & \text { FALSE } \\ \text { GM } & 0.00413 & 2 & 0.002065 & 3.404762 & 0.04423 & 5.247898 & \text { FALSE } \\ \text { G } & 0.000723 & 1 & 0.000723 & 1.192335 & 0.282113 & 7.395556 & \text { FALSE } \\ \text { OM } & 0.005496 & 4 & 0.001374 & 2.265165 & 0.081222 & 3.890307 & \text { FALSE } \\ \text { O } & 0.000357 & 2 & 0.000178 & 0.294183 & 0.746914 & 5.247898 & \text { FALSE } \\ \text { M } & 0.020773 & 2 & 0.010386 & 17.12334 & 5.95 E-06 & 5.247898 & \text { TRUE } \\ \text { Error } & 0.021836 & 36 & 0.000607 & & & & \\ & & & & & & & \end{array}$

Number of Replicates $=\mathbf{3}$

Levels

2

3

3

$\begin{array}{cc}\mathbf{0 . 0 5} & \text { significant? } \\ 2.633534 & \text { FALSE } \\ 3.259444 & \text { FALSE } \\ 3.259444 & \text { TRUE } \\ 4.113161 & \text { FALSE } \\ 2.633534 & \text { FALSE } \\ 3.259444 & \text { FALSE } \\ 3.259444 & \text { TRUE }\end{array}$


Table B.14 Factorial Analysis of Variance: Left Casting Angle (all 0.50 inch castings)

\author{
Number of Factors $=3$ \\ Factor \\ $G=$ Gating System \\ $\mathrm{O}=$ Orientation \\ $M=$ Sand Type
}

\section{Number of Replicates $=3$}

Levels

$$
2
$$

3

3

F $\quad F$ 0.01 significant? 0.05 significant?

$\begin{array}{lccccccccc}\text { Source } & \text { SS } & \text { DF } & \text { MS } & \mathbf{F} & \mathbf{P} & \mathbf{0 . 0 1} & \text { significant? } & \mathbf{0 . 0 5} & \text { significant? } \\ \text { GOM } & 1.71 E-05 & 4 & 4.28 E-06 & 0.226785 & 0.921616 & 3.890307 & \text { FALSE } & 2.633534 & \text { FALSE } \\ \text { GO } & 4.41 E-05 & 2 & 2.21 E-05 & 1.170021 & 0.321883 & 5.247898 & \text { FALSE } & 3.259444 & \text { FALSE } \\ \text { GM } & 5.28 E-05 & 2 & 2.64 E-05 & 1.400412 & 0.259602 & 5.247898 & \text { FALSE } & 3.259444 & \text { FALSE } \\ \text { G } & 3.51 E-05 & 1 & 3.51 E-05 & 1.863787 & 0.18066 & 7.395556 & \text { FALSE } & 4.113161 & \text { FALSE } \\ \text { OM } & 0.000171 & 4 & 4.26 E-05 & 2.261245 & 0.081644 & 3.890307 & \text { FALSE } & 2.633534 & \text { FALSE } \\ \text { O } & 4.49 E-05 & 2 & 2.24 E-05 & 1.190387 & 0.31579 & 5.247898 & \text { FALSE } & 3.259444 & \text { FALSE } \\ \text { M } & 0.000855 & 2 & 0.000427 & 22.67399 & 4.24 E-07 & 5.247898 & \text { TRUE } & 3.259444 & \text { TRUE } \\ \text { Error } & 0.000679 & 36 & 1.89 E-05 & & & & & & \end{array}$

Total $\quad 0.001898 \quad 53$ 


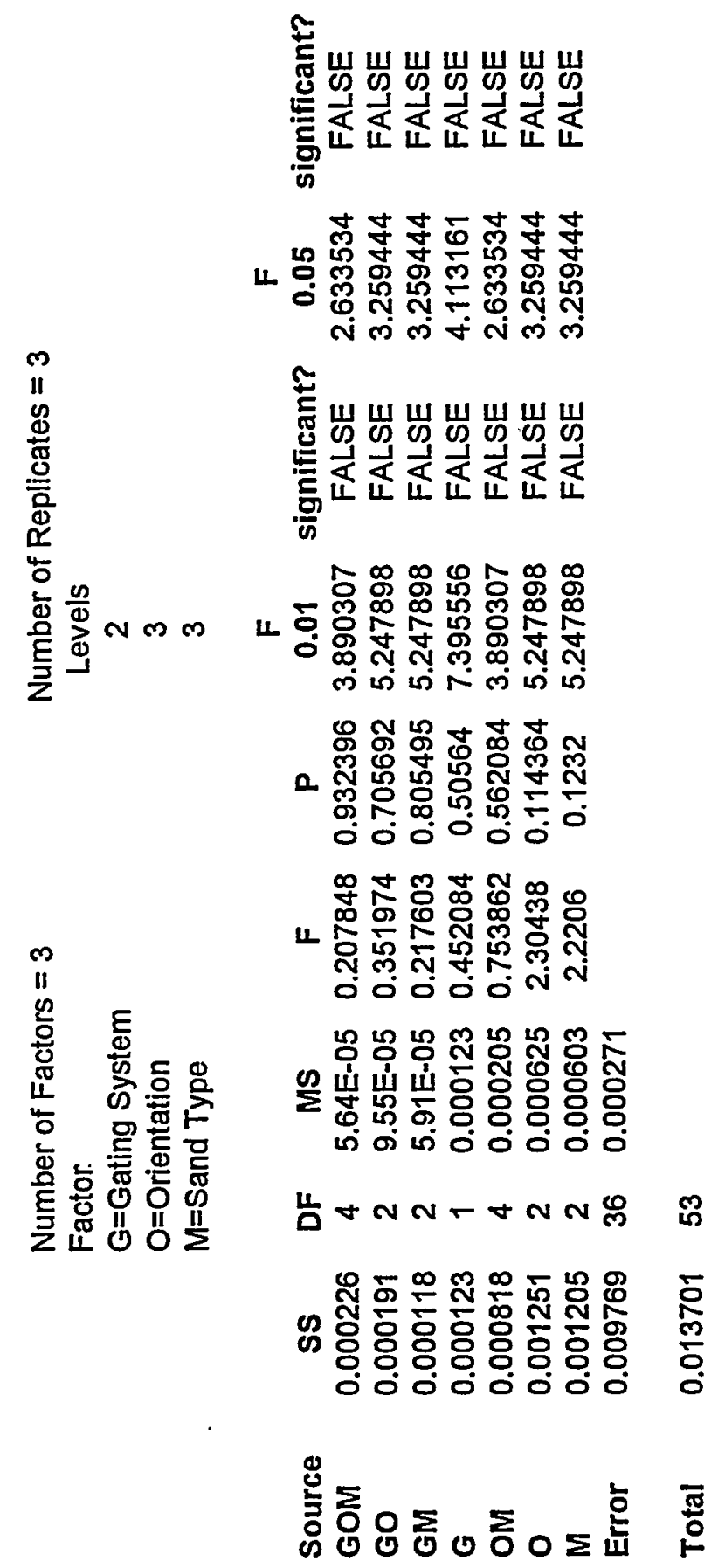


Table B.16 Factorial Analysis of Variance: Left Flange Flatness

(all 0.50 inch castings)

\begin{tabular}{|c|c|c|c|c|c|c|c|c|c|}
\hline & & $\begin{array}{l}\text { Numb } \\
\text { Facto } \\
G=G \\
O=O r \\
M=S e\end{array}$ & $\begin{array}{l}\text { er of Factor } \\
\text { ting System } \\
\text { entation } \\
\text { nd Type }\end{array}$ & $s=3$ & & $\begin{array}{c}\text { Number of } \\
\text { Levels } \\
2 \\
3 \\
3\end{array}$ & Replicates $=3$ & - & \\
\hline Source & SS & DF & MS & $\mathbf{F}$ & $\mathbf{P}$ & 0.01 & significant? & 0.05 & significant? \\
\hline GOM & 0.000811 & 4 & 0.000203 & 2.951794 & 0.033007 & 3.890307 & FALSE & 2.633534 & TRUE \\
\hline GO & 0.00055 & 2 & 0.000275 & 4.003906 & 0.02691 & 5.247898 & FALSE & 3.259444 & TRUE \\
\hline GM & 0.000444 & 2 & 0.000222 & 3.233633 & 0.051105 & 5.247898 & FALSE & 3.259444 & FALSE \\
\hline G & 0.000261 & 1 & 0.000261 & 3.799656 & 0.059087 & 7.395556 & FALSE & 4.113161 & FALSE \\
\hline OM & 0.001535 & 4 & 0.000384 & 5.590194 & 0.00132 & 3.890307 & TRUE & 2.633534 & TRUE \\
\hline 0 & 8.16E-05 & 2 & 4.08E-05 & 0.594425 & 0.557206 & 5.247898 & FALSE & 3.259444 & FALSE \\
\hline M & 0.000178 & 2 & 8.92E-05 & 1.298523 & 0.285412 & 5.247898 & FALSE & 3.259444 & FALSE \\
\hline Error & 0.002472 & 36 & 6.87E-05 & & & & & & \\
\hline Total & 0.006333 & 53 & & & & & & & \\
\hline
\end{tabular}


Table B.17 Factorial Analysis of Variance: Right Flange Flatness (all 0.50 inch castings)

Number of Factors $=3$
Factor
$G=$ Gating System
$O=$ Orientation
$M=$ Sand Type

Number of Replicates $=3$

Levels

2
3
3

\begin{tabular}{|c|c|c|c|c|c|c|c|c|c|}
\hline \multirow{2}{*}{ Source } & \multirow[b]{2}{*}{ SS } & \multirow[b]{2}{*}{ DF } & \multirow[b]{2}{*}{ MS } & \multirow[b]{2}{*}{$F$} & & $\mathbf{F}$ & \multicolumn{3}{|c|}{$F$} \\
\hline & & & & & $\mathbf{P}$ & 0.01 & significant? & 0.05 & significant? \\
\hline GOM & 0.003325 & 4 & 0.000831 & 4.326239 & 0.005847 & 3.890307 & TRUE & 2.633534 & TRUE \\
\hline GO & 0.000703 & 2 & 0.000351 & 1.828916 & 0.175196 & 5.247898 & FALSE & 3.259444 & FALSE \\
\hline GM & 0.003044 & 2 & 0.001522 & 7.92174 & 0.001409 & 5.247898 & TRUE & 3.259444 & TRUE \\
\hline $\mathbf{G}$ & 0.001391 & 1 & 0.001391 & 7.238098 & 0.010753 & 7.395556 & FALSE & 4.113161 & TRUE \\
\hline OM & 0.00329 & 4 & 0.000823 & 4.281142 & 0.006177 & 3.890307 & TRUE & 2.633534 & TRUE \\
\hline 0 & 0.001173 & 2 & 0.000586 & 3.05134 & 0.059686 & 5.247898 & FALSE & 3.259444 & FALSE \\
\hline M & 0.007276 & 2 & 0.003638 & 18.93426 & 2.41E-06 & 5.247898 & TRUE & 3.259444 & TRUE \\
\hline Error & 0.006917 & 36 & 0.000192 & & & & & & \\
\hline tal & 0.027118 & 53 & & & & & & . & \\
\hline
\end{tabular}



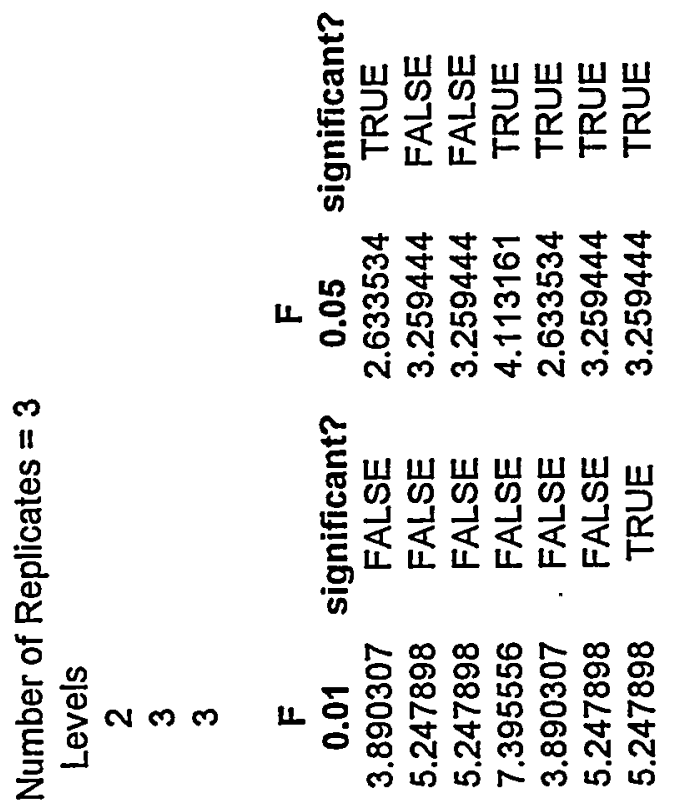

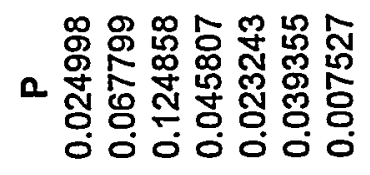
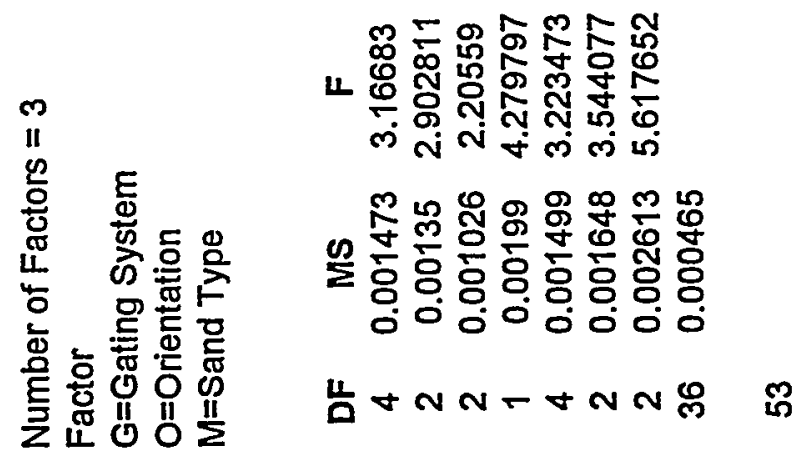

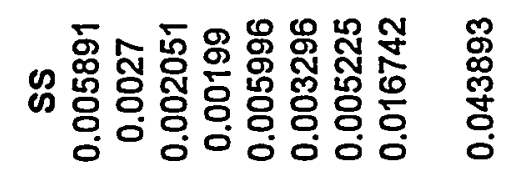

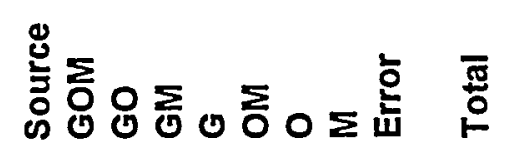


Table B.19 Factorial Analysis of Variance: Right Wall Flatness

(all 0.50 inch castings)

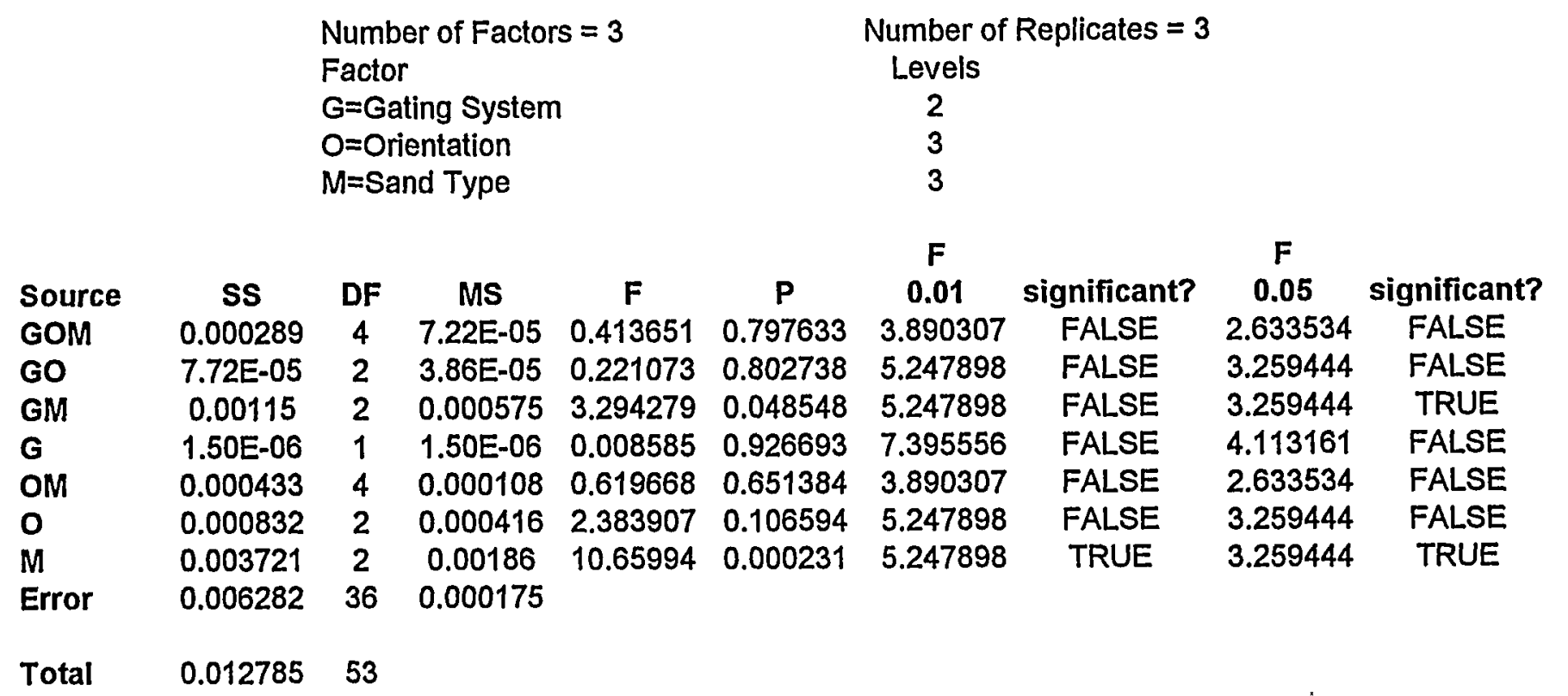


Table B.20 Factorial Analysis of Variance: Thickness

(all 0.75 inch castings)

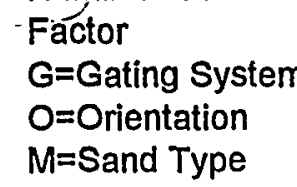

Number of Replicates $=3$

Levels

2
3
3

$F$

0.01 significant?

3.890307

5.247898

5.247898

7.395556

3.890307

5.247898

5.247898

\section{TRUE}

FALSE

FALSE

FALSE

FALSE

FALSE

TRUE

0.000251

$\begin{array}{lllll}0.05225 & 2 & 0.026125 & 104.2333 & 1.06 \mathrm{E}-15\end{array}$

0.009023

36

Total

0.075934

53 
Table B.21 Factorial Analysis of Variance: Length

(all 0.75 inch castings)

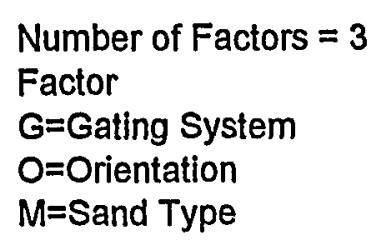

Number of Replicates $=3$ Levels

2
3
3

\begin{tabular}{|c|c|c|c|c|c|c|c|c|c|}
\hline & & & & & & $\mathbf{F}$ & & $F$ & \\
\hline Source & SS & DF & MS & $F$ & $\mathbf{P}$ & 0.01 & significant? & 0.05 & significant? \\
\hline GOM & 0.000718 & 4 & 0.00018 & 0.393437 & 0.811956 & 3.890307 & FALSE & 2.633534 & FALSE \\
\hline GO & 0.00028 & 2 & 0.00014 & 0.30688 & 0.737644 & 5.247898 & FALSE & 3.259444 & FALSE \\
\hline GM & 0.000262 & 2 & 0.000131 & 0.287217 & 0.752052 & 5.247898 & FALSE & 3.259444 & FALSE \\
\hline G & 1.95E-05 & 1 & 1.95E-05 & 0.042681 & 0.837492 & 7.395556 & FALSE & 4.113161 & FALSE \\
\hline OM & 0.002552 & 4 & 0.000638 & 1.397732 & 0.254364 & 3.890307 & FALSE & 2.633534 & FALSE \\
\hline 0 & 0.000107 & 2 & 5.33E-05 & 0.116804 & 0.890095 & 5.247898 & FALSE & 3.259444 & FALSE \\
\hline M & 20.90547 & 2 & 10.45273 & $2.29 E+04$ & 0 & 5.247898 & TRUE & 3.259444 & TRUE \\
\hline Error & 0.016432 & 36 & 0.000456 & & & & & & \\
\hline Total & 20.92584 & 53 & & & & & & & \\
\hline
\end{tabular}


Table B.22 Factorial Analysis of Variance: Height

(all 0.75 inch castings)

Number of Factors $=3$
Factor
$G=$ Gating System
$O=$ Orientation
$M=$ Sand Type

Number of Replicates $=3$

Levels

$O=$ Orientation

2

3

3

\begin{tabular}{lccccccccc} 
& & & \multicolumn{1}{c}{$\mathbf{F}$} & \multicolumn{3}{c}{$\mathbf{F}$} & \\
Source & SS & DF & MS & $\mathbf{F}$ & $\mathbf{P}$ & $\mathbf{0 . 0 1}$ & significant? & $\mathbf{0 . 0 5}$ & significant? \\
GOM & 0.011518 & 4 & 0.00288 & 16.89049 & $7.00 E-08$ & 3.890307 & TRUE & 2.633534 & TRUE \\
GO & 0.002317 & 2 & 0.001159 & 6.796832 & 0.003132 & 5.247898 & TRUE & 3.259444 & TRUE \\
GM & 0.002286 & 2 & 0.001143 & 6.704251 & 0.00335 & 5.247898 & TRUE & 3.259444 & TRUE \\
G & 0.000149 & 1 & 0.000149 & 0.87269 & 0.356436 & 7.395556 & FALSE & 4.113161 & FALSE \\
OM & 0.002985 & 4 & 0.000746 & 4.377251 & 0.005495 & 3.890307 & TRUE & 2.633534 & TRUE \\
O & 0.005185 & 2 & 0.002592 & 15.20546 & $1.63 E-05$ & 5.247898 & TRUE & 3.259444 & TRUE \\
M & 0.859507 & 2 & 0.429753 & $2.52 E+03$ & 0 & 5.247898 & TRUE & 3.259444 & TRUE \\
Error & 0.006137 & 36 & 0.00017 & & & & & &
\end{tabular}

Total $0.890084 \quad 53$ 
Table B.23 Factorial Analysis of Variance: Parallelism

(all 0.75 inch castings)

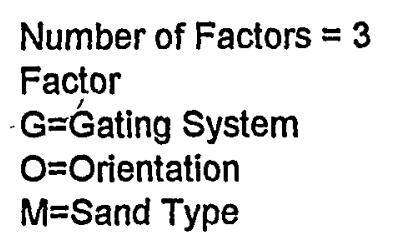

Number of Replicates $=\mathbf{3}$ Levels

$$
\begin{aligned}
& 2 \\
& 3 \\
& 3
\end{aligned}
$$

$\begin{array}{lccccccccc} & & & & & & \mathbf{F} & & -\mathbf{F} & \\ \text { Source } & \mathbf{S S} & \mathbf{D F} & \mathbf{M S} & \mathbf{F} & \mathbf{P} & \mathbf{0 . 0 1} & \text { significant? } & \mathbf{0 . 0 5} & \text { significant? } \\ \text { GOM } & 0.005731 & 4 & 0.001433 & 0.32429 & 0.859821 & 3.890307 & \text { FALSE } & 2.633534 & \text { FALSE } \\ \text { GO } & 0.003531 & 2 & 0.001766 & 0.399631 & 0.673506 & 5.247898 & \text { FALSE } & 3.259444 & \text { FALSE } \\ \text { GM } & 0.004004 & 2 & 0.002002 & 0.453211 & 0.63916 & 5.247898 & \text { FALSE } & 3.259444 & \text { FALSE } \\ \text { G } & 0.00674 & 1 & 0.00674 & 1.525725 & 0.224756 & 7.395556 & \text { FALSE } & 4.113161 & \text { FALSE } \\ \text { OM } & 0.006379 & 4 & 0.001595 & 0.360973 & 0.834696 & 3.890307 & \text { FALSE } & 2.633534 & \text { FALSE } \\ \text { O } & 0.004495 & 2 & 0.002247 & 0.50871 & 0.605529 & 5.247898 & \text { FALSE } & 3.259444 & \text { FALSE } \\ \text { M } & 0.062341 & 2 & 0.03117 & 7.0555 & 0.002598 & 5.247898 & \text { TRUE } & 3.259444 & \text { TRUE } \\ \text { Error } & 0.159044 & 36 & 0.004418 & & & & & & \end{array}$


Table B.24 Factorial Analysis of Variance: Left Casting Angle

(all 0.75 inch castings)

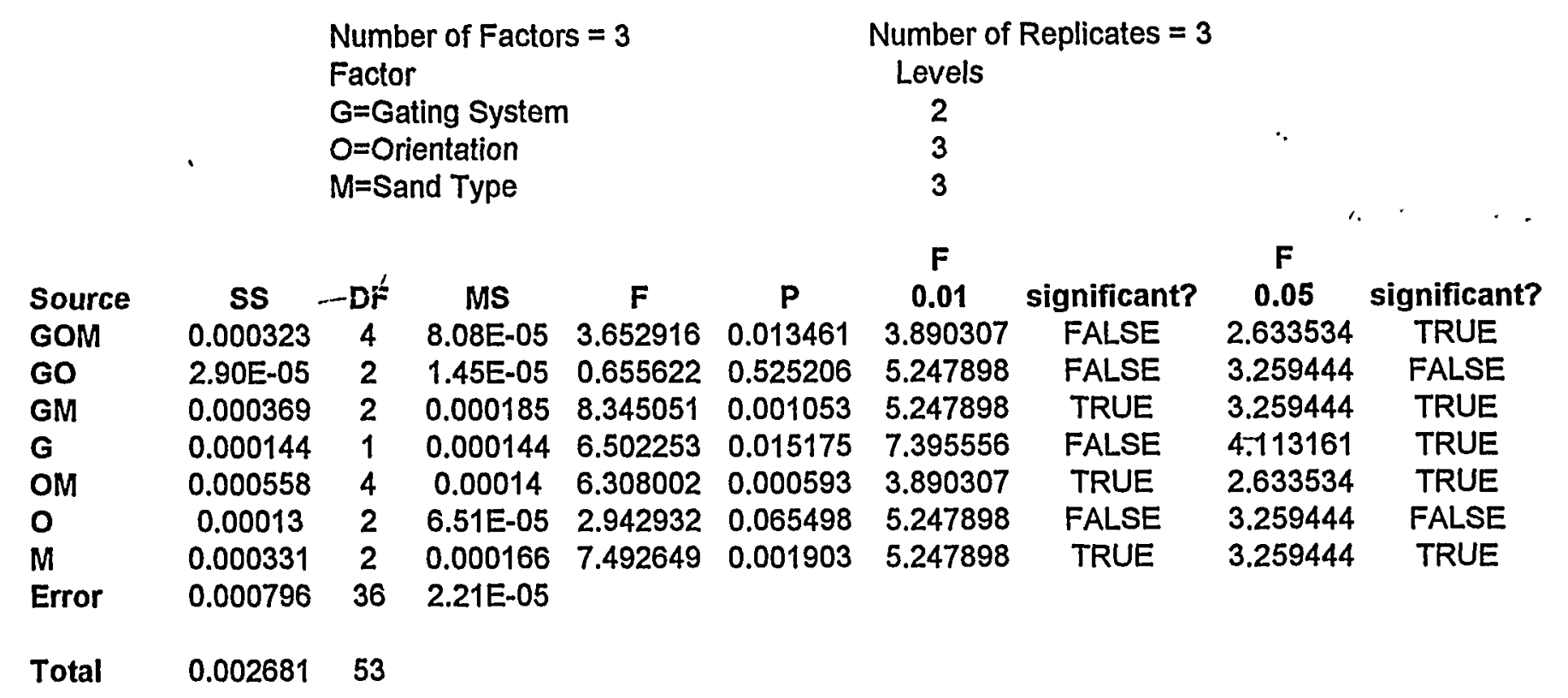


Table B.25 Factorial Analysis of Variance: Right Casting Angle

(all 0.75 inch castings)

\begin{tabular}{|c|c|c|c|c|c|c|c|c|c|}
\hline & & \multicolumn{3}{|c|}{$\begin{array}{l}\text { Number of Factors = } 3 \\
\text { Factor } \\
G=\text { Gating System } \\
O=\text { Orientation } \\
M=\text { Sand Type }\end{array}$} & & $\begin{array}{c}\text { Number of } \\
\text { Levels } \\
2 \\
3 \\
3\end{array}$ & Replicates $=3$ & & \\
\hline & & & & & & $\mathbf{F}$ & & $\mathbf{F}$ & \\
\hline Source & SS & DF & MS & $\mathbf{F}$ & $\mathbf{P}$ & 0.01 & significant? & 0.05 & significant? \\
\hline GOM & 0.000839 & 4 & 0.00021 & 4.175475 & 0.00703 & 3.890307 & TRUE & 2.633534 & TRUE \\
\hline GO & 0.000426 & 2 & 0.000213 & 4.235734 & 0.022283 & 5.247898 & FALSE & 3.259444 & TRUE \\
\hline GM & 0.00016 & 2 & 8.01E-05 & 1.594003 & 0.217114 & 5.247898 & FALSE & 3.259444 & FALSE \\
\hline G & 0.000117 & 1 & 0.000117 & 2.328554 & 0.135758 & 7.395556 & FALSE & 4.113161 & FALSE \\
\hline OM & 0.000633 & 4 & 0.000158 & 3.149391 & 0.025566 & 3.890307 & FALSE & 2.633534 & TRUE \\
\hline 0 & 0.000613 & 2 & 0.000306 & 6.094134 & 0.005254 & 5.247898 & TRUE & 3.259444 & TRUE \\
\hline & 0.000977 & 2 & 0.000489 & 9.723177 & 0.00042 & 5.247898 & TRUE & 3.259444 & TRUE \\
\hline Error & 0.001809 & 36 & 5.03E-05 & & & & & & \\
\hline Total & 0.005574 & 53 & & & & & & & \\
\hline
\end{tabular}


Table B.26 Factorial Analysis of Variance: Left Flange Flatness

(all 0.75 inch castings)

\begin{tabular}{|c|c|c|c|c|c|c|c|}
\hline \multicolumn{4}{|c|}{ Number of Factors $=3$} & \multicolumn{4}{|c|}{ Number of Replicates $=3$} \\
\hline \multicolumn{4}{|c|}{ Factor } & Levels & & & \\
\hline \multicolumn{4}{|c|}{$\mathrm{G}=$ Gating System } & 2 & & & \\
\hline \multicolumn{3}{|c|}{$\mathrm{O}=$ Orientation } & & 3 & & & \\
\hline \multicolumn{2}{|c|}{$M=$ Sand Type } & & & 3 & & & \\
\hline DF & MS & $\mathbf{F}$ & $\mathbf{P}$ & $\begin{array}{c}F \\
0.01\end{array}$ & significant? & $\begin{array}{c}F \\
0.05\end{array}$ & significant? \\
\hline 4 & 5.61E-05 & 1.978235 & 0.118715 & 3.890307 & FALSE & 2.633534 & FALSE \\
\hline 2 & 0.000211 & 7.455045 & 0.001954 & 5.247898 & TRUE & 3.259444 & TRUE \\
\hline 2 & 0.000201 & 7.088215 & 0.002538 & 5.247898 & TRUE & 3.259444 & TRUE \\
\hline 1 & $1.43 E-06$ & 0.050435 & 0.823577 & 7.395556 & FALSE & 4.113161 & FALSE \\
\hline 4 & 0.000186 & 6.541942 & 0.00046 & 3.890307 & TRUE & 2.633534 & TRUE \\
\hline 2 & $1.25 \mathrm{E}-05$ & 0.439946 & 0.647487 & 5.247898 & FALSE & 3.259444 & FALSE \\
\hline 2 & $1.25 E-05$ & 0.440723 & 0.646997 & 5.247898 & FALSE & 3.259444 & FALSE \\
\hline 36 & 2.84E-05 & & & & & & \\
\hline
\end{tabular}

Total $\quad 0.002864 \quad 53$ 
Table B.27 Factorial Analysis of Variance: Right Flange Flatness

(all 0.75 inch castings)

\begin{tabular}{|c|c|c|c|c|c|c|c|c|c|}
\hline & & $\begin{array}{l}\text { Num } \\
\text { Factc } \\
G=G \\
O=O \\
M=S\end{array}$ & $\begin{array}{l}\text { er of Factor } \\
\text { ting System } \\
\text { entation } \\
\text { nd Type }\end{array}$ & & & $\begin{array}{c}\text { Number of } \\
\text { Levels } \\
2 \\
3 \\
3\end{array}$ & Replicates $=3$ & - & \\
\hline Source & SS & DF & MS & $\mathbf{F}$ & $\mathbf{P}$ & 0.01 & significant? & 0.05 & significant? \\
\hline GOM & 0.103194 & 4 & 0.025799 & 3.03331 & 0.029699 & 3.890307 & FALSE & 2.633534 & TRUE \\
\hline GO & 0.040046 & 2 & 0.020023 & 2.354238 & 0.109425 & 5.247898 & FALSE & 3.259444 & FALSE \\
\hline GM & 0.057977 & 2 & 0.028988 & 3.408348 & 0.044097 & 5.247898 & FALSE & 3.259444 & TRUE \\
\hline $\mathbf{G}$ & 0.032852 & 1 & 0.032852 & 3.862613 & 0.057125 & 7.395556 & FALSE & 4.113161 & FALSE \\
\hline OM & 0.056323 & 4 & 0.014081 & 1.655556 & 0.181718 & 3.890307 & FALSE & 2.633534 & FALSE \\
\hline 0 & 0.06128 & 2 & 0.03064 & 3.602579 & 0.03748 & 5.247898 & FALSE & 3.259444 & TRUE \\
\hline M & 0.115735 & 2 & 0.057868 & 6.803896 & 0.003116 & 5.247898 & TRUE & 3.259444 & TRUE \\
\hline Error & 0.306183 & 36 & 0.008505 & & & & & & \\
\hline Total & 0.773589 & 53 & & & & & & & \\
\hline
\end{tabular}


Table B.28 Factorial Analysis of Variance: Left Wall Flatness (all 0.75 inch castings)

\begin{tabular}{|c|c|c|c|c|c|c|c|}
\hline \multicolumn{4}{|c|}{ Number of Factors $=3$} & \multicolumn{4}{|c|}{ Number of Replicates $=3$} \\
\hline \multicolumn{4}{|c|}{ Faćtor } & Levels & & & \\
\hline \multicolumn{4}{|c|}{$G=$ Gating System } & 2 & & & \\
\hline \multicolumn{4}{|c|}{$O=$ Orientation } & 3 & & & \\
\hline \multicolumn{4}{|c|}{$M=$ Sand Type } & 3 & & & \\
\hline DF & MS & $\mathbf{F}$ & $\mathbf{P}$ & 0.01 & significant? & 0.05 & significant? \\
\hline 4 & 0.000216 & 2.845882 & 0.037881 & 3.890307 & FALSE & 2.633534 & TRUE \\
\hline 2 & 0.000373 & 4.927512 & 0.012838 & 5.247898 & FALSE & 3.259444 & TRUE \\
\hline 2 & 6.31E-05 & 0.832483 & 0.443168 & 5.247898 & FALSE & 3.259444 & FALSE \\
\hline 1 & 2.13E-06 & 0.028164 & 0.867663 & 7.395556 & FALSE & 4.113161 & FALSE \\
\hline 4 & 0.000105 & 1.380432 & 0.260113 & 3.890307 & FALSE & 2.633534 & FALSE \\
\hline 2 & 4.91E-05 & 0.648011 & 0.529078 & 5.247898 & FALSE & 3.259444 & FALSE \\
\hline 2 & 0.000984 & 12.98991 & 5.66E-05 & 5.247898 & TRUE & 3.259444 & TRUE \\
\hline 36 & 7.57E-05 & & & & & & \\
\hline
\end{tabular}


Table B.29 Factorial Analysis of Variance: Right Wall Flatness

(all 0.75 inch castings)

Number of Factors $=3$

Factor

$G=$ Gating System

$\mathrm{O}=$ Orientation

$M=S a n d$ Type
Number of Replicates $=3$ Levels

$$
\begin{aligned}
& 2 \\
& 3 \\
& 3
\end{aligned}
$$

\begin{tabular}{lccccccccc} 
& & & & & \multicolumn{2}{c}{$\mathbf{F}$} & & $\mathbf{F}$ \\
Source & $\mathbf{S S}$ & $\mathbf{D F}$ & $\mathbf{M S}$ & $\mathbf{F}$ & $\mathbf{P}$ & $\mathbf{0 . 0 1}$ & significant? & $\mathbf{0 . 0 5}$ & significant? \\
GOM & 0.002587 & 4 & 0.000647 & 0.655664 & 0.626738 & 3.890307 & FALSE & 2.633534 & FALSE \\
GO & 0.001544 & 2 & 0.000772 & 0.782955 & 0.46468 & 5.247898 & FALSE & 3.259444 & FALSE \\
GM & 0.004642 & 2 & 0.002321 & 2.353463 & 0.1095 & 5.247898 & FALSE & 3.259444 & FALSE \\
G & 0.000209 & 1 & 0.000209 & 0.211477 & 0.648378 & 7.395556 & FALSE & 4.113161 & FALSE \\
OM & 0.001018 & 4 & 0.000255 & 0.25812 & 0.902777 & 3.890307 & FALSE & 2.633534 & FALSE \\
O & 0.000567 & 2 & 0.000284 & 0.287588 & 0.751777 & 5.247898 & FALSE & 3.259444 & FALSE \\
M & 0.025794 & 2 & 0.012897 & 13.07711 & $5.38 E-05$ & 5.247898 & TRUE & 3.259444 & TRUE \\
Error & 0.035505 & 36 & 0.000986 & & & & & &
\end{tabular}

Total $\quad 0.071866 \quad 53$ 
Table B.30 Factorial Analysis of Variance: Thickness of Resin Bonded Castings (all)

$\begin{array}{lc}\text { Number of Factors } & \begin{array}{c}\text { Number of Replicates } \\ \text { Levels }\end{array} \\ \text { Factor } & 2 \\ G=\text { Gating } & 3 \\ O=\text { Orientation } & 3 \\ T=\text { Thickness } & \end{array}$

\begin{tabular}{|c|c|c|c|c|c|c|c|c|c|}
\hline & & & & & & $\mathbf{F}$ & & $\mathbf{F}$ & \\
\hline Source & SS & DF & MS & $F$ & $\mathbf{P}$ & 0.01 & significant? & 0.05 & significant? \\
\hline GOT & 0.0027 & 4 & 0.0007 & 2.1275 & 0.0974 & 3.890307 & FALSE & 2.633534 & FALSE \\
\hline GO & 0.0006 & 2 & 0.0003 & 0.9464 & 0.3976 & 5.247898 & FALSE & 3.259444 & FALSE \\
\hline GT & 0.0001 & 2 & 5.86E-05 & 0.1868 & 0.8304 & 5.247898 & FALSE & 3.259444 & FALSE \\
\hline $\mathbf{G}$ & 0.0004 & 1 & 0.0004 & 1.3099 & 0.26 & 7.395556 & FALSE & 4.113161 & FALSE \\
\hline OT & 0.0015 & 4 & 0.0004 & 1.181 & 0.3356 & 3.890307 & FALSE & 2.633534 & FALSE \\
\hline 0 & 0.0037 & 2 & 0.0018 & 5.8593 & 0.0063 & 5.247898 & TRUE & 3.259444 & TRUE \\
\hline $\mathbf{T}$ & 2.4372 & 2 & 1.2186 & 3885.551 & 0 & 5.247898 & TRUE & 3.259444 & TRUE \\
\hline Error & 0.0113 & 36 & 0.0003 & & & & & & \\
\hline Total & 2.4575 & 53 & & & & & & & \\
\hline
\end{tabular}



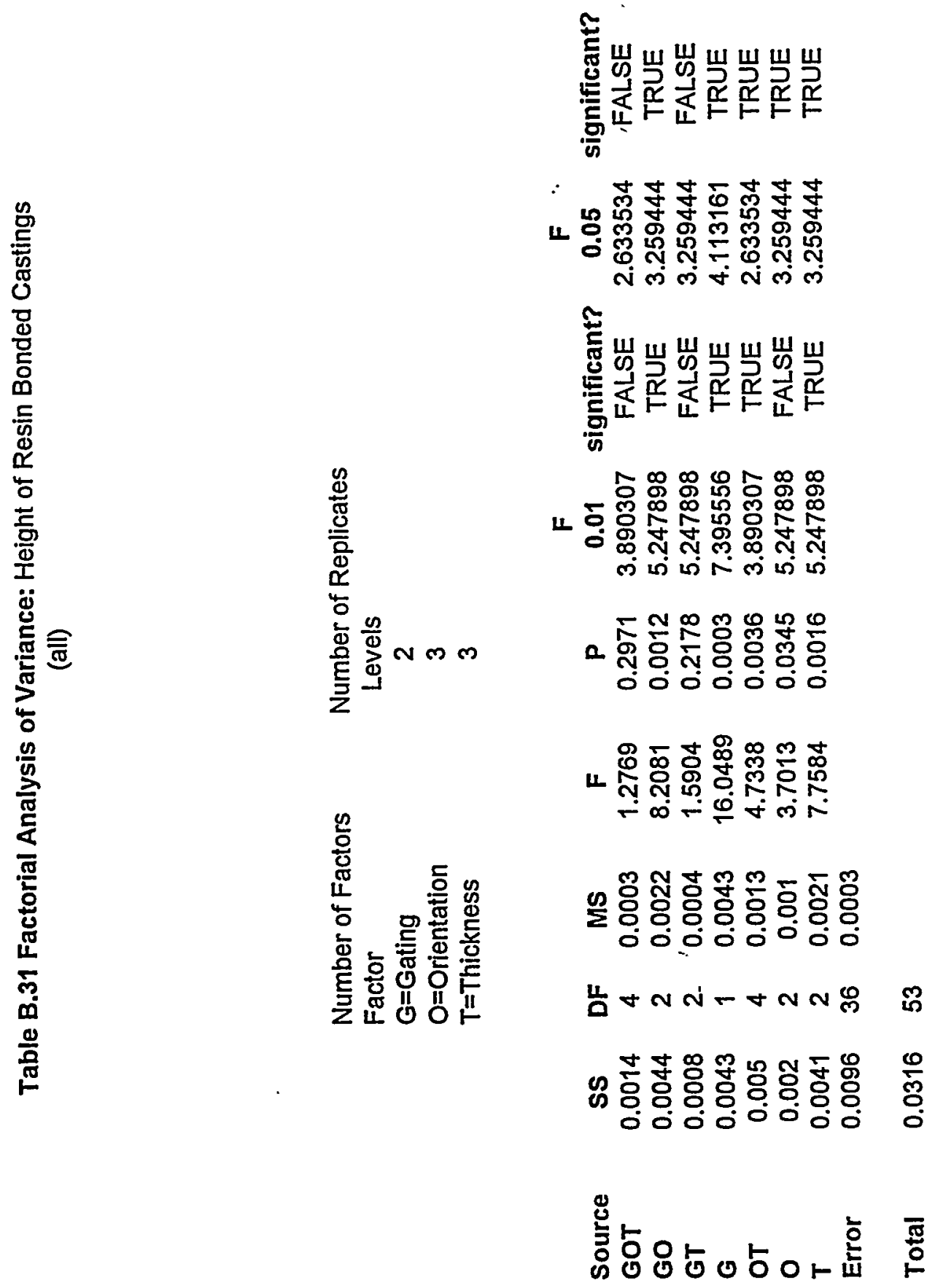
Table B.32 Factorial Analysis of Variance: Length of Resin Bonded Castings (all)

$\begin{array}{lc}\begin{array}{l}\text { Number of Factors } \\ \text { Factor }\end{array} & \begin{array}{c}\text { Number of Replicates } \\ \text { Levels }\end{array} \\ G=\text { Gating } & 2 \\ O=\text { Orientation } & 3 \\ T=\text { Thickness } & 3\end{array}$

\begin{tabular}{|c|c|c|c|c|c|c|c|c|c|}
\hline & & & & & & F & & $\mathbf{F}$ & \\
\hline Source & SS & DF & MS & $\mathbf{F}$ & $\mathbf{P}$ & 0.01 & significant? & 0.05 & siǵnificant? \\
\hline GOT & 0.0005 & 4 & 0.0001 & 0.9149 & 0.4658 & 3.890307 & FALSE & 2.633534 & FALSE \\
\hline GO & 0.0002 & 2 & 0.0001 & 0.9595 & 0.3927 & 5.247898 & FALSE & 3.259444 & FALSE \\
\hline GT & 0.0006 & 2 & 0.0003 & 2.3997 & 0.1051 & 5.247898 & FALSE & 3.259444 & FALSE \\
\hline G & 0.0004 & 1 & 0.0004 & 3.1504 & 0.0844 & 7.395556 & FALSE & 4.113161 & FALSE \\
\hline OT & 0.0011 & 4 & 0.0003 & 2.1631 & 0.093 & 3.890307 & FALSE & 2.633534 & FALSE \\
\hline 0 & 0.0044 & 2 & 0.0022 & 17.0789 & 6.08E-06 & 5.247898 & TRUE & $3.259 \overline{4} 44$ & TRUE \\
\hline$T$ & 0.0263 & 2 & 0.0131 & 102.6046 & $1.35 E-15$ & 5.247898 & TRUE & 3.259444 & TRUE \\
\hline Error & 0.0046 & 36 & 0.0001 & & & & & & \\
\hline Total & 0.0381 & 53 & & & & & & & \\
\hline
\end{tabular}


Table B.33 Factorial Analysis of Variance: Parallelism of Resin Bonded Castings (all)

$\begin{array}{lc}\text { Number of Factors } & \begin{array}{c}\text { Number of Replicates } \\ \text { Levels }\end{array} \\ \text { Factor } & 2 \\ G=\text { Gating } & 3 \\ O=\text { Orientation } & 3 \\ \text { T=Thickness } & \end{array}$

\begin{tabular}{|c|c|c|c|c|c|c|c|c|c|}
\hline & & & & & & $\mathbf{F}$ & & $\mathbf{F}$ & \\
\hline Source & SS & DF & MS & $\mathbf{F}$ & $\mathbf{P}$ & 0.01 & significant? & 0.05 & significant? \\
\hline GOT & 0.0549 & 4 & 0.0137 & 2.8689 & 0.0368 & 3.890307 & FALSE & 2.633534 & TRUE \\
\hline GO & 0.0445 & 2 & 0.0223 & 4.6524 & 0.016 & 5.247898 & FALSE & 3.259444 & TRUE \\
\hline GT & 0.0532 & 2 & 0.0266 & 5.557 & 0.0079 & 5.247898 & TRUE & 3.259444 & TRUE \\
\hline G & 0.013 & 1 & 0.013 & 2.7127 & 0.1083 & 7.395556 & FALSE & 4.113161 & FALSE \\
\hline OT & 0.0054 & 4 & 0.0013 & 0.2797 & 0.8892 & 3.890307 & FALSE & 2.633534 & FALSE \\
\hline 0 & 0.0141 & 2 & 0.0071 & 1.4735 & 0.2426 & 5.247898 & FALSE & 3.259444 & FALSE \\
\hline $\mathbf{T}$ & 0.0599 & 2 & 0.03 & 6.2606 & 0.0046 & 5.247898 & TRUE & 3.259444 & TRUE \\
\hline Error & 0.1723 & 36 & 0.0048 & & & & & & \\
\hline Total & 0.4174 & 53 & & & & & & & \\
\hline
\end{tabular}


Table B.34 Factorial Analysis of Variance: Left Casting Angle of Resin Bonded Castings (all)

$\begin{array}{lc}\begin{array}{l}\text { Number of Factors } \\ \text { Factor }\end{array} & \begin{array}{c}\text { Number of Replicates } \\ \text { Levels }\end{array} \\ G=\text { Gating } & 2 \\ O=\text { Orientation } & 3 \\ T=\text { Thickness } & 3\end{array}$

$\begin{array}{lccccccccc} & & & & & & \mathbf{F} & & \mathbf{F} & \\ \text { Source } & \text { SS } & \text { DF } & \text { MS } & \mathbf{F} & \mathbf{P} & \mathbf{0 . 0 1} & \text { significant? } & \mathbf{0 . 0 5} & \text { significant? } \\ \text { GOT } & 0.0006 & 4 & 0.0002 & 2.2933 & 0.0783 & 3.890307 & \text { FALSE } & 2.633534 & \text { FALSE } \\ \text { GO } & 0.0005 & 2 & 0.0002 & 3.4268 & 0.0434 & 5.247898 & \text { FALSE } & 3.259444 & \text { TRUE } \\ \text { GT } & 0.0002 & 2 & 8.36 E-05 & 1.2268 & 0.3052 & 5.247898 & \text { FALSE } & 3.259444 & \text { FALSE } \\ \text { G } & 0.0004 & 1 & 0.0004 & 6.4986 & 0.0152 & 7.395556 & \text { FALSE } & 4.113161 & \text { TRUE } \\ \text { OT } & 0.0005 & 4 & 0.0001 & 1.9545 & 0.1225 & 3.890307 & \text { FALSE } & 2.633534 & \text { FALSE } \\ \text { O } & 0.0005 & 2 & 0.0002 & 3.5362 & 0.0396 & 5.247898 & \text { FALSE } & 3.259444 & \text { TRUE } \\ \text { T } & 0.0014 & 2 & 0.0007 & 10.5837 & 0.0002 & 5.247898 & \text { TRUE } & 3.259444 & \text { TRUE } \\ \text { Error } & 0.0025 & 36 & 6.81 E-05 & & & & & & \end{array}$

Total $\quad 0.0066 \quad 53$ 
Table B.35 Factorial Analysis of Variance: Right Casting Angle of Resin Bonded Castings

(all)

$\begin{array}{lc}\begin{array}{l}\text { Number of Factors } \\ \text { Factor }\end{array} & \begin{array}{c}\text { Number of Replicates } \\ \text { Levels }\end{array} \\ G=\text { Gating } & 2 \\ O=\text { Orientation } & 3 \\ T=\text { Thickness } & 3\end{array}$

\begin{tabular}{|c|c|c|c|c|c|c|c|c|c|}
\hline & & & & & & $\mathbf{F}$ & & $\mathbf{F}$ & \\
\hline Source & SS & DF & MS & $\mathbf{F}$ & $\mathbf{P}$ & 0.01 & significant? & 0.05 & significant? \\
\hline GOT & 0.0534 & 4 & 0.0134 & 2.272 & 0.0805 & 3.890307 & FALSE & 2.633534 & FALSE \\
\hline GO & 0.0403 & 2 & 0.0201 & 3.4242 & 0.0435 & 5.247898 & FALSE & 3.259444 & TRUE \\
\hline GT & 0.0069 & 2 & 0.0034 & 0.5868 & 0.5614 & 5.247898 & FALSE & 3.259444 & FALSE \\
\hline $\mathbf{G}$ & 0.0023 & 1 & 0.0023 & 0.3966 & 0.5328 & 7.395556 & FALSE & 4.113161 & FALSE \\
\hline OT & 0.0076 & 4 & 0.0019 & 0.3252 & 0.8592 & 3.890307 & FALSE & 2.633534 & FALSE \\
\hline 0 & 0.0017 & 2 & 0.0008 & 0.1427 & 0.8675 & 5.247898 & FALSE & 3.259444 & FALSE \\
\hline$T$ & 0.0783 & 2 & 0.0391 & 6.658 & 0.0035 & 5.247898 & TRUE & 3.259444 & TRUE \\
\hline Error & 0.2116 & 36 & 0.0059 & & & & & & \\
\hline Total & 0.4021 & 53 & & & & & & & \\
\hline
\end{tabular}


Table B.36 Factorial Analysis of Variance: Left Flange Flatness of Resin Bonded Castings

(ali)

$\begin{array}{lc}\text { Number of Factors } & \begin{array}{c}\text { Number of Replicates } \\ \text { Levels }\end{array} \\ \text { Factor } & 2 \\ G=\text { Gating } & 3 \\ O=\text { Orientation } & 3 \\ \text { T=Thickness } & \end{array}$

\begin{tabular}{|c|c|c|c|c|c|c|c|c|c|}
\hline & & & & & & $\mathbf{F}$ & & $F_{-}$ & \\
\hline Source & SS & DF & MS & $\mathbf{F}$ & $\mathbf{P}$ & 0.01 & significant? & $0 . \overline{5}$ & significant? \\
\hline GOT & 0.0005 & 4 & 0.0001 & 2.1872 & 0.09 & 3.890307 & FALSE & 2.633534 & FALSE \\
\hline GO & 0.0011 & 2 & 0.0006 & 9.5365 & 0.0005 & 5.247898 & TRUE & 3.259444 & TRUE \\
\hline GT & 0.0002 & 2 & 8.03E-05 & 1.3571 & 0.2703 & 5.247898 & FALSE & 3.259444 & FALSE \\
\hline $\mathbf{G}$ & 0.0008 & 1 & 0.0008 & 12.898 & 0.001 & 7.395556 & TRUE & 4.113161 & TRUE \\
\hline OT & 0.0003 & 4 & 7.81E-05 & 1.3205 & 0.281 & 3.890307 & FALSE & 2.633534 & FALSE \\
\hline 0 & 0.001 & 2 & 0.0005 & 8.7834 & 0.0008 & 5.247898 & TRUE & 3.259444 & TRUE \\
\hline$T$ & 0.0002 & 2 & 8.39E-05 & 1.4172 & 0.2556 & 5.247898 & FALSE & 3.259444 & FALSE \\
\hline Error & 0.0021 & 36 & 5.92E-05 & & & & & & \\
\hline Total & 0.0062 & 53 & & & & & & & \\
\hline
\end{tabular}


Table B.37 Factorial Analysis of Variance: Right Flange Flatness of Resin Bonded Castings (all)

$\begin{array}{lc}\begin{array}{l}\text { Number of Factors } \\ \text { Factor }\end{array} & \begin{array}{c}\text { Number of Replicates } \\ \text { Levels }\end{array} \\ G=\text { Gating } & 2 \\ O=\text { Orientation } & 3 \\ T=\text { Thickness } & 3\end{array}$

\begin{tabular}{|c|c|c|c|c|c|c|c|c|c|}
\hline & & & & & & $\mathbf{F}$ & & $\mathbf{F}$ & \\
\hline Source & SS & DF & MS & $\mathbf{F}$ & $\mathbf{P}$ & 0.01 & significant? & 0.05 & significant? \\
\hline GOT & $3.79 E-05$ & 4 & 9.46E-06 & 0.123 & 0.9733 & 3.890307 & FALSE & 2.633534 & FALSE \\
\hline GO & 0.0005 & 2 & 0.0002 & 3.0883 & 0.0578 & 5.247898 & FALSE & 3.259444 & FALSE \\
\hline GT & 6.26E-06 & 2 & 3.13E-06 & 0.0407 & 0.9602 & 5.247898 & FALSE & 3.259444 & FALSE \\
\hline $\mathbf{G}$ & 0.0001 & 1 & 0.0001 & 1.6584 & 0.206 & 7.395556 & FALSE & 4.113161 & FALSE \\
\hline OT & $3.03 E-05$ & 4 & 7.57E-06 & 0.0985 & 0.9823 & 3.890307 & FALSE & 2.633534 & FALSE \\
\hline 0 & 0.0003 & 2 & 0.0001 & 1.8519 & 0.1716 & 5.247898 & FALSE & 3.259444 & FALSE \\
\hline$T$ & 0.0002 & 2 & 9.87E-05 & 1.2829 & 0.2896 & 5.247898 & FALSE & 3.259444 & FALSE \\
\hline Error & 0.0028 & 36 & 7.69E-05 & & & & & & \\
\hline Total & 0.0039 & 53 & & & & & & & \\
\hline
\end{tabular}


Table B.38 Factorial Analysis of Variance: Left Wall Flatness of Resin Bonded Castings

(all)

$\begin{array}{lc}\begin{array}{l}\text { Number of Factors } \\ \text { Factor }\end{array} & \begin{array}{c}\text { Number of Replicates } \\ \text { Levels }\end{array} \\ \text { G=Gating } & 2 \\ \text { O=Orientation } & 3 \\ \text { T=Thickness } & 3\end{array}$

\begin{tabular}{lccccccccc} 
& & & & & & $\mathbf{F}$ & & \multicolumn{1}{c}{} \\
Source & $\mathbf{S S}$ & $\mathrm{DF}$ & $\mathbf{M S}$ & $\mathbf{F}$ & $\mathbf{P}$ & $\mathbf{0 . 0 1}$ & significant? & $\mathbf{0 . 0 5}$ & significant? \\
GOT & 0.0011 & 4 & 0.0003 & 3.3953 & 0.0187 & 3.890307 & FALSE & 2.633534 & TRUE \\
GO & 0.0005 & 2 & 0.0003 & 3.2831 & 0.049 & 5.247898 & FALSE & 3.259444 & TRUE \\
GT & 0.0004 & 2 & 0.0002 & 2.1825 & 0.1275 & 5.247898 & FALSE & 3.259444 & FALSE \\
G & 0.0003 & 1 & 0.0003 & 3.5081 & 0.0692 & 7.395556 & FALSE & 4.113161 & FALSE \\
OT & 0.0009 & 4 & 0.0002 & 2.7857 & 0.041 & 3.890307 & FALSE & 2.633534 & TRUE \\
O & 0.0003 & 2 & 0.0002 & 1.8673 & 0.1692 & 5.247898 & FALSE & 3.259444 & FALSE \\
T & 0.0001 & 2 & $5.17 E-05$ & 0.6266 & 0.5401 & 5.247898 & FALSE & 3.259444 & FALSE \\
Error & 0.003 & 36 & $8.25 E-05$ & & & & & &
\end{tabular}


Table B.39 Factorial Analysis of Variance: Right Wall Flatness of Resin Bonded Castings (all)

$\begin{array}{lc}\text { Number of Factors } & \begin{array}{c}\text { Number of Replicates } \\ \text { Levels }\end{array} \\ \text { Factor } & 2 \\ G=\text { Gating } & 3 \\ O=\text { Orientation } & 3 \\ T=\text { Thickness } & \end{array}$

\begin{tabular}{lccccccccc} 
& & & & & \multicolumn{3}{c}{$\mathbf{F}$} & & $\mathbf{F}$ \\
Source & SS & DF & MS & $\mathbf{F}$ & $\mathbf{P}$ & $\mathbf{0 . 0 1}$ & significant? & $\mathbf{0 . 0 5}$ & significant? \\
GOT & 0.0003 & 4 & $8.18 E-05$ & 0.2999 & 0.8761 & 3.890307 & FALSE & 2.633534 & FALSE \\
GO & 0.0005 & 2 & 0.0002 & 0.8513 & 0.4353 & 5.247898 & FALSE & 3.259444 & FALSE \\
GT & 0.0051 & 2 & 0.0025 & 9.2658 & 0.0006 & 5.247898 & TRUE & 3.259444 & TRUE \\
G & $7.41 E-06$ & 1 & $7.41 E-06$ & 0.0271 & 0.8701 & 7.395556 & FALSE & 4.113161 & FALSE \\
OT & 0.0007 & 4 & 0.0002 & 0.6568 & 0.626 & 3.890307 & FALSE & 2.633534 & FALSE \\
O & 0.0001 & 2 & $5.36 E-05$ & 0.1965 & 0.8224 & 5.247898 & FALSE & 3.259444 & FALSE \\
T & 0.0003 & 2 & 0.0002 & 0.5754 & 0.5676 & 5.247898 & FALSE & 3.259444 & FALSE \\
Error & 0.0098 & 36 & 0.0003 & & & & & &
\end{tabular}


Table B.40 Factorial Analysis of Variance

Resin Bonded Castings

(0.25 inch)

Number of Factors $=2$

Number of Replicates $=3$

Factor

$\mathrm{G}=$ Gating System

Levels

$\mathrm{O}=$ Orientation

2

3

Left Flange Flatness

\begin{tabular}{|c|c|c|c|c|c|c|c|c|c|}
\hline \multicolumn{5}{|c|}{$\therefore$} & \multicolumn{3}{|c|}{$\mathbf{F}$} & \multicolumn{2}{|l|}{$\mathbf{F}$} \\
\hline Source & SS & DF & MS & $\mathbf{F}$ & $\mathbf{P}$ & 0.01 & significant? & 0.05 & significant? \\
\hline GO & $4.3 \mathrm{IE}-05$ & 2 & $2.16 \mathrm{E}-05$ & 0.728 & 0.503 & 6.926598 & FALSE & 3.88529 & FALSE \\
\hline$G$ & $4.67 \mathrm{E}-05$ & 1 & $4.67 \mathrm{E}-05^{\prime}$ & 1.5779 & 0.233 & 9.330279 & FALSE & 4.747221 & FALSE \\
\hline 0 & 0.0002 & 2 . & $9.96 \mathrm{E}-05$ & 3.3621 & 0.0693 & 6.926598 & FALSE & 3.88529 & FALSE \\
\hline Error & 0.0004 & 12 & $2.96 \mathrm{E}-05$ & & & & & & \\
\hline tal & 0.0006 & 17 & & & & & & & \\
\hline
\end{tabular}

Right Flange Flatness

\begin{tabular}{lccccccccc} 
& & & & & \multicolumn{5}{c}{$\mathbf{F}$} \\
Source & SS & DF & MS & F & P & $\mathbf{0 . 0 1}$ & significant? & $\mathbf{0 . 0 5}$ & significant? \\
GO & $2.00 E-04$ & 2 & $1.00 \mathrm{E}-04$ & 1.8406 & 0.2008 & 6.926598 & FALSE & 3.88529 & FALSE \\
G & $2.01 \mathrm{E}-05$ & 1 & $2.01 \mathrm{E}-05$ & 0.3406 & 0.5703 & 9.330279 & FALSE & 4.747221 & FALSE \\
O & 0.0001 & 2 & $6.95 \mathrm{E}-05$ & 1.1802 & 0.3405 & 6.926598 & FALSE & 3.88529 & FALSE \\
Error & 0.0007 & 12 & $5.89 \mathrm{E}-05$ & & & & & &
\end{tabular}

Total $\quad 0.0011 \quad 17$

\section{Left Wall Flatness}

\begin{tabular}{|c|c|c|c|c|c|c|c|c|c|}
\hline Source & SS & DF & MS & $\mathbf{F}$ & $\mathbf{P}$ & $\begin{array}{c}\mathbf{F} \\
\mathbf{0 . 0 1}\end{array}$ & significant? & $\begin{array}{c}\mathbf{F} \\
0.05\end{array}$ & significant? \\
\hline GO & 0.0001 & 2 & $5.07 \mathrm{E}-05$ & 0.958 & 0.4112 & 6.926598 & FALSE & 3.88529 & FALSE \\
\hline G & 0.0003 & 1 & 0.0003 & 5.4454 & 0.0378 & 9.330279 & FALSE & 4.747221 & TRUE \\
\hline 0 & 0.0005 & 2 & 0.0003 & 5.0294 & 0.0259 & 6.926598 & FALSE & 3.88529 & TRUE \\
\hline Error & 0.0006 & 12. & $5.29 E-05$ & & & , & & & \\
\hline Total & 0.0016 & 17 & & & & & & & \\
\hline
\end{tabular}

Right Wall Flatness

$\begin{array}{lccccccccc} & & & & & & \mathbf{F} & & \mathbf{F} & \\ \text { Source } & \text { SS } & \text { DF } & \text { MS } & \mathbf{F} & \mathbf{P} & \mathbf{0 . 0 1} & \text { significant? } & \mathbf{0 . 0 5} & \text { significant? } \\ \text { GO } & 2.10 \mathrm{E}-05 & 2 & 1.05 \mathrm{E}-05 & 0.3474 & 0.7134 & 6.926598 & \text { FALSE } & 3.88529 & \text { FALSE } \\ \text { G } & 8.00 \mathrm{E}-04 & 1 & \mathbf{8 . 0 0 \mathrm { E } - 0 4} & 25.1636 & 0.0003 & 9.330279 & \text { TRUE } & 4.747221 & \text { TRUE } \\ \text { O } & 5.83 \mathrm{E}-05 & 2 & 2.92 \mathrm{E}-05 & 0.9651 & 0.4087 & 6.926598 & \text { FALSE } & 3.88529 & \text { FALSE } \\ \text { Error } & 0.0004 & 12 & 3.02 \mathrm{E}-05 & & & & & & \\ & & & & & & & & & \end{array}$


Table B.41 Factorial Analysis of Variance

Resin Bonded Castings

. (0.25 inch)

\author{
Number of Factors $=2$ \\ Factor \\ $\mathrm{G}=$ Gating System \\ $\mathrm{O}=$ Orientation
}

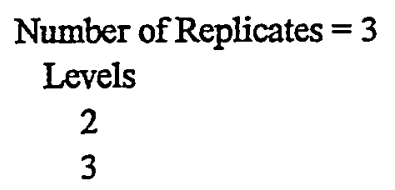

Height

$\begin{array}{lccccccccc} & & & & & & \mathbf{F} & & \mathbf{F} & \text { I } \\ \text { Source } & \text { SS } & \text { DF } & \text { MS } & \mathbf{F} & \mathbf{P} & \mathbf{0 . 0 1} & \text { significant? } & \mathbf{0 . 0 5} & \text { significant? } \\ \text { GO } & 0.0033 & 2 & 1.70 \mathrm{E}-03 & 3.1661 & 0.0787 & 6.926598 & \text { FALSE } & 3.88529 & \text { FALSE } \\ \text { G } & 0.0038 & 1 & 0.0038 & 7.1934 & 0.02 & 9.330279 & \text { FALSE } & 4.747221 & \text { TRUE } \\ \text { O } & 0.0035 & 2 & 0.0018 & 3.3673 & 0.0691 & 6.926598 & \text { FALSE } & 3.88529 & \text { FALSE } \\ \text { Error } & 0.0063 & 12 & 0.0005 & & & & & & \\ \text { Total } & 0.0169 & 17 & & & & & & & \end{array}$

\section{Length}

\begin{tabular}{lccccccccc} 
& & & & & & $\mathbf{F}$ & \multicolumn{3}{c}{$\mathbf{F}$} \\
Source & SS & DF & MS & $\mathbf{F}$ & $\mathbf{P}$ & $\mathbf{0 . 0 1}$ & significant? & $\mathbf{0 . 0 5}$ & significant? \\
GO & 0.0002 & 2 & $7.98 E-05$ & 0.3691 & 0.6989 & 6.926598 & FALSE & 3.88529 & FALSE \\
G & 0.0008 & 1 & 0.0008 & 3.7416 & 0.077 & 9.330279 & FALSE & 4.747221 & FALSE \\
O & $\mathbf{0 . 0 0 2 4}$ & 2 & 0.0012 & 5.493 & 0.0202 & 6.926598 & FALSE & 3.88529 & TRUE \\
Error & 0.0026 & 12 & 0.0002 & & & & & &
\end{tabular}

$\begin{array}{lll}\text { Total } & 0.0059 & 17\end{array}$

\begin{tabular}{lccccccccc} 
& \multicolumn{8}{c}{ Left Casting Angle } \\
Source & SS & DF & MS & F & P & $\mathbf{~ F ~}$ & significant? & 0.05 & significant? \\
GO & 0.0008 & 2 & $4.00 \mathrm{E}-04$ & 3.7114 & 0.0556 & 6.926598 & FALSE & 3.88529 & FALSE \\
G & $2.84 \mathrm{E}-05$ & 1 & $2.84 \mathrm{E}-05$ & $\mathbf{0 . 2 7 8 9}$ & 0.6071 & 9.330279 & FALSE & 4.747221 & FALSE \\
O & 0.0002 & 2 & 0.0001 & 1.1281 & 0.3557 & 6.926598 & FALSE & 3.88529 & FALSE \\
Error & 0.0012 & 12 & 0.0001 & & & & & &
\end{tabular}

$\begin{array}{lll}\text { Total } & 0.0022 & 17\end{array}$

Right Casting Angle

$\begin{array}{lccccccccc} & & & & & & \mathbf{F} & & \text { F } & \\ \text { Source } & \text { SS } & \text { DF } & \text { MS } & \text { F } & \text { P } & \mathbf{0 . 0 1} & \text { significant? } & \mathbf{0 . 0 5} & \text { significant? } \\ \text { GO } & 0.0922 & 2 & 4.61 \mathrm{E}-02 & 2.6597 & 0.1106 & 6.926598 & \text { FALSE } & 3.88529 & \text { FALSE } \\ \text { G } & 9.10 \mathrm{E}-03 & 1 & 9.10 \mathrm{E}-03 & 0.5254 & 0.4824 & 9.330279 & \text { FALSE } & 4.747221 & \text { FALSE } \\ \text { O } & 0.007 & 2 & 0.0035 & 0.2007 & 0.8208 & 6.926598 & \text { FALSE } & 3.88529 & \text { FALSE } \\ \text { Error } & 0.208 & 12 & 0.0173 & & & & & & \\ & & & & & & & & & \end{array}$


Table B.42 Factorial Analysis of Variance Resin Bonded Castings

(0.25 inch)

Number of Factors $=2$

Factor

$\mathrm{G}=$ Gating System

$\mathrm{O}=$ Orientation
Number of Replicates $=3$

Levels

2

3

Parallelism

$\begin{array}{lccccccccc} & & & & & & \text { F } & & \text { F } & \\ \text { Source } & \text { SS } & \text { DF } & \text { MS } & \text { F } & \text { P } & \mathbf{0 . 0 1} & \text { significant? } & \mathbf{0 . 0 5} & \text { significant? } \\ \text { GO } & 0.0957 & 2 & 0.0478 & 3.4831 & 0.0642 & 6.926598 & \text { FALSE } & 3.88529 & \text { FALSE } \\ \text { G } & 0.0646 & 1 & 0.0646 & 4.7028 & 0.0509 & 9.330279 & \text { FALSE } & 4.747221 & \text { FALSE } \\ \text { O } & 0.0129 & 2 & 0.0065 & 0.4714 & 0.6352 & 6.926598 & \text { FALSE } & 3.88529 & \text { FALSE } \\ \text { Error } & 0.1648 & 12 & 0.0137 & & & & & & \\ \text { Total } & 0.3379 & 17 & & & & & & & \end{array}$

Thickness

$\begin{array}{lccccccccc} & & & & & & \mathbf{F} & & \mathbf{F} & \\ \text { Source } & \text { SS } & \mathbf{D F} & \text { MS } & \mathbf{F} & \mathbf{P} & \mathbf{0 . 0 1} & \text { significant? } & \mathbf{0 . 0 5} & \text { significant? } \\ \text { GO } & 1.18 \mathrm{E}-05 & 2 & 5.89 \mathrm{E}-06 & 0.0341 & 0.9666 & 6.926598 & \text { FALSE } & 3.88529 & \text { FALSE } \\ \text { G } & 1.80 \mathrm{E}-05 & 1 & 1.80 \mathrm{E}-05 & 0.1044 & 0.7522 & 9.330279 & \text { FALSE } & 4.747221 & \text { FALSE } \\ \text { O } & 0.0025 & 2 & 0.0013 & 7.3535 & 0.0082 & 6.926598 & \text { TRUE } & 3.88529 & \text { TRUE } \\ \text { Error } & 0.0021 & 12 & 0.0002 & & & & & & \\ & & & & & & & & & \end{array}$


Table B.43 Factorial Analysis of Variance:

Resin Bonded Castings

(0.50 inch)

Number of Factors $=2$

Number of Replicates $=3$

Factor .

Levels

$\mathrm{G}=$ Gating System

2

$\mathrm{O}=$ Orientation

3

Left Flange Flatness

$\begin{array}{lccccccccc} & & & & & & \mathbf{F} & & \mathbf{F} & \\ \text { Source } & \text { SS } & \text { DF } & \text { MS } & \mathbf{F} & \mathbf{P} & \mathbf{0 . 0 1} & \text { significant? } & \mathbf{0 . 0 5} & \text { significant? } \\ \text { GO } & 0.0013 & \therefore 2 & 0.0006 & 5.8537 & 0.0168 & 6.926598 & \text { FALSE } & 3.88529 & \text { TRUE } \\ \text { G } & 0.0006 & 1 & 0.0006 & 5.6597 & 0.0348 & 9.330279 & \text { FALSE } & 4.747221 & \text { TRUE } \\ \text { O } & 0.0008 & 2 & 0.0004 & 3.7161 & 0.0555 & 6.926598 & \text { FALSE } & 3.88529 & \text { FALSE } \\ \text { Error } & \mathbf{0 . 0 0 1 3} & 12 & 0.0001 & & & & & & \\ & & & & & & & & & \end{array}$

Right Flange Flatness

$\begin{array}{lccccccccc} & & & & & & \mathbf{F} & & \mathbf{F} & \\ \text { Source } & \text { SS } & \text { DF } & \text { MS } & \text { F } & \mathbf{P} & \mathbf{0 . 0 1} & \text { significant? } & \mathbf{0 . 0 5} & \text { significant? } \\ \text { GO } & 0.0001 & 2 & 7.41 \mathrm{E}-05 & 0.8617 & 0.447 & 6.926598 & \text { FALSE } & 3.88529 & \text { FALSE } \\ \text { G } & 5.69 \mathrm{E}-05 & 1 & 5.69 \mathrm{E}-05 & 0.6619 & 0.4317 & 9.330279 & \text { FALSE } & 4.747221 & \text { FALSE } \\ \text { O } & 8.81 \mathrm{E}-05 & 2 & 4.41 \mathrm{E}-05 & 0.5126 & 0.6115 & 6.926598 & \text { FALSE } & 3.88529 & \text { FALSE } \\ \text { Error } & 0.001 & 12 & 8.59 \mathrm{E}-05 & & & & & & \\ & & & & & & & & & \\ \text { Total } & \mathbf{0 . 0 0 1 3} & 17 & & & & & & & \end{array}$

Left Wall Flatness

\begin{tabular}{|c|c|c|c|c|c|c|c|c|c|}
\hline \multirow[b]{2}{*}{ Source } & \multirow[b]{2}{*}{ SS } & \multirow[b]{2}{*}{ DF } & \multirow[b]{2}{*}{ MS } & \multirow[b]{2}{*}{$\mathbf{F}$} & \multicolumn{2}{|r|}{$\mathbf{F}$} & \multicolumn{3}{|c|}{$\mathbf{F}$} \\
\hline & & & & & $\mathbf{P}$ & 0.01 & significant? & 0.05 & significant \\
\hline GO & 0.0001 & 2 & $5.07 \mathrm{E}-05$ & 0.958 & 0.4112 & 6.926598 & FALSE & 3.88529 & FALSE \\
\hline G & 0.0003 & 1 & 0.0003 & 5.4454 & 0.0378 & 9.330279 & FALSE & 4.747221 & TRUE \\
\hline 0 & 0.0005 & 2 & 0.0003 & 5.0294 & 0.0259 & 6.926598 & FALSE & 3.88529 & TRUE \\
\hline Error & 0.0006 & 12 & $5.29 \mathrm{E}-\mathrm{d}-5$ & & & & & & \\
\hline otal & 0.0016 & 17 & & & & & & & \\
\hline
\end{tabular}

Right Wall Flatness

\begin{tabular}{|c|c|c|c|c|c|c|c|c|c|}
\hline & & & & & & $\mathbf{F}$ & & $\mathbf{F}$ & \\
\hline Source & SS & DF & MS & $\mathbf{F}$ & $\mathbf{P}$ & 0.01 & significant? & 0.05 & significant? \\
\hline GO & $3.21 E-05$ & 2 & $1.61 \mathrm{E}-05$ & 0.3345 & 0.7222 & 6.926598 & FALSE & 3.88529 & FALSE \\
\hline $\mathrm{G}$ & 0.0007 & 1 & 0.0007 & 15.5741 & 0.0019 & 9.330279 & TRUE & 4.747221 & TRUE \\
\hline O & 0.0006 & 2 & 0.0003 & 5.7789 & 0.0175 & 6.926598 & FALSE & 3.88529 & TRUE \\
\hline Error & 0.0006 & 12 & $4.80 \mathrm{E}-05$ & & & & & & \\
\hline Total & 0.0019 & 17 & & & & & & & \\
\hline
\end{tabular}


Table B.44 Factorial Analysis of Variance:

Resin Bonded Castings

(0.50 inch)

Number of Factors $=2$

Factor

$\mathrm{G}=$ Gating System

$\mathrm{O}=$ Orientation
Number of Replicates $=3$

Levels

2

3

: Height

\begin{tabular}{|c|c|c|c|c|c|c|c|c|c|}
\hline \multirow{3}{*}{ Source } & \multirow[b]{2}{*}{ SS } & \multirow[b]{2}{*}{ DF } & \multirow[b]{2}{*}{ MS } & \multirow[b]{2}{*}{$\mathbf{F}$} & \multirow[b]{2}{*}{$\mathbf{P}$} & $\mathbf{F}$ & \multicolumn{3}{|c|}{$\mathbf{F}$} \\
\hline & & & & & & 0.01 & significant? & 0.05 & significant? \\
\hline & 0.0009 & 2 & $5.00 \mathrm{E}-04$ & 3.6468 & 0.0579 & 6.926598 & FAISE & 3.88529 & FALSE \\
\hline G & 0.0006 & 1 & 0.0006 & 4.4439 & 0.0567 & 9.330279 & FALSE & 4.747221 & FALSE \\
\hline 0 & 0.0013 & 2 & 0.0006 & 5.1016 & 0.0249 & 6.926598 & FALSE & 3.88529 & TRUE \\
\hline Error & 0.0015 & 12 & 0.0001 & & & & & & \\
\hline \multirow[t]{3}{*}{ Total } & 0.0043 & 17 & & & & & & & \\
\hline & & & & & Length & & & & \\
\hline & & & & & & $\mathbf{F}$ & & $\mathbf{F}$ & \\
\hline Source & SS & DF & MS & $\mathbf{F}$ & $\mathbf{P}$ & 0.01 & significant? & 0.05 & significant? \\
\hline GO & 0.0005 & 2 & 0.0003 & 6.627 & 0.0115 & 6.926598 & FALSE & 3.88529 & TRUE \\
\hline $\mathrm{G}$ & 0.0002 & 1 & 0.0002 & 4.0794 & 0.0663 & 9.330279 & FALSE & 4.747221 & FALSE \\
\hline $\mathrm{O}$ & 0.0029 & 2 & 0.0014 & 35.2632 & $9.45 E-06$ & 6.926598 & TRUE & 3.88529 & TRUE \\
\hline Error & 0.0005 & 12 & $4.08 \mathrm{E}-05$ & & & & & & \\
\hline Total & 0.0041 & 17 & & & & & & & \\
\hline
\end{tabular}

Left Casting Angle

\begin{tabular}{|c|c|c|c|c|c|c|c|c|c|}
\hline \multirow[b]{2}{*}{ Source } & \multirow{3}{*}{ SS } & \multirow[b]{2}{*}{ DF } & \multirow[b]{2}{*}{ MS } & \multirow[b]{2}{*}{$\mathbf{F}$} & \multirow[b]{2}{*}{$\mathbf{P}$} & \multirow{2}{*}{$F$} & \multicolumn{3}{|c|}{$\mathbf{F}$} \\
\hline & & & & & & & significant? & 0.05 & significant? \\
\hline GO & & 2 & $1.80 E-05$ & 0.3681 & 0.6996 & 6.926598 & FALSE & 3.88529 & FALSE \\
\hline G & $7.36 \mathrm{E}-05$ & 1 & 7.36E-05 & 1.5065 & 0.2432 & 9.330279 & FALSE & 4.747221 & FALSE \\
\hline 0 & 0.0001 & 2 & $6.75 \mathrm{E}-05$ & 1.3824 & 0.2882 & 6.926598 & FALSE & 3.88529 & FALSE \\
\hline Error & 0.0006 & 12 & $4.89 \mathrm{E}-05$ & & & & & & \\
\hline
\end{tabular}

Total . $0.0008 \quad 17$

\section{Right Casting Angle}

$\begin{array}{lccccccccc} & & & & & & \mathbf{F} & & \mathbf{F} & \\ \text { Source } & \text { SS } & \text { DF } & \text { MS } & \text { F } & \text { P } & \mathbf{0 . 0 1} & \text { significant? } & \mathbf{0 . 0 5} & \text { significant? } \\ \text { GO } & 0.0002 & 2 & 0.0001 & 0.5 & 0.6186 & 6.926598 & \text { FALSE } & 3.88529 & \text { FALSE } \\ \text { G } & 6.81 E-07 & 1 & 6.81 E-07 & 0.0028 & 0.9583 & 9.330279 & \text { FALSE } & 4.747221 & \text { FALSE } \\ \text { O } & 0.0012 & 2 & 0.0006 & 2.5105 & 0.1228 & 6.926598 & \text { FALSE } & 3.88529 & \text { FALSE } \\ \text { Error } & 0.0029 & 12 & 0.0002 & & & & & & \end{array}$

Total $\quad 0.0043 \quad 17$ 
Table B.45 Factorial Analysis of Variance:

Resin Bonded Castings

(0.50 inch) .

\author{
Number of Factors $=2$ \\ Factor \\ $\mathrm{G}=$ Gating System \\ $\mathrm{O}=$ Orientation
}

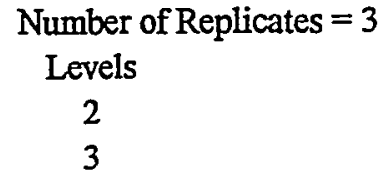

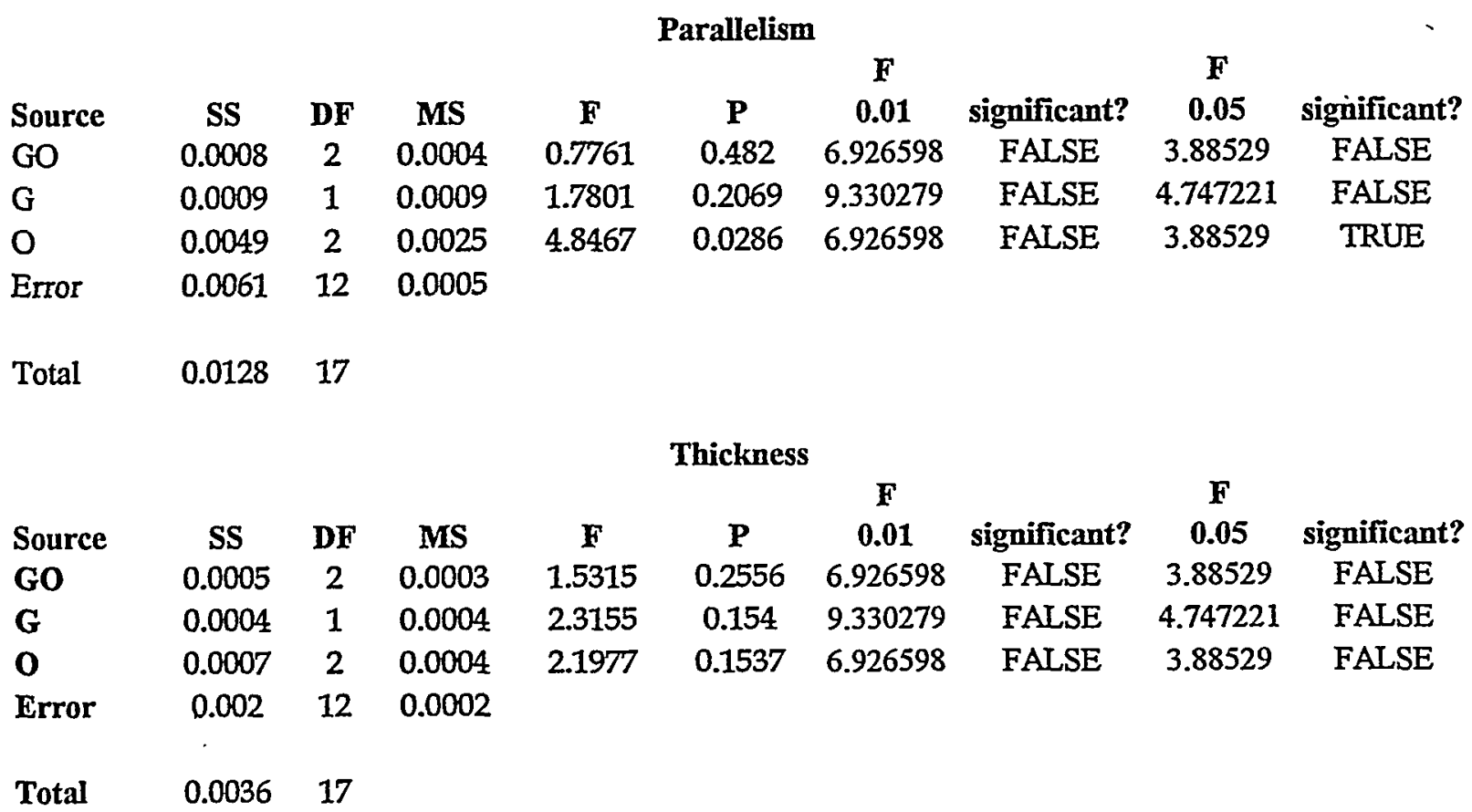


Table B.46 Factorial Analysis of Variance:

Resin Bonded Castings

(0.75 inch)

Number of Factors $=2$

Number of Replicates $=3$

Factor

Levels

$\mathrm{G}=$ Gating System

2

$\mathrm{O}=$ Orientation

3

\section{Left Flange Flatness}

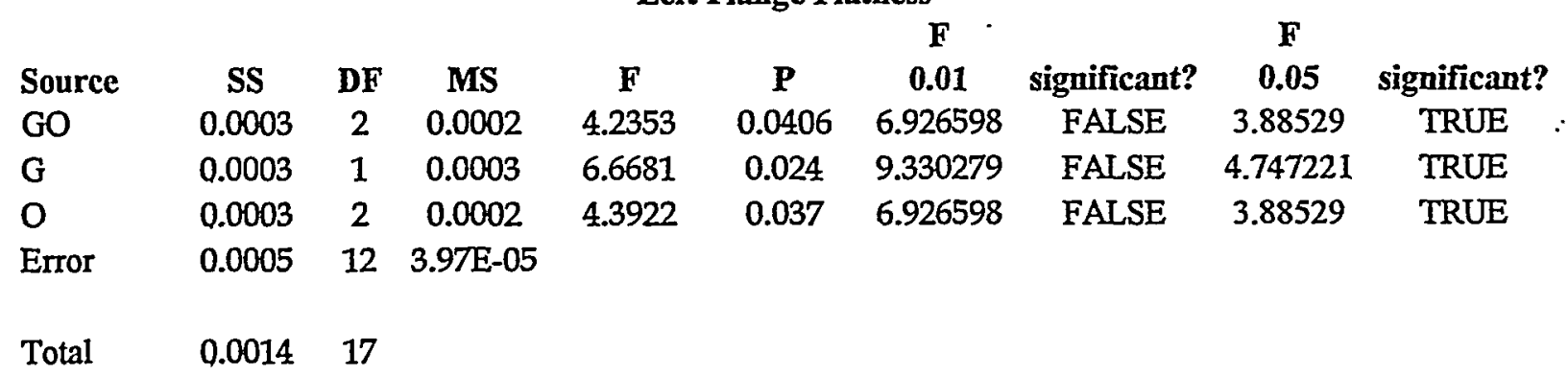

Right Flange Flatness

$\begin{array}{lccccccccc} & & & & & & \mathbf{F} & & \mathbf{F} & \\ \text { Source } & \text { SS } & \text { DF } & \text { MS } & \mathbf{F} & \mathbf{P} & \mathbf{0 . 0 1} & \text { significant? } & \mathbf{0 . 0 5} & \text { significant? } \\ \text { GO } & 0.0001 & 2 & 7.41 E-05 & 0.8617 & 0.447 & 6.926598 & \text { FALSE } & 3.88529 & \text { FALSE } \\ \text { G } & 5.69 \mathrm{E}-05 & 1 & 5.69 \mathrm{E}-05 & 0.6619 & 0.4317 & 9.330279 & \text { FALSE } & 4.747221 & \text { FALSE } \\ \text { O } & 8.81 \mathrm{E}-05 & 2 & 4.41 \mathrm{E}-05 & 0.5126 & 0.6115 & 6.926598 & \text { FALSE } & 3.88529 & \text { FALSE } \\ \text { Error } & 0.001 & 12 & 8.59 E-05 & & & & & & \end{array}$

Total $\quad 0.0013 \quad 17$

Left Wall Flatness

\begin{tabular}{|c|c|c|c|c|c|c|c|c|c|}
\hline \multirow{3}{*}{$\begin{array}{l}\text { Source } \\
\text { GO }\end{array}$} & \multirow{3}{*}{$\begin{array}{c}\text { SS } \\
0.0015\end{array}$} & \multirow[b]{2}{*}{ DF } & \multirow[b]{2}{*}{ MS } & \multirow[b]{2}{*}{$F$} & \multicolumn{2}{|r|}{$\mathbf{F}$} & \multicolumn{3}{|c|}{$\mathbf{F}$} \\
\hline & & & & & $\mathbf{P}$ & 0.01 & significant? & 0.05 & significant? \\
\hline & & 2 & 0.0008 & 4.4897 & 0.035 & 6.926598 & FALSE & 3.88529 & TRUE \\
\hline G & $3.20 \mathrm{E}-05$ & 1 & $3.20 \mathrm{E}-05$ & 0.1874 & 0.6727 & 9.330279 & FALSE & 4.747221 & FALSE \\
\hline 0 & 0.0002 & 2 & $9.82 \mathrm{E}-05$ & 0.575 & 0.5775 & 6.926598 & FALSE & 3.88529 & FALSE \\
\hline Error & 0.002 & 12 & 0.0002 & & & & & & \\
\hline Total & 0.0038 & 17 & & & & & & & \\
\hline
\end{tabular}

Right Wall Flatness

\begin{tabular}{|c|c|c|c|c|c|c|c|c|c|}
\hline \multirow{2}{*}{ Source } & & \multirow[b]{2}{*}{ DF } & \multirow[b]{2}{*}{ MS } & \multirow[b]{2}{*}{$\mathbf{F}$} & \multicolumn{2}{|r|}{$\mathbf{F}$} & \multicolumn{3}{|c|}{$\mathbf{F}$} \\
\hline & & & & & $\mathbf{P}$ & 0.01 & significant? & 0.05 & significant? \\
\hline GO & 0.0007 & 2 & 0.0004 & 0.4989 & 0.6192 & 6.926598 & FALSE & 3.88529 & FALSE \\
\hline G & 0.0036 & 1 & 0.0036 & 4.803 & 0.0489 & 9.330279 & FALSE & 4.747221 & TRUE \\
\hline $\mathrm{O}$ & 0.0002 & 2 & 0.0001 & 0.1425 & 0.8686 & 6.926598 & FALSE & 3.88529 & FALSE \\
\hline Error & 0.0089 & 12 & 0.0007 & & & & & & \\
\hline Total & 0.0134 & 17 & & & & & & & \\
\hline
\end{tabular}


Table B.47 Factorial Analysis of Variance:

Resin Bonded Castings

(0.75 inch)

Number of Factors $=2$

Factor

$\mathrm{G}=$ Gating System

$\mathrm{O}=$ Orientation
Number of Replicates $=3$

Levels

2

3

Height

\begin{tabular}{|c|c|c|c|c|c|c|c|c|c|}
\hline \multirow[b]{2}{*}{ Source } & \multirow[b]{2}{*}{ SS } & \multirow[b]{2}{*}{ DF } & \multirow[b]{2}{*}{ MS } & \multirow[b]{2}{*}{$\mathbf{F}$} & & \multicolumn{3}{|c|}{ 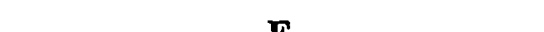 } \\
\hline & & & & & $\mathbf{P}$ & 0.01 & significant? & 0.05 & significant? \\
\hline GO & 0.0015 & 2 & 0.0007 & 5.019 & 0.0261 & 6.926598 & FALSE & 3.88529 & TRUE \\
\hline G & 0.0008 & 1 & 0.0008 & 5.3251 & 0.0396 & 9.330279 & FALSE & 4.747221 & TRUE \\
\hline 0 & 0.0022 & 2 & 0.0011 & 7.3818 & 0.0081 & 6.926598 & TRUE & 3.88529 & TRUE \\
\hline Error & 0.0018 & 12 & 0.0001 & & & & & & \\
\hline Total & 0.0063 & 17 & & & & & & & \\
\hline
\end{tabular}

Length

$\begin{array}{lccccccccc} & & & & & & \mathbf{F} & & \text { F } & \\ \text { Source } & \text { SS } & \text { DF } & \text { MS } & \text { F } & \mathbf{P} & \mathbf{0 . 0 1} & \text { significant? } & \mathbf{0 . 0 5} & \text { significant? } \\ \text { GO } & 1.42 E-05 & 2 & 7.12 E-06 & 0.056 & 0.9458 & 6.926598 & \text { FALSE } & 3.88529 & \text { FALSE } \\ \text { G } & 4.29 \mathrm{E}-05 & 1 & 4.29 \mathrm{E}-05 & 0.337 & 0.5723 & 9.330279 & \text { FALSE } & 4.747221 & \text { FALSE } \\ \text { O } & 0.0002 & 2 & 0.0001 & 0.908 & 0.4293 & 6.926598 & \text { FALSE } & 3.88529 & \text { FALSE } \\ \text { Error } & 0.0015 & \mathbf{1 2} & \mathbf{0 . 0 0 0 1} & & & & & & \end{array}$

$\begin{array}{lll}\text { Total } & 0.0018 \quad 17\end{array}$

\section{Left Casting Angle}

\begin{tabular}{lcccccccccc} 
& & & & & \multicolumn{4}{c}{$\mathbf{F}$} & & \\
Source & SS & DF & MS & F & $\mathbf{P}$ & $\mathbf{0 . 0 1}$ & significant? & $\mathbf{0 . 0 5}$ & significant? \\
GO & 0.0003 & 2 & 0.0002 & 2.7953 & 0.1008 & 6.926598 & FALSE & 3.88529 & FALSE \\
G & 0.0005 & 1 & 0.0005 & 9.4517 & 0.0096 & 9.330279 & TRUE & 4.747221 & TRUE \\
O & 0.0006 & 2 & 0.0003 & 6.0455 & 0.0153 & 6.926598 & FALSE & 3.88529 & TRUE \\
Error & $\mathbf{0 . 0 0 0 6}$ & 12 & $5.37 \mathrm{E}-05$ & & & & & &
\end{tabular}

$\begin{array}{lll}\text { Total } & \mathbf{0 . 0 0 2 1} \quad 17\end{array}$

\section{Right Casting Angle}

$\begin{array}{lccccccccc} & & & & & & \mathbf{F} & & \mathbf{F} & \\ \text { Source } & \text { SS } & \text { DF } & \text { MS } & \mathbf{F} & \mathbf{P} & \mathbf{0 . 0 1} & \text { significant? } & \mathbf{0 . 0 5} & \text { significant? } \\ \text { GO } & \mathbf{0 . 0 0 1 2} & 2 & 0.0006 & 10.0607 & \mathbf{0 . 0 0 2 7} & 6.926598 & \text { TRUE } & 3.88529 & \text { TRUE } \\ \text { G } & 0.0001 & 1 & 0.0001 & 1.98 & 0.1848 & 9.330279 & \text { FALSE } & 4.747221 & \text { FALSE } \\ \text { O } & 0.0012 & 2 & 0.0006 & 9.5522 & 0.0033 & 6.926598 & \text { TRUE } & 3.88529 & \text { TRUE } \\ \text { Error } & 0.0007 & 12 & 6.09 \mathrm{E}-05 & & & & & & \\ & & & & & & & & & \end{array}$


Table B.48 Factorial Analysis of Variance:

Resin Bonded Castings

(0.75 inch)

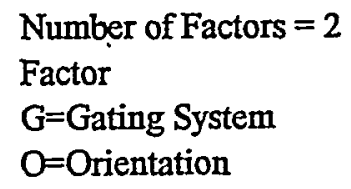

Number of Replicates $=3$

Levels

2

3

\section{Parallelism}

\begin{tabular}{lccccccccc} 
& & & \multicolumn{8}{c}{$\mathbf{c}$} & $\mathbf{F}$ & \multicolumn{2}{c}{$\mathbf{F}$} \\
Source & SS & DF & MS & $\mathbf{F}$ & $\mathbf{P}$ & $\mathbf{0 . 0 1}$ & significant? & $\mathbf{0 . 0 5}$ & significant? \\
GO & 0.003 & 2 & 0.0015 & 12.6439 & 0.0011 & 6.926598 & TRUE & 3.88529 & TRUE \\
G & 0.0007 & 1 & 0.0007 & 5.896 & 0.0318 & 9.330279 & FALSE & 4.747221 & TRUE \\
O & 0.0016 & 2 & 0.0008 & 6.5426 & 0.012 & 6.926598 & FALSE & 3.88529 & TRUE \\
Error & 0.0014 & 12 & 0.0001 & & & & & & .
\end{tabular}

$\begin{array}{lll}\text { Total } & 0.0067 \quad 17\end{array}$

Thickness

$\begin{array}{lccccccccc} & & & & & & \mathbf{F} & & \mathbf{F} & \\ \text { Source } & \text { SS } & \text { DF } & \text { MS } & \text { F } & \mathbf{P} & \mathbf{0 . 0 1} & \text { significant? } & \mathbf{0 . 0 5} & \text { significant? } \\ \text { GO } & 0.0027 & 2 & 0.0014 & 2.275 & 0.1453 & 6.926598 & \text { FALSE } & 3.88529 & \text { FALSE } \\ \text { G } & 0.0001 & 1 & 0.0001 & 0.2125 & 0.653 & 9.330279 & \text { FALSE } & 4.747221 & \text { FALSE } \\ \text { O } & 0.0019 & 2 & 0.0009 & 1.5697 & 0.248 & 6.926598 & \text { FALSE } & 3.88529 & \text { FALSE } \\ \text { Error } & 0.0072 & 12 & 0.0006 & & & & & & \\ & & & & & & & & & \end{array}$


Table B.49 Factorial Analysis of Variance: Thickness of Lost Foam Low Expansion Castings

(ali)

$\begin{array}{lc}\begin{array}{l}\text { Number of Factors } \\ \text { Factor }\end{array} & \begin{array}{c}\text { Number of Replicates } \\ \text { Levels }\end{array} \\ G=\text { Gating } & 2 \\ O=\text { Orientation } & 3 \\ T=\text { Thickness } & 3\end{array}$

\begin{tabular}{|c|c|c|c|c|c|c|c|c|c|}
\hline \multirow{3}{*}{$\begin{array}{l}\text { Source } \\
\text { GOT }\end{array}$} & \multirow{3}{*}{$\begin{array}{c}\text { SS } \\
0.0005\end{array}$} & \multirow{3}{*}{ DF } & \multirow{3}{*}{ MS } & \multirow{3}{*}{$\begin{array}{c}F \\
0.2179\end{array}$} & \multirow[b]{2}{*}{$\mathbf{P}$} & & \multicolumn{3}{|c|}{$\mathbf{F}$} \\
\hline & & & & & & 0.01 & significant? & 0.05 & significant? \\
\hline & & & & & 0.9267 & 3.890307 & FALSE & 2.633534 & FALSE \\
\hline GO & 0.0203 & 2 & 0.0101 & 16.6954 & 7.41E-06 & 5.247898 & TRUE & 3.259444 & TRUE \\
\hline GT & 0.0005 & 2 & 0.0002 & 0.3939 & 0.6773 & 5.247898 & FALSE & 3.259444 & FALSE \\
\hline G & 0.0003 & 1 & 0.0003 & 0.4514 & 0.506 & 7.395556 & FALSE & 4.113161 & FALSE \\
\hline OT & 0.0014 & 4 & 0.0003 & 0.5604 & 0.6928 & 3.890307 & FALSE & 2.633534 & FALSE \\
\hline 0 & 0.0075 & 2 & 0.0037 & 6.1471 & 0.0051 & 5.247898 & TRUE & 3.259444 & TRUE \\
\hline$T$ & 2.4048 & 2 & 1.2024 & 1982.49 & 0 & 5.247898 & TRUE & 3.259444 & TRUE \\
\hline Error & 0.0218 & 36 & 0.0006 & & & & & & \\
\hline Total & 2.457 & 53 & & & & & & & \\
\hline
\end{tabular}


Table B.50 Factorial Analysis of Variance: Height of Lost Foam Low Expansion Castings (all)

$\begin{array}{lc}\begin{array}{l}\text { Number of Factors } \\ \text { Factor }\end{array} & \begin{array}{c}\text { Number of Replicates } \\ \text { Levels }\end{array} \\ G=\text { Gating } & 2 \\ O=\text { Orientation } & 3 \\ T=\text { Thickness } & 3\end{array}$

\begin{tabular}{|c|c|c|c|c|c|c|c|c|c|}
\hline \multirow{3}{*}{$\begin{array}{l}\text { Source } \\
\text { GOT }\end{array}$} & \multirow{3}{*}{$\begin{array}{c}\text { SS } \\
0.0179\end{array}$} & \multirow{3}{*}{ DF } & \multirow[b]{2}{*}{ MS } & \multirow{3}{*}{$\begin{array}{c}F \\
2.5664\end{array}$} & \multirow{3}{*}{$\begin{array}{c}P \\
0.0546\end{array}$} & $\mathbf{F}$ & \multicolumn{3}{|c|}{$\mathbf{F}$} \\
\hline & & & & & & 0.01 & significant? & 0.05 & significant? \\
\hline & & & 0.0045 & & & 3.890307 & FALSE & 2.633534 & FALSE \\
\hline GO & 0.0149 & 2 & 0.0075 & 4.2839 & 0.0214 & 5.247898 & FALSE & 3.259444 & TRUE \\
\hline GT & 0.0069 & 2 & 0.0034 & 1.9726 & 0.1539 & 5.247898 & FALSE & 3.259444 & FALSE \\
\hline $\mathbf{G}$ & 0.0036 & 1 & 0.0036 & 2.0684 & 0.159 & 7.395556 & FALSE & 4.113161 & FALSE \\
\hline OT & 0.0058 & 4 & 0.0014 & 0.827 & 0.5168 & 3.890307 & FALSE & 2.633534 & FALSE \\
\hline 0 & 0.0089 & 2 & 0.0045 & 2.5538 & 0.0918 & 5.247898 & FALSE & 3.259444 & FALSE \\
\hline $\mathrm{T}$ & 0.0125 & 2 & 0.0063 & 3.5968 & 0.0377 & 5.247898 & FALSE & 3.259444 & TRUE \\
\hline Error & 0.0627 & 36 & 0.0017 & & & & & & \\
\hline Total & 0.1332 & 53 & & & & & & & \\
\hline
\end{tabular}


Table B.51 Factorial Analysis of Variance: Length of Lost Foam Low Expansion Castings

(all)

$\begin{array}{lc}\begin{array}{l}\text { Number of Factors } \\ \text { Factor }\end{array} & \begin{array}{c}\text { Number of Replicates } \\ \text { Levels }\end{array} \\ G=\text { Gating } & 2 \\ O=\text { Orientation } & 3 \\ T=\text { Thickness } & 3\end{array}$

\begin{tabular}{|c|c|c|c|c|c|c|c|c|c|}
\hline & & & & & & $\mathbf{F}$ & & $F$ & \\
\hline Source & ss & DF & MS & $\mathbf{F}$ & $\mathbf{P}$ & 0.01 & significant? & 0.05 & significant? \\
\hline GOT & 0.0005 & 4 & 0.0001 & 0.9149 & 0.4658 & 3.890307 & FALSE & 2.633534 & FALSE \\
\hline GO & 0.0002 & 2 & 0.0001 & 0.9595 & 0.3927 & 5.247898 & FALSE & 3.259444 & FALSE \\
\hline GT & 0.0006 & 2 & 0.0003 & 2.3997 & 0.1051 & 5.247898 & FALSE & 3.259444 & FALSE \\
\hline $\mathbf{G}$ & 0.0004 & 1 & 0.0004 & 3.1504 & 0.0844 & 7.395556 & FALSE & 4.113161 & FALSE \\
\hline OT & 0.0011 & 4 & 0.0003 & 2.1631 & 0.093 & 3.890307 & FALSE & 2.633534 & FALSE \\
\hline 0 & 0.0044 & 2 & 0.0022 & 17.0789 & 6.08E-06 & 5.247898 & TRUE & 3.259444 & TRUE \\
\hline$T$ & 0.0263 & 2 & 0.0131 & 102.6046 & 1.35E-15 & 5.247898 & TRUE & 3.259444 & TRUE \\
\hline Error & 0.0046 & 36 & 0.0001 & & & & & & \\
\hline Total & 0.0381 & 53 & & & & & & & \\
\hline
\end{tabular}


Table B.52 Factorial Analysis of Variance: Parallelism of Lost Foam Low Expansion Castings (all)

$\begin{array}{lc}\begin{array}{l}\text { Number of Factors } \\ \text { Factor }\end{array} & \begin{array}{c}\text { Number of Replicates } \\ \text { Levels }\end{array} \\ G=\text { Gating } & 2 \\ O=\text { Orientation } & 3 \\ T=\text { Thickness } & 3\end{array}$

\begin{tabular}{|c|c|c|c|c|c|c|c|c|c|}
\hline \multirow{3}{*}{ Source } & \multirow{3}{*}{ Ss } & \multirow{3}{*}{ DF } & \multirow{3}{*}{ MS } & \multirow{3}{*}{$\begin{array}{c}\mathbf{F} \\
13167\end{array}$} & \multicolumn{2}{|r|}{$\mathbf{F}$} & \multicolumn{3}{|c|}{$\mathbf{F}$} \\
\hline & & & & & $\mathbf{P}$ & 0.01 & significant? & 0.05 & signnificant? \\
\hline & & & & & 0.2823 & 3.890307 & FALSE & 2.633534 & FALSE \\
\hline GO & 0.0049 & 2 & 0.0024 & 2.0834 & 0.1393 & 5.247898 & FALSE & 3.259444 & FALSE \\
\hline GT & 0.0025 & 2 & 0.0012 & 1.0706 & 0.3535 & 5.247898 & FALSE & 3.259444 & FALSE \\
\hline $\mathbf{G}$ & 0.0013 & 1 & 0.0013 & 1.0948 & 0.3024 & 7.395556 & FALSE & 4.113161 & FALSE \\
\hline OT & 0.0037 & 4 & 0.0009 & 0.8013 & 0.5324 & 3.890307 & FALSE & 2.633534 & FALSE \\
\hline 0 & 0.003 & 2 & 0.0015 & 1.2954 & 0.2862 & 5.247898 & FALSE & $3.25 \overline{9444}$ & FALSE \\
\hline $\mathbf{T}$ & 0.0189 & 2 & 0.0094 & 8.0851 & 0.0013 & 5.247898 & TRUE & 3.259444 & TRUE \\
\hline Error & 0.042 & 36 & 0.0012 & & & & & & \\
\hline Total & 0.0825 & 53 & & & & & & & \\
\hline
\end{tabular}


Table B.53 Factorial Analysis of Variance: Left Casting Angle of Lost Foam Low Expansion Castings (all)

$\begin{array}{lc}\begin{array}{l}\text { Number of Factors } \\ \text { Factor }\end{array} & \begin{array}{c}\text { Number of Replicates } \\ \text { Levels }\end{array} \\ G=\text { Gating } & 2 \\ \text { O=Orientation } & 3 \\ \text { T=Thickness } & 3\end{array}$

\begin{tabular}{|c|c|c|c|c|c|c|c|c|c|}
\hline & & & & & & $\mathbf{F}$ & & $F$ & \\
\hline Source & SS & DF & MS & $F$ & $P$ & 0.01 & significant? & 0.05 & significant? \\
\hline GOT & 0.0002 & 4 & 4.53E-05 & 2.1153 & 0.099 & 3.890307 & FALSE & 2.633534 & FALSE \\
\hline GO & 0.0002 & 2 & 8.91E-05 & 4.159 & 0.0237 & 5.247898 & FALSE & 3.259444 & TRUE \\
\hline GT & 3.10E-05 & 2 & 1.55E-05 & 0.7226 & 0.4924 & 5.247898 & FALSE & 3.259444 & FALSE \\
\hline $\mathbf{G}$ & 2.67E-07 & 1 & 2.67E-07 & 0.0125 & 0.9117 & 7.395556 & FALSE & 4.113161 & FALSE \\
\hline OT & $6.42 E-05$ & 4 & $1.61 \mathrm{E}-05$ & 0.7491 & 0.5651 & 3.890307 & FALSE & 2.633534 & FALSE \\
\hline 0 & 1.90E-07 & 2 & $9.50 \mathrm{E}-08$ & 0.0044 & 0.9956 & 5.247898 & FALSE & 3.259444 & FALSE \\
\hline$T$ & 0.0013 & 2 & 0.0006 & 30.238 & 1.97E-08 & 5.247898 & TRUE & 3.259444 & TRUE \\
\hline Error & 0.0008 & 36 & 2.14E-05 & & & & & & \\
\hline Total & 0.0025 & 53 & & & & & & & \\
\hline
\end{tabular}



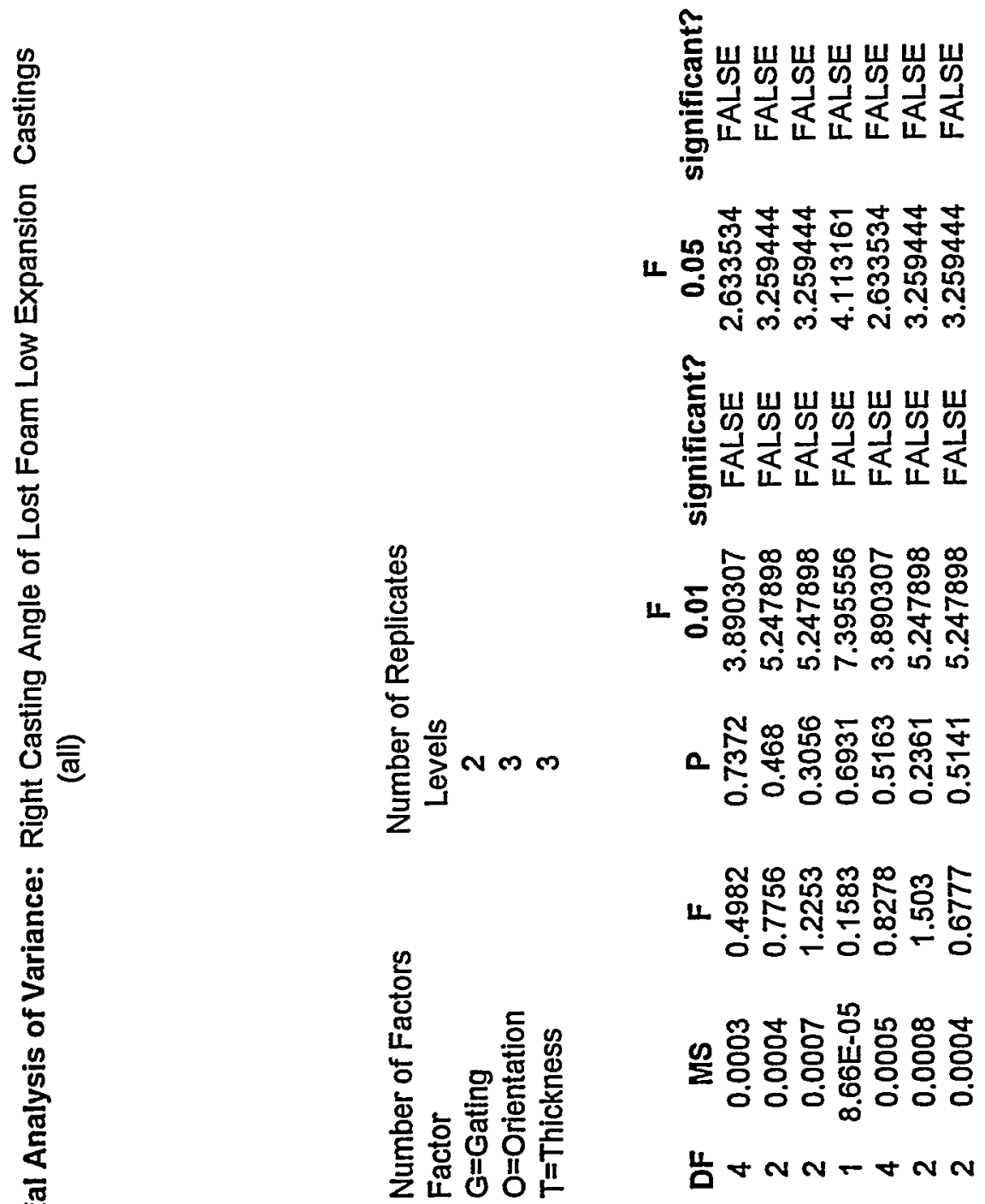

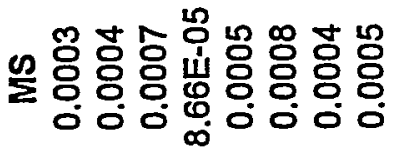

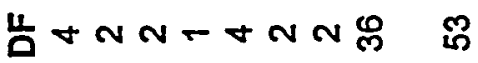

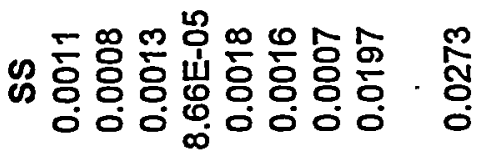

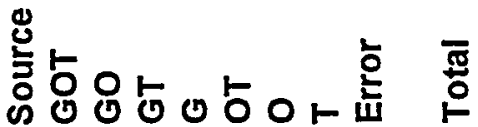


Table B.55 Factorial Analysis of Variance: Left Flange Flatness of Lost Foam Low Expansion Castings

(all)

$\begin{array}{lc}\begin{array}{l}\text { Number of Factors } \\ \text { Factor }\end{array} & \begin{array}{c}\text { Number of Replicates } \\ \text { Levels }\end{array} \\ \text { G=Gating } & 2 \\ O=\text { Orientation } & 3 \\ T=\text { Thickness } & 3\end{array}$

\begin{tabular}{|c|c|c|c|c|c|c|c|c|c|}
\hline & & & & & & $\mathbf{F}$ & & $\mathbf{F}$ & \\
\hline Source & SS & DF & MS & $\mathbf{F}$ & $\mathbf{P}$ & 0.01 & significant? & 0.05 & significant? \\
\hline GOT & 0.0001 & 4 & 2.71E-05 & 0.7888 & 0.5401 & 3.890307 & FALSE & 2.633534 & FALSE \\
\hline GO & $7.81 \mathrm{E}-05$ & 2 & $3.91 \mathrm{E}-05$ & 1.1363 & 0.3322 & 5.247898 & FALSE & 3.259444 & FALSE \\
\hline GT & 0.0002 & 2 & $9.09 \mathrm{E}-05$ & 2.6444 & 0.0848 & 5.247898 & FALSE & 3.259444 & FALSE \\
\hline G & 4.27E-05 & 1 & 4.27E-05 & 1.2414 & 0.2726 & 7.395556 & FALSE & 4.113161 & FALSE \\
\hline OT & $9.23 E-05$ & 4 & 2.31E-05 & 0.6713 & 0.6162 & 3.890307 & FALSE & 2.633534 & FALSE \\
\hline 0 & 0.0006 & 2 & 0.0003 & 9.2365 & 0.0006 & 5.247898 & TRUE & 3.259444 & TRUE \\
\hline $\mathrm{T}$ & $6.86 \mathrm{E}-05$ & 2 & $3.43 \mathrm{E}-05$ & 0.9978 & 0.3786 & 5.247898 & FALSE & 3.259444 & FALSE \\
\hline Error & 0.0012 & 36 & 3.44E-05 & & & & & & \\
\hline Total & 0.0024 & 53 & & & & & & & \\
\hline
\end{tabular}


Table B.56 Factorial Analysis of Variance: Right Flange Flatness of Lost Foam Low Expansion Castings (ali)

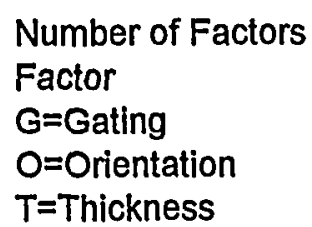

Number of Factors

Factor

$G=$ Gating

$\mathrm{O}=$ Orientation

$T=$ Thickness

$\begin{array}{lccc} & & & \\ \text { Source } & \text { SS } & \text { DF } & \text { MS } \\ \text { GOT } & 0.0805 & 4 & 0.020 \\ \text { GO } & 0.063 & 2 & 0.031 \\ \text { GT } & 0.0483 & 2 & 0.024 \\ \text { G } & 0.0455 & 1 & 0.045 \\ \text { OT } & 0.0516 & 4 & 0.012 \\ \text { O } & 0.0653 & 2 & 0.0327 \\ \text { T } & 0.1307 & 2 & 0.065 \\ \text { Error } & 0.2645 & 36 & 0.007 \\ \text { Total } & 0.7495 & 53 & \end{array}$

Number of Replicates

Levels

$$
2
$$

3

3

\begin{tabular}{cccccc} 
& \multicolumn{3}{c}{$\mathbf{F}$} & $\mathbf{F}$ & \\
$\mathbf{F}$ & $\mathbf{P}$ & $\mathbf{0 . 0 1}$ & significant? & $\mathbf{0 . 0 5}$ & significant? \\
2.74 & $\mathbf{0 . 0 4 3 5}$ & 3.890307 & FALSE & 2.633534 & TRUE \\
4.2875 & 0.0214 & 5.247898 & FALSE & 3.259444 & TRUE \\
3.2857 & 0.0489 & 5.247898 & FALSE & 3.259444 & TRUE \\
6.1969 & 0.0176 & 7.395556 & FALSE & 4.113161 & TRUE \\
1.7574 & 0.1589 & 3.890307 & FALSE & 2.633534 & FALSE \\
4.4467 & 0.0188 & 5.247898 & FALSE & 3.259444 & TRUE \\
8.8957 & 0.0007 & 5.247898 & TRUE & 3.259444 & TRUE
\end{tabular}

$0.0007 \quad 5.247898$

TRUE $\quad 3.259444$ 
Table B.57 Factorial Analysis of Variance: Left Wall Flatness of Lost Foam Low Expansion Castings

(all)

$\begin{array}{lc}\begin{array}{l}\text { Number of Factors } \\ \text { Factor }\end{array} & \begin{array}{c}\text { Number of Replicates } \\ \text { Levels }\end{array} \\ G=\text { Gating } & 2 \\ O=\text { Orientation } & 3 \\ T=\text { Thickness } & 3\end{array}$

\begin{tabular}{|c|c|c|c|c|c|c|c|c|c|}
\hline & & & & & & $\mathbf{F}$ & & $\mathbf{F}$ & \\
\hline Source & SS & DF & MS & $\mathbf{F}$ & $\mathbf{P}$ & 0.01 & significant? & 0.05 & significant? \\
\hline GOT & 0.0002 & 4 & 4.69E-05 & 1.9366 & 0.1254 & 3.890307 & FALSE & 2.633534 & FALSE \\
\hline GO & 8.81E-05 & 2 & 4.41E-05 & 1.8174 & 0.177 & 5.247898 & FALSE & 3.259444 & FALSE \\
\hline GT & 6.74E-05 & 2 & 3.37E-05 & 1.3911 & 0.2618 & 5.247898 & FALSE & 3.259444 & FALSE \\
\hline $\mathbf{G}$ & 1.50E-06 & 1 & 1.50E-06 & 0.0619 & 0.805 & 7.395556 & FALSE & 4.113161 & FALSE \\
\hline OT & 0.0002 & 4 & 5.93E-05 & 2.4461 & 0.064 & 3.890307 & FALSE & 2.633534 & FALSE \\
\hline 0 & 0.0001 & 2 & 5.61E-05 & 2.3155 & 0.1132 & 5.247898 & FALSE & 3.259444 & FALSE \\
\hline $\mathbf{T}$ & 0.0004 & 2 & 0.0002 & 7.2429 & 0.0023 & 5.247898 & TRUE & 3.259444 & TRUE \\
\hline Error & 0.0009 & 36 & 2.42E-05 & & & & & & \\
\hline Total & 0.0019 & 53 & & & & & & & \\
\hline
\end{tabular}


Table B.58 Factorial Analysis of Variance: Right Wall Flatness of Lost Foam Low Expansion Castings (all)

$\begin{array}{lc}\text { Number of Factors } & \begin{array}{c}\text { Number of Replicates } \\ \text { Levels }\end{array} \\ \text { Factor } & 2 \\ \text { G=Gating } & 3 \\ O=\text { Orientation } & 3 \\ \text {-T=Thickness } & 3\end{array}$

\begin{tabular}{|c|c|c|c|c|c|c|c|c|c|}
\hline \multirow{3}{*}{ Source } & \multirow{3}{*}{ Ss } & \multirow[b]{2}{*}{ DF } & \multirow[b]{2}{*}{ MS } & \multirow[b]{2}{*}{$F$} & \multicolumn{2}{|r|}{$F$} & \multicolumn{3}{|c|}{$\mathbf{F}$} \\
\hline & & & & & $\mathbf{P}$ & 0.01 & significant? & 0.05 & significant? \\
\hline & & 4 & 0.0006 & 0.516 & 0.7244 & 3.890307 & FALSE & 2.633534 & FALSE \\
\hline GO & 0.0021 & 2 & 0.001 & 0.8295 & 0.4444 & 5.247898 & FALSE & 3.259444 & FALSE \\
\hline GT & 0.0017 & 2 & 0.0008 & 0.6774 & 0.5143 & 5.247898 & FALSE & 3.259444 & FALSE \\
\hline G & 0.0005 & 1 & 0.0005 & 0.4222 & 0.52 & 7.395556 & FALSE & 4.113161 & FALSE \\
\hline OT & 0.0039 & 4 & 0.001 & 0.772 & 0.5506 & 3.890307 & FALSE & 2.633534 & FALSE \\
\hline 0 & 0.0016 & 2 & 0.0008 & 0.6393 & 0.5336 & 5.247898 & FALSE & 3.259444 & FALSE \\
\hline$T$ & 0.0133 & 2 & 0.0067 & 5.308 & 0.0095 & 5.247898 & TRUE & 3.259444 & TRUE \\
\hline Error & 0.0451 & 36 & 0.0013 & & & & & & \\
\hline Total & 0.0708 & 53 & & & & & & & \\
\hline
\end{tabular}


Table B.59 Factorial Analysis of Variance:

Lost Foam Low Expansion Castings

(0.25 inch)

Number of Factors

Number of Replicates

Factor

$\mathrm{G}=$ Gating

Levels

$\mathrm{O}=$ Orientation

2

3

Left Flange Flatness

\begin{tabular}{lccccccccc} 
& & & \multicolumn{1}{c}{} & \multicolumn{1}{c}{$\mathbf{F}$} & & F & \\
Source & SS & DF & MS & F & P & $\mathbf{0 . 0 1}$ & significant? & $\mathbf{0 . 0 5}$ & significant? \\
G0 & $4.21 \mathrm{E}-05$ & 2 & $2.11 \mathrm{E}-05$ & 1.1147 & 0.3597 & 6.926598 & FALSE & 3.8852903 & FALSE \\
G & $5.69 \mathrm{E}-05$ & 1 & $5.69 \mathrm{E}-05$ & $3 ! .0118$ & 0.1082 & 9.3302788 & FALSE & 4.7472213 & FALSE \\
$\mathbf{O}$ & $6.54 \mathrm{E}-05$ & 2 & $3.27 \mathrm{E}-05$ & 1.7324 & 0.2183 & 6.926598 & FALSE & 3.8852903 & FALSE \\
Error & 0.0002 & 12 & $1.89 \mathrm{E}-05$ & & & & & &
\end{tabular}

Total $\quad 0.0004 \quad 17$

Right Flange Flatness

\begin{tabular}{lccccccccc} 
& & & & & & $\mathbf{F}$ & \multicolumn{3}{c}{$\mathbf{F}$} \\
Source & SS & DF & MS & F & $\mathbf{P}$ & $\mathbf{0 . 0 1}$ & significant? & $\mathbf{0 . 0 5}$ & significant? \\
GO & 0.0001 & 2 & $6.04 \mathrm{E}-05$ & 0.3291 & 0.7259 & 6.926598 & FALSE & 3.8852903 & FALSE \\
G & $2.22 \mathrm{E}-05$ & 1 & $2.22 \mathrm{E}-05$ & $\mathbf{0 . 1 2 1 1}$ & 0.7339 & 9.3302788 & FALSE & 4.7472213 & FALSE \\
$\mathbf{O}$ & 0.0031 & 2 & 0.0016 & $\mathbf{8 . 4 5 0 8}$ & 0.0051 & 6.926598 & TRUE & 3.8852903 & TRUE \\
Error & 0.0022 & 12 & 0.0002 & & & & & &
\end{tabular}

Total $\quad 0.0054 \quad 17$

Left Wall Flatness

\begin{tabular}{|c|c|c|c|c|c|c|c|c|c|}
\hline \multirow[b]{2}{*}{ Source } & \multirow[b]{2}{*}{ SS } & \multirow[b]{2}{*}{ DF } & \multirow[b]{2}{*}{ MS } & \multirow[b]{2}{*}{$\mathbf{F}$} & & \multicolumn{3}{|c|}{$\mathbf{F}$} \\
\hline & & & & & $\mathbf{P}$ & 0.01 & significant? & 0.05 & significant? \\
\hline GO & 0.0002 & 2 & 0.0001 & 5.4367 & 0.0209 & 6.926598 & FALSE & 3.8852903 & TRUE \\
\hline $\mathbf{G}$ & 4.67E-05 & 1 & $4.67 \mathrm{E}-05$ & 2.2668 & 0.158 & 9.3302788 & FALSE & 4.7472213 & FALSE \\
\hline 0 & $2.33 \mathrm{E}-06$ & 2 & $1.17 \mathrm{E}-06$ & 0.0566 & 0.9452 & 6.926598 & FALSE & 3.8852903 & FALSE \\
\hline Error & 0.0002 & 12 & $2.06 \mathrm{E}-05$ & & & & & & \\
\hline otal & 0.0005 & $\cdot 17$ & & & & & & & \\
\hline
\end{tabular}

Right Wall Flatness

\begin{tabular}{lccccccccc} 
& & & \multicolumn{1}{c}{$\mathbf{F}$} & \multicolumn{3}{c}{$\mathbf{F}$} \\
Source & SS & DF & MS & $\mathbf{F}$ & $\mathbf{P}$ & $\mathbf{0 . 0 1}$ & significant? & $\mathbf{0 . 0 5}$ & significant? \\
G0 & $\mathbf{0 . 0 0 1}$ & 2 & 0.0005 & 0.4538 & 0.6457 & 6.926598 & FALSE & 3.8852903 & FALSE \\
G & 0.0006 & 1 & 0.0006 & 0.5482 & 0.4733 & 9.3302788 & FALSE & 4.7472213 & FALSE \\
O & 0.0035 & 2 & 0.0018 & 1.6136 & 0.2395 & 6.926598 & FALSE & 3.8852903 & FALSE \\
Error & 0.0132 & 12 & 0.0011 & & & & & &
\end{tabular}

Total $\quad 0.0183 \quad 17$ 
Table B.60 Factorial Analysis of Variance:

Lost Foam Low Expansion Castings

(0.25 inch)

\begin{tabular}{lccccccccc} 
& & & \multicolumn{8}{c}{ Height } & $\mathbf{F}$ & & F \\
Source & SS & DF & MS & F & P & $\mathbf{0 . 0 1}$ & significant? & $\mathbf{0 . 0 5}$ & significant? \\
GO & 0.0096 & 2 & 0.0048 & 2.2083 & 0.1525 & 6.926598 & FALSE & 3.8852903 & FALSE \\
G & 0.0083 & 1 & 0.0083 & 3.809 & 0.0747 & 9.3302788 & FALSE & 4.7472213 & FALSE \\
O & 0.0088 & 2 & 0.0044 & 2.0252 & $\mathbf{0 . 1 7 4 7}$ & 6.926598 & FALSE & 3.8852903 & FALSE \\
Error & 0.026 & 12 & 0.0022 & $:$ & & & & &
\end{tabular}

Total $\quad 0.0526 \quad 17$

\begin{tabular}{|c|c|c|c|c|c|c|c|c|c|}
\hline & & & & & Lengt & & & & \\
\hline & & & & & & $F$ & & $\mathbf{F}$ & \\
\hline Source & SS & DF & MS & $\mathbf{F}$ & $\mathbf{P}$ & 0.01 & significant? & 0.05 & significant? \\
\hline GO & 0.0002 & 2 & $7.98 \mathrm{E}-05$ & 0.3691 & 0.6989 & 6.926598 & FALSE & 3.8852903 & FALSE \\
\hline $\mathbf{G}$ & 0.0008 & 1 & 0.0008 & 3.7416 & 0.077 & 9.3302788 & FALSE & 4.7472213 & FALSE \\
\hline $\mathbf{0}$ & 0.0024 & 2 & 0.0012 & 5.493 & 0.0202 & 6.926598 & FALSE & 3.8852903 & TRUE \\
\hline Error & 0.0026 & 12 & 0.0002 & & & & & & \\
\hline
\end{tabular}

$\begin{array}{lll}\text { Total } & 0.0059 & 17\end{array}$

Left Casting Angle

\begin{tabular}{|c|c|c|c|c|c|c|c|c|c|}
\hline \multirow{3}{*}{$\begin{array}{l}\text { Source } \\
\text { GO }\end{array}$} & \multirow[b]{2}{*}{ SS } & \multirow[b]{2}{*}{ DF } & \multirow[b]{2}{*}{ MS } & \multirow[b]{2}{*}{$\mathbf{F}$} & \multicolumn{2}{|c|}{$\mathrm{F}$} & \multicolumn{3}{|c|}{$\mathbf{F}$} \\
\hline & & & & & $\mathbf{P}$ & 0.01 & significant? & 0.05 & significant? \\
\hline & 0.0003 & 2 & 0.0002 & 2.5673 & 0.118 & 6.926598 & FALSE & 3.8852903 & FALSE \\
\hline G & $2.33 \mathrm{E}-05$ & 1 & $2.33 \mathrm{E}-05$ & 0.3754 & 0.5515 & 9.3302788 & FALSE & 4.7472213 & FALSE \\
\hline 0 & $4.19 \mathrm{E}-05$ & 2 & $2.09 \mathrm{E}-05$ & 0.3365 & 0.7208 & 6.926598 & FALSE & 3.8852903 & FALSE \\
\hline Error & 0.0007 & 12 & $6.22 \mathrm{E}-05$ & & & & & & \\
\hline
\end{tabular}

Total $\quad 0.0011 \quad 17$

Right Casting Angle

\begin{tabular}{lcccc|ccccc} 
Source & SS & DF & MS & $\mathbf{F}$ & $\mathbf{P}$ & $\mathbf{0 . 0 1}$ & significant? & $\mathbf{0 . 0 5}$ & significant? \\
GO & 0.0018 & 2 & 0.0009 & 0.8531 & 0.4504 & 6.926598 & FALSE & 3.8852903 & FALSE \\
G & 0.0012 & 1 & 0.0012 & 1.169 & 0.3009 & 9.3302788 & FALSE & 4.7472213 & FALSE \\
O & 0.0026 & 2 & 0.0013 & 1.2592 & 0.3188 & 6.926598 & FALSE & 3.8852903 & FALSE \\
Error & 0.0123 & 12 & 0.001 & & & & & &
\end{tabular}

$\begin{array}{lll}\text { Total } & 0.0179 \quad 17\end{array}$ 
Table B.61 Factorial Analysis of Variance:

Lost Foam Low Expansion Castings

(0.25 inch)

\author{
Number of Factors \\ Factor \\ $\mathrm{G}=$ Gating \\ $\mathrm{O}=$ Orientation
}
Number of Replicates
Levels
2
3

\title{
Parallelism
}

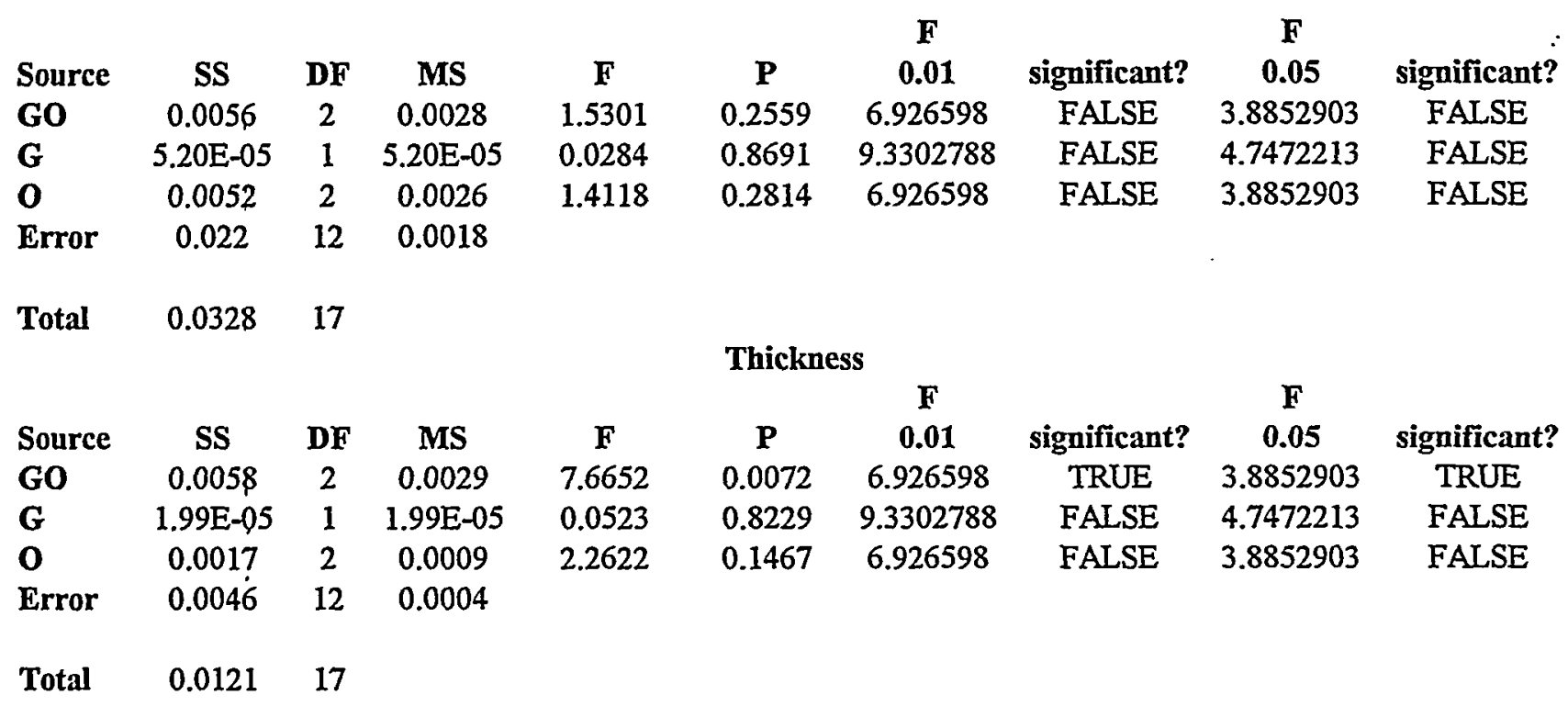


Table B.62 Factorial Analysis of Variance:

Lost Foam Low Expansion Castings

(0.50 inch)

Number of Factors

Number of Replicates

Factor

$\mathrm{G}=$ Gating

Levels

$\mathrm{O}=$ Orientation

2

3

\section{Left Flange Flatness}

$\begin{array}{lccccccccc} & & & & & & \mathbf{F} & & \text { F } & \\ \text { Source } & \text { SS } & \text { DF } & \text { MS } & \text { F } & \text { P } & \mathbf{0 . 0 1} & \text { significant? } & \mathbf{0 . 0 5} & \text { significant? } \\ \text { GO } & 8.33 E-06 & 2 & 4.17 E-06 & \mathbf{0 . 0 7 0 7} & 0.9321 & 6.926598 & \text { FALSE } & 3.8852903 & \text { FALSE } \\ \text { G } & 5.00 E-05 & 1 & 5.00 E-05 & 0.8483 & 0.3752 & 9.3302788 & \text { FALSE } & 4.7472213 & \text { FALSE } \\ \text { O } & 0.0004 & 2 & 0.0002 & 3.0094 & 0.0872 & 6.926598 & \text { FALSE } & 3.8852903 & \text { FALSE } \\ \text { Error } & 0.0007 & 12 & 5.89 E-05 & & & & & & \\ & & & & & & & & & \end{array}$

Right Flange Flatness

\begin{tabular}{lccccccccc} 
& & & \multicolumn{1}{c}{$\mathbf{F}$} & \multicolumn{3}{c}{$\mathbf{F}$} & \\
Source & SS & DF & MS & F & P & $\mathbf{0 . 0 1}$ & significant? & $\mathbf{0 . 0 5}$ & significant? \\
GO & 0.0035 & 2 & 0.0017 & 4.9576 & 0.027 & 6.926598 & FALSE & 3.8852903 & TRUE \\
G & 0.0043 & 1 & 0.0043 & 12.3498 & 0.0043 & 9.3302788 & TRUE & 4.7472213 & TRUE \\
O & 0.004 & 2 & 0.002 & 5.6589 & 0.0186 & 6.926598 & FALSE & 3.8852903 & TRUE \\
Error & $\mathbf{0 . 0 0 4 2}$ & 12 & 0.0004 & & & & & &
\end{tabular}

$\begin{array}{lll}\text { Total } & 0.016 & 17\end{array}$

Left Wall Flatness

$\begin{array}{lccccccccc} & & & & & & \mathbf{F} & & \mathbf{F} & \\ \text { Source } & \text { SS } & \text { DF } & \text { MS } & \mathbf{F} & \mathbf{P} & \mathbf{0 . 0 1} & \text { significant? } & \mathbf{0 . 0 5} & \text { significant? } \\ \text { GO } & 1.30 \mathrm{E}-05 & \mathbf{2} & 6.50 \mathrm{E}-06 & \mathbf{0 . 5 0 4 3} & 0.6162 & 6.926598 & \text { FALSE } & 3.8852903 & \text { FALSE } \\ \text { G } & 0 & 1 & \mathbf{0} & \mathbf{8 . 1 1 E - 2 8} & 1 & 9.3302788 & \text { FALSE } & 4.7472213 & \text { FALSE } \\ \mathbf{O} & 8.33 \mathrm{E}-06 & 2 & 4.17 \mathrm{E}-06 & 0.3233 & 0.7299 & 6.926598 & \text { FALSE } & 3.8852903 & \text { FALSE } \\ \text { Error } & 0.0002 & 12 & 1.29 E-05 & & & & & & \end{array}$

Total $\quad 0.0002 \quad 17$

Right Wall Flatness

$\begin{array}{lccccccccc} & & & & & & \mathbf{F} & & \text { F } & \\ \text { Source } & \text { SS } & \text { DF } & \text { MS } & \text { F } & \text { P } & \mathbf{0 . 0 1} & \text { significant? } & \mathbf{0 . 0 5} & \text { significant? } \\ \text { GO } & 0.0003 & 2 & 0.0001 & 0.2815 & 0.7595 & 6.926598 & \text { FALSE } & 3.8852903 & \text { FALSE } \\ \text { G } & 0.0004 & 1 & 0.0004 & 0.8413 & 0.3771 & 9.3302788 & \text { FALSE } & 4.7472213 & \text { FALSE } \\ \text { O } & 0.0007 & 2 & 0.0003 & 0.7022 & 0.5148 & 6.926598 & \text { FALSE } & 3.8852903 & \text { FALSE } \\ \text { Error } & \mathbf{0 . 0 0 5 6} & 12 & 0.0005 & & & & & & \\ & & & & & & & & & \end{array}$


Table B.63 Factorial Analysis of Variance:

Lost Foam Low Expansion Castings

(0.50 inch)

Number of Factors

Number of Replicates

Factor

$\mathrm{G}=$ Gating

Levels

$\mathrm{O}=$ Orientation

2

3

Height

$\begin{array}{lccccccccc} & & & & & & & \mathbf{F} & & \mathbf{F} \\ \text { Source } & \text { SS } & \text { DF } & \text { MS } & \mathbf{F} & \mathbf{P} & \mathbf{0 . 0 1} & \text { significant? } & \mathbf{0 . 0 5} & \text { significant? } \\ \text { GO } & 0.0096 & 2 & 0.0048 & 2.2083 & 0.1525 & 6.926598 & \text { FALSE } & 3.8852903 & \text { FALSE } \\ \text { G } & 0.0083 & 1 & 0.0083 & 3.809 & 0.0747 & 9.3302788 & \text { FALSE } & 4.7472213 & \text { FALSE } \\ \text { O } & 0.0088 & 2 & 0.0044 & 2.0252 & 0.1747 & 6.926598 & \text { FALSE } & 3.8852903 & \text { FALSE } \\ \text { Error } & 0.026 & 12 & 0.0022 & & & & & & \end{array}$

$\begin{array}{lll}\text { Total } & 0.0526 & 17\end{array}$

Length

$\begin{array}{lcc}\text { Source } & \text { SS } & \text { DF } \\ \text { GO } & 0.0005 & 2 \\ \text { G } & 0.0002 & 1 \\ \text { O } & 0.0029 & 2 \\ \text { Error } & 0.0005 & 12 \\ & & \\ \text { Total } & 0.0041 & 17\end{array}$

Left Casting Angle

\begin{tabular}{lccccccccc} 
& & & & & & $\mathbf{F}$ & \multicolumn{3}{c}{$\mathbf{F}$} \\
Source & SS & DF & MS & $\mathbf{F}$ & $\mathbf{P}$ & $\mathbf{0 . 0 1}$ & significant? & $\mathbf{0 . 0 5}$ & significant? \\
GO & $2.70 \mathrm{E}-07$ & 2 & $1.35 \mathrm{E}-07$ & $\mathbf{0 . 1 6 0 6}$ & $\mathbf{0 . 8 5 3 4}$ & 6.926598 & FALSE & 3.8852903 & FALSE \\
$\mathbf{G}$ & $2.88 \mathrm{E}-06$ & 1 & $2.88 \mathrm{E}-06$ & 3.4263 & 0.0889 & 9.3302788 & FALSE & 4.7472213 & FALSE \\
$\mathbf{0}$ & $5.50 \mathrm{E}-06$ & 2 & $2.75 \mathrm{E}-06$ & 3.2736 & 0.0734 & 6.926598 & FALSE & 3.8852903 & FALSE \\
Error & $1.01 \mathrm{E}-05$ & 12 & $8.41 \mathrm{E}-07$ & & & & & &
\end{tabular}

Total 1.87E-0. 17

Right Casting Angle

\begin{tabular}{|c|c|c|c|c|c|c|c|c|c|}
\hline \multirow[b]{2}{*}{ Source } & \multirow[b]{2}{*}{ SS } & \multirow[b]{2}{*}{ DF } & \multirow[b]{2}{*}{ MS } & \multirow[b]{2}{*}{$\mathbf{F}$} & \multicolumn{2}{|r|}{$\mathbf{F}$} & \multicolumn{3}{|c|}{$\mathbf{F}$} \\
\hline & & & & & $\mathbf{P}$ & 0.01 & significant? & 0.05 & significant? \\
\hline GO & 0.0002 & 2 & 8.52E-05 & 0.1578 & 0.8558 & 6.926598 & FALSE & 3.8852903 & FALSE \\
\hline $\mathbf{G}$ & 0.0002 & 1 & 0.0002 & 0.3881 & 0.545 & 9.3302788 & FALSE & 4.7472213 & FALSE \\
\hline $\mathbf{O}$ & 0.0008 & 2 & 0.0004 & 0.7449 & 0.4955 & 6.926598 & FALSE & 3.8852903 & FALSE \\
\hline Error & 0.0066 & 12 & 0.0005 & & & & & & \\
\hline
\end{tabular}


Table B.64 Factorial Analysis of Variance:

Lost Foam Low Expansion Castings

(0.50 inch)

Number of Factors
Factor
$G=$ Gating
$O=$ Orientation

Number of Replicates Levels

$$
2
$$

3

\section{Parallelism :}

\begin{tabular}{lccccccccc} 
& & & & & \multicolumn{3}{c}{$\mathbf{F}$} & & $\mathbf{F}$ \\
Source & SS & DF & MS & $\mathbf{F}$ & $\mathbf{P}$ & $\mathbf{0 . 0 1}$ & significant? & $\mathbf{0 . 0 5}$ & significant? \\
GO & 0.0049 & 2 & $\mathbf{0 . 0 0 2 5}$ & 2.0413 & 0.1726 & 6.926598 & FALSE & 3.8852903 & FALSE \\
G & 0.0037 & 1 & 0.0037 & 3.0491 & 0.1063 & 9.3302788 & FALSE & 4.7472213 & FALSE \\
O & 0.0007 & 2 & 0.0004 & 0.3074 & 0.741 & 6.926598 & FALSE & 3.8852903 & FALSE \\
Error & 0.0145 & 12 & 0.0012 & & & & & &
\end{tabular}

Total $\quad 0.0239 \quad 17$

Thickness

\begin{tabular}{|c|c|c|c|c|c|c|c|c|c|}
\hline \multirow[b]{2}{*}{ Source } & \multirow{3}{*}{ SS } & \multirow[b]{2}{*}{ DF } & \multirow[b]{2}{*}{ MS } & \multirow[b]{2}{*}{$\mathbf{F}$} & \multicolumn{2}{|r|}{$\mathbf{F}$} & \multicolumn{3}{|c|}{$\mathbf{F}$} \\
\hline & & & & & $\mathbf{P}$ & 0.01 & significant? & 0.05 & significant? \\
\hline GO & & 2 & 0.0044 & 3.3953 & 0.0678 & 6.926598 & FALSE & 3.8852903 & FALSE \\
\hline & 7.36E-06 & 1 & 7.36E-06 & 0.0057 & 0.9413 & 9.3302788 & FALSE & 4.7472213 & FALSE \\
\hline & 0.0042 & 2 & 0.0021 & 1.613 & 0.2396 & 6.926598 & FALSE & 3.8852903 & FALSE \\
\hline ror & 0.0156 & 12 & 0.0013 & & & & & & \\
\hline
\end{tabular}

Total $\quad 0.0287 \quad 17$ 
Table B.65 Factorial Analysis of Variance:

Lost Foam Low Expansion Castings

(0.75 inch)

Number of Factors

Factor

$\mathrm{G}=$ Gating

$\mathrm{O}=$ Orientation

Number of Replicates

Levels

2

3

Left Flange Flatness

\begin{tabular}{lccccccccc} 
& & & & \multicolumn{1}{c}{$\mathbf{F}$} & & $\mathbf{F}$ & \\
Source & SS & DF & MS & $\mathbf{F}$ & $\mathbf{P}$ & $\mathbf{0 . 0 1}$ & significant? & $\mathbf{0 . 0 5}$ & significant? \\
GO & 0.0001 & 2 & $6.81 E-05$ & 2.6923 & 0.1082 & 6.926598 & FALSE & 3.8852903 & FALSE \\
G & 0.0001 & 1 & 0.0001 & 4.6505 & 0.052 & 9.3302788 & FALSE & 4.7472213 & FALSE \\
O & 0.0003 & 2 & 0.0002 & 6.0725 & 0.0151 & 6.926598 & FALSE & 3.8852903 & TRUE \\
Error & 0.0003 & 12 & $2.53 E-05$ & & & & & &
\end{tabular}

Total $\quad 0.0009 \quad 17$

Right Flange Flatness

\begin{tabular}{lccccccccc} 
& & & & \multicolumn{2}{c}{$\mathbf{F}$} & & $\mathbf{F}$ & \\
Source & SS & DF & MS & F & $\mathbf{P}$ & $\mathbf{0 . 0 1}$ & significant? & $\mathbf{0 . 0 5}$ & significant? \\
GO & 0.1399 & 2 & 0.07 & 3.2531 & 0.0743 & 6.926598 & FALSE & 3.8852903 & FALSE \\
G & 0.0895 & 1 & 0.0895 & 4.1596 & 0.064 & 9.3302788 & FALSE & 4.7472213 & FALSE \\
O & 0.1099 & 2 & 0.055 & 2.5554 & 0.119 & 6.926598 & FALSE & 3.8852903 & FALSE \\
Error & 0.2581 & 12 & 0.0215 & & & & & &
\end{tabular}

$\begin{array}{lll}\text { Total } & 0.5974 & 17\end{array}$

Left Wall Flatness

\begin{tabular}{|c|c|c|c|c|c|c|c|c|c|}
\hline \multirow{4}{*}{$\begin{array}{l}\text { Source } \\
\text { GO }\end{array}$} & \multirow{4}{*}{$\underset{3.88 \mathrm{ES}-05}{\text { SS }}$} & \multirow[b]{3}{*}{ DF } & \multirow[b]{3}{*}{ MS } & \multirow{2}{*}{\multicolumn{3}{|c|}{$\mathbf{F}$}} & \multirow{2}{*}{\multicolumn{3}{|c|}{$\mathbf{F}$}} \\
\hline & & & & & & $\mathbf{F}$ & & & \\
\hline & & & & $\mathbf{F}$ & $\mathbf{P}$ & 0.01 & significant? & 0.05 & significant? \\
\hline & & 2 & 1.94E-05 & 0.4943 & 0.6219 & 6.926598 & FALSE & 3.8852903 & FAISE \\
\hline $\mathbf{G}$ & 2.22E-05 & 1 & 2.22E-05 & 0.5666 & 0.4661 & 9.3302788 & FALSE & 4.7472213 & FALSE \\
\hline 0 & 0.0003 & 2 & 0.0002 & 4.3187 & 0.0387 & 6.926598 & FALSE & 3.8852903 & TRUE \\
\hline Error & 0.0005 & 12 & 3.92E-05 & & & & & & \\
\hline Cotal & 0.0009 & 17 & & & & & & & \\
\hline
\end{tabular}

Right Wall Flatness

$\begin{array}{lccccccccc} & & & & & & \mathbf{F} & & \text { F } & \\ \text { Source } & \text { SS } & \text { DF } & \text { MS } & \text { F } & \mathbf{P} & \mathbf{0 . 0 1} & \text { significant? } & \mathbf{0 . 0 5} & \text { significant? } \\ \text { G0 } & 0.0034 & 2 & 0.0017 & 0.7757 & 0.4822 & 6.926598 & \text { FALSE } & 3.8852903 & \text { FALSE } \\ \text { G } & 0.0012 & 1 & 0.0012 & 0.5616 & 0.4681 & 9.3302788 & \text { FALSE } & 4.7472213 & \text { FALSE } \\ \text { O } & 0.0013 & 2 & 0.0006 & 0.2912 & 0.7525 & 6.926598 & \text { FALSE } & 3.8852903 & \text { FALSE } \\ \text { Error } & 0.0264 & 12 & 0.0022 & & & & & & \\ & & & & & & & & & \end{array}$


Table B.66 Factorial Analysis of Variance:

Lost Foam Low Expansion Castings

(0.75 inch)

Height

$\begin{array}{lccccccccc} & & & & & & \mathbf{F} & & \mathbf{F} & \\ \text { Source } & \text { SS } & \text { DF } & \text { MS } & \mathbf{F} & \mathbf{P} & \mathbf{0 . 0 1} & \text { significant? } & \mathbf{0 . 0 5} & \text { significant? } \\ \text { GO } & 0.0119 & 2 & 0.006 & 24.5254 & 5.77 \mathrm{E}-05 & 6.926598 & \text { TRUE } & 3.8852903 & \text { TRUE } \\ \text { G } & 0.0015 & 1 & 0.0015 & 6.3565 & 0.0268 & 9.3302788 & \text { FALSE } & 4.7472213 & \text { TRUE } \\ \text { O } & 0.0047 & 2 & 0.0023 & 9.6696 & 0.0032 & 6.926598 & \text { TRUE } & 3.8852903 & \text { TRUE } \\ \text { Error } & \mathbf{0 . 0 0 2 9} & \mathbf{1 2} & \mathbf{0 . 0 0 0 2} & & & & & & \end{array}$

Total $\quad 0.021 \quad 17$

\begin{tabular}{lccccccccc} 
& & & & & & $\mathbf{F}$ & \multicolumn{3}{c}{$\mathbf{F}$} \\
Source & SS & DF & MS & F & $\mathbf{P}$ & $\mathbf{0 . 0 1}$ & significant? & $\mathbf{0 . 0 5}$ & significant? \\
G0 & $1.42 E-05$ & 2 & $7.12 E-06$ & 0.056 & 0.9458 & 6.926598 & FALSE & 3.8852903 & FALSE \\
G & $4.29 E-05$ & 1 & $4.29 E-05$ & 0.337 & 0.5723 & 9.3302788 & FALSE & 4.7472213 & FALSE \\
O & 0.0002 & 2 & 0.0001 & 0.908 & 0.4293 & 6.926598 & FALSE & 3.8852903 & FALSE \\
Error & 0.0015 & 12 & 0.0001 & & & & & &
\end{tabular}

Total $\quad 0.0018 \quad 17$

Left Casting Angle

\begin{tabular}{|c|c|c|c|c|c|c|c|c|c|}
\hline \multirow[b]{2}{*}{ Source } & \multirow[b]{2}{*}{ SS } & \multirow[b]{2}{*}{ DF } & \multirow[b]{2}{*}{ MS } & \multirow[b]{2}{*}{$\mathbf{F}$} & \multicolumn{2}{|c|}{$\mathbf{F}$} & \multicolumn{3}{|c|}{$\mathbf{F}$} \\
\hline & & & & & $\mathbf{P}$ & 0.01 & significant? & 0.05 & significant? \\
\hline GO & $4.00 E-05$ & 2 & $2.00 E-05$ & 15.8325 & 0.0004 & 6.926598 & TRUE & 3.8852903 & TRUE \\
\hline $\mathbf{G}$ & 5.01E-06 & 1 & 5.01E-06 & 3.967 & 0.0697 & 9.3302788 & FALSE & 4.7472213 & FALSE \\
\hline 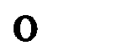 & 1.71E-05 & 2 & 8.53E-06 & 6.7468 & 0.0109 & 6.926598 & FALSE & 3.8852903 & TRUE \\
\hline rror & 1.52E-05 & 12 & $1.26 E-06$ & & & & & & \\
\hline
\end{tabular}

Total 7.73E-0. 17

Right Casting Angle

\begin{tabular}{|c|c|c|c|}
\hline Source & SS & DF & MS \\
\hline GO & 1.52E-05 & 2 & 7.59E-06 \\
\hline G & 1.66E-05 & 1 & 1.66E-05 \\
\hline 0 & 6.45E-@05 & 2 & 3.22E-05 \\
\hline Error & 0.0009 & 12 & 7.43E-05 \\
\hline Tot & 0.001 & 17 & \\
\hline
\end{tabular}


Table B.67 Factorial Analysis of Variance:

Lost Foam Low Expansion Castings

(0.75 inch)

Number of Factors
Factor
$\mathrm{G}=$ Gating
$\mathrm{O}=$ Orientation

Number of Replicates

Levels

2

3

Parallelism

\begin{tabular}{|c|c|c|c|c|c|c|c|c|c|}
\hline & & & & & & & & & \\
\hline Source & SS & $\mathbf{D F}$ & MS & $\mathbf{F}$ & $\mathbf{P}$ & $\begin{array}{c} \\
0.01\end{array}$ & significant? & $\stackrel{ }{0.05}$ & significant? \\
\hline GO & 0.0005 & 2 & 0.0002 & 0.5048 & 0.6159 & 6.926598 & FALSE & 3.8852903 & FALSE \\
\hline $\mathbf{G}$ & $3.64 \mathrm{E}-05$ & 1 & 3.64E-05 & 0.0792 & 0.7832 & 9.3302788 & FALSE & 4.7472213 & FALSE \\
\hline $\mathbf{0}$ & 0.0008 & 2 & 0.0004 & 0.9219 & 0.4242 & 6.926598 & FALSE & 3.8852903 & FALSE \\
\hline Error & 0.0055 & 12 & 0.0005 & & & & & & \\
\hline Total & 0.0069 & 17 & & & & & & & \\
\hline & & & & & Thickn & & & & \\
\hline & & & & & & $\mathbf{F}$ & & $F$ & \\
\hline Source & SS & DF & MS & $\mathbf{F}$ & $\mathbf{P}$ & 0.01 & significant? & 0.05 & significant? \\
\hline GO & 0.0061 & 2 & 0.0031 & 22.3388 & 0.0072 & 6.926598 & TRUE & 3.8852903 & TRUE \\
\hline $\mathbf{G}$ & 0.0007 & 1 & 0.0007 & 5.2988 & 0.8229 & 9.3302788 & FALSE & 4.7472213 & TRUE \\
\hline $\mathbf{0}$ & 0.0029 & 2 & 0.0014 & 10.5852 & 0.1467 & 6.926598 & TRUE & 3.8852903 & TRUE \\
\hline Error & 0.0016 & 12 & 0.0001 & & & & & & \\
\hline Total & 0.0114 & 17 & & & & & & & \\
\hline
\end{tabular}


Table B.69 Factorial Analysis of Variance: Height of Lost Foam Silica Sand Castings

(all)

$\begin{array}{lc}\begin{array}{l}\text { Number of Factors } \\ \text { Factor }\end{array} & \begin{array}{c}\text { Number of Replicates } \\ \text { Levels }\end{array} \\ G=\text { Gating } & 2 \\ O=\text { Orientation } & 3 \\ \text { T=Thickness } & 3\end{array}$

\begin{tabular}{|c|c|c|c|c|c|c|c|c|c|}
\hline \multirow{3}{*}{$\begin{array}{l}\text { Source } \\
\text { GOT }\end{array}$} & \multirow{3}{*}{$\begin{array}{c}\text { SS } \\
0177\end{array}$} & \multirow[b]{2}{*}{ DF } & \multirow[b]{2}{*}{ MS } & \multirow[b]{2}{*}{$\mathbf{F}$} & \multicolumn{2}{|r|}{$F$} & \multicolumn{3}{|c|}{$\mathbf{F}$} \\
\hline & & & & & $\mathbf{P}$ & 0.01 & significant? & 0.05 & significant? \\
\hline & & 4 & 0.0044 & 7.7947 & 0.0001 & 3.890307 & TRUE & 2.633534 & TRUE \\
\hline GO & 0.013 & 2 & 0.0065 & 11.4652 & 0.0001 & 5.247898 & TRUE & 3.259444 & TRUE \\
\hline GT & 0.0008 & 2 & 0.0004 & 0.7462 & 0.4813 & 5.247898 & FALSE & 3.259444 & 'FALSE \\
\hline $\mathbf{G}$ & 0.0015 & 1 & 0.0015 & 2.7087 & 0.1085 & 7.395556 & FALSE & 4.113161 & FALSE \\
\hline OT & 0.0068 & 4 & 0.0017 & 3.0185 & 0.0303 & 3.890307 & FALSE & 2.633534 & TRUE \\
\hline 0 & 0.0061 & 2 & 0.003 & 5.3521 & 0.0092 & 5.247898 & TRUE & 3.259444 & TRUE \\
\hline$T$ & 0.1018 & 2 & 0.0509 & 89.8565 & $1.01 E-14$ & 5.247898 & TRUE & 3.259444 & TRUE \\
\hline Error & 0.0204 & 36 & 0.0006 & & & & & & \\
\hline Total & 0.1332 & 53 & & & & & & & \\
\hline
\end{tabular}


Table B.71 Factorial Analysis of Variance: Parallelism of Lost Foam Silica Sand Castings

(all)

$\begin{array}{lc}\begin{array}{l}\text { Number of Factors } \\ \text { Factor }\end{array} & \begin{array}{c}\text { Number of Replicates } \\ \text { Levels }\end{array} \\ G=\text { Gating } & 2 \\ O=\text { Orientation } & 3 \\ T=\text { Thickness } & 3\end{array}$

\begin{tabular}{|c|c|c|c|c|c|c|c|c|c|}
\hline & & & & & & $\mathbf{F}$ & & $\mathbf{F}$ & \\
\hline Source & SS & DF & MS & $\mathbf{F}$ & $\mathbf{P}$ & 0.01 & significant? & 0.05 & significant? \\
\hline GOT & 0.0029 & 4 & 0.0007 & 1.4093 & 0.2506 & 3.890307 & FALSE & 2.633534 & FALSE \\
\hline GO & 0.0034 & 2 & 0.0017 & 3.2427 & 0.0507 & 5.247898 & FALSE & 3.259444 & FALSE \\
\hline GT & 0.0151 & 2 & 0.0075 & 14.4775 & 2.43E-05 & 5.247898 & TRUE & 3.259444 & TRUE \\
\hline $\mathbf{G}$ & 0.0074 & 1 & 0.0074 & 14.2065 & 0.0006 & 7.395556 & TRUE & 4.113161 & TRUE \\
\hline OT & 0.0149 & 4 & 0.0037 & 7.1702 & 0.0002 & 3.890307 & TRUE & 2.633534 & TRUE \\
\hline 0 & 0.0022 & 2 & 0.0011 & 2.1543 & 0.1307 & 5.247898 & FALSE & 3.259444 & FALSE \\
\hline $\mathrm{T}$ & 0.1887 & 2 & 0.0944 & 181.2317 & $1.61 \mathrm{E}-19$ & 5.247898 & TRUE & 3.259444 & TRUE \\
\hline Error & 0.0187 & 36 & 0.0005 & & & & & & \\
\hline Total & 0.2534 & 53 & & & & & & & \\
\hline
\end{tabular}


Table B.72 Factorial Analysis of Variance: Left Casting Angle of Lost Foam Silica Sand Castings

(all)

$\begin{array}{lc}\begin{array}{l}\text { Number of Factors } \\ \text { Factor }\end{array} & \begin{array}{c}\text { Number of Replicates } \\ \text { Levels }\end{array} \\ G=\text { Gating } & 2 \\ O=\text { Orientation } & 3 \\ \text { T=Thickness } & 3\end{array}$

\begin{tabular}{|c|c|c|c|c|c|c|c|c|c|}
\hline \multirow{3}{*}{$\begin{array}{l}\text { Source } \\
\text { GOT }\end{array}$} & \multirow{3}{*}{$\begin{array}{c}\text { SS } \\
0.0001\end{array}$} & \multirow[b]{2}{*}{ DF } & \multirow[b]{2}{*}{ MS } & \multirow[b]{2}{*}{$F$} & \multicolumn{2}{|r|}{$\mathbf{F}$} & \multicolumn{3}{|c|}{$\mathbf{F}$} \\
\hline & & & & & $\mathbf{P}$ & 0.01 & significant? & 0.05 & significant? \\
\hline & & 4 & 3.39E-05 & 2.3151 & 0.076 & 3.890307 & FALSE & 2.633534 & FALSE \\
\hline GO & 5.32E-05 & 2 & 2.66E-05 & 1.8161 & 0.1772 & 5.247898 & FALSE & 3.259444 & FALSE \\
\hline GT & 2.58E-05 & 2 & 1.29E-05 & 0.8798 & 0.4236 & 5.247898 & FALSE & 3.259444 & FALSE \\
\hline $\mathbf{G}$ & 4.39E-05 & 1 & 4.39E-05 & 2.9985 & 0.0919 & 7.395556 & FALSE & 4.113161 & FALSE \\
\hline OT & 5.51E-05 & 4 & $1.38 \mathrm{E}-05$ & 0.941 & 0.4515 & 3.890307 & FALSE & 2.633534 & FALSE \\
\hline 0 & 8.25E-05 & 2 & 4.12E-05 & 2.8148 & 0.0731 & 5.247898 & FALSE & 3.259444 & FALSE \\
\hline$T$ & 0.0001 & 2 & 7.13E-05 & 4.8647 & 0.0135 & 5.247898 & FALSE & 3.259444 & TRUE \\
\hline Error & 0.0005 & 36 & 1.47E-05 & & & & & & \\
\hline Total & 0.0011 & 53 & & & & & & & \\
\hline
\end{tabular}


Table B.73 Factorial Analysis of Variance: Right Casting Angle of Lost Foam Silica Sand Castings (all)

\section{Number of Factors \\ Factor \\ $G=$ Gating \\ $O=$ Orientation \\ $T=$ Thickness}

Number of Replicates

Levels

2
3
3

\begin{tabular}{|c|c|c|c|c|c|c|c|c|c|}
\hline & & & & & & $\mathbf{F}$ & & $\mathbf{F}$ & \\
\hline Source & SS & DF & MS & $\mathbf{F}$ & $\mathbf{P}$ & 0.01 & significant? & 0.05 & significant? \\
\hline GOT & 0.0184 & 4 & 0.0046 & 22.3856 & 2.38E-09 & 3.890307 & TRUE & 2.633534 & TRUE \\
\hline GO & 0.009 & 2 & 0.0045 & 21.7871 & $6.30 \mathrm{E}-07$ & 5.247898 & TRUE & 3.259444 & TRUE \\
\hline GT & 0.0014 & 2 & 0.0007 & 3.5252 & 0.04 & 5.247898 & FALSE & 3.259444 & TRUE \\
\hline $\mathbf{G}$ & 0.0004 & 1 & 0.0004 & 1.9512 & 0.171 & 7.395556 & FALSE & 4.113161 & FALSE \\
\hline OT & 0.0026 & 4 & 0.0006 & 3.1607 & 0.0252 & 3.890307 & FALSE & 2.633534 & TRUE \\
\hline 0 & 0.0016 & 2 & 0.0008 & 3.7821 & 0.0323 & 5.247898 & FALSE & 3.259444 & TRUE \\
\hline$T$ & 0.1021 & 2 & 0.051 & 248.5019 & $8.56 E-22$ & 5.247898 & TRUE & 3.259444 & TRUE \\
\hline Error & 0.0074 & 36 &, 0.0002 & & & & & & \\
\hline Total & 0.1428 & 53 & & & & & & & \\
\hline
\end{tabular}


Table B.74 Factorial Analysis of Variance: Left Flange Flatness of Lost Foam Silica Sand Castings

(all)

$\begin{array}{lc}\begin{array}{l}\text { Number of Factors } \\ \text { Factor }\end{array} & \begin{array}{c}\text { Number of Replicates } \\ \text { Levels }\end{array} \\ G=\text { Gating } & 2 \\ O=\text { Orientation } & 3 \\ T=\text { Thickness } & 3\end{array}$

\begin{tabular}{|c|c|c|c|c|c|c|c|c|c|}
\hline & & & & & & $\mathbf{F}$ & & $\mathbf{F}$ & \\
\hline Source & ss & DF & MS & $\mathbf{F}$ & $\mathbf{P}$ & 0.01 & significant? & 0.05 & significant? \\
\hline GOT & 0.0001 & 4 & 2.71E-05 & 0.7888 & 0.5401 & 3.890307 & FALSE & 2.633534 & FALSE \\
\hline GO & 7.81E-05 & 2 & 3.91E-05 & 1.1363 & 0.3322 & 5.247898 & FALSE & 3.259444 & FALSE \\
\hline GT & 0.0002 & 2 & $9.09 E-05$ & 2.6444 & 0.0848 & 5.247898 & FALSE & 3.259444 & FALSE \\
\hline $\mathbf{G}$ & 4.27E-05 & 1 & 4.27E-05 & 1.2414 & 0.2726 & 7.395556 & FALSE & 4.113161 & FALSE \\
\hline OT & 9.23E-05 & 4 & 2.31E-05 & 0.6713 & 0.6162 & 3.890307 & FALSE & 2.633534 & FALSE \\
\hline 0 & 0.0006 & 2 & 0.0003 & 9.2365 & 0.0006 & 5.247898 & TRUE & 3.259444 & TRUE \\
\hline$T$ & 6.86E-05 & 2 & $3.43 E-05$ & 0.9978 & 0.3786 & 5.247898 & FALSE & 3.259444 & FALSE \\
\hline Error & 0.0012 & 36 & 3.44E-05 & & & & & & \\
\hline Total & 0.0024 & 53 & & & & & & & \\
\hline
\end{tabular}


Table B.75 Factorial Analysis of Variance: Right Flange Flatness of Lost Foam Silica Sand Castings (all)

$\begin{array}{lc}\begin{array}{l}\text { Number of Factors } \\ \text { Factor }\end{array} & \begin{array}{c}\text { Number of Replicates } \\ \text { Levels }\end{array} \\ \text { G=Gating } & 2 \\ O=\text { Orientation } & 3 \\ T=\text { Thickness } & 3\end{array}$

\begin{tabular}{|c|c|c|c|c|c|c|c|c|c|}
\hline & & & & & & $\mathbf{F}$ & & $\mathbf{F}$ & \\
\hline Source & ss & DF & MS & $\mathbf{F}$ & $\mathbf{P}$ & 0.01 & significant? & 0.05 & significant? \\
\hline GOT & 0.0805 & 4 & 0.0201 & 2.74 & 0.0435 & 3.890307 & FALSE & 2.633534 & TRUE \\
\hline GO & 0.063 & 2 & 0.0315 & 4.2875 & 0.0214 & 5.247898 & FALSE & 3.259444 & TRUE \\
\hline GT & 0.0483 & 2 & 0.0241 & 3.2857 & 0.0489 & 5.247898 & FALSE & 3.259444 & TRUE \\
\hline $\mathbf{G}$ & 0.0455 & 1 & 0.0455 & 6.1969 & 0.0176 & 7.395556 & FALSE & 4.113161 & TRUE \\
\hline OT & 0.0516 & 4 & 0.0129 & 1.7574 & 0.1589 & 3.890307 & FALSE & 2.633534 & FALSE \\
\hline 0 & 0.0653 & 2 & 0.0327 & 4.4467 & 0.0188 & 5.247898 & FALSE & 3.259444 & TRUE \\
\hline $\mathrm{T}$ & 0.1307 & 2 & 0.0654 & 8.8957 & 0.0007 & 5.247898 & TRUE & 3.259444 & TRUE \\
\hline Error & 0.2645 & 36 & 0.0073 & & & & & & \\
\hline
\end{tabular}

Total $\quad 0.7495 \quad 53$ 
Table B.76 Factorial Analysis of Variance: Left Wall Flatness of Lost Foam Silica Sand Castings

(all)

$\begin{array}{lc}\begin{array}{l}\text { Number of Factors } \\ \text { Factor }\end{array} & \begin{array}{c}\text { Number of Replicates } \\ \text { Levels }\end{array} \\ G=\text { Gating } & 2 \\ O=\text { Orientation } & 3 \\ T=\text { Thickness } & 3\end{array}$

\begin{tabular}{|c|c|c|c|c|c|c|c|c|c|}
\hline & & & & & & $\mathbf{F}$ & & $\mathbf{F}$ & \\
\hline Source & SS & DF & MS & $\mathbf{F}$ & $\mathbf{P}$ & 0.01 & significant? & 0.05 & significant? \\
\hline GOT & 0.0002 & 4 & 4.69E-05 & 1.9366 & 0.1254 & 3.890307 & FALSE & 2.633534 & FALSE \\
\hline GO & 8.81E-05 & 2 & 4.41E-05 & 1.8174 & 0.177 & 5.247898 & FALSE & 3.259444 & FALSE \\
\hline GT & 6.74E-05 & 2 & 3.37E-05 & 1.3911 & 0.2618 & 5.247898 & FALSE & 3.259444 & FALSE \\
\hline $\mathbf{G}$ & 1.50E-06 & 1 & 1.50E-06 & 0.0619 & 0.805 & 7.395556 & FALSE & 4.113161 & FALSE \\
\hline OT & 0.0002 & 4 & 5.93E-05 & 2.4461 & 0.064 & 3.890307 & FALSE & 2.633534 & FALSE \\
\hline 0 & 0.0001 & 2 & 5.61E-05 & 2.3155 & 0.1132 & 5.247898 & FALSE & 3.259444 & FALSE \\
\hline $\mathrm{T}$ & 0.0004 & 2 & 0.0002 & 7.2429 & 0.0023 & 5.247898 & TRUE & 3.259444 & TRUE \\
\hline Error & 0.0009 & 36 & 2.42E-05 & & & & & & \\
\hline Total & 0.0019 & 53 & & & & & & & \\
\hline
\end{tabular}


Table B.77 Factorial Analysis of Variance: Right Wall Flatness of Lost Foam Silica Sand Castings (all)

Number of Factors
Factor
$G=$ Gating
$O=$ Orientation
$T=$ Thickness

$\begin{array}{lccccccccc} & & & & & & \mathbf{F} & & \mathbf{F} & \\ \text { Source } & \text { SS } & \text { DF } & \text { MS } & \mathbf{F} & \mathbf{P} & \mathbf{0 . 0 1} & \text { significant? } & \mathbf{0 . 0 5} & \text { significant? } \\ \text { GOT } & 0.0026 & 4 & 0.0006 & 0.516 & 0.7244 & 3.890307 & \text { FALSE } & 2.633534 & \text { FALSE } \\ \text { GO } & 0.0021 & 2 & 0.001 & 0.8295 & 0.4444 & 5.247898 & \text { FALSE } & 3.259444 & \text { FALSE } \\ \text { GT } & 0.0017 & 2 & 0.0008 & 0.6774 & 0.5143 & 5.247898 & \text { FALSE } & 3.259444 & \text { FALSE } \\ \text { G } & 0.0005 & 1 & 0.0005 & 0.4222 & 0.52 & 7.395556 & \text { FALSE } & 4.113161 & \text { FALSE } \\ \text { OT } & 0.0039 & 4 & 0.001 & 0.772 & 0.5506 & 3.890307 & \text { FALSE } & 2.633534 & \text { FALSE } \\ \text { O } & 0.0016 & 2 & 0.0008 & 0.6393 & 0.5336 & 5.247898 & \text { FALSE } & 3.259444 & \text { FALSE } \\ \text { T } & 0.0133 & 2 & 0.0067 & 5.308 & 0.0095 & 5.247898 & \text { TRUE } & 3.259444 & \text { TRUE } \\ \text { Error } & 0.0451 & 36 & 0.0013 & & & & & & \end{array}$


Table B.78 Factorial Analysis of Variance:

Lost Foam Silica Sand Castings

(0.25 inch)

Number of Factors

Number of Replicates

Factor

$\mathrm{G}=$ Gating

$\mathrm{O}=$ Orientation

Levels

2

3

\section{Left Flange Flatness}

\begin{tabular}{llllcccccc} 
& & & & & & $\mathbf{F}$ & & F & \\
Source & \multicolumn{1}{c}{ SS } & DF & MS & $\mathbf{F}$ & $\mathbf{P}$ & $\mathbf{0 . 0 1}$ & significant? & $\mathbf{0 . 0 5}$ & significant? \\
GO & 0.0001 & 4 & $3.64 \mathrm{E}-05$ & 2.2837 & 0.1002 & 4.579022 & FALSE & 2.927749 & FALSE \\
G & $6.90 \mathrm{E}-05$ & 2 & $3.45 \mathrm{E}-05$ & 2.1651 & 0.1437 & 6.012897 & FALSE & 3.554561 & FALSE \\
O & 0.0002 & 2 & $8.14 \mathrm{E}-05$ & 5.1093 & 0.0175 & 6.012897 & FALSE & 3.554561 & TRUE \\
Error & 0.0003 & 18 & $1.59 \mathrm{E}-05$ & & & &. & &
\end{tabular}

Total $\quad 0.0007 \quad 26$

Right Flange Flatness

\begin{tabular}{lccccccccc} 
& & & & & & $\mathbf{F}$ & \multicolumn{3}{c}{$\mathbf{F}$} \\
Source & SS & DF & MS & $\mathbf{F}$ & $\mathbf{P}$ & $\mathbf{0 . 0 1}$ & significant? & $\mathbf{0 . 0 5}$ & significant? \\
GO & 0.0053 & 4 & 0.0013 & 0.5215 & 0.7211 & 4.579022 & FALSE & 2.927749 & FALSE \\
G & 0.0035 & 2 & 0.0018 & 0.6934 & 0.5128 & 6.012897 & FALSE & 3.554561 & FALSE \\
O & 0.0034 & 2 & 0.0017 & 0.6727 & 0.5227 & 6.012897 & FALSE & 3.554561 & FALSE \\
Error & 0.046 & 18 & 0.0026 & & & & & &
\end{tabular}

Total $\quad 0.0583 \quad 26$

\section{Left Wall Flatness}

\begin{tabular}{|c|c|c|c|c|c|c|c|c|c|}
\hline & & & & & & $\mathbf{F}$ & & $\mathbf{F}$ & \\
\hline Source & SS & DF & MS & $\mathbf{F}$ & $\mathbf{P}$ & 0.01 & significant? & 0.05 & significant? \\
\hline GO & $2.77 \mathrm{E}-05$ & 4 & $6.93 \mathrm{E}-06$ & 0.3929 & 0.811 & 4.579022 & FALSE & 2.927749 & FALSE \\
\hline G & $9.19 \mathrm{E}-06$ & 2 & $4.59 \mathrm{E}-06$ & 0.2605 & 0.7735 & 6.012897 & FALSE & 3.554561 & FALSE \\
\hline 0 & $2.96 \mathrm{E}-05$ & 2 & $1.48 \mathrm{E}-05$ & 0.8403 & 0.4478 & 6.012897 & FALSE & 3.554561 & FALSE \\
\hline Error & 0.0003 & 18 & $1.76 \mathrm{E}-05$ & & & & & & \\
\hline
\end{tabular}

Total $\quad 0.0004 \quad 26$

Right Wall Flatness

$\begin{array}{lccccccccc} & & & & & & \mathbf{F} & & \mathbf{F} & \\ \text { Source } & \text { SS } & \text { DF } & \text { MS } & \text { F } & \mathbf{P} & \mathbf{0 . 0 1} & \text { significant? } & \mathbf{0 . 0 5} & \text { 'significant? } \\ \text { GO } & 0.0019 & 4 & 0.0005 & 1.5623 & 0.2271 & 4.579022 & \text { FALSE } & 2.927749 & \text { FALSE } \\ \text { G } & 0.0007 & 2 & 0.0003 & 1.0697 & 0.364 & 6.012897 & \text { FALSE } & 3.554561 & \text { FALSE } \\ \text { O } & 0.0015 & 2 & 0.0007 & 2.3988 & 0.1192 & 6.012897 & \text { FALSE } & 3.554561 & \text { FALSE } \\ \text { Error } & 0.0056 & 18 & 0.0003 & & & & & & \\ & & & & & & & & & \end{array}$


Table B.79 Factorial Analysis of Variance:

Lost Foam Silica Sand Castings

(0.25 inch)

$\begin{array}{lc}\text { Number of Factors } & \text { Number of Replicates } \\ \text { Factor } & \text { Levels } \\ \text { G=Gating } & 2 \\ \text { O=Orientation } & 3\end{array}$

\begin{tabular}{|c|c|c|c|c|c|c|c|c|c|}
\hline & & & & & Heigh & & & & \\
\hline & & & & & & $\mathbf{F}$ & & $F$ & \\
\hline Source & SS & DF & MS & $\mathbf{F}$ & $\mathbf{P}$ & 0.01 & significant? & 0.05 & significant? \\
\hline GO & 0.0316 & 4 & 0.0079 & 6.5195 & 0.002 & 4.579022 & TRUE & 2.927749 & TRUE \\
\hline $\mathrm{G}$ & 0.0086 & 2 & 0.0043 & 3.5415 & 0.0505 & 6.012897 & FALSE & 3.554561 & FALSE \\
\hline 0 & 0.0141 & 2 & 0.0071 & 5.8292 & 0.0112 & 6.012897 & FALSE & 3.554561 & TRUE \\
\hline Error & 0.0218 & 18 & 0.0012 & & & & & & \\
\hline Total & 0.0762 & 26 & & & & & & & \\
\hline
\end{tabular}

\section{Length}

\begin{tabular}{lccccccccc} 
& & & & & & $\mathbf{F}$ & & \multicolumn{1}{c}{$\mathbf{c}$} \\
Source & SS & DF & MS & $\mathbf{F}$ & $\mathbf{P}$ & $\mathbf{0 . 0 1}$ & significant? & $\mathbf{0 . 0 5}$ & significant? \\
GO & 0.3633 & 4 & 0.0908 & 3.0186 & 0.0455 & 4.579022 & FALSE & 2.927749 & TRUE \\
G & 0.0137 & 2 & 0.0068 & 0.2272 & 0.799 & 6.012897 & FALSE & 3.554561 & FALSE \\
O & 0.2588 & 2 & 0.1294 & 4.3 & 0.0298 & 6.012897 & FALSE & 3.554561 & TRUE \\
Error & 0.5417 & 18 & 0.0301 & & & & & &
\end{tabular}

Total $\quad 1.1775 \quad 26$

\section{Left Casting Angle}

\begin{tabular}{|c|c|c|c|c|c|c|c|c|c|}
\hline \multirow[b]{2}{*}{ Source } & \multirow[b]{2}{*}{ SS } & \multirow[b]{2}{*}{ DF } & \multirow[b]{2}{*}{ MS } & \multicolumn{3}{|r|}{ n } & \multicolumn{3}{|c|}{$\mathbf{F}$} \\
\hline & & & & $\mathbf{F}$ & $\mathbf{P}$ & 0.01 & significant? & 0.05 & significant? \\
\hline GO & 0.0002 & 4 & $4.17 \mathrm{E}-05$ & 2.2304 & 0.1063 & 4.579022 & FALSE & 2.927749 & FALSE \\
\hline G & $6.20 \mathrm{E}-05$ & 2 & $3.10 \mathrm{E}-05$ & 1.6569 & 0.2185 & 6.012897 & FALSE & 3.554561 & FALSE \\
\hline 0 & $4.93 E-05$ & 2 & $2.46 \mathrm{E}-05$ & 1.3169 & 0.2926 & 6.012897 & FALSE & 3.554561 & FALSE \\
\hline Errot & 0.0003 & 18 & $1.87 \mathrm{E}-05$ & & & & & & \\
\hline Total & 0.0006 & 26 & & & & & & & \\
\hline
\end{tabular}

\section{Right Casting Angle}

\begin{tabular}{|c|c|c|c|c|c|c|c|c|c|}
\hline \multirow[b]{2}{*}{ Source } & \multirow[b]{2}{*}{ SS } & \multirow[b]{2}{*}{ DF } & \multirow[b]{2}{*}{ MS } & \multicolumn{3}{|r|}{$\mathbf{F}$} & \multicolumn{3}{|c|}{$\mathbf{F}$} \\
\hline & & & & $\mathbf{F}$ & $\mathbf{P}$ & 0.01 & significant? & 0.05 & significant? \\
\hline GO & 0.0331 & 4 & 0.0083 & 15.0229 & $1.46 \mathrm{E}-05$ & 4.579022 & TRUE & 2.927749 & TRUE \\
\hline $\mathrm{G}$ & 0.0031 & 2 & 0.0016 & 2.8134 & 0.0865 & 6.012897 & FALSE & 3.554561 & FALSE \\
\hline 0 & 0.001 & 2 & 0.0005 & 0.8909 & 0.4276 & 6.012897 & FALSE & 3.554561 & FALSE \\
\hline Error & 0.0099 & 18 & 0.0006 & & & & & & \\
\hline Cotal & 0.0471 & 26 & & & & & & & \\
\hline
\end{tabular}


Table B.80 Factorial Analysis of Variance: Lost Foam Silica Sand Castings (0.25 inch)

$\begin{array}{lc}\text { Number of Factors } & \begin{array}{c}\text { Number of Replicates } \\ \text { Levels }\end{array} \\ \text { Factor } & 2 \\ \mathrm{G}=\text { Gating } & 3 \\ \mathrm{O}=\text { Orientation } & \end{array}$

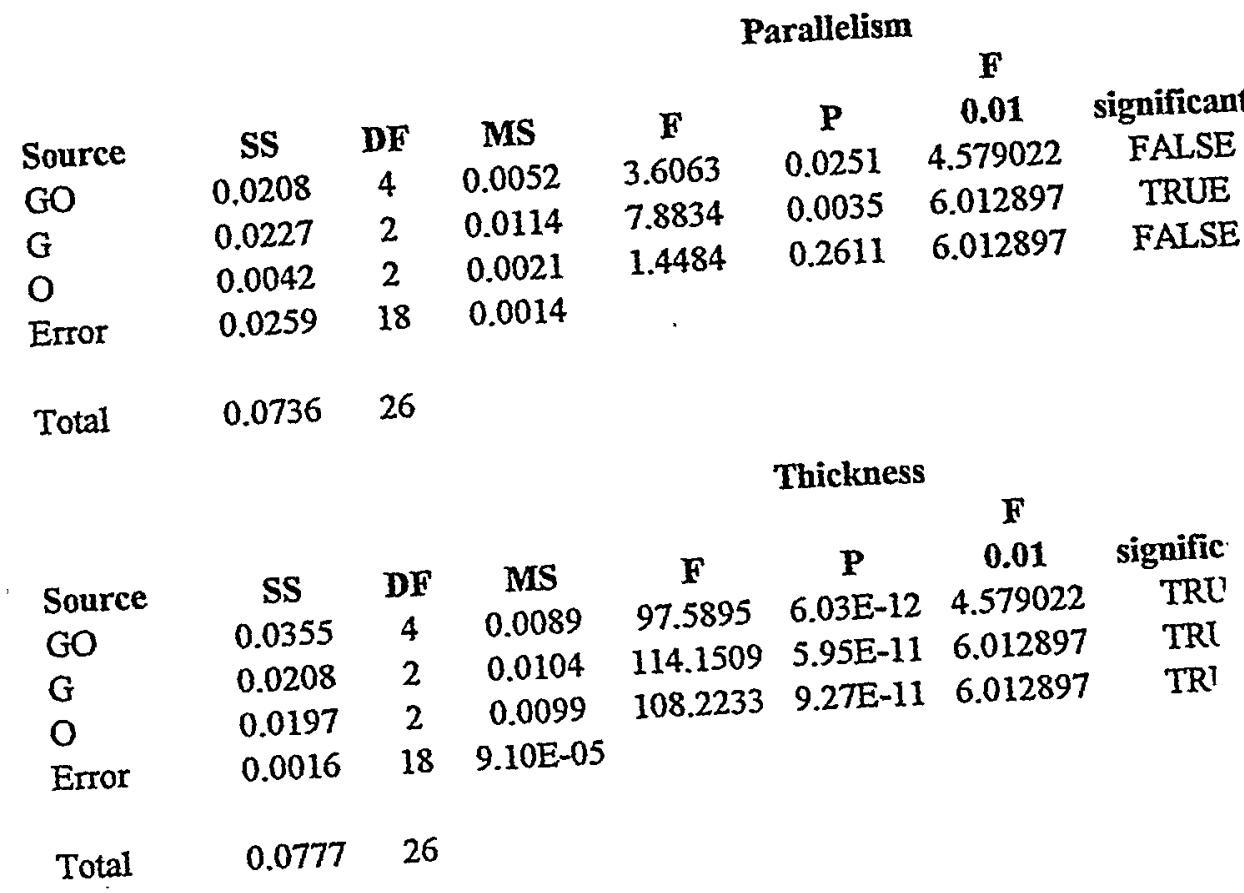


Table B.81 Factorial Analysis of Variance:

Lost Foam Silica Sand Castings

(0.50 inch)

$\begin{array}{lc}\begin{array}{l}\text { Number of Factors } \\ \text { Factor }\end{array} & \begin{array}{c}\text { Number of Replicates } \\ \text { Levels }\end{array} \\ G=\text { Gating } & 2 \\ O=\text { Orientation } & 3\end{array}$

$\begin{array}{lccccccc} & & & & & & \text { F } & \\ \text { Source } & \text { SS } & \text { DF } & \text { MS } & \text { F } & \text { P } & 0.01 & \text { significant? } \\ \text { GO } & 0.0002 & 2 & 7.65 E-05 & 2.8389 & 0.0978 & 6.926598 & \text { FALSE } \\ \text { G } & 0.0001 & 1 & 0.0001 & 4.916 & 0.0467 & 9.330279 & \text { FALSE } \\ \text { O } & 0.0002 & 2 & 7.99 E-05 & 2.9682 & 0.0897 & 6.926598 & \text { FALSE } \\ \text { Error } & 0.0003 & 12 & 2.69 E-05 & & & & \\ \text { Total } & 0.0008 & 17 & & & & & \end{array}$

\begin{tabular}{lccccccc} 
& & & \multicolumn{5}{c}{ Length } \\
Source & SS & DF & MS & F & P & O.01 & significant \\
GO & $6.15 E-05$ & 2 & $3.07 E-05$ & 0.2451 & 0.7865 & 6.926598 & FALSE \\
G & 0.0005 & 1 & 0.0005 & 3.6286 & 0.081 & 9.330279 & FALSE \\
O & 0.0002 & 2 & 0.0001 & 0.919 & $4.25 E-01$ & 6.926598 & FALSE \\
Error & 0.0015 & 12 & $1.00 E-04$ & & & & \\
& & & & & & &
\end{tabular}

\begin{tabular}{lccccccc} 
& & \multicolumn{8}{c}{ Parallelism } \\
Source & SS & DF & MS & F & P & O.01 & significe \\
GO & 0.0006 & 2 & 0.0003 & 3.123 & 0.0809 & 6.926598 & FALS \\
G & 0.0003 & 1 & 0.0003 & 2.5752 & 0.1345 & 9.330279 & FALS \\
O & 0.0002 & 2 & $8.03 E-05$ & 0.8097 & 0.4679 & 6.926598 & FALS \\
Error & 0.0012 & 12 & $9.92 E-05$ & & & &
\end{tabular}

$\begin{array}{lll}\text { Total } & 0.0022 \quad 17\end{array}$

Thickness

\begin{tabular}{lccccccc} 
& & & & & & \multicolumn{2}{c}{} \\
Source & SS & DF & MS & F & $\mathbf{P}$ & $\mathbf{0 . 0 1}$ & signif \\
GO & $2.37 \mathrm{E}-05$ & 2 & $1.18 \mathrm{E}-05$ & 0.3544 & 0.7087 & 6.926598 & FAl \\
G & $2.56 E-05$ & 1 & $2.56 E-05$ & 0.7657 & 0.3987 & 9.330279 & FA \\
O & $8.82 E-05$ & 2 & $4.41 E-05$ & 1.3207 & 0.3031 & 6.926598 & FA \\
Error & 0.0004 & 12 & $3.34 E-05$ & & & & \\
& & & & & & & \\
Total & 0.0005 & 17 & & & & &
\end{tabular}


Table B.82 Factorial Analysis of Variance:

Lost Foam Silica Sand Castings

(0.50 inch)

$\begin{array}{lc}\text { Number of Factors } & \begin{array}{c}\text { Number of Replicates } \\ \text { Levels }\end{array} \\ \text { Factor } & 2 \\ G=\text { Gating } & 3 \\ \text { O=Orientation } & \end{array}$

Left Flange Flatness

\begin{tabular}{|c|c|c|c|c|c|c|c|c|}
\hline Source & SS & DF & MS & $\mathbf{F}$ & $\mathbf{P}$ & $\begin{array}{c}F \\
0.01\end{array}$ & significant? & $\begin{array}{c}F \\
0.05\end{array}$ \\
\hline GO & 8.33E-06 & 2 & 4.17E-06 & 0.0707 & 0.9321 & 6.926598 & FALSE & 3.885 \\
\hline $\mathbf{G}$ & 5.00E-05 & 1 & 5.00E-05 & 0.8483 & 0.3752 & 9.330279 & FALSE & $4.747 \%$ \\
\hline 0 & 0.0004 & 2 & 0.0002 & 3.0094 & 0.0872 & 6.926598 & FALSE & 3.885 \\
\hline Error & 0.0007 & 12 & 5.89E-05 & & & & & \\
\hline Total & 0.0011 & 17 & & & & & & \\
\hline
\end{tabular}

$\begin{array}{lcccccccc} & & & & & & \mathbf{F} & & \mathbf{F} \\ \text { Source } & \text { SS } & \text { DF } & \text { MS } & \text { F } & \mathbf{P} & \mathbf{0 . 0 1} & \text { significant? } & \mathbf{0 . 0} \\ \text { Go } & 0.0035 & 2 & 0.0017 & 4.9576 & 0.027 & 6.926598 & \text { FALSE } & 3.885 \\ \text { G } & 0.0043 & 1 & 0.0043 & 12.3498 & 0.0043 & 9.330279 & \text { TRUE } & 4.747 \\ \text { O } & 0.004 & 2 & 0.002 & 5.6589 & 0.0186 & 6.926598 & \text { FALSE } & 3.885 \\ \text { Error } & 0.0042 & 12 & 0.0004 & & & & & \\ & & & & & & & & \end{array}$

$\begin{array}{lcccccccc} & & & & & & \text { F } & & \text { F } \\ \text { Source } & \text { SS } & \text { DF } & \text { MS } & \text { F } & \text { P } & 0.01 & \text { significant? } & \mathbf{0 . C} \\ \text { Go } & 1.30 E-05 & 2 & 6.50 E-06 & 0.5043 & 0.6162 & 6.926598 & \text { FALSE } & 3.88 \\ \text { G } & 0 & 1 & 0 & 8.11 E-28 & 1 & 9.330279 & \text { FALSE } & 4.74 i \\ \text { O } & 8.33 E-06 & 2 & 4.17 E-06 & 0.3233 & 0.7299 & 6.926598 & \text { FALSE } & 3.88 \\ \text { Error } & 0.0002 & 12 & 1.29 E-05 & & & & & \\ & & & & & & & & \end{array}$

Right Wall Flatness

\begin{tabular}{|c|c|c|c|c|c|c|c|c|}
\hline \multirow[b]{2}{*}{ Source } & \multirow[b]{2}{*}{ Ss } & \multirow[b]{2}{*}{ DF } & \multirow[b]{2}{*}{ MS } & \multirow[b]{2}{*}{$\mathbf{F}$} & \multicolumn{3}{|c|}{$F$} & \multirow{2}{*}{$0^{1}$} \\
\hline & & & & & $\mathbf{P}$ & 0.01 & significant? & \\
\hline GO & 0.0003 & 2 & 0.0001 & 0.2815 & 0.7595 & 6.926598 & FALSE & .3 .81 \\
\hline G & 0.0004 & 1 & 0.0004 & 0.8413 & 0.3771 & 9.330279 & FALSE & 4.74 \\
\hline 0 & 0.0007 & 2 & 0.0003 & 0.7022 & 0.5148 & 6.926598 & FALSE & $3.8:$ \\
\hline Error & 0.0056 & 12 & 0.0005 & & & & & \\
\hline Total & 0.0069 & 17 & & & & & & \\
\hline
\end{tabular}


Table B.83 Factorial Analysis of Variance: Lost Foam Silica Sand Castings

(0.50 inch)

$\begin{array}{lc}\text { Number of Factors } & \begin{array}{c}\text { Number of Replicates } \\ \text { Levels }\end{array} \\ \text { Factor } & 2 \\ O=\text { Gating } & 3\end{array}$

Left Casting Angle

\begin{tabular}{|c|c|c|c|c|c|c|c|}
\hline \multirow[b]{2}{*}{ Source } & & \multicolumn{6}{|c|}{ Left Casting Angle $F$} \\
\hline & SS & DF & MS & $F$ & $P$ & 0.01 & $\begin{array}{c}\text { significant? } \\
\text { FAl SE }\end{array}$ \\
\hline GO & $2.50 E-05$ & 2 & 1.25E-05 & 1.8202 & 0.204 & 6.926598 & $\begin{array}{l}\text { FALSE } \\
\text { FAI SF }\end{array}$ \\
\hline $\begin{array}{l}\mathbf{G} \\
\mathbf{0}\end{array}$ & 1.15E-05 & 1 & 1.15E-05 & 1.6696 & 0.2206 & 9.330279 & $\begin{array}{l}\text { FALSE } \\
\text { FAISE }\end{array}$ \\
\hline $\begin{array}{l}\text { O } \\
\text { Error }\end{array}$ & $\begin{array}{l}7.48 E-05 \\
833 E-05\end{array}$ & 2 & 3.74E-05 & 5.4527 & 0.0207 & 6.926598 & FALSE \\
\hline & 8.23E-05 & 12 & 6.86E-06 & & & & \\
\hline Totạl & $2.00 E-04$ & 17 & & & & & \\
\hline & & & & Rig & Casting & $\begin{array}{l}\text { Angle } \\
F\end{array}$ & \\
\hline $\begin{array}{l}\text { Source } \\
\text { GO }\end{array}$ & $\begin{array}{c}\text { SS } \\
7.52 E-06\end{array}$ & $\begin{array}{c}\text { DF } \\
2\end{array}$ & $\begin{array}{c}\text { MS } \\
3.76 E-06\end{array}$ & $\begin{array}{c}F \\
0.1056\end{array}$ & $\begin{array}{c}P \\
0.9006\end{array}$ & $\begin{array}{c}0.01 \\
6.926598\end{array}$ & $\begin{array}{c}\text { significar } \\
3 \quad \text { FALSE }\end{array}$ \\
\hline $\begin{array}{l}\text { GO } \\
G\end{array}$ & $\begin{array}{l}\text { 7.52E-06 } \\
3.07 E-05\end{array}$ & $\begin{array}{l}2 \\
1\end{array}$ & $\begin{array}{l}3.76 E-06 \\
3.07 E-05\end{array}$ & $\begin{array}{l}0.7450 \\
0.8606\end{array}$ & 0.3718 & 9.330279 & $9 \quad$ FALSE \\
\hline & $6.58 E-05$ & 2 & $3.29 E-05$ & 0.9236 & 0.4236 & 6.926598 & FALSE \\
\hline Error & 0.0004 & 12 & $3.56 E-05$ & & & & \\
\hline & 0.0005 & & & & & & \\
\hline & & 17 & & & & & \\
\hline
\end{tabular}


Table B.84 Factorial Analysis of Variance: Lost Foam Silica Sand Castings

$$
\text { (0.75 inch) }
$$

Factor

$\mathrm{G}=$ Gating

$0=$ Orientation
Number of Factors

Number of Replicates Levels

$$
2
$$

3

\section{Height}

$\begin{array}{lccccccc} & & & & & & \text { F } & \\ \text { Source } & \text { SS } & \text { DF } & \text { MS } & \text { F } & \text { P } & \text { 0.01 } & \text { significant? } \\ \text { GO } & 0.0004 & 2 & 0.0002 & 1.8332 & 2.02 E-01 & 6.926598 & \text { FALSE } \\ \text { G } & 0.0001 & 1 & 0.0001 & 0.8333 & 0.3793 & 9.330279 & \text { FALSE } \\ \text { O } & 0.0013 & 2 & 0.0006 & 5.3338 & 0.022 & 6.926598 & \text { FALSE } \\ \text { Error } & 0.0014 & 12 & 0.0001 & & & & \\ & & & & & & & \end{array}$

\section{Thickness}

$\begin{array}{lccc}\text { Source } & \text { SS } & \text { DF } & \text { MS } \\ \text { GO } & 9.42 E-05 & 2 & 4.71 E-05 \\ \text { G } & 6.78 E-05 & 1 & 6.78 E-05 \\ \text { O } & 5.26 E-06 & 2 & 2.63 E-06 \\ \text { Error } & 0.0001 & 12 & 1.17 E-05 \\ \text { Total } & 0.0003 & 17\end{array}$

$\begin{array}{cccr} & & \mathbf{F} & \\ \mathbf{F} & \mathbf{P} & \mathbf{0 . 0 1} & \text { significar } \\ 4.0103 & 4.64 \mathrm{E}-02 & 6.926598 & \text { FALSE } \\ 5.7757 & 0.0333 & 9.330279 & \text { FALSE } \\ 0.2241 & 0.8025 & 6.926598 & \text { FALSI }\end{array}$

$\begin{array}{lcccc} & & & & \\ \text { Source } & \text { SS } & \text { DF } & \text { MS } & F \\ \text { GO } & 5.60 E-06 & 2 & 2.80 E-06 & 0.0398 \\ \text { G } & 3.00 E-04 & 1 & 3.00 E-04 & 4.0336 \\ \text { O } & 0.0023 & 2 & 0.0012 & 16.6647 \\ \text { Error } & 0.0008 & 12 & 7.04 E-05 & \\ & & & & \\ \text { Total } & 0.0035 & 17 & & \end{array}$

Parallelism

$\begin{array}{cccr} & \text { Parallelism } & \\ \text { F } & \text { P } & \mathbf{0 . 0 1} & \text { signifi } \\ 0.0398 & 0.9611 & 6.926598 & \text { FAl } \\ 4.0336 & 0.0677 & 9.330279 & \text { FA }^{\prime} \\ 16.6647 & 0.0003 & 6.926598 & \text { TF }\end{array}$

Length

$\begin{array}{lccccccc} & & & & & \mathbf{F} & \mathbf{0 . 0 1} & \text { sig } \\ \text { Source } & \text { SS } & \text { DF } & \text { MS } & \text { F } & \text { P } & 6.926598 \\ \text { GO } & 0.0002 & 2 & 7.55 E-05 & 0.6796 & 0.5253 & 6.3449 & 9.330279 \\ \text { G } & 0.0001 & 1 & 0.0001 & 0.9669 & 0.3449 \\ \text { O } & 0.0014 & 2 & 0.0007 & 6.4315 & 0.0126 & 6.926598 \\ \text { Error } & 0.0013 & 12 & 0.0001 & & & \\ \text { Total } & 0.003 & 17 & & & & & \end{array}$


Table B.85 Factorial Analysis of Variance: Lost Foam Silica Sand Castings

( 0.75 inch)

$$
\begin{aligned}
& \begin{array}{lc}
\text { Number of Factors } & \text { Number } \\
\text { Lactor } & 2
\end{array} \\
& G=\text { Gating } \\
& \mathrm{O}=\text { Orientation }
\end{aligned}
$$

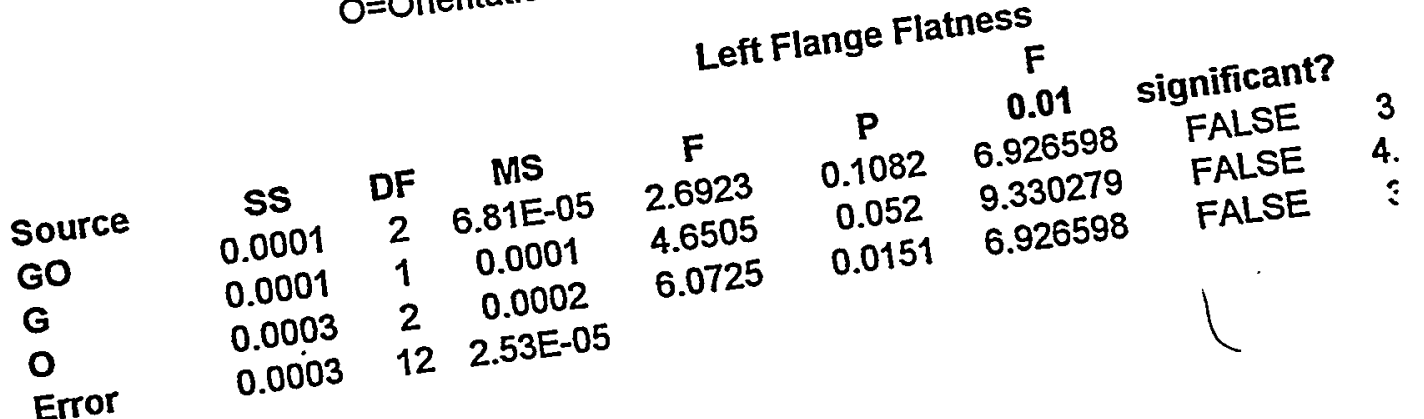

$$
\begin{aligned}
& \text { Error } \\
& \text { source } \\
& \text { GO } \\
& \text { G } \\
& \text { Error } \\
& \text { Total } \\
& 0.5974 \quad 17 \\
& \begin{array}{ccccc} 
& & & & \\
\text { SS } & \text { DF } & \text { MS } & \text { F } & \text { P } \\
& 2 & 0.07 & 3.2531 & 0.0743
\end{array} \\
& \begin{array}{llll}
0.1399 & 2 & 0.0795 & 4.1596
\end{array} \\
& \begin{array}{llll}
0.0895 & 1 & 0.055 & 2.5554
\end{array} \\
& 0.2581 \\
& 12 \\
& 0.0215 \\
& \begin{array}{lllllllll}
\text { GO } & 0.0034 & 2 & 0.0017 & 0.5616 & 0.4681 & 9.33026598 & \text { Ff } \\
\text { G } & 0.0012 & 1 & 0.0012 & 0.2912 & 0.7525 & 6.9265 & \\
\text { O } & 0.0013 & 2 & 0.0006 & 0.0022 & & & & \\
\text { Eror } & 0.0264 & 12 & 0.0022 & & & &
\end{array} \\
& 0.0323 \quad 17
\end{aligned}
$$


Table B.86 Factorial Analysis of Variance:

Lost Foam Silica Sand Castings

(0.75 inch)

Left Casting Angle

$\begin{array}{lcccccccc} & & & & & & F & & F \\ \text { Source } & \text { SS } & \text { DF } & \text { MS } & \text { F } & \text { P } & 0.01 & \text { significant? } & 0.05 \\ \text { Go } & 1.19 E-05 & 2 & 5.93 E-06 & 0.521 & 0.6067 & 6.926598 & \text { FALSE } & 3.885: \\ \text { G } & 2.28 E-07 & 1 & 2.28 E-07 & 0.0201 & 0.8896 & 9.330279 & \text { FALSE } & 4.7472 \\ \text { O } & 2.17 E-05 & 2 & 1.09 E-05 & 0.9543 & 0.4125 & 6.926598 & \text { FALSE } & 3.885: \\ \text { Error } & 1.00 E-04 & 12 & 1.14 E-05 & & & & & \\ & & & & & & & & \end{array}$

Right Casting Angle

$\begin{array}{lcccccccrr} & & & & & & \mathbf{F} & & \mathbf{F} \\ \text { Source } & \text { SS } & \text { DF } & \text { MS } & \text { F } & \text { P } & \mathbf{0 . 0 1} & \text { significant? } & \mathbf{0 . 0 !} \\ \text { Go } & 2.38 E-05 & 2 & 1.19 E-05 & 0.7692 & 0.4849 & 6.926598 & \text { FALSE } & 3.885 \\ \text { G } & 1.00 E-04 & 1 & 1.00 E-04 & 9.031 & 0.011 & 9.330279 & \text { FALSE } & 4.747: \\ \text { O } & 1.70 E-05 & 2 & 8.52 E-06 & 0.55 & 0.5909 & 6.926598 & \text { FALSE } & 3.885 \\ \text { Error } & 0.0002 & 12 & 1.55 E-05 & & & & & \\ & & & & & & & & & \end{array}$


Newman Keuls Test of Means on Mold Type of Thickness Data (all 0.50 inch)

\begin{tabular}{lrrrrr} 
ColName & Count & Mean & Std.Dev. & Std.Err. \\
\hline M1 & 18 & 10.1106 & 0.0111 & 0.0026 \\
M2 & 18 & 10.1151 & 0.0021 & 0.0005 \\
M3 & 18 & 10.1163 & 0.0032 & 0.0007 \\
\hline
\end{tabular}

Newman-Keuls' Test

Significance Level: 0.05

\begin{tabular}{lcccccc}
\multicolumn{3}{c}{ Variables } & Mean Diff $-f$ & Critical Value / Significant \\
M1 & Vs. & M2 & 0.0046 & 0.0058 & NO \\
M1 & Vs. & M3 & 0.0057 & 0.0074 & NO \\
M2 & Vs. & M3 & 0.0012 & 0.0058 & NO
\end{tabular}

Newman Keuls Test of Means on Mold Type of Height Data (all 0.50 inch)

ColName Count Mean Std.Dev. Std.Err.

\begin{tabular}{lrrrr} 
M1 & 18 & 8.8712 & 0.0159 & 0.0037 \\
M2 & 18 & 8.6424 & 0.0556 & 0.0131 \\
M3 & 18 & 8.6024 & 0.0067 & 0.0016 \\
\hline
\end{tabular}

Newman-Keuls' Test

Significance Level: 0.05

\begin{tabular}{cccccc}
\multicolumn{3}{c}{ Variables } & Mean Diff | Critical Value | Significant \\
M1 & Vs. & M2 & 0.2288 & 0.0288 & YES \\
M1 & Vs. & M3 & 0.2688 & 0.0365 & YES \\
M2 & Vs. & M3 & 0.0400 & 0.0288 & YES
\end{tabular}

Newman Keuls Test of Means on Orientation of Length Data (all 0.50 inch)

ColName Count Mean Std.Dev. Std.Err.

$\begin{array}{ccccc}\text { O1 } & 18 & 6.9081 & 0.6502 & 0.1532 \\ \text { O2 } & 18 & 6.9111 & 0.6594 & 0.1554 \\ \text { O3 } & 18 & 6.8947 & 0.6462 & 0.1523 \\ - & - & & & \\ - & & \end{array}$

Newman-Keuls' Test

Significance Level: 0.05

\begin{tabular}{lcc|ccc}
\multicolumn{3}{c}{ Variables | } & Mean Diff | Critical Value | Significant \\
O1 & Vs. & O2 & 0.0030 & 0.5594 & NO \\
O1 & Vs. & O3 & 0.0134 & 0.5594 & NO \\
O2 & Vs. & O3 & 0.0164 & 0.7069 & NO
\end{tabular}


Newman Keuls Test of Means on Mold Type of Length Data ( 0.50 inch)

\begin{tabular}{lrrrr} 
ColName & Count & Mean & Std.Dev. Std.Err. \\
\hline M1 & 18 & 7.8000 & 0.0292 & 0.0069 \\
M2 & 18 & 6.4748 & 0.0155 & 0.0036 \\
M3 & 18 & 6.4390 & 0.0115 & 0.0027 \\
\hline & - & &
\end{tabular}

Newman-Keuls' Test

Significance Level: 0.05

\begin{tabular}{lccccc}
\multicolumn{3}{c|}{ Variables } & Mean Diff | Critical Value | Significant \\
M1 & Vs. & M2 & 1.3252 & 0.0173 & YES \\
M1 & Vs. & M3 & 1.3611 & 0.0219 & YES \\
M2 & Vs. & M3 & 0.0358 & 0.0173 & YES
\end{tabular}

Newman Keuls Test of Means on Mold Type of Parallelism Data (0.50 inch)

\begin{tabular}{lllll} 
ColName & Count & Mean & Std.Dev. Std.Err \\
\hline M1 & 18 & 0.0874 & 0.0274 & 0.0065 \\
M2 & 18 & 0.0663 & 0.0375 & 0.0088 \\
M3 & 18 & 0.0394 & 0.0114 & 0.0027 \\
\hline & & &
\end{tabular}

Newman-Keuls' Test

Significance Level: 0.05

\begin{tabular}{lllllll}
\multicolumn{3}{c}{ Variables } & Mean Diff | Critical Value | Significant \\
M1 & Vs. & M2 & 0.0211 & 0.0237 & NO \\
M1 & Vs. & M3 & 0.0479 & 0.0299 & YES \\
M2 & Vs. & M3 & 0.0268 & 0.0237 & YES
\end{tabular}

Newman Keuls Test of Means on Mold Type of Left Casting Angle Data (0.50 inch)

\begin{tabular}{lrrrr} 
ColName & \multicolumn{1}{c}{ Count } & Mean & Std.Dev. & Std.Err. \\
\hline M1 & 18 & 10.1086 & 0.0070 & 0.0016 \\
M2 & 18 & 10.1165 & 0.0010 & 0.0002 \\
M3 & 18 & 10.1175 & 0.0034 & 0.0008 \\
\hline & + & &
\end{tabular}

Newman-Keuls' Test

Significance Level: 0.05

\begin{tabular}{lccccc|c}
\multicolumn{3}{c|}{ Variables } & Mean Diff | Critical Value | Significant \\
M1 & Vs. & M2 & 0.0079 & 0.0039 & YES \\
M1 & Vs. & M3 & 0.0089 & 0.0049 & YES \\
M2 & Vs. & M3 & 0.0010 & 0.0039 & NO
\end{tabular}


Newman Keuls Test of Means on Gating of Right Flange Flatness Data (0.50 inch)

\begin{tabular}{lrrrr} 
ColName & \multicolumn{1}{c}{ Count } & Mean & Std.Dev. & Std.Err. \\
\hdashline G1 & 27 & 0.0221 & 0.0113 & 0.0022 \\
G2 & 27 & 0.0323 & 0.0294 & 0.0056 \\
\hline
\end{tabular}

Newman-Keuls' Test

Significance Level: 0.05

$$
\begin{array}{c|ccc}
\text { Variables | } & \text { Mean Diff | Critical Value | Significant } \\
\text { G1 Vs. G2 } & 0.0101 & 0.0156 \text { NO }
\end{array}
$$

\begin{tabular}{|c|c|c|c|c|}
\hline ColName & Count & Mean & Std.Dev. & Std.Err. \\
\hline M1 & 18 & 0.0124 & 0.0088 & 0.0021 \\
\hline M2 & 18 & 0.0407 & 0.0307 & 0.0072 \\
\hline M3 & 18 & 0.0284 & 0.0121 & 0.0029 \\
\hline
\end{tabular}

Newman Keuls Test of Means on Mold Type of Right Flange Flatness Data(0.50 inch)

Newman-Keuls' Test

Significance Level: 0.05

\begin{tabular}{lccccc}
\multicolumn{3}{c}{ Variables $\cdot \mid$} & Mean:Diff | Critical Value | Significant \\
M1 & Vs. & M2 & 0.0283 & 0.0214 & YES \\
M1 & Vs. & M3 & 0.0161 & 0.0169 & NO \\
M2 & Vs. & M3 & 0.0123 & 0.0169 & NO
\end{tabular}

Newman Keuls Test of Means on Gating of Left Wall Flatness Data(0.50 inch)

ColName Count Mean Std.Dev. Std.Err.

\begin{tabular}{lrrrr}
$-G 1$ & 27 & 0.0185 & 0.0089 & 0.0017 \\
G2 & 27 & 0.0306 & 0.0391 & 0.0075 \\
\hdashline & -
\end{tabular}

Newman-Keuls' Test

Significance Level: 0.05

$$
\begin{array}{cccc}
\text { Variables } & \text { Mean Diff | Critical Value | Significant } \\
\text { G1 Vs. G2 } & 0.0121 & 0.0199 & \text { NO }
\end{array}
$$


Newman Keuls Test of Means on Orientation of Left Wall Flatness Data(0.50 inch)

ColName Count Mean Std.Dev. Std.Err.

\begin{tabular}{lrrrr} 
O1 & 18 & 0.0352 & 0.0464 & 0.0109 \\
O2 & 18 & 0.0219 & 0.0134 & 0.0031 \\
O3 & 18 & 0.0166 & 0.0076 & 0.0018 \\
\hline
\end{tabular}

Newman-Keuls' Test

Significance Level: 0.05

\begin{tabular}{lll|ccl}
\multicolumn{3}{c|}{ Variables | } & Mean Diff | Critical Value | Significant \\
O1 & Vs. & O2 & 0.0133 & 0.0242 & NO \\
O1 & Vs. & O3 & 0.0186 & 0.0306 & NO \\
O2 & Vs. & O3 & $0.0053 \mid 0.0242$ & NO
\end{tabular}

Newman Keuls Test of Means on Mold Type of Left Wall Flatness Data(0.50 inch)

\begin{tabular}{llllll} 
ColName & Count & Mean & Std.Dev. Std.Err \\
\hline M1 & 18 & 0.0319 & 0.0095 & 0.0022 \\
M2 & 18 & 0.0106 & 0.0032 & 0.0008 \\
M3 & 18 & 0.0311 & 0.0466 & 0.0110 \\
\hline
\end{tabular}

Newman-Keuls' Test

Significance Level: 0.05

\begin{tabular}{llllll}
\multicolumn{3}{c|}{ Variables } & Mean Diff | Critical Value | Significant \\
M1 & Vs. & M2 & 0.0213 & 0.0299 & NO \\
M1 & Vs. & M3 & 0.0009 & 0.0236 & NO \\
M2 & Vs. & M3 & 0.0204 & 0.0236 & NO
\end{tabular}

Newman Keuls Test of Means on Mold Type of Right Wall Flatness Data(0.50 inch)

\begin{tabular}{lrrrrr} 
& ColName & Count & Mean & Std.Dev. Std.Err. \\
\hdashline M1 & 18 & 0.0316 & 0.0106 & 0.0025 \\
M2 & 18 & 0.0357 & 0.0202 & 0.0048 \\
M3 & 18 & 0.0164 & 0.0038 & 0.0009 & \\
\hdashline
\end{tabular}

Newman-Keuls' Test

Significance Level: 0.05

\begin{tabular}{lcl|cll}
\multicolumn{3}{c|}{ Variables } & Mean Diff | Critical Value | Significant \\
M1 & Vs. & M2 & 0.0041 & 0.0114 & NO \\
M1 & Vs. & M3 & 0.0152 & 0.0114 & YES \\
M2 & Vs. & M3 & 0.0193 & 0.0145 & YES
\end{tabular}


Newman Keuls Test of Means on Mold Type of Thickness Data(0.75 inch)

\begin{tabular}{lllll} 
ColName & Count & Mean & Std.Dev. Std.Err \\
\hdashline M1 & 18 & 0.8153 & 0.0266 & 0.0063 \\
M2 & 18 & 0.7661 & 0.0259 & 0.0061 \\
M3 & 18 & 0.7403 & 0.0043 & 0.0010 \\
\hline
\end{tabular}

Newman-Keuls' Test

Significance Level: 0.05

\begin{tabular}{lll|lll}
\multicolumn{3}{c}{ Variables } & Mean Diff | Critical Value | Significant \\
M1 & Vs. & M2 & 0.0492 & 0.0185 & YES \\
M1 & Vs. & M3 & 0.0750 & 0.0234 & YES \\
M2 & Vs. & M3 & 0.0257 & 0.0185 & YES
\end{tabular}

Newman Keuls Test of Means on Orientation of Height Data(0.75 inch)

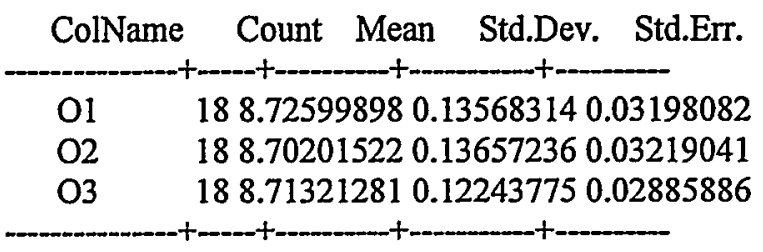

Newman-Keuls' Test

Significance Level: 0.05

\begin{tabular}{lllllll}
\multicolumn{3}{c}{ Variables } & Mean Diff | Critical Value | Significant \\
O1 & Vs. & O2 & 0.02398376 & 0.14281819 & NO \\
O1 & Vs. & O3 & 0.01278617 & 0.11301266 & NO \\
O2 & Vs. & O3 & 0.01119759 & 0.11301266 & NO
\end{tabular}

Newman Keuls Test of Means on Mold Type of Height Data( 0.75 inch)

ColName Count Mean Std.Dev. Std.Err.

$\begin{array}{lr}\text { M1 } & 188.891977780 .019201260 .00452578 \\ \text { M2 } & 188.631645340 .035187750 .00829383 \\ \text { M3 } & 188.617603900 .013849230 .00326430\end{array}$

Newman-Keuls' Test

Significance Level: 0.05

\begin{tabular}{lllllll}
\multicolumn{3}{c}{ Variables } & Mean Diff | Critical Value | Significant \\
M1 & Vs. & M2 & 0.26033244 & 0.02100777 & YES \\
M1 & Vs. & M3 & 0.27437388 & 0.02654828 & YES \\
M2 & Vs. & M3 & $\mathbf{0 . 0 1 4 0 4 1 4 4}$ & 0.02100777 & NO
\end{tabular}


Newman Keuls Test of Means on Mold Type of Length Data(0.75 inch)

\begin{tabular}{crr} 
ColName & Count Mean Std.Dev. Std.Err. \\
\hdashline M1 & 187.77364889 & 0.030227630 .00712472 \\
M2 & 186.471750560 .010335370 .00243607 \\
M3 & 186.436465330 .013330970 .00314214 \\
\hline & +
\end{tabular}

Newman-Keuls' Test

Significance Level: 0.05

\begin{tabular}{lllllll}
\multicolumn{3}{c}{ Variables } & Mean Diff | Critical Value | Significant \\
M1 & Vs. & M2 & 1.30189833 & 0.01714657 & YES \\
M1 & Vs. & M3 & 1.33718356 & 0.02166874 & YES \\
M2 & Vs. & M3 & 0.03528522 & 0.01714657 & YES
\end{tabular}

Newman Keuls Test of Means on Mold Type of Parallelism Data(0.75 inch)

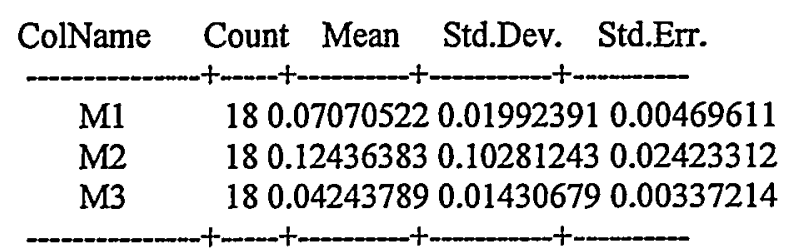

Newman-Keuls' Test

Significance Level: 0.05

\begin{tabular}{lll|lll}
\multicolumn{3}{c|}{ Variables } & Mean Diff | Critical Value | Significant \\
M1 & Vs. & M2 & 0.05365861 & 0.05235652 & YES \\
M1 & Vs. & M3 & 0.02826733 & 0.05235652 & NO \\
M2 & Vs. & M3 & 0.08192594 & 0.06616483 & YES
\end{tabular}

Newman Keuls Test of Means on Gating of Left Casting Angle Data(0.75 inch)

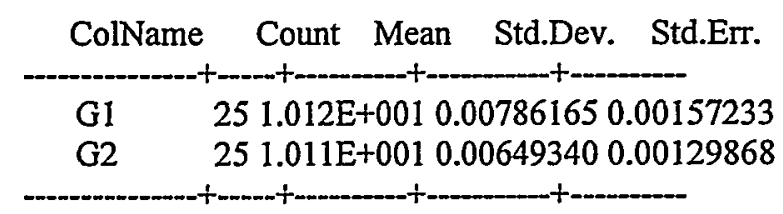

Newman-Keuls' Test

Significance Level: 0.05

$$
\begin{array}{ccccc}
\multicolumn{2}{c}{\text { Variables | Mean Diff | Critical Value | Significant }} \\
\text { G1 Vs. G2 } & 0.00369429 & 0.00412415 & \text { NO }
\end{array}
$$


Newman Keuls Test of Means on Mold Type of Left Casting Angle Data(0.75 inch)

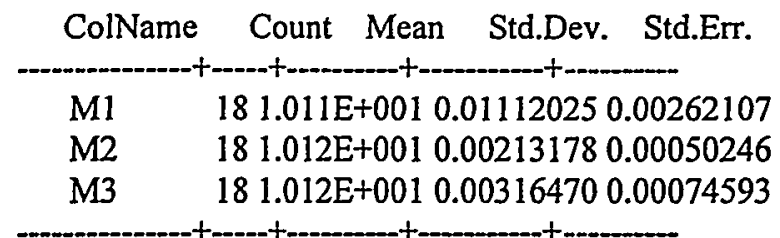

Newman-Keuls' Test

Significance Level: 0.05

\begin{tabular}{llllll}
\multicolumn{3}{c}{ Variables } & Mean Diff $=$ Critical Value | Significant \\
M1 & Vs. & M2 & 0.00456111 & 0.00582358 & NO \\
M1 & Vs. & M3 & 0.00574732 & 0.00735946 & NO \\
M2 & Vs. & M3 & 0.00118621 & 0.00582358 & NO
\end{tabular}

Newman Keuls Test of Means on Orientation of Right Casting Angle Data(0.75 inch)

ColName Count Mean Std.Dev. Std.Err.

$\begin{array}{lrl}\mathrm{O} 1 & 181.011 \mathrm{E}+0010.007777040 .00183307 \\ \mathrm{O} 2 & 181.012 \mathrm{E}+0010.009222930 .00217387 \\ \mathrm{O} 3 & 181.012 \mathrm{E}+0010.012096420 .00285115\end{array}$

Newman-Keuls' Test

Significance Level: 0.05

\begin{tabular}{lllllll}
\multicolumn{3}{c}{ Variables } & Mean Diff | Critical Value | Significant \\
O1 & Vs. & O2 & & 0.00125561 & 0.00846248 & NO \\
O1 & Vs. & O3 & 0.00768896 & 0.01069435 & NO \\
O2 & Vs. & O3 & & 0.00643335 & 0.00846248 & NO
\end{tabular}

Newman Keuls Test of Means on Mold Type of Right Casting Angle Data(0.75 inch)

\begin{tabular}{lllll} 
ColName & Count & Mean & Std.Dev. Std.Err. \\
\hdashline M1 & 18 & $1.012 \mathrm{E}+001$ & 0.01380946 & 0.00325492 \\
M2 & 18 & $1.011 \mathrm{E}+001$ & 0.00762451 & 0.00179711 \\
M3 & 18 & $1.011 \mathrm{E}+001$ & 0.00464524 & 0.00109489
\end{tabular}

Newman-Keuls' Test

Significance Level: 0.05

\begin{tabular}{lllllll}
\multicolumn{3}{c}{ Variables } & Mean Diff | Critical Value | Significant \\
M1 & Vs. & M2 & 0.00922778 & 0.01029377 & NO \\
M1 & Vs. & M3 & 0.00880602 & 0.00814550 & YES \\
M2 & Vs. & M3 & $\mathbf{0 . 0 0 0 4 2 1 7 5}$ & 0.00814550 & NO
\end{tabular}


Newman Keuls Test of Means on Orientation of Right Flange Flatness Data(0.75 inch)

\begin{tabular}{llll} 
ColName & Count Mean & Std.Dev. Std.Err. \\
\hline O1 & 180.060094270 .051371710 .01210843 \\
O2 & 180.117660730 .196800230 .04638626 \\
O3 & 180.037679070 .023046660 .00543215
\end{tabular}

Newman-Keuls' Test

Significance Level: 0.05

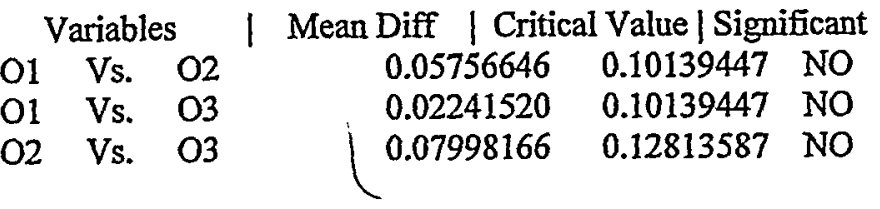

Newman Keuls Test of Means on Mold Type of Right Flange Flatness Data(0.75 inch)

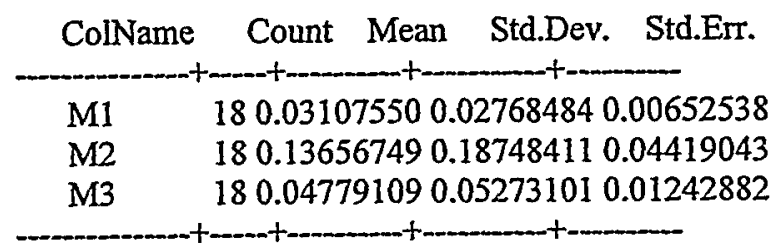

Newman-Keuls' Test

Significance Level: 0.05

\begin{tabular}{lcccccc}
\multicolumn{3}{c}{ Variables } & Mean Diff | Critical Value | Significant \\
M1 & Vs. & M2 & 0.10549199 & 0.12314061 & NO \\
M1 & Vs. & M3 & 0.01671560 & 0.09744170 & NO \\
M2 & Vs. & M3 & 0.08877639 & 0.09744170 & NO
\end{tabular}

Newman Keuls Test of Means on Mold Type of Left Wall Flatness Data(0.75 inch)

ColName Count Mean Std.Dev. Std.Err.

$\begin{array}{lll}M 1 & 180.030369070 .014973230 .00352923 \\ M 2 & 180.016434610 .007178420 .00169197 \\ M 3 & 180.019116880 .004150480 .00097828\end{array}$

Newman-Keuls: Test

Significance Level: 0.05

\begin{tabular}{ccc|ccc}
\multicolumn{3}{c}{ Variables } & Mean Diff | Critical Value | Significant \\
M1 & Vs. & M2 & 0.01393446 & 0.01071422 & YES \\
M1 & Vs. & M3 & 0.01125219 & 0.00847821 & YES \\
M2 & Vs. & M3 & 0.00268227 & 0.00847821 & NO
\end{tabular}


Newman Keuls Test of Means on Mold Type of Right Wall Flatness Data ( 0.75 inch)

ColName Count Mean Std.Dev. Std.Err.

$\begin{array}{ll}\text { M1 } & 180.035758410 .028147530 .00663444 \\ \text { M2 } & 180.069483640 .043578280 .01027150 \\ M 3 & 180.016614180 .004332210 .00102111\end{array}$

Newman-Keuls' Test

Significance Level: 0.05

\begin{tabular}{lll|lll}
\multicolumn{3}{c|}{ Variables } & Mean Diff | Critical Value | Significant \\
M1 & Vs. & M2 & 0.03372522 & 0.02578687 & YES \\
M1 & Vs. & M3 & 0.01914424 & 0.02578687 & NO \\
M2 & Vs. & M3 & 0.05286946 & 0.03258780 & YES
\end{tabular}

Newman Keuls Test of Means on Gating for Lost Foam Silica Sand Height Data(0.50 inch)

ColName Count Mean Std.Dev. Std.Err.

\begin{tabular}{lrrrr} 
G1 & 9 & 8.6051 & 0.0059 & 0.0020 \\
G2 & 9 & 8.5997 & 0.0067 & 0.0022 \\
\hline
\end{tabular}

Newman-Keuls' Test

Significance Level: 0.05

$\begin{array}{ccc}\text { Variables } \\ \text { G1 Vs. G2 } & 0.0054 \quad 0.0076 \text { No No }\end{array}$

Newman Keuls Test of Means on Orientation for Lost Foam Silica Sar Left Casting Angle Data (0.50 inch)

\begin{tabular}{lrrrrr} 
ColName & \multicolumn{2}{c}{ Count } & Mean & Std.Dev. Std.Err. \\
\hline O1 & 6 & 10.1178 & 0.0037 & 0.0015 \\
O2 & 6 & 10.1198 & 0.0028 & 0.0011 \\
O3 & 6 & 10.1148 & 0.0014 & 0.0006 \\
\hline
\end{tabular}

Newman-Keuls' Test

Significance Level: 0.05

\begin{tabular}{lll|cc|c}
\multicolumn{3}{c}{ Variables | } & Mean Diff | Critical Value | Significant \\
$\mathrm{O} 1$ & Vs. & O2 & 0.0020 & 0.0000 & YES \\
$\mathrm{O} 1$ & Vs. & $\mathrm{O} 3$ & 0.0030 & 0.0000 & YES \\
$\mathrm{O} 2$ & Vs. & $\mathrm{O} 3$ & 0.0050 & 0.0000 & YES
\end{tabular}


Newman Keuls Test of Means on Gating for Lost Foam Silica Sand Thickness Data ( 0.75 inch)

ColName Count Mean Std.Dev. Std.Err.

\begin{tabular}{lrrrr}
- & 9 & 0.7423 & 0.0037 & 0.0012 \\
G2 & 9 & 0.7384 & 0.0040 & 0.0013 \\
\hdashline
\end{tabular}

Newman-Keuls' Test

Significance Level: 0.05

$$
\begin{aligned}
& \text { Variables | Mean Diff | Critical Value | Significant } \\
& \text { G1 Vs. G2 } \\
& \text { V. } 0.0039 \quad 0.0000 \text { YES }
\end{aligned}
$$

Newman Keuls Test of Means on Orientation for Lost Foam Silica Sand Height Data (0.75 inch)

\begin{tabular}{lrrrr} 
ColName & \multicolumn{2}{c}{ Count } & Mean Std.Dev. Std \\
\hline O1 & 6 & 8.6205 & 0.0125 & 0.0051 \\
O2 & 6 & 8.6061 & 0.0143 & 0.0058 \\
O3 & 6 & 8.6261 & 0.0060 & 0.0025 \\
\hline
\end{tabular}

Newman-Keuls' Test

Significance Level: 0.05

\begin{tabular}{lcc|ccc}
\multicolumn{3}{c}{ Variables } & Mean Diff | Critical Value | Significant \\
O1 & Vs. & O2 & 0.0144 & 0.0000 & YES \\
O1 & Vs. & O3 & 0.0056 & 0.0000 & YES \\
O2 & Vs. & O3 & 0.0200 & 0.0000 & YES
\end{tabular}

Newman Keuls Test of Means on Orientation for Lost Foam Silica Sand Length Data (0.75 inch)

\begin{tabular}{crrrr} 
ColName & Count & Mean & Std.Dev. Std.Err. \\
\hline O1 & 6 & 6.4402 & 0.0067 & 0.0027 \\
$\mathrm{O} 2$ & 6 & 6.4242 & 0.0162 & 0.0066 \\
$\mathrm{O} 3$ & 6 & 6.4450 & 0.0032 & 0.0013 \\
\hline
\end{tabular}

Newman-Keuls' Test

Significance Level: 0.05

\begin{tabular}{lcc|ccc}
\multicolumn{3}{c}{ Variables } & Mean Diff | Critical Value | Significant \\
$\mathrm{O} 1$ & Vs. & O2 & 0.0160 & 0.0000 & YES \\
$\mathrm{O} 1$ & Vs. & O3 & 0.0049 & 0.0000 & YES \\
$\mathrm{O} 2$ & Vs. & O3 & 0.0209 & 0.0000 & YES \\
\hline
\end{tabular}


Newman Keuls Test of Means on Orientation for Lost Foam Silica Sand Parallelism Data (0.75 inch)

ColName Count Mean Std.Dev. Std.Err.

\begin{tabular}{rrrrr} 
& 6 & 0.0380 & 0.0090 & 0.0037 \\
O1 & 6 & 0.0581 & 0.0102 & 0.0042 \\
O3 & 6 & 0.0312 & 0.0064 & 0.0026 \\
\hline
\end{tabular}

Newman-Keuls' Test

Significance Level: 0.05

\begin{tabular}{lcc|cc|c}
\multicolumn{3}{c}{ Variables | } & Mean Diff | Critical Value | Significant \\
O1 & Vs. & O2 & 0.0201 & 0.0000 & YES \\
O1 & Vs. & O3 & 0.0068 & 0.0000 & YES \\
O2 & Vs. & O3 & 0.6269 & 0.0000 & YES
\end{tabular}

Newman Keuls Test of Means on Gating for Lost Foam Silica Sand

Right Casting Angle Data (0.75 inch)

Newman-Keuls' Test

Significance Level: 0.05

$$
\begin{array}{c|cc|c|c|c|}
\multicolumn{2}{c}{\text { Variables | Mean Diff | Critical Value | Significant }} \\
\text { G1 Vs. G2 } & 0.0056 & 0.0000 & \text { YES }
\end{array}
$$

\section{Newman Keuls Test of Means on Gating for Lost Foam Silica Sand Thickness Data (all)}

ColName Count Mean Std.Dev. Std.Err.

\begin{tabular}{lllll} 
G1 & 27 & 0.4949 & 0.2094 & 0.0403 \\
G2 & $27 \mid$ & 0.5127 & 0.1889 & 0.0364 \\
\hdashline
\end{tabular}

Newman-Keuls' Test

Significance Level: 0.05

$$
\begin{array}{c|c|c|c|c|c}
\multicolumn{2}{c}{\text { Variables | Mean Diff | Critical Value | Significant }} \\
\text { G1 Vs. G2 } & 0.0178 \cdot 0.0000 \text { YES } \\
\hline
\end{array}
$$




\section{Newman Keuls Test of Means on Orientation for Lost Foam Silica Sand}

Thickness Data (all)

\begin{tabular}{lrrrr} 
ColName & Count & Mean & Std.Dev. Std.Err. \\
\hline O1 & 18 & 0.4953 & 0.2094 & 0.0494 \\
O2 & 18 & 0.4946 & 0.2103 & 0.0496 \\
O3 & 18 & 0.5214 & 0.1825 & 0.0430 \\
\hline & - & &
\end{tabular}

Newman-Keuls' Test

Significance Level: 0.05

\begin{tabular}{lccccc}
\multicolumn{2}{c}{ Variables | } & Mean Diff | Critical Value | Significant \\
$\mathrm{O} 1$ & Vs. & O2 & 0.0007 & 0.0000 & YES \\
$\mathrm{O} 1$ & Vs. & $\mathrm{O} 3$ & 0.0261 & 0.0000 & YES \\
$\mathrm{O} 2$ & Vs. & $\mathrm{O} 3$ & 0.0268 & 0.0000 & YES
\end{tabular}

\section{Newman Keuls Test of Means on Thickness for Lost Foam Silica Sand Thickness Data (all)}

\begin{tabular}{lrrrrr} 
& ColName & Count & Mean & Std.De \\
\hline T1 & 18 & 0.7403 & 0.0043 & 0.0010 \\
T2 & 18 & 0.5020 & 0.0056 & 0.0013 \\
T3 & 18 & 0.2691 & 0.0654 & 0.0154 \\
\hline
\end{tabular}

Newman-Keuls' Test

Significance Level: 0.05

\begin{tabular}{lcc|cc|c}
\multicolumn{3}{c}{ Variables | } & Mean Diff | Critical Value | Significant \\
T1 & Vs. & T2 & 0.2384 & 0.0000 & YES \\
T1 & Vs. & T3 & 0.4712 & 0.0000 & YES \\
T2 & Vs. & T3 & 0.2329 & 0.0000 & YES \\
\hline
\end{tabular}

Newman Keuls Test of Means on Orientation for Lost Foam Silica Sand Height Data (all)

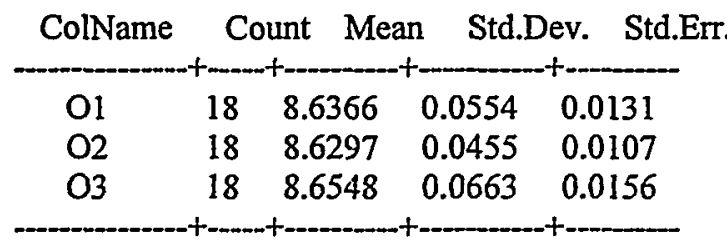

Newman-Keuls' Test

Significance Level: 0.05

\begin{tabular}{lcc|ccc}
\multicolumn{3}{c}{ Variables | } & Mean Diff | Critical Value | Significant \\
O1 & Vs. & O2 & 0.0069 & 0.0000 & YES \\
O1 & Vs. & O3 & 0.0183 & 0.0000 & YES \\
O2 & Vs. & O3 & 0.0251 & 0.0000 & YES \\
\hline
\end{tabular}




\section{Newman Keuls Test of Means on Thickness for Lost Foam Silica Sand \\ Height Data (all)}

ColName Count Mean Std.Dev. Std.Err.

\begin{tabular}{lrrrr}
$\mathrm{T} 1$ & 18 & 8.6176 & 0.0138 & 0.0033 \\
$\mathrm{~T} 2$ & 18 & 8.6024 & 0.0067 & 0.0016 \\
$\mathrm{~T} 3$ & 18 & 8.7012 & 0.0606 & 0.0143 \\
\hdashline
\end{tabular}

Newman-Keuls' Test

Significance Level: 0.05

\begin{tabular}{lcc|ccc}
\multicolumn{3}{c}{ Variables } & Mean Diff | Critical Value | Significant \\
T1 & Vs. & T2 & 0.0152 & 0.0000 & YES \\
T1 & Vs. & T3 & 0.0836 & 0.0000 & YES \\
T2 & Vs. & T3 & 0.0988 & 0.0000 & YES \\
\hline
\end{tabular}

\section{Newman Keuls Test of Means on Orientation for Lost Foam Silica Sand \\ Length Data (all)}

Newman-Keuls' Test

\begin{tabular}{lrrrr} 
ColName & Count & Mean & Std.Dev. & Std.Err. \\
\hline O1 & 18 & 6.3741 & 0.1607 & 0.0379 \\
O2 & 18 & 6.4754 & 0.1528 & 0.0360 \\
O3 & 18 & 6.3853 & 0.1281 & 0.0302 \\
\hline
\end{tabular}

Significance Level: 0.05

\begin{tabular}{llllll}
\multicolumn{3}{c}{ Variables } & Mean Diff | Critical Value | Significant \\
$\mathrm{O} 1$ & Vs. & O2 & 0.1013 & 0.0000 & YES \\
$\mathrm{O} 1$ & Vs. & O3 & 0.0111 & 0.0000 & YES \\
$\mathrm{O} 2$ & Vs. & O3 & 0.0901 & 0.0000 & YES \\
\hline
\end{tabular}

Newman Keuls Test of Means on Thickness for Lost Foam Silica Sand

Parallelism Data (all)

ColName Count Mean Std.Dev. Std.Err.

\begin{tabular}{|c|c|c|c|c|}
\hline $\mathrm{T} 1$ & 18 & 0.0424 & 0.0143 & 0.0034 \\
\hline$T 2$ & 18 & 0.0394 & 0.0114 & 0.0027 \\
\hline T3 & 18 & 0.2922 & 0.3042 & 0.0717 \\
\hline
\end{tabular}

Newman-Keuls' Test

Significance Level: 0.05

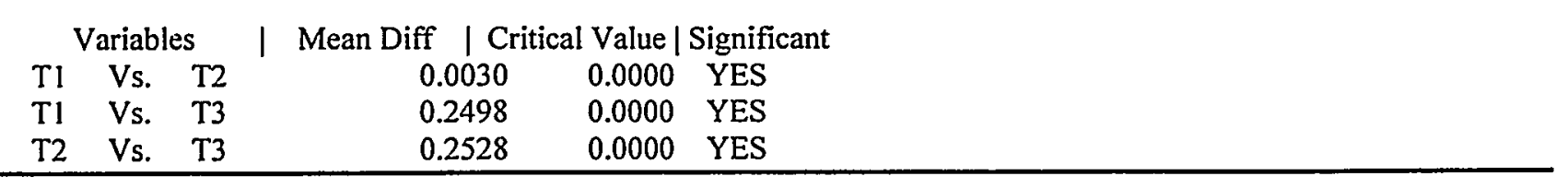




\section{Newman Keuls Test of Means on Gating for Lost Foam Silica Sand}

Parallelism Data (all)

ColName Count Mean Std.Dev. Std.Err.

\begin{tabular}{lrrrr} 
& 18 & 10.1163 & 0.0032 & 0.0007 \\
$\mathrm{~T} 1$ & 18 & 10.1175 & 0.0034 & 0.0008 \\
$\mathrm{~T} 3$ & 18 & 10.1192 & 0.0075 & 0.0018 \\
\hline
\end{tabular}

Newman-Keuls' Test

Significance Level: 0.05

\begin{tabular}{|c|c|c|c|}
\hline \multicolumn{2}{|c|}{ Variables } & Mean Diff & I Value | Significant \\
\hline $\mathrm{T} 1$ & Vs. T2 & 0.0012 & $0.0000 \mid$ YES \\
\hline T1 & Vs. & 0.0028 & 0.0000 YES \\
\hline $\mathrm{T} 2$ & Vs. & 0.0017 & 0.0000 \\
\hline
\end{tabular}

\section{Newman Keuls Test of Means on Thickness for Lost Foam Silica Sand} Left Casting Angle Data (all)

\begin{tabular}{cccccc} 
ColName & Count & Mean & Std.Dev. & Std.Err. \\
\hdashline T1 & 18 & 10.1163 & 0.0032 & 0.0007 \\
T2 & 18 & 10.1175 & 0.0034 & 0.0008 \\
T3 & 18 & 10.1192 & 0.0075 & 0.0018 \\
\hline \\
\hdashline
\end{tabular}

Newman Keuls Test of Means on Orientation for Lost Foam Silica Sand Right Casting Angle Data (all)

\begin{tabular}{|c|c|c|c|c|}
\hline ColName & Count & Mean & Std.Deqv. & Std.Err \\
\hline 01 & 18 & 10.1548 & 0.0623 & 0.0147 \\
\hline $\mathrm{O} 2$ & 18 & 10.1428 & 0.0484 & 0.0114 \\
\hline $\mathrm{O} 3$ & 18 & 10.1421 & 0.0421 & 0.0099 \\
\hline
\end{tabular}

Newman-Keuls' Test

Significance Level: 0.05

\begin{tabular}{lcc|ccc}
\multicolumn{3}{c}{ Variables | } & Mean Diff | Critical Value | Significant \\
O1 & Vs. & O2 & 0.0120 & 0.0000 & YES \\
O1 & Vs. & O3 & 0.0127 & 0.0000 & YES \\
O2 & Vs. & O3 & 0.0007 & 0.0000 & YES \\
\hline
\end{tabular}




\section{Newman Keuls Test of Means on Thickness for Lost Foam Silica Sand}

Right Casting Angle Data (all)

\begin{tabular}{lrrrr}
\multicolumn{2}{c}{ ColName } & \multicolumn{1}{c}{ Count } & Mean & Std.Dev. Std. \\
\hdashline T1 & 18 & 10.1143 & 0.0046 & 0.0011 \\
T2 & 18 & 10.1188 & 0.0056 & 0.0013 \\
T3 & 18 & 10.2066 & 0.0479 & 0.0113 \\
\hline
\end{tabular}

Newman-Keuls' Test

Significance Level: 0.05

\begin{tabular}{|c|c|c|c|c|c|}
\hline \multicolumn{3}{|c|}{ Variables } & Mean Diff | Cri & Value & ignificant \\
\hline $\mathrm{T} 1$ & Vs. & $\mathrm{T} 2$ & 0.0045 & 0.0000 & YES \\
\hline $\mathrm{T} 1$ & Vs. & T3 & 0.0924 & 0.0000 & YES \\
\hline $\mathrm{T} 2$ & Vs. & T3 & 0.0879 & 0.0000 & YES \\
\hline
\end{tabular}

Newman Keuls Test of Means on Orientation for Lost Foam Silica Sand

Left Flange Flatness Data (all)

ColName Count Mean Std.Dev. Std.Err.

Keuls' Test

Significance Level: 0.05

\begin{tabular}{lll|ccl}
\multicolumn{3}{c}{ Variables | } & Mean Diff | Critical Value | Significant \\
O1 & Vs. & O2 & 0.0073 & 0.0055 & YES \\
O1 & Vs. & O3 & 0.0038 & 0.0044 & NO \\
O2 & Vs. & O3 & 0.0036 & 0.0044 & NO
\end{tabular}

Newman Keuls Test of Means on Orientation for Lost Foam Silica Sand Right Flange Flatness Data (all)

\begin{tabular}{lrrrrr} 
ColName & \multicolumn{2}{c}{ Count } & Mean & Std.Dev. Std.Err. \\
\hline $\mathrm{O} 1$ & 18 & 0.0446 & 0.0316 & 0.0074 \\
$\mathrm{O} 2$ & 18 & 0.0621 & 0.0638 & 0.0150 \\
$\mathrm{O} 3$ & 18 & 0.0402 & 0.0290 & 0.0068 \\
\hline
\end{tabular}

Newman-Keuls' Test

Significance Level: 0.05

\begin{tabular}{lcc|ccc}
\multicolumn{3}{c}{ Variables } & Mean Diff | Critical Value | Significant \\
O1 & Vs. & O2 & 0.0174 & 0.0381 & NO \\
O1 & Vs. & O3 & 0.0044 & 0.0381 & NO \\
O2 & Vs. & O3 & 0.0218 & 0.0481 & NO \\
\hline
\end{tabular}


Newman Keuls Test of Means on Orientation for Lost Foam Silica Sand Left Flange Flatness Data (0.75 inch)

ColName Count Mean Std.Dev. Std.Err.

\begin{tabular}{rrrrr} 
O1 & 6 & 0.0071 & 0.0022 & 0.0009 \\
O2 & 6 & 0.0139 & 0.0085 & 0.0035 \\
O3 & 6 & 0.0100 & 0.0037 & 0.0015 \\
\hline
\end{tabular}

Newman-Keuls' Test

Significance Level: 0.05

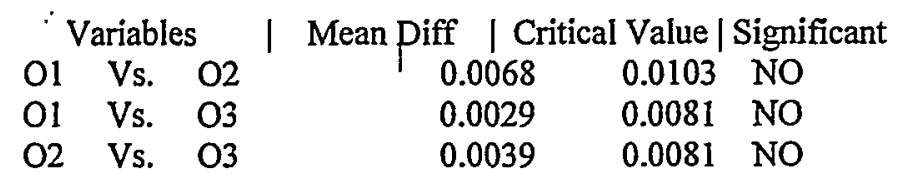

Newman Keuls Test of Means on Orientation for Lost Foam Silica Sand Left Wall Flatness Data (0.75 inch)

\begin{tabular}{lrrrr} 
& ColName & Count & Mean & Std.De \\
\hline O1 & 6 & 0.0200 & 0.0048 & 0.0020 \\
O2 & 6 & 0.0177 & 0.0027 & 0.0011 \\
O3 & 6 & 0.0197 & 0.0049 & 0.0020 \\
\hline
\end{tabular}

Newman-Keuls' Test

Significance Level: 0.05

\begin{tabular}{lll|ccc}
\multicolumn{3}{c}{ Variables | } & Mean Diff | Critical Value | Significant \\
O1 & Vs. & O2 & 0.0023 & 0.0080 & NO \\
O1 & Vs. & O3 & 0.0003 & 0.0064 & NO \\
O2 & Vs. & O3 & 0.0020 & 0.0064 & NO
\end{tabular}

\begin{tabular}{llllll}
\hline \multicolumn{6}{c}{ Newman Keuls Test of Means on Orientation for Lost Foam Silica Sand } \\
Right Flange Flatness Data (0.50 inch)
\end{tabular}


Newman Keuls Test of Means on Thickness for Lost Foam Silica Sand

Right Flange Flatness Data (all)

\begin{tabular}{lllll} 
ColName & Count & Mean Std.Dev. Std \\
\hdashline T1 & 18 & 0.0477 & 0.0528 & 0 \\
T2 & 18 & 0.0296 & 0.0114 & 0.0027 \\
T3 & 18 & 0.0707 & 0.0481 & 0.0113 \\
\hline
\end{tabular}

Newman-Keuls' Test

Significance Level: 0.05

\begin{tabular}{lcc|ccc}
\multicolumn{3}{c|}{ Variables } & Mean Diff | Critical Value | Significant \\
T1 & Vs. & T2 & 0.0182 & 0.0358 & NO \\
T1 & Vs. & T3 & 0.0229 & 0.0358 & NO \\
T2 & Vs. & T3 & 0.0411 & 0.0452 & NO
\end{tabular}

Newman Keuls Test of Means on Thickness for Lost Foam Silica Sand

Left Wall Flatness Data (all)

\begin{tabular}{|c|c|c|c|c|}
\hline & ColName & Count & Mean & Std.Dev. \\
\hline $\mathrm{T} 1$ & 18 & 0.0191 & 0.0042 & 0.0010 \\
\hline $\mathrm{T} 2$ & 18 & 0.0311 & 0.0466 & 0.0110 \\
\hline T3 & 18 & 0.0111 & 0.0034 & 0.0008 \\
\hline
\end{tabular}

Newman-Keuls' Test

Significance Level: 0.05

\begin{tabular}{lcc|cc|c}
\multicolumn{3}{c}{ Variables } & Mean Diff | Critical Value | Significant \\
T1 & Vs. & T2 & 0.0119 & 0.0232 & NO \\
T1 & Vs. & T3 & 0.0080 & 0.0232 & NO \\
T2 & Vs. & T3 & 0.0200 & 0.0294 & NO \\
\hline
\end{tabular}

Newman Keuls Test of Means on Thickness for Lost Foam Silica Sand

Right Wall Flatness Data (all)

\begin{tabular}{lllll} 
ColName & Count & Mean & Std.Dev. Std.Err. \\
\hdashline T1 & 18 & 0.0166 & 0.0043 & 0.0010 \\
T2 & 18 & 0.0164 & 0.0038 & 0.0009 \\
T3 & 18 & 0.0293 & 0.0218 & 0.0051 \\
\hline & - & - & -
\end{tabular}

Newman-Keuls' Test

Significance Level: 0.05

\begin{tabular}{llllll}
\multicolumn{3}{c}{ Variables } & | Mean Diff | Critical Value | Significant \\
$\mathrm{T} 1$ & Vs. & T2 & 0.0002 & 0.0112 & NO \\
T1 & Vs. & T3 & 0.0127 & 0.0112 & YES \\
T2 & Vs. & T3 & 0.0129 & 0.0141 & NO \\
\hline
\end{tabular}




\section{Newman Keuls Test of Means on Gating for Lost Foam Silica Sand}

Right Flange Flatness Data (all)

ColName Count Mean Std.Dev. Std.Err.

\begin{tabular}{|c|c|c|c|c|}
\hline Gl & 27 & 0.0452 & 0.0474 & 0.0091 \\
\hline G2 & 27 & 0.0527 & 0.0421 & 0.0081 \\
\hline
\end{tabular}

Newman-Keuls' Test

Significance Level: 0.05

\begin{tabular}{c|ccc}
\multicolumn{2}{c}{ Variables } \\
G1 Vs. G2 & 0.0075 & 0.0314 & No
\end{tabular}

Newman Keuls Test of Means on Gating for Lost Foam Silica Sand

Right Flange Flatness Data (0.50 inch)

\begin{tabular}{lrrrrr} 
ColName & Count & Mean & Std.Dev. & Std.Err. \\
\hdashline G1 & 9 & 0.0270 & 0.0096 & 0.0032 \\
G2 & 9 & 0.0299 & 0.0147 & 0.0049 \\
\hline
\end{tabular}

Newman-Keuls' Test

Significance Level:.0.05:-: :-

Variables_. I Mean:Diff: | Critical:Valne.| Significant
G. Vs: G2
0.0029 .
0.0151 NO 
APPENDIX C.

(Bullseye Plots)

$\therefore$

$\therefore$

1

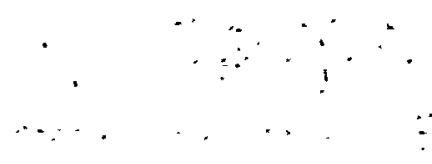




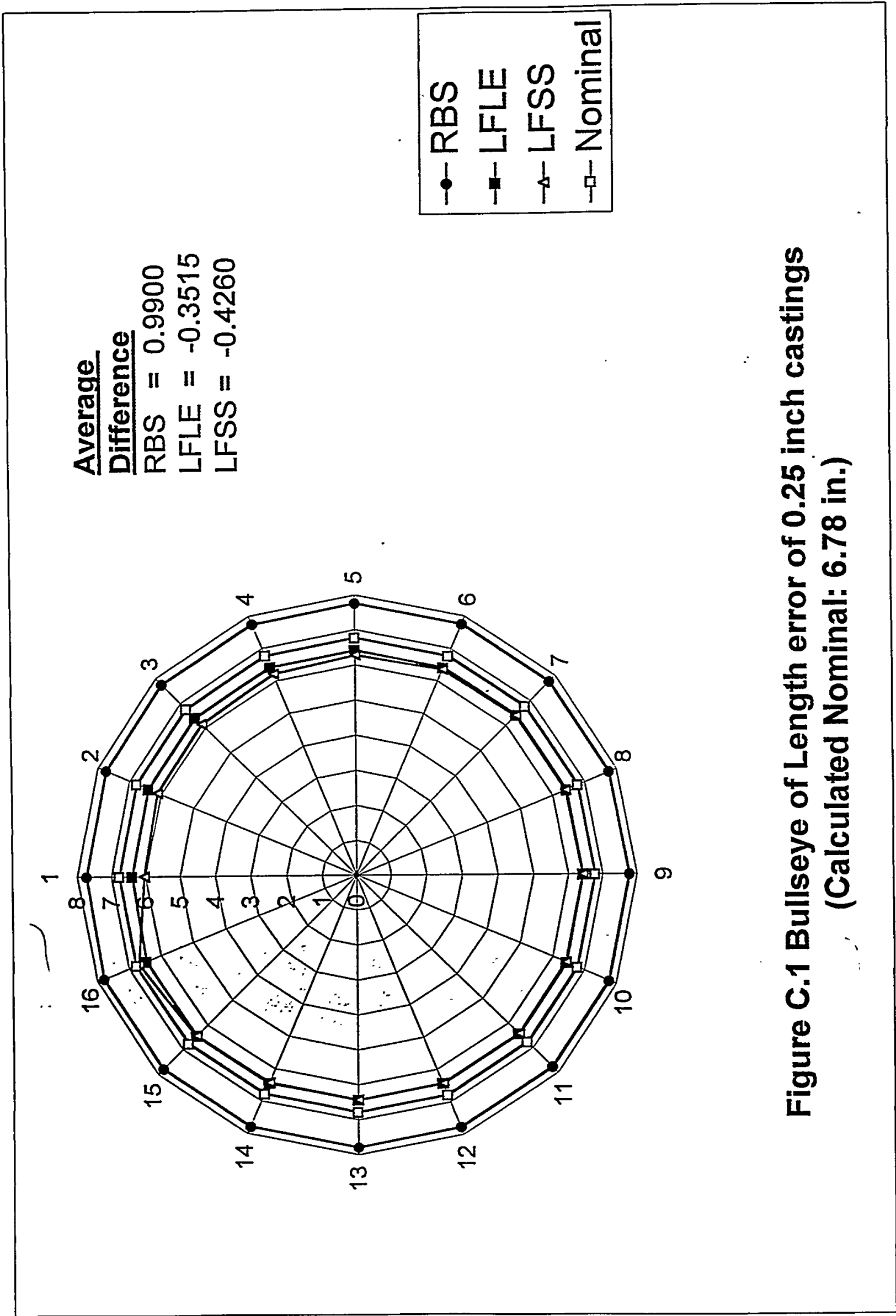




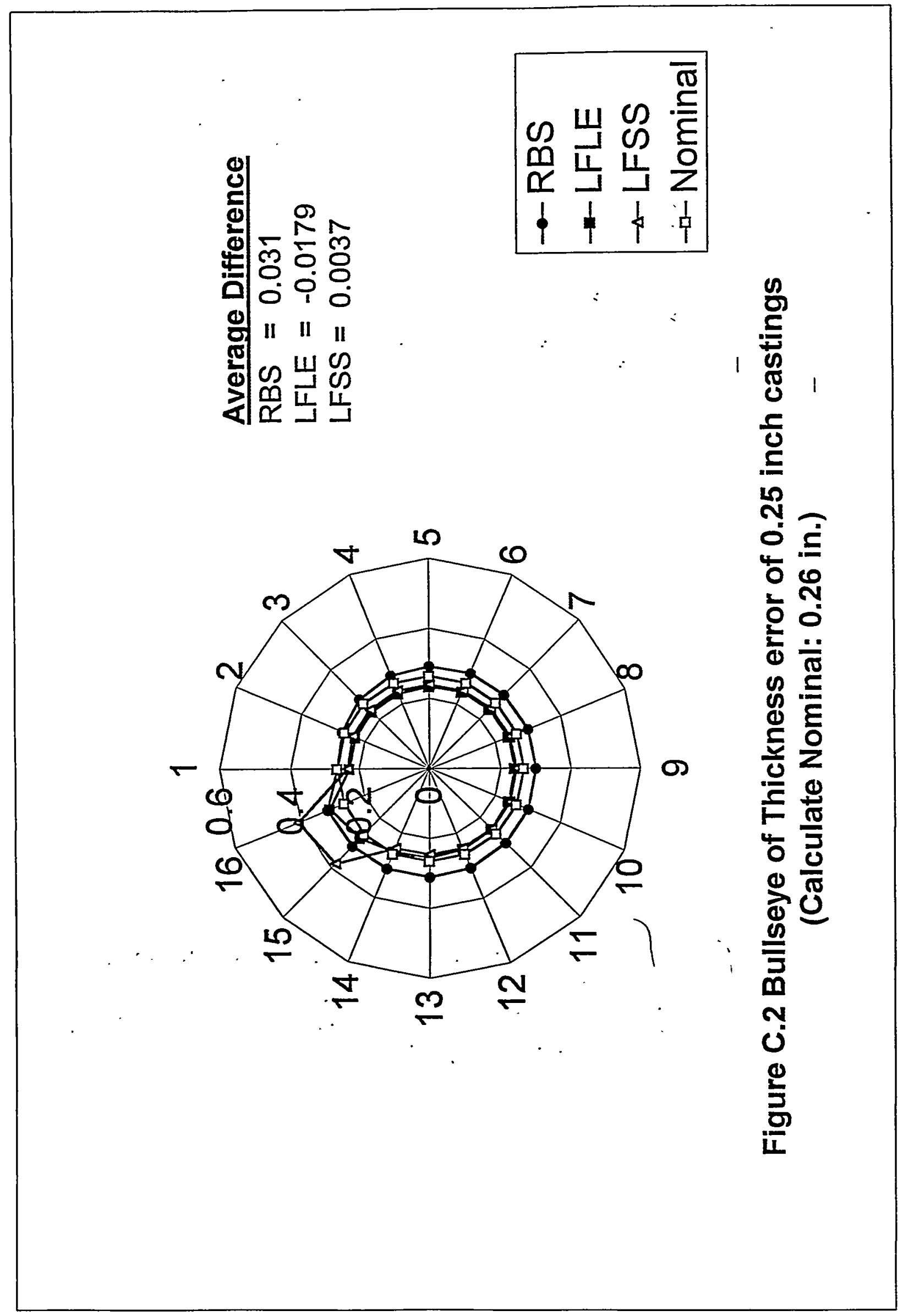




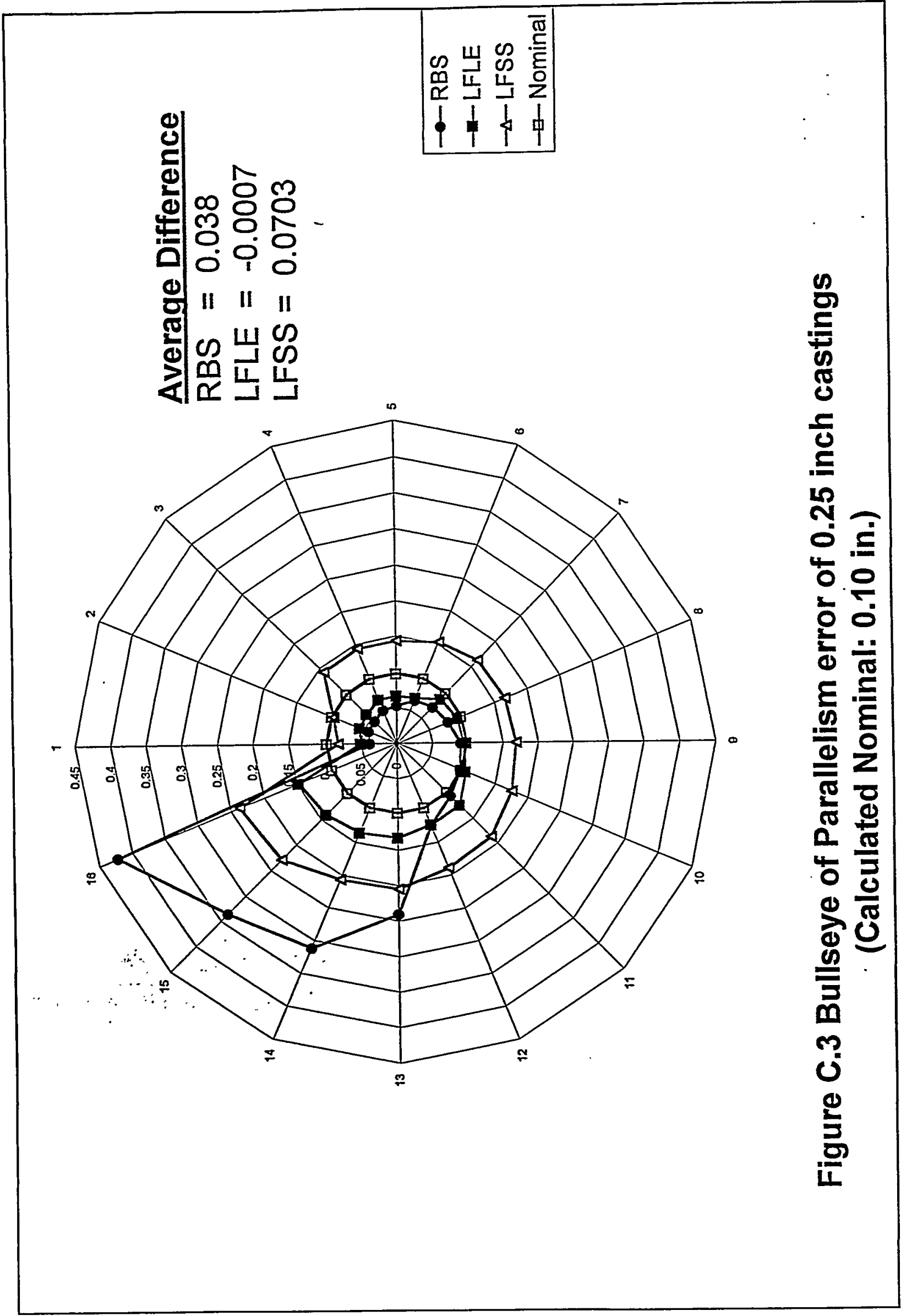




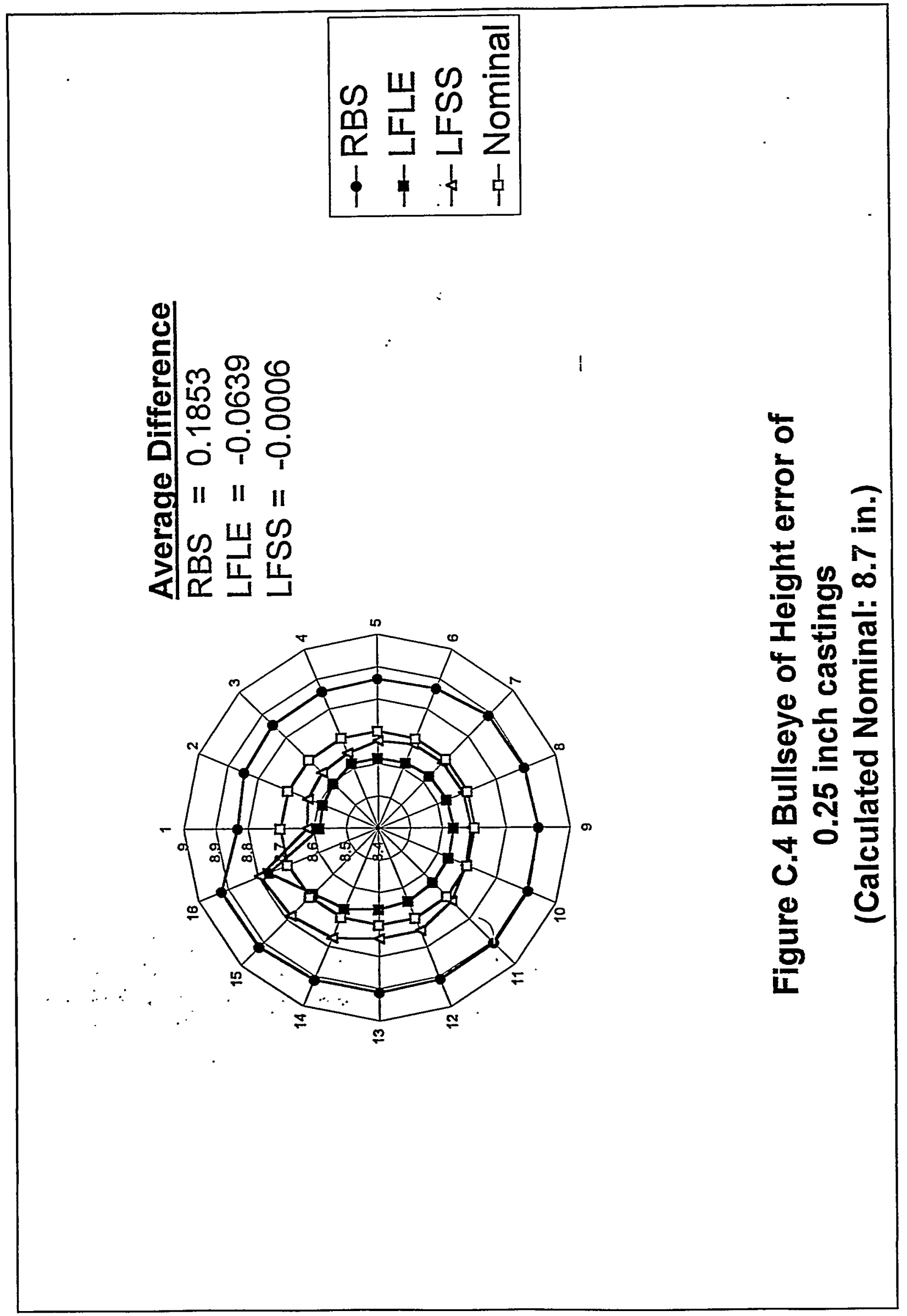




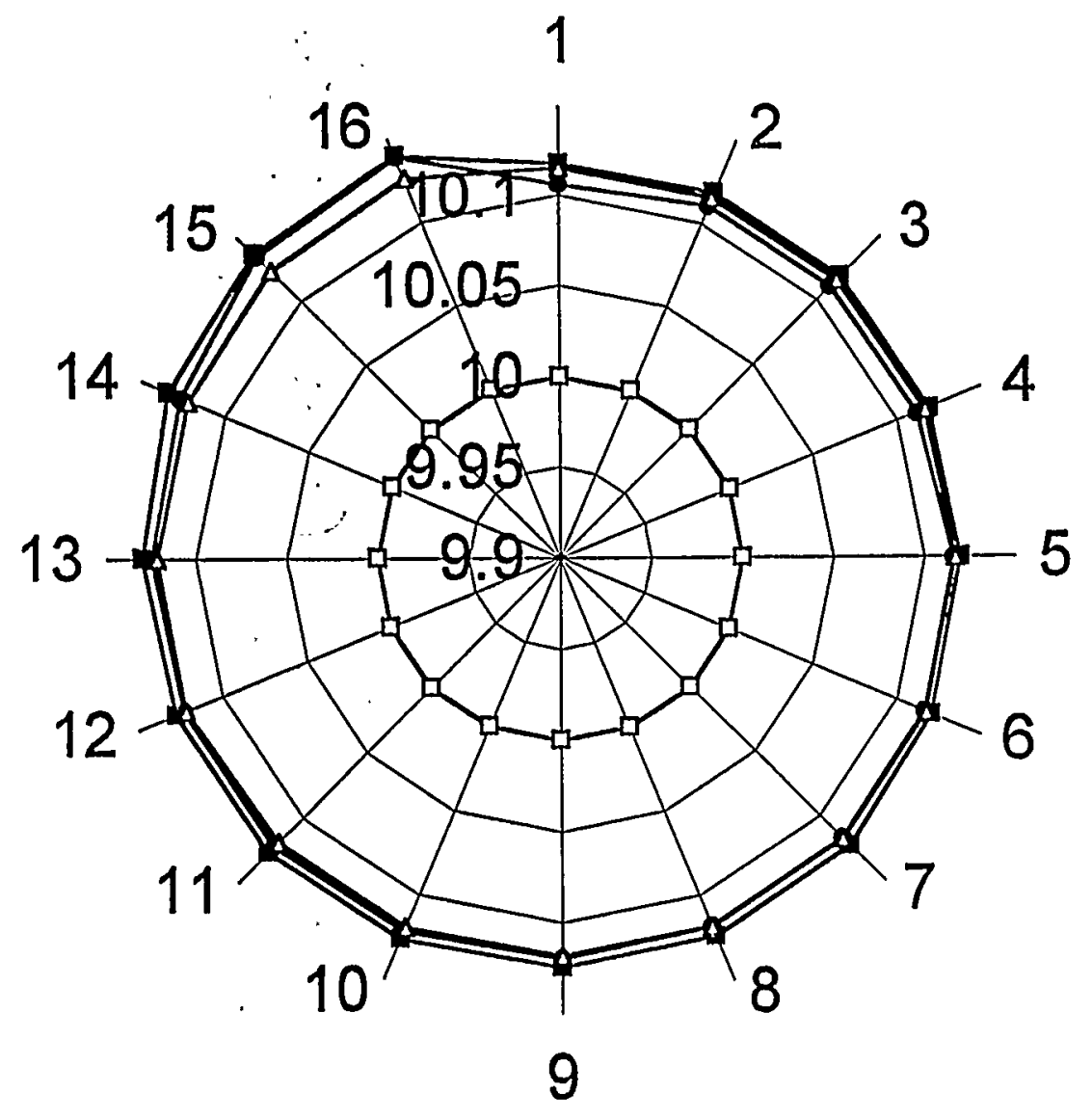

Average

Difference

RBS $=0: 1201$

LFLE $=0.1255$

LFSS $=0.1193$

Figure C.5 Bullseye of Left Casting Angle error of 0.25 inch castings

\section{(Calculated Nominal: 10 degrees)}




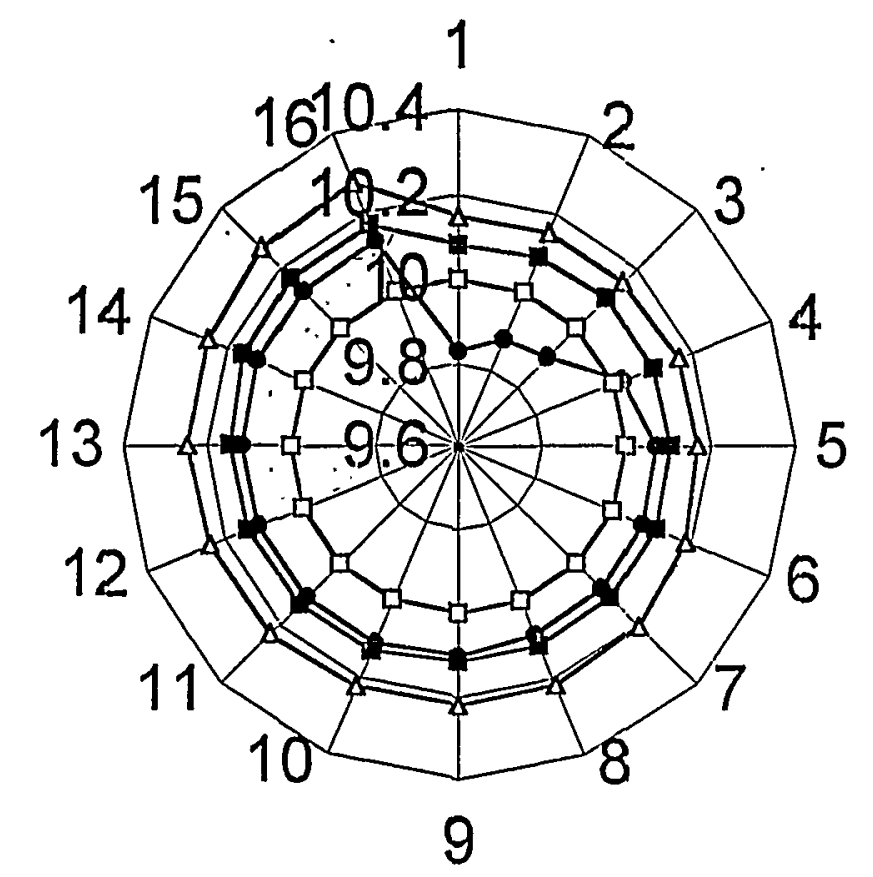

\section{Average Difference}

RBS $=0.0545$

LFLE $=0.1228$

LFSS $=0.2091$

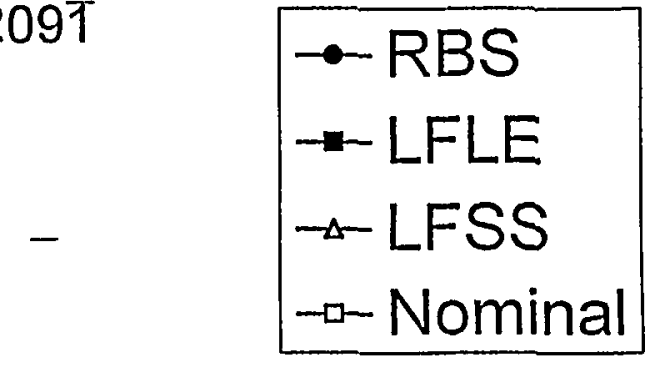

Figure C. 6 Bullseye of Right Casting Angle error of

0.25 inch castings (Calculated Nominal: 10 degrees) 


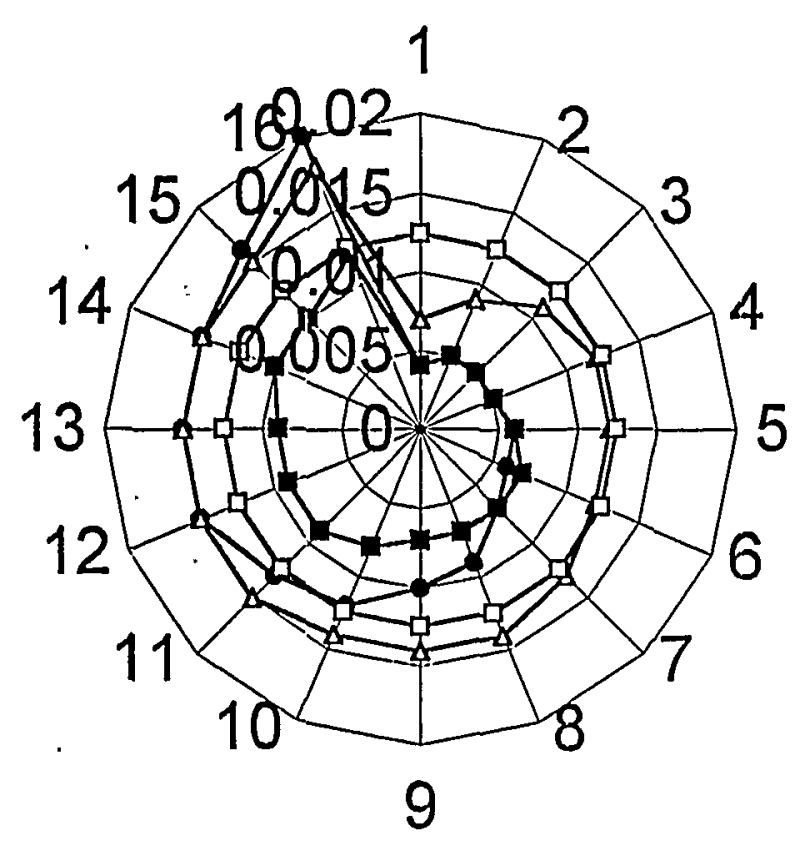

$$
\begin{aligned}
& \text { Average Difference } \\
& \text { RBS }=-0.00224 \\
& \text { LFLE }=-0.0049 \\
& \text { LFSS }=0.00076
\end{aligned}
$$$$
\rightarrow \text { RBS }
$$$$
\rightarrow \text { LFLE }
$$$$
\rightarrow-\text { LFSS }
$$$$
\rightarrow-\text { Nominal }
$$

\section{Figure C.7 Bullseye of Left Flange Flatness error of 0.25 inch castings (Calculated Nominal: 0.01 in.)}




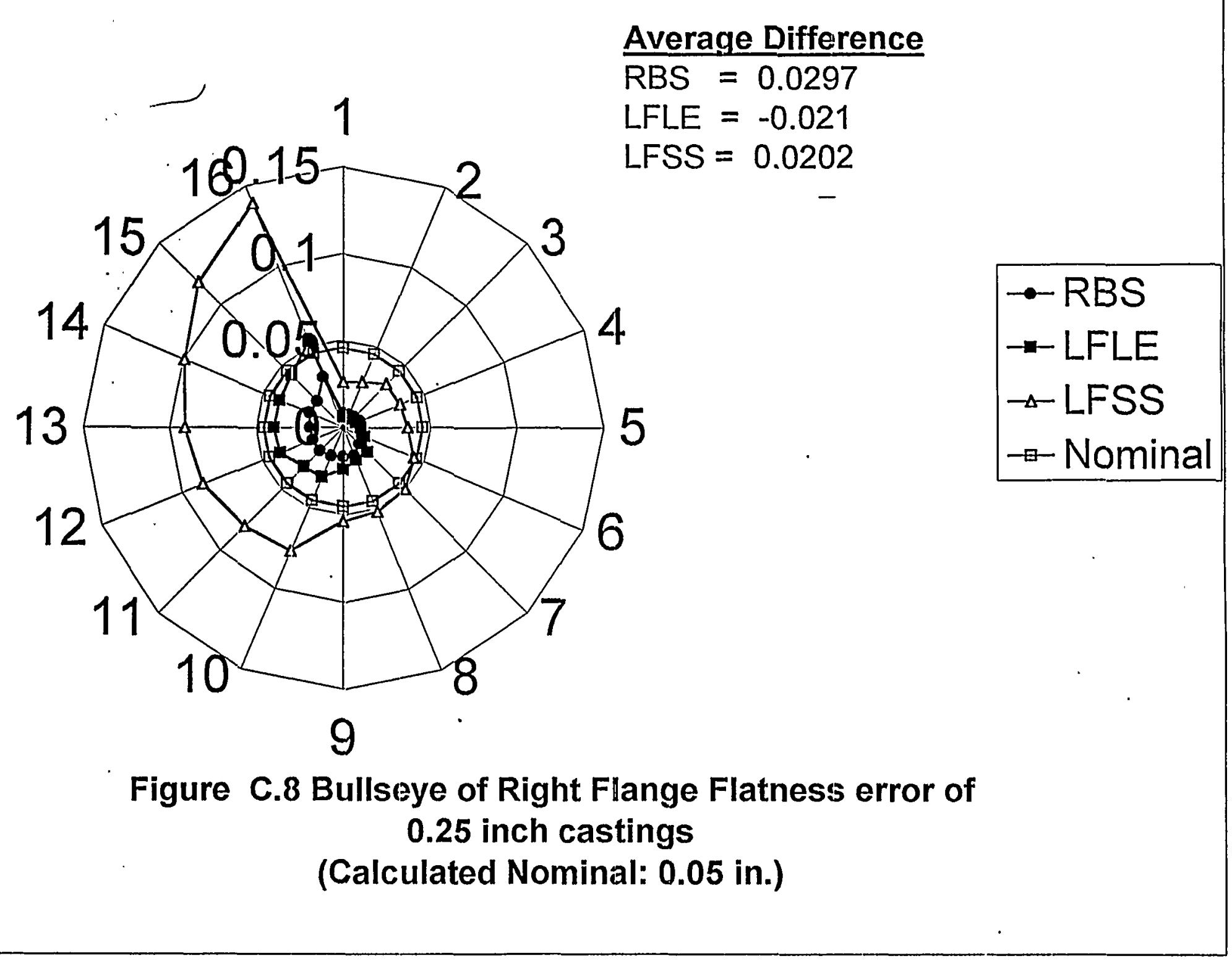




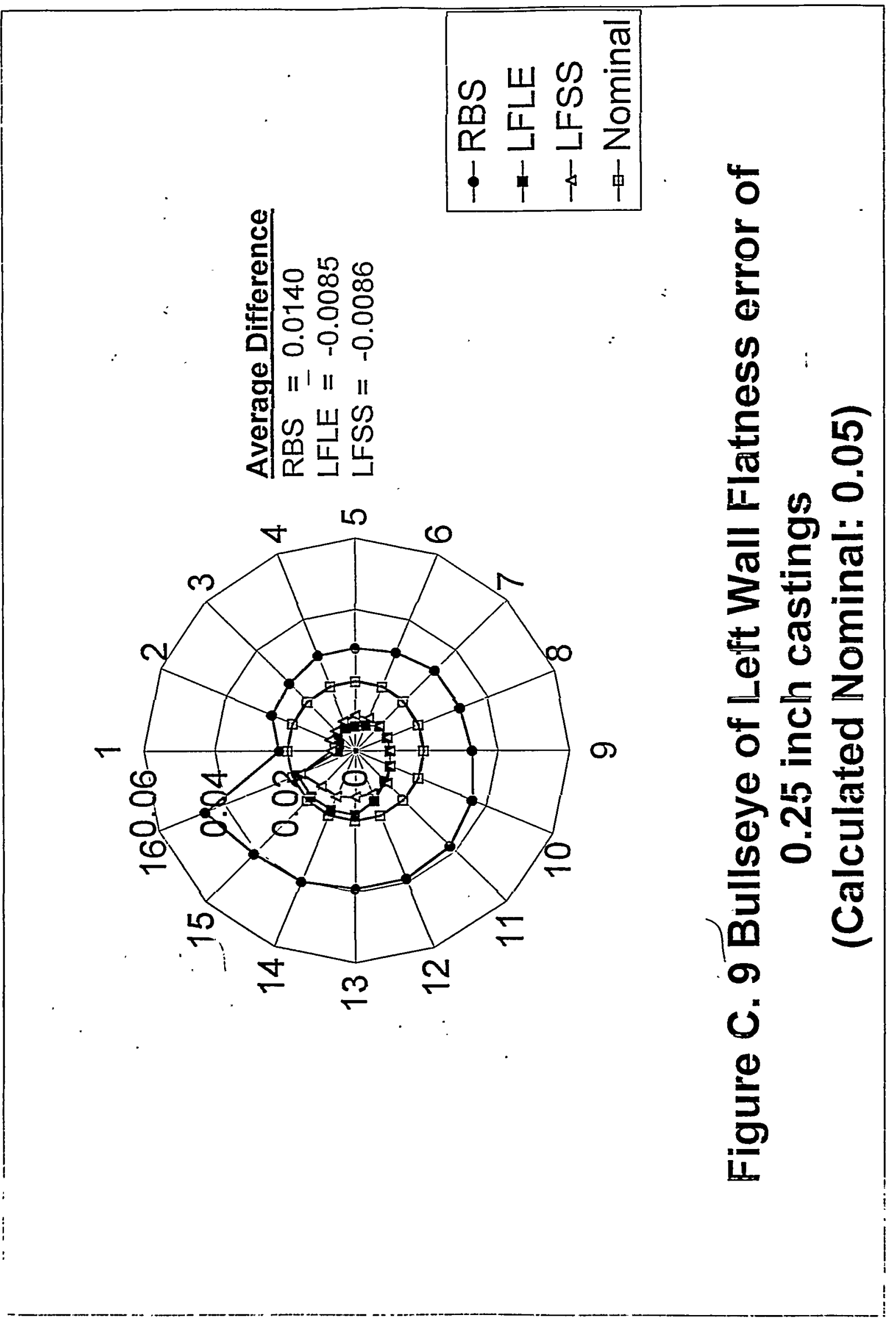




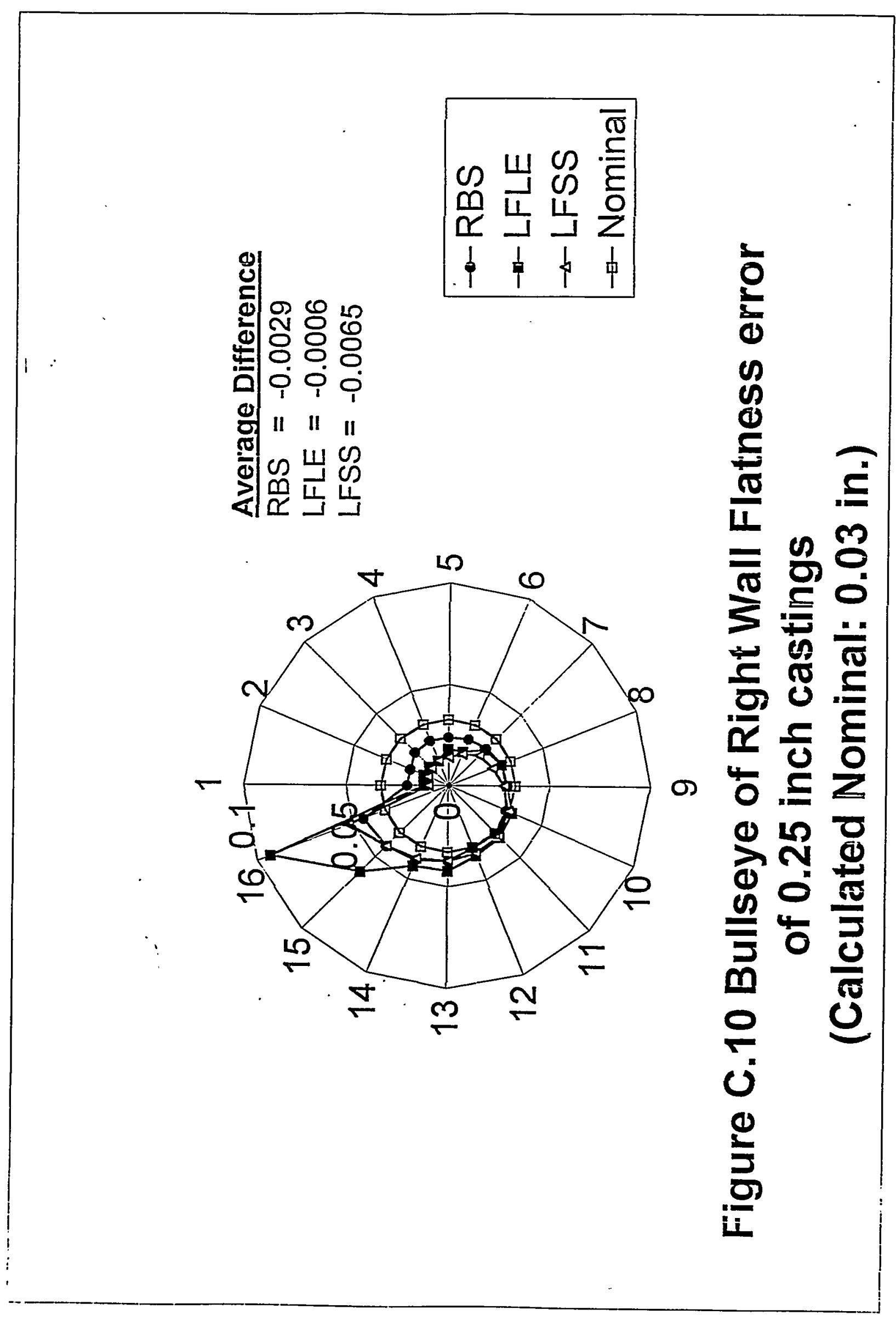




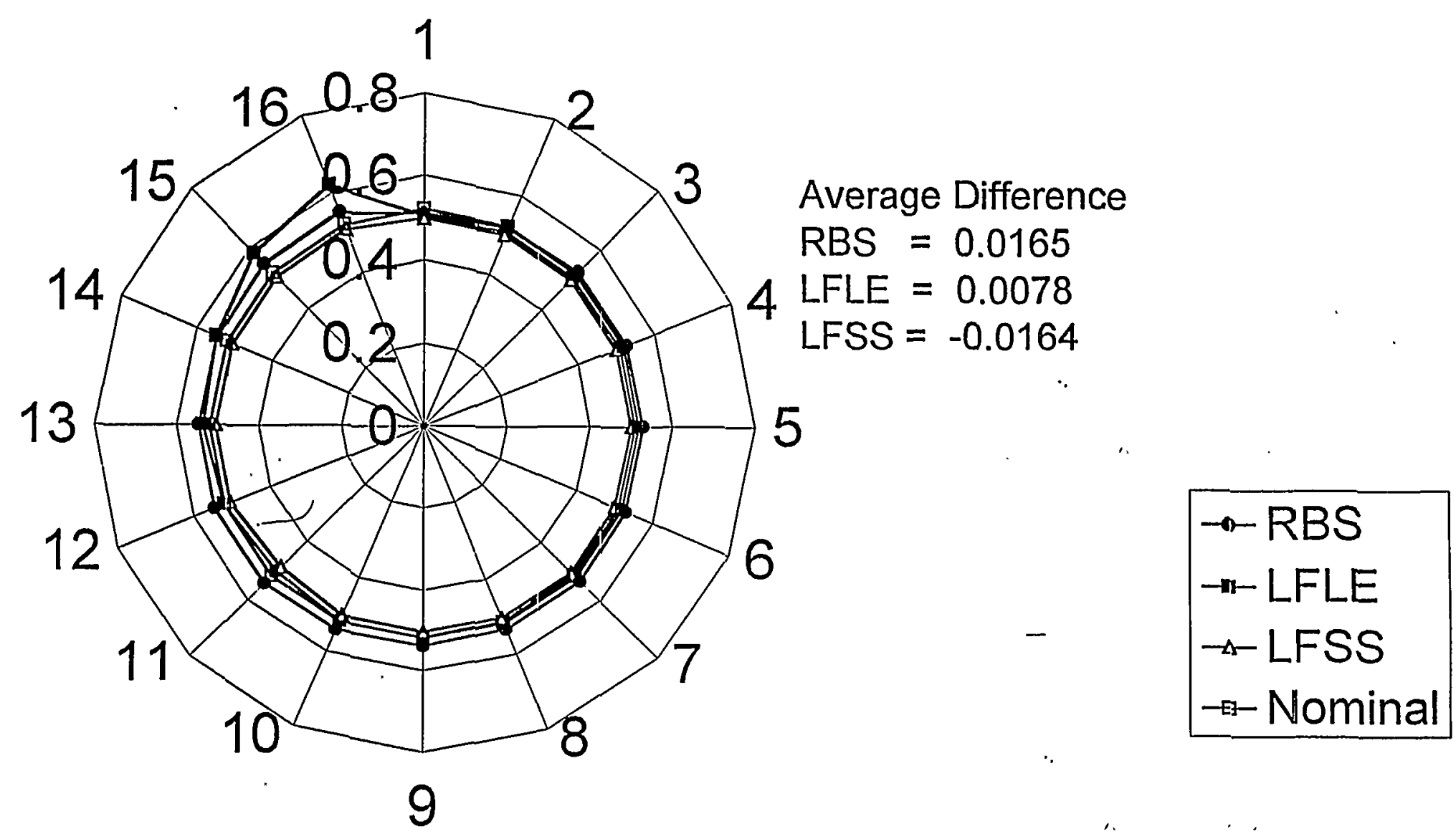

Figure C.1.1 Bullseye of Thickness error of 0.50 inch castings

(Calculated Nominal: 0.52 in-) 

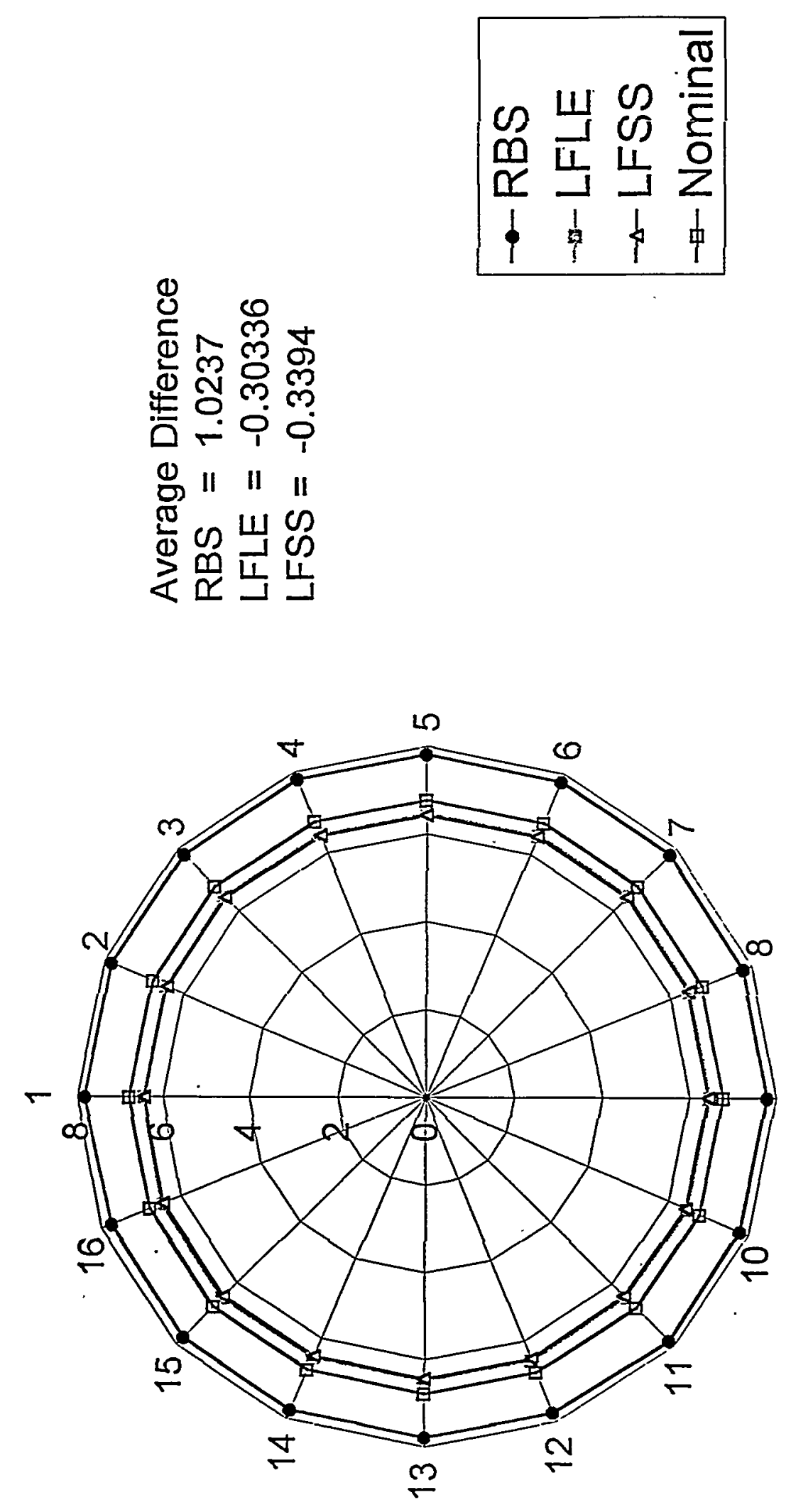


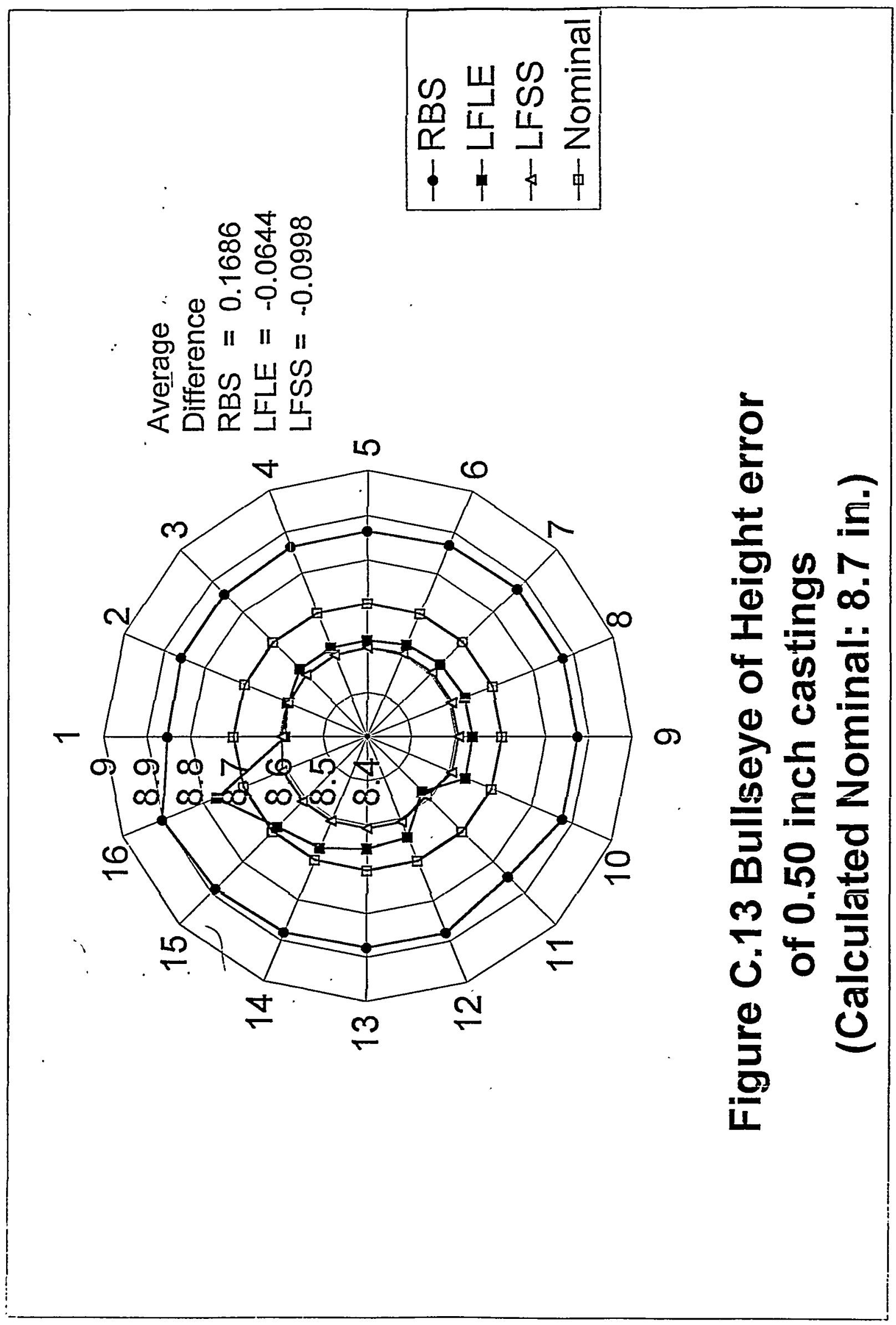




\section{Average Difference}

RBS $=-0.0103$

LFLE $=-0.0331$

LFSS $=-0.059$

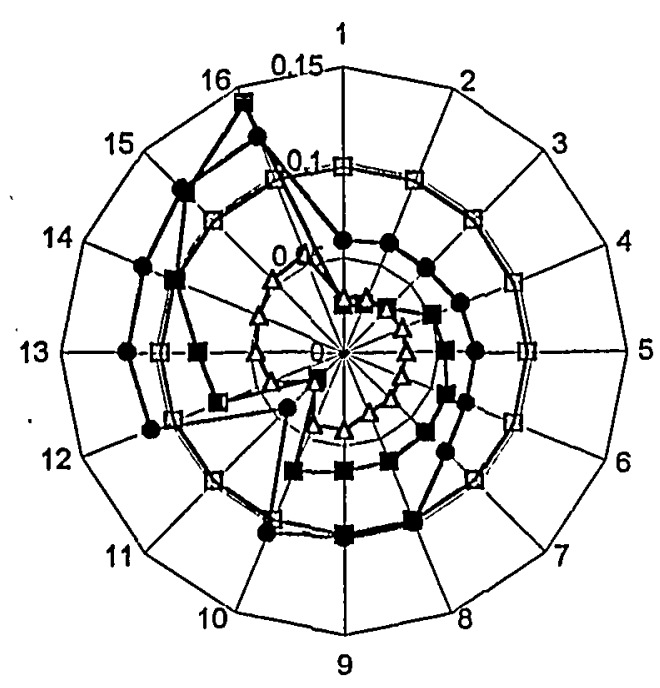

Figure C.14 Bullseye of Parallelism error of $\mathbf{0 . 5 0}$ inch castings 


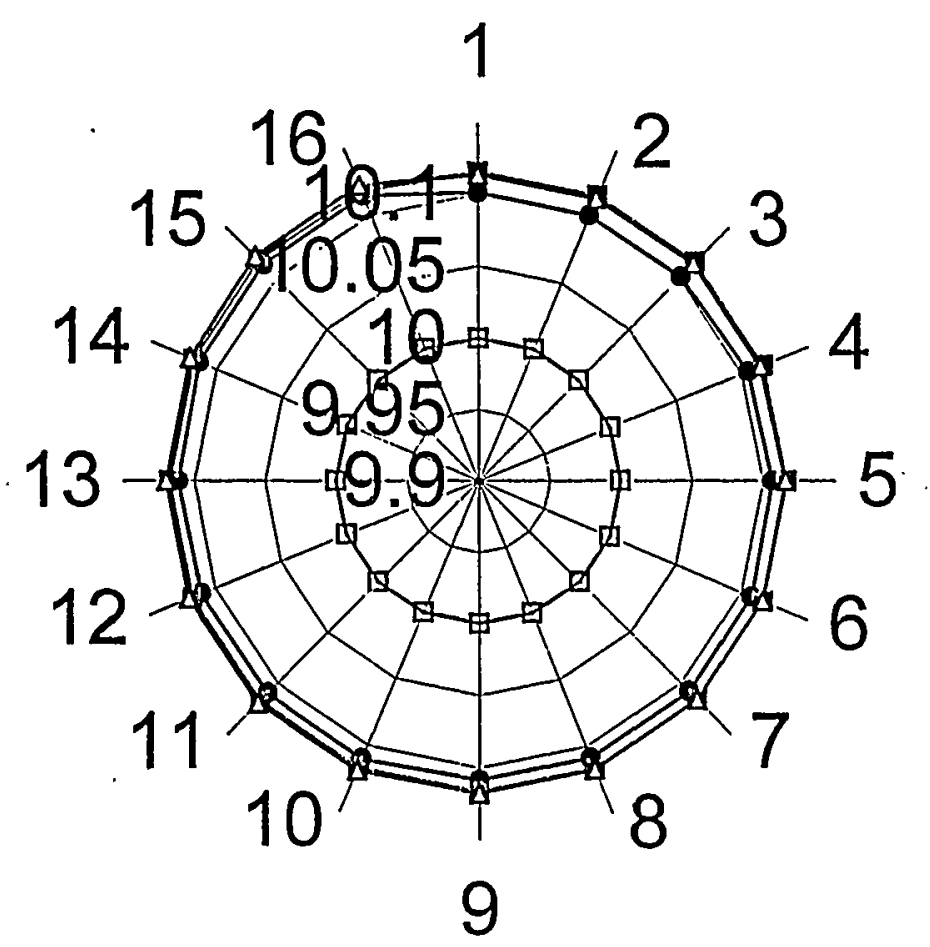

Average Difference

RBS $=0.1081$

LFLE $=0.1164$

LFSS $=0.1173$

$\rightarrow-R B S$

-1- LFLE

$-\Delta$ LFSS

$\rightarrow-$ Nominal

Table C.15 Bullseye of Left Casting Angle-error of

0.50 inch castings

(Calculated Nominal: 10 degrees) 


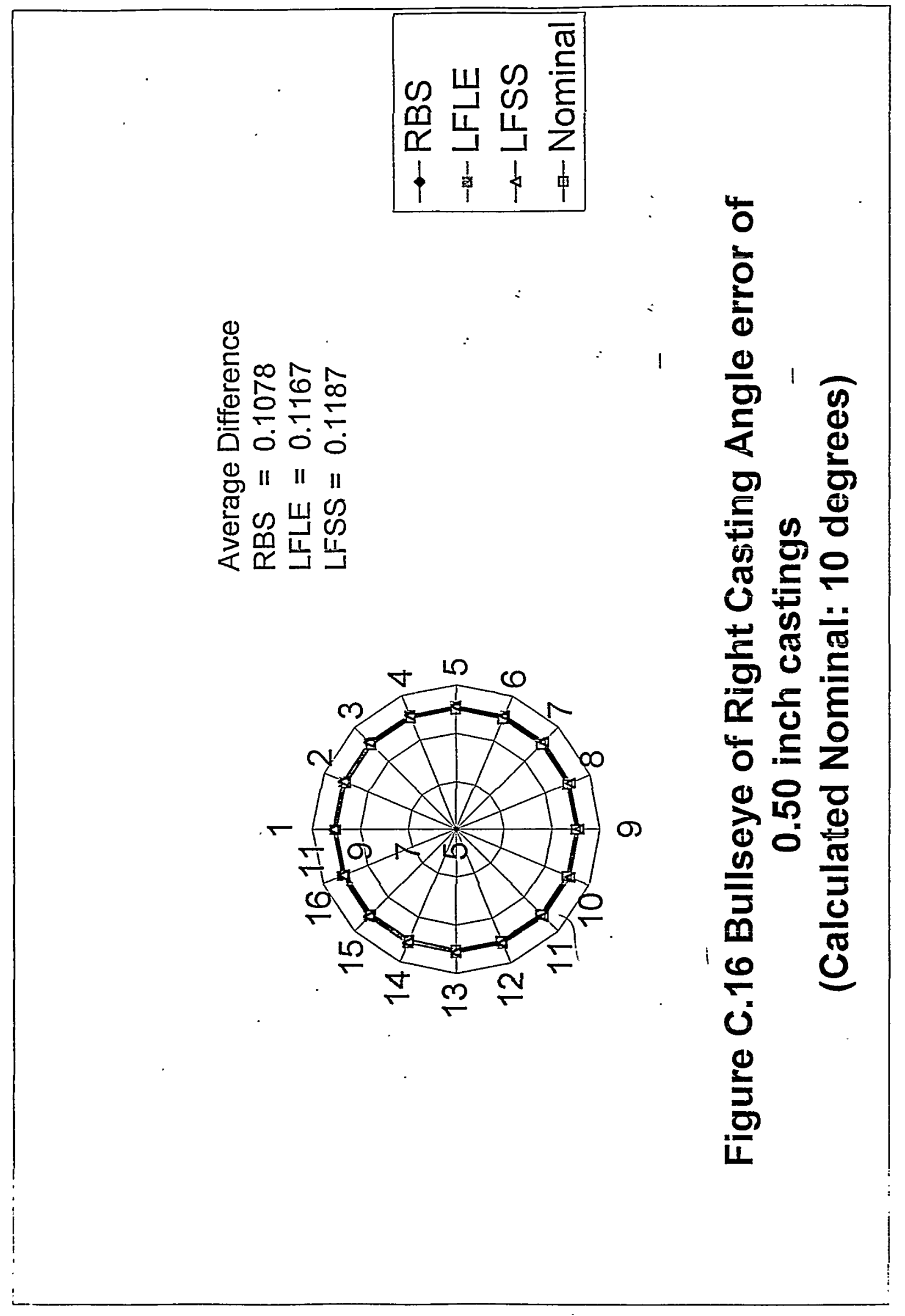




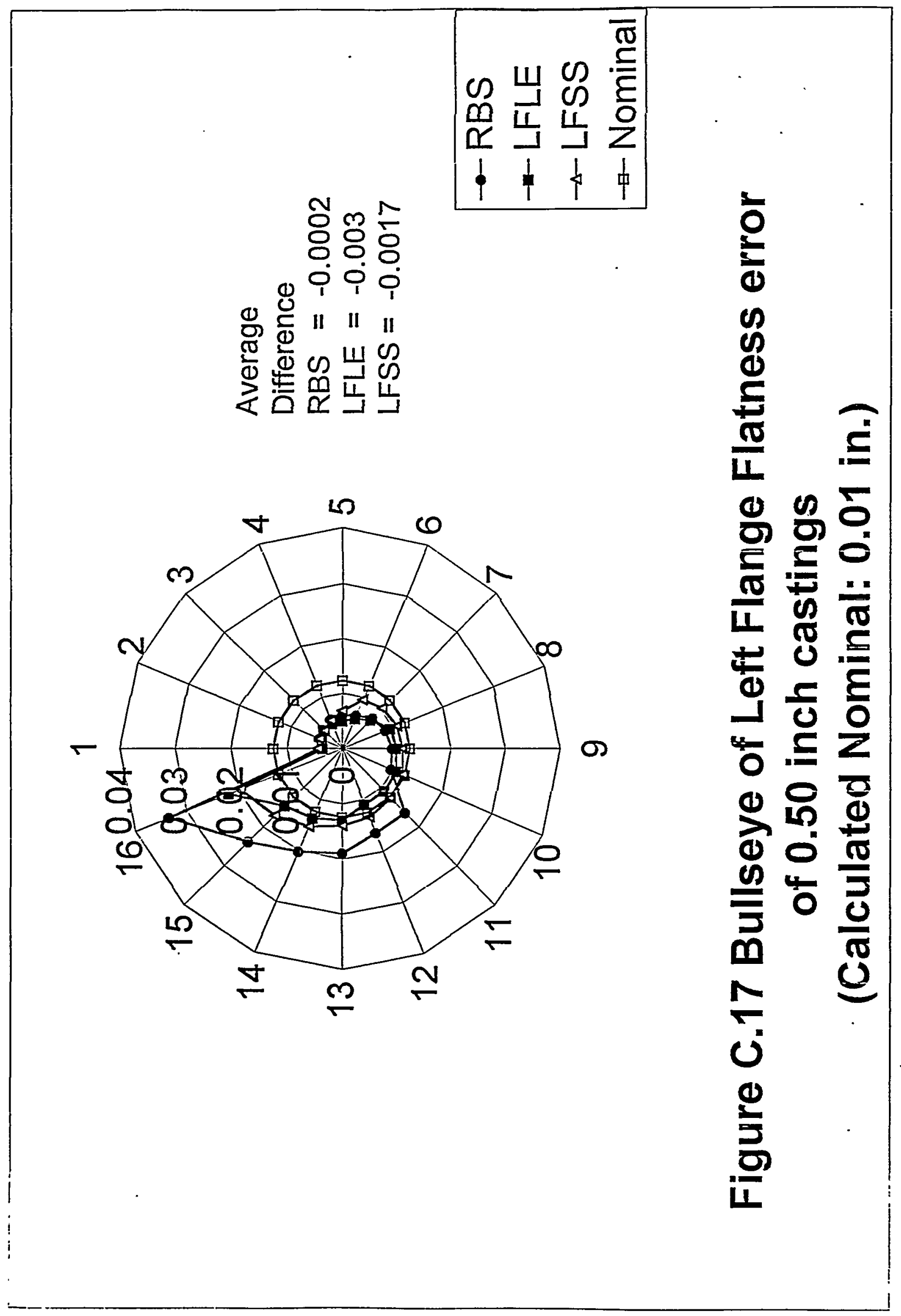



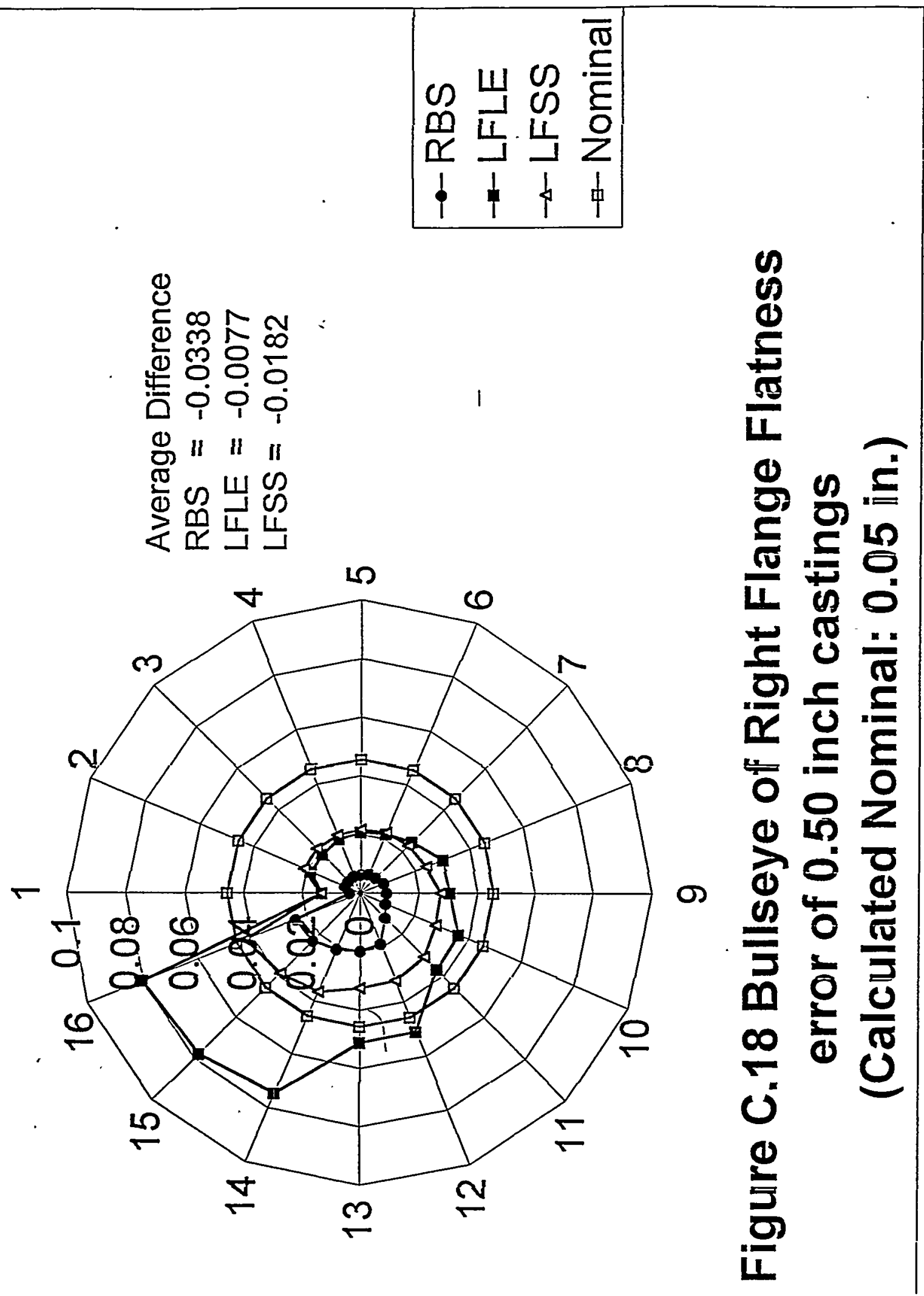


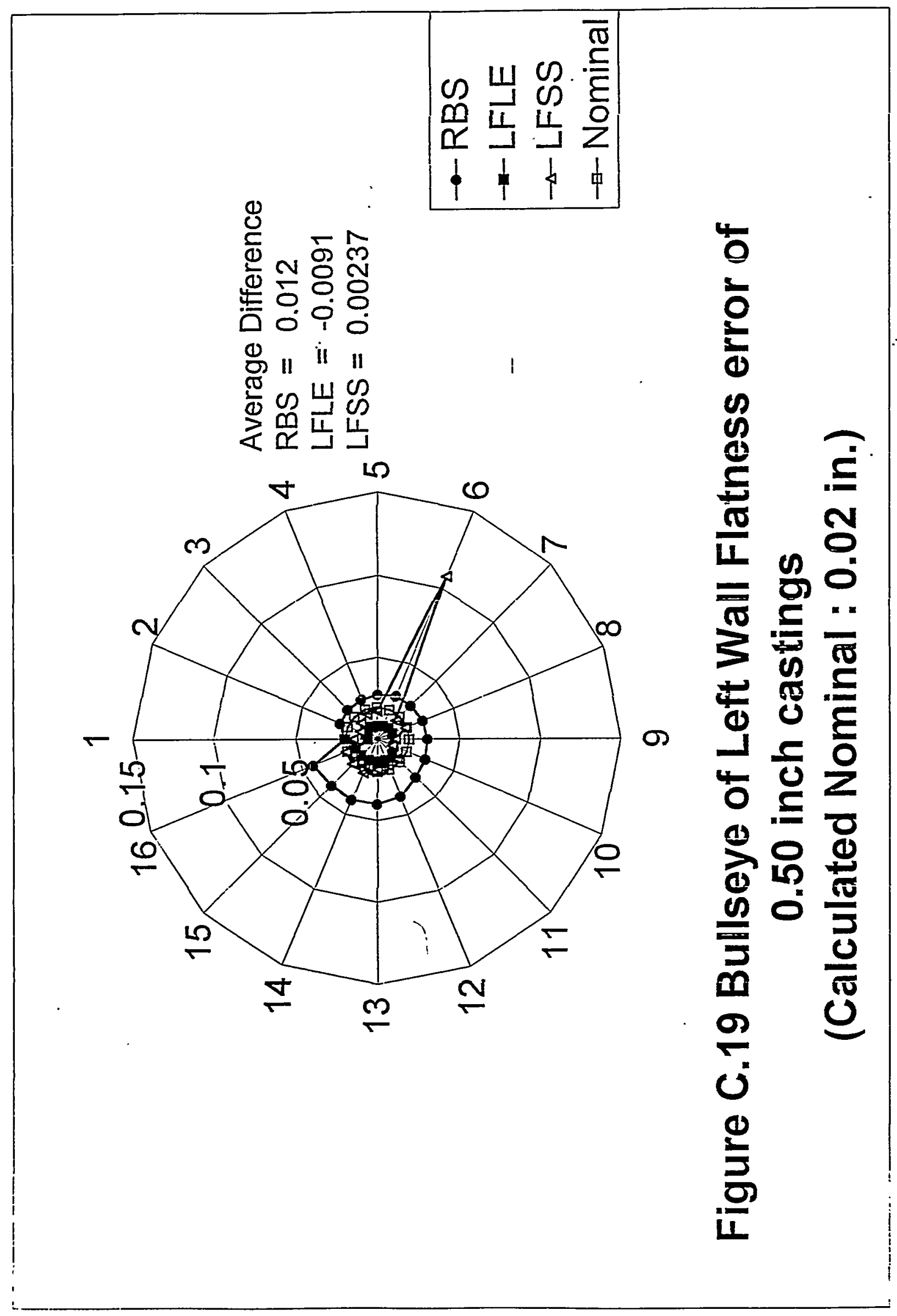




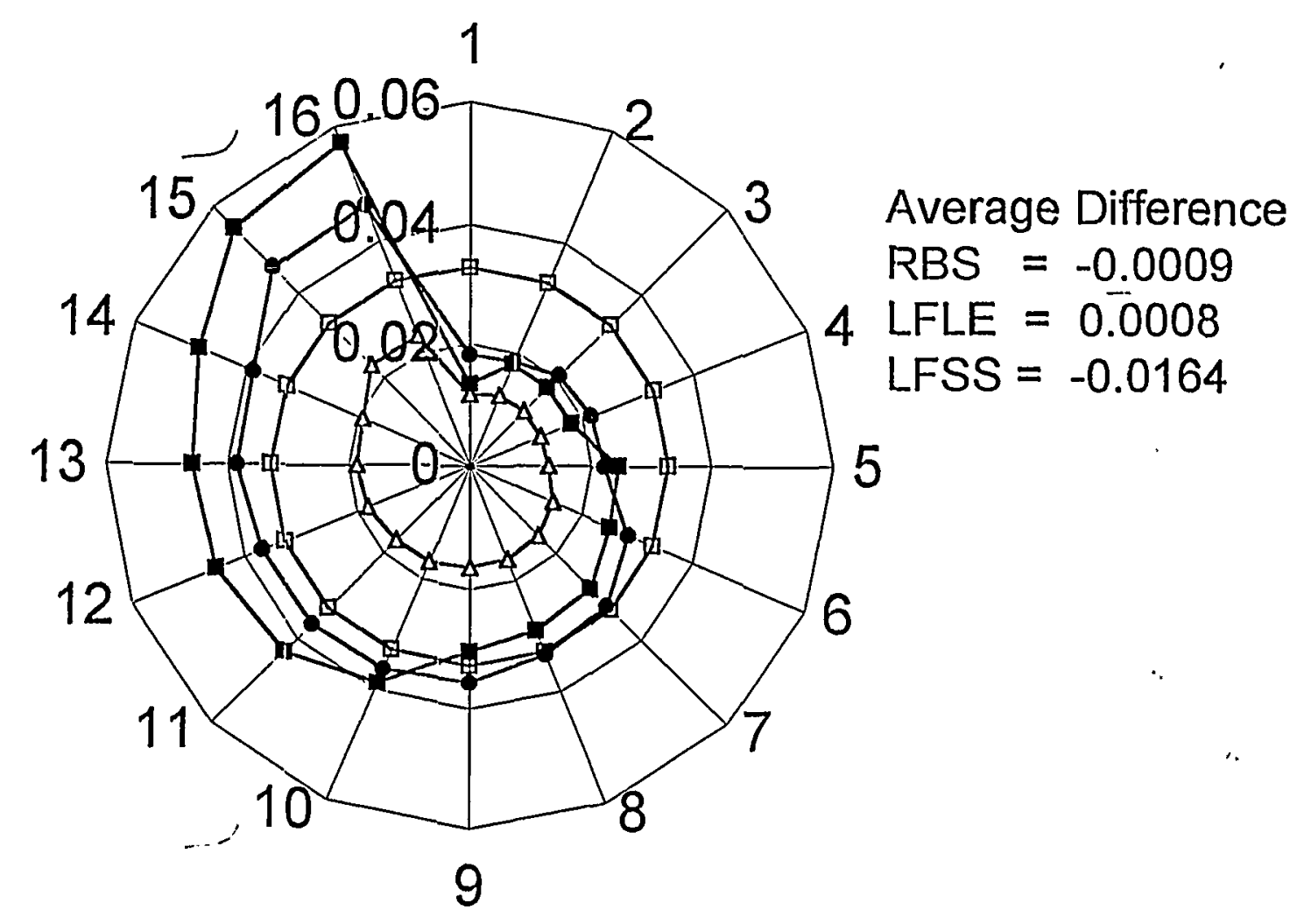

- - RBS
- - LFLE
$-\Delta-$ LFSS
- - Nominal

Figure C.20 Bullseye of Right Wall Flatness error of 0.50 inch castings

(Calculated Nominal: 0.03 in.) 
Average

Difference

RBS $=0.0443$

LFLE $=-0.0042$

LFSS $=-0.0269$

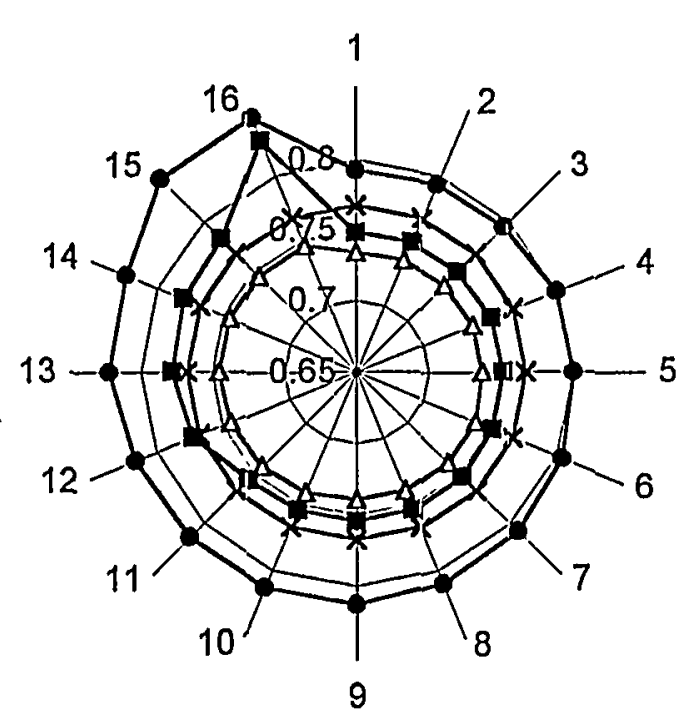

$\rightarrow-$ RBS

$\rightarrow-$ LFLE

$\rightarrow-$ LFSS

$\rightarrow$ Nominal

Figure C.21 Bullseye of Thickness error of 0.75 inch castings

(Calculated Nominal: 0.77 in.) 


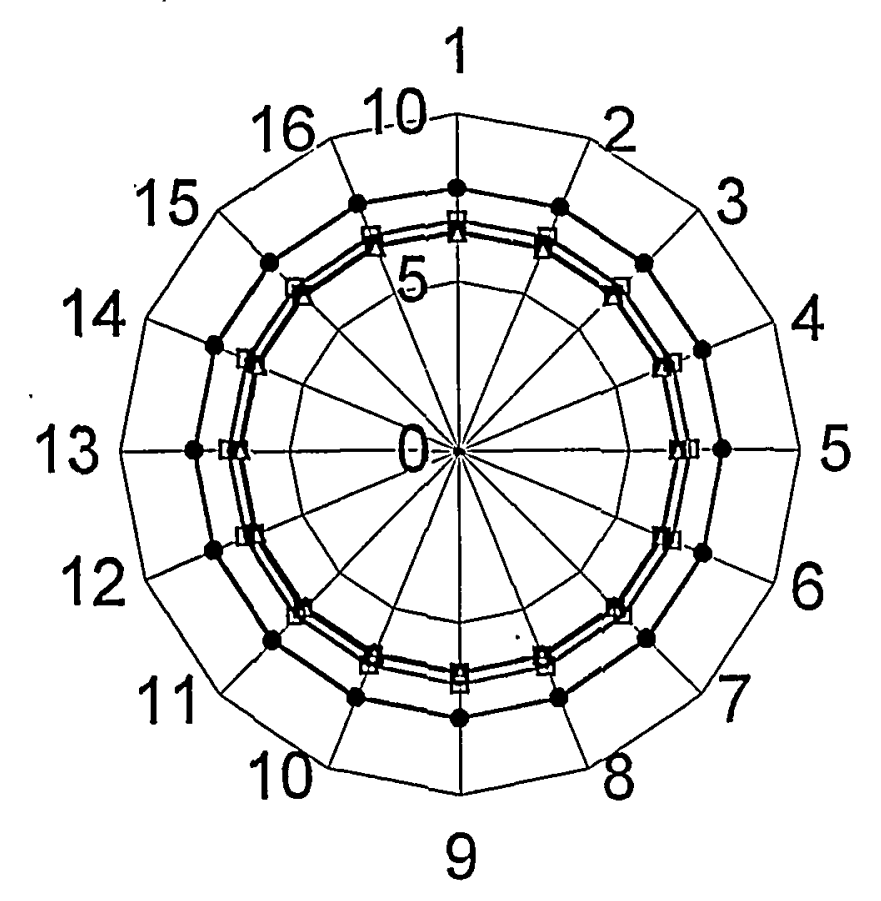

\section{Average \\ Difference \\ RBS $=0.9941$ \\ LFLE $=-0.3065$ \\ LFSS $=-0.3404$}

Figure C.22 Bullseye of Length error of 0.75 inch castings (Calculated Nominal: 6.78 in.) 


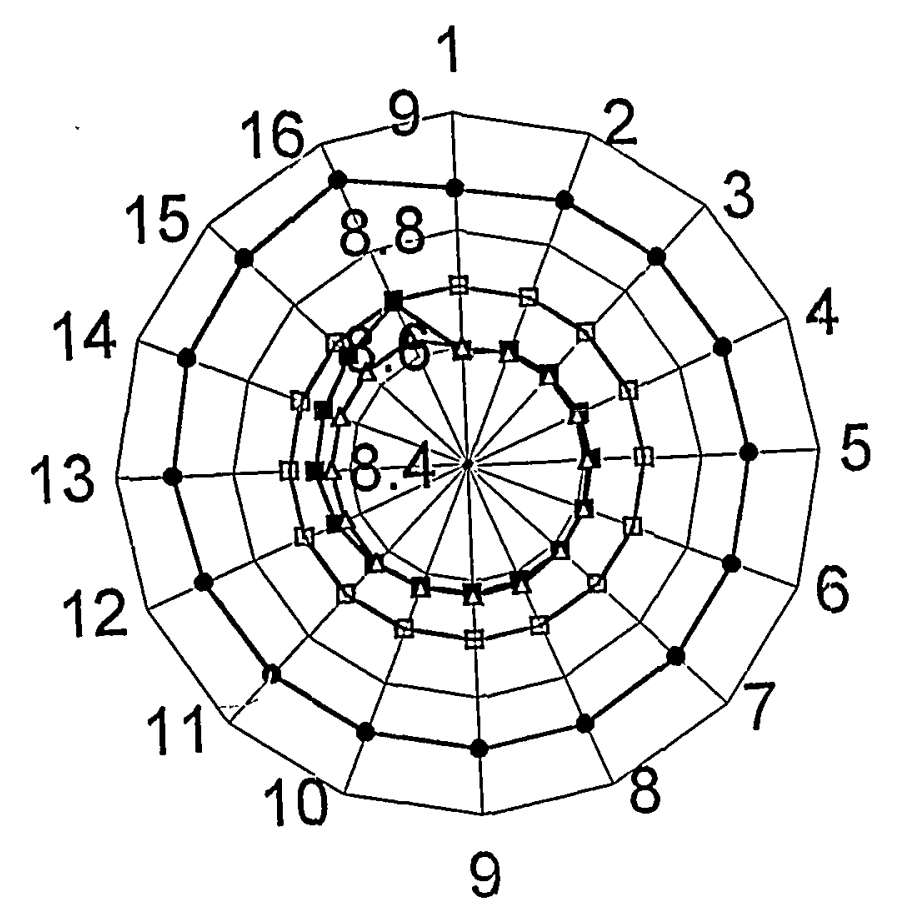

Average Difference

$\mathrm{RBS}=0.1888$

LFLE $=-0.0717$

LFSS $=-0.0834$

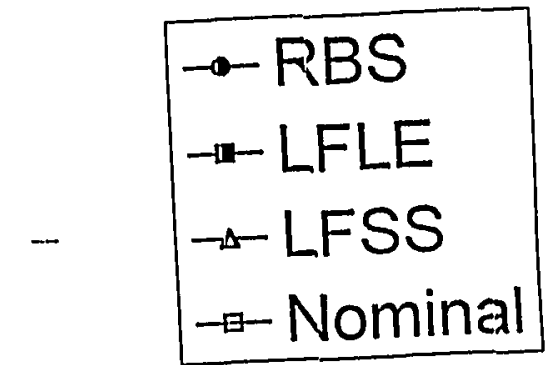

Figure C.23 Bullseye of Height error of

\subsection{5 inch castings}

(Calculated Nominal: 8.7 in.) 

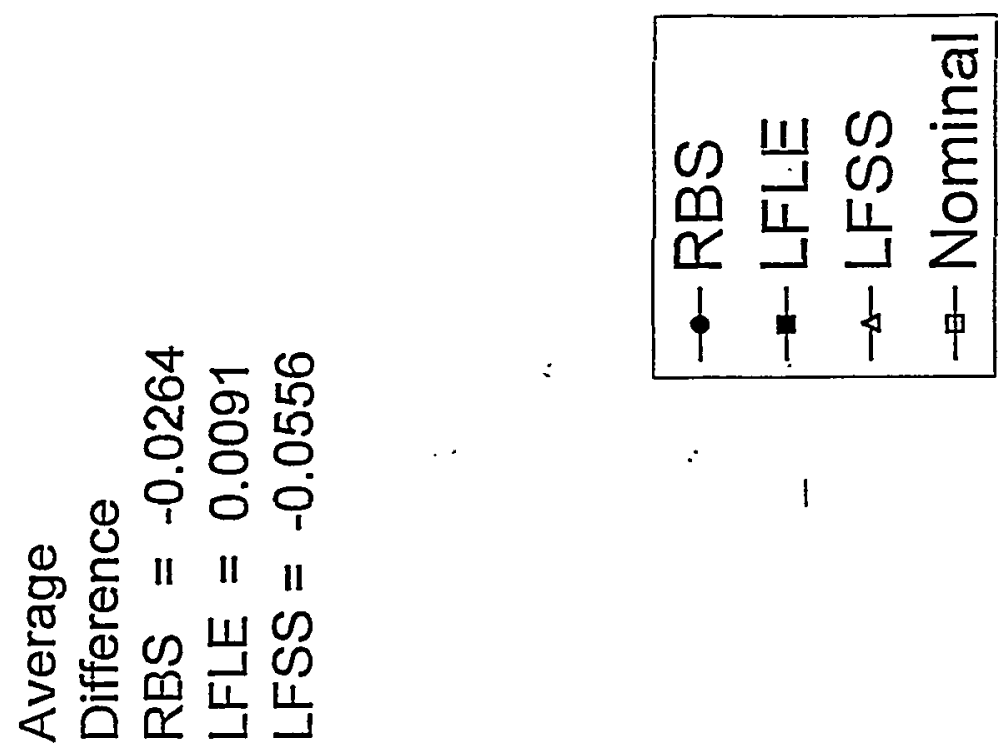

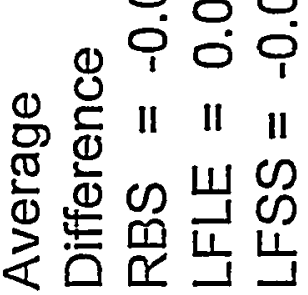

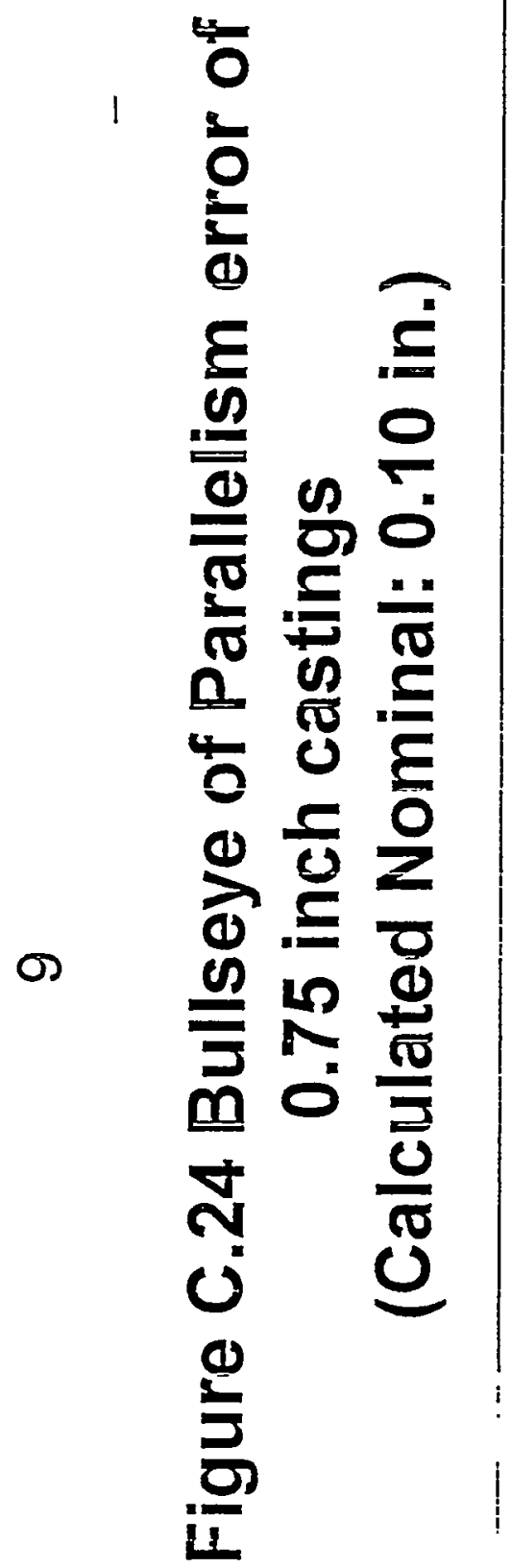




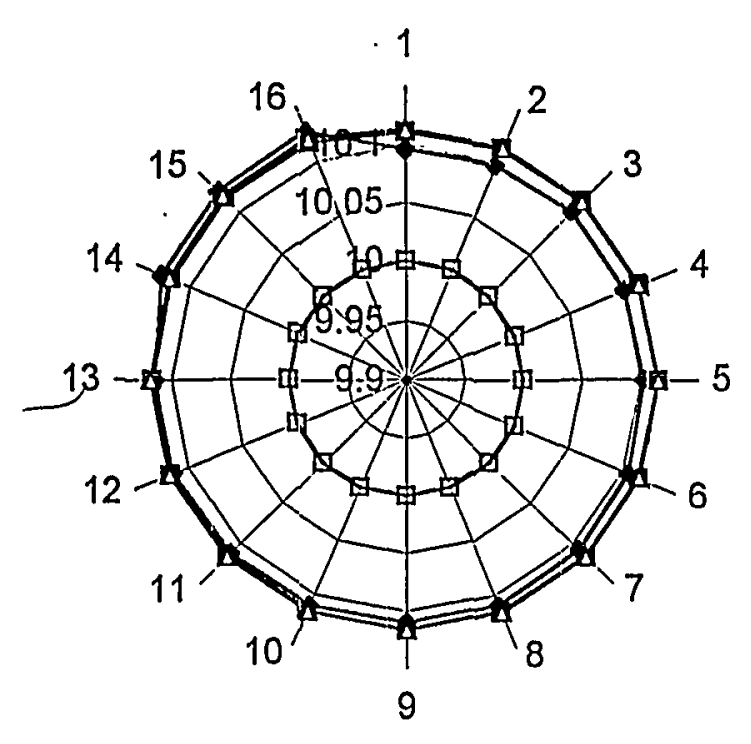

$$
\begin{aligned}
& \text { Average } \\
& \text { Difference } \\
& \text { RBS }=0.1103 \\
& \text { LFLE }=0.1153 \\
& \text { LFSS }=0.1164
\end{aligned}
$$

Figure C.25 Bullseye of Left Casting Angle error of

0.75 inch castings

(Calculated Nominal: 10 degrees) 


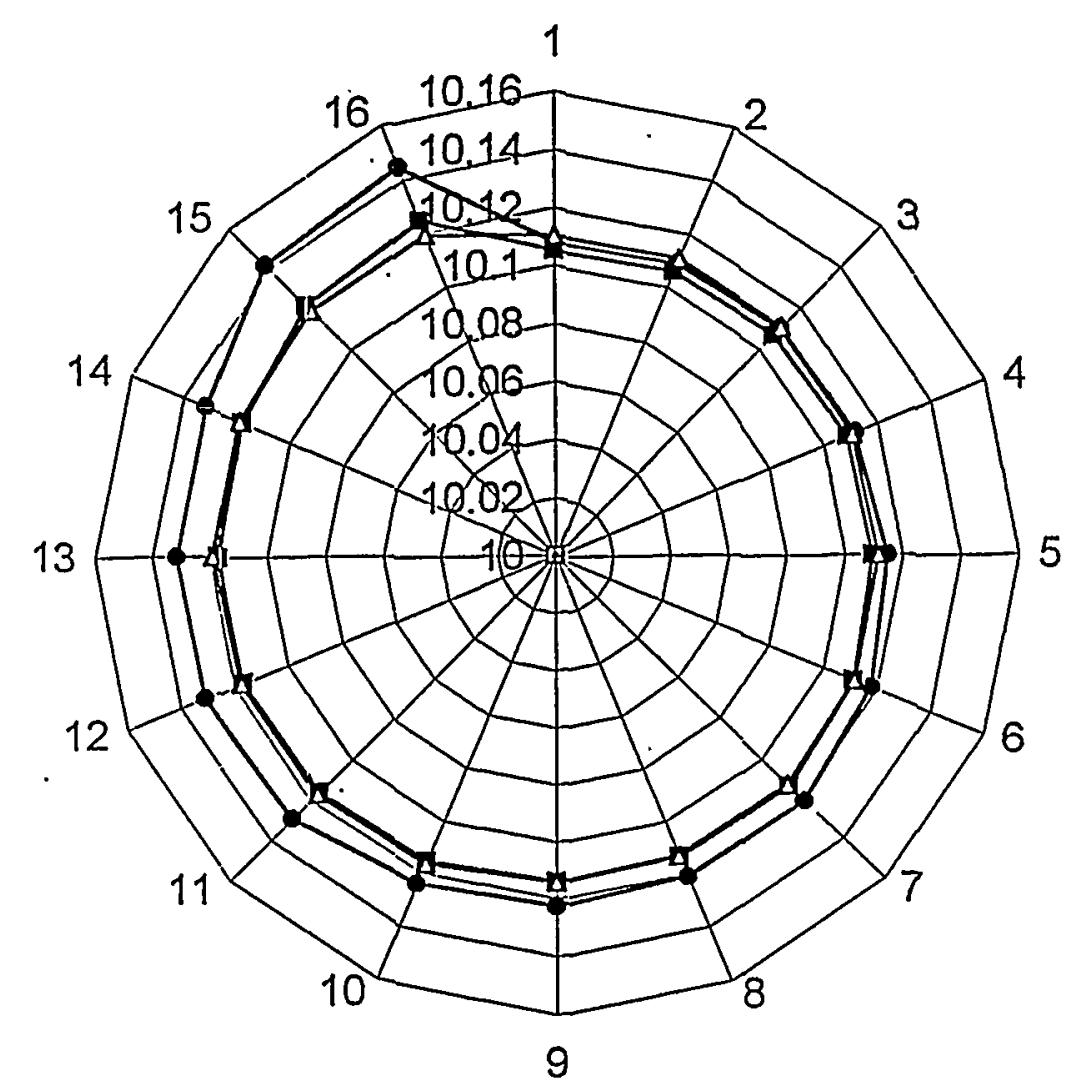

Average Difference

$\mathrm{RBS}=0.1230$

LFLE $=0.1137$

LFSS $=0.1145$

$\rightarrow-\operatorname{RBS}$

$\rightarrow-$ LFLE

$\rightarrow-$ LFSS

- - Nominal

Figure C.26 Bullseye of Right Casting Angle error of

0.75 inch castings -(Nominal Value: 10 degrees) 


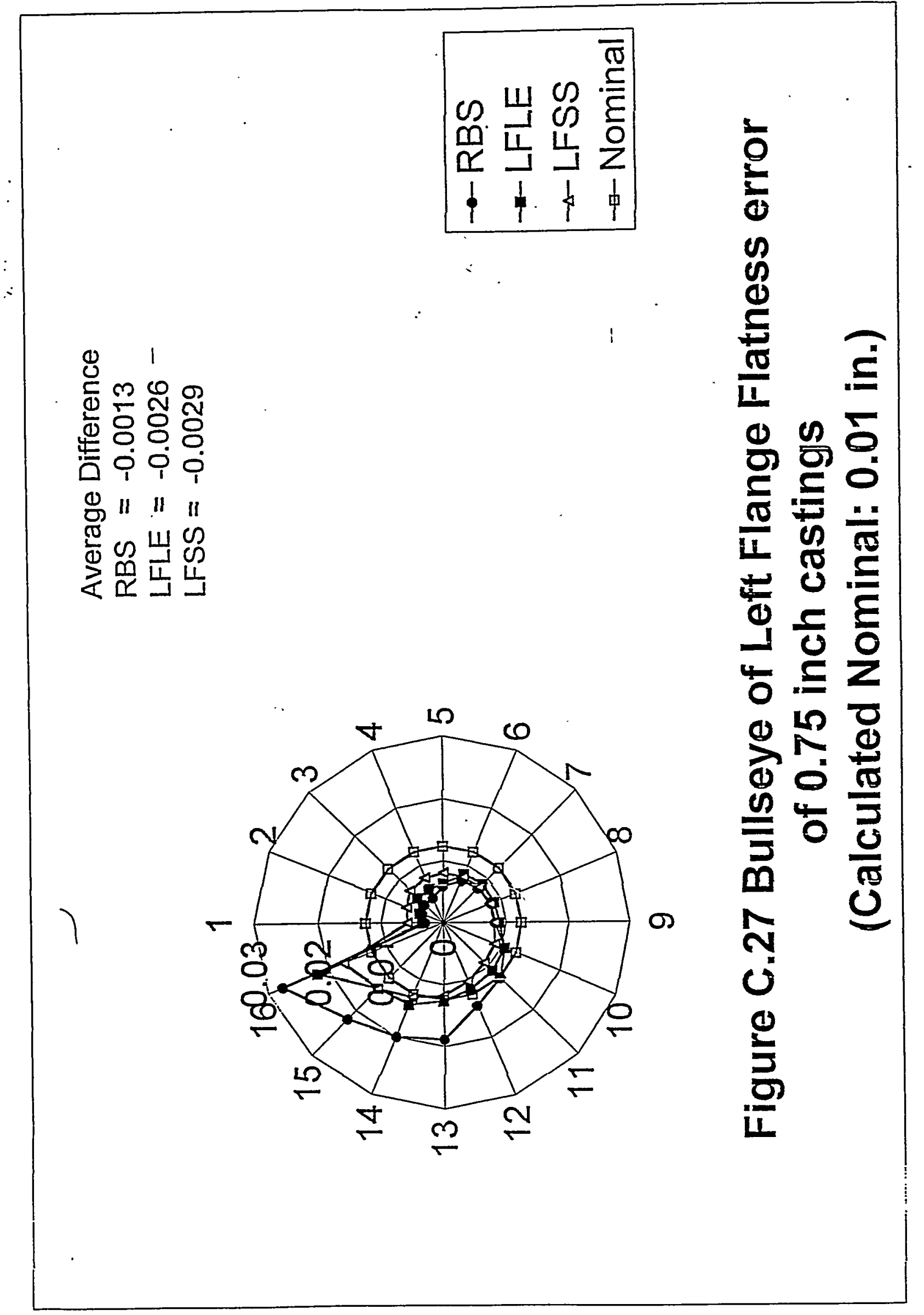




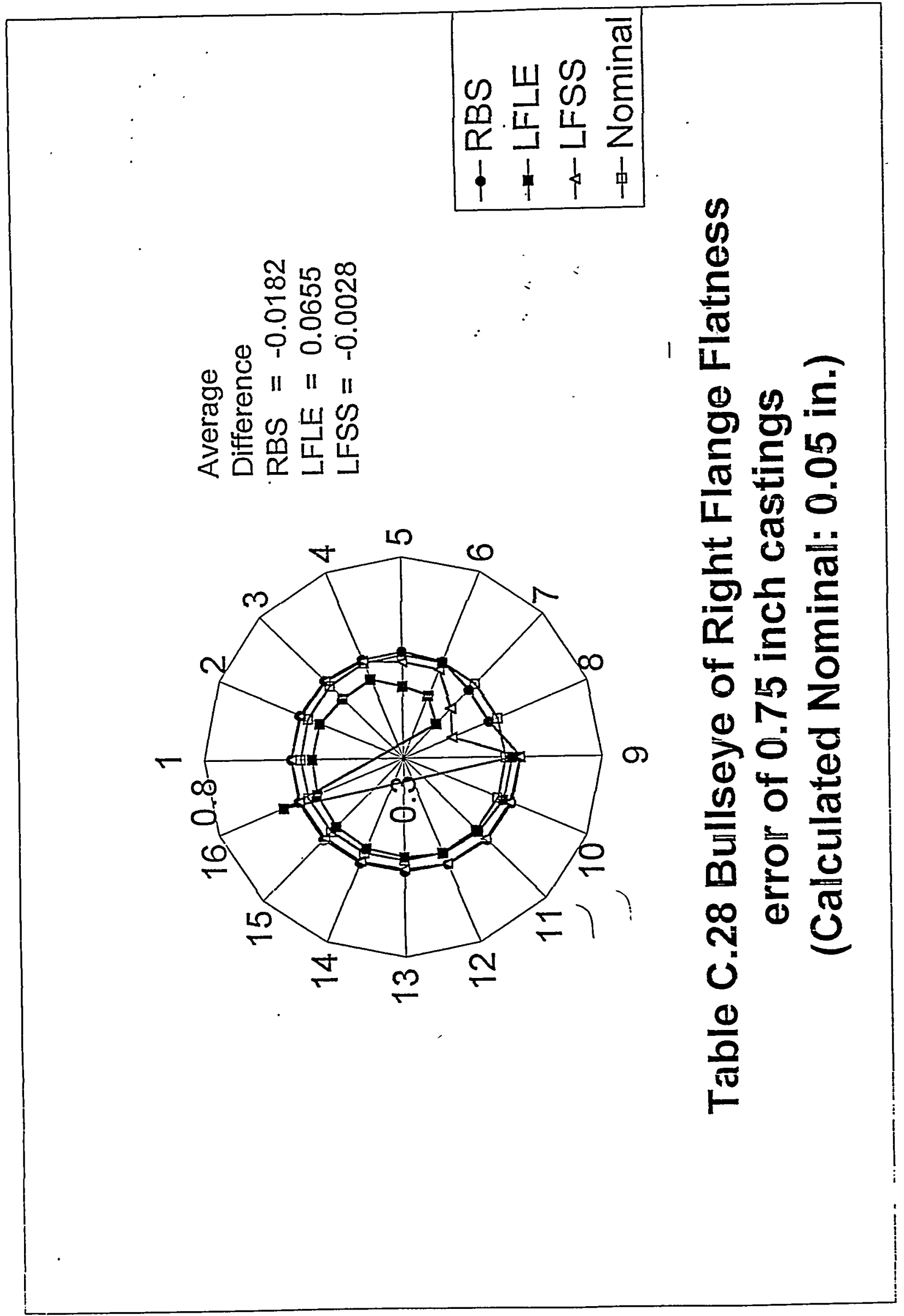



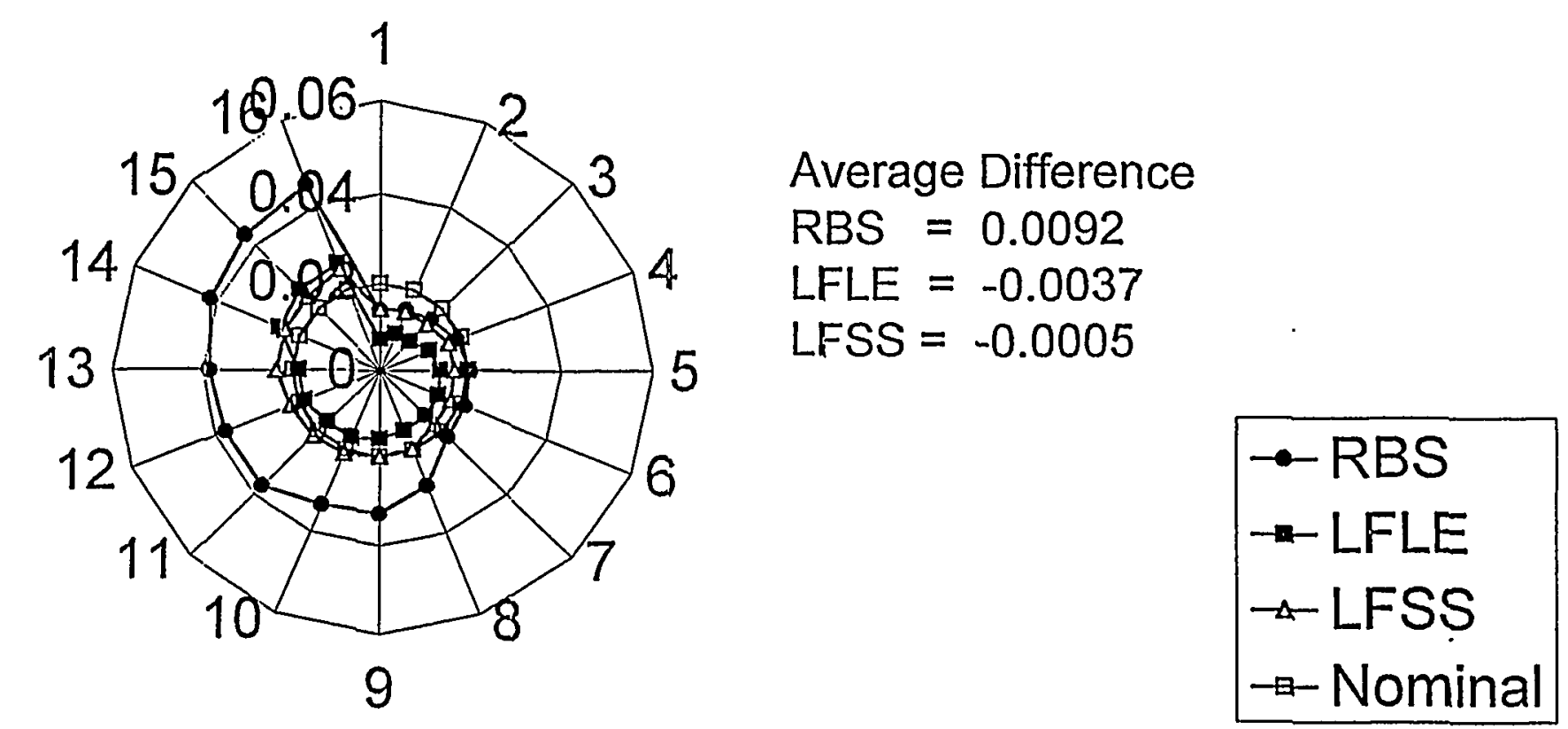

Figure C.29 Bullseye of Left Wall Flatness error of 0.75 inch castings

Calculated Nominal: 0.02 in.) 


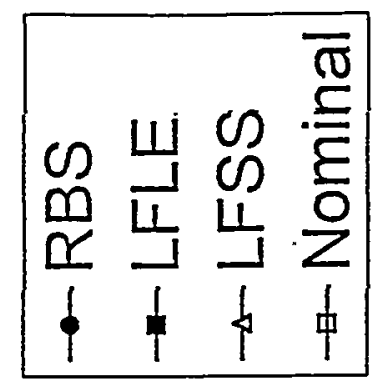

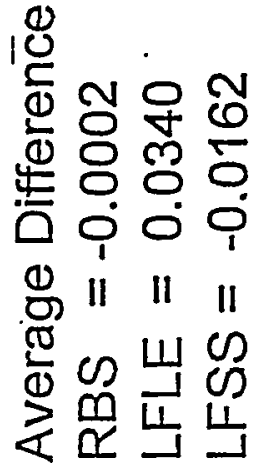
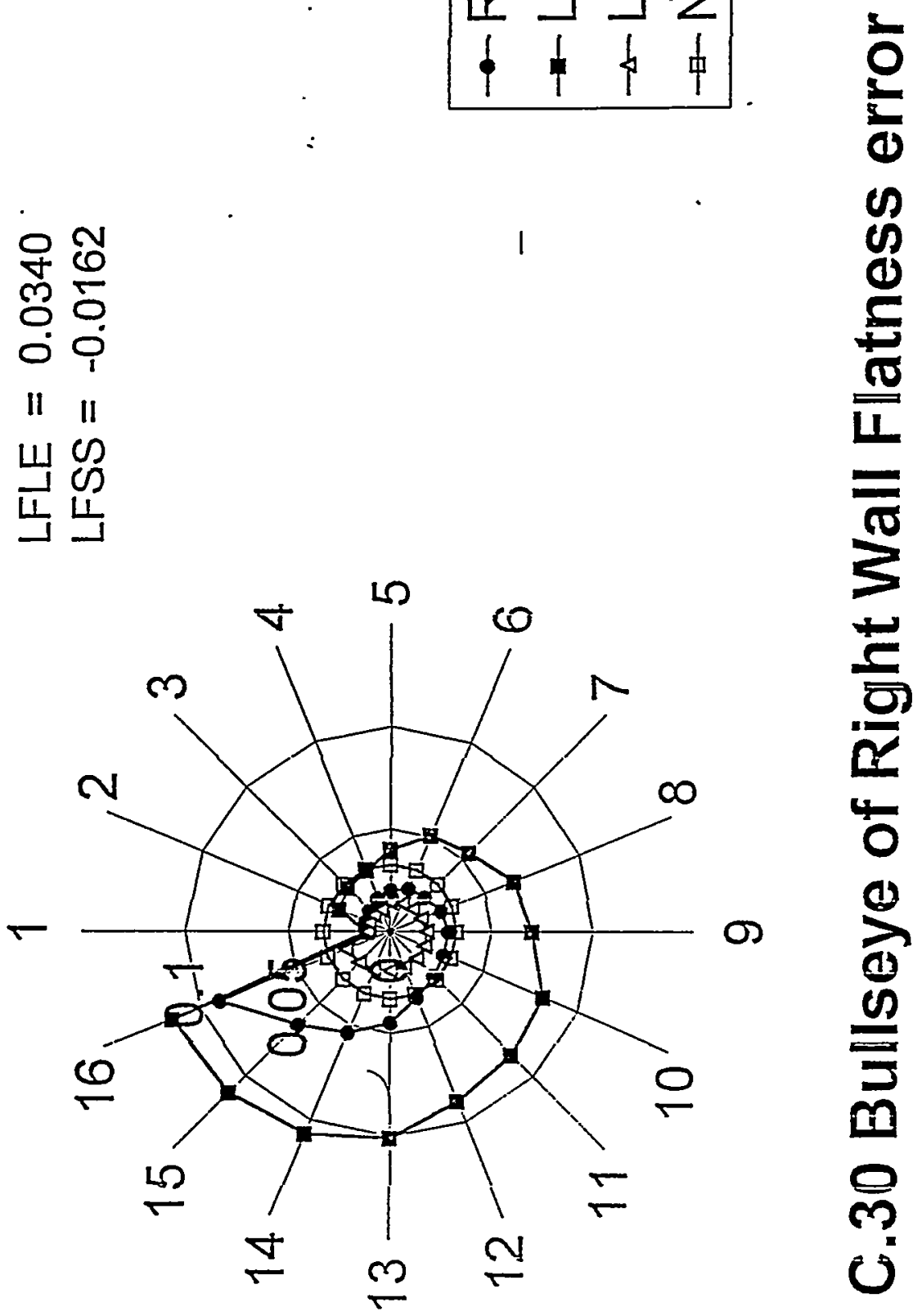

o

(5)

E)

ㄴ.

10 05

$\geqslant 0$

$\geq$

ID $=$

\pm op

()ㅜ를

D E E

$40 \geq 2$

(1) 100

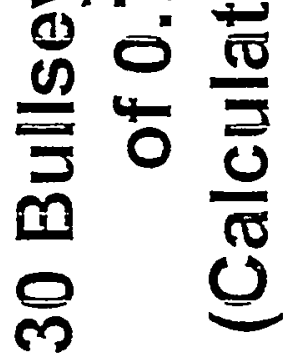

15

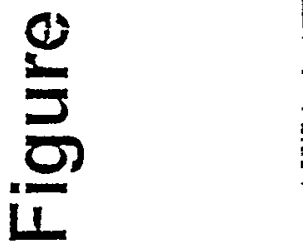


DOE/ID/13363-F

Part IV

\title{
The Relationship between Casting Distortion, Mold Filling and Interfacial Heat Transfer in Sand Molds
}

DE-FC07-95ID13363

FINAL REPORT - PART IV

\begin{abstract}
NEUTRON DIFFRACTION RESIDUAL STRAIN MEASUREMENTS ON ALUMINUM CASTING GATING SYSTEM
\end{abstract}

(MPLUS proposal \#96-018)

September 30, 1999

C.M. Hoffman, E.A. Payzant and C.R. Hubbard

High Temperature Materials Laboratory

Oak Ridge National Laboratory

Oak Ridge, TN 


\subsection{Background}

Residual stress measurements by X-ray diffraction is a well-established technique, but it is practically limited to near-surface stresses, whereas deeply penetrating neutron radiation permits non-destructive diffraction measurement of lattice strain within the bulk of large specimens.

Neutron scattering experiments were carried out on the HB-2 spectrometer at the High Flux Isotope Reactor (HFIR) of the Oak Ridge National Laboratory. The incident neutron beam wavelength was set by diffracting from the (331) planes of a double focusing silicon monochromator. Calibration of the diffractometer and the position-sensitive detector was determined using a nickel powder standard.

Macro-residual strain is determined from the shift in lattice d-spacing of diffracting grains relative to the d-spacing of a strain-free reference material. The strain is an average of the strains in the large number of diffracting grains within the sampling volume. Bragg's law relates the angular location of the diffraction peak determined in the scattering measurement to the lattice dspacing by:

$$
d_{h k l}=\frac{\lambda}{2 \sin \theta_{h k l}}
$$

where $\lambda$ is the wavelength of the neutrons and $\theta_{b k l}$ is one-half the scattering angle for a diffraction peak corresponding to the crystallographic Miller indices $h, k, l$. The residual strain component is related to the shift in d-spacing by:

$$
\varepsilon=\frac{d_{h k l}-d_{h k l}^{0}}{d_{h k l}^{0}}
$$

where $d^{0}{ }_{h k l}$ is the d-spacing of the stress-free reference. The direction of the measured strain bisects the incident and scattered beams. Substitution of Bragg's law into this strain equation gives, equivalently:

$$
\varepsilon=\frac{\sin \theta_{h k l}^{0}}{\sin \theta_{h k l}}-1
$$

which shows how a shift in the Bragg diffraction angle is used to calculate strain. The Bragg peak shift form a reference Bragg peak position is measured with an array of three fixed positionsensitive proportional counters (PSDs). The stability of the Bragg peak measurements in the course of a measurement campaign was monitored by repeated measurement of a fixed point in an aluminum reference sample attached to the casting. An accurate Bragg peak measurement from a stress-free reference sample is required for accurate residual strain determination. In this 
investigation, no stress-free reference sample was provided, so all strains were referenced to an ORNL Al specimen of dimensions $10.0 \times 12.5 \times 20.0 \mathrm{~mm}$. Errors will be discussed below.

\subsection{Experimental}

With the neutron wavelength 1.65 angstroms, the aluminum (311) peak was located at around 85.0 degrees 2-theta. Consequently the diffracting volume was defined by a parallelepiped $2 \mathrm{~mm}$ high with a $1.5 \mathrm{~mm} \times 1.5 \mathrm{~mm}$ sides, for a volume of approximately $4.5 \mathrm{~mm}^{3}$ (schematically shown in Figure 1).

The sample was mounted on an automated $\mathrm{X}-\mathrm{Y}-\mathrm{Z}$ translation table and the coordinates of the sampling positions were referenced with respect to the surfaces of the segment. The locations of the external surfaces of the segment were determined by making fitting an intensity-position scan using a non-linear fit to the intensity. The coordinate system and origin used for mapping is shown in Figure 2a. Note that in Figure 2, gates are referred to as "webs".

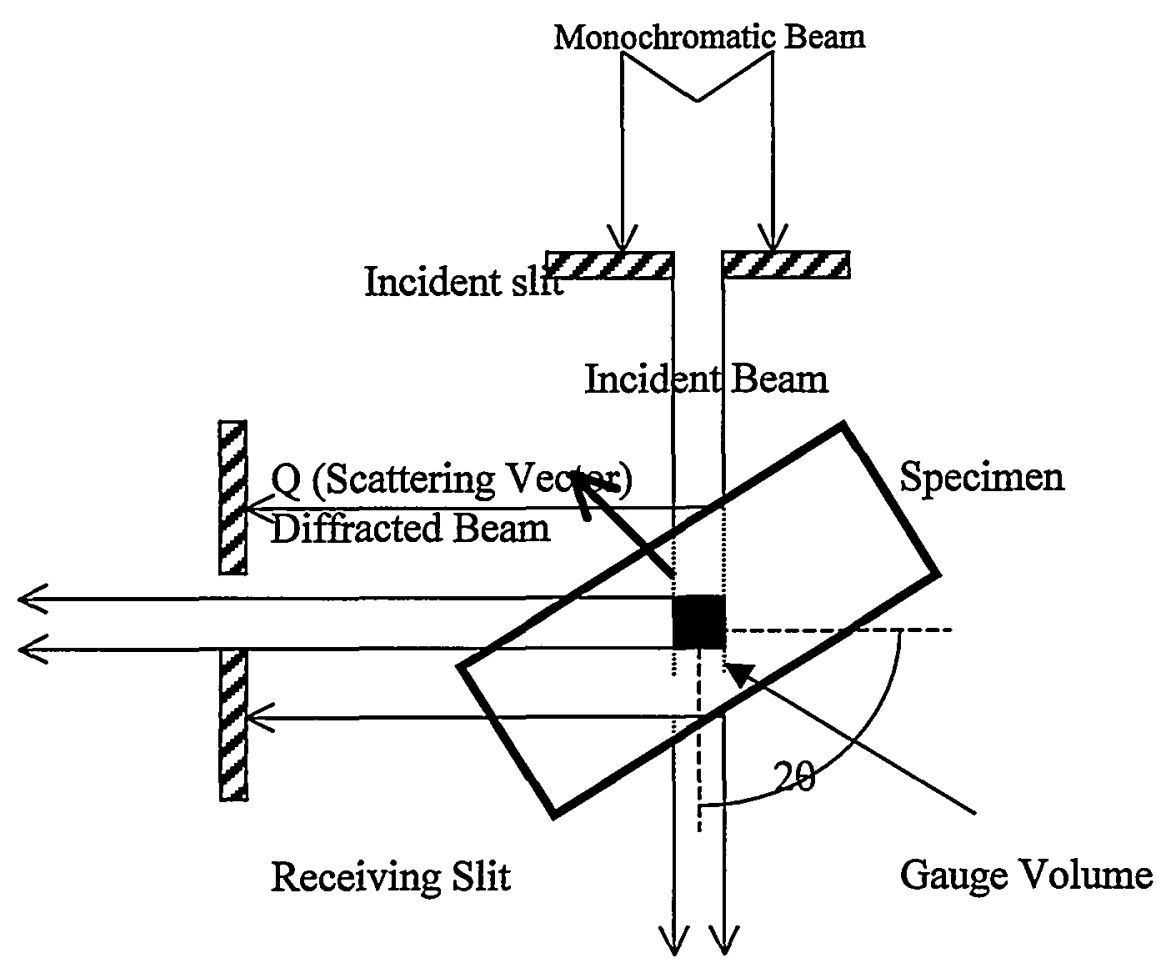

Transmitted Beam

Figure 1. Schematic of the neutron diffraction method, showing the gauge volume cross section as defined by the slits (shown at $90^{\circ}$ for convenience) and also the direction of the measured strain component (given by $\mathrm{Q}$ ). 


\subsection{Measurements Made}

The residual strains were mapped at three locations corresponding to the crown, the side and one of the webs of the casting. Mapping involved three to ten locations on the web, five locations on the side, and from 7 to 70 locations on the crown. This exploratory mapping was intended to determine if there were regions of significant change in strain upon removal of the gates. Where possible, residual strains were determined in three orthogonal directions at each location to permit calculation of stress. However, this was not possible for the normal direction in the web.

The casting posed a challenge for the neutron diffraction strain mapping because the strain mapping method relies on having a large number of randomly oriented grains distributed evenly throughout the gauge volume to avoid diffraction peak shift artifacts. For the $4.5 \mathrm{~mm}^{3}$ gauge volume the diffraction peaks were not of smooth Gaussian shape, indicating the existence of large grains and hence poor grain averaging. The peak shapes were significantly improved by modifying the data collection software to implement oscillating two to three of the translation and goniometer axes $(\mathrm{x}, \mathrm{z}$, and $\mathrm{psi})$ to increase the number of diffracting grains within the gauge volume.

Further grain and hence strain averaging was accomplished by using three detectors: one in the horizontal plane and the other two inclined above and below the horizontal plane by 7 degrees. The strain measurements from each detector were averaged. The root mean square deviation from the average was calculated and is given as a variance to the reported strain.

The cast specimen was first studied in the as-cast condition, with gating and runner in place. After these measurements the runner was removed. The changes on dimension of the cast part are recorded in Appendix B. These correspond to an opening of the gate region of the casting by approximately 0.06 ", indicating that the as-cast specimen is under residual stress due tot the constraint imposed by the gates and runner.

Altogether approximately 300 measurements were made for the specimen in each condition. Each measurement of strain required 10 to 20 minutes of neutron diffraction collection, depending on measurement location. Altogether the total specimen mounting, aligning, and residual strain mapping measurement time was approximately 3.5 days per specimen condition. The measure tangential strain data is presented in Appendices $\mathrm{C}$ and $\mathrm{D}$.

\subsection{Possible Measurement Limitations}

The strain values represent the average strain in the subset of grains within the gauge volume that are oriented to diffract. If a strain gradient exists within the gauge volume then the measured average will be underestimated. However, the data indicates that there are no large strain gradients and hence this is not a serous issue for this specimen.

The stress-free reference d-spacing was chosen to be the d-spacing value at the East corner, $\mathrm{E}$, of the specimen (Figure 2a). If there were chemical differences in the cast alloy 
between this location and the measured locations this could lead to a shift in d-spacing that we would interpret as a residual strain. We also assume that there is no residual strain at location $\mathrm{E}$ as it is well removed from the regions affected by the constraints of the webs and runner.

We believe that the possible uncertainty or bias resulting from these limitations is small. It would be possible to quantify the effects if further studies are desired.

\subsection{Results}

The tangential strain values are tabulated separately for each measurement location in Appendix $\mathrm{C}$ and $\mathrm{D}$. Positive values indicate tensile strain and negative values compressive strain. The tangential strains, as well as the axial and normal strains in the crown location are quite small and are essentially zero within experimental error along the length of the crown and at three depths within the crown.

The tangential component of strain in the crown was investigated at three different depths to examine the effect of the opening of the web (gate) upon removing the runner (Appendix C). In the as-cast specimen the strains went from slightly compressive at the inside to slightly tensile at the outside. The data from the casting where the runner had been removed showed a different trend, with the more compressive behavior at the outside and the more relaxed strains closer to the inside of the sample. This is consistent with the measured opening of the casting when the runner was removed. Thus the strain distribution ins modified (it was actually relieved or shifted to the more compressive in the direction we examined) by the gate removal, particularly in the crown.

It is likely safe to assume that the tangential, axial, and normal strains at any location along the length of the crown are nearly identical. Thus in Table 1 and Table 2 below we present the averaged strain for the three regions of the casting for each strain component. The reported variance is the rms deviation from the average for the number of locations measured.

Table 1. Average Strains in the As-cast Specimen

\section{Crown}

\begin{tabular}{|c|c|c|}
\hline & Strain $(\mathrm{ppm})$ & Variance \\
\hline Tangential & 60 & 120 \\
\hline Axial & 110 & 170 \\
\hline Normal & -80 & 190 \\
\hline
\end{tabular}

Side

\begin{tabular}{|c|c|c|}
\hline & Strain $(\mathrm{ppm})$ & Variance \\
\hline Tangential & 19 & 111 \\
\hline Axial & -35 & 138 \\
\hline Normal & 120 & 79 \\
\hline
\end{tabular}




\section{Web (Gate)}

\begin{tabular}{|c|c|c|}
\hline & Strain $(\mathrm{ppm})$ & Variance \\
\hline Tangential & -142 & 88 \\
\hline Axial & -293 & 143 \\
\hline Normal & NA & NA \\
\hline
\end{tabular}

In the web (gate) region, closes to the constraining forces imposed by the runner, there is a small shift toward less compressive strain for the tangential and axial strains. However, the variability of strain values as shown by the variance suggests that these shifts may not be significant. In the side and crown region the changes in strain were also quite small and not significant relative to the calculated variance.

Table 2. Average Strains in the Casting After Runner Removal

\section{Crown}

\begin{tabular}{|c|c|c|}
\hline & Strain $(\mathrm{ppm})$ & Variance \\
\hline Tangential & -70 & 150 \\
\hline Axial & -40 & 60 \\
\hline Normal & -110 & 190 \\
\hline
\end{tabular}

Side

\begin{tabular}{|c|c|c|}
\hline & Strain $(\mathrm{ppm})$ & Variance \\
\hline Tangential & -114 & 131 \\
\hline Axial & 43 & 93 \\
\hline Normal & 218 & 261 \\
\hline
\end{tabular}

Web (Gate)

\begin{tabular}{|c|c|c|}
\hline & Strain $(\mathrm{ppm})$ & Variance \\
\hline Tangential & -30 & 196 \\
\hline Axial & -28 & 138 \\
\hline Normal & NA & NA \\
\hline
\end{tabular}

\subsection{Conclusions}

1.0 The slightly compressive strains in tangential and axial directions in the web (gate) were released after the runner was removed from the casting. The fact that we measured a compressive strain in the gate closest to the fill of the runner suggests that this gate solidified second. This hypothesis is supported by the increased opining on cutting at the location of the other gates.

2.0 The tangential component of strain in the crown was investigated at three different depths to examine the effect of the opening of the gate on removing the runner. In the as-cast specimen the strains went from slightly compressive at the inside to slightly tensile at the outside. The 
data after the runner was removed showed a different trend, with the more relaxed strains closer to the inside of the casting. This is consistent with the measured opening of the casting when the runner was removed. Thus the strain distribution is modified (it was actually relieved or shifted to more compressive in the direction we examined) by the runner removal, particularly in the crown.

3.0 The overall low measured value of the strains in the casting are likely a consequence of a strain-relief mechanism at work during solidification. One possibility is that there is a thermal stress relief occurring during the slow cooling in the mold. 